\title{
Pd-catalyzed Perfluoroalkylative Aryloxycarbonylation of Alkynes with Formates as CO Surrogates.
}

\author{
Beata Gatlik and Wojciech Chaładaj* \\ Institute of Organic Chemistry, Polish Academy of Sciences, Kasprzaka 44/52, o1-224 Warsaw, Poland. \\ E-mail: wojciech.chaladaj@icho.edu.pl
}

\section{Table of content}

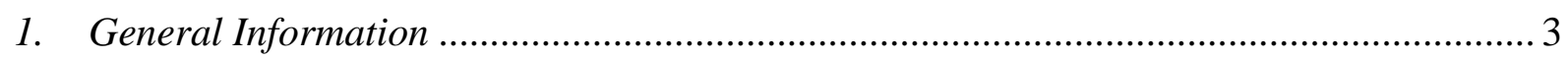

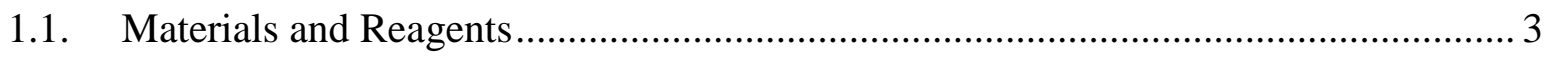

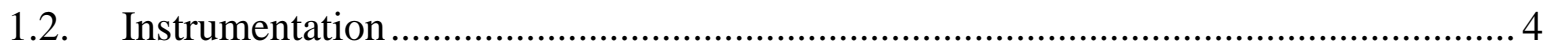

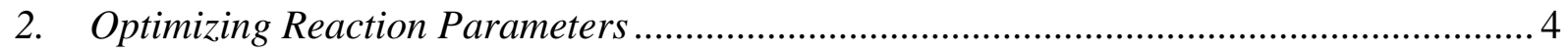

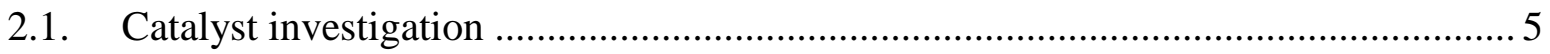

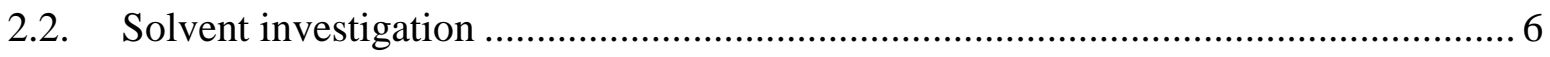

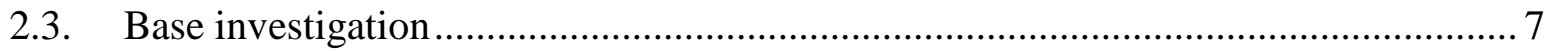

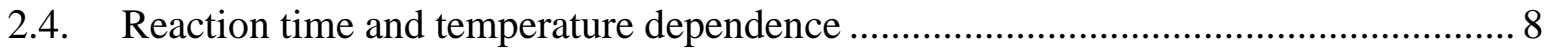

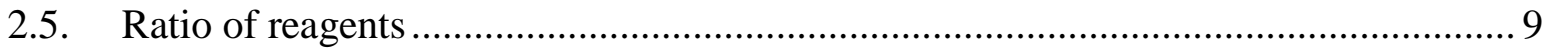

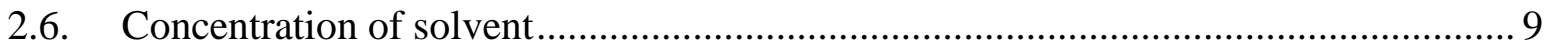

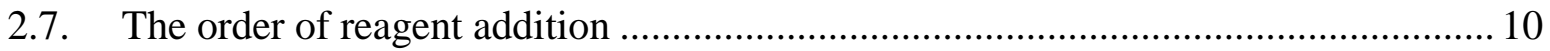

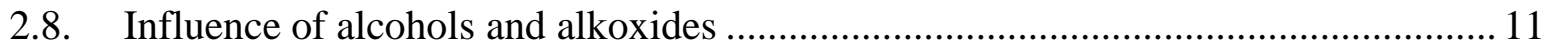

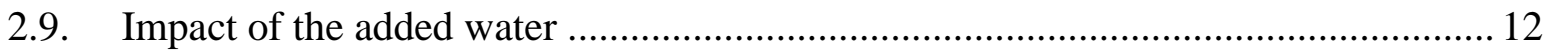

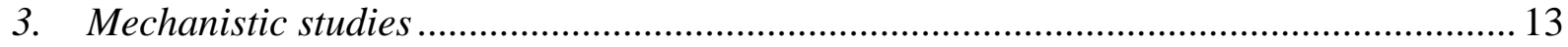

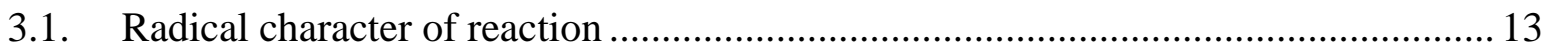

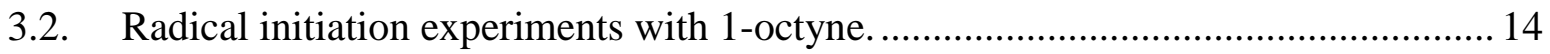

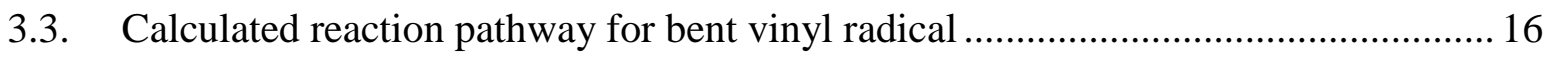

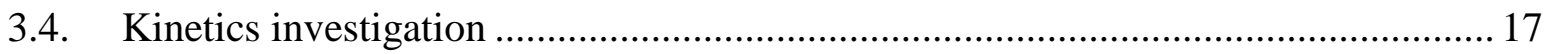

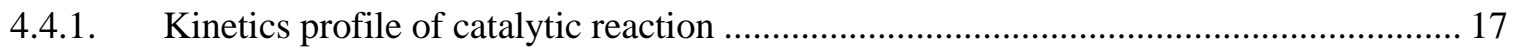

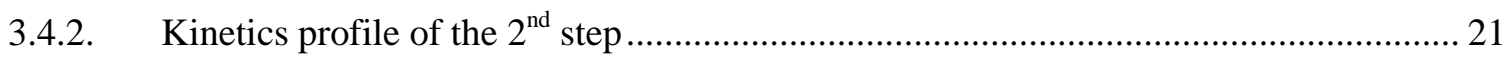

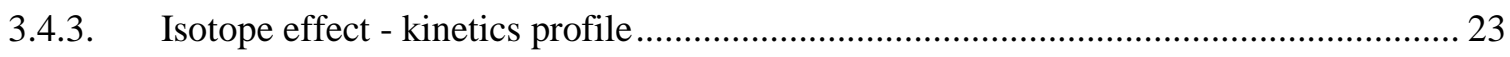




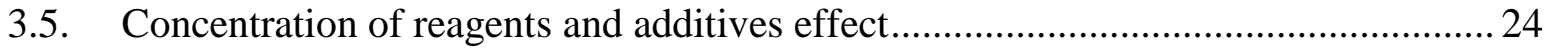

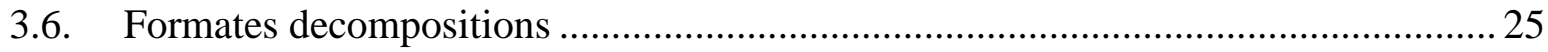

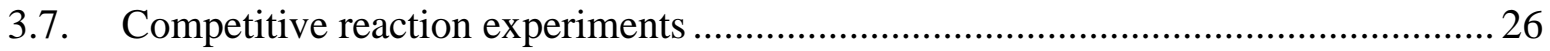

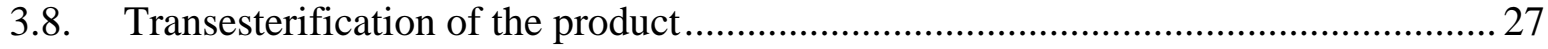

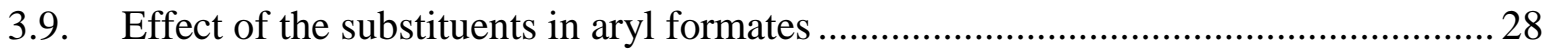

3.9.1. Effect of the electronic nature of aryl formate rate of aryloxycarbonylation of 1 ......... 28

3.9.2. Gibbs free energy profile for aryloxycarbonylation of $\mathbf{1}$ with electronically varied aryl formates. 28

4. Synthesis and characterization of isolated compounds ...............................................29

4.1. Non-commercially available starting materials - synthesis and analytical data .......29

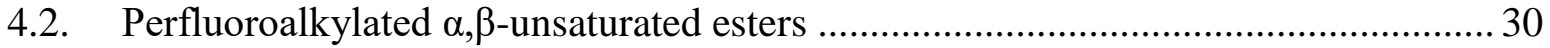

4.2.1. General procedure for perfluoroalkylative aryloxycarbonylation of alkyne ................. 30

4.2.2. Perfluorosubstituted $\alpha, \beta$-unsaturated esters - analytical data ......................................... 31

4.3. Synthetic utility of perfluorosubstituted $\alpha, \beta$-unsaturated esters ............................. 45

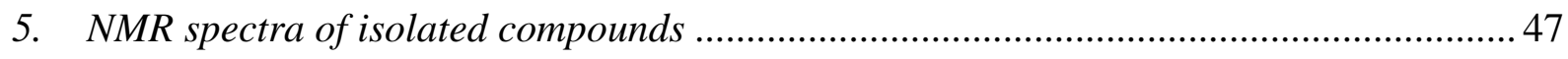

5.1. Non-commercially available starting materials ................................................. 47

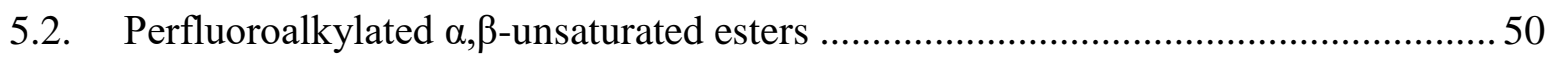

5.3. Synthetic utility of perfluorosubstituted $\alpha, \beta$-unsaturated esters .............................. 126

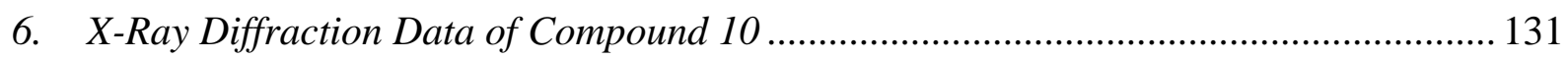

7. Optimized geometries, energies and corrections to thermodynamic functions. .............. 132 


\section{General Information}

\subsection{Materials and Reagents}

Unless otherwise noted, commercial reagents were purchased from ABCR, Acros, Fluorochem, SigmaAldrich, STREM, TCI or other commercial suppliers and were used as received. Buchwald type $3^{\text {rd }}$ generation palladacyclic precatalysts (Ligand Pd G3) were prepared following literature procedure, using phosphine ligands (from Aldrich or Fluorochem) and $\mathrm{Pd}(\mathrm{OAc})_{2}$ (from STREM) and showed similar reactivity to the commercial precatalysts counterparts (complexes with BINAP, RuPhos and XPhos ligands were compared with commercial samples).

Caution: efficiency and reproducibility of the reactions strongly depend on the purity of the reagents.

The following points were identified as significant and the following procedure were applied to ensure reproducibility of the results:

- Dry dichloroethane (dry DCE) - the solvent was dried by distillation over $\mathrm{CaH}_{2}$ and stored over $4 \AA$ molecular sieves.

- Dry cesium carbonate (dry $\mathrm{Cs}_{2} \mathrm{CO}_{3}$ ) - the base was dried at $180^{\circ} \mathrm{C}$ under vacuum (ca. 1 mbar) overnight and stored in a nitrogen-filled glovebox.

- Perfluoroalkyl iodides - in most cases iodides from commercial suppliers exhibit sufficient purity. Pink, orange or brownish color may indicate decomposition (good quality reagents are colorless in most cases) caused by, for e.g. exposure to light. Employment of the decomposed reagent may cause decrease reaction yield or even completely shutdown of the reactivity. The reagents were purified by filtration through a anhydrous sodium thiosulfate $\left(\mathrm{Na}_{2} \mathrm{~S}_{2} \mathrm{O}_{3}\right)$ pad under inert atmosphere.

All operations were carried out in a nitrogen-filled glove box or by using standard high vacuum and Schlenk techniques unless otherwise noted. Standard chromatography was performed using Merck silica gel 60 (230-400 mesh), for reversed phase chromatography Merck LiChroprep RP-18 (40-63 $\mu \mathrm{m}$ ) was used. All reactions and column chromatographical purifications were followed by thin-layer chromatography TLC when practical, performed on Merck TLC silica gel $60 F_{254}$ TLC plates which were visualized under UV light or by staining with $p$-anisaldehyde, cerium molybdate or potassium permanganate. For reversed phase TLC chromatography Merck TLC Silica gel $60 R P-18 F_{254 S}$ was used.

Deactivated silica gel was prepared by addition of $12.5 \mathrm{~g}$ of deionized water to $100 \mathrm{~g}$ of silica gel Merck silica gel 60 (230-400 mesh) and mixing the resulting material for $2 \mathrm{~h}$. 


\subsection{Instrumentation}

Optimization was based primarily on gas chromatography application (Agilent 7890B GC System) using calibration curves. Spectroscopic, spectrometric and chromatographic techniques were used for the analysis of obtained compounds. ${ }^{1} \mathrm{H},{ }^{13} \mathrm{C}\{1 \mathrm{H}\}$, and ${ }^{19} \mathrm{~F}$ NMR spectra were recorded with a Bruker $A V 400$ spectrometer. ${ }^{1} \mathrm{H}$ and ${ }^{13} \mathrm{C}$ chemical shifts are given in ppm relative to TMS. The solvent signals were used as references $\left(\mathrm{CDCl}_{3} \delta_{\mathrm{H}}=7.26 \mathrm{ppm}, \delta_{\mathrm{C}}=77.16 \mathrm{ppm}\right)$ and the chemical shift converted to the TMS scale. Coupling constants $(J)$ are reported in Hertz $(\mathrm{Hz})$, and the following abbreviations were used to denote multiplets: $\mathrm{s}=$ singlet, $\mathrm{d}=$ doublet, $\mathrm{t}=$ triplet, $\mathrm{q}=$ quartet, quint $=$ quintet, $\mathrm{m}=$ multiplet $($ denotes complex pattern), $\mathrm{dd}=$ doublet of doublets, $\mathrm{dt}=$ doublet of triplets and $\mathrm{br}=$ broad signal. Infrared spectra were recorded with a Jasco FTIR-6200 spectrometer. Electron ionization high-resolution mass spectra (EI-HR) were recorded with an Autospec Premier (Waters Inc) mass spectrometer using the narrow-range highvoltage scan technique with low-boiling perfluorokerosene (PFK) as internal standard. Samples were introduced by using a heated direct insertion probe. Electrospray ionization high-resolution mass spectra (ESI-HR) were recorded with MALDISynapt G2-S HDMS (Waters Inc) mass spectrometer equipped with an electrospray ion source and q-TOF type mass analyzer. ESI-MS spectra were recorded in the positive ion mode (the source parameters: capillary voltage $3.15 \mathrm{kV}$, sampling cone $25 \mathrm{~V}$, source temperature $120^{\circ} \mathrm{C}$, desolvation temperature $150^{\circ} \mathrm{C}$ ). For obtained crystals, $X$-ray diffraction analysis has been done using Bruker AXS diffractometer with area detector APEX II.

\section{Optimizing Reaction Parameters}

All reactions were performed in $4 \mathrm{~mL}$ dark glass screw-capped vials under a nitrogen $\left(\mathrm{N}_{2}\right)$ atmosphere unless otherwise noted. Stainless steel gas-tight syringes were used to transfer liquids.

Optimization was based primarily on the employment of gas chromatography using calibration curves prepared by the internal standard method with $n$-dodecane (as an internal standard).

Bases described as "dry base" were additionally dried at $180^{\circ} \mathrm{C}$ under vacuum (ca. 1 mbar) overnight.

Caution: Sequence of addition of reagents is crucial for repeatability of results.

\section{Example of typical reaction conditions for optimization:}

To a $4 \mathrm{~mL}$ dark glass screw-capped vial RuPhos Pd G3 (1.25 $\mu$ mol, 1 mol\%) was added. The vial was moved to a glovebox, then reagents were sequentially added: $\mathbf{d r y} \mathbf{C s}_{\mathbf{2}} \mathbf{C O}_{\mathbf{3}}(0.250 \mathrm{mmol}, 2.0$ equiv), formate $(0.188 \mathrm{mmol}$, 1.5 equiv), acetylene $(0.125 \mathrm{mmol})$, perfluorinated iodide $(0.375 \mathrm{mmol}, 3.0$ equiv), dry DCE $(0.5 \mathrm{~mL})$. Then, a magnetic stirring bar was placed in the reaction mixture. The vial was sealed with a cap. The reaction mixture was stirred at $60^{\circ} \mathrm{C}$ for $22 \mathrm{~h}$ and then cooled down. The mixture was diluted with MTBE (2 mL) quenched with sat. aqueous $\mathrm{NH}_{4} \mathrm{Cl}(0.5 \mathrm{~mL})$ and $n$-dodecane $(25 \mu \mathrm{l})$ was added as an internal standard. 


\subsection{Catalyst investigation}
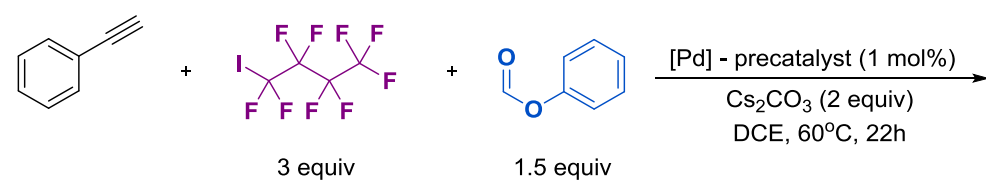

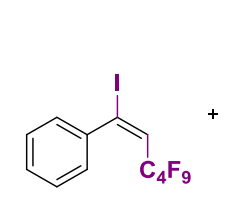

(1)

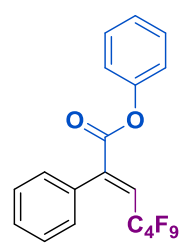

(2)

\begin{tabular}{|c|c|c|c|c|}
\hline Entry & Ligand Pd G3 & Conversion [\%] & Yield 1 [\%] & Yield $2[\%]$ \\
\hline 1 & BINAP & 100 & 32 & 30 \\
\hline 2 & Tol-BINAP & 100 & 55 & 8 \\
\hline 3 & $(R)$-DM-BINAP & 100 & - & 62 \\
\hline 4 & MonoPhos & 100 & - & 5 \\
\hline 5 & RuPhos & 100 & 16 & 58 \\
\hline 6 & Xphos & 100 & 38 & 33 \\
\hline 7 & $t$-BuXphos & 100 & 58 & - \\
\hline 8 & BrettPhos & 100 & 83 & 3 \\
\hline 9 & JackiePhos & 100 & 41 & 3 \\
\hline 10 & Sphos & 100 & 22 & 23 \\
\hline 11 & DavePhos & 100 & - & 19 \\
\hline 12 & CyJohnPhos & 100 & - & 40 \\
\hline 13 & XantPhos & 100 & 14 & 17 \\
\hline 14 & APhos & 100 & - & 20 \\
\hline 15 & DPPF & 100 & - & 55 \\
\hline 16 & DCyPF & 100 & 49 & 19 \\
\hline 17 & DPPE & 100 & 27 & 16 \\
\hline 18 & DPPB & 100 & 3 & 44 \\
\hline 19 & DPPPY & 100 & - & - \\
\hline 20 & CataCXium & 100 & - & 51 \\
\hline 21 & $\mathrm{PPh}_{3}$ & 100 & - & 7 \\
\hline 22 & $(o-\mathrm{Tol})_{3} \mathrm{P}$ & 100 & 80 & - \\
\hline 23 & $\mathrm{P}(\mathrm{Cy})_{3}$ & 100 & - & 7 \\
\hline 24 & $\mathrm{P}(t-\mathrm{Bu})_{3}$ & 100 & - & 17 \\
\hline
\end{tabular}

Reaction conditions: Ligand Pd G3 (1 mol\%), $\mathrm{C}_{4} \mathrm{~F}_{9} \mathrm{I}(0.375 \mathrm{mmol}, 3$ equiv), phenylacetylene (0.125 mmol), phenyl formate (0.188 mmol, 1.5 equiv), dry $\mathrm{Cs}_{2} \mathrm{CO}_{3}\left(0.251 \mathrm{mmol}, 2\right.$ equiv), dry DCE $(0.5 \mathrm{~mL}), 60^{\circ} \mathrm{C}, 22 \mathrm{~h}$. Determined by GC with $n$ dodecane as an internal standard. 


\subsection{Solvent investigation}
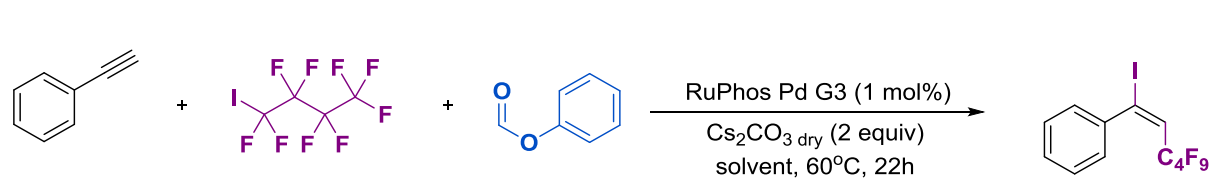

3 equiv

1.5 equiv

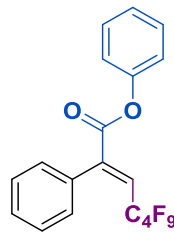

(2)

\begin{tabular}{ccccc}
\hline Entry & Solvent & Conversion [\%] & Yield 1 [\%] & Yield 2 [\%] \\
\hline 1 & Hexane & 100 & - & 42 \\
2 & Benzene & 100 & - & 18 \\
3 & Xylene & 100 & - & 16 \\
4 & Mesitylene & 100 & - & 24 \\
5 & DCB & 100 & - & 36 \\
6 & Chlorobenzene & 100 & - & 28 \\
7 & Bromobenzene & 100 & - & 19 \\
8 & Fluorobenzene & 100 & - & 3 \\
9 & Hexafluorobenzene & 100 & - & 27 \\
10 & dry DCM & 100 & 30 & 26 \\
11 & dry DCE & 100 & 19 & - \\
12 & Formamide & 100 & - & - \\
13 & DMF & 100 & - & 30 \\
14 & $1,4-$ dioksane & 100 & - & 11 \\
15 & THF & 100 & - & - \\
16 & DMSO & 100 & - & 2 \\
17 & Acetonitrile & 100 & - & 41 \\
18 & Acetone & 100 & -1 & - \\
\hline
\end{tabular}

Reaction conditions: RuPhos Pd G3 (1 mol\%), $\mathrm{C}_{4} \mathrm{~F}_{9} \mathrm{I}(0.375 \mathrm{mmol}, 3$ equiv), solvent $(0.5 \mathrm{~mL})$, phenylacetylene $(0.125 \mathrm{mmol})$, phenyl formate $\left(0.188 \mathrm{mmol}, 1.5\right.$ equiv), dry $\mathrm{Cs}_{2} \mathrm{CO}_{3}\left(0.251 \mathrm{mmol}, 2\right.$ equiv), $60^{\circ} \mathrm{C}, 22 \mathrm{~h}$. Determined by $\mathrm{GC}$ with $n$-dodecane as an internal standard. 
2.3. Base investigation
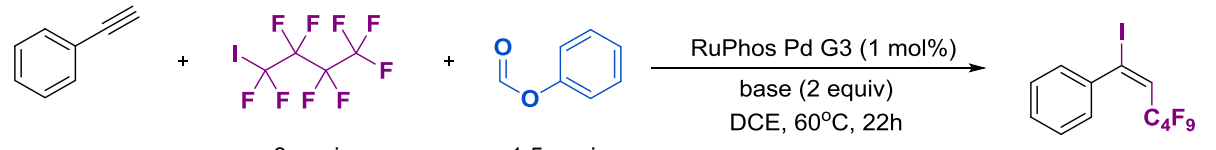

(1)

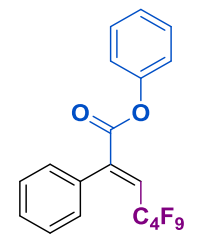

(2)

\begin{tabular}{|c|c|c|c|c|}
\hline Entry & Base & Conversion [\%] & Yield 1 [\%] & Yield 2 [\%] \\
\hline 1 & dry $\mathrm{Cs}_{2} \mathrm{CO}_{3}$ & 100 & 19 & 43 \\
\hline 2 & $\mathrm{Cs}_{2} \mathrm{CO}_{3}$ & 100 & 27 & 30 \\
\hline 3 & $2 \mathrm{M}$ aqueous $\mathrm{Cs}_{2} \mathrm{CO}_{3}$ & 100 & - & - \\
\hline 4 & dry $\mathrm{K}_{3} \mathrm{PO}_{4}$ & 100 & - & 42 \\
\hline 5 & $\mathrm{~K}_{3} \mathrm{PO}_{4}$ & 100 & 60 & - \\
\hline 6 & $\mathrm{~K}_{2} \mathrm{HPO}_{4}$ & - & - & - \\
\hline 7 & $\mathrm{NaHCO}_{3}$ & 51 & 43 & - \\
\hline 8 & $\mathrm{KHCO}_{3}$ & 89 & - & - \\
\hline 9 & $\mathrm{BaCO}_{3}$ & 28 & 26 & - \\
\hline 10 & $\mathrm{NaOH}$ & 100 & 66 & - \\
\hline 11 & $\mathrm{LiOH}$ & 79 & 55 & - \\
\hline 12 & $\mathrm{KOH}$ & 100 & 67 & - \\
\hline 13 & $\mathrm{Ba}(\mathrm{OH})_{2} \cdot 8 \mathrm{H}_{2} \mathrm{O}$ & 32 & 29 & - \\
\hline 14 & $\mathrm{NaOAc}$ & 35 & 36 & - \\
\hline 15 & $\mathrm{LiNH}_{2}$ & 100 & - & - \\
\hline 16 & LiHMDS & 89 & - & - \\
\hline 17 & KHMDS & 13 & - & - \\
\hline 18 & NaHMDS & 28 & - & - \\
\hline 19 & Triethylamine & 100 & - & - \\
\hline 20 & Pyridine & - & - & - \\
\hline
\end{tabular}

Reaction conditions: RuPhos Pd G3 (1 mol\%), $\mathrm{C}_{4} \mathrm{~F}_{9} \mathrm{I}(0.375 \mathrm{mmol}, 3$ equiv), dry DCE $(0.5 \mathrm{~mL})$, phenylacetylene $(0.125$ mmol), phenyl formate $\left(0.188 \mathrm{mmol}, 1.5\right.$ equiv), base $\left(0.251 \mathrm{mmol}, 2\right.$ equiv), $60^{\circ} \mathrm{C}, 22 \mathrm{~h}$. Determined by GC with $n$-dodecane as an internal standard. 


\subsection{Reaction time and temperature dependence}

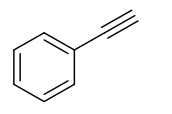

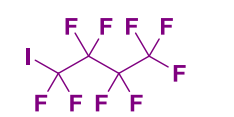

3 equiv

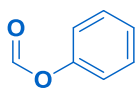

1.5 equiv

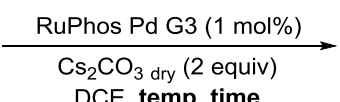

DCE, temp, time

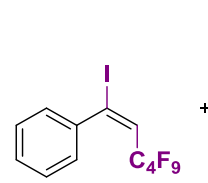

(1)

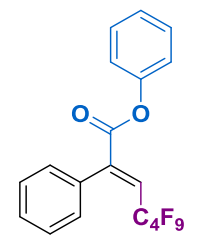

(2)

\begin{tabular}{cccccc}
\hline Entry & Temperature & Time & Conversion [\%] & Yield 1 [\%] & Yield 2 [\%] \\
\hline 1 & $40^{\circ} \mathrm{C}$ & $2 \mathrm{~h}$ & 100 & 53 & 27 \\
2 & $40^{\circ} \mathrm{C}$ & $4 \mathrm{~h}$ & 100 & 40 & 40 \\
3 & $50^{\circ} \mathrm{C}$ & $2 \mathrm{~h}$ & 100 & 36 & 39 \\
4 & $50^{\circ} \mathrm{C}$ & $4 \mathrm{~h}$ & 100 & 21 & 48 \\
5 & $60^{\circ} \mathrm{C}$ & $4 \mathrm{~h}$ & 100 & 16 & 53 \\
6 & $60^{\circ} \mathrm{C}$ & $5 \mathrm{~h}$ & 100 & 20 & 52 \\
7 & $70^{\circ} \mathrm{C}$ & $3 \mathrm{~h}$ & 100 & 22 & 41 \\
8 & $70^{\circ} \mathrm{C}$ & $18 \mathrm{~h}$ & 100 & 15 & 39 \\
9 & $70^{\circ} \mathrm{C}$ & $26 \mathrm{~h}$ & 100 & 17 & 42 \\
10 & $80^{\circ} \mathrm{C}$ & $2 \mathrm{~h}$ & 100 & 17 & 46 \\
11 & $80^{\circ} \mathrm{C}$ & $3 \mathrm{~h}$ & 100 & 13 & 41 \\
12 & $80^{\circ} \mathrm{C}$ & $18 \mathrm{~h}$ & 100 & 8 & 46 \\
13 & $80^{\circ} \mathrm{C}$ & $26 \mathrm{~h}$ & 100 & 6 & \\
\hline
\end{tabular}

Reaction conditions: RuPhos Pd G3 (1 mol\%), $\mathrm{C}_{4} \mathrm{~F}_{9} \mathrm{I}(0.375 \mathrm{mmol}, 3$ equiv), dry DCE $(0.5 \mathrm{~mL})$, phenylacetylene $(0.125$ mmol), phenyl formate ( $0.188 \mathrm{mmol}, 1.5$ equiv), dry $\mathrm{Cs}_{2} \mathrm{CO}_{3}(0.251 \mathrm{mmol}, 2$ equiv), temp, time. Determined by GC with $n$ dodecane as an internal standard.

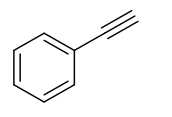

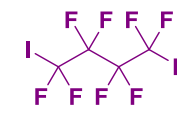

3 equiv

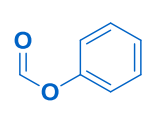

2 equiv

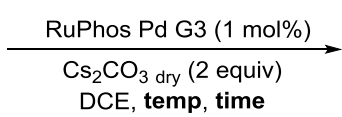

DCE, temp, time

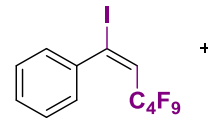

(1)

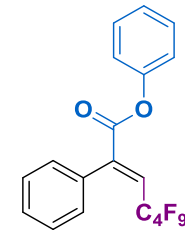

(2)

\begin{tabular}{cccccc}
\hline Entry & Temperature & Time & Conversion [\%] & Yield 1 [\%] & Yield 2 [\%] \\
\hline 1 & $40^{\circ} \mathrm{C}$ & $4 \mathrm{~h}$ & 100 & 20 & 65 \\
2 & $50^{\circ} \mathrm{C}$ & $4 \mathrm{~h}$ & 100 & - & 84 \\
3 & $60^{\circ} \mathrm{C}$ & $4 \mathrm{~h}$ & 100 & - & 80 \\
4 & $70^{\circ} \mathrm{C}$ & $4 \mathrm{~h}$ & 100 & - & 72 \\
5 & $40^{\circ} \mathrm{C}$ & $5 \mathrm{~h}$ & 100 & - & 68 \\
6 & $50^{\circ} \mathrm{C}$ & $5 \mathrm{~h}$ & 100 & - & 70 \\
7 & $70^{\circ} \mathrm{C}$ & $5 \mathrm{~h}$ & 100 & - & 62 \\
8 & $80^{\circ} \mathrm{C}$ & $5 \mathrm{~h}$ & 100 & 47 & 35 \\
9 & $\mathrm{rt}$ & $6 \mathrm{~h}$ & 100 & 9 & 72 \\
10 & $\mathrm{rt}$ & overnight & 100 & & \\
\hline
\end{tabular}

Reaction conditions: RuPhos Pd G3 (1 mol\%), $\mathrm{C}_{4} \mathrm{~F}_{9} \mathrm{I}(0.375 \mathrm{mmol}, 3$ equiv), dry DCE $(0.5 \mathrm{~mL})$, phenylacetylene $(0.125$ $\mathrm{mmol})$, phenyl formate $\left(0.25 \mathrm{mmol}, 2\right.$ equiv), dry $\mathrm{Cs}_{2} \mathrm{CO}_{3}(0.251 \mathrm{mmol}, 2$ equiv), temp, time. Determined by $\mathrm{GC}$ with $n$ dodecane as an internal standard. 


\subsection{Ratio of reagents}
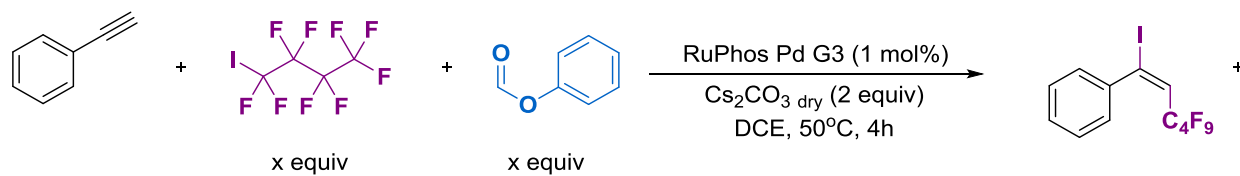

(1)

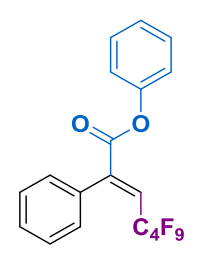

(2)

\begin{tabular}{cccccc}
\hline Entry & Reagent & Amount [equiv] & Conversion [\%] & Yield 1 [\%] & Yield 2 [\%] \\
\hline 1 & Phenyl formate $^{a}$ & 2.0 & 100 & - & 84 \\
2 & Phenyl formate $^{a}$ & 2.5 & 100 & - & 72 \\
3 & $\mathrm{C}_{4} \mathrm{~F}_{9} \mathrm{I}^{b}$ & 1.5 & 100 & - & 62 \\
4 & $\mathrm{C}_{4} \mathrm{~F}_{9} \mathrm{I}^{b}$ & 2.0 & 100 & - & 69 \\
5 & $\mathrm{C}_{4} \mathrm{~F}_{9} \mathrm{I}^{b}$ & 2.5 & 100 & - & 72 \\
6 & $\mathrm{C}_{4} \mathrm{~F}_{9} \mathrm{I}^{b}$ & 3.0 & 100 & - & 84 \\
\hline
\end{tabular}

Reaction conditions: ${ }^{a}$ RuPhos Pd G3 (1 mol\%), $\mathrm{C}_{4} \mathrm{~F}_{9} \mathrm{I}(0.375 \mathrm{mmol}, 3$ equiv), dry DCE (0.5 mL), phenylacetylene $(0.125 \mathrm{mmol})$, phenyl formate (x equiv), dry $\mathrm{Cs}_{2} \mathrm{CO}_{3}\left(0.251 \mathrm{mmol}, 2\right.$ equiv), 50 ${ }^{\circ} \mathrm{C}, 4 \mathrm{~h} ;{ }^{b}$ RuPhos Pd G3 (1 mol\%), $\mathrm{C}_{4} \mathrm{~F}_{9} \mathrm{I}$ (x equiv), dry DCE $(0.5 \mathrm{~mL})$, phenylacetylene $(0.125 \mathrm{mmol})$, phenyl formate $\left(0.25 \mathrm{mmol}, 2\right.$ equiv), dry $\mathrm{Cs}_{2} \mathrm{CO}_{3}(0.251 \mathrm{mmol}$, 2 equiv), $50^{\circ} \mathrm{C}, 4 \mathrm{~h}$. Determined by GC with $n$-dodecane as an internal standard.

\subsection{Concentration of solvent}
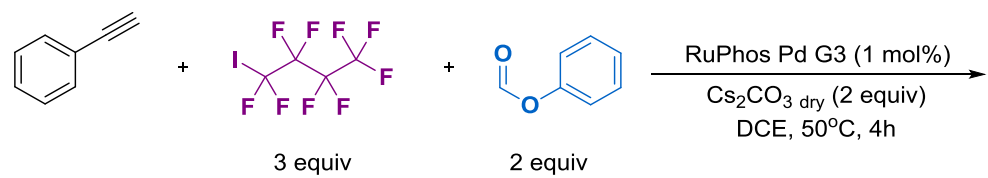

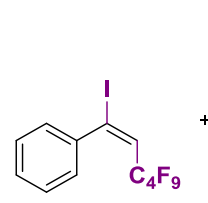

(1)

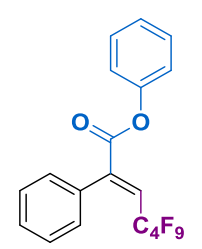

(2)

\begin{tabular}{ccccc}
\hline Entry & DCE $[\mathrm{mL}]$ & Conversion [\%] & Yield 1 [\%] & Yield 2 [\%] \\
\hline 1 & 0.25 & 100 & - & - \\
2 & 0.50 & 100 & - & 85 \\
3 & 0.75 & 100 & 75 \\
\hline
\end{tabular}

Reaction conditions: RuPhos Pd G3 (1 mol\%), dry $\mathrm{Cs}_{2} \mathrm{CO}_{3}(0.251 \mathrm{mmol}, 2$ equiv), phenylacetylene (0.125 mmol), phenyl formate $\left(0.25 \mathrm{mmol}, 2\right.$ equiv), $\mathrm{C}_{4} \mathrm{~F}_{9} \mathrm{I}\left(0.375 \mathrm{mmol}, 3\right.$ equiv), dry DCE $(\mathrm{x} \mathrm{mL}), 50^{\circ} \mathrm{C}, 4 \mathrm{~h}$. Determined by GC with $n$-dodecane as an internal standard. 

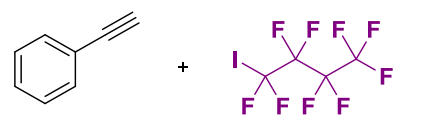

3 equiv

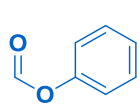

2 equiv

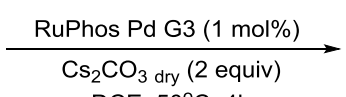

$\mathrm{DCE}, 50^{\circ} \mathrm{C}, 4 \mathrm{~h}$

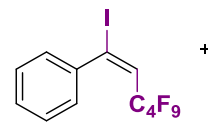

(1)

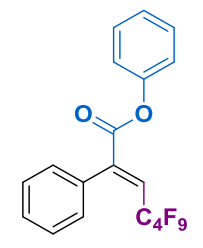

(2)

\begin{tabular}{|c|c|c|c|c|c|c|c|c|}
\hline \multirow{2}{*}{ Entry } & \multicolumn{5}{|c|}{ The order of reagent addition } & \multirow{2}{*}{$\begin{array}{c}\text { Conversion } \\
{[\%]}\end{array}$} & \multicolumn{2}{|c|}{ Yield [\%] } \\
\hline & 1 & 2 & 3 & 4 & 5 & & 1 & 2 \\
\hline 1 & $\begin{array}{c}\text { RuPhos Pd G3, } \\
\text { dry } \mathrm{Cs}_{2} \mathrm{CO}_{3}\end{array}$ & Phenyl formate & Phenylacetylene & $\mathrm{C}_{4} \mathrm{~F}_{9} \mathrm{I}$ & dry DCE & 100 & - & 85 \\
\hline 2 & $\begin{array}{c}\text { RuPhos Pd G3 } \\
\text { dry } \mathrm{Cs}_{2} \mathrm{CO}_{3}\end{array}$ & Phenyl formate & Phenylacetylene & dry DCE & $\mathrm{C}_{4} \mathrm{~F}_{9} \mathrm{I}$ & 100 & 7 & 69 \\
\hline 3 & $\begin{array}{c}\text { RuPhos Pd G3 } \\
\text { dry } \mathrm{Cs}_{2} \mathrm{CO}_{3}\end{array}$ & Phenyl formate & $\mathrm{C}_{4} \mathrm{~F}_{9} \mathrm{I}$ & dry DCE & Phenylacetylene & 100 & - & 75 \\
\hline 4 & $\begin{array}{c}\text { RuPhos Pd G3, } \\
\text { dry } \mathrm{Cs}_{2} \mathrm{CO}_{3}\end{array}$ & Phenyl formate & $\mathrm{C}_{4} \mathrm{~F}_{9} \mathrm{I}$ & Phenylacetylene & dry DCE & 100 & - & 69 \\
\hline 5 & $\begin{array}{l}\text { RuPhos Pd G3, } \\
\text { dry } \mathrm{Cs}_{2} \mathrm{CO}_{3}\end{array}$ & Phenyl formate & dry DCE & $\mathrm{C}_{4} \mathrm{~F}_{9} \mathrm{I}$ & Phenylacetylene & 100 & 4 & 68 \\
\hline 6 & $\begin{array}{c}\text { RuPhos Pd G3, } \\
\text { dry } \mathrm{Cs}_{2} \mathrm{CO}_{3}\end{array}$ & Phenyl formate & dry DCE & Phenylacetylene & $\mathrm{C}_{4} \mathrm{~F}_{9} \mathrm{I}$ & 100 & 5 & 66 \\
\hline 7 & $\begin{array}{l}\text { RuPhos Pd G3, } \\
\text { dry } \mathrm{Cs}_{2} \mathrm{CO}_{3}\end{array}$ & $\mathrm{C}_{4} \mathrm{~F}_{9} \mathrm{I}$ & Phenylacetylene & Phenyl formate & dry DCE & 100 & - & 67 \\
\hline 8 & $\begin{array}{c}\text { RuPhos Pd G3, } \\
\text { dry } \mathrm{Cs}_{2} \mathrm{CO}_{3}\end{array}$ & $\mathrm{C}_{4} \mathrm{~F}_{9} \mathrm{I}$ & Phenyl formate & dry DCE & Phenylacetylene & 100 & - & 72 \\
\hline 9 & $\begin{array}{c}\text { RuPhos Pd G3, } \\
\text { dry } \mathrm{Cs}_{2} \mathrm{CO}_{3}\end{array}$ & $\mathrm{C}_{4} \mathrm{~F}_{9} \mathrm{I}$ & Phenyl formate & Phenylacetylene & dry DCE & 100 & - & 76 \\
\hline 10 & $\begin{array}{l}\text { RuPhos Pd G3, } \\
\text { dry } \mathrm{Cs}_{2} \mathrm{CO}_{3}\end{array}$ & $\mathrm{C}_{4} \mathrm{~F}_{9} \mathrm{I}$ & Phenylacetylene & dry DCE & Phenyl formate & 100 & - & 68 \\
\hline 11 & $\begin{array}{l}\text { RuPhos Pd G3, } \\
\text { dry } \mathrm{Cs}_{2} \mathrm{CO}_{3}\end{array}$ & Phenylacetylene & dry DCE & Phenyl formate & $\mathrm{C}_{4} \mathrm{~F}_{9} \mathrm{I}$ & 100 & - & 60 \\
\hline 12 & $\begin{array}{c}\text { RuPhos Pd G3, } \\
\text { dry } \mathrm{Cs}_{2} \mathrm{CO}_{3}\end{array}$ & Phenylacetylene & dry DCE & $\mathrm{C}_{4} \mathrm{~F}_{9} \mathrm{I}$ & Phenyl formate & 100 & - & 64 \\
\hline 13 & $\begin{array}{l}\text { RuPhos Pd G3, } \\
\text { dry } \mathrm{Cs}_{2} \mathrm{CO}_{3}\end{array}$ & Phenylacetylene & $\mathrm{C}_{4} \mathrm{~F}_{9} \mathrm{I}$ & Phenyl formate & dry DCE & 100 & 5 & 58 \\
\hline 14 & $\begin{array}{l}\text { RuPhos Pd G3, } \\
\text { dry } \mathrm{Cs}_{2} \mathrm{CO}_{3}\end{array}$ & Phenylacetylene & $\mathrm{C}_{4} \mathrm{~F}_{9} \mathrm{I}$ & dry DCE & Phenyl formate & 100 & - & 68 \\
\hline 15 & $\begin{array}{l}\text { RuPhos Pd G3, } \\
\text { dry } \mathrm{Cs}_{2} \mathrm{CO}_{3}\end{array}$ & Phenylacetylene & Phenyl formate & $\mathrm{C}_{4} \mathrm{~F}_{9} \mathrm{I}$ & dry DCE & 100 & 9 & 52 \\
\hline 16 & $\begin{array}{l}\text { RuPhos Pd G3, } \\
\text { dry } \mathrm{Cs}_{2} \mathrm{CO}_{3}\end{array}$ & Phenylacetylene & Phenyl formate & dry DCE & $\mathrm{C}_{4} \mathrm{~F}_{9} \mathrm{I}$ & 100 & 8 & 59 \\
\hline 17 & $\begin{array}{l}\text { RuPhos Pd G3, } \\
\text { dry } \mathrm{Cs}_{2} \mathrm{CO}_{3}\end{array}$ & dry DCE & $\mathrm{C}_{4} \mathrm{~F}_{9} \mathrm{I}$ & Phenyl formate & Phenylacetylene & 100 & 6 & 62 \\
\hline 18 & $\begin{array}{l}\text { RuPhos Pd G3, } \\
\text { dry } \mathrm{Cs}_{2} \mathrm{CO}_{3}\end{array}$ & dry DCE & $\mathrm{C}_{4} \mathrm{~F}_{9} \mathrm{I}$ & Phenylacetylene & Phenyl formate & 100 & 28 & 40 \\
\hline 19 & $\begin{array}{c}\text { RuPhos Pd G3, } \\
\text { dry } \mathrm{Cs}_{2} \mathrm{CO}_{3}\end{array}$ & dry DCE & Phenylacetylene & $\mathrm{C}_{4} \mathrm{~F}_{9} \mathrm{I}$ & Phenyl formate & 100 & - & 62 \\
\hline 20 & $\begin{array}{c}\text { RuPhos Pd G3, } \\
\text { dry } \mathrm{Cs}_{2} \mathrm{CO}_{3}\end{array}$ & dry DCE & Phenylacetylene & Phenyl formate & $\mathrm{C}_{4} \mathrm{~F}_{9} \mathrm{I}$ & 100 & 5 & 57 \\
\hline 21 & $\begin{array}{l}\text { RuPhos Pd G3, } \\
\text { dry } \mathrm{Cs}_{2} \mathrm{CO}_{3}\end{array}$ & dry DCE & Phenyl formate & $\mathrm{C}_{4} \mathrm{~F}_{9} \mathrm{I}$ & Phenylacetylene & 100 & - & 60 \\
\hline 22 & $\begin{array}{c}\text { RuPhos Pd G3, } \\
\text { dry } \mathrm{Cs}_{2} \mathrm{CO}_{3}\end{array}$ & dry DCE & Phenyl formate & Phenylacetylene & $\mathrm{C}_{4} \mathrm{~F}_{9} \mathrm{I}$ & 100 & 5 & 59 \\
\hline
\end{tabular}

Reaction conditions: RuPhos Pd G3 (1 mol\%), dry $\mathrm{Cs}_{2} \mathrm{CO}_{3}\left(0.251 \mathrm{mmol}, 2\right.$ equiv), phenylacetylene $(0.125 \mathrm{mmol}), \mathrm{C}_{4} \mathrm{~F}_{9} \mathrm{I}$ ( $0.375 \mathrm{mmol}, 3$ equiv), phenyl formate $\left(0.25 \mathrm{mmol}, 2\right.$ equiv), dry DCE $(0.5 \mathrm{~mL}), 50^{\circ} \mathrm{C}, 4 \mathrm{~h}$. Determined by $\mathrm{GC}$ with $n$ dodecane as an internal standard. 


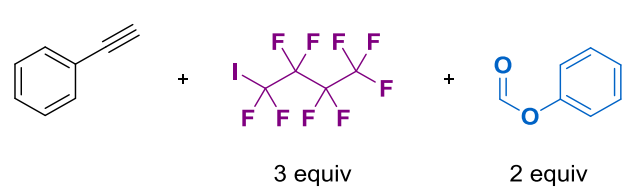
RuPhos Pd G3 (1 mol\%) $\mathrm{Cs}_{2} \mathrm{CO}_{3}$ dry (2 equiv), alcohol $(10 \% \mathrm{~mol})$, DCE, $50^{\circ} \mathrm{C}, 4 \mathrm{~h}$

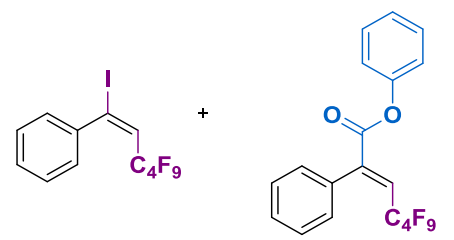

(1)

(2)

\begin{tabular}{ccccc}
\hline Entry & Alcohol & Conversion [\%] & Yield 1 [\%] & Yield 2 [\%] \\
\hline 1 & Methanol & 100 & 3 & 57 \\
2 & n-Butanol & 100 & 1 & 62 \\
\hline 4 & - & 100 & 0 & 63 \\
\hline
\end{tabular}

Reaction conditions: RuPhos Pd G3 (1 mol\%), dry $\mathrm{Cs}_{2} \mathrm{CO}_{3}(1.0 \mathrm{mmol}, 2$ equiv), phenylacetylene (0.5 mmol), phenyl formate (1.0 mmol, 2 equiv), $\mathrm{C}_{4} \mathrm{~F}_{9} \mathrm{I}$ (1.5 mmol, 3 equiv), dry DCE $(2 \mathrm{~mL})$, alcohol $(10 \mathrm{~mol} \%), 50^{\circ} \mathrm{C}, 4 \mathrm{~h}$. Determined by GC with $n$ dodecane as an internal standard.

Raction with alkoxide

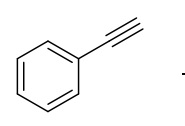

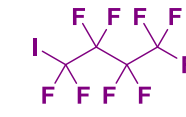

3 equiv<smiles>CCOc1ccccc1COCCO</smiles>

2 equiv
RuPhos Pd G3 (1 mol\%) NaOMe (2 equiv), DCE, $50^{\circ} \mathrm{C}, 4 \mathrm{~h}$

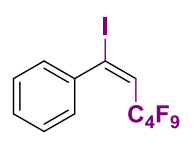

$40 \%$<smiles>CC(C)C=C(C(=O)Oc1ccccc1)c1ccccc1</smiles>

2

Reaction conditions: RuPhos Pd G3 (1 mol\%), NaOMe (1.0 mmol, 2 equiv), phenylacetylene (0.5 mmol), phenyl formate (1.0 mmol, 2 equiv), $\mathrm{C}_{4} \mathrm{~F}_{9} \mathrm{I}\left(1.5 \mathrm{mmol}, 3\right.$ equiv), dry DCE $(2 \mathrm{~mL}), 50^{\circ} \mathrm{C}$, 4h. Determined by $\mathrm{GC}$ with $n$-dodecane as an internal standard. 


\subsection{Impact of the added water}
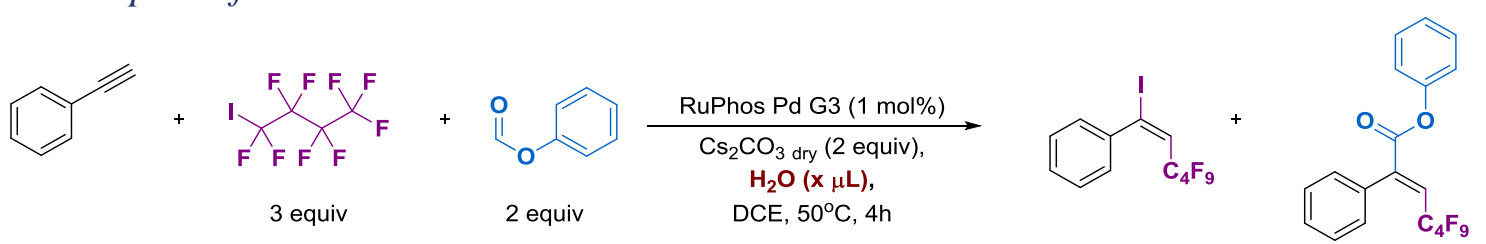

(1)

(2)

\begin{tabular}{ccccc}
\hline Entry & Amount of water $[\mu \mathrm{L}]$ & Conversion $[\%]$ & Yield 1 [\%] & Yield 2 [\%] \\
\hline 1 & 1 & 100 & 0 & 60 \\
2 & 10 & 100 & 55 & 13 \\
3 & 100 & 100 & 68 & 0 \\
\hline 4 & - & 100 & 0 & 59
\end{tabular}

Reaction conditions: RuPhos Pd G3 (1 mol\%), dry $\mathrm{Cs}_{2} \mathrm{CO}_{3}(1.0 \mathrm{mmol}, 2$ equiv), phenylacetylene (0.5 mmol), phenyl formate (1.0 mmol, 2 equiv), $\mathrm{C}_{4} \mathrm{~F}_{9} \mathrm{I}\left(1.5 \mathrm{mmol}, 3\right.$ equiv), dry DCE $(2 \mathrm{~mL})$, water $(\mathrm{x} \mu \mathrm{L}), 50^{\circ} \mathrm{C}$, 4h. Determined by GC with $n$ dodecane as an internal standard.

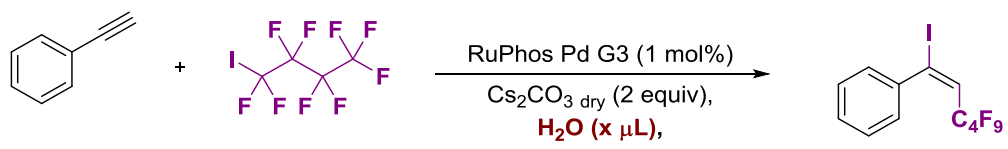

3 equiv $\mathrm{DCE}, 50^{\circ} \mathrm{C}, 4 \mathrm{~h}$

(1)

\begin{tabular}{cccc}
\hline Entry & Amount of water $[\mu \mathrm{L}]$ & Conversion [\%] & Yield 1 [\%] \\
\hline 1 & 1 & 99 & 72 \\
2 & 10 & 99 & 73 \\
3 & 100 & 97 & 73 \\
\hline 4 & - & 98 & 73 \\
\hline
\end{tabular}

Reaction conditions: RuPhos Pd G3 (1 mol\%), dry $\mathrm{Cs}_{2} \mathrm{CO}_{3}\left(1.0 \mathrm{mmol}, 2\right.$ equiv), phenylacetylene $(0.5 \mathrm{mmol}), \mathrm{C}_{4} \mathrm{~F}_{9} \mathrm{I}(1.5$ mmol, 3 equiv), dry DCE $(2 \mathrm{~mL})$, water $(\mathrm{x} \mu \mathrm{L}), 50^{\circ} \mathrm{C}, 4 \mathrm{~h}$. Determined by GC with $n$-dodecane as an internal standard.

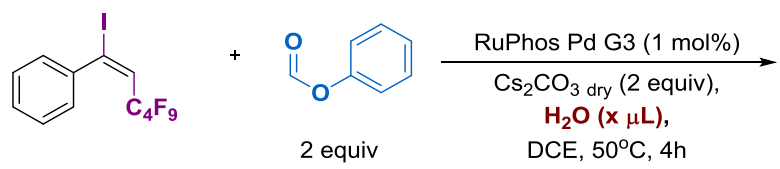

(1)

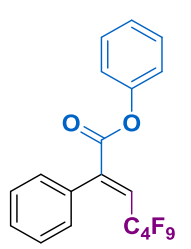

(2)

\begin{tabular}{cccc}
\hline Entry & Amount of water $[\mu \mathrm{L}]$ & Conversion [\%] & Yield 2 [\%] \\
\hline 1 & 1 & 100 & 79 \\
2 & 10 & 41 & 22 \\
3 & 100 & 18 & 0 \\
\hline 4 & - & 100 & 79 \\
\hline
\end{tabular}

Reaction conditions: RuPhos Pd G3 (1 mol\%), dry $\mathrm{Cs}_{2} \mathrm{CO}_{3}(1.0 \mathrm{mmol}, 2$ equiv), vinyl iodide 1 (0.5 mmol), phenyl formate (1.0 mmol, 2 equiv), dry DCE $(2 \mathrm{~mL})$, water $(\mathrm{x} \mu \mathrm{L}), 50^{\circ} \mathrm{C}$, $4 \mathrm{~h}$. Determined by GC with $n$-dodecane as an internal standard.

The results demonstrates that water exerts strong inhibitory effect on the $2^{\text {nd }}$ step of the reaction. Although, $1 \mu \mathrm{L}$ of $\mathrm{H}_{2} \mathrm{O}$ ( $\sim .1$ equiv) do not influence the yield of both steps, $10 \mu \mathrm{L}$ (1.1 equiv) of water hampered $2^{\text {nd }}$ step of reaction efficiently and $100 \mu \mathrm{L}$ (11 equiv) stopped aryloxycarbonylation step completely. 


\section{Mechanistic studies}

\subsection{Radical character of reaction}

Reactions were performed following typical reaction condition, modified by addition of radical scavenger as the third component in the order of reagent addition.
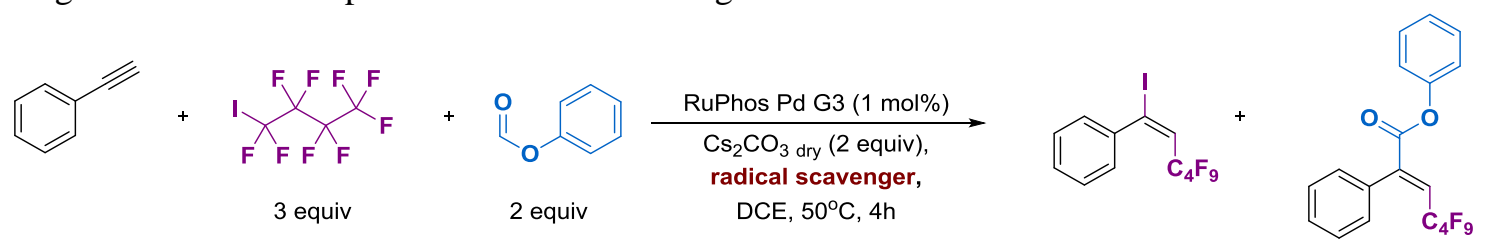

(1)

(2)

\begin{tabular}{cccccc}
\hline Entry & Radical scavenger & Amount [equiv] & Conversion [\%] & Yield 1 [\%] & Yield 2 [\%] \\
\hline 1 & TEMPO & 0.5 & $38(\downarrow)$ & $-(\sim)$ & $-(\downarrow)$ \\
2 & TEMPO & 1.0 & $49(\downarrow)$ & $-(\sim)$ & $-(\downarrow)$ \\
3 & TEMPO & 2.0 & $57(\downarrow)$ & $-(\sim)$ & $-(\downarrow)$ \\
4 & BHT & 0.5 & $100(\sim)$ & $5(\uparrow)$ & $74(\downarrow)$ \\
5 & BHT & 1.0 & $100(\sim)$ & $11(\uparrow)$ & $65(\downarrow)$ \\
6 & BHT & 2.0 & $100(\sim)$ & $19(\uparrow)$ & $55(\downarrow)$ \\
\hline 7 & - & - & 100 & - & 85 \\
\hline
\end{tabular}

Reaction conditions: RuPhos Pd G3 (1 mol\%), dry $\mathrm{Cs}_{2} \mathrm{CO}_{3}(1.0 \mathrm{mmol}, 2$ equiv), radical scavenger (x equiv), phenylacetylene $(0.5 \mathrm{mmol})$, phenyl formate $\left(1.0 \mathrm{mmol}, 2\right.$ equiv), $\mathrm{C}_{4} \mathrm{~F}_{9} \mathrm{I}\left(1.5 \mathrm{mmol}, 3\right.$ equiv), dry DCE $(2 \mathrm{~mL}), 50^{\circ} \mathrm{C}$, $4 \mathrm{~h}$. Determined by $\mathrm{GC}$ with $n$-dodecane as an internal standard.

All reactions were completely shut down in the presence of TEMPO (0.5 - 2.0 equiv). In the case of BHT, the reactions were hampered but not that efficient as in the case of TEMPO. The results may also indicate that the second step is not radical reaction and the $2^{\text {nd }}$ step is slower than the $1^{\text {st }}$ one or the catalyst is poisoned during the reaction. To prove this thesis additional experiments was carried out.

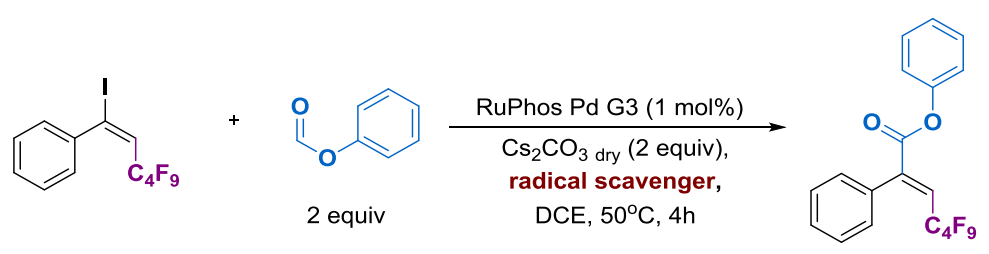

(1)

(2)

\begin{tabular}{ccccc}
\hline Entry & Radical scavenger & Amount [equiv] & Conversion [\%] & Yield 2 [\%] \\
\hline 1 & TEMPO & 0.5 & $100(\sim)$ & $99(\uparrow)$ \\
2 & TEMPO & 1.0 & $100(\sim)$ & $100(\uparrow)$ \\
3 & TEMPO & 2.0 & $100(\sim)$ & $96(\uparrow)$ \\
4 & BHT & 0.5 & $95(\downarrow)$ & $90(\uparrow)$ \\
5 & BHT & 1.0 & $88(\downarrow)$ & $87(\uparrow)$ \\
6 & BHT & 2.0 & $70(\downarrow)$ & $66(\downarrow)$ \\
\hline 7 & - & - & 100 & 75 \\
\hline
\end{tabular}

Reaction conditions: RuPhos Pd G3 (1 mol\%), dry $\mathrm{Cs}_{2} \mathrm{CO}_{3}(1.0 \mathrm{mmol}, 2$ equiv), radical scavenger (x equiv), phenyl iodide $1(0.5 \mathrm{mmol})$, phenyl formate $\left(1.0 \mathrm{mmol}, 2\right.$ equiv), dry DCE $(2.0 \mathrm{~mL}), 50^{\circ} \mathrm{C}, 4 \mathrm{~h}$. Determined by $\mathrm{GC}$ with $n$-dodecane as an internal standard.

The experiments proved that the $2^{\text {nd }}$ step is not a radical reaction. The $2^{\text {nd }}$ step reaction is even more efficient with TEMPO compared to the standard procedure. Presumably, radical scavengers suppress the radical site reactions. 
3.2. Radical initiation experiments with 1-octyne.
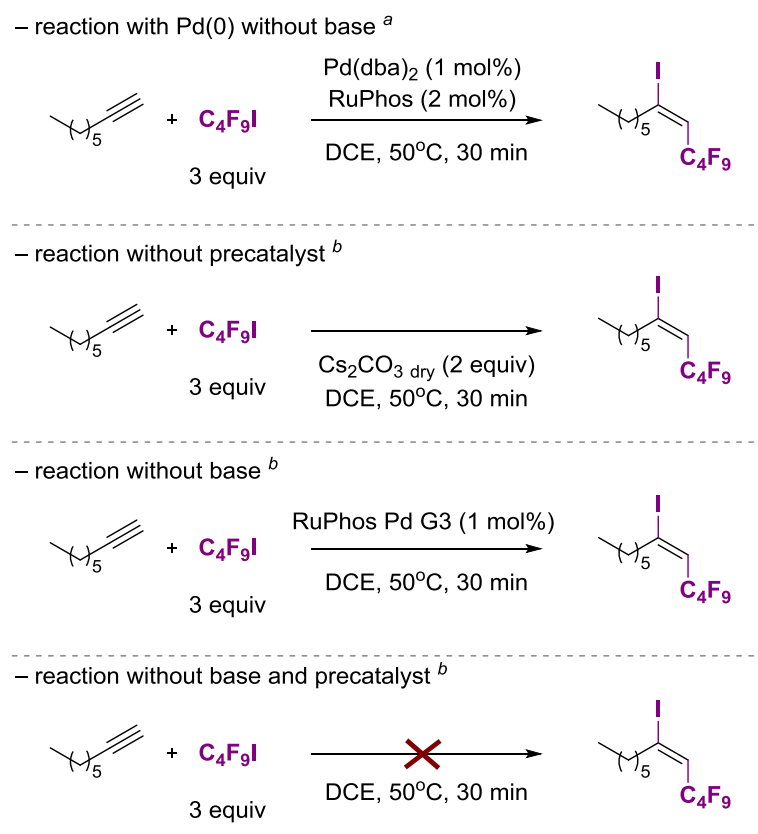
- reaction with $\mathrm{Pd}(\mathrm{II})$ without base ${ }^{a}$

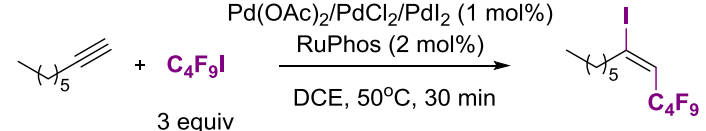

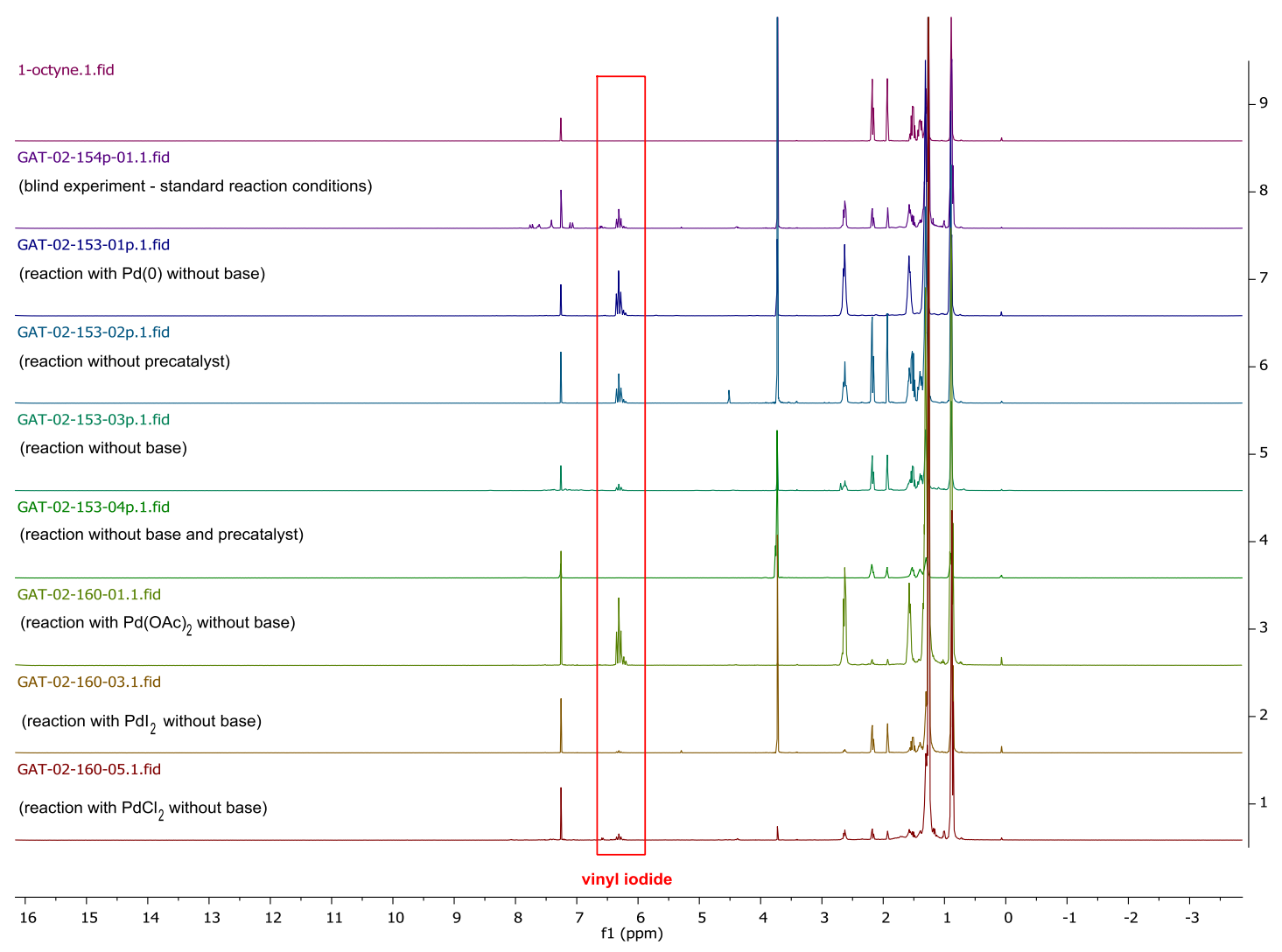

${ }^{a}$ Reaction conditions: Pd-complex ( $\left.1 \mathrm{~mol} \%\right)$, RuPhos (2 mol\%), 1-octyne (0.5 mmol), $\mathrm{C}_{4} \mathrm{~F}_{9} \mathrm{I}$ (1.5 mmol, 3 equiv), dry DCE (0.5 mL), 50 C, 4h. ${ }^{b}$ Reaction conditions: RuPhos Pd G3 (1 mol\%), dry $\mathrm{Cs}_{2} \mathrm{CO}_{3}(1.0 \mathrm{mmol}, 2$ equiv), 1 -octyne $(0.5 \mathrm{mmol})$,

$\mathrm{C}_{4} \mathrm{~F}_{9} \mathrm{I}\left(1.5 \mathrm{mmol}, 3\right.$ equiv), dry DCE $(0.5 \mathrm{~mL}), 50^{\circ} \mathrm{C}$, $4 \mathrm{~h}$. Blind experiment conditions - standard reaction conditions: RuPhos Pd G3 (1 mol\%), dry $\mathrm{Cs}_{2} \mathrm{CO}_{3}\left(1.0 \mathrm{mmol}, 2\right.$ equiv), 1-octyne (0.5 mmol), $\mathrm{C}_{4} \mathrm{~F}_{9} \mathrm{I}\left(1.5 \mathrm{mmol}, 3\right.$ equiv), dry DCE $(0.5 \mathrm{~mL}), 50^{\circ} \mathrm{C}$, 4h. Determined by NMR spectra. 
The radical addition of $\mathrm{C}_{4} \mathrm{~F}_{9} \mathrm{I}$ to unfunctionalized alkyl-substituted alkynes as 1-octyne can be promoted by $\mathrm{Pd}(0)$ complex or $\mathrm{Cs}_{2} \mathrm{CO}_{3}$ dry as well as $\mathrm{Pd}(\mathrm{OAc})_{2}$ complex without base. The $\mathrm{Pd}(\mathrm{II})$ complexes as $\mathrm{PdCl}_{2}$ or $\mathrm{PdI}_{2}$ did not initiate the radical reaction efficiently. 


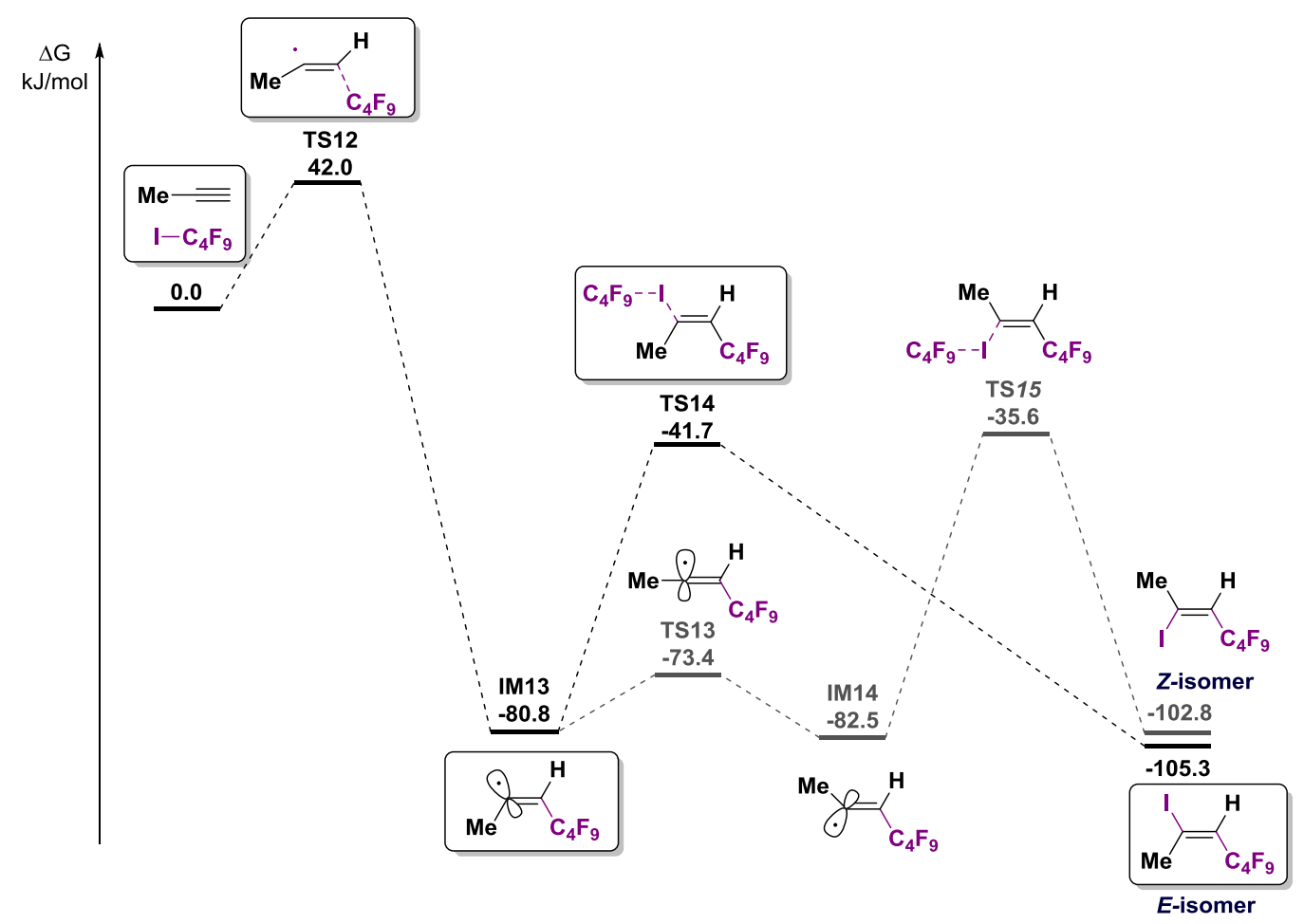

We also performed calculations for bent $\alpha$-methylvinyl radical to examine possible reaction pathway. The computed values are in agreement with anticipated preferences of anti addition pathway. The difference between intermediate IM13 and energetically lower IM14 is $1.7 \mathrm{~kJ} / \mathrm{mol}$, energy barrier between this two forms is $9.1 \mathrm{~kJ} / \mathrm{mol}$. The anti-addition pathway through transition state TS14 proceed with energy barrier $39.1 \mathrm{~kJ} / \mathrm{mol}$. Alternatively, forming of Z-isomer can take place via TS15 with energy barrier 46.9 $\mathrm{kJ} / \mathrm{mol}$. Moreover, forming $E$-isomer is thermodynamically favored than $Z$-isomer $(2.5 \mathrm{~kJ} / \mathrm{mol}$ energy difference). Calculation performed on the basis of Boltzmann distribution pointed out tendency toward forming $E$-isomer in ratio ( $E: Z, 90.6: 9.4$, calculated value for reaction conducted in $\left.50^{\circ} \mathrm{C}\right)$. 


\subsection{Kinetics investigation}

\section{An example of typical reaction conditions for kinetics experiments:}

A set of eight $4 \mathrm{~mL}$ dark glass screw-capped vials was prepared and RuPhos Pd G3 (5.0 $\mu \mathrm{mol}, 1 \mathrm{~mol} \%)$ was added to each vial. The whole set of vials was moved to a glovebox. The reactions were set up separately. Reagents were sequentially added: dry $\mathrm{Cs}_{2} \mathrm{CO}_{3}$ (1.0 mmol, 2.0 equiv), phenylacetylene $(0.5 \mathrm{mmol})$, phenyl formate (1.0 mmol, 2.0 equiv), perfluorobutyl iodide (1.5 mmol, 3.0 equiv), dry DCE $(2 \mathrm{~mL})$. Then, a magnetic stirring bar was placed in the reaction mixture. The vials were sealed with a cap. The reaction mixtures were stirred at $50^{\circ} \mathrm{C}$ for a given time period and then cooled down. The mixture was diluted with $\mathrm{CHCl}_{3}(1 \mathrm{~mL})$ quenched with sat. aquous $\mathrm{NH}_{4} \mathrm{Cl}(0.5 \mathrm{~mL})$ and analyzed by $\mathrm{GC}$ with $n$-dodecane $(25 \mu \mathrm{l})$ as an internal standard. The values given are the average of at least two independent experts.

\subsubsection{Kinetics profile of catalytic reaction}

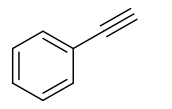

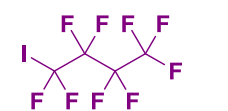

3 equiv

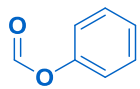

2 equiv

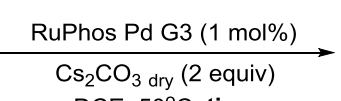

DCE, $50^{\circ} \mathrm{C}$, time

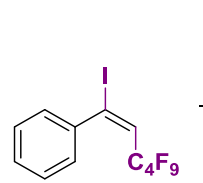

(1)

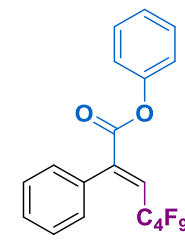

(2)

\begin{tabular}{ccccc}
\hline Entry & Time & Conversion [\%] & Yield 1 [\%] & Yield 2 [\%] \\
\hline 1 & $5 \mathrm{~min}$ & 16 & 7 & 2 \\
2 & $10 \mathrm{~min}$ & 33 & 18 & 7 \\
3 & $15 \mathrm{~min}$ & 69 & 40 & 17 \\
4 & $30 \mathrm{~min}$ & 97 & 48 & 35 \\
5 & $1 \mathrm{~h}$ & 100 & 23 & 60 \\
6 & $1.5 \mathrm{~h}$ & 100 & 15 & 68 \\
7 & $2 \mathrm{~h}$ & 100 & 8 & 69 \\
8 & $3 \mathrm{~h}$ & 100 & 3 & 71 \\
\hline
\end{tabular}

Reaction conditions: RuPhos Pd G3 (1 mol\%), dry $\mathrm{Cs}_{2} \mathrm{CO}_{3}(1.0 \mathrm{mmol}, 2$ equiv), phenylacetylene (0.5 mmol), phenyl formate (1.0 mmol, 2 equiv), $\mathrm{C}_{4} \mathrm{~F}_{9} \mathrm{I}\left(1.5 \mathrm{mmol}, 3\right.$ equiv), dry DCE $(2 \mathrm{~mL}), 50^{\circ} \mathrm{C}$, time. Determined by $\mathrm{GC}$ with $n$-dodecane as an internal standard.

Figure S1. Reaction profile

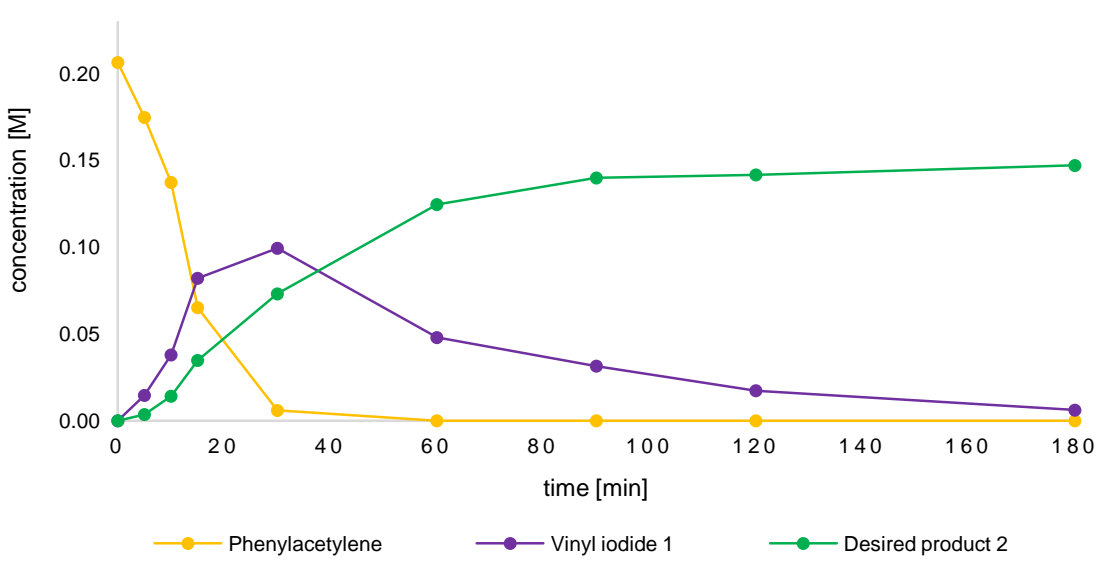


Figure S2. Phenyl formate - plot of ln(concentration) vs. $t$

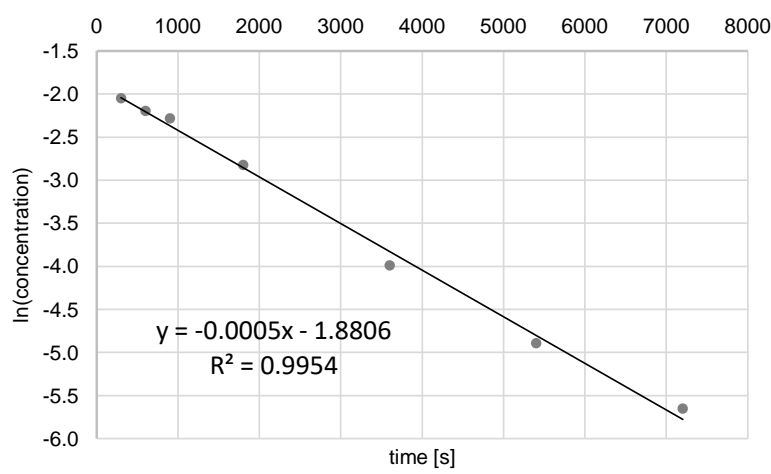


Kinetics profile of the $1^{\text {st }}$ step

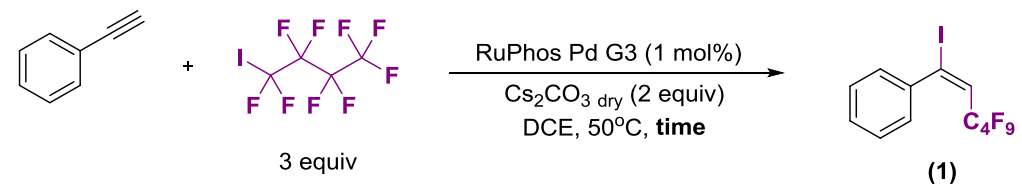

\begin{tabular}{cccc}
\hline Entry & Time & Conversion [\%] & Yield 1 [\%] \\
\hline 1 & $5 \mathrm{~min}$ & 68 & 55 \\
2 & $10 \mathrm{~min}$ & 76 & 62 \\
3 & $15 \mathrm{~min}$ & 79 & 63 \\
4 & $30 \mathrm{~min}$ & 87 & 69 \\
5 & $1 \mathrm{~h}$ & 92 & 72 \\
6 & $1.5 \mathrm{~h}$ & 94 & 74 \\
7 & $2 \mathrm{~h}$ & 96 & 75 \\
8 & $3 \mathrm{~h}$ & 97 & 75 \\
\hline
\end{tabular}

Reaction conditions: RuPhos Pd G3 (1 mol\%), dry $\mathrm{Cs}_{2} \mathrm{CO}_{3}\left(1.0 \mathrm{mmol}, 2\right.$ equiv), phenylacetylene $(0.5 \mathrm{mmol}), \mathrm{C}_{4} \mathrm{~F}_{9} \mathrm{I}(1.5$ mmol, 3 equiv), dry DCE $(2.0 \mathrm{~mL}), 50^{\circ} \mathrm{C}$, time. Determined by GC with $n$-dodecane as an internal standard.

Figure S3. Reaction profile $-1^{\text {st }}$ step $\left(50^{\circ} \mathrm{C}\right)$

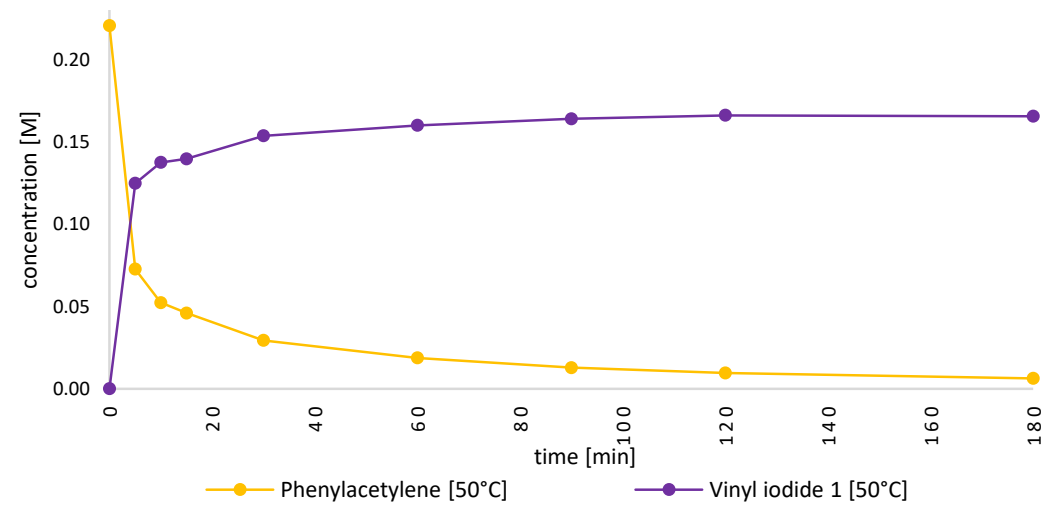



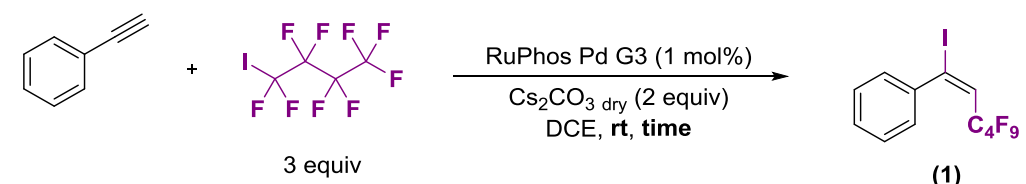

(1)

\begin{tabular}{cccc}
\hline Entry & Time & Conversion [\%] & Yield 1 [\%] \\
\hline 1 & $5 \mathrm{~min}$ & 45 & 39 \\
2 & $10 \mathrm{~min}$ & 49 & 42 \\
3 & $15 \mathrm{~min}$ & 64 & 53 \\
4 & $30 \mathrm{~min}$ & 69 & 57 \\
5 & $1 \mathrm{~h}$ & 75 & 61 \\
6 & $1.5 \mathrm{~h}$ & 77 & 62 \\
7 & $2 \mathrm{~h}$ & 79 & 63 \\
8 & $3 \mathrm{~h}$ & 82 & 65 \\
\hline
\end{tabular}

Reaction conditions: RuPhos Pd G3 (1 mol\%), dry $\mathrm{Cs}_{2} \mathrm{CO}_{3}\left(1.0 \mathrm{mmol}, 2\right.$ equiv), phenylacetylene $(0.5 \mathrm{mmol}), \mathrm{C}_{4} \mathrm{~F}_{9} \mathrm{I}(1.5$ mmol, 3 equiv), dry DCE (2.0 mL), rt, time. Determined by GC with $n$-dodecane as an internal standard.

Figure S4. Reaction profile $-1^{\text {st }}$ step $(r t)$

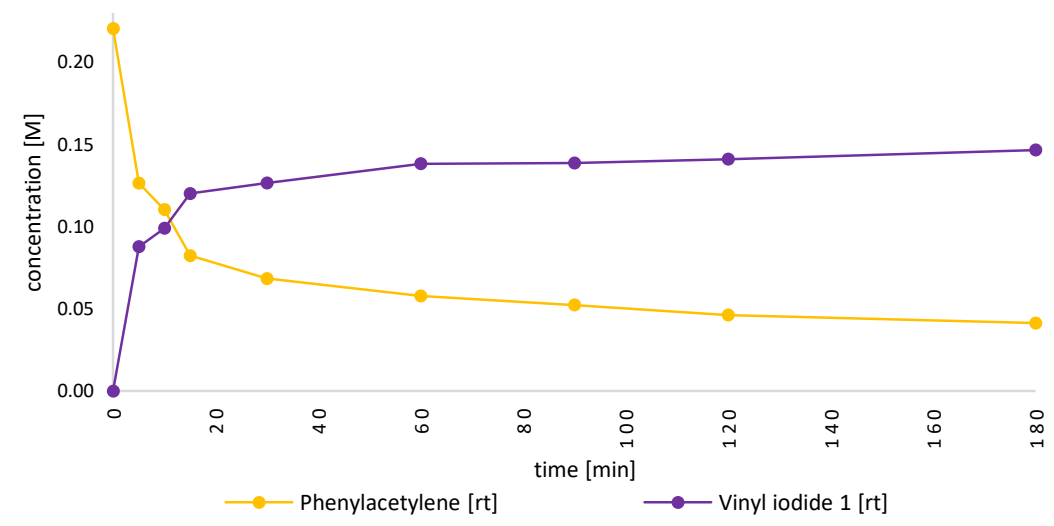


3.4.2. Kinetics profile of the $2^{\text {nd }}$ step<smiles>CC(=CC(I)c1ccccc1)C(F)(F)F</smiles>

(1)

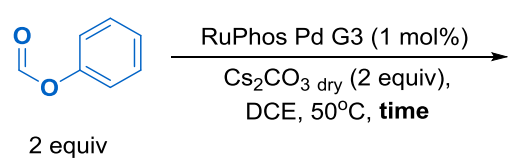

2 equiv

Time

$5 \mathrm{~min}$

$10 \min$

$15 \min$

$30 \mathrm{~min}$

$1 \mathrm{~h}$

$1.5 \mathrm{~h}$

$\begin{array}{ll}7 & 2 \mathrm{~h} \\ 8 & 3 \mathrm{~h}\end{array}$

$\begin{array}{ll}7 & 2 \mathrm{~h} \\ 8 & 3 \mathrm{~h}\end{array}$

Reaction conditions: RuPhos Pd G3 (1 mol\%), dry $\mathrm{Cs}_{2} \mathrm{CO}_{3}\left(1.0 \mathrm{mmol}, 2\right.$ equiv), phenylacetylene $(0.5 \mathrm{mmol}), \mathrm{C}_{4} \mathrm{~F}_{9} \mathrm{I}(1.5$ mmol, 3 equiv), dry DCE $(2.0 \mathrm{~mL})$, rt, time. Determined by GC with $n$-dodecane as an internal standard.

Figure S5. Reaction profile $-2^{\text {nd }}$ step

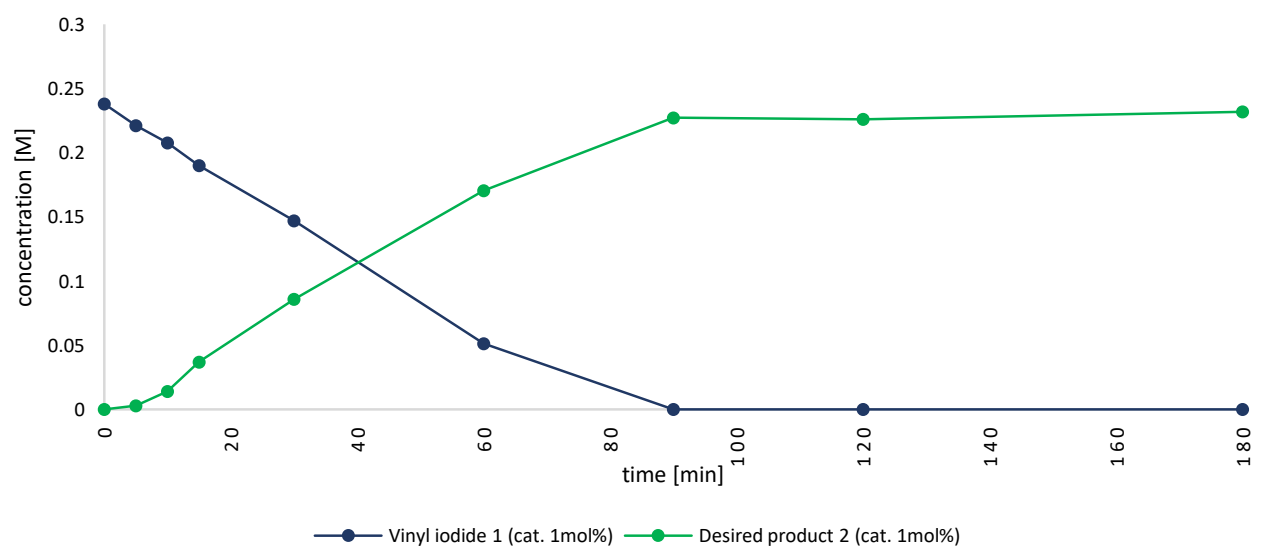

Figure S6. Vinyl iodide 1 - plot of concentration vs. $t$

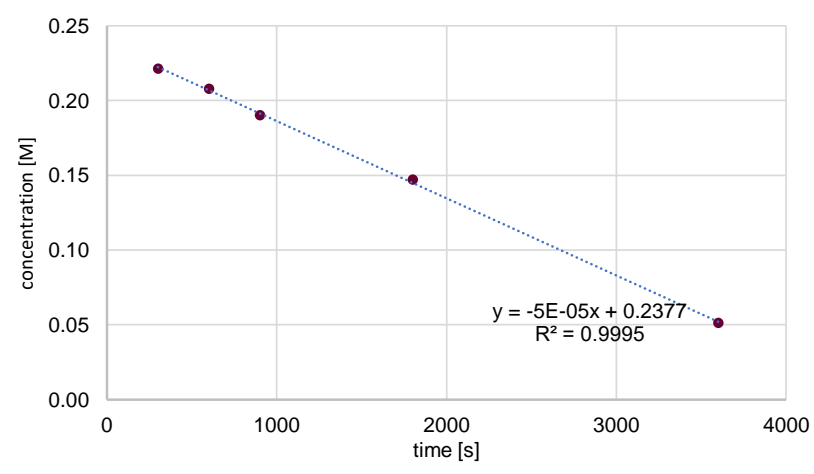


Figure S7. Phenyl formate - plot of concentration vs. $t$

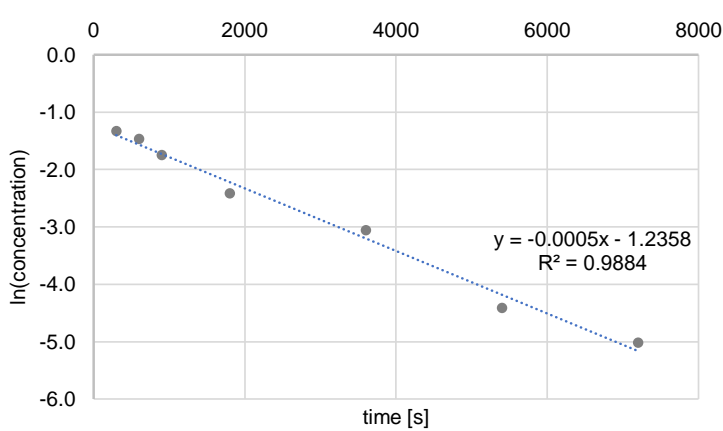

Figure S8. Phenol - plot of concentration vs. $t$

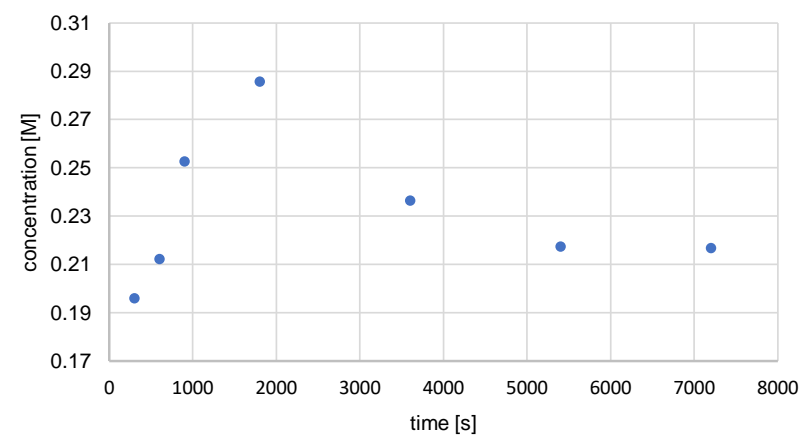

Figure S9. Desired product $\mathbf{2}$ - plot of concentration vs. $t$

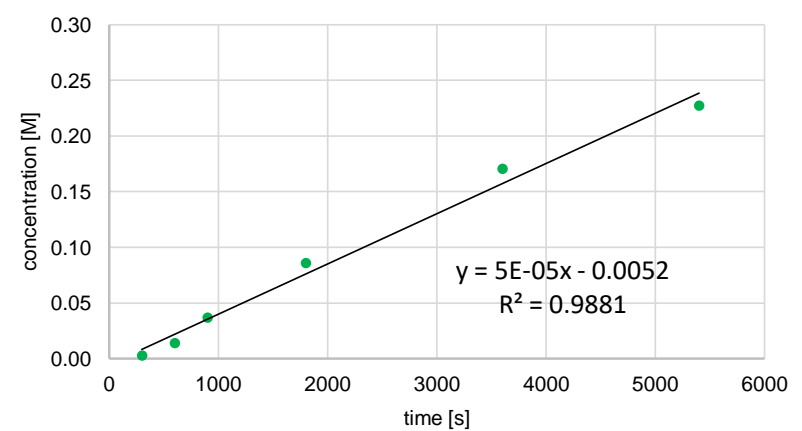




\subsubsection{Isotope effect - kinetics profile}

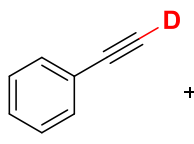

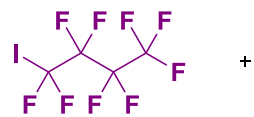

3 equiv

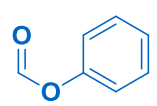

2 equiv

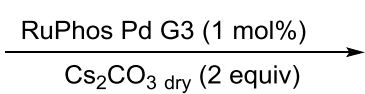

DCE, $50^{\circ} \mathrm{C}$, time

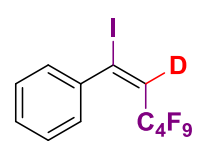

(1-D)

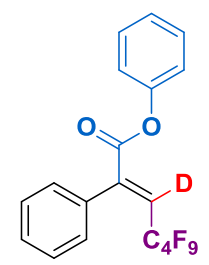

(2-D)

\begin{tabular}{ccccc}
\hline Entry & Time & Conversion [\%] & Yield 1-D [\%] & Yield 2-D [\%] \\
\hline 1 & $5 \mathrm{~min}$ & 10 & 12 & 4 \\
2 & $10 \mathrm{~min}$ & 37 & 25 & 8 \\
3 & $15 \mathrm{~min}$ & 69 & 39 & 16 \\
4 & $30 \mathrm{~min}$ & 96 & 42 & 37 \\
5 & $1 \mathrm{~h}$ & 100 & 24 & 61 \\
6 & $1.5 \mathrm{~h}$ & 100 & 11 & 74 \\
7 & $2 \mathrm{~h}$ & 100 & 6 & 82 \\
8 & $3 \mathrm{~h}$ & 100 & - & 86 \\
\hline
\end{tabular}

Reaction conditions: RuPhos Pd G3 (1 mol\%), dry $\mathrm{Cs}_{2} \mathrm{CO}_{3}(1.0 \mathrm{mmol}, 2$ equiv), phenylacetylene- $d$ (0.5 mmol), phenyl formate (1.0 mmol, 2 equiv), $\mathrm{C}_{4} \mathrm{~F}_{9} \mathrm{I}\left(1.5 \mathrm{mmol}, 3\right.$ equiv), dry DCE $(2 \mathrm{~mL}), 50^{\circ} \mathrm{C}$, time. Determined by $\mathrm{GC}$ with $n$-dodecane as an internal standard.

Figure S10. Reaction profile - phenylacetylene-d

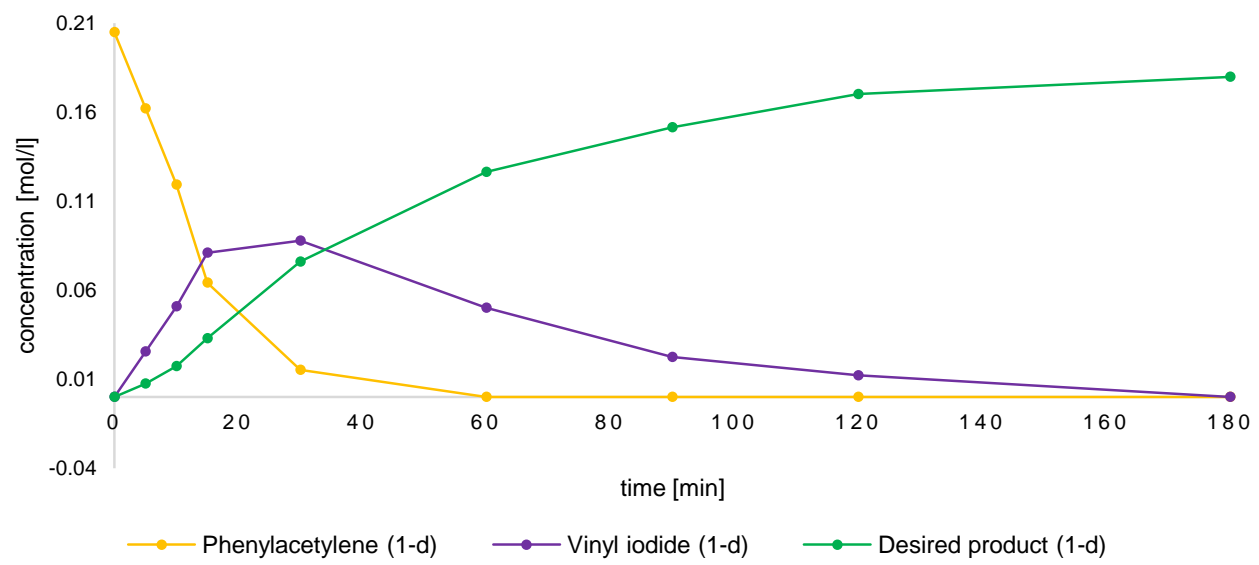

Application of deuterium labelled phenylacetylene did not affect the kinetic profile of the tandem reaction, which can indicate that deuterium did not involve in the rate limiting step of this reaction. 


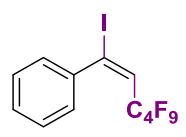

(1)

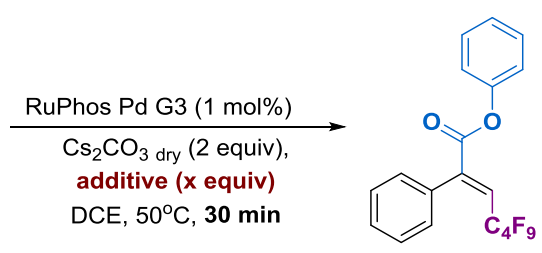

(2)

\begin{tabular}{|c|c|c|c|c|}
\hline Entry & Reagent & Amount [mol \%] & Conversion $[\%]$ & Yield 2 [\%] \\
\hline 1 & Phenylacetylene & 2 & $51(\uparrow)$ & $42(\uparrow)$ \\
\hline 2 & Perfluorobutyl iodide & 2 & $49(\uparrow)$ & $43(\uparrow)$ \\
\hline 3 & Isopropanol & 10 & $49(\uparrow)$ & $42(\uparrow)$ \\
\hline 4 & Phenylboronic Acid & 1 & $47(\sim)$ & $38(\sim)$ \\
\hline 5 & Dimethyl acetylenedicarboxylate & 2 & $50(\uparrow)$ & $41(\uparrow)$ \\
\hline 6 & Referential reaction & - & 47 & 37 \\
\hline
\end{tabular}

Reaction conditions: RuPhos Pd G3 (1 mol\%), dry $\mathrm{Cs}_{2} \mathrm{CO}_{3}$ (1.0 mmol, 2 equiv), additive (x mol \%), vinyl iodide 1 (0.5 $\mathrm{mmol})$, phenyl formate (1.0 mmol, 2 equiv), dry DCE $(2 \mathrm{~mL}), 50^{\circ} \mathrm{C}, 30 \mathrm{~min}$

Almost all additives slightly increased the yield of the reaction. 


\subsection{Formates decompositions}

For a few reaction with formates that the desired product was not detected, the decomposition experiments were performed. The reactions was setting up under reaction conditions described below with 4-methoxyphenylacetylene, perfluorobutyl iodide and formate, after $4 \mathrm{~h}$ the reaction vessel was cooled down to rt. Both the vessel and the carbon monoxide detector were put in a ziplock bag. The bag was closed and the cap was unscrewed carefully, after 10 minutes waiting, no detection of $\mathrm{CO}$ was verified.

$$
\text { (n) }
$$

Reaction conditions: RuPhos Pd G3 (1 mol\%), dry $\mathrm{Cs}_{2} \mathrm{CO}_{3}(1.0 \mathrm{mmol}, 2$ equiv), 4-methoxyphenylacetylene $(0.5 \mathrm{mmol})$, formate (1.0 mmol, 2 equiv), $\mathrm{C}_{4} \mathrm{~F}_{9} \mathrm{I}\left(1.5 \mathrm{mmol}, 3\right.$ equiv), dry DCE $(2 \mathrm{~mL}), 50^{\circ} \mathrm{C}, 4 \mathrm{~h}$.

Analogous procedure were performed for formates decomposition reactions.

$$
\begin{aligned}
& \text { 共 } \\
& \text { detected } \\
& \text { (29 ppm) }
\end{aligned}
$$

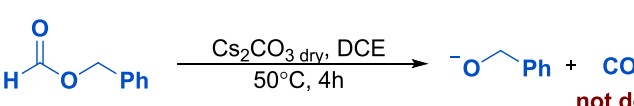

$$
\begin{aligned}
& \text { not detected }
\end{aligned}
$$

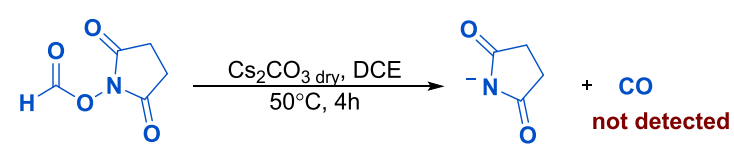

Reaction conditions: dry $\mathrm{Cs}_{2} \mathrm{CO}_{3}\left(1.0 \mathrm{mmol}, 1\right.$ equiv), formate $(1.0 \mathrm{mmol})$, dry DCE $(2 \mathrm{~mL}), 50^{\circ} \mathrm{C}, 4 \mathrm{~h}$.

Formates decomposed with formation of poorly stabilized alkoxide were not detected or detected in very low concentration under reaction conditions. 


\subsection{Competitive reaction experiments}

A few sets of experiments were conducted to investigate general reaction mechanism: charge impact experiments - competitive reaction with phenyl formate and phenol bearing fluorine substituents and transesterification reaction as well as competitive reaction with phenylacetylene derivatives bearing electron withdrawing and donating groups in para position.

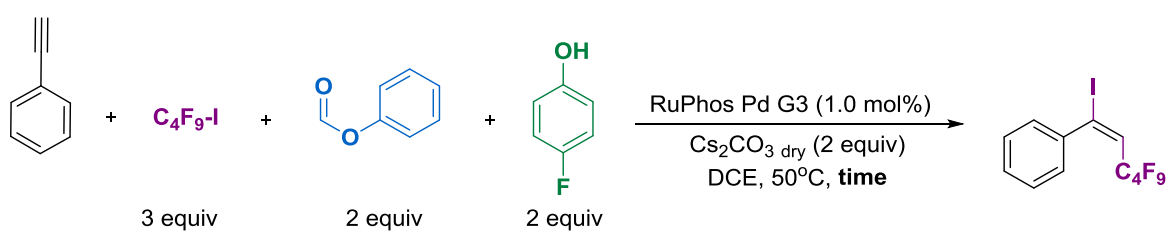

(1)

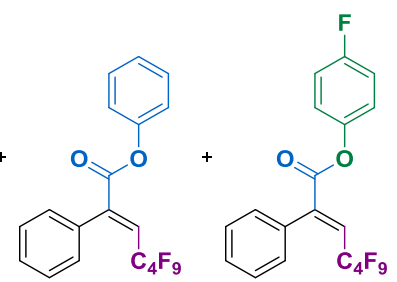

(2)

(39)

\begin{tabular}{cccccc}
\hline Entry & Time & Conversion [\%] & Yield 1 [\%] & Yield 2 [\%] & Yield 39 [\%] \\
\hline 1 & $5 \mathrm{~min}$ & 5 & 2 & 1 & 1 \\
2 & $10 \mathrm{~min}$ & 7 & 4 & 2 & 3 \\
3 & $15 \mathrm{~min}$ & 20 & 5 & 5 & 12 \\
4 & $30 \mathrm{~min}$ & 51 & 4 & 9 & 22 \\
5 & $1 \mathrm{~h}$ & 62 & 3 & 17 & 23 \\
6 & $1.5 \mathrm{~h}$ & 71 & 1 & 21 & 26 \\
7 & $2 \mathrm{~h}$ & 78 & 0 & 24 & 25 \\
8 & $3 \mathrm{~h}$ & 82 & 0 & 23 & \\
\hline
\end{tabular}

Reaction conditions: RuPhos Pd G3 (1 mol\%), dry $\mathrm{Cs}_{2} \mathrm{CO}_{3}(1.0 \mathrm{mmol}, 2$ equiv), 4-fluorophenol (1.0 mmol, 2 equiv), phenyl formate $\left(1.0 \mathrm{mmol}, 2\right.$ equiv), phenylacetylene $(0.5 \mathrm{mmol}), \mathrm{C}_{4} \mathrm{~F}_{9} \mathrm{I}\left(1.5 \mathrm{mmol}, 3\right.$ equiv), dry DCE $(2 \mathrm{~mL}), 50^{\circ} \mathrm{C}$, time. Determined by GC with $n$-dodecane as an internal standard.

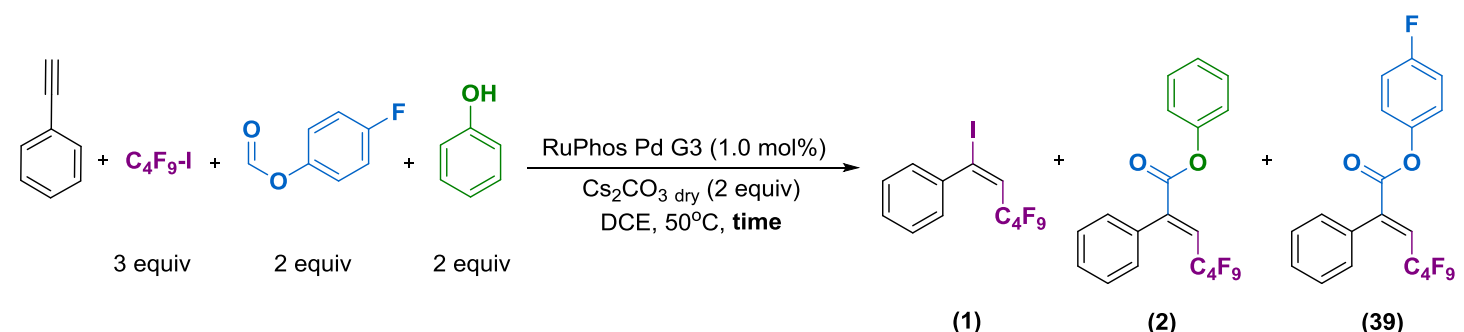

\begin{tabular}{cccccc}
\hline Entry & Time & Conversion [\%] & Yield 1 [\%] & Yield 2 [\%] & Yield 39 [\%] \\
\hline 1 & $5 \mathrm{~min}$ & 4 & 3 & 1 & 1 \\
2 & $10 \mathrm{~min}$ & 16 & 10 & 3 & 5 \\
3 & $15 \mathrm{~min}$ & 27 & 13 & 5 & 7 \\
4 & $30 \mathrm{~min}$ & 71 & 25 & 13 & 19 \\
5 & $1 \mathrm{~h}$ & 100 & 31 & 19 & 26
\end{tabular}

Reaction conditions: RuPhos Pd G3 (1 mol\%), dry $\mathrm{Cs}_{2} \mathrm{CO}_{3}(1.0 \mathrm{mmol}, 2$ equiv), phenol (1.0 mmol, 2 equiv), 4-fluorophenyl formate ( $1.0 \mathrm{mmol}, 2$ equiv), phenylacetylene $(0.5 \mathrm{mmol}), \mathrm{C}_{4} \mathrm{~F}_{9} \mathrm{I}\left(1.5 \mathrm{mmol}, 3\right.$ equiv), dry $\mathrm{DCE}(2 \mathrm{~mL}), 50^{\circ} \mathrm{C}$, time. Determined by GC with $n$-dodecane as an internal standard.

The conversion of phenylacetylene differ for both experiments, the efficiency is hampered for reaction with 4-fluorophenol and phenyl formate after $30 \mathrm{~min}, 51 \%$ conversion were observed, in corresponding reaction with phenol and 4-fluorophenyl formate $71 \%$ conversion were noticed. In the case of first two experiments, product 39 with fluorine substituent were preferentially formed. One explanation can be that in undercurrent of base conditions 4-fluorophonoxy anion is formed preferentially due to better stabilization of anion which results on better yield of product 39. But this assumption can be call into question by the transesterification experiment results revealing the possible transesterification reaction under reaction condition leading to product 39 (see next chapter 3.8.). 


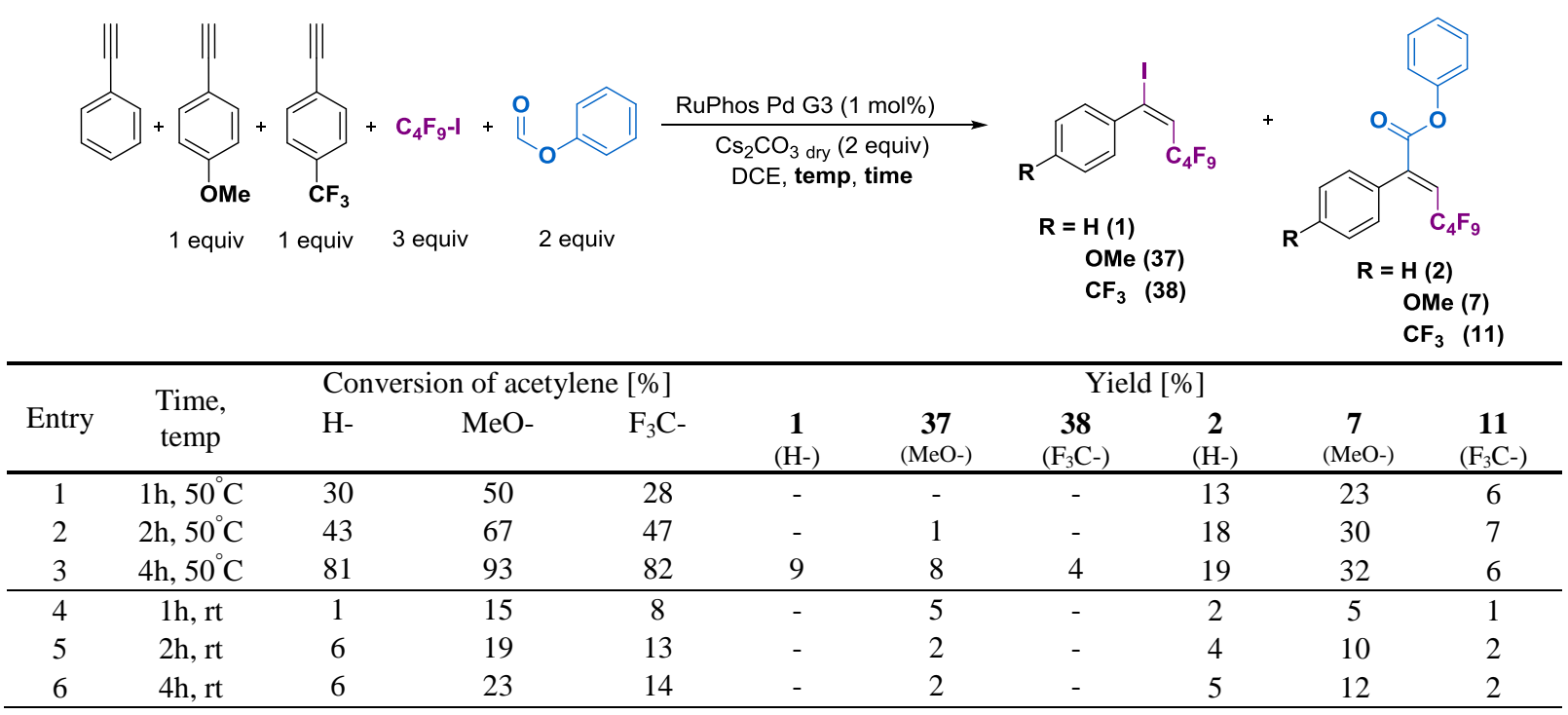

Reaction conditions: RuPhos Pd G3 (1 mol\%), dry $\mathrm{Cs}_{2} \mathrm{CO}_{3}(1.0 \mathrm{mmol}, 2$ equiv), phenyl formate (1.0 mmol, 2 equiv), 4-ethynylanisole $(0.5 \mathrm{mmol}, 1$ equiv), phenylacetylene $(0.5 \mathrm{mmol}), 4$ '-trifluoromethylphenyl acetylene $(0.5 \mathrm{mmol}, 1$ equiv), $\mathrm{C}_{4} \mathrm{~F}_{9} \mathrm{I}\left(1.5 \mathrm{mmol}, 3\right.$ equiv), dry DCE $(2 \mathrm{~mL}), 50^{\circ} \mathrm{C}$, time. Determined by GC with $n$-dodecane as an internal standard.

In the case of reaction with various phenylacetylene derivatives, perfluoroalkylative phenyloxycarbonylation of alkynes worked preferably for 4-methoxyphenylacetylene leading to product 7 and proceed poorly for 4'-trifluoromethylphenyl acetylene forming product $\mathbf{1 1}$. The same tendency was observed for vinyl iodides $\mathbf{3 7}$ and $\mathbf{3 8}$ formation. The obtained results reflected in the substrate scope, the best results were obtained for phenylacetylenes with electron donating group and phenyl formate with electron withdriving substituent.

\subsection{Transesterification of the product}

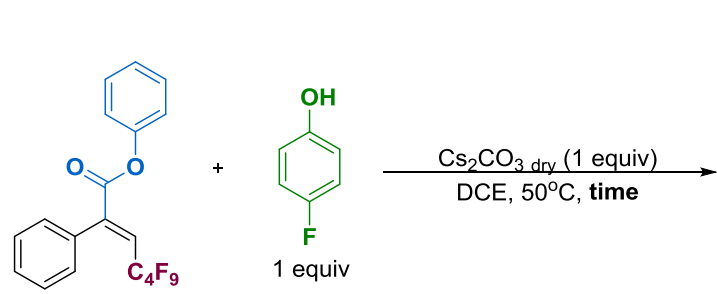

(2)

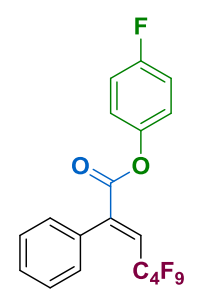

(39)

\begin{tabular}{cccc}
\hline Entry & Time & Conversion of 2 [\%] & Yield 39 [\%] \\
\hline 1 & $5 \mathrm{~min}$ & 75 & 62 \\
2 & $10 \mathrm{~min}$ & 76 & 58 \\
3 & $15 \mathrm{~min}$ & 77 & 56 \\
4 & $30 \mathrm{~min}$ & 79 & 50 \\
5 & $1 \mathrm{~h}$ & 83 & 40 \\
\hline
\end{tabular}

Reaction conditions: dry $\mathrm{Cs}_{2} \mathrm{CO}_{3}$ (1.0 mmol, 1 equiv), compound 2 (1.0 mmol, 1 equiv), 4-fluorophenol (1.0 mmol, 1 equiv), dry DCE $(2 \mathrm{~mL}), 50^{\circ} \mathrm{C}$, time. Determined by GC with $n$-dodecane as an internal standard. 


\subsection{Effect of the substituents in aryl formates}

3.9.1. Effect of the electronic nature of aryl formate rate of aryloxycarbonylation of $\mathbf{1}$

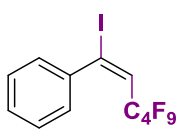

1

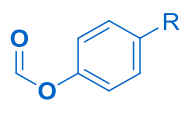

2 equiv

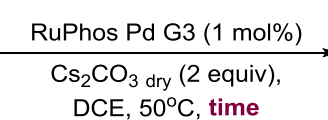

DCE, $50^{\circ} \mathrm{C}$, time

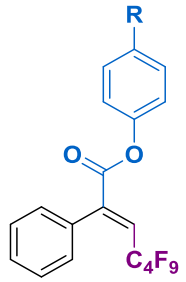

\begin{tabular}{|c|c|c|c|c|}
\hline \multirow[t]{2}{*}{ Entry } & \multirow[t]{2}{*}{ Time } & \multicolumn{3}{|c|}{ Conversion of $\mathbf{1}[\%]$} \\
\hline & & $\mathrm{R}=\mathrm{H}$ & $\mathrm{R}=\mathrm{OMe}$ & $\mathrm{R}=\mathrm{NO}_{2}$ \\
\hline 1 & $30 \mathrm{~min}$ & 40 & 35 & 19 \\
\hline 2 & $1 \mathrm{~h}$ & 78 & 50 & 25 \\
\hline 3 & $3 h$ & 100 & 100 & 59 \\
\hline
\end{tabular}

Reaction conditions: RuPhos Pd G3 (1 mol\%), dry $\mathrm{Cs}_{2} \mathrm{CO}_{3}(1.0 \mathrm{mmol}, 2$ equiv), vinyl iodide 1 (0.5 mmol), 4-methoxyphenyl formate $\left(1.0 \mathrm{mmol}, 2\right.$ equiv), dry DCE $(2 \mathrm{~mL}), 50^{\circ} \mathrm{C}$, time. Determined by GC with $n$-dodecane as an internal standard.

\subsubsection{Gibbs free energy profile for aryloxycarbonylation of $\mathbf{1}$ with electronically varied} aryl formates.

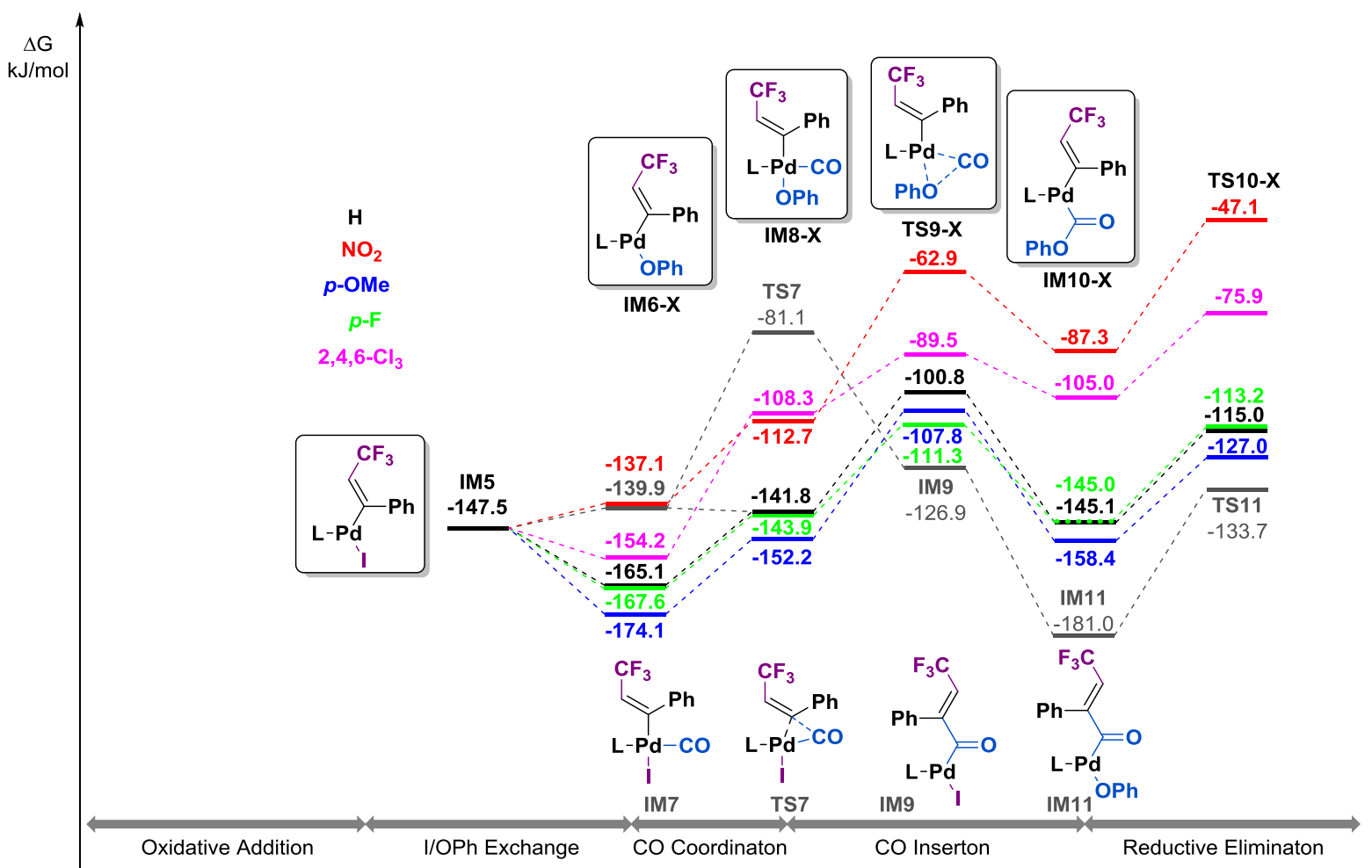




\section{4. $\quad$ Synthesis and characterization of isolated compounds}

\subsection{Non-commercially available starting materials - synthesis and analytical data}

\section{4-fluorophenyl formate}

F In a $100 \mathrm{~mL}$ flask equipped with a condenser acetic anhydride (29.1g, $0.285 \mathrm{~mol}, 8.00$ equiv) was cooled to $0^{\circ} \mathrm{C}$. Then formic acid $(16.4 \mathrm{~g}, 0.357 \mathrm{~mol}, 10.00$ equiv) was added slowly. The solution was warmed up to room temperature and stirred for 10 minutes. Then, the mixture was heated to $60^{\circ} \mathrm{C}$ and stirred for $1 \mathrm{~h}$. After cooling to the room temperature, $p$-fluorophenol $(4.0 \mathrm{~g}, 0.036 \mathrm{~mol}, 1.00 \mathrm{eq})$ $\mathrm{O} \Xi \mathrm{O}$ and $\mathrm{NaHCO}_{3}(6.0 \mathrm{~g}, 0.071 \mathrm{~mol}, 2.00$ equiv $)$ were added. The mixture was stirred at room temperature for $4 \mathrm{~h}$. DCM an water were added to the mixture. The crude was extracted with DCM (three times). Organic layers were combined and dried over anhydrous $\mathrm{Na}_{2} \mathrm{SO}_{4}$. After filtration, the filtrate was concentrated under reduced pressure. The residue was purified by distillation performed under reduced pressure: temperature of the vapor $44^{\circ}-46^{\circ} \mathrm{C}$, pressure 4.3 mbar. Pale yellow oil was obtained ( $3.206 \mathrm{~g}, 0.023 \mathrm{~mol}, 64 \%$ ).

${ }^{1} \mathrm{H}$ NMR (400 MHz, Chloroform- $d$ ) $\delta 8.27$ (s, 1H), $7.18-7.00(\mathrm{~m}, 4 \mathrm{H})$.

${ }^{13} \mathrm{C}$ NMR $(101 \mathrm{MHz}$, Chloroform- $d) \delta 160.66\left(\mathrm{~d},{ }^{1} J_{C-F}=245.1 \mathrm{~Hz}\right), 159.18,145.79\left(\mathrm{~d},{ }^{4} J_{C-F}=2.9 \mathrm{~Hz}\right), 122.78\left(\mathrm{~d},{ }^{3} J_{C-F}=8.5\right.$ $\mathrm{Hz}), 116.48\left(\mathrm{~d},{ }^{2} J_{C-F}=23.5 \mathrm{~Hz}\right)$.

\section{3-methoxyphenyl formate- ${ }^{-1} d$}

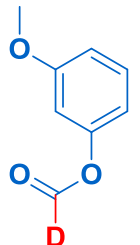

In a $25 \mathrm{~mL}$ two neck flask equipped with a condenser and septum acetic anhydride $(4.63 \mathrm{~g}, 0.045$ mol, 8.00 equiv) was cooled to $0^{\circ} \mathrm{C}$. Then formic acid- $\mathrm{d}_{2}(2.72 \mathrm{~g}, 0.057 \mathrm{~mol}, 10.00$ equiv) was added slowly. The solution was warmed up to room temperature and stirred for 10 minutes. Then, the mixture was heated to $60^{\circ} \mathrm{C}$ and stirred for $1 \mathrm{~h}$. After cooling to the room temperature, 3methoxyphenol $(0.70 \mathrm{~g} 0.006 \mathrm{~mol}, 1.00 \mathrm{eq})$ and $\mathrm{NaHCO}_{3}(0.95 \mathrm{~g}, 0.011 \mathrm{mmol}, 2.00$ equiv) were added. The mixture was stirred at room temperature overnight. DCM an water were added to the mixture. The crude was extracted with DCM (three times). Organic layers were combined and dried over anhydrous $\mathrm{Na}_{2} \mathrm{SO}_{4}$. After filtration, the filtrate was concentrated under reduced pressure. The crude was purified via flash chromatography on silica gel using MTBE:Hexane (95:5) as a eluent. Colorless oil was obtained (0.734 g, $0.005 \mathrm{~mol}, 85 \%$ yield).

${ }^{1} \mathrm{H}$ NMR $(400 \mathrm{MHz}$, Chloroform- $d$ ) $\delta 7.30(\mathrm{t}, J=8.2 \mathrm{~Hz}, 1 \mathrm{H}), 6.82(\mathrm{ddd}, J=8.4,2.4,0.9 \mathrm{~Hz}, 1 \mathrm{H}), 6.73$ (ddd, $J=8.1,2.2,0.9$ $\mathrm{Hz}, 1 \mathrm{H}), 6.69(\mathrm{t}, J=2.3 \mathrm{~Hz}, 1 \mathrm{H}), 3.81(\mathrm{~s}, 3 \mathrm{H})$.

${ }^{13} \mathrm{C}$ NMR (101 MHz, Chloroform- $d$ ) $\delta$ 160.9, 151.0, 130.3, 113.3, 112.3, 107.4, 55.6.

\section{3-methoxyphenyl formate- ${ }^{13} \mathrm{C}$}

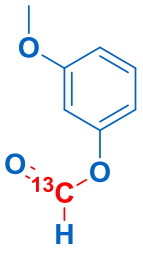

In a $25 \mathrm{~mL}$ two neck flask equipped with a condenser and septum acetic anhydride $(1.64 \mathrm{~g}, 0.016$ mol, 4.00 equiv) was cooled to $0^{\circ} \mathrm{C}$. Then formic acid- ${ }^{13} \mathrm{C}(0.95 \mathrm{~g}, 0.020 \mathrm{~mol}, 5.00$ equiv $)$ was added slowly. The solution was warmed up to room temperature and stirred for 10 minutes. Then, the mixture was heated to $65^{\circ} \mathrm{C}$ and stirred for $3 \mathrm{~h}$. After cooling to the room temperature, 3methoxyphenol $(0.50 \mathrm{~g}, 0.004 \mathrm{~mol})$ and $\mathrm{NaHCO}_{3}(0.67 \mathrm{~g}, 0.008 \mathrm{mmol}, 2.00$ equiv) were added. The mixture was stirred at room temperature for 2 days. DCM an water were added to the mixture. The crude was extracted with DCM (three times). Organic layers were combined and dried over anhydrous $\mathrm{Na}_{2} \mathrm{SO}_{4}$. After filtration, the filtrate was concentrated under reduced pressure. The crude was purified via flash chromatography on silica gel using MTBE:Hexane (95:5) as a eluent. Colorless oil was obtained (0.374 g, 0.002 mol $61 \%$ yield).

${ }^{1} \mathrm{H}$ NMR (400 MHz, Chloroform- $d$ ) $\delta 8.29(\mathrm{~d}, J=231.5 \mathrm{~Hz}, 1 \mathrm{H}), 7.30(\mathrm{t}, J=8.2 \mathrm{~Hz}, 1 \mathrm{H}), 6.82(\mathrm{dd}, J=8.3,2.1 \mathrm{~Hz}, 1 \mathrm{H}), 6.73$ (dd, $J=8.1,2.2 \mathrm{~Hz}, 1 \mathrm{H}), 6.69(\mathrm{t}, J=2.4 \mathrm{~Hz}, 1 \mathrm{H}), 3.81$ (s, $3 \mathrm{H})$.

${ }^{13} \mathrm{C}$ NMR (101 MHz, Chloroform- $d$ ) $\delta 160.8,159.3,151.0(\mathrm{~d}, J=3.3 \mathrm{~Hz}), 130.3,113.3(\mathrm{~d}, J=1.8 \mathrm{~Hz}), 112.3,107.3(\mathrm{~d}, J=$ $1.8 \mathrm{~Hz}), 55.6$. 


\subsection{Perfluoroalkylated $\alpha, \beta$-unsaturated esters}

\subsubsection{General procedure for perfluoroalkylative aryloxycarbonylation of alkyne}

Caution!: Sequence of addition of reagents is important and guarantee reproducibility of results.

\section{Standard reaction conditions:}

To a 4 mL dark glass screw-capped vial RuPhos Pd G3 ( 0.005 mmol, 1 mol\%) was added. The vial was moved to a glovebox, then reagents were sequentially added: dry $\mathbf{C s}_{\mathbf{2}} \mathbf{C O}_{\mathbf{3}}$ (1.0 mmol, 2.0 equiv), formate (1.0 mmol, 2.0 equiv), acetylene $(0.5 \mathrm{mmol})$, perfluorinated iodide $(1.5 \mathrm{mmol}, 3.0$ equiv), dry DCE $(2.0 \mathrm{~mL})$. Then, a magnetic stirring bar was placed in the reaction mixture. The vial was sealed with a cap. The reaction mixture was stirred at $50^{\circ} \mathrm{C}$ for $4 \mathrm{~h}$ and then cooled down. The mixture was filtrated through a Celite pad using $\mathrm{CHCl}_{3}$. The filtrate was concentrated under reduced pressure.

\section{Conditions B}

To a 4 mL dark glass screw-capped vial RuPhos Pd G3 ( 0.010 mmol, 2 mol\%) was added. The vial was moved to a glovebox, then reagents were sequentially added: dry $\mathbf{C s}_{\mathbf{2}} \mathbf{C O}_{\mathbf{3}}$ (1.0 mmol, 2.0 equiv), formate (1.0 mmol, 2.0 equiv), acetylene $(0.5 \mathrm{mmol})$, perfluorobutyl iodide $(1.5 \mathrm{mmol}, 3.0$ equiv), dry DCE $(2.0 \mathrm{~mL})$. Then, a magnetic stirring bar was placed in the reaction mixture. The vial was sealed with a cap. The reaction mixture was stirred at $50^{\circ} \mathrm{C}$ for $4 \mathrm{~h}$ and then cooled down. The mixture was filtrated through a Celite pad using $\mathrm{CHCl}_{3}$. The filtrate was concentrated under reduced pressure.

\section{Conditions C}

To a 4 mL dark glass screw-capped vial RuPhos Pd G3 ( $0.025 \mathrm{mmol}, 5 \mathrm{~mol} \%$ ) was added. The vial was moved to a glovebox, then reagents were sequentially added: $\mathbf{d r y} \mathbf{C s}_{\mathbf{2}} \mathbf{C O}_{\mathbf{3}}(1.0 \mathrm{mmol}, 2.0$ equiv), formate (1.0 mmol, 2.0 equiv), acetylene $(0.5 \mathrm{mmol})$, perfluorobutyl iodide $(1.5 \mathrm{mmol}, 3.0$ equiv), dry DCE $(2.0 \mathrm{~mL})$. Then, a magnetic stirring bar was placed in the reaction mixture. The vial was sealed with a cap. The reaction mixture was stirred at $50^{\circ} \mathrm{C}$ for $4 \mathrm{~h}$ and then cooled down. The mixture was filtrated through a Celite pad using $\mathrm{CHCl}_{3}$. The filtrate was concentrated under reduced pressure.

\section{Conditions D}

To a 4 mL dark glass screw-capped vial RuPhos Pd G3 ( 0.005 mmol, 1 mol\%) was added. The vial was moved to a glovebox, then reagents were sequentially added: $\mathbf{d r y} \mathbf{C s}_{\mathbf{2}} \mathbf{C O}_{\mathbf{3}}$ (2.0 mmol, 4.0 equiv), formate (2.0 mmol, 4.0 equiv), acetylene $(0.5 \mathrm{mmol})$, perfluorobutyl iodide $(1.5 \mathrm{mmol}, 3.0$ equiv), dry DCE $(2.0 \mathrm{~mL})$. Then, a magnetic stirring bar was placed in the reaction mixture. The vial was sealed with a cap. The reaction mixture was stirred at $50^{\circ} \mathrm{C}$ for $4 \mathrm{~h}$ and then cooled down. The mixture was filtrated through a Celite pad using $\mathrm{CHCl}_{3}$. The filtrate was concentrated under reduced pressure. 


\subsubsection{Perfluorosubstituted $\alpha, \beta$-unsaturated esters - analytical data}

Phenyl (2E)-4,4,5,5,6,6,7,7,7-nonafluoro-2-phenylhept-2-enoate (2)<smiles>O=C(Oc1ccccc1)/C(=C/C(F)(F)F)c1ccccc1</smiles>

Prepared in reaction of phenylacetylene $(51.1 \mathrm{mg})$ with perfluorobutyl iodide and phenyl formate under standard reaction conditions (yield: $68 \%, 150.5 \mathrm{mg}, 0.340 \mathrm{mmol}$ ). The title compound was isolated as yellow oil after chromatography on silica gel [the crude mass $\mathrm{x}$ 50] eluted with hexane $\rightarrow \mathrm{Hex}: \mathrm{CHCl}_{3}$ (9:1). The contaminated product was filtered through $\mathrm{C} 18$ reversed phase silica gel, eluted with acetonitrile.

${ }^{1} \mathrm{H}$ NMR (400 MHz, Chloroform- $d$ ) $\delta 7.46-7.29(\mathrm{~m}, 7 \mathrm{H}), 7.25(\mathrm{tt}, J=6.9,1.2 \mathrm{~Hz}, 1 \mathrm{H}), 7.15-7.05(\mathrm{~m}$, $3 \mathrm{H})$.

${ }^{13} \mathrm{C}$ NMR (101 MHz, Chloroform- $d$ ) $\delta 164.1,150.7,144.5(\mathrm{t}, J=4.3 \mathrm{~Hz}), 132.2,129.7,129.1,128.8(\mathrm{t}, J=2.5 \mathrm{~Hz}), 128.0$, $126.5,126.0(\mathrm{t}, J=21.8 \mathrm{~Hz}), 121.3$.

${ }^{19}$ F NMR (376 MHz, Chloroform- $d$ ) $\delta-80.99$ (m, 3F), -107.01 (m, 2F), -123.64 (m, 2F), -125.70 (m, 2F).

IR $\left(\mathrm{CH}_{2} \mathrm{Cl}_{2}\right): 3475,3065,2929,2856,1746,1493,1223,1194,1135\left(\mathrm{~cm}^{-1}\right)$

HRMS, m/z: calc'd for $\mathrm{C}_{19} \mathrm{H}_{11} \mathrm{~F}_{9} \mathrm{O}_{2}$ : 442.0615, found 442.0625.

Phenyl (2E)-4,4,5,5,6,6,7,7,7-nonafluoro-2-(naphthalen-2-yl)hept-2-enoate (3a)<smiles>O=C(Oc1ccccc1)C(=CC(F)(F)F)c1ccc2ccccc2c1</smiles>

Prepared in reaction of 2-ethynylnaphthalene $(76.3 \mathrm{mg})$ with perfluorobutyl iodide and phenyl formate under standard reaction conditions (yield: $30 \%, 73.6 \mathrm{mg}, 0.149 \mathrm{mmol}$ ). The title compound was isolated as yellowish oil after chromatography on silica gel [the crude mass x 50] eluted with hexane $\rightarrow$ hexane: $\mathrm{CHCl}_{3}(9: 1)$. The contaminated product was filtered through $\mathrm{C} 18$-reversed phase silica gel, eluted with acetonitrile.

Prepared in reaction of 2-ethynylnaphthalene $(76.1 \mathrm{mg})$ with perfluorobutyl iodide and phenyl formate conditions B (yield: $39 \%, 95.7 \mathrm{mg}, 0.194 \mathrm{mmol}$ ). The title compound was isolated as yellowish oil after chromatography on silica gel [the crude mass x 50] eluted with hexane $\rightarrow$ hexane: $\mathrm{CHCl}_{3}$ (9:1). The contaminated product was filtered through $\mathrm{C} 18$-reversed phase silica gel, eluted with acetonitrile.

${ }^{1} \mathrm{H}$ NMR $(400 \mathrm{MHz}$, Chloroform- $d$ ) $\delta 7.93-7.86(\mathrm{~m}, 3 \mathrm{H}), 7.84(\mathrm{~s}, 1 \mathrm{H}), 7.58-7.51(\mathrm{~m}, 2 \mathrm{H}), 7.44(\mathrm{~d}, J=8.5 \mathrm{~Hz}, 1 \mathrm{H}), 7.39(\mathrm{t}$, $J=7.8 \mathrm{~Hz}, 2 \mathrm{H}), 7.28-7.22(\mathrm{~m}, 1 \mathrm{H}), 7.22-7.07(\mathrm{~m}, 3 \mathrm{H})$.

${ }^{13}$ C NMR (101 MHz, Chloroform- $d$ ) $\delta$ 164.2, 150.7, 144.6, 133.4, 132.7, 129.9, 129.7, 129.6, 128.6, 128.5, 127.9, 127.8, $127.1,126.7,126.5,126.5,126.3,126.2,126.0,121.3$.

${ }^{19}$ F NMR (376 MHz, Chloroform- $d$ ) $\delta-80.95$ (m, 3F), -106.82 (t, 2F), -123,58 (m, 2F), -125.66 (m, 2F).

IR $\left(\mathrm{CH}_{2} \mathrm{Cl}_{2}\right): 3062,1743,1224,1196,1134\left(\mathrm{~cm}^{-1}\right)$.

HRMS, m/z: calc'd for $\mathrm{C}_{23} \mathrm{H}_{13} \mathrm{~F}_{9} \mathrm{O}_{2}$ : 492.0772, found 492.0776.

(E)-4-fluorophenyl 4,4,5,5,6,6,7,7,7-nonafluoro-2-(naphthalen-2-yl)hept-2-enoate (3b)<smiles>O=C(Oc1ccc(F)cc1)/C(=C/C(F)(F)F)c1ccc2ccccc2c1</smiles>

Prepared in reaction of 2-Ethynylnaphthalene $(76.3 \mathrm{mg})$ with perfluorobutyl iodide and 4fluorophenyl formate under conditions B (yield: $32 \%, 82.7 \mathrm{mg}, 0.162 \mathrm{mmol}$ ). The title compound was isolated as yellowish oil after chromatography on silica gel [the crude mass $x$ 50] eluted with hexane $\rightarrow$ hexane: $\mathrm{CHCl}_{3}$ (95:5). The contaminated product was filtered through C18-reversed phase silica gel, eluted with acetonitrile.

${ }^{1} \mathrm{H}$ NMR (400 MHz, Chloroform- $d$ ) $\delta 7.93-7.86(\mathrm{~m}, 3 \mathrm{H}), 7.83(\mathrm{~s}, 1 \mathrm{H}), 7.60-7.51(\mathrm{~m}, 2 \mathrm{H}), 7.43$ (dt, $J=8.5,2.2 \mathrm{~Hz}, 1 \mathrm{H}), 7.19(\mathrm{td}, J=14.0,3.4 \mathrm{~Hz}, 1 \mathrm{H}), 7.14-7.01(\mathrm{~m}, 4 \mathrm{H})$. 
${ }^{13}$ C NMR (101 MHz, Chloroform- $d$ ) $\delta 164.17,161.89,159.45,146.46(\mathrm{~d}, J=3.0 \mathrm{~Hz}), 144.38,144.34,144.30,133.41$, $132.73,129.50,128.90,128.54(\mathrm{t}, J=2.6 \mathrm{~Hz}), 128.45,128.01,127.95,127.81,127.39,127.13,126.79,126.48(\mathrm{t}, J=21.8$ Hz), 126.13, 122.80, 122.72, 116.48, 116.24.

${ }^{19}$ F NMR (376 MHz, Chloroform- $d$ ) $\delta-80.97(\mathrm{~m}, 3 \mathrm{~F}),-106,85(\mathrm{~m}, 2 \mathrm{~F}),-115.95(\mathrm{~s}, 1 \mathrm{~F}),-123,58(\mathrm{~m}, 2 \mathrm{~F}),-125,66(\mathrm{~m}, 2 \mathrm{~F})$

Phenyl (2E)-4,4,5,5,6,6,7,7,7-nonafluoro-2-(naphthalen-1-yl)hept-2-enoate (4)<smiles>O=C(Oc1ccccc1)/C(=C\C(F)(F)F)c1cccc2ccccc12</smiles>

Prepared in reaction of 1-ethynylnaphthalene $(75.8 \mathrm{mg})$ with perfluorobutyl iodide and phenyl formate under standard reaction conditions (yield: $21 \%, 52.0 \mathrm{mg}, 0.105 \mathrm{mmol}$ ). The title compound was isolated as yellowish oil after chromatography on silica gel [the crude mass $\mathrm{x}$ 100] eluted with hexane $\rightarrow$ hexane: $\mathrm{CHCl}_{3}(85: 15)$. The contaminated product was filtered through $\mathrm{C} 18$-reversed phase silica gel, eluted with acetonitrile.

Prepared in reaction of 1-ethynylnaphthalene $(76.2 \mathrm{mg})$ with perfluorobutyl iodide and phenyl formate under conditions B (yield: $45 \%, 111.5 \mathrm{mg}, 0.226 \mathrm{mmol}$ ). The title compound was isolated as yellowish oil after chromatography on silica gel [the crude mass x 50] eluted with hexane $\rightarrow$ hexane: $\mathrm{CHCl}_{3}$ (8:2). The contaminated product was filtered through $\mathrm{C} 18$-reversed phase silica gel, eluted with acetonitrile.

${ }^{1} \mathrm{H}$ NMR (400 MHz, Chloroform- $d$ ) $\delta 7.96-7.86(\mathrm{~m}, 2 \mathrm{H}), 7.74(\mathrm{~d}, J=7.8 \mathrm{~Hz}, 1 \mathrm{H}), 7.61-7.49$ (m, 3H), 7.45 (d, $J=7.0 \mathrm{~Hz}$, $1 \mathrm{H}), 7.42-7.27(\mathrm{~m}, 3 \mathrm{H}), 7.20(\mathrm{t}, J=7.4 \mathrm{~Hz}, 1 \mathrm{H}), 6.97(\mathrm{~d}, J=7.8 \mathrm{~Hz}, 2 \mathrm{H})$.

${ }^{13} \mathrm{C}$ NMR (101 MHz, Chloroform- $d$ ) $\delta 164.3,150.6,143.8(\mathrm{t}, J=4.1 \mathrm{~Hz}), 133.4,131.6,129.9,129.7,129.6,128.8,128.0(\mathrm{t}, J$ $=21.8 \mathrm{~Hz}), 127.0(\mathrm{t}, J=2.8 \mathrm{~Hz}), 127.0,126.5,126.4,125.0,124.7,121.2$.

${ }^{19} \mathrm{~F}$ NMR $(376 \mathrm{MHz}$, Chloroform- $d$ ) $\delta-80.98(\mathrm{tt}, J=9.6,2.9 \mathrm{~Hz}, 3 \mathrm{~F}),-107.45(\mathrm{~m}, 1 \mathrm{~F}),-109.27(\mathrm{~m}, 1 \mathrm{~F}),-123.60(\mathrm{~m}, 2 \mathrm{~F}),-$ $125.70(\mathrm{~m}, 2 \mathrm{~F})$.

IR $\left(\mathrm{CH}_{2} \mathrm{Cl}_{2}\right): 3063,1743,1492,1231,1134\left(\mathrm{~cm}^{-1}\right)$.

HRMS, m/z: calc'd for $\mathrm{C}_{23} \mathrm{H}_{13} \mathrm{~F}_{9} \mathrm{O}_{2}: 492.0772$, found 492.0773 .

\section{Phenyl (2E)-2-(4-tert-butylphenyl)-4,4,5,5,6,6,7,7,7-nonafluorohept-2-enoate (5)}<smiles>CC(C)(C)c1ccc(C(=CC(F)(F)F)C(=O)Oc2ccccc2)cc1</smiles>

Prepared in reaction of 4-tert-butylphenylacetylene $(79.0 \mathrm{mg})$ with perfluorobutyl iodide and phenyl formate under standard reaction conditions (yield: $81 \%, 202.2 \mathrm{mg}, 0.405$ $\mathrm{mg}$ ). The title compound was isolated as yellow oil after chromatography on silica gel [the crude mass x 100] eluted with hexane $\rightarrow$ Hex:AcOEt (8:2).

${ }^{1} \mathrm{H}$ NMR (400 MHz, Chloroform- $d$ ) $\delta 7.46-7.32(\mathrm{~m}, 4 \mathrm{H}), 7.30-7.22(\mathrm{~m}, 3 \mathrm{H}), 7.16-7.10(\mathrm{~m}, 2 \mathrm{H})$, $7.06(\mathrm{t}, J=14.2 \mathrm{~Hz}, 1 \mathrm{H}), 1.35(\mathrm{~d}, J=0.7 \mathrm{~Hz}, 9 \mathrm{H})$.

${ }^{13} \mathrm{C}$ NMR (101 MHz, Chloroform- $d$ ) $\delta 164.4,152.3,150.8,144.6,129.7,129.1,128.7(\mathrm{t}, J=2.6 \mathrm{~Hz})$, $126.5,125.4(\mathrm{t}, J=21.6 \mathrm{~Hz}), 125.0,121.4,34.9,31.4$

${ }^{19}$ F NMR (376 MHz, Chloroform- $d$ ) $\delta-80.97(\mathrm{t}, J=10.0 \mathrm{~Hz}, 3 \mathrm{~F}),-106.81(\mathrm{~m}, 2 \mathrm{~F}),-123.71(\mathrm{~m}, 2 \mathrm{~F}),-125.68(\mathrm{~m}, 2 \mathrm{~F})$.

IR $\left(\mathrm{CH}_{2} \mathrm{Cl}_{2}\right): 3478,3071,2965,2909,2872,1747,1490,1228,1196,1134\left(\mathrm{~cm}^{-1}\right)$.

HRMS, m/z: calc'd for $\mathrm{C}_{23} \mathrm{H}_{19} \mathrm{~F}_{9} \mathrm{O}_{2}$ : 498.1241, found 498.1235.

Phenyl (2E)-2-(4-(benzyloxy)phenyl)-4,4,5,5,6,6,7,7,7-nonafluorohept-2-enoate (6)<smiles>O=C(Oc1ccccc1)/C(=C\C(F)(F)F)c1ccc(OCc2ccccc2)cc1</smiles>

Prepared in reaction of 4'-benzyloxyphenyl acetylene $(104.4 \mathrm{mg})$ with perfluorobutyl iodide and phenyl formate under standard reaction conditions (yield: $61 \%, 168.3 \mathrm{mg}$, $0.307 \mathrm{mmol}$ ). The title compound was isolated as yellow oil after chromatography on silica gel [the crude mass x 100] eluted with hexane $\rightarrow$ Hex:AcOEt (99.5:0.5).

${ }^{1} \mathrm{H}$ NMR (400 MHz, Chloroform- $d$ ) $\delta 7.49-7.33$ (m, 7H), $7.33-7.22(\mathrm{~m}, 3 \mathrm{H}), 7.17-7.09$ (m, $2 \mathrm{H}), 7.09-6.98(\mathrm{~m}, 3 \mathrm{H}), 5.10(\mathrm{~d}, J=4.0 \mathrm{~Hz}, 2 \mathrm{H})$.

${ }^{13} \mathrm{C}$ NMR (101 MHz, Chloroform- $d$ ) $\delta 164.4,159.7,150.7,144.2,136.8,130.5$ (t, $\left.J=2.7 \mathrm{~Hz}\right), 129.7,128.8,128.3,127.7$, $126.5,125.3(\mathrm{t}, J=21.7 \mathrm{~Hz}), 124.5,121.3,114.4,70.3$. 
${ }^{19} \mathrm{~F}$ NMR (376 MHz, Chloroform- $d$ ) $\delta-80.95$ (m, 3F), -106.66 (m, 2F), -123.64 (m, 2F), -125.66 (m, 2F).

IR $\left(\mathrm{CH}_{2} \mathrm{Cl}_{2}\right): 3474,3067,3037,2929,1744,1607,1512,1235,1194,1134\left(\mathrm{~cm}^{-1}\right)$.

HRMS, m/z: calc'd for $\mathrm{C}_{26} \mathrm{H}_{17} \mathrm{~F}_{9} \mathrm{O}_{3}: 548.1034$, found 548.1033 .

Phenyl (2E)-4,4,5,5,6,6,7,7,7-nonafluoro-2-(4-methoxyphenyl)hept-2-enoate (7)<smiles>COc1ccc(/C(=C\C(F)(F)F)C(=O)Oc2ccccc2)cc1</smiles>

Prepared in reaction of 1-ethynyl-4-methoxybenzene $(67.5 \mathrm{mg})$ with perfluorobutyl iodide and phenyl formate under standard reaction conditions (yield: $80 \%, 194.0 \mathrm{mg}, 0.411$ $\mathrm{mmol})$. The title compound was isolated as yellow oil after chromatography on silica gel [the crude mass x 100] eluted with hexane $\rightarrow$ Hex:AcOEt (99.5:0.5).

${ }^{1} \mathrm{H}$ NMR $(400 \mathrm{MHz}$, Chloroform- $d$ ) $\delta 7.44-7.35(\mathrm{~m}, 2 \mathrm{H}), 7.32-7.22(\mathrm{~m}, 3 \mathrm{H}), 7.16-7.10(\mathrm{~m}, 2 \mathrm{H})$, $7.05(\mathrm{t}, J=14.4 \mathrm{~Hz}, 1 \mathrm{H}), 6.97-6.91(\mathrm{~m}, 2 \mathrm{H}), 3.84(\mathrm{~s}, 3 \mathrm{H})$.

${ }^{13} \mathrm{C}$ NMR (101 MHz, Chloroform- $d$ ) $\delta 164.5,160.4,150.8,144.3,130.5(\mathrm{t}, J=2.4 \mathrm{~Hz}), 129.7,126.5,125.3(\mathrm{t}, J=21.5 \mathrm{~Hz})$, 124.3, 121.3, 113.6, 55.4.

${ }^{19} \mathrm{~F}$ NMR (376 MHz, Chloroform- $d$ ) $\delta-80.99(\mathrm{~m}, 3 \mathrm{~F}),-106.71(\mathrm{~m}, 2 \mathrm{~F}),-123.68(\mathrm{~m}, 2 \mathrm{~F}),-125.69$ (m, 2F).

IR $\left(\mathrm{CH}_{2} \mathrm{Cl}_{2}\right): 3472,3071,3007,2961,2937,2842,2548,2056,1889,1745,1609,1514,1223,1195,1134\left(\mathrm{~cm}^{-1}\right)$.

HRMS, m/z: calc'd for $\mathrm{C}_{20} \mathrm{H}_{13} \mathrm{~F}_{9} \mathrm{O}_{3}$ : 472.0721, found 472.0718 .

Phenyl(2E)-4,4,5,5,6,6,7,7,7-nonafluoro-2-(3,4,5-trimethoxyphenyl)hept-2-enoate (8)<smiles>COc1cc(/C(=C\C(F)(F)F)C(=O)Oc2ccccc2)cc(OC)c1OC</smiles>

Prepared in reaction of 3,4,5-trimethoxyphenylacetylene $(96.0 \mathrm{mg})$ with perfluorobutyl iodide and phenyl formate under standard reaction conditions (yield: $40 \%, 107.2 \mathrm{mg}$, $0.201 \mathrm{mmol}$ ). The title compound was isolated as yellowish solid after chromatography on silica gel [the crude mass x 50] eluted with hexane $\rightarrow$ hexane:AcOEt (95:5 - slow gradient). The contaminated product was filtered through C18-reversed phase silica gel, eluted with acetonitrile.

${ }^{1} \mathrm{H}$ NMR (400 MHz, Chloroform- $d$ ) $\delta 7.45-7.35(\mathrm{~m}, 2 \mathrm{H}), 7.31-7.24(\mathrm{~m}, 1 \mathrm{H}), 7.18-7.08$ (m, $2 \mathrm{H}), 7.05(\mathrm{t}, J=14.1 \mathrm{~Hz}, 1 \mathrm{H}), 6.55(\mathrm{~d}, J=1.2 \mathrm{~Hz}, 2 \mathrm{H}), 3.90(\mathrm{~s}, 3 \mathrm{H}), 3.86(\mathrm{~s}, 6 \mathrm{H})$

${ }^{13} \mathrm{C}$ NMR $(101 \mathrm{MHz}$, Chloroform- $d$ ) $\delta 164.1,152.9,150.6,144.3(\mathrm{t}, J=4.2 \mathrm{~Hz}), 139.0,129.7,127.2,126.6,125.6(\mathrm{t}, J=21.6$ $\mathrm{Hz}), 121.3,106.6,61.1,56.3$.

${ }^{19}$ F NMR (376 MHz, Chloroform- $d$ ) $\delta-80.97$ (m, 3F), -107.12 (t, $\left.J=12.2 \mathrm{~Hz}, 2 \mathrm{~F}\right),-123.55(\mathrm{~m}, 2 \mathrm{~F}),-125.67(\mathrm{~m}, 2 \mathrm{~F})$.

IR $\left(\mathrm{CH}_{2} \mathrm{Cl}_{2}\right): 3461,3076,2963,2944,2837,1741,1584,1415,1223,1216,1196,1131\left(\mathrm{~cm}^{-1}\right)$.

HRMS, m/z: calc'd for $\mathrm{C}_{22} \mathrm{H}_{17} \mathrm{~F}_{9} \mathrm{O}_{5}$ : 532.0932, found 532.0942 .

Phenyl (2E)-4,4,5,5,6,6,7,7,7-nonafluoro-2-(2-methoxyphenyl)hept-2-enoate (9)<smiles>COc1ccccc1/C(=C\C(F)(F)F)C(=O)Oc1ccccc1</smiles>

Prepared in reaction of 2-ethynylanisole $(65.0 \mathrm{mg})$ with perfluorobutyl iodide and phenyl formate under standard reaction conditions (yield: $38 \%, 88.6 \mathrm{mg}, 0.188 \mathrm{mmol}$ ). The title compound was isolated as yellowish oil after chromatography on silica gel [the crude mass x 50] eluted with hexane: $\mathrm{CHCl}_{3}$ (99:1) $\rightarrow$ hexane: $\mathrm{CHCl}_{3}$ (1:1). The contaminated product was filtered through $\mathrm{C} 18$-reversed phase silica gel, eluted with acetonitrile.

${ }^{1} \mathrm{H}$ NMR (400 MHz, Chloroform- $\left.d\right) \delta 7.43-7.33(\mathrm{~m}, 3 \mathrm{H}), 7.28-7.20(\mathrm{~m}, 2 \mathrm{H}), 7.11-7.03(\mathrm{~m}, 3 \mathrm{H})$, 7.01 (tdd, $J=7.5,1.9,1.0 \mathrm{~Hz}, 1 \mathrm{H}), 6.94(\mathrm{~d}, J=8.3 \mathrm{~Hz}, 1 \mathrm{H}), 3.86(\mathrm{~s}, 3 \mathrm{H})$.

${ }^{13} \mathrm{C}$ NMR $(101 \mathrm{MHz}$, Chloroform- $d$ ) $\delta 164.4,157.1,151.0,142.3,130.9,130.5(\mathrm{t}, J=3.1 \mathrm{~Hz}), 129.6,126.3,125.9(\mathrm{t}, J=22.0$ $\mathrm{Hz}), 121.9,121.4,120.3,110.6,55.8$.

${ }^{19}$ F NMR (376 MHz, Chloroform- $d$ ) $\delta-81.00$ (m, 3F), -107.96 (m, 2F), - 123.82 (m, 2F), -125.74 (m, 2F). 
IR $\left(\mathrm{CH}_{2} \mathrm{Cl}_{2}\right): 3070,2943,2842,1747,1493,1223,1195,1134\left(\mathrm{~cm}^{-1}\right)$.

HRMS, m/z: calc'd for $\mathrm{C}_{20} \mathrm{H}_{13} \mathrm{~F}_{9} \mathrm{O}_{3}$ 472.0721, found 472.0727 .

Phenyl (2E)-4,4,5,5,6,6,7,7,7-nonafluoro-2-(4-(4,4,5,5-tetramethyl-1,3,2-dioxaborolan-2-yl)phenyl)hept-2-enoate (10)<smiles>CC1(C)OB(c2ccc(/C(=C\C(F)(F)F)C(=O)Oc3ccccc3)cc2)OC1(C)C</smiles>

Prepared in reaction of 2-(4-ethynylphenyl)-4,4,5,5-tetramethyl-1,3,2-dioxaborolane $(114.5 \mathrm{mg})$ with perfluorobutyl iodide and phenyl formate under standard reaction conditions (yield: $51 \%, 146.7 \mathrm{mg}, 0.258 \mathrm{mmol}$ ). The title compound was isolated as white solid after chromatography on deactivated silica gel $\left(11 \% \mathrm{H}_{2} \mathrm{O}\right)$ [the crude mass $x$ 50] eluted with hexane $\rightarrow$ hexane: MTBE (97:3). The contaminated product was filtered through $\mathrm{C} 18$-reversed phase silica gel, eluted with acetonitrile.

${ }^{1} \mathrm{H}$ NMR (400 MHz, Chloroform- $d$ ) $\delta 7.91-7.83(\mathrm{~m}, 2 \mathrm{H}), 7.42-7.31(\mathrm{~m}, 4 \mathrm{H}), 7.28-7.21(\mathrm{~m}$, $1 \mathrm{H}), 7.15-7.05(\mathrm{~m}, 3 \mathrm{H}), 1.36(\mathrm{~s}, 12 \mathrm{H})$.

${ }^{13} \mathrm{C}$ NMR (101 MHz, Chloroform- $d$ ) $\delta 163.9,150.7,144.5(\mathrm{t}, J=4.6 \mathrm{~Hz}), 134.9,134.4,129.7,128.1(\mathrm{t}, J=2.6 \mathrm{~Hz}), 126.5$, $126.1(\mathrm{t}, J=21.9 \mathrm{~Hz}), 121.3,84.2,25.0$.

${ }^{19}$ F NMR (376 MHz, Chloroform- $d$ ) $\delta-81.00(\mathrm{~m}, 3 \mathrm{~F}),-107.05(\mathrm{t}, J=12.2 \mathrm{~Hz}, 2 \mathrm{~F}),-123.62(\mathrm{~m}, 2 \mathrm{~F}),-125.71(\mathrm{~m}, 2 \mathrm{~F})$.

IR $\left(\mathrm{CH}_{2} \mathrm{Cl}_{2}\right): 3073,2981,2934,1746,1361,1222,1194,1135\left(\mathrm{~cm}^{-1}\right)$.

HRMS, m/z: calc'd for $\mathrm{C}_{25} \mathrm{H}_{22} \mathrm{BF}_{9} \mathrm{O}_{4}: 568.1467$, found: 568.1491 .

\section{Phenyl (2E)-4,4,5,5,6,6,7,7,7-nonafluoro-2-(4-(trifluoromethyl)phenyl)hept-2-enoate (11)}<smiles>O=C(Oc1ccccc1)C(=CC(F)(F)F)c1ccc(C(F)(F)F)cc1</smiles>

Prepared in reaction of 4'-trifluoromethylphenyl $(86.2 \mathrm{mg})$ acetylene with perfluorobutyl iodide and phenyl formate under standard reaction conditions (yield: $22 \%, 55.9 \mathrm{mg}$, $0.110 \mathrm{mmol}$ ). The title compound was isolated as yellowish oil after chromatography on silica gel [the crude mass $x$ 50] eluted with hexane $\rightarrow$ hexane: $\mathrm{CHCl}_{3}$ (9:1). The contaminated product was filtered through $\mathrm{C} 18$-reversed phase silica gel, eluted with acetonitrile.

Prepared in reaction of 4'-trifluoromethylphenyl $(85.2 \mathrm{mg})$ acetylene with perfluorobutyl iodide and phenyl formate under conditions B (yield: $35 \%, 89.5 \mathrm{mg}, 0.175 \mathrm{mmol}$ ). The title compound was isolated as yellowish oil after chromatography on silica gel [the crude mass x 50] eluted with hexane $\rightarrow$ hexane: $\mathrm{CHCl}_{3}$ (9:1). The contaminated product was filtered through C18-reversed phase silica gel, eluted with acetonitrile.

${ }^{1} \mathrm{H}$ NMR (400 MHz, Chloroform- $d$ ) $\delta 7.69(\mathrm{~d}, J=8.1 \mathrm{~Hz}, 2 \mathrm{H}), 7.46(\mathrm{~d}, J=8.0 \mathrm{~Hz}, 2 \mathrm{H}), 7.43-7.36(\mathrm{~m}, 2 \mathrm{H}), 7.30-7.24(\mathrm{~m}$, $1 \mathrm{H}), 7.19(\mathrm{t}, J=14.2 \mathrm{~Hz}, 1 \mathrm{H}), 7.14-7.08(\mathrm{~m}, 2 \mathrm{H})$.

${ }^{13}$ C NMR (101 MHz, Chloroform- $d$ ) $\delta 163.4,150.5,143.2,135.9,131.4(\mathrm{q}, J=32.5 \mathrm{~Hz}), 129.9,129.8,129.3(\mathrm{t}, J=2.6 \mathrm{~Hz})$, $127.4,127.3(\mathrm{t}, J=22.1 \mathrm{~Hz}), 126.9,126.7,125.4,125.13(\mathrm{q}, J=3.8 \mathrm{~Hz}), 122.7,121.2,121.2$.

${ }^{19}$ F NMR (376 MHz, Chloroform- $d$ ) $\delta$-62.92 (m, 3F), -81.00 (m, 3F), -107.15 (m, 2F), -123.62 (m, 2F), -125.71 (m, 2F).

IR $\left(\mathrm{CH}_{2} \mathrm{Cl}_{2}\right): 3475,3074,1746,1493,1327,1225,1195,1134\left(\mathrm{~cm}^{-1}\right)$.

HRMS, m/z: calc'd for $\mathrm{C}_{20} \mathrm{H}_{10} \mathrm{~F}_{12} \mathrm{O}_{2}: 510.0489$, found 510.0508.

\section{Phenyl (2E)-4,4,5,5,6,6,7,7,7-nonafluoro-2-(4-fluorophenyl)hept-2-enoate (12)}<smiles>O=C(Oc1ccccc1)/C(=C/C(F)(F)F)c1ccc(F)cc1</smiles>

Prepared in reaction of 1-ethynyl-4-fluorobenzene $(60.0 \mathrm{mg})$ with perfluorobutyl iodide and phenyl formate under standard reaction conditions (yield: $55 \%, 126.8 \mathrm{mg}, 0.275 \mathrm{mmol}$ ). The title compound was isolated as yellowish oil after chromatography on silica gel [the crude mass x 50] eluted with hexane $\rightarrow$ hexane: $\mathrm{CHCl}_{3}(8: 2)$. The contaminated product was filtered through $\mathrm{C} 18$-reversed phase silica gel, eluted with acetonitrile.

\footnotetext{
${ }^{1} \mathrm{H}$ NMR (400 MHz, Chloroform- $d$ ) $\delta 7.43-7.36(\mathrm{~m}, 2 \mathrm{H}), 7.34-7.23(\mathrm{~m}, 3 \mathrm{H}), 7.15-7.05(\mathrm{~m}, 5 \mathrm{H})$.
} 
${ }^{13} \mathrm{C}$ NMR (101 MHz, Chloroform- $d$ ) $\delta 164.5,163.9,162.1,150.6,143.6(\mathrm{t}, J=4.1 \mathrm{~Hz}), 131.0,130.9(\mathrm{dt}, J=8.3,2.4 \mathrm{~Hz})$, $129.7,128.0(\mathrm{~d}, J=3.63 \mathrm{~Hz}), 126.6,126.5(\mathrm{t}, J=21.7 \mathrm{~Hz}), 121.2,115.3(\mathrm{~d}, J=22.0 \mathrm{~Hz})$.

${ }^{19}$ F NMR (376 MHz, Chloroform- $d$ ) $\delta-80.98$ (m, 3F), -106.97 (m, 2F), -112.00 (s, 1F), -123.64 (m, 2F), -125.72 (m, 2F).

IR $\left(\mathrm{CH}_{2} \mathrm{Cl}_{2}\right): 3475,3076,1745,1512,1229,1194,1134\left(\mathrm{~cm}^{-1}\right)$.

HRMS, m/z: calc'd for $\mathrm{C}_{10} \mathrm{H}_{10} \mathrm{~F}_{10} \mathrm{O}_{2}: 460.0521$, found 460.0524 .

Phenyl (2E)-2-(4-chlorophenyl)-4,4,5,5,6,6,7,7,7-nonafluorohept-2-enoate (13)<smiles>O=C(Oc1ccccc1)/C(=C/C(F)(F)F)c1ccc(Cl)cc1</smiles>

Prepared in reaction of (4-chlorophenyl)acetylene $(68.6 \mathrm{mg})$ with perfluorobutyl iodide and phenyl formate under standard reaction conditions (yield: $47 \%, 111.9 \mathrm{mg}, 0.234 \mathrm{mmol}$ ). The title compound was isolated as yellow oil after chromatography on silica gel [the crude mass x 50] eluted with hexane $\rightarrow$ hexane: $\mathrm{CHCl}_{3}$ (7:3). The contaminated product was filtered through $\mathrm{C} 18$-reversed phase silica gel, eluted with acetonitrile.

${ }^{13} \mathrm{C}$ NMR $(101 \mathrm{MHz}$, Chloroform- $d$ ) $\delta 163.7,150.6,143.4(\mathrm{t}, J=3.7 \mathrm{~Hz}), 135.5,130.5,130.3(\mathrm{t}, J=2.6 \mathrm{~Hz}), 129.7,128.4$, $126.7(\mathrm{t}, J=22.1 \mathrm{~Hz}), 126.7,121.2$.

${ }^{19}$ F NMR (376 MHz, Chloroform- $d$ ) $\delta-80.96$ (m, 3F), -106.99 (m, 2F), -123.61 (m, 2F), -125.70 (m, 2F).

IR $\left(\mathrm{CH}_{2} \mathrm{Cl}_{2}\right): 3476,3073,1745,1493,1223,1194,1135\left(\mathrm{~cm}^{-1}\right)$.

HRMS, m/z: calc'd for $\mathrm{C}_{19} \mathrm{H}_{10} \mathrm{~F}_{9} \mathrm{O}_{2} \mathrm{Cl}$ : 476.0226, found 476.0229.

\section{Phenyl (2E)-2-(4-bromophenyl)-4,4,5,5,6,6,7,7,7-nonafluorohept-2-enoate (14)}<smiles>O=C(Oc1ccccc1)/C(=C/C(F)(F)F)c1ccc(Br)cc1</smiles>

Prepared in reaction of 1-bromo-4-ethynylbenzene $(90.6 \mathrm{mg})$ with perfluorobutyl iodide and phenyl formate under standard reaction conditions (yield: $55 \%, 143.7 \mathrm{mg}, 0.276 \mathrm{mmol}$ ). The title compound was isolated as yellowish oil after chromatography on silica gel [the crude mass x 50] eluted with hexane $\rightarrow$ hexane: $\mathrm{CHCl}_{3}$ (9:1). The contaminated product was filtered through $\mathrm{C} 18$-reversed phase silica gel, eluted with acetonitrile.

$7.24-7.19(\mathrm{~m}, 2 \mathrm{H}), 7.18-7.09(\mathrm{~m}, 3 \mathrm{H})$.

${ }^{13} \mathrm{C}$ NMR $(101 \mathrm{MHz}$, Chloroform- $d$ ) $\delta 163.6,150.6,143.4(\mathrm{t}, J=3.9 \mathrm{~Hz}), 131.4,131.0,130.5(\mathrm{t}, J=2.6 \mathrm{~Hz}), 129.7,126.9$, 126.7, 126.4, 123.7, 121.2.

${ }^{19}$ F NMR (376 MHz, Chloroform- $d$ ) $\delta-80.96$ (m, 3F), -106.99 (m, 2F), -123.61 (m, 2F), -125.69 (m, 2F).

IR $\left(\mathrm{CH}_{2} \mathrm{Cl}_{2}\right): 3475,3071,1744,1491,1222,1193,1134\left(\mathrm{~cm}^{-1}\right)$.

HRMS, m/z: calc'd for $\mathrm{C}_{19} \mathrm{H}_{10} \mathrm{BrF}_{9} \mathrm{O}_{2}$ : 519.9720, found 519.9724.

\section{Phenyl (2E)-2-(2-bromophenyl)-4,4,5,5,6,6,7,7,7-nonafluorohept-2-enoate (15)}

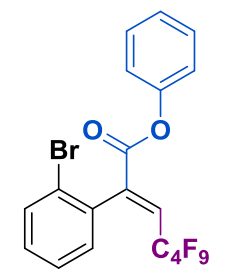

Prepared in reaction of 1-bromo-2-ethynylbenzene $(90.2 \mathrm{mg})$ with perfluorobutyl iodide and phenyl formate under standard reaction conditions (yield: $11 \%, 27.7 \mathrm{mg}, 0.053 \mathrm{mmol}$ ). The title compound was isolated as colorless oil after chromatography on silica gel [the crude mass $\mathrm{x}$ 50] eluted with hexane $\rightarrow$ hexane: $\mathrm{CHCl}_{3}$ (98:2). The contaminated product was filtered through C18-reversed phase silica gel, eluted with acetonitrile.

${ }^{1} \mathrm{H}$ NMR (400 MHz, Chloroform- $d$ ) $\delta 7.68-7.61(\mathrm{~m}, 1 \mathrm{H}), 7.41-7.15(\mathrm{~m}, 7 \mathrm{H}), 7.15-7.09(\mathrm{~m}, 2 \mathrm{H})$.

${ }^{13} \mathrm{C}$ NMR $(101 \mathrm{MHz}$, Chloroform- $d$ ) $\delta 163.0,150.7,143.6(\mathrm{t}, J=4.6 \mathrm{~Hz}), 133.9,132.5,130.7,130.7,129.7,127.5(\mathrm{t}, J=22.0$ $\mathrm{Hz}), 127.2,126.6,122.8,121.3$.

${ }^{19} \mathrm{~F}$ NMR (376 MHz, Chloroform- $d$ ) $\delta 80.95$ (m, 3F), -107.50 (m, 1F), -109.71 (m, 1F), -123.70 (m, 2F), -125.71 (m, 2F). 
IR $\left(\mathrm{CH}_{2} \mathrm{Cl}_{2}\right): 3068,1746,1492,1225,1194,1134\left(\mathrm{~cm}^{-1}\right)$.

HRMS, m/z: calc'd for $\mathrm{C}_{19} \mathrm{H}_{10} \mathrm{BrF}_{9} \mathrm{O}_{2}$ : 519.9720, found 519.9712.

\section{(E)-4-fluorophenyl 2-(2-bromophenyl)-4,4,5,5,6,6,7,7,7-nonafluorohept-2-enoate (15b)}

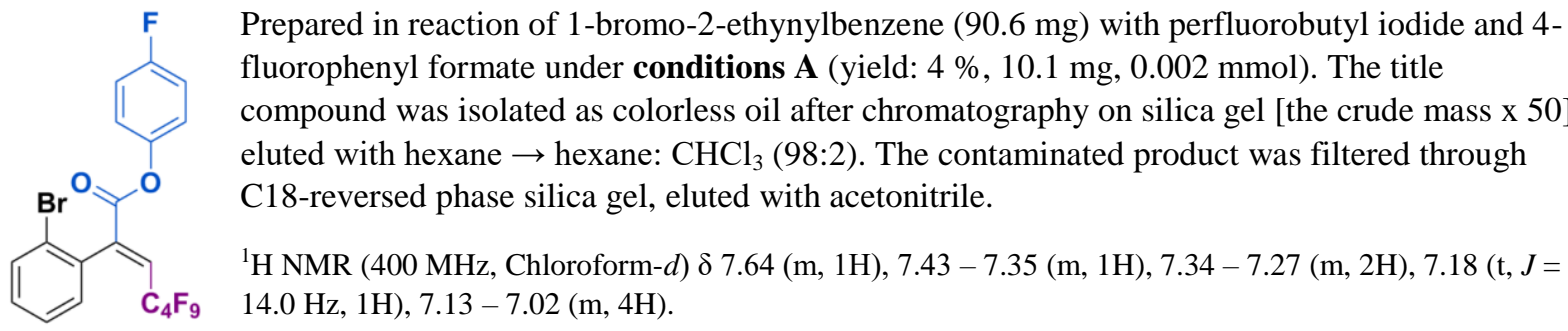

${ }^{13}$ C NMR (101 MHz, Chloroform- $d$ ) $\delta$ 146.50, 133.70, 132.53, 130.78, 127.61, 127.21, 122.85, 122.76, 116.48, 116.25.

${ }^{19}$ F NMR (376 MHz, Chloroform- $d$ ) $\delta-80.94$ (m, 3F), -108.34 (m, 2F), -115.97 (s, 1F), -123.69 (m, 2F), -125.70 (m, 2F).

\section{Phenyl (2E)-4,4,5,5,6,6,7,7,7-nonafluoro-2-(thiophen-3-yl)hept-2-enoate (16a)}<smiles>O=C(Oc1ccccc1)/C(=C\C(F)(F)F)c1ccsc1</smiles>

Prepared in reaction of 3-ethynylthiophene $(53.9 \mathrm{mg})$ with perfluorobutyl iodide and phenyl formate under standard reaction conditions (yield: $23 \%, 50.4 \mathrm{mg}, 0.112 \mathrm{mmol}$ ). The title compound was isolated as yellow oil after chromatography on silica gel [the crude mass $\mathrm{x}$ 50] eluted with hexane $\rightarrow$ hexane: $\mathrm{CHCl}_{3}$ (95:5). The contaminated product was filtered through C18-reversed phase silica gel, eluted with acetonitrile. phenyl formate under conditions B (yield: $39 \%, 87.5 \mathrm{mg}, 0.195 \mathrm{mmol}$ ). The title compound was isolated as yellow oil after chromatography on silica gel [the crude mass x 50] eluted with hexane $\rightarrow$ hexane: $\mathrm{CHCl}_{3}(95: 5)$. The contaminated product was filtered through C18-reversed phase silica gel, eluted with acetonitrile.

${ }^{1} \mathrm{H}$ NMR (400 MHz, Chloroform- $d$ ) $\delta 7.49-7.44(\mathrm{~m}, 1 \mathrm{H}), 7.44-7.37(\mathrm{~m}, 2 \mathrm{H}), 7.36(\mathrm{dd}, J=5.0,3.0 \mathrm{~Hz}, 1 \mathrm{H}), 7.30-7.24(\mathrm{~m}$, $1 \mathrm{H}), 7.21-7.11(\mathrm{~m}, 3 \mathrm{H}), 7.06(\mathrm{t}, J=14.2 \mathrm{~Hz}, 1 \mathrm{H})$.

${ }^{13} \mathrm{C}$ NMR (101 MHz, Chloroform- $d$ ) $\delta$ 163.9, 150.7, 139.7, 131.2, 129.7, 128.7 (t, $\left.J=2.1 \mathrm{~Hz}\right), 126.9(\mathrm{t}, J=3.5 \mathrm{~Hz}), 126.6$, $125.7(\mathrm{t}, J=21.7 \mathrm{~Hz}), 125.3,121.3$.

${ }^{19}$ F NMR (376 MHz, Chloroform- $d$ ) $\delta-80.97$ (m, 3F), -106.92 (m, 2F), -123.68 (m, 2F), -125.67 (m, 2F).

IR $\left(\mathrm{CH}_{2} \mathrm{Cl}_{2}\right): 3111,1746,1492,1233,1135\left(\mathrm{~cm}^{-1}\right)$.

HRMS, m/z: calc'd for $\mathrm{C}_{17} \mathrm{H}_{9} \mathrm{~F}_{9} \mathrm{O}_{2} \mathrm{~S}: 448.0180$, found: 448.0184 .

(E)-4-fluorophenyl 4,4,5,5,6,6,7,7,7-nonafluoro-2-(thiophen-3-yl)hept-2-enoate (16b)<smiles>O=C(Oc1ccc(F)cc1)/C(=C/C(F)(F)F)c1ccsc1</smiles>

Prepared in reaction of 3-Ethynylthiophene $(53.8 \mathrm{mg})$ with perfluorobutyl iodide and 4fluorophenyl formate under conditions B (yield: $40 \%, 93.7 \mathrm{mg}, 0.201 \mathrm{mmol}$,). The title compound was isolated as yellow oil after chromatography on silica gel [the crude mass x 50] eluted with hexane $\rightarrow$ hexane: $\mathrm{CHCl}_{3}$ (95:5). The contaminated product was filtered through C18-reversed phase silica gel, eluted with acetonitrile.

${ }^{1} \mathrm{H}$ NMR (400 MHz, Chloroform- $d$ ) $\delta 7.44(\mathrm{~m}, 1 \mathrm{H}), 7.36(\mathrm{~m}, 1 \mathrm{H}), 7.17-7.00(\mathrm{~m}, 6 \mathrm{H})$.

${ }^{13} \mathrm{C}$ NMR (101 MHz, Chloroform- $d$ ) $\delta 163.94,160.70(\mathrm{~d}, J=245.4 \mathrm{~Hz}), 146.43(\mathrm{~d}, J=3.0 \mathrm{~Hz}), 139.50,131.03,128.63(\mathrm{t}, J$ $=2.0 \mathrm{~Hz}), 126.94(\mathrm{t}, J=3.3 \mathrm{~Hz}), 125.98(\mathrm{t}, J=21.8 \mathrm{~Hz}), 125.39,122.80(\mathrm{~d}, J=8.6 \mathrm{~Hz}), 116.42(\mathrm{~d}, J=23.6 \mathrm{~Hz})$.

${ }^{19}$ F NMR (376 MHz, Chloroform- $d$ ) $\delta-80.90--81.09(\mathrm{~m}),-106.88--107.08(\mathrm{~m}),-115.91,-123.51--123.79(\mathrm{~m}),-125.57-$ $-125.81(\mathrm{~m})$. 


\section{Phenyl (2E)-2-ferrocenyl-4,4,5,5,6,6,7,7,7-nonafluorohept-2-enoate $(E$-17a)}

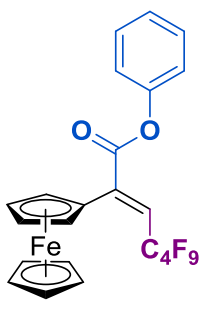

Prepared in reaction of ethynylferrocene $(105.3 \mathrm{mg})$ with perfluorobutyl iodide and phenyl formate under standard reaction conditions (yield: $28 \%, 76.2 \mathrm{mg}, 0.138 \mathrm{mmol}$ ). The title compound was isolated as dark red oil after chromatography on silica gel [the crude mass $\mathrm{x}$ 50] eluted with hexane $\rightarrow$ hexane: $\mathrm{CHCl}_{3}$ (92:8). The contaminated product was filtered through C18-reversed phase silica gel, eluted with acetonitrile.

${ }^{1} \mathrm{H}$ NMR (400 MHz, Chloroform- $d$ ) $\delta 7.53-7.44(\mathrm{~m}, 2 \mathrm{H}), 7.37-7.30(\mathrm{~m}, 1 \mathrm{H}), 7.30-7.23(\mathrm{~m}, 2 \mathrm{H}), 6.46$ $(\mathrm{t}, J=15.5 \mathrm{~Hz}, 1 \mathrm{H}), 4.86-4.78(\mathrm{~m}, 2 \mathrm{H}), 4.48(\mathrm{t}, J=2.0 \mathrm{~Hz}, 2 \mathrm{H}), 4.23(\mathrm{~s}, 5 \mathrm{H})$.

${ }^{13} \mathrm{C}$ NMR (101 MHz, Chloroform- $d$ ) $\delta 164.5,150.5,145.0(\mathrm{t}, J=3.6 \mathrm{~Hz}), 130.0,126.6,121.5,116.7(\mathrm{t}, J=21.7 \mathrm{~Hz}), 74.2$, $71.0,71.0,70.9,70.1$.

${ }^{19}$ F NMR (376 MHz, Chloroform- $d$ ) $\delta-80.96(\mathrm{~m}, 3 \mathrm{~F}),-106.16(\mathrm{~m}, 2 \mathrm{~F}),-123.86(\mathrm{~m}, 2 \mathrm{~F}),-125.58(\mathrm{~m}, 2 \mathrm{~F})$.

IR $\left(\mathrm{CH}_{2} \mathrm{Cl}_{2}\right): 3099,1754,1493,1235,1187,1134\left(\mathrm{~cm}^{-1}\right)$.

HRMS, m/z: calc'd for $\mathrm{C}_{23} \mathrm{H}_{15} \mathrm{~F}_{9} \mathrm{FeO}_{2}$ : 550.0278, found 550.0284 .

Phenyl (2Z)-2-ferrocenyl-4,4,5,5,6,6,7,7,7-nonafluorohept-2-enoate ( $Z$-17a)

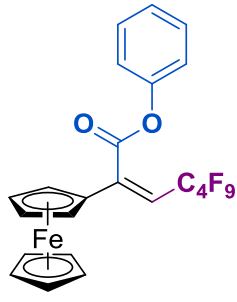

Prepared in reaction of ethynylferrocene $(105.3 \mathrm{mg}$ ) with perfluorobutyl iodide and phenyl formate under standard reaction conditions (yield: $8 \%, 21.7 \mathrm{mg}, 0.039 \mathrm{mmol}$ ). The title compound was isolated as dark red oil after chromatography on silica gel [the crude mass x 50] eluted with hexane $\rightarrow$ hexane: $\mathrm{CHCl}_{3}$ (9:1). The contaminated product was filtered through C18-reversed phase silica gel, eluted with acetonitrile.

${ }^{1} \mathrm{H}$ NMR (400 MHz, Chloroform- $d$ ) $\delta 7.54-7.39(\mathrm{~m}, 2 \mathrm{H}), 7.37-7.27(\mathrm{~m}, 1 \mathrm{H}), 7.31-7.19(\mathrm{~m}, 2 \mathrm{H})$, $5.81(\mathrm{t}, J=14.9 \mathrm{~Hz}, 1 \mathrm{H}), 4.56(\mathrm{t}, J=1.9 \mathrm{~Hz}, 2 \mathrm{H}), 4.49(\mathrm{t}, J=1.9 \mathrm{~Hz}, 2 \mathrm{H}), 4.28(\mathrm{~s}, 5 \mathrm{H})$.

${ }^{13} \mathrm{C}$ NMR (101 MHz, Chloroform- $d$ ) $\delta 164.4,150.4,145.8,129.9,126.6,121.4,107.6(\mathrm{t}, J=23.0 \mathrm{~Hz}), 71.3,70.5,67.2$.

${ }^{19}$ F NMR (376 MHz, Chloroform- $d$ ) $\delta-80.90$ (m, 3F), -107.72 (m, 2F), -123.68 (m, 2F), -125.65 (m, 2F).

IR $\left(\mathrm{CH}_{2} \mathrm{Cl}_{2}\right): 3097,1761,1491,1236,1188,1132\left(\mathrm{~cm}^{-1}\right)$.

HRMS, m/z: calc'd for $\mathrm{C}_{23} \mathrm{H}_{15} \mathrm{~F}_{9} \mathrm{FeO}_{2}$ : 550.0278, found 550.0282.

\section{Phenyl (2E)-2-(2,2,3,3,4,4,5,5,5-nonafluoropentylidene)octanoate (18)}

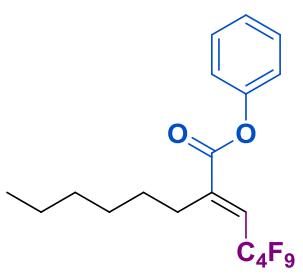

Prepared in reaction of 1-octyne (56.9 $\mathrm{mg})$ with perfluorobutyl iodide and phenyl formate under standard reaction conditions (yield: $61 \%, 140.9 \mathrm{mg}, 0.313$ mmol). The title compound was isolated as yellowish oil after chromatography on silica gel [the crude mass x 50] eluted with hexane $\rightarrow$ hexane: $\mathrm{CHCl}_{3}$ (95:5). The contaminated product was filtered through $\mathrm{C} 18$-reversed phase silica gel, eluted with acetonitrile.

${ }^{1} \mathrm{H}$ NMR $(400 \mathrm{MHz}$, Chloroform- $d) \delta 7.50-7.36(\mathrm{~m}, 2 \mathrm{H}), 7.36-7.23(\mathrm{~m}, 1 \mathrm{H}), 7.20-7.08(\mathrm{~m}, 2 \mathrm{H}), 6.80(\mathrm{t}, J=15.3 \mathrm{~Hz}$, $1 \mathrm{H}), 2.71-2.58(\mathrm{~m}, 2 \mathrm{H}), 1.63-1.51(\mathrm{~m}, 2 \mathrm{H}), 1.46-1.36(\mathrm{~m}, 2 \mathrm{H}), 1.36-1.26(\mathrm{~m}, 4 \mathrm{H}), 0.95-0.82(\mathrm{~m}, 3 \mathrm{H})$.

${ }^{13} \mathrm{C}$ NMR $(101 \mathrm{MHz}$, Chloroform- $d) \delta 164.7,150.7,146.1(\mathrm{t}, J=4.3 \mathrm{~Hz}), 129.8,126.5,124.5(\mathrm{t}, J=23.3 \mathrm{~Hz}), 121.5,31.5$, $29.6,29.5,28.5,22.6,14.1$.

${ }^{19}$ F NMR (376 MHz, Chloroform- $d$ ) $\delta-80.98$ (m, 3F), -108.14 (m, 2F), -124,05 (m, 2F), -125.68 (m, 2F).

IR $\left(\mathrm{CH}_{2} \mathrm{Cl}_{2}\right): 3077,2959,2933,2862,1747,1494,1235,1195,1136\left(\mathrm{~cm}^{-1}\right)$.

HRMS, m/z: calc'd for $\mathrm{C}_{19} \mathrm{H}_{19} \mathrm{~F}_{9} \mathrm{O}_{2}$ : 450.1241, found 450.1247. 


\section{Phenyl (2E)-2-cyclopropyl-4,4,5,5,6,6,7,7,7-nonafluorohept-2-enoate (19a)}<smiles>O=C(Oc1ccccc1)C(=CC(F)(F)C(F)(F)C(F)(F)C(F)(F)C(F)(F)C(F)(F)F)C1CC1</smiles>

Prepared in reaction of cyclopropylacetylene $(34.3 \mathrm{mg})$ with perfluorobutyl iodide and phenyl formate under standard reaction conditions (yield: $36 \%, 76.6 \mathrm{mg}, 0.189 \mathrm{mmol}$ ). The title compound was isolated as yellowish oil after chromatography on silica gel [the crude mass $\mathrm{x}$ 50] eluted with hexane $\rightarrow$ hexane: $\mathrm{CHCl}_{3}$ (95:5). The contaminated product was filtered through $\mathrm{C} 18$ reversed phase silica gel, eluted with acetonitrile.

${ }^{1} \mathrm{H}$ NMR $(400 \mathrm{MHz}$, Chloroform- $d) \delta 7.47-7.36(\mathrm{~m}, 2 \mathrm{H}), 7.32-7.23(\mathrm{~m}, 1 \mathrm{H}), 7.15-7.07(\mathrm{~m}, 2 \mathrm{H}), 6.57(\mathrm{t}$, $J=15.3 \mathrm{~Hz}, 1 \mathrm{H}), 2.13-2.01(\mathrm{~m}, 1 \mathrm{H}), 1.26-1.16(\mathrm{~m}, 2 \mathrm{H}), 1.04-0.94(\mathrm{~m}, 2 \mathrm{H})$.

${ }^{13} \mathrm{C}$ NMR (101 MHz, Chloroform- $d$ ) $\delta$ 163.6, 150.3, $147.3(\mathrm{t}, J=4.3 \mathrm{~Hz}), 129.8,126.6,123.1(\mathrm{t}, J=23.8 \mathrm{~Hz}), 121.5,11.2(\mathrm{t}$, $J=3.5 \mathrm{~Hz}), 8.5$.

${ }^{19} \mathrm{~F}$ NMR (376 MHz, Chloroform- $d$ ) $\delta-81.00(\mathrm{~m}, 3 \mathrm{~F}),-107.02(\mathrm{~m}, 2 \mathrm{~F}),-124.00$ (m, 2F), -125.70 (m, 2F).

IR $\left(\mathrm{CH}_{2} \mathrm{Cl}_{2}\right): 3083,3023,1749,1493,1235,1192,1135\left(\mathrm{~cm}^{-1}\right)$.

HRMS, m/z: calc'd for $\mathrm{C}_{16} \mathrm{H}_{11} \mathrm{~F}_{9} \mathrm{O}_{2}$ : 406.0615, found 406.0612.

Phenyl (2E)-2-(cyclohex-1-en-1-yl)-4,4,5,5,6,6,7,7,7-nonafluorohept-2-enoate (20a)<smiles>O=C(Oc1ccccc1)C(=CC(F)(F)F)C1=CCCCC1</smiles>

Prepared in reaction of 1-ethynyl-1-cyclohexene $(52.9 \mathrm{mg})$ with perfluorobutyl iodide and phenyl formate under standard reaction conditions (yield: $17 \%, 38.2 \mathrm{mg}, 0.086 \mathrm{mmol}$,). The title compound was isolated as yellowish oil after chromatography on silica gel [the crude mass $x$ 50] eluted with hexane $\rightarrow$ hexane: $\mathrm{CHCl}_{3}$ (95:5). The contaminated product was filtered through C18-reversed phase silica gel, eluted with acetonitrile.

Prepared in reaction of 1-ethynyl-1-cyclohexene $(53.1 \mathrm{mg})$ with perfluorobutyl iodide and phenyl formate under conditions C (yield: $22 \%, 49.0 \mathrm{mg}, 0.109 \mathrm{mmol}$,). The title compound was isolated as yellowish oil after chromatography on silica gel [the crude mass x 50] eluted with hexane $\rightarrow$ hexane: $\mathrm{CHCl}_{3}$ (95:5). The contaminated product was filtered through C18-reversed phase silica gel, eluted with acetonitrile.

${ }^{1} \mathrm{H}$ NMR (400 MHz, Chloroform- $d$ ) $\delta 7.45-7.36(\mathrm{~m}, 2 \mathrm{H}), 7.27(\mathrm{t}, J=7.5 \mathrm{~Hz}, 1 \mathrm{H}), 7.15(\mathrm{~d}, J=8.2 \mathrm{~Hz}, 2 \mathrm{H}), 6.77(\mathrm{t}, J=14.2$ $\mathrm{Hz}, 1 \mathrm{H}), 5.83-5.68(\mathrm{~m}, 1 \mathrm{H}), 2.25-2.11(\mathrm{~m}, 4 \mathrm{H}), 1.79-1.61(\mathrm{~m}, 4 \mathrm{H})$.

${ }^{13} \mathrm{C}$ NMR (101 MHz, Chloroform- $d$ ) $\delta 164.1,150.8,147.0,130.9,129.7,129.4(\mathrm{t}, J=3.1 \mathrm{~Hz}), 126.4,124.5(\mathrm{t}, J=21.6 \mathrm{~Hz})$, $121.4,28.7,25.3,22.5,21.6$.

${ }^{19}$ F NMR (376 MHz, Chloroform- $d$ ) $\delta-81.03(\mathrm{~m}, 3 \mathrm{~F}),-107.68(\mathrm{t}, J=12.4 \mathrm{~Hz}, 2 \mathrm{~F}),-123.78(\mathrm{~m}, 2 \mathrm{~F}),-125.72(\mathrm{~m}, 2 \mathrm{~F})$.

IR $\left(\mathrm{CH}_{2} \mathrm{Cl}_{2}\right): 3070,3044,2936,2863,1744,1493,1233,1216,1194,1135\left(\mathrm{~cm}^{-1}\right)$.

HRMS, m/z: calc'd for $\mathrm{C}_{19} \mathrm{H}_{15} \mathrm{~F}_{9} \mathrm{O}_{2}$ : 446.0928, found 446.0937.

Phenyl (2E)-2-(3-chloropropyl)-4,4,5,5,6,6,7,7,7-nonafluorohept-2-enoate (21)<smiles>O=C(Oc1ccccc1)/C(=C/C(F)(F)F)CCCCl</smiles>

Prepared in reaction of 5-chloro-1-pentyne $(52.2 \mathrm{mg})$ with perfluorobutyl iodide and phenyl formate under standard reaction conditions (yield: $26 \%, 57.6 \mathrm{mg}, 0.130 \mathrm{mmol}$ ). The title compound was isolated as yellowish oil after chromatography on silica gel [the crude mass x 50] eluted with hexane $\rightarrow$ hexane: $\mathrm{CHCl}_{3}$ (95:5). The contaminated product was filtered through C18-reversed phase silica gel, eluted with acetonitrile.

Prepared in reaction of 5-chloro-1-pentyne $(53.0 \mathrm{mg})$ with perfluorobutyl iodide and phenyl formate under standard reaction conditions $\mathbf{C}$ (yield: $49 \%, 112.7 \mathrm{mg}, 0.255 \mathrm{mmol}$ ). The title compound was isolated as yellowish oil after chromatography on silica gel [the crude mass x 50] eluted with hexane $\rightarrow$ hexane: $\mathrm{CHCl}_{3}$ (95:5). The contaminated product was filtered through $\mathrm{C} 18$-reversed phase silica gel, eluted with acetonitrile. 
${ }^{1} \mathrm{H}$ NMR (400 MHz, Chloroform- $d$ ) $\delta 7.48-7.38(\mathrm{~m}, 2 \mathrm{H}), 7.34-7.26(\mathrm{~m}, 1 \mathrm{H}), 7.20-7.11(\mathrm{~m}, 2 \mathrm{H}), 6.91(\mathrm{t}, J=15.1 \mathrm{~Hz}$, $1 \mathrm{H}), 3.61(\mathrm{t}, J=6.5 \mathrm{~Hz}, 2 \mathrm{H}), 2.82(\mathrm{tt}, J=7.9,2.3 \mathrm{~Hz}, 2 \mathrm{H}), 2.13-2.01(\mathrm{~m}, 2 \mathrm{H})$.

${ }^{13} \mathrm{C}$ NMR (101 MHz, Chloroform- $d$ ) $\delta 164.2,150.5,144.4(\mathrm{t}, J=4.2 \mathrm{~Hz}), 129.8,126.6,126.0(\mathrm{t}, J=23.5 \mathrm{~Hz}), 121.4,44.3$, $32.3,26.2$.

${ }^{19}$ F NMR (376 MHz, Chloroform- $d$ ) $\delta-80.95$ (m, 3F), -108.25 (m, 2F), -124.00 (m, 2F), -125.66 (m, 2F).

IR $\left(\mathrm{CH}_{2} \mathrm{Cl}_{2}\right): 3077,2963,1744,1493,1234,1196,1134\left(\mathrm{~cm}^{-1}\right)$.

HRMS, m/z: calc'd for $\mathrm{C}_{16} \mathrm{H}_{12} \mathrm{ClF}_{9} \mathrm{O}_{2}$ : 442.0382, found 442.0397 .

Phenyl (2E)-4,4,5,5,6,6,7,7,7-nonafluoro-2-(4-(formyloxy)butyl)hept-2-enoate (22)

Prepared in reaction of 5-hexyn-1-ol $(49.2 \mathrm{mg})$ with perfluorobutyl iodide and phenyl
formate under conditions $\mathbf{D}$ (yield: $53 \%, 124.0 \mathrm{mg}, 0.266 \mathrm{mmol})$. The title compound
was isolated as yellowish oil after chromatography on silica gel [the crude mass x 50]
eluted with hexane $\rightarrow$ hexane:AcOEt $(95: 5)$. The contaminated product was filtered
through C18-reversed phase silica gel, eluted with acetonitrile. $-7.10(\mathrm{~m}, 2 \mathrm{H}), 6.86(\mathrm{t}, J=15.2 \mathrm{~Hz}, 1 \mathrm{H}), 4.21(\mathrm{t}, J=6.1 \mathrm{~Hz}, 2 \mathrm{H}), 2.74-2.62(\mathrm{~m}, 2 \mathrm{H}), 1.84-1.73(\mathrm{~m}, 2 \mathrm{H}), 1.72-1.62(\mathrm{~m}$, $2 \mathrm{H})$.

${ }^{13} \mathrm{C}$ NMR (101 MHz, Chloroform- $d$ ) $\delta$ 164.3, 161.1, 150.6, $145.2(\mathrm{t}, J=4.3 \mathrm{~Hz}), 129.8,126.6,125.3(\mathrm{t}, J=23.4 \mathrm{~Hz}), 121.4$, 63.4, 28.6, $27.9(\mathrm{t}, J=2.5 \mathrm{~Hz}), 25.9$.

${ }^{19} \mathrm{~F}$ NMR (376 MHz, Chloroform- $d$ ) $\delta-80.00$ (m, 3F), -108.20 (m, 2F), -124.06 (m, 2F), -125.69 (m, 2F).

IR $\left(\mathrm{CH}_{2} \mathrm{Cl}_{2}\right): 3077,2957,1730,1494,1235,1195,1136\left(\mathrm{~cm}^{-1}\right)$.

HRMS, m/z: calc'd for $\mathrm{C}_{18} \mathrm{H}_{15} \mathrm{~F}_{9} \mathrm{O}_{4}: 466.0827$, found 466.0833 .

2-Methoxyphenyl (2E)-4,4,5,5,6,6,7,7,7-nonafluoro-2-(4-methoxyphenyl)hept-2-enoate (23)<smiles>COc1ccc(/C(=C\C(F)(F)F)C(=O)Oc2ccccc2OC)cc1</smiles>

Prepared in reaction of 4'-methoxyphenyl acetylene $(66.0 \mathrm{mg})$ with perfluorobutyl iodide and 2-methoxyphenyl formate under standard reaction conditions (yield: $80 \%, 200.9$ $\mathrm{mg}, 0.400 \mathrm{mmol}$ ). The title compound was isolated as colorless oil after chromatography on silica [the crude mass $x$ 100] eluted with hexane $\rightarrow$ hexane: $\mathrm{CHCl}_{3}$ (1:1) The contaminated product was filtered through $\mathrm{C} 18$-reversed phase silica gel, eluted with acetonitrile.

$7.01-6.89(\mathrm{~m}, 4 \mathrm{H}), 3.84(\mathrm{~s}, 6 \mathrm{H})$

H NMR (400 MHz, Chloroform- $d$ ) $\delta 7.37-7.29(\mathrm{~m}, 2 \mathrm{H}), 7.27-7.17(\mathrm{~m}, 1 \mathrm{H}), 7.10-6.99(\mathrm{~m}, 2 \mathrm{H})$,

${ }^{13}$ C NMR (101 MHz, Chloroform- $d$ ) $\delta 164.3,160.4,151.0,144.1(\mathrm{t}, J=3.9 \mathrm{~Hz}), 139.9,130.6(\mathrm{t}, J=2.7 \mathrm{~Hz}), 127.5,124.8(\mathrm{t}$, $J=21.3 \mathrm{~Hz}), 124.4,122.6,120.9,113.5,112.7,56.1,55.3$.

${ }^{19} \mathrm{~F}$ NMR (376 MHz, Chloroform- $d$ ) $\delta-81.00(\mathrm{~m}, 3 \mathrm{~F}),-106.55(\mathrm{~m}, 2 \mathrm{~F}),-123.76(\mathrm{~m}, 2 \mathrm{~F}),-125.69$ (m, 2F).

IR $\left(\mathrm{CH}_{2} \mathrm{Cl}_{2}\right): 3073,3008,2962,2945,2912,2842,1749,1609,1502,1234,1172,1134\left(\mathrm{~cm}^{-1}\right)$.

HRMS, m/z: calc'd for $\mathrm{C}_{21} \mathrm{H}_{15} \mathrm{~F}_{9} \mathrm{O}_{4}:$ 502.0827, found 502.0819.

3-Methoxyphenyl (2E)-4,4,5,5,6,6,7,7,7-nonafluoro-2-(4-methoxyphenyl)hept-2-enoate (24)<smiles>COc1ccc(C(=CC(F)(F)F)C(=O)Oc2cccc(OC)c2)cc1</smiles>

Prepared in reaction of 4'-methoxyphenyl acetylene $(66.3 \mathrm{mg})$ with perfluorobutyl iodide and 3-methoxyphenyl formate under standard reaction conditions (yield: $64 \%, 162.2 \mathrm{mg}$, $0.323 \mathrm{mmol})$. The title compound was isolated as yellow oil after chromatography on silica gel [the crude mass x 50] eluted with hexane $\rightarrow$ hexane: $\mathrm{CHCl}_{3}(1: 1)$. The contaminated product was filtered through $\mathrm{C} 18$-reversed phase silica gel, eluted with acetonitrile.

${ }^{1} \mathrm{H}$ NMR (400 MHz, Chloroform- $d$ ) $\delta 7.32-7.23(\mathrm{~m}, 3 \mathrm{H}), 7.04(\mathrm{t}, J=14.3 \mathrm{~Hz}, 1 \mathrm{H}), 6.97-6.91(\mathrm{~m}$, $2 \mathrm{H}), 6.81(\mathrm{ddd}, J=8.4,2.5,0.9 \mathrm{~Hz}, 1 \mathrm{H}), 6.71(\mathrm{ddd}, J=8.1,2.2,0.9 \mathrm{~Hz}, 1 \mathrm{H}), 6.67(\mathrm{t}, J=2.3 \mathrm{~Hz}, 1 \mathrm{H})$, 
$3.84(\mathrm{~s}, 3 \mathrm{H}), 3.80(\mathrm{~s}, 3 \mathrm{H})$.

${ }^{13} \mathrm{C}$ NMR (101 MHz, Chloroform- $d$ ) $\delta$ 164.4, 160.7, 160.4, 151.6, 144.2, 130.5 (t, $\left.J=2.7 \mathrm{~Hz}\right), 130.1,125.3(\mathrm{t}, J=21.5 \mathrm{~Hz})$, 124.2, 113.6, 113.5, 112.4, 107.4, 55.6, 55.4.

${ }^{19} \mathrm{~F}$ NMR $(376 \mathrm{MHz}$, Chloroform- $d$ ) $\delta-80.97$ (tt, $J=9.9,2.9 \mathrm{~Hz}, 3 \mathrm{~F}),-106.69(\mathrm{~m}, 2 \mathrm{~F}),-123.66(\mathrm{~m}, 2 \mathrm{~F}),-125.69(\mathrm{~m}, 2 \mathrm{~F})$.

IR $\left(\mathrm{CH}_{2} \mathrm{Cl}_{2}\right): 3007,2961,2841,1745,1610,1514,1491,1220,1171,1134\left(\mathrm{~cm}^{-1}\right)$.

HRMS, m/z: calc'd for $\mathrm{C}_{21} \mathrm{H}_{15} \mathrm{~F}_{9} \mathrm{O}_{4}$ : 502.0827, found 502.0825 .

\section{4-Methoxyphenyl (2E)-4,4,5,5,6,6,7,7,7-nonafluoro-2-(4-methoxyphenyl)hept-2-enoate (25)}<smiles>COc1ccc(OC(=O)C(=CC(F)(F)C(F)(F)F)c2ccc(OC)cc2)cc1</smiles>

Prepared in reaction of 4'-methoxyphenyl acetylene $(66.4 \mathrm{mg})$ with perfluorobutyl iodide and 4-methoxyphenyl formate under standard reaction conditions (yield: $35 \%, 89.5 \mathrm{mg}$, $0.178 \mathrm{mmol}$ ). The title compound was isolated as yellow oil after chromatography on silica gel [the crude mass x 50] eluted with hexane $\rightarrow$ hexane: AcOEt (95:5). The contaminated product was filtered through C18-reversed phase silica gel, eluted with acetonitrile.

${ }^{1} \mathrm{H}$ NMR (400 MHz, Chloroform- $d$ ) $\delta 7.30-7.23(\mathrm{~m}, 2 \mathrm{H}), 7.08-6.98(\mathrm{~m}, 3 \mathrm{H}), 6.95-6.91(\mathrm{~m}, 2 \mathrm{H})$, $6.91-6.86(\mathrm{~m}, 2 \mathrm{H}), 3.84(\mathrm{~s}, 3 \mathrm{H}), 3.79(\mathrm{~s}, 3 \mathrm{H})$.

${ }^{13} \mathrm{C}$ NMR (101 MHz, Chloroform- $d$ ) $\delta 164.8,160.3,157.8,144.3(\mathrm{t}, J=4.0 \mathrm{~Hz}), 144.2,130.4(\mathrm{t}, J=$ $2.6 \mathrm{~Hz}), 125.1(\mathrm{t}, J=21.3 \mathrm{~Hz}), 124.3,122.1,114.7,113.5,55.7,55.4$.

${ }^{19} \mathrm{~F}$ NMR (376 MHz, Chloroform- $d$ ) $\delta-81.00$ (m, 3F), -106.68 (m, 2F), -123.68 (m, 2F), -125.70 (m, 2F).

IR $\left(\mathrm{CH}_{2} \mathrm{Cl}_{2}\right): 3006,2960,2841,1742,1508,1224,1194,1134\left(\mathrm{~cm}^{-1}\right)$.

HRMS, m/z: calc'd for $\mathrm{C}_{21} \mathrm{H}_{15} \mathrm{~F}_{9} \mathrm{O}_{4}$ : 502.0827, found 502.0824.

\section{4-Methylphenyl (2E)-4,4,5,5,6,6,7,7,7-nonafluoro-2-(4-methoxyphenyl)hept-2-enoate (26)}<smiles>COc1ccc(/C(=C\C(F)(F)F)C(=O)Oc2ccc(C)cc2)cc1</smiles>

Prepared in reaction of 4'-methoxyphenyl acetylene $(66.6 \mathrm{mg})$ with perfluorobutyl iodide and $p$-tolyl formate under standard reaction conditions (yield: 69 \%, $169.6 \mathrm{mg}, 0.349$ mmol). The title compound was isolated as yellow oil after chromatography on silica [the crude mass x 50] eluted with hexane $\rightarrow$ hexane: $\mathrm{CHCl}_{3}(1: 1)$ The contaminated product was filtered through C18-reversed phase silica gel, eluted with acetonitrile

${ }^{1} \mathrm{H}$ NMR (400 MHz, Chloroform- $d$ ) $\delta 7.31-7.25(\mathrm{~m}, 2 \mathrm{H}), 7.21-7.15(\mathrm{~m}, 2 \mathrm{H}), 7.10-6.97(\mathrm{~m}, 3 \mathrm{H})$, $6.97-6.91(\mathrm{~m}, 2 \mathrm{H}), 3.84(\mathrm{~s}, 3 \mathrm{H}), 2.35(\mathrm{~s}, 3 \mathrm{H})$.

${ }^{13} \mathrm{C}$ NMR (101 MHz, Chloroform- $d$ ) $\delta 164.6,160.4,148.5,144.3(\mathrm{t}, J=4.2 \mathrm{~Hz}), 136.2,130.5(\mathrm{t}, J=2.3 \mathrm{~Hz}), 130.1,125.1(\mathrm{t}$, $J=21.5 \mathrm{~Hz}), 124.3,121.0,113.5,55.4,21.0$.

${ }^{19}$ F NMR (376 MHz, Chloroform- $d$ ) $\delta-80.99(\mathrm{~m}, 3 \mathrm{~F}),-106.66(\mathrm{~m}, 2 \mathrm{~F}),-123.68(\mathrm{~m}, 2 \mathrm{~F}),-125.70(\mathrm{~m}, 2 \mathrm{~F})$.

IR $\left(\mathrm{CH}_{2} \mathrm{Cl}_{2}\right): 3039,3006,2960,2937,2841,1744,1609,1509,1224,1196,1134\left(\mathrm{~cm}^{-1}\right)$.

ESIHR, m/z: calc'd for $\mathrm{NaC}_{21} \mathrm{H}_{15} \mathrm{~F}_{9} \mathrm{O}_{3}:$ 509.0775, found: 509.0772.

\section{4-Nitrophenyl (2E)-4,4,5,5,6,6,7,7,7-nonafluoro-2-(4-methoxyphenyl)hept-2-enoate (27)}

Prepared in reaction of 4'-methoxyphenyl acetylene $(66.1 \mathrm{mg})$ with perfluorobutyl iodide
and $p$-nitrophenyl formate under standard reaction conditions (yield: $38 \%, 97.7 \mathrm{mg}$,
$0.189 \mathrm{mmol})$. The title compound was isolated as yellow oil after chromatography on silica
gel [the crude mass x 50] eluted with hexane $\rightarrow$ hexane: $\mathrm{CHCl}_{3}(1: 1)$. The contaminated
product was filtered through C18-reversed phase silica gel, eluted with acetonitrile.

${ }^{13} \mathrm{C}$ NMR (101 MHz, Chloroform- $d$ ) $\delta 163.6,160.6,155.2,145.9,143.5,130.4(\mathrm{t}, J=2.6 \mathrm{~Hz}), 126.4(\mathrm{t}, J=21.8 \mathrm{~Hz}), 125.4$, $123.6,122.4,113.7,55.4$.

${ }^{19}$ F NMR (376 MHz, Chloroform- $d$ ) $\delta-80.96$ (m, 3F), -106.89 (t, $\left.J=12.0 \mathrm{~Hz}, 2 \mathrm{~F}\right),-123.61$ (m, 2F), -125.68 (m, 2F). 
IR $\left(\mathrm{CH}_{2} \mathrm{Cl}_{2}\right): 3116,3084,3007,2962,2940,2843,2184,1750,1609,1528,1515,1349,1201,1162,1135\left(\mathrm{~cm}^{-1}\right)$.

HRMS, m/z: calc'd for $\mathrm{C}_{20} \mathrm{H}_{12} \mathrm{NF}_{9} \mathrm{O}_{5}$ : 517.0572, found 517.0573.

\section{2,4,6-Trichlorophenyl (2E)-4,4,5,5,6,6,7,7,7-nonafluoro-2-(4-methoxyphenyl)hept-2-enoate (28)}<smiles>COc1ccc(/C(=C\C(F)(F)C(F)(F)F)C(=O)Oc2c(Cl)cc(Cl)cc2Cl)cc1</smiles>

Prepared in reaction of 4'-methoxyphenyl acetylene $(66.3 \mathrm{mg})$ with perfluorobutyl iodide and 2,4,6-trichlorophenyl formate under standard reaction conditions (yield: $20 \%, 56.5 \mathrm{mg}, 0.098 \mathrm{mmol}$ ). The title compound was isolated as yellowish oil after chromatography on silica gel [the crude mass $x$ 50] eluted with hexane $\rightarrow$ hexane: $\mathrm{CHCl}_{3}$ (8:2). The contaminated product was filtered through $\mathrm{C} 18$-reversed phase silica gel, eluted with acetonitrile.

Prepared in reaction of 4'-methoxyphenyl acetylene $(67.8 \mathrm{mg})$ with perfluorobutyl iodide and 2,4,6-trichlorophenyl formate under standard reaction conditions $\mathbf{C}$ (yield: $24 \%, 71.1 \mathrm{mg}, 0.124 \mathrm{mmol})$. The title compound was isolated as yellowish oil after chromatography on silica gel [the crude mass x 50] eluted with hexane $\rightarrow$ hexane: $\mathrm{CHCl}_{3}(8: 2)$. The contaminated product was filtered through C18-reversed phase silica gel, eluted with acetonitrile.

${ }^{1} \mathrm{H}$ NMR (400 MHz, Chloroform- $d$ ) $\delta 7.39$ (s, 2H), $7.35-7.27$ (m, 2H), $7.11(\mathrm{t}, J=14.0 \mathrm{~Hz}, 1 \mathrm{H}), 6.99-6.90$ (m, $\left.2 \mathrm{H}\right), 3.84$ $(\mathrm{s}, 3 \mathrm{H})$.

${ }^{13} \mathrm{C}$ NMR (101 MHz, Chloroform- $d$ ) $\delta 162.4,160.6,142.9,142.8,132.7,130.5(\mathrm{t}, J=2.6 \mathrm{~Hz}), 129.5,128.9,126.4(\mathrm{t}, J=21.7$ $\mathrm{Hz}), 123.6,113.7,55.4$.

${ }^{19} \mathrm{~F}$ NMR (376 MHz, Chloroform- $d$ ) $\delta-80.97$ (m, 3F), -106.87 (m, 2F), -123.76 (m, 2F), -125.69 (m, 2F).

IR $\left(\mathrm{CH}_{2} \mathrm{Cl}_{2}\right): 3500,3083,3007,2961,2939,2841,1762,1515,1448,1237,1135\left(\mathrm{~cm}^{-1}\right)$.

HRMS, m/z: calc'd for $\mathrm{C}_{20} \mathrm{H}_{10} \mathrm{Cl}_{3} \mathrm{~F}_{9} \mathrm{O}_{3}: 573.9552$, found 573.9555 .

4-Bromophenyl (2E)-4,4,5,5,6,6,7,7,7-nonafluoro-2-(4-methoxyphenyl)hept-2-enoate (29)<smiles>COc1ccc(C(=CC(F)(F)F)C(=O)Oc2ccc(Br)cc2)cc1</smiles>

Prepared in reaction of 4'-methoxyphenyl acetylene $(66.4 \mathrm{mg})$ with perfluorobutyl iodide and 4-bromophenyl formate under standard reaction conditions (yield: $37 \%, 103.5 \mathrm{mg}$, $0.188 \mathrm{mmol}$ ). The title compound was isolated as colorless oil after chromatography on silica [the crude mass x 50] eluted with hexane $\rightarrow$ hexane: $\mathrm{CHCl}_{3}$ (95:5) The contaminated product was filtered through $\mathrm{C} 18$-reversed phase silica gel, eluted with acetonitrile.

${ }^{1} \mathrm{H}$ NMR (400 MHz, Chloroform- $d$ ) $\delta 7.53-7.48(\mathrm{~m}, 2 \mathrm{H}), 7.29-7.23(\mathrm{~m}, 2 \mathrm{H}), 7.09-6.98(\mathrm{~m}, 3 \mathrm{H})$, $6.98-6.91(\mathrm{~m}, 2 \mathrm{H}), 3.84(\mathrm{~s}, 3 \mathrm{H})$.

${ }^{13} \mathrm{C}$ NMR (101 MHz, Chloroform- $d$ ) $\delta 164.2,160.5,149.7,143.9(\mathrm{t}, J=3.8 \mathrm{~Hz}), 132.7,130.4(\mathrm{t}, J=$ $2.7 \mathrm{~Hz}), 125.6(\mathrm{t}, J=21.7 \mathrm{~Hz}), 124.0,123.1,119.6,113.6,55.4$.

${ }^{19}$ F NMR (376 MHz, Chloroform- $d$ ) $\delta-80.99$ (m, 3F), -106.77 (m, 2F), -123.66 (m, 2F), -125.70 (m, 2F).

IR $\left(\mathrm{CH}_{2} \mathrm{Cl}_{2}\right): 3072,3007,2962,2841,2201,2057,1888,1746,1609,1513,1484,1199,1134\left(\mathrm{~cm}^{-1}\right)$.

HRMS, m/z: calc'd for $\mathrm{C}_{20} \mathrm{H}_{12} \mathrm{~F}_{9} \mathrm{O}_{3} \mathrm{Br}$ : 549.9826, found: 549.9822 .

4-Fluorophenyl (2E)-4,4,5,5,6,6,7,7,7-nonafluoro-2-(4-methoxyphenyl)hept-2-enoate (30)<smiles>COc1ccc(C(=CC(F)(F)F)C(=O)Oc2ccc(F)cc2)cc1</smiles>

55.4 .

Prepared in reaction of 4'-methoxyphenyl acetylene $(67.6 \mathrm{mg})$ with perfluorobutyl iodide and 4-fluorophenyl formate under standard reaction conditions (yield: $86 \%, 216.0 \mathrm{mg}$, $0.441 \mathrm{mmol})$. The title compound was isolated as yellow oil after chromatography on silica gel [the crude mass x 100] eluted with hexane $\rightarrow$ Hex:AcOEt (99.5:0.5).

${ }^{1} \mathrm{H}$ NMR (400 MHz, Chloroform- $d$ ) $\delta 7.33-7.17$ (m, 2H), $7.17-6.98$ (m, 5H), $6.98-6.85$ (m, 2H), $3.84(\mathrm{~s}, 3 \mathrm{H})$.

${ }^{13} \mathrm{C}$ NMR (101 MHz, Chloroform- $d$ ) $\delta 164.5,161.9,160.4,159.4,146.5,146.5,144.0$ (t, $\left.J=4.0 \mathrm{~Hz}\right)$, $130.4(\mathrm{t}, J=2.6 \mathrm{~Hz}), 125.50(\mathrm{t}, J=21.6 \mathrm{~Hz}), 124.1,122.9,122.8,116.5,116.2,114.9,114.3,113.6$,

\footnotetext{
${ }^{19} \mathrm{~F}$ NMR (376 MHz, Chloroform- $d$ ) $\delta-80.99$ (m, 3F), -106.76 (m, 2F), -116.08 (1F), -123.68 (m, 2F), -125.69 (m, 2F).

IR $\left(\mathrm{CH}_{2} \mathrm{Cl}_{2}\right): 3080,3007,2938,2843,2549,2054,1879,1746,1609,1504,1235,1184,1134\left(\mathrm{~cm}^{-1}\right)$.
} 
HRMS, m/z: calc'd for $\mathrm{C}_{20} \mathrm{H}_{12} \mathrm{~F}_{10} \mathrm{O}_{3}: 490.0627$, found 490.0635 .

\section{Phenyl (2E)-4,4,5,5,6,6,7,7,8,8,9,9,9-tridecafluoro-2-(4-methoxyphenyl)non-2-enoate (31)}<smiles>COc1ccc(/C(=C\C(=O)OCc2ccccc2)C(=O)Oc2ccccc2)cc1</smiles>

Prepared in reaction of 4'-methoxyphenyl acetylene $(66.9 \mathrm{mg})$ with perfluoro- $n$-hexyl iodide and phenyl formate under standard reaction conditions (yield: $70 \%, 203.3 \mathrm{mg}$, $0.355 \mathrm{mmol})$. The title compound was isolated as yellow oil after chromatography on silica gel [the crude mass $x$ 50] eluted with hexane $\rightarrow$ hexane: $\mathrm{CHCl}_{3}$ (7:3). The contaminated product was filtered through C18-reversed phase silica gel, eluted with acetonitrile.

${ }^{1} \mathrm{H}$ NMR (400 MHz, Chloroform- $d$ ) $\delta 7.44-7.34$ (m, 2H), $7.32-7.20(\mathrm{~m}, 3 \mathrm{H}), 7.18-7.08$ (m, 2H), $7.05(\mathrm{t}, J=14.2 \mathrm{~Hz}$, $1 \mathrm{H}), 6.98-6.88(\mathrm{~m}, 2 \mathrm{H}), 3.84(\mathrm{~s}, 3 \mathrm{H})$.

${ }^{13} \mathrm{C}$ NMR (101 MHz, Chloroform- $d$ ) $\delta 164.5,160.4,150.8,144.2(\mathrm{t}, J=4.0 \mathrm{~Hz}), 130.5(\mathrm{t}, J=2.6 \mathrm{~Hz}), 129.7,126.5,125.4(\mathrm{t}$, $J=21.6 \mathrm{~Hz}), 124.3,121.3,113.6,55.4$.

${ }^{19}$ F NMR (376 MHz, Chloroform- $d$ ) $\delta$-80.82 (m, 3F), -106.47 (m, 2F), -121.55 (m,2F), -122.75 (m, 4F), -126.09 (m, 2F).

IR $\left(\mathrm{CH}_{2} \mathrm{Cl}_{2}\right): 3073,2962,2842,1745,1514,1241,1197,1145\left(\mathrm{~cm}^{-1}\right)$.

HRMS, m/z: calc'd for $\mathrm{C}_{22} \mathrm{H}_{13} \mathrm{~F}_{13} \mathrm{O}_{3}: 572.0657$, found 572.0649 .

Phenyl (2E)-4,4,5,5,6,6,7,7,8,8,9,9,10,10,11,11,12,12,13,13,13-henicosafluoro-2-(4-methoxyphenyl)tridec-2-enoate (32)<smiles>COc1ccc(/C(=C\C(F)F)C(=O)Oc2ccccc2)cc1</smiles>

Prepared in reaction of 4'-methoxyphenyl acetylene $(66.4 \mathrm{mg})$ with perfluorodecyl iodide and phenyl formate under standard reaction conditions (yield: $68 \%, 247.5 \mathrm{mg}, 0.343$ $\mathrm{mmol}$ ). The title compound was isolated as white solid after chromatography on silica gel [the crude mass x 30] eluted with hexane $\rightarrow$ hexane: $\mathrm{CHCl}_{3}$ (7:3). The contaminated product was filtered through $\mathrm{C} 18$-reversed phase silica gel, eluted with acetonitrile.

${ }^{1} \mathrm{H}$ NMR (400 MHz, Chloroform- $d$ ) $\delta 7.43-7.35(\mathrm{~m}, 2 \mathrm{H}), 7.32-7.23(\mathrm{~m}, 3 \mathrm{H}), 7.15-7.10(\mathrm{~m}$, $2 \mathrm{H}), 7.05(\mathrm{t}, J=14.2 \mathrm{~Hz}, 1 \mathrm{H}), 6.98-6.90(\mathrm{~m}, 2 \mathrm{H}), 3.84(\mathrm{~s}, 3 \mathrm{H})$.

${ }^{13} \mathrm{C}$ NMR (101 MHz, Chloroform- $d$ ) $\delta 164.5,160.4,150.8,144.2,130.5(\mathrm{t}, J=2.6 \mathrm{~Hz}), 129.7,126.5,125.4(\mathrm{t}, J=21.3 \mathrm{~Hz})$, 124.3, 121.3, 113.6, 55.4.

${ }^{19} \mathrm{~F}$ NMR $(376 \mathrm{MHz}$, Chloroform- $d$ ) $\delta-80.82(\mathrm{t}, J=10.0 \mathrm{~Hz}, 3 \mathrm{~F}),-106.48(\mathrm{t}, J=12.9 \mathrm{~Hz}, 2 \mathrm{~F}),-121.35(\mathrm{~m}, 2 \mathrm{~F}),-121.78(\mathrm{~m}$, $8 \mathrm{~F}),-122.70(4 \mathrm{~F}),-126.10(\mathrm{~m}, 2 \mathrm{~F})$.

IR $\left(\mathrm{CH}_{2} \mathrm{Cl}_{2}\right): 3066,2964,2643,1739,1514,1204,1149\left(\mathrm{~cm}^{-1}\right)$.

HRMS, m/z: calc'd for $\mathrm{C}_{26} \mathrm{H}_{13} \mathrm{~F}_{21} \mathrm{O}_{3}:$ 772.0529, found 772.0521 .

Phenyl (2E)-4,4,5,5,6,6,7,7,8,9,9,9-dodecafluoro-2-(4-methoxyphenyl)-8-(trifluoromethyl)non-2-enoate (33)

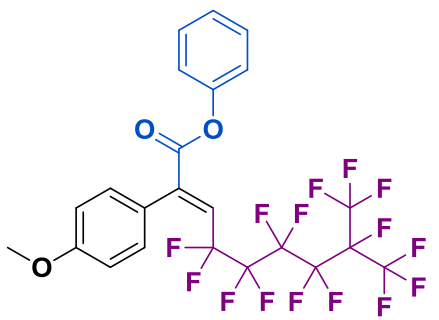

Prepared in reaction of 4'-methoxyphenyl acetylene $(66.2 \mathrm{mg})$ with perfluoroisoheptyl iodide and phenyl formate under standard reaction conditions (yield: $62 \%, 192.0 \mathrm{mg}, 0.309 \mathrm{mmol}$ ). The title compound was isolated as yellow oil after chromatography on silica gel [the crude mass $\mathrm{x}$ 50] eluted with hexane $\rightarrow$ hexane: $\mathrm{CHCl}_{3}$ (7:3). The contaminated product was filtered through $\mathrm{C} 18$-reversed phase silica gel, eluted with acetonitrile.

${ }^{1} \mathrm{H}$ NMR (400 MHz, Chloroform- $d$ ) $\delta 7.42-7.35$ (m, 2H), 7.30 - $7.22(\mathrm{~m}, 3 \mathrm{H}), 7.14-$ $7.09(\mathrm{~m}, 2 \mathrm{H}), 7.04(\mathrm{t}, J=14.2 \mathrm{~Hz}, 1 \mathrm{H}), 6.96-6.90(\mathrm{~m}, 2 \mathrm{H}), 3.84(\mathrm{~s}, 3 \mathrm{H})$.

${ }^{13} \mathrm{C}$ NMR (101 MHz, Chloroform- $d$ ) $\delta 164.5,160.4,150.8,144.2(\mathrm{t}, J=4.0 \mathrm{~Hz}), 130.5(\mathrm{t}, J=2.7 \mathrm{~Hz}), 129.7,126.5,125.5(\mathrm{t}$, $J=21.6 \mathrm{~Hz}), 124.3,121.3,113.6,55.4$.

${ }^{19}$ F NMR (376 MHz, Chloroform- $d$ ) $\delta$-71.82 (m, 6F), -106.42 (m, 2F), -115.00 (m, 2F), -120.36 (m, 2F), -122.21 (m, 2F), $185.99(\mathrm{~m}, 1 \mathrm{~F})$.

IR $\left(\mathrm{CH}_{2} \mathrm{Cl}_{2}\right): 3072,2962,2842,1745,1609,1514,1492,1253,1193,1123\left(\mathrm{~cm}^{-1}\right)$. 
HRMS, m/z: calc'd for $\mathrm{C}_{23} \mathrm{H}_{13} \mathrm{~F}_{15} \mathrm{O}_{3}: 622.0625$, found 622.0621 .

Phenyl (2E)-4,4,5,5,6,6,7,7-octafluoro-7-iodo-2-(4-methoxyphenyl)hept-2-enoate (34)

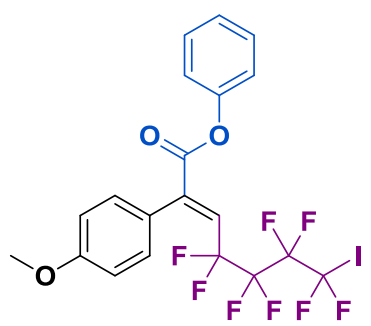

Prepared in reaction of 4'-methoxyphenyl acetylene $(66.0 \mathrm{mg})$ with 1,4-diiodoperfluorobutane and phenyl formate under standard reaction conditions (yield: $43 \%, 125.1 \mathrm{mg}, 0.216 \mathrm{mmol}$ ). The title compound was isolated as yellow oil after chromatography on silica gel [the crude mass x 30] eluted with hexane $\rightarrow$ hexane: $\mathrm{CHCl}_{3}$ (7:3). The contaminated product was filtered through $\mathrm{C} 18$-reversed phase silica gel, eluted with acetonitrile.

$(\mathrm{m}, 2 \mathrm{H}), 7.05(\mathrm{t}, J=14.2 \mathrm{~Hz}, 1 \mathrm{H}), 6.97-6.90(\mathrm{~m}, 2 \mathrm{H}), 3.84(\mathrm{~s}, 3 \mathrm{H})$

${ }^{13} \mathrm{C}$ NMR (101 MHz, Chloroform- $d$ ) $\delta 164.5,160.3,150.8,143.9,130.5(\mathrm{t}, J=2.6 \mathrm{~Hz}), 129.7,126.5,125.7(\mathrm{t}, J=21.5 \mathrm{~Hz})$, 124.3, 121.3, 113.5, 55.4.

${ }^{19} \mathrm{~F}$ NMR (376 MHz, Chloroform- $d$ ) $\delta-58.51(\mathrm{~m}, 2 \mathrm{~F}),-106.53(\mathrm{~m}, 2 \mathrm{~F}),-112.50(\mathrm{~m}, 2 \mathrm{~F}),-121.85(\mathrm{~m}, 2 \mathrm{~F})$.

IR $\left(\mathrm{CH}_{2} \mathrm{Cl}_{2}\right): 3071,2961,2840,1743,1608,1513,1492,1252,1188,1124\left(\mathrm{~cm}^{-1}\right)$.

HRMS, m/z: calc'd for $\mathrm{C}_{20} \mathrm{H}_{13} \mathrm{~F}_{8} \mathrm{IO}_{3}$ : 579.9782, found 579.9796 .

Phenyl (2E)-4,4,5,5,6,6,7,7,7-nonafluoro-2-phenylhept-2-enoate-d (35)<smiles>[2H]/C(C(=O)Oc1ccccc1)=C(\c1ccccc1)C(F)(F)F</smiles>

Prepared in reaction of phenylacetylene-d $(51.5 \mathrm{mg})$ with perfluorobutyl iodide and phenyl formate under standard reaction conditions (yield: $74 \%, 163.2 \mathrm{mg}, 0.368 \mathrm{mmol}$ ). The title compound was isolated as yellow oil after chromatography on silica gel [the crude mass $\mathrm{x}$ 50] eluted with hexane $\rightarrow \mathrm{Hex}: \mathrm{CHCl}_{3}$ (9:1). The contaminated product was filtered through $\mathrm{C} 18$ reversed phase silica gel, eluted with acetonitrile.

${ }^{1} \mathrm{H}$ NMR (400 MHz, Chloroform- $d$ ) $\delta 7.47-7.30(\mathrm{~m}, 7 \mathrm{H}), 7.31-7.21(\mathrm{~m}, 1 \mathrm{H}), 7.14-7.10(\mathrm{~m}, 2 \mathrm{H})$.

${ }^{13}$ C NMR (101 MHz, Chloroform- $d$ ) $\delta$ 164.1, 150.7, 144.5, 132.2, 129.7, 129.1, 128.8, 128.0, 126.5, 126.0, 121.3.

${ }^{19} \mathrm{~F}$ NMR (376 MHz, Chloroform- $d$ ) $\delta-81.00(\mathrm{~m}, 3 \mathrm{~F}),-107.05(\mathrm{~m}, 2 \mathrm{~F}),-123.66(\mathrm{~m}, 2 \mathrm{~F}),-125.73(\mathrm{~m}, 2 \mathrm{~F})$.

IR $\left(\mathrm{CH}_{2} \mathrm{Cl}_{2}\right): 3473,3064,1746,1493,1224,1195,1135\left(\mathrm{~cm}^{-1}\right)$.

HRMS, m/z: calc'd for $\mathrm{C}_{19} \mathrm{H}_{10} \mathrm{DF}_{9} \mathrm{O}_{2}: 443.0678$, found 443.0690 .

3-Methoxyphenyl (2E)-4,4,5,5,6,6,7,7,7-nonafluoro-2-(4-methoxyphenyl)hept-2-enoate $-{ }^{13} \mathrm{C}(36)$<smiles>COc1ccc(C(=CC(F)(F)F)C(=O)Oc2cccc(OC)c2)cc1</smiles>

Prepared in reaction of 4'-methoxyphenyl acetylene $(65.9 \mathrm{mg})$ with perfluorobutyl iodide and 3-methoxyphenyl formate- ${ }^{13} \mathrm{C}$ under standard reaction conditions (yield: $64 \%, 159.7$ $\mathrm{mg}, 0.317 \mathrm{mmol})$. The title compound was isolated as yellow oil after chromatography on silica gel [the crude mass $\mathrm{x}$ 50] eluted with hexane $\rightarrow$ hexane: $\mathrm{CHCl}_{3}$ (1:1). The contaminated product was filtered through C18-reversed phase silica gel, eluted with acetonitrile. $6.90(\mathrm{~m}, 2 \mathrm{H}), 6.80(\mathrm{dd}, J=8.1,2.2 \mathrm{~Hz}, 1 \mathrm{H}), 6.71(\mathrm{dd}, J=8.0,2.1 \mathrm{~Hz}, 1 \mathrm{H}), 6.67(\mathrm{t}, J=2.3 \mathrm{~Hz}, 1 \mathrm{H}), 3.84(\mathrm{~s}, 3 \mathrm{H}), 3.79(\mathrm{~s}, 3 \mathrm{H})$.

${ }^{13} \mathrm{C}$ NMR (101 MHz, Chloroform- $d$ ) $\delta 164.4(\mathrm{t}, J=1.7 \mathrm{~Hz}), 160.7,160.4,151.6(\mathrm{~d}, J=3.34 \mathrm{~Hz}), 130.5(\mathrm{~m}), 130.1,125.3(\mathrm{t}$, $J=20.2 \mathrm{~Hz}), 124.2(\mathrm{~d}, J=1.8 \mathrm{~Hz}), 113.6,113.5(\mathrm{~d}, J=1.4 \mathrm{~Hz}), 112.4,107.4$ (d, $J=1.5 \mathrm{~Hz}), 55.6,55.4$.

${ }^{19}$ F NMR (376 MHz, Chloroform- $d$ ) ) $\delta-80.99$ (m, 3F), -106.70 (m, 2F), -123.67 (m, 2F), -125.69 (m, 2F).

IR $\left(\mathrm{CH}_{2} \mathrm{Cl}_{2}\right): 3075,3007,2962,2841,1705,1610,1514,1491,1238,1204,1157,1134\left(\mathrm{~cm}^{-1}\right)$. 
HRMS, m/z: calc'd for ${ }^{13} \mathrm{CC}_{20} \mathrm{H}_{15} \mathrm{~F}_{9} \mathrm{O}_{4}$ : 503.0860, found 503.0853. 


\subsection{Synthetic utility of perfluorosubstituted $\alpha, \beta$-unsaturated esters}

\section{1,1-dimethyl 3-phenyl 3-(4-fluorophenyl)-2-(perfluorobutyl)propane-1,1,3-tricarboxylate}

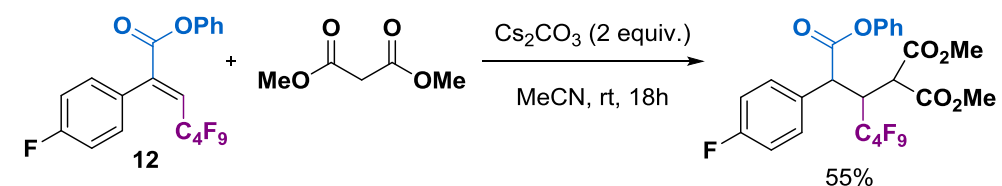

In a $4 \mathrm{~mL}$ vial phenyl (2E)-4,4,5,5,6,6,7,7,7-nonafluoro-2-(4-fluorophenyl)hept-2-enoate (12) (250.4 mg, 0.5 mmol) and dimethyl malonate $(0.7 \mathrm{mmol}, 1.2$ equiv) were dissolved and mixed in acetonitrile (2.5 mL). After stirring, $\mathrm{Cs}_{2} \mathrm{CO}_{3}(1.1 \mathrm{mmol}, 2.0$ equiv) was added and the reaction mixture was carried out overnight at rt. After the reaction was completed, $5 \mathrm{~mL}$ of water was added to quench the reaction, and the mixture was extracted with ethyl acetate $(8 \mathrm{~mL} \times 3)$. The organic phase was dried over anhydrous sodium sulfate and concentrated under reduced pressure. The crude was purified via column chromatography on silica gel using Hexane $\rightarrow$ AcOEt:Hexane (95:5) as a eluent. The title compound was isolated as white solid (177.6 mg, $30.0 \mathrm{mmol}, 55 \%$ ).

${ }^{1} \mathrm{H}$ NMR (400 MHz, Chloroform- $d$ ) $\delta 7.57(\mathrm{~m}, 2 \mathrm{H}), 7.33(\mathrm{t}, J=7.8 \mathrm{~Hz}, 2 \mathrm{H}), 7.20(\mathrm{t}, J=7.5 \mathrm{~Hz}, 1 \mathrm{H}), 7.08-6.99(\mathrm{~m}, 2 \mathrm{H})$, $6.92-6.85(\mathrm{~m}, 2 \mathrm{H}), 5.00(\mathrm{dd}, J=11.4,3.2 \mathrm{~Hz}, 1 \mathrm{H}), 4.50-4.32(\mathrm{~m}, 1 \mathrm{H}), 3.74(\mathrm{~s}, 4 \mathrm{H}), 3.39(\mathrm{~s}, 3 \mathrm{H})$.

${ }^{13} \mathrm{C}$ NMR $\left(101 \mathrm{MHz}, \mathrm{CDCl}_{3}\right) \delta 170.79,167.33,166.71,164.26,161.79,150.60,132.78(\mathrm{~d}, J=8.3 \mathrm{~Hz}), 129.59,128.48(\mathrm{~d}, J$ $=3.3 \mathrm{~Hz}), 126.27,121.17,115.60(\mathrm{~d}, J=21.6 \mathrm{~Hz}), 52.96,52.84,48.61(\mathrm{~d}, J=4.5 \mathrm{~Hz}), 47.20(\mathrm{~d}, J=9.1 \mathrm{~Hz}), 43.96(\mathrm{t}, J=$ $20.3 \mathrm{~Hz})$.

${ }^{19}$ F NMR (376 MHz, Chloroform- $d$ ) $\delta-80.85$ (m, 3F), -108.20 (m, 2F), -112.46 (s, 1F), -121.93 (m, 2F), -125.78 (m, 2F).

\section{(E)-4,4,5,5,6,6,7,7,7-nonafluoro-2-(4-fluorophenyl)-1-morpholinohept-2-en-1-one}<smiles>O=C(Oc1ccccc1)C(=CC(F)(F)F)c1ccc(F)cc1</smiles>

12<smiles>C1COCCN1</smiles>

2 equiv
$\mathrm{MeCN}, 60^{\circ} \mathrm{C}, 48 \mathrm{~h}$<smiles>Fc1ccc(/C=C\C(F)(F)F)cc1</smiles>

In a $4 \mathrm{~mL}$ vial phenyl (2E)-4,4,5,5,6,6,7,7,7-nonafluoro-2-(4-fluorophenyl)hept-2-enoate (12) (202.1 mg, 0.4 mmol) and morpholine ( $0.9 \mathrm{mmol}, 2.0$ equiv) were dissolved and mixed in acetonitrile $(2.0 \mathrm{~mL})$. The reaction mixture was carried at $60^{\circ} \mathrm{C}$ for $48 \mathrm{~h}$. After the reaction was completed, $5 \mathrm{~mL}$ of brine was added and the mixture was extracted with ethyl acetate $(8 \mathrm{~mL} \times 3)$. The organic phase was dried over anhydrous sodium sulfate and concentrated under reduced pressure. The crude was purified via column chromatography on silica gel using AcOEt:Hexane (95:5) $\rightarrow$ AcOEt:Hexane (1:1) as a eluent. The title compound was isolated as yellow oil (121.7 $\mathrm{mg}, 0.268 \mathrm{mmol}, 61 \%)$.

${ }^{1} \mathrm{H}$ NMR (400 MHz, Chloroform- $d$ ) $\delta 7.49-7.31(\mathrm{~m}, 2 \mathrm{H}), 7.20-6.97(\mathrm{~m}, 2 \mathrm{H}), 5.83(\mathrm{t}, J=14.2 \mathrm{~Hz}, 1 \mathrm{H}), 3.64(\mathrm{~s}, 4 \mathrm{H}), 3.56-$ $3.30(\mathrm{~m}, 4 \mathrm{H})$.

${ }^{13} \mathrm{C}$ NMR (101 MHz, Chloroform- $d$ ) $\delta$ 166.97, 164.73, 162.24, $148.53(\mathrm{t}, J=5.0 \mathrm{~Hz}), 130.03(\mathrm{dt}, J=8.3,2.9 \mathrm{~Hz}), 128.64(\mathrm{~d}$, $J=3.7 \mathrm{~Hz}), 115.89,115.67,66.61,47.32,42.33$.

${ }^{19} \mathrm{~F}$ NMR (470 MHz, Chloroform- $d$ ) $\delta-81.08$ (m, 3F), -105.67 (m, 2F), -111.03 (m, 1F), -123.81 (m, 2F), -125.80 (m, 2F).

(E)-butyl 4,4,5,5,6,6,7,7,7-nonafluoro-2-phenylhept-2-enoate

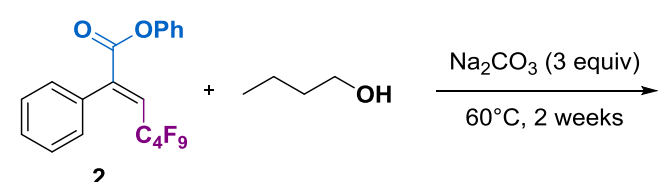

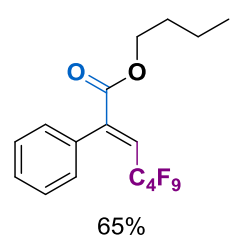

In a $4 \mathrm{~mL}$ vial, $\mathrm{Na}_{2} \mathrm{CO}_{3}(0.3 \mathrm{mmol}, 3.0$ equiv) was added to a solution of phenyl phenyl $(2 E)-4,4,5,5,6,6,7,7,7$ nonafluoro-2-phenylhept-2-enoate $(2)(50.0 \mathrm{mg}, 0.1 \mathrm{mmol})$ in $n$-butanol $(1.0 \mathrm{~mL})$. The reaction mixture was 
carried out at $60^{\circ} \mathrm{C}$ for 2 weeks. After the reaction was completed, $10 \mathrm{~mL}$ of water was added and the mixture was extracted with DCM $(20 \mathrm{~mL} \times 3)$. The organic phase was dried over anhydrous sodium sulfate and concentrated under reduced pressure. The crude was purified via column chromatography on silica gel using hexane as a eluent. The title compound was isolated as yellow oil (30.90 mg, $0.073 \mathrm{mmol}, 65 \%)$.

${ }^{1} \mathrm{H}$ NMR (400 MHz, Chloroform- $d$ ) $\delta 7.39-7.35(\mathrm{~m}, 2 \mathrm{H}), 7.24-7.17(\mathrm{~m}, 2 \mathrm{H}), 6.89(\mathrm{t}, J=14.4 \mathrm{~Hz}, 1 \mathrm{H}), 4.21(\mathrm{t}, J=6.6 \mathrm{~Hz}$, 2H), $1.67-1.57(\mathrm{~m}, 2 \mathrm{H}), 1.37-1.25(\mathrm{~m}, 2 \mathrm{H}), 0.90(\mathrm{t}, J=7.4 \mathrm{~Hz}, 3 \mathrm{H})$.

${ }^{13} \mathrm{C}$ NMR (101 MHz, Chloroform- $d$ ) $\delta 165.59,145.10,132.64,128.79,128.71(\mathrm{t}, J=2.7 \mathrm{~Hz}), 127.82,124.44(\mathrm{t}, J=21.4 \mathrm{~Hz})$, $66.41,30.53,19.15,13.69$. 


\section{NMR spectra of isolated compounds}

\subsection{Non-commercially available starting materials}

\section{4-fluorophenyl formate}

GAT-01-103-F2.1.fid<smiles>O=COc1ccc(F)cc1</smiles>
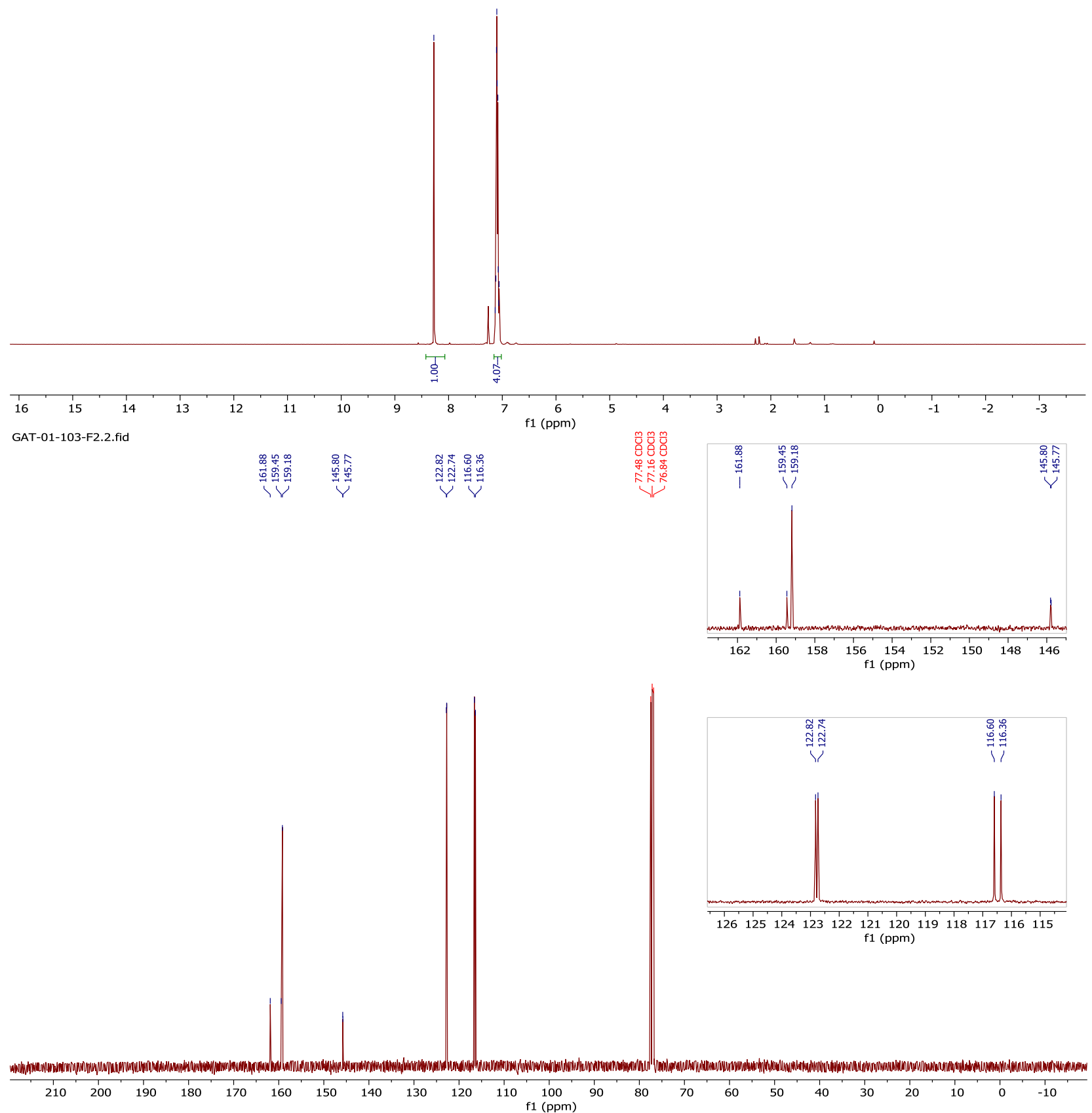
3-methoxyphenyl formate- ${ }^{1} d$ GAT-02-141dp.1.fid<smiles>[2H]C(=O)Oc1cccc(OC)c1</smiles>
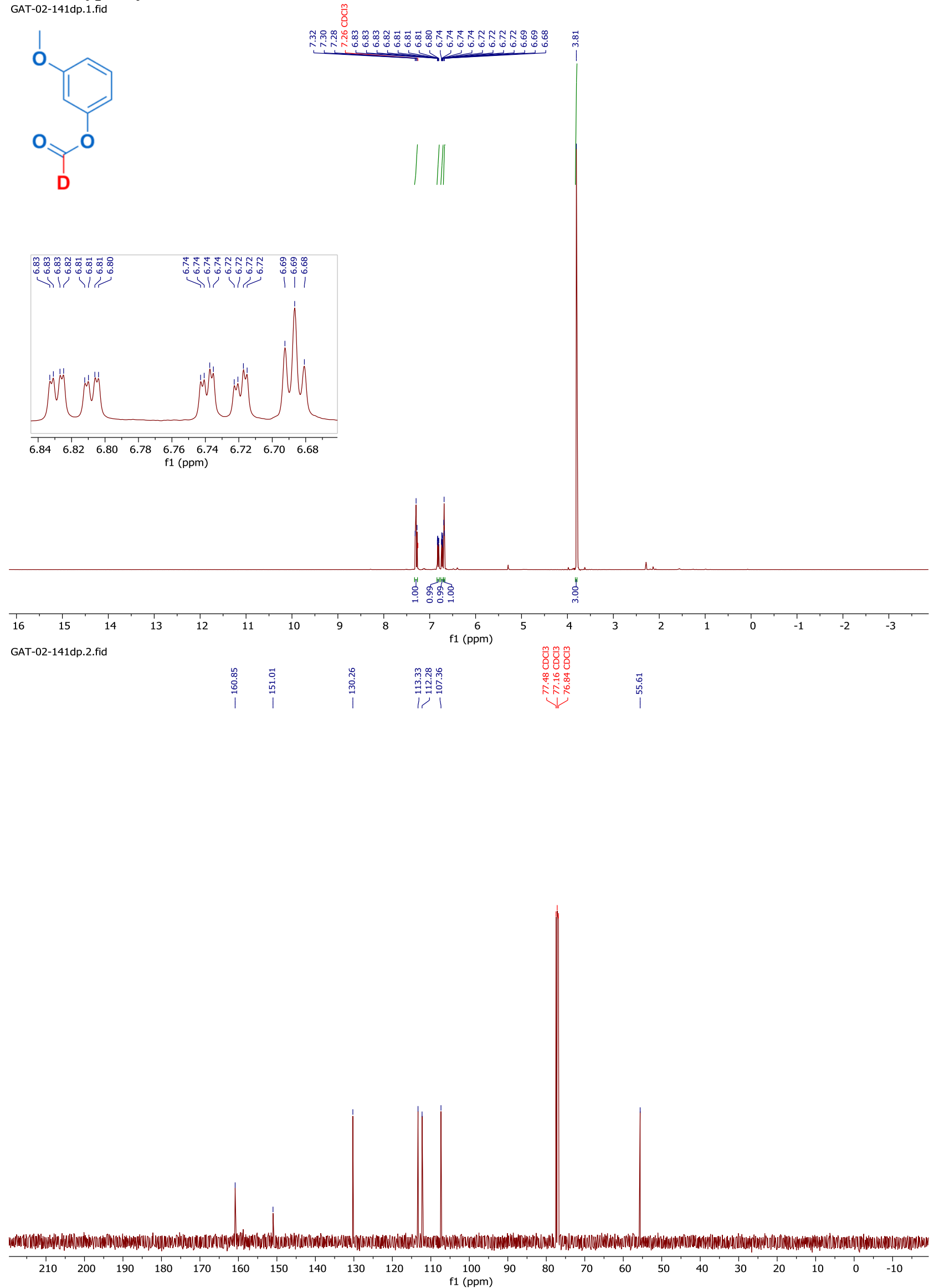


\section{3-methoxyphenyl formate- ${ }^{13} \mathrm{C}$}
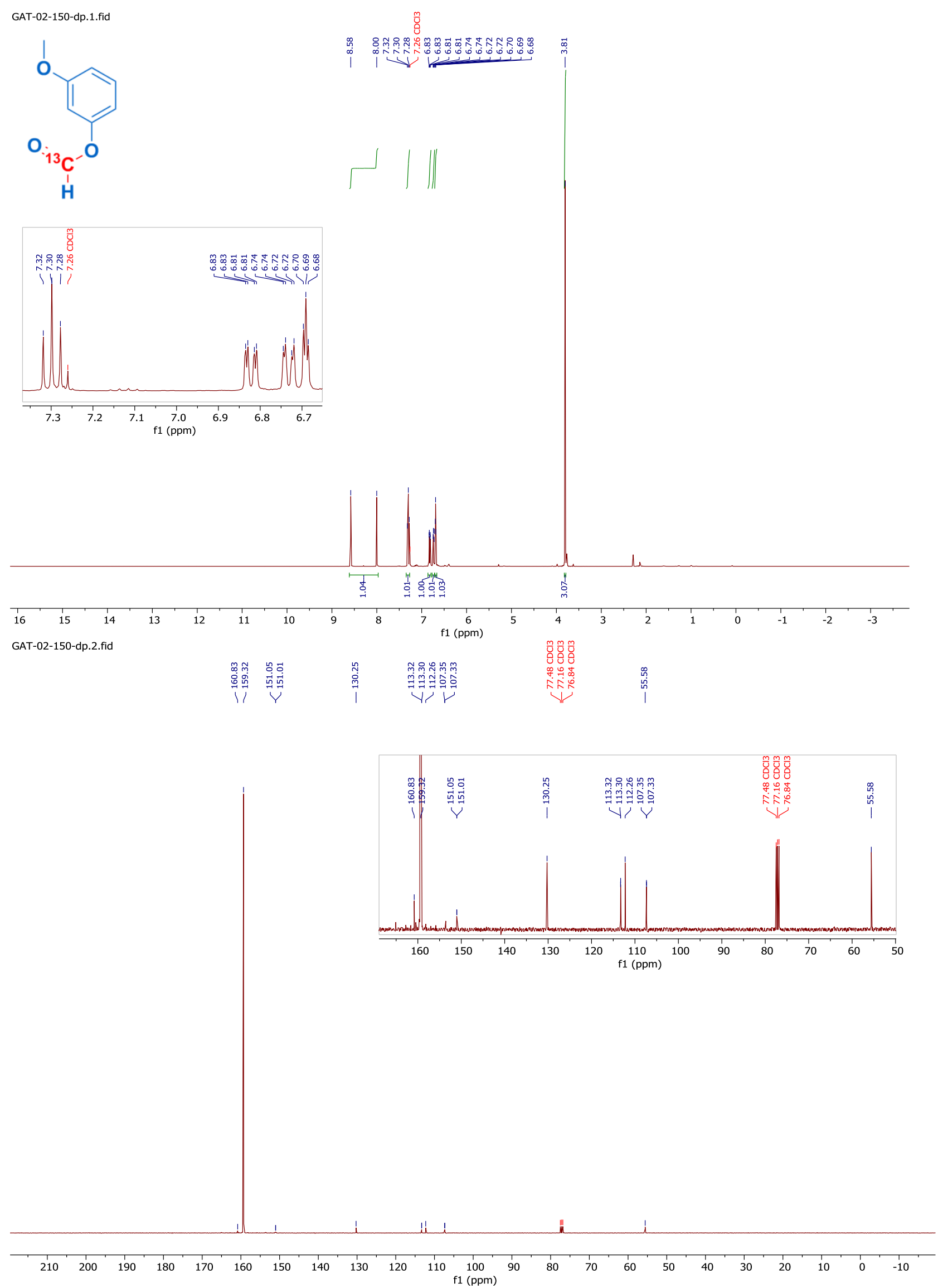


\subsection{Perfluoroalkylated $\alpha, \beta$-unsaturated esters}

Phenyl (2E)-4,4,5,5,6,6,7,7,7-nonafluoro-2-phenylhept-2-enoate (2)<smiles>O=C([18OH])/C=C(\C(=O)Oc1ccccc1)c1ccccc1</smiles>

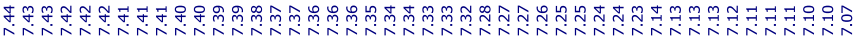

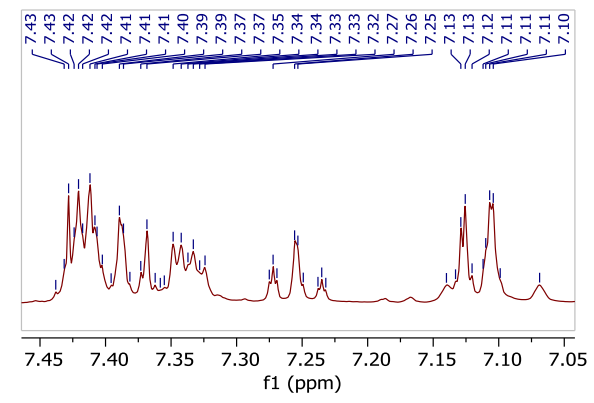

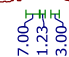

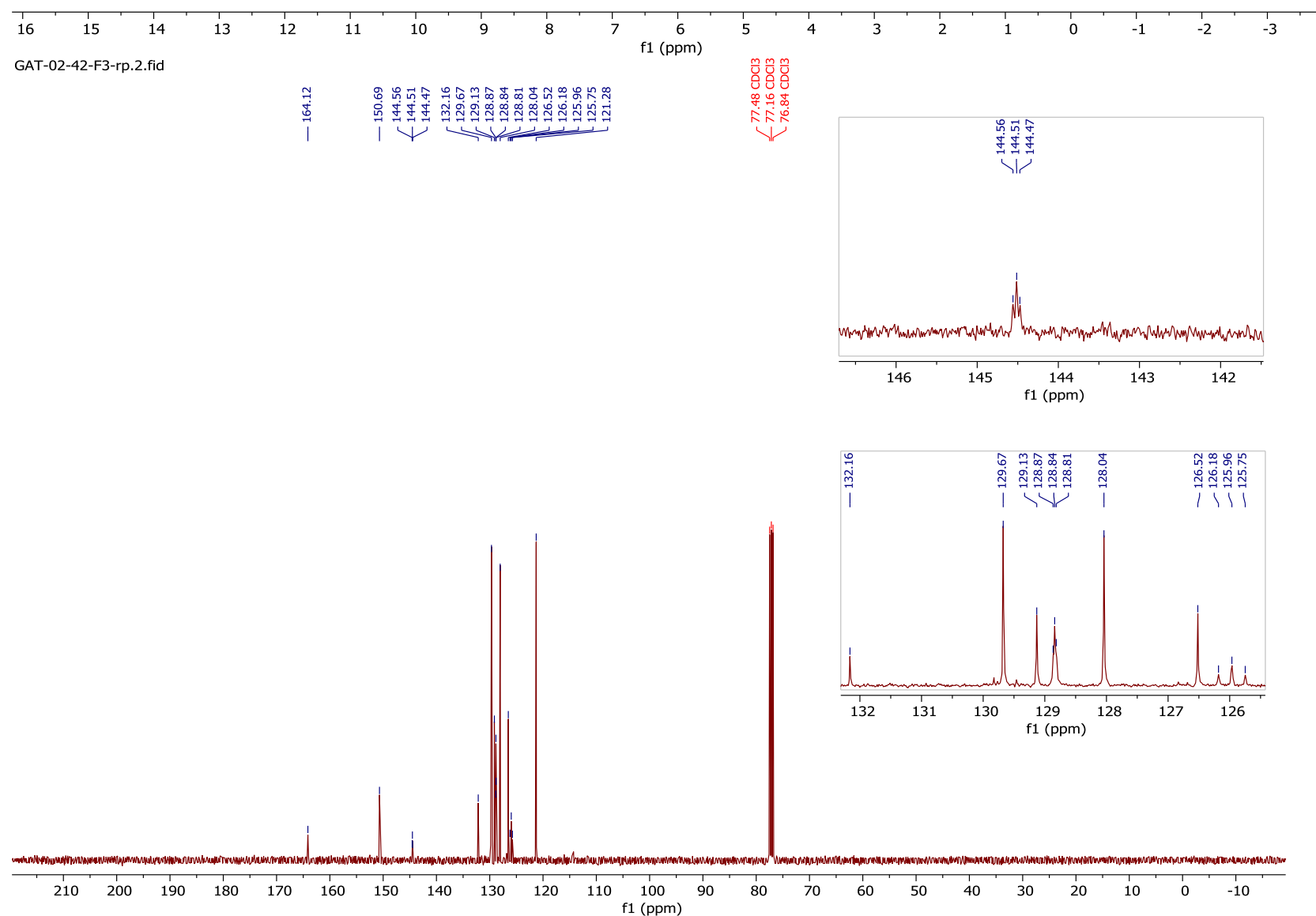




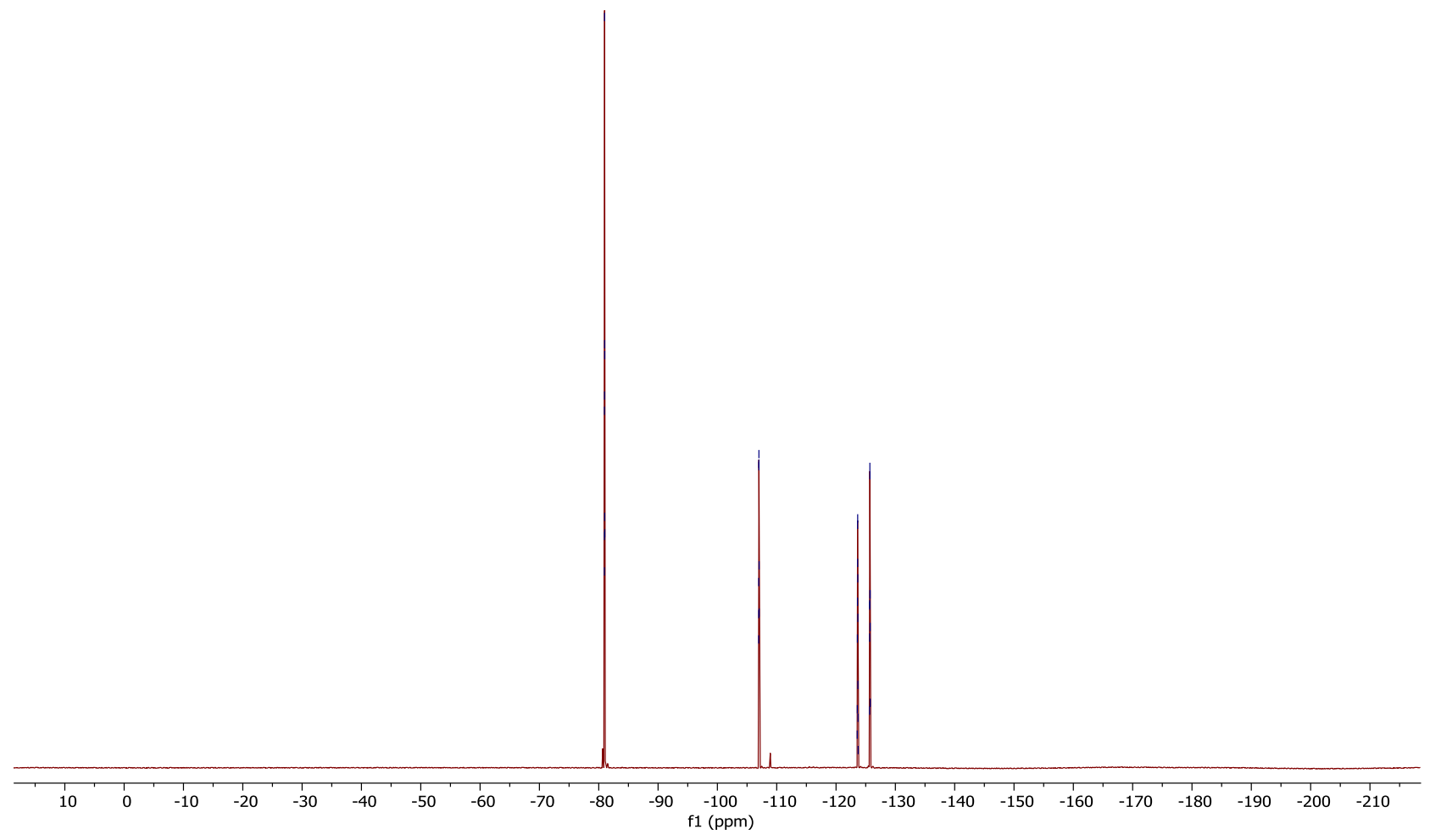


Phenyl (2E)-4,4,5,5,6,6,7,7,7-nonafluoro-2-(naphthalen-2-yl)hept-2-enoate (3a) GAT-02-33-rp-F1.1.fid<smiles>O=C(Oc1ccccc1)/C(=C/C(F)(F)F)c1ccc2ccccc2c1</smiles>

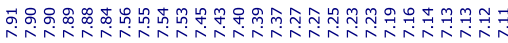
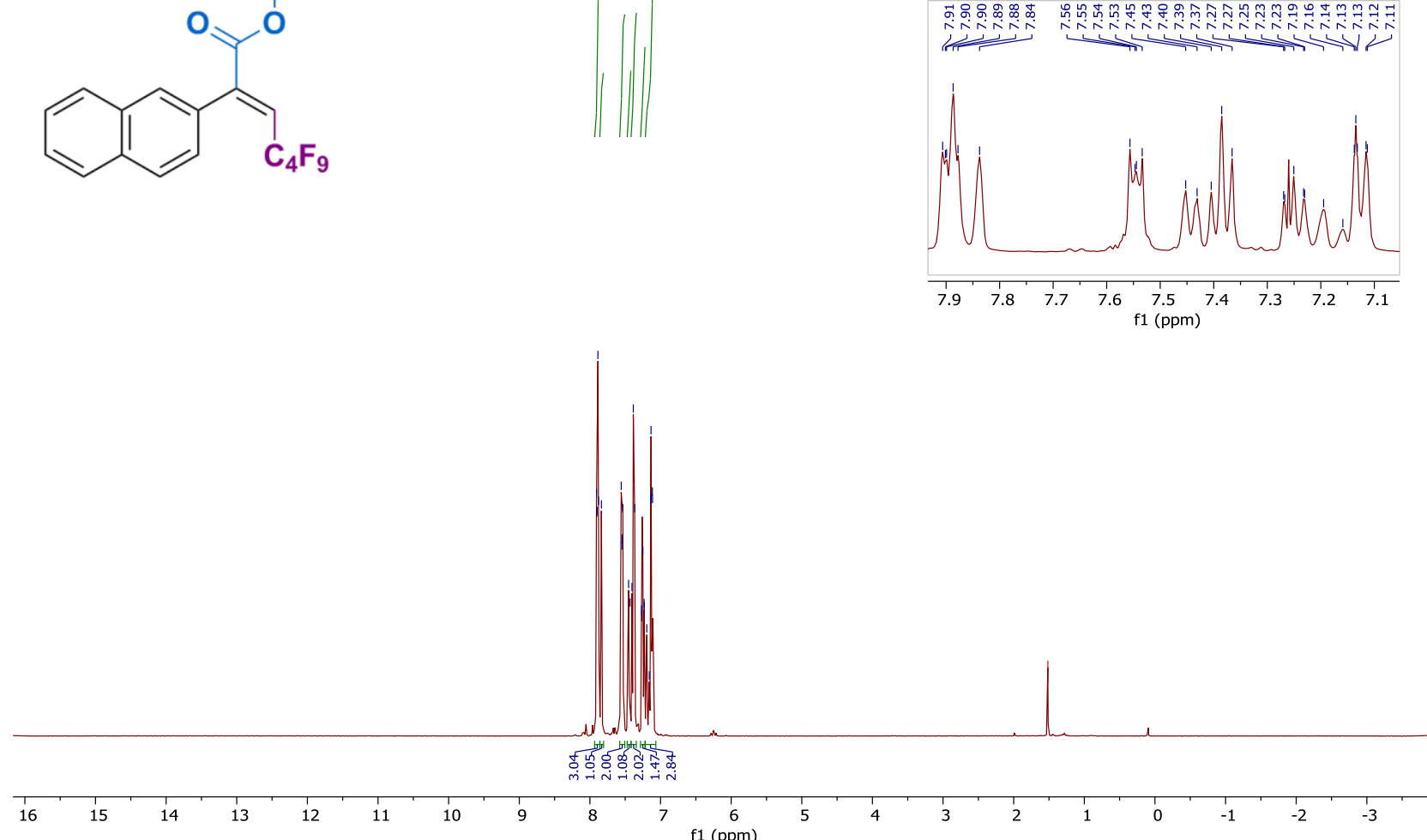

GAT-02-33-rp-F1.2.fid
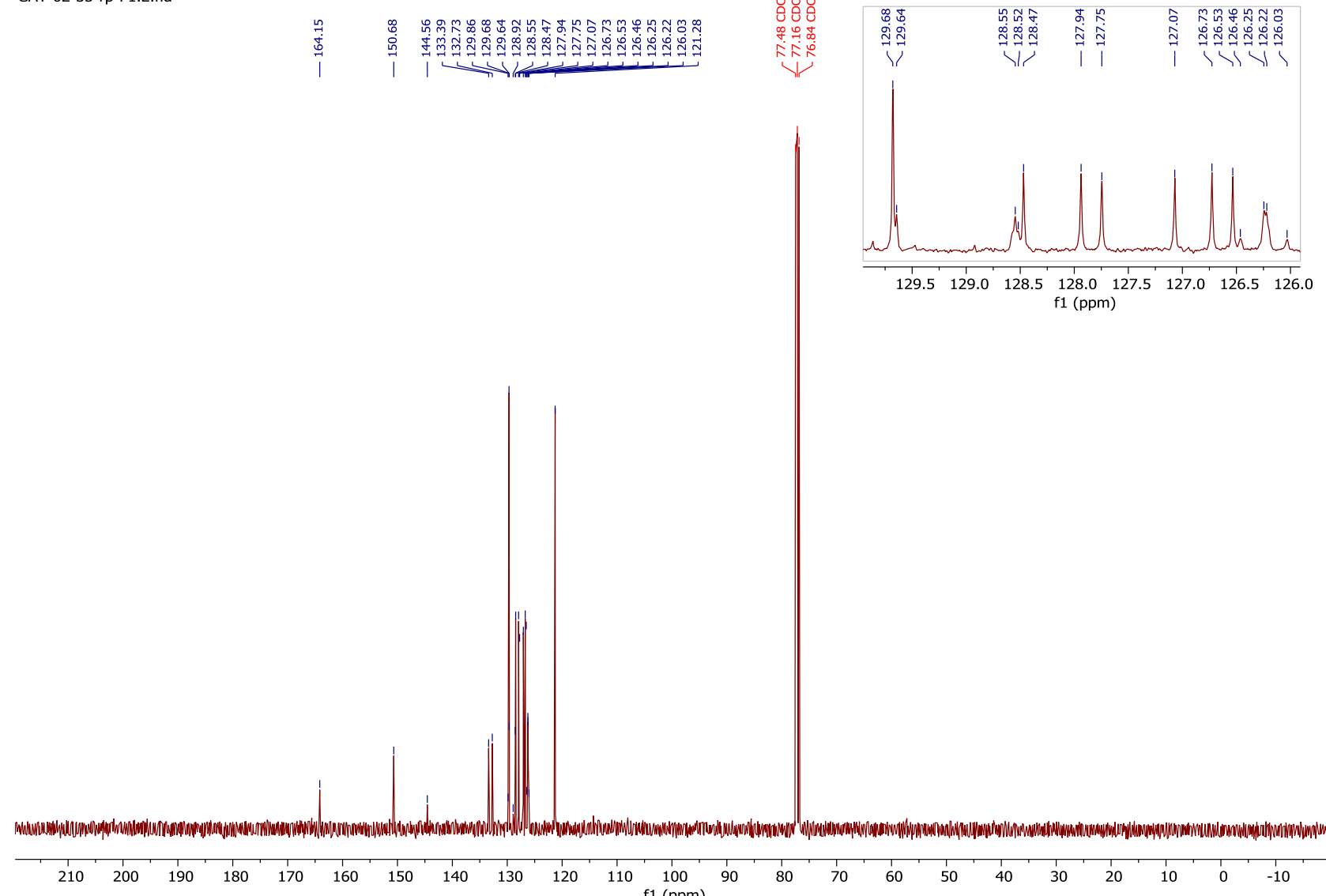

$\begin{array}{lllllllllll}210 & 200 & 190 & 180 & 170 & 160 & 150 & 140 & 130 & 120 & 110 \\ \mathrm{f} 1(\mathrm{ppm})\end{array}$ 


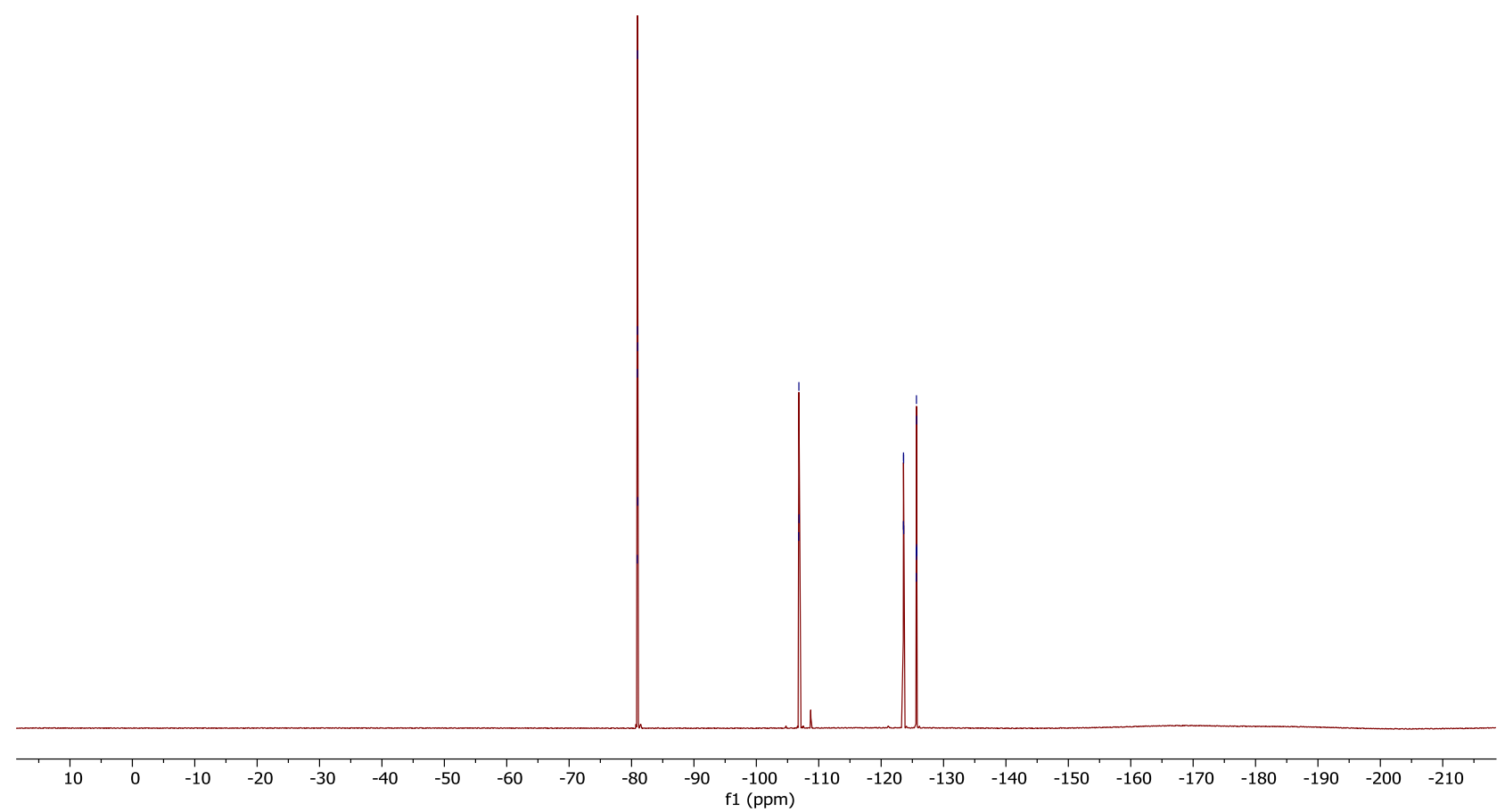


(E)-4-fluorophenyl 4,4,5,5,6,6,7,7,7-nonafluoro-2-(naphthalen-2-yl)hept-2-enoate (3b)

GAT-02-186-rp.1.fid<smiles>O=C(Oc1ccc(F)cc1)/C(=C/C(F)(F)F)c1ccc2ccccc2c1</smiles>
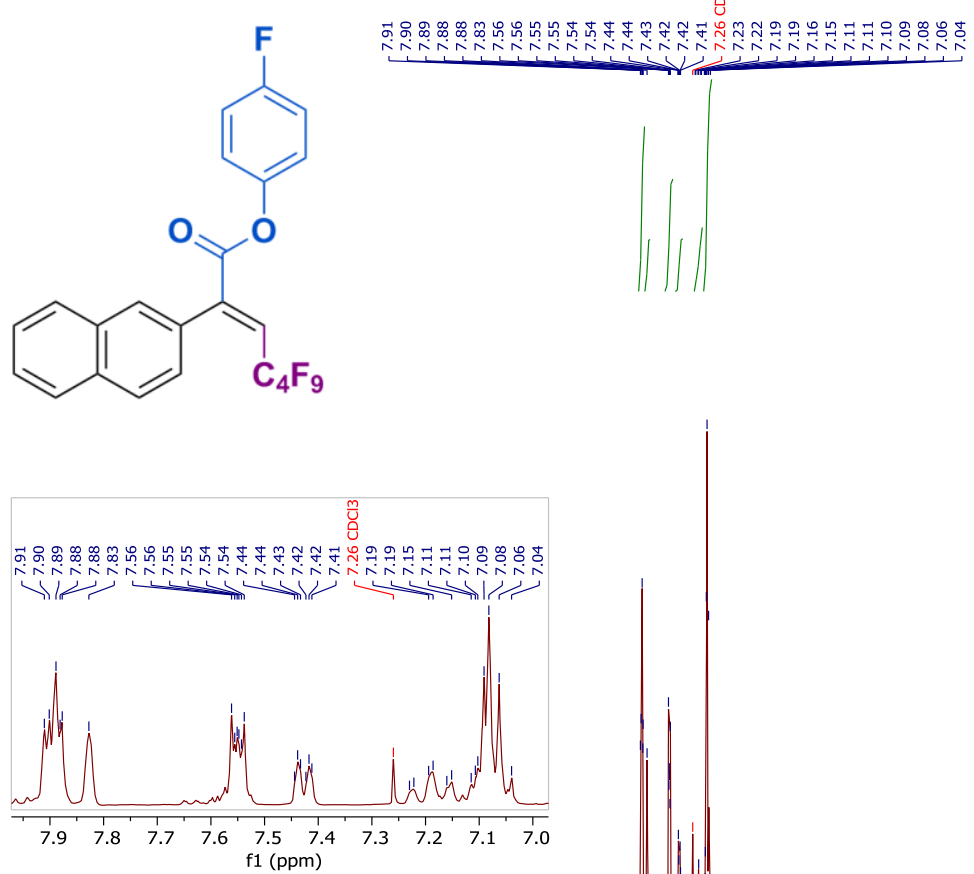

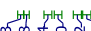

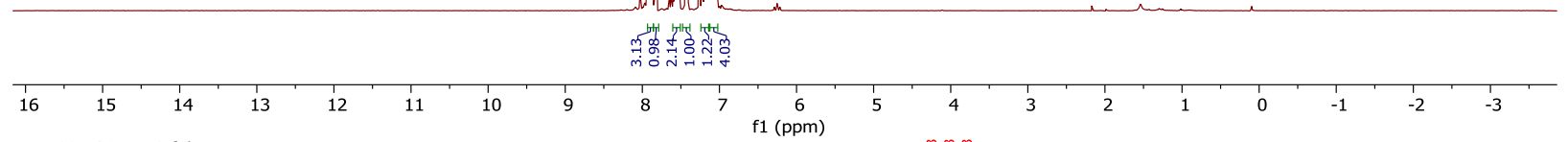

GAT-02-186-rpp.2.fid

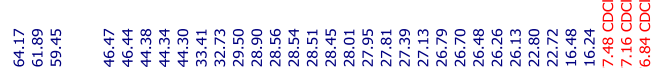

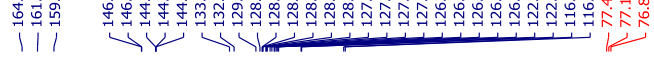
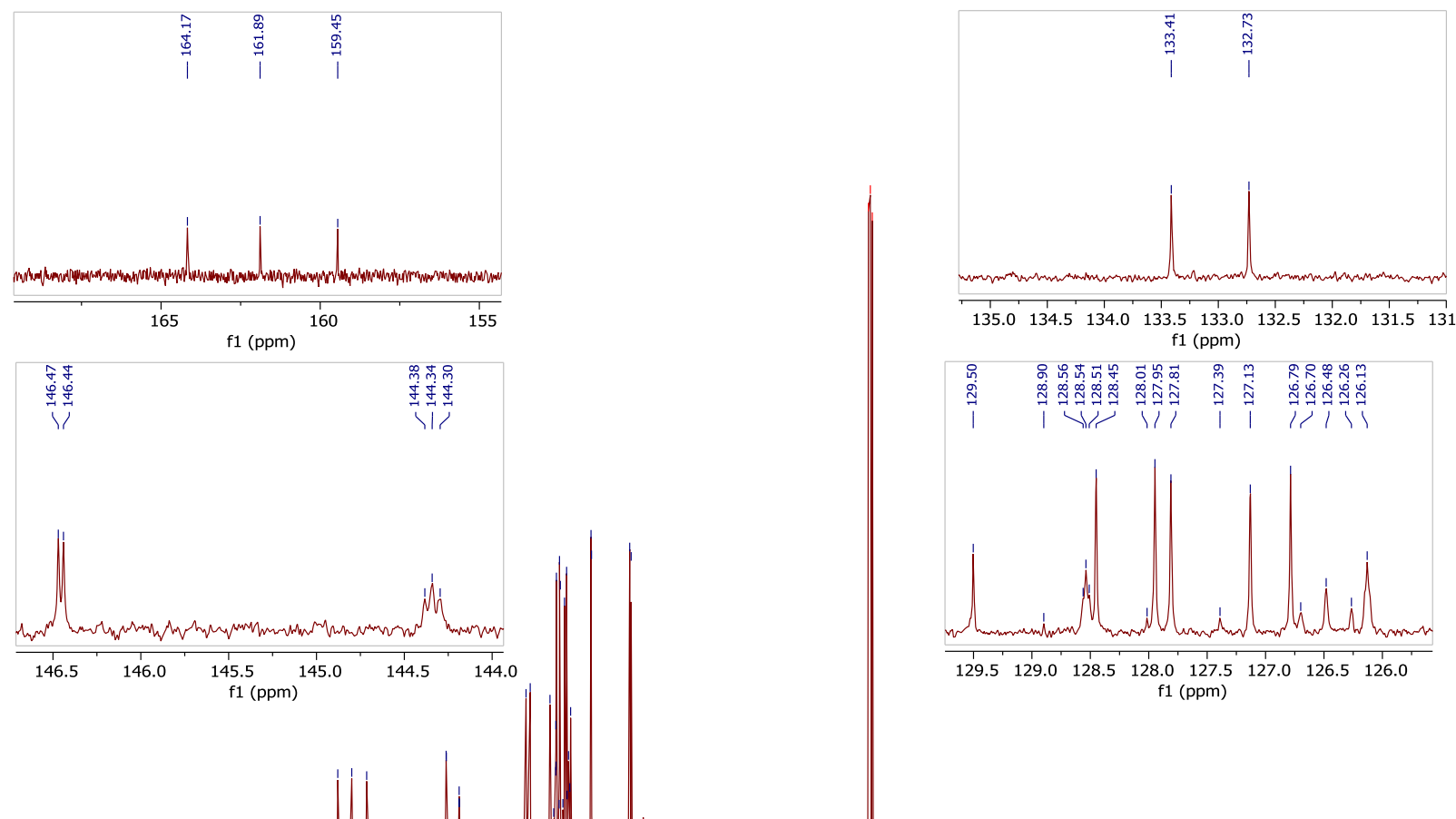

$\begin{array}{llllllllll}135.0 & 134.5 & 134.0 & 133.5 & 133.0 & 132.5 & 132.0 & 131.5 & 131\end{array}$ f1 $(\mathrm{ppm})$

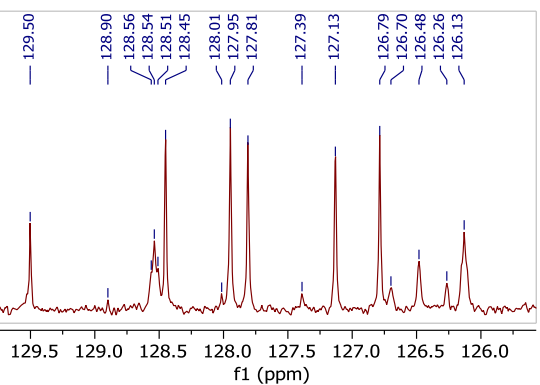

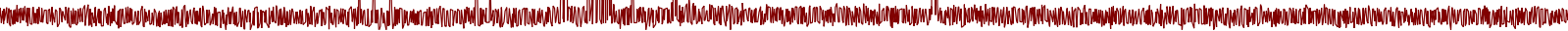

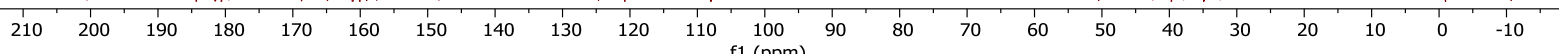




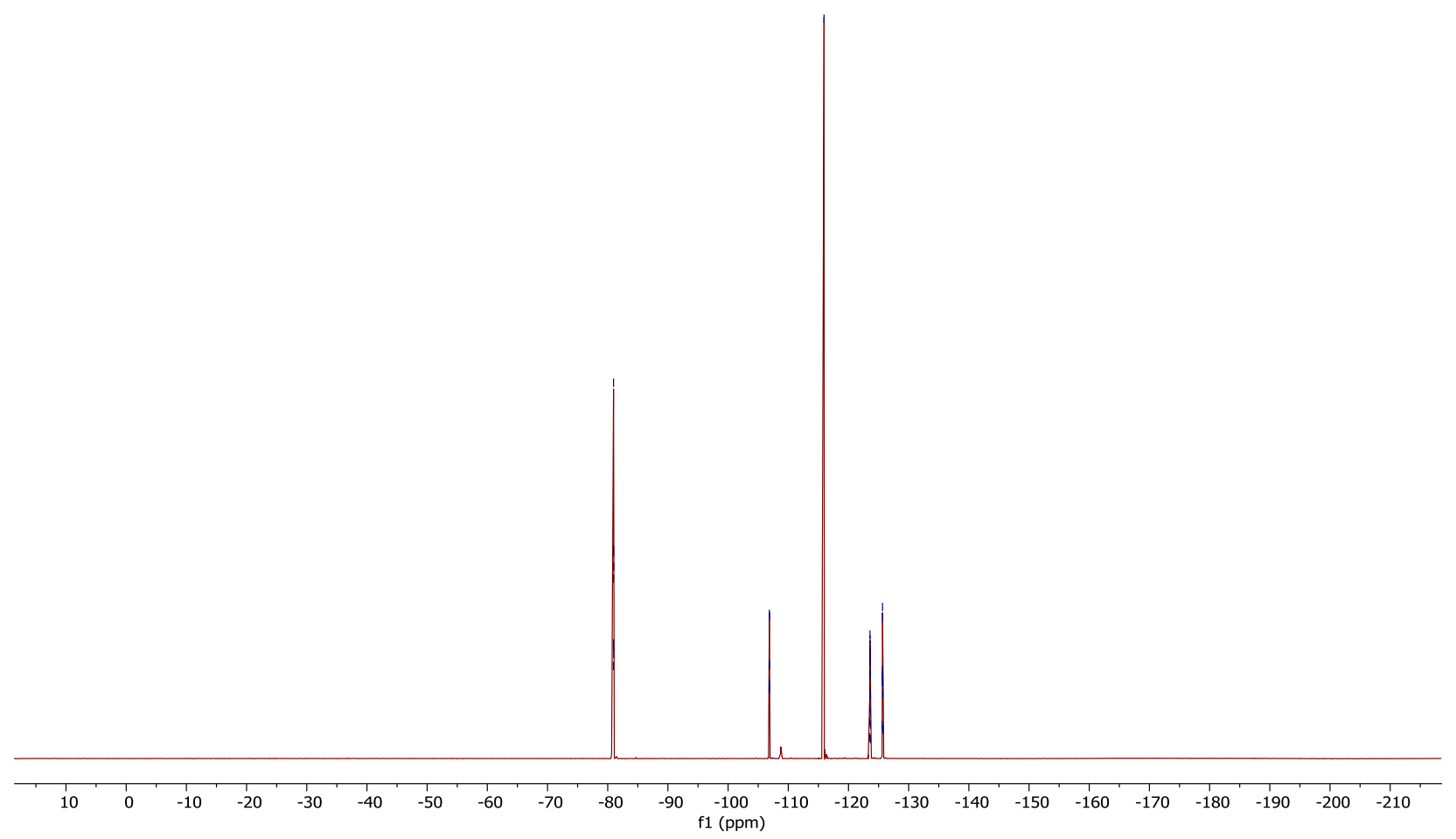


Phenyl (2E)-4,4,5,5,6,6,7,7,7-nonafluoro-2-(naphthalen-1-yl)hept-2-enoate (4)<smiles>O=C(Oc1ccccc1)/C(=C\C(F)(F)F)c1cccc2ccccc12</smiles>
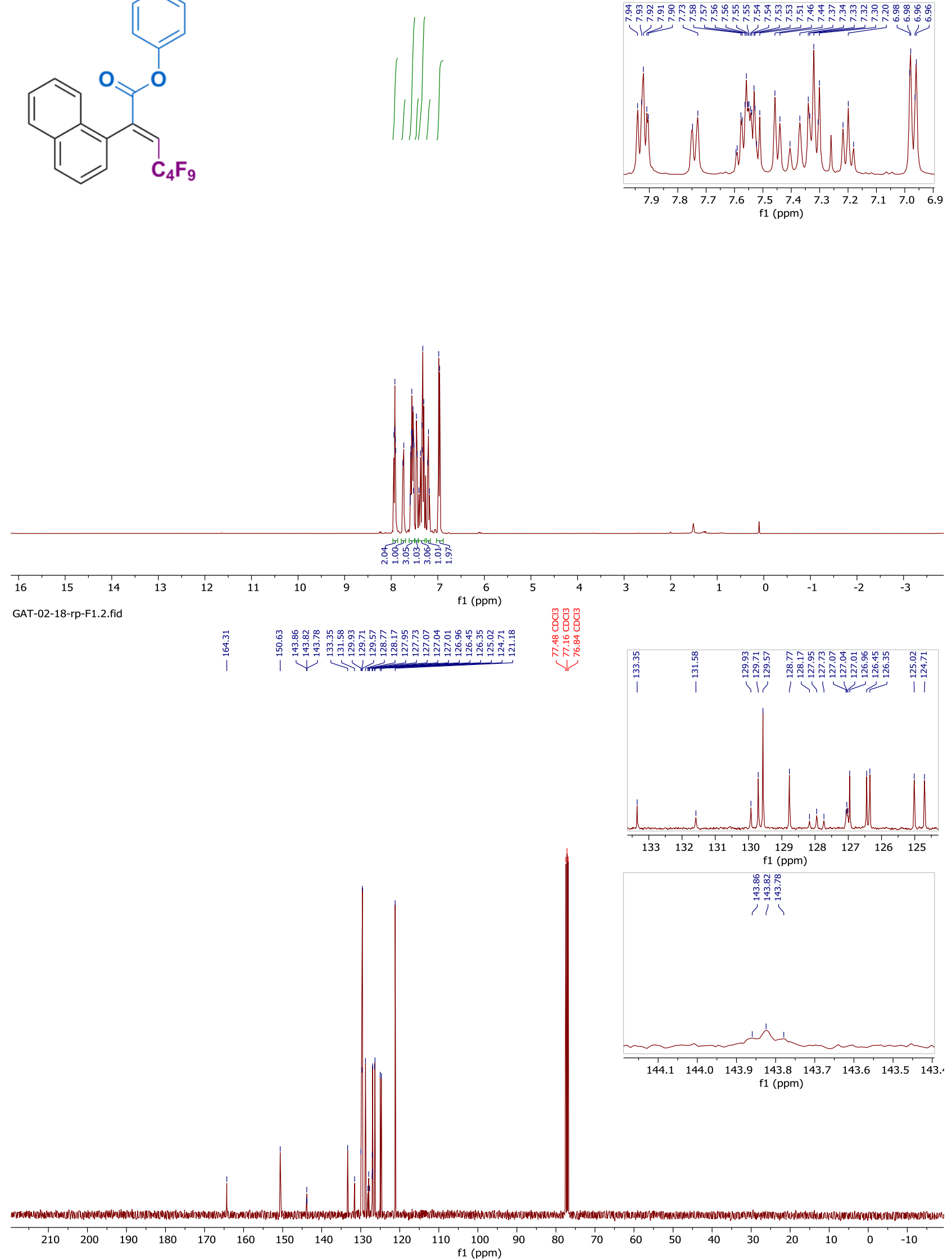


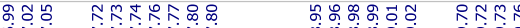

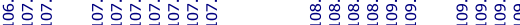

诵调

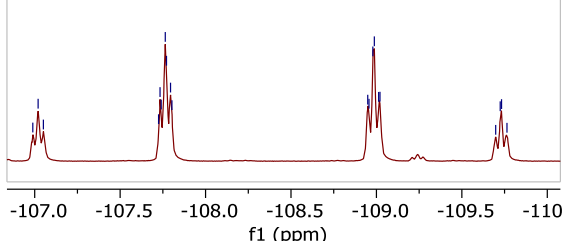

$\begin{array}{lllllll}-107.0 & -107.5 & -108.0 & -108.5 & -109.0 & -109.5 & -110\end{array}$

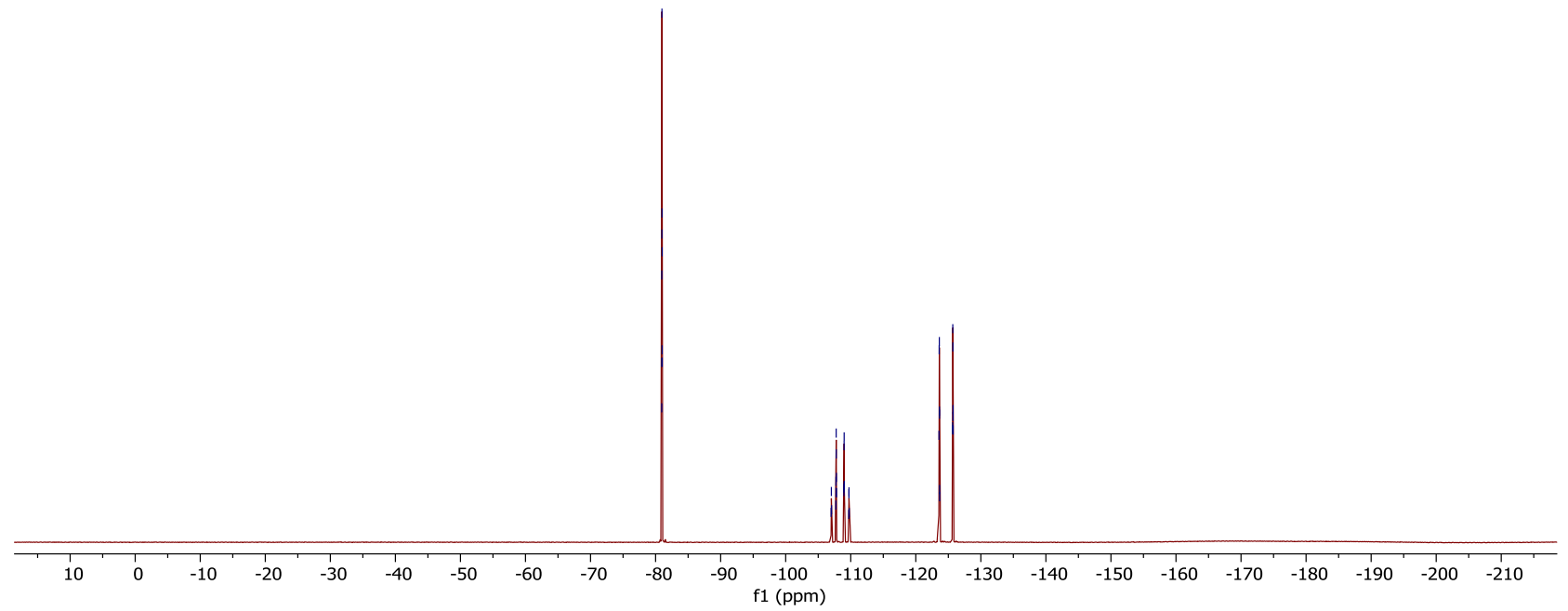


Phenyl (2E)-2-(4-tert-butylphenyl)-4,4,5,5,6,6,7,7,7-nonafluorohept-2-enoate (5) GAT-02-21:1<smiles>CC(C)(C)c1ccc(C(=CC(F)(F)F)C(=O)Oc2ccccc2)cc1</smiles>

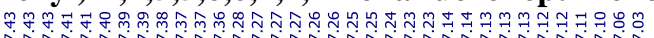
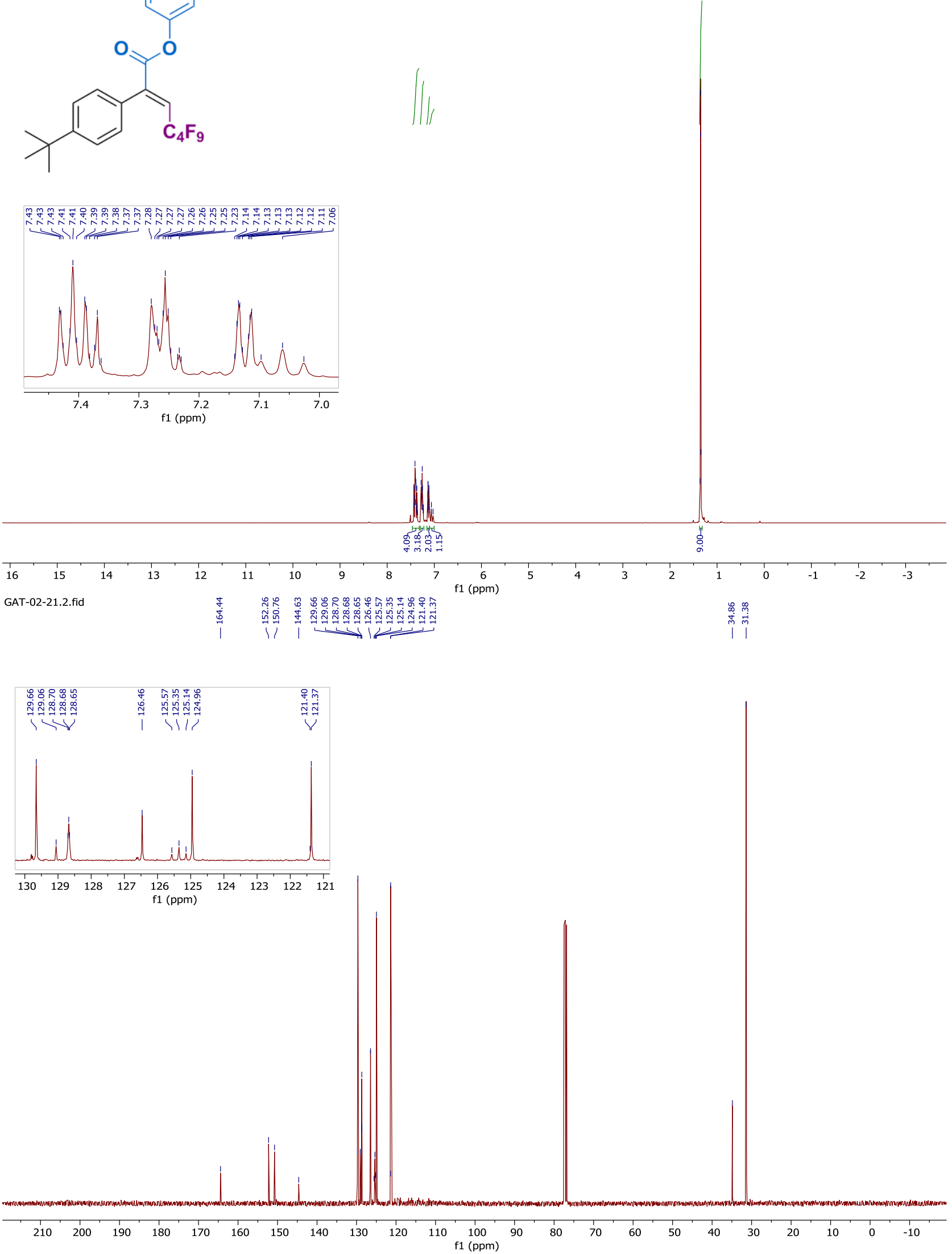


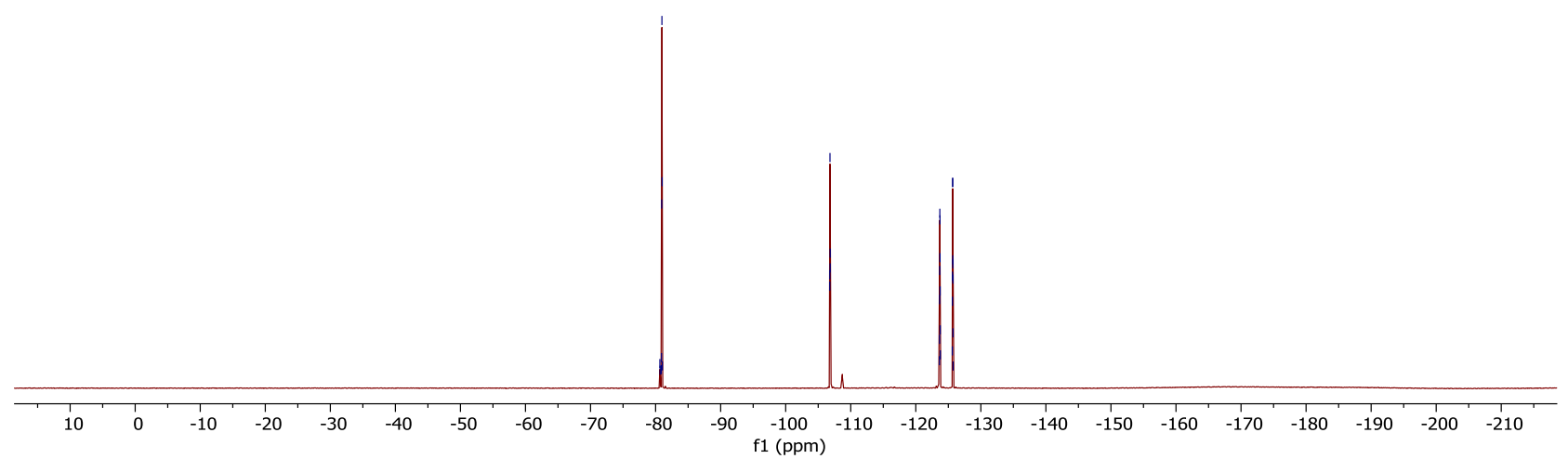


Phenyl (2E)-2-(4-(benzyloxy)phenyl)-4,4,5,5,6,6,7,7,7-nonafluorohept-2-enoate (6)

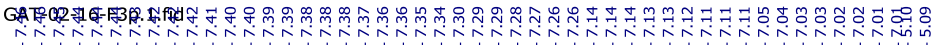<smiles>O=C(Oc1ccccc1)/C(=C/C(F)(F)F)c1ccc(OCc2ccccc2)cc1</smiles>
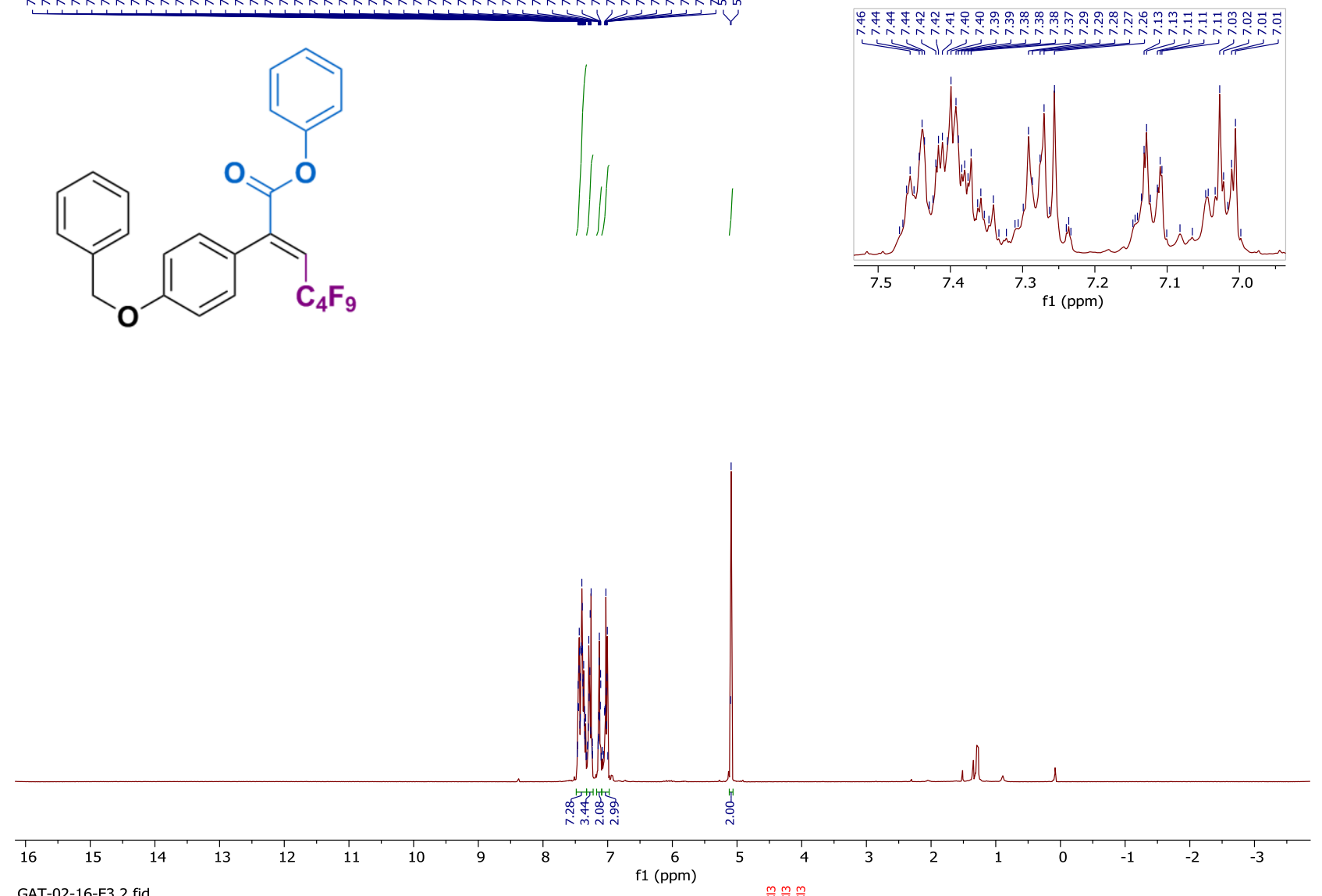

GAT-02-16-F3.2.fid

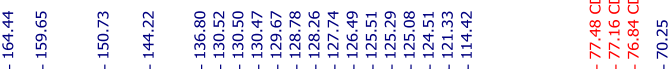

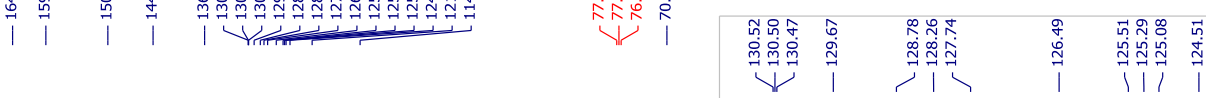
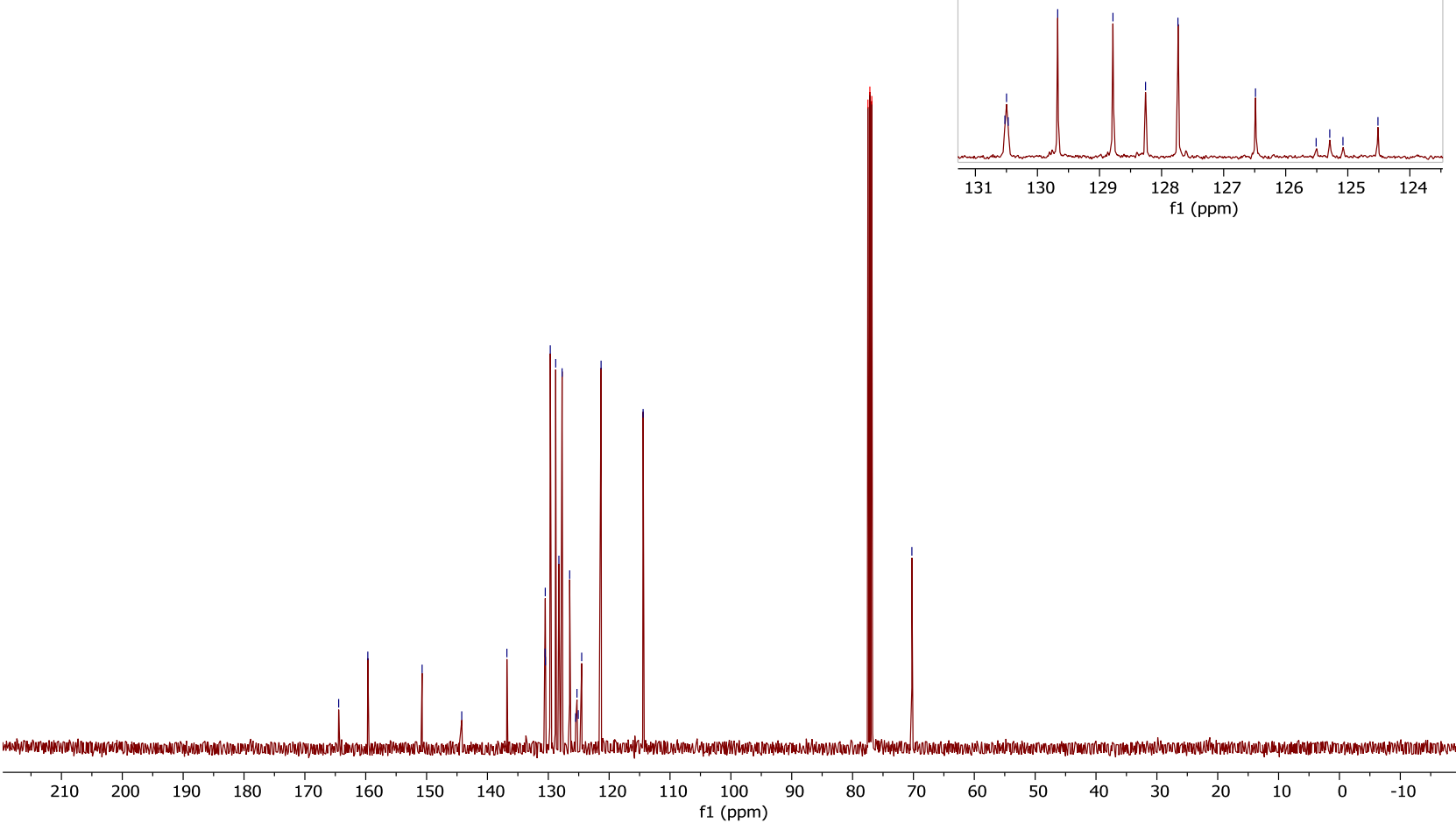


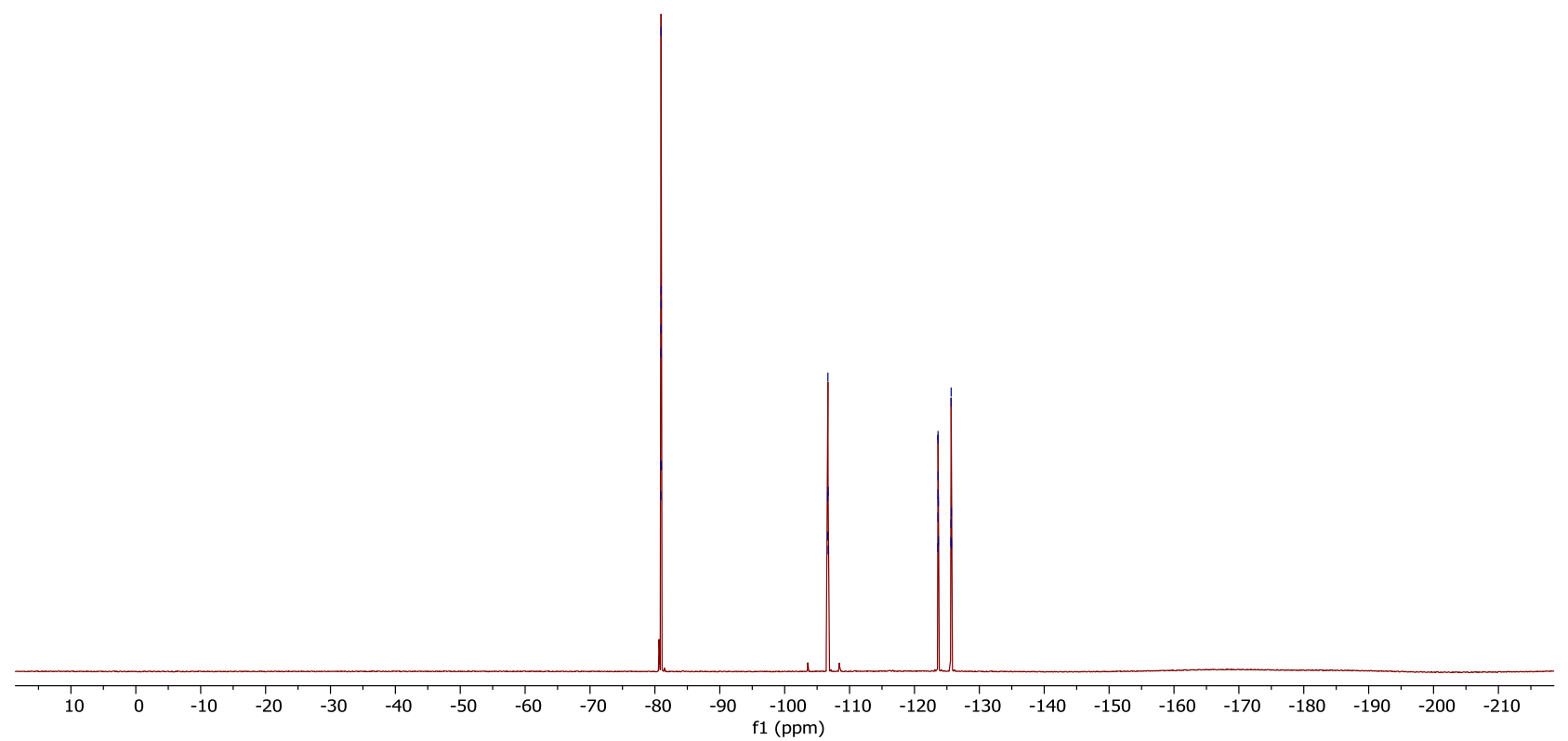


Phenyl (2E)-4,4,5,5,6,6,7,7,7-nonafluoro-2-(4-methoxyphenyl)hept-2-enoate (7)

GAT-02-12-F3.1.fid

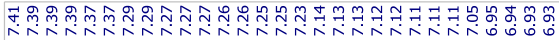<smiles>COc1ccc(/C(=C\C(F)(F)F)C(=O)Oc2ccccc2)cc1</smiles>
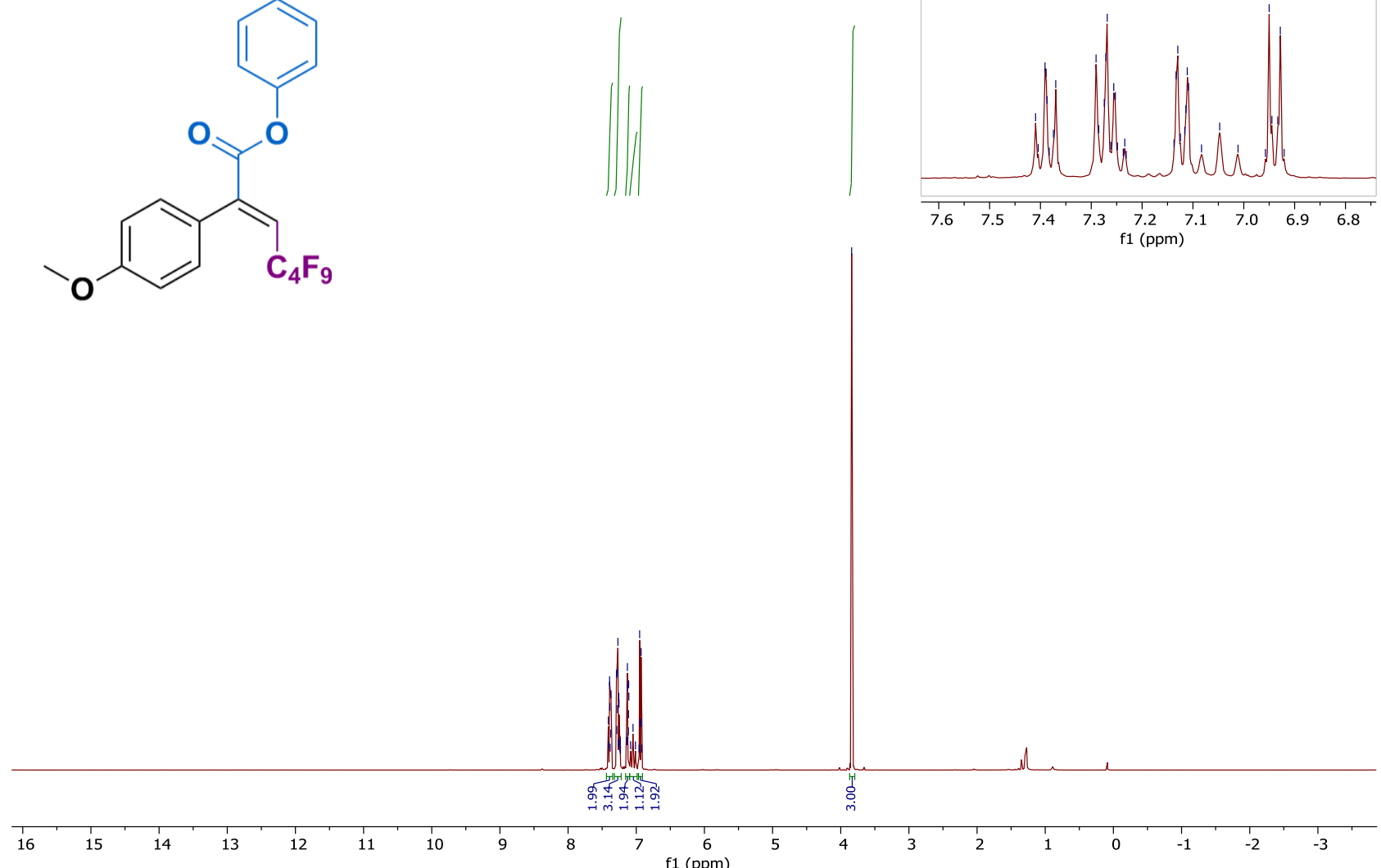

GAT-02-12-F3p.2.fid

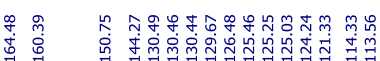

|

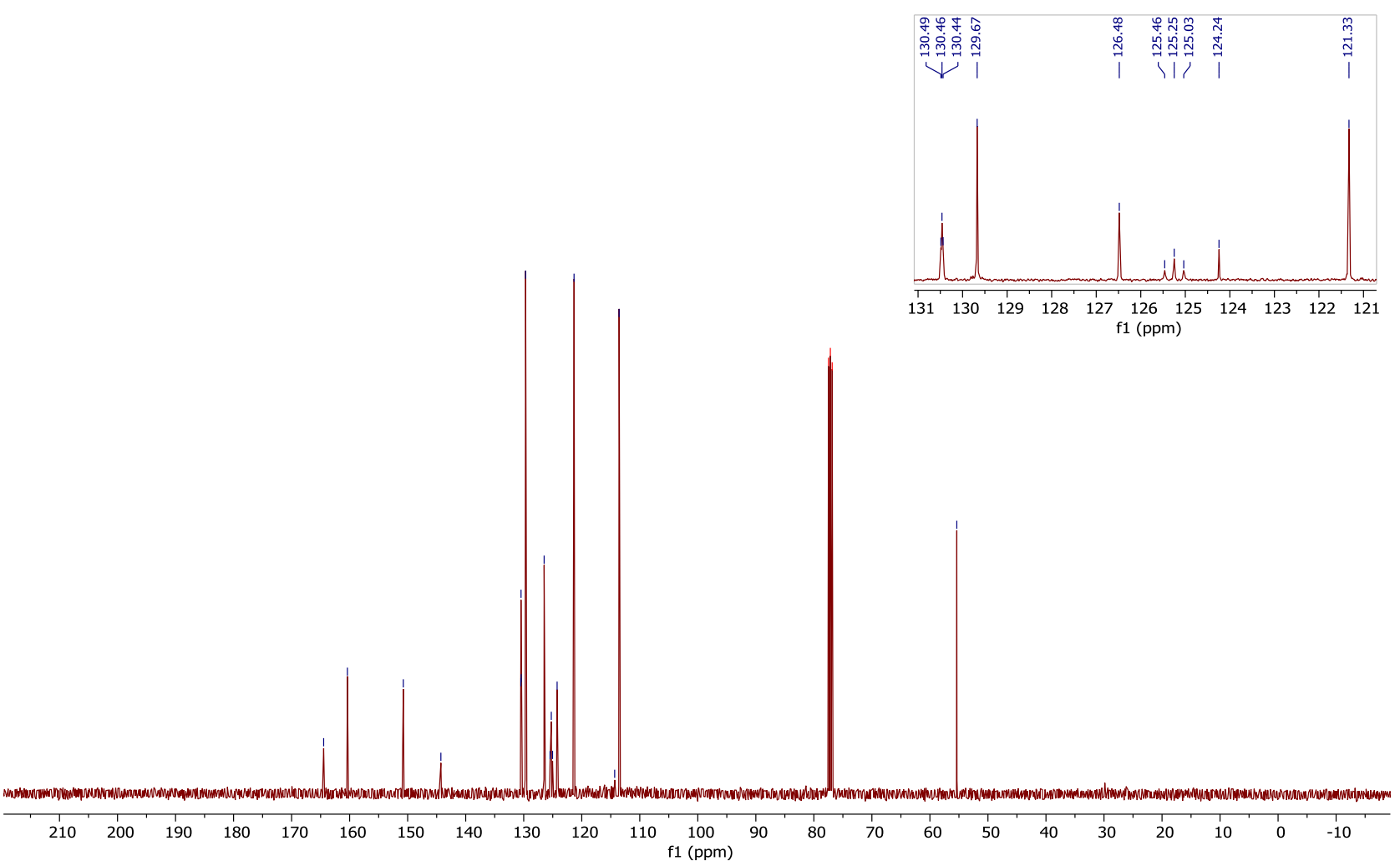




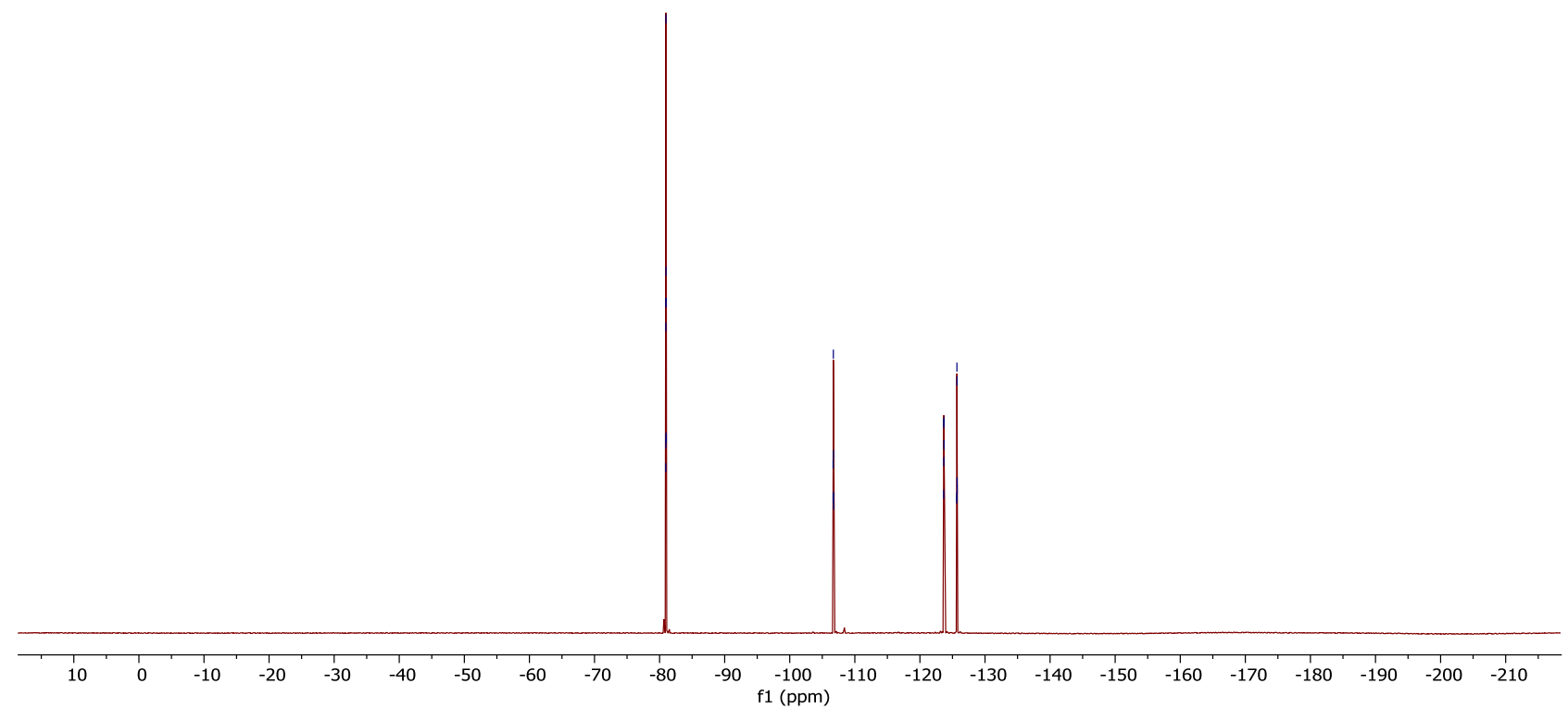


Phenyl (2E)-4,4,5,5,6,6,7,7,7-nonafluoro-2-(3,4,5-trimethoxyphenyl)hept-2-enoate (8) GAT-02-50-F2-rp.1.fid<smiles>COc1cc(/C(=C\C(F)(F)F)C(=O)Oc2ccccc2)cc(OC)c1OC</smiles>

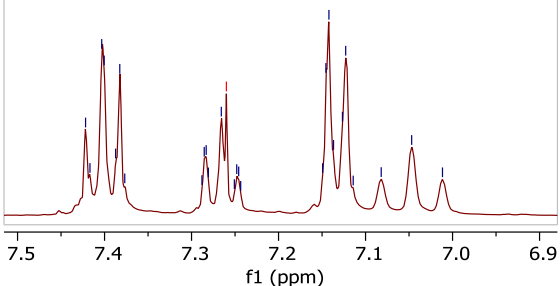

Whim

至

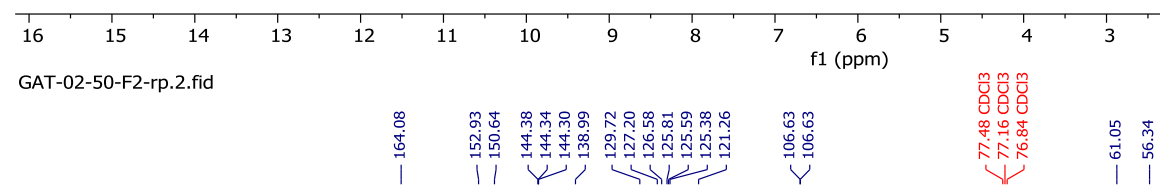
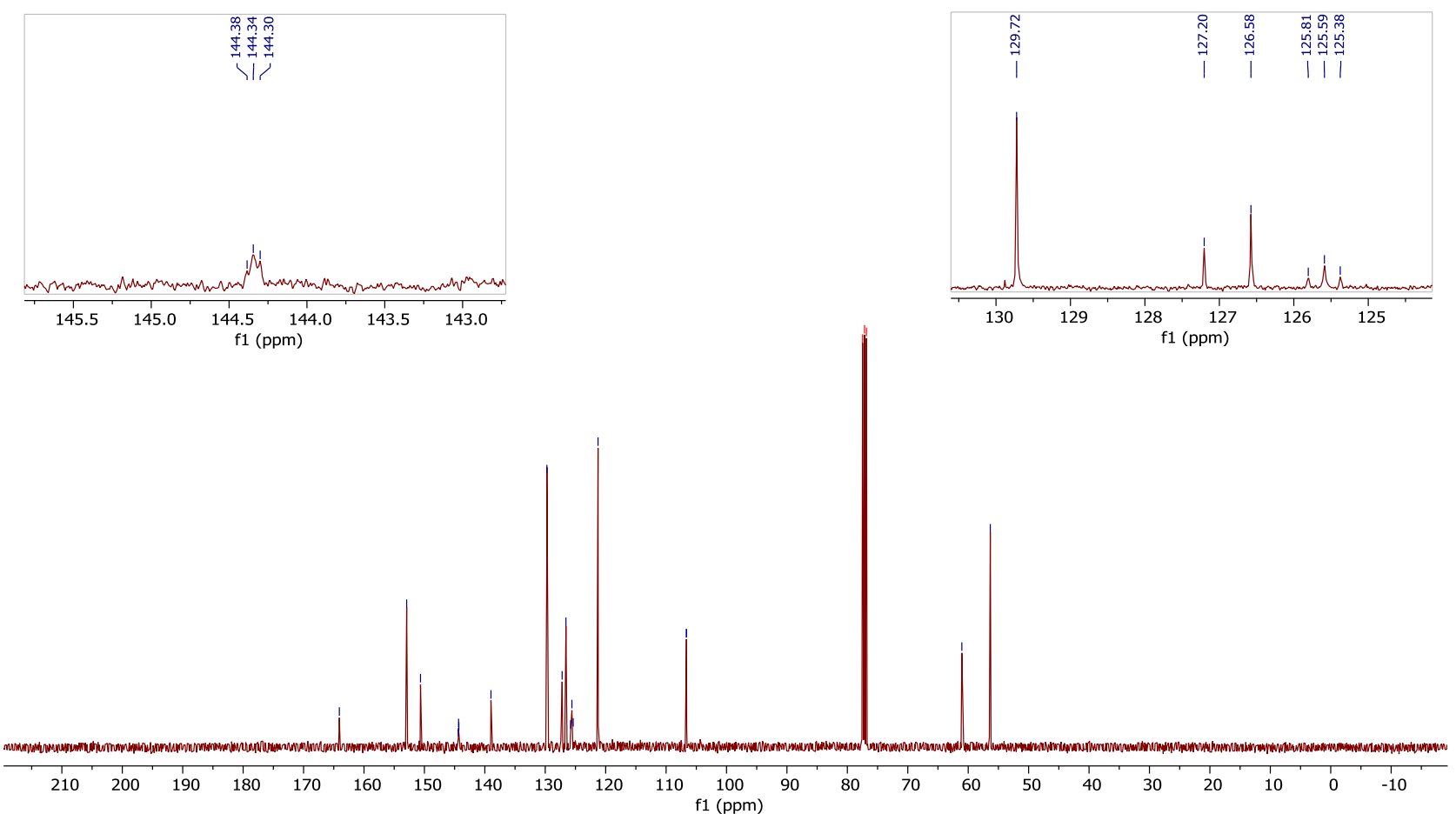


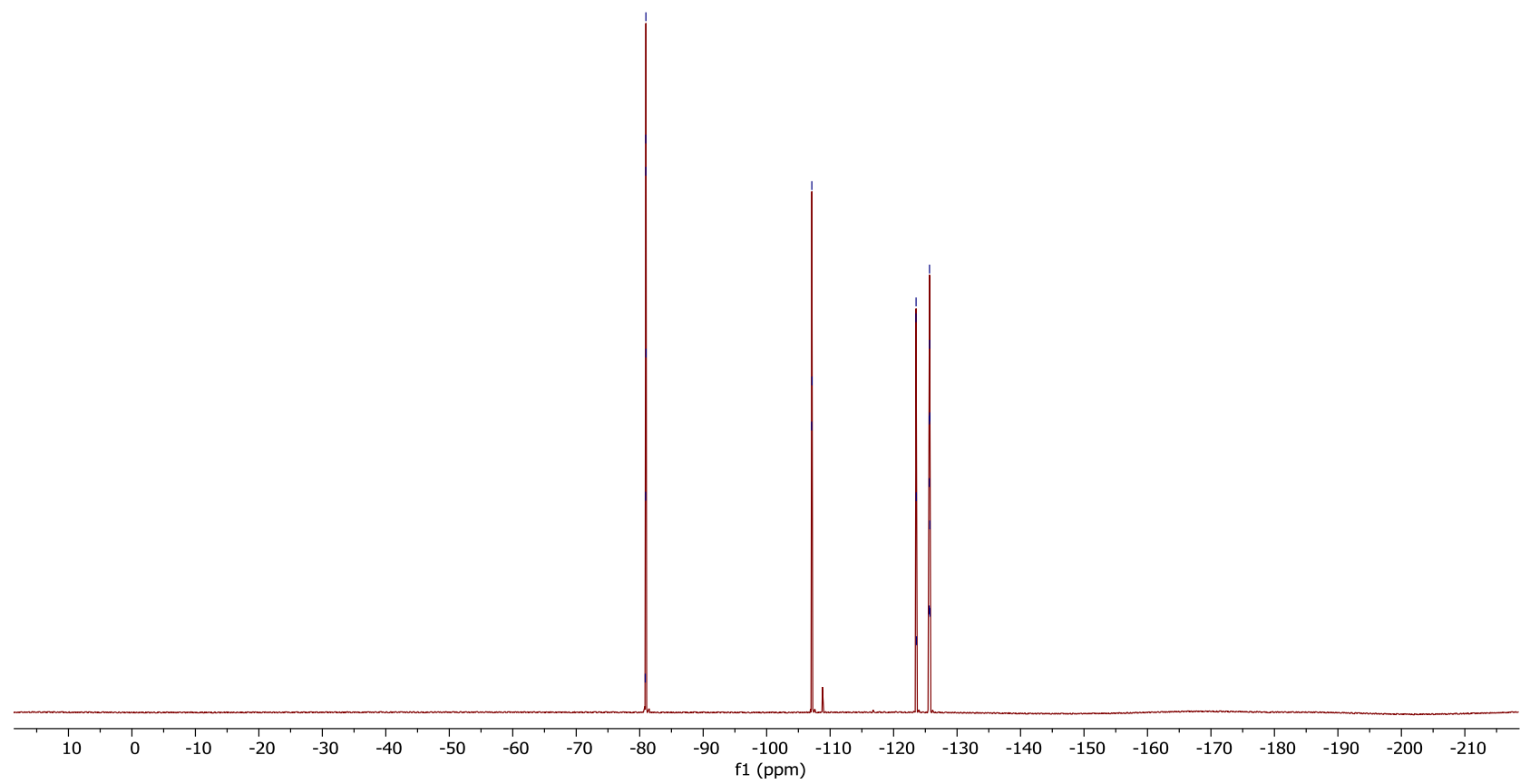


Phenyl (2E)-4,4,5,5,6,6,7,7,7-nonafluoro-2-(2-methoxyphenyl)hept-2-enoate (9a) GAT-02-25-rp-F1.1.fid

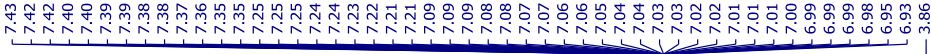<smiles>COc1ccccc1/C(=C\C(F)(F)F)C(=O)Oc1ccccc1</smiles>

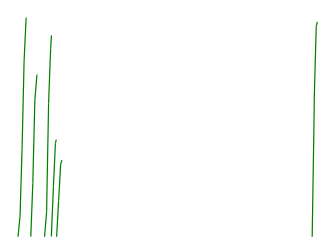

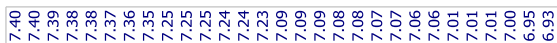
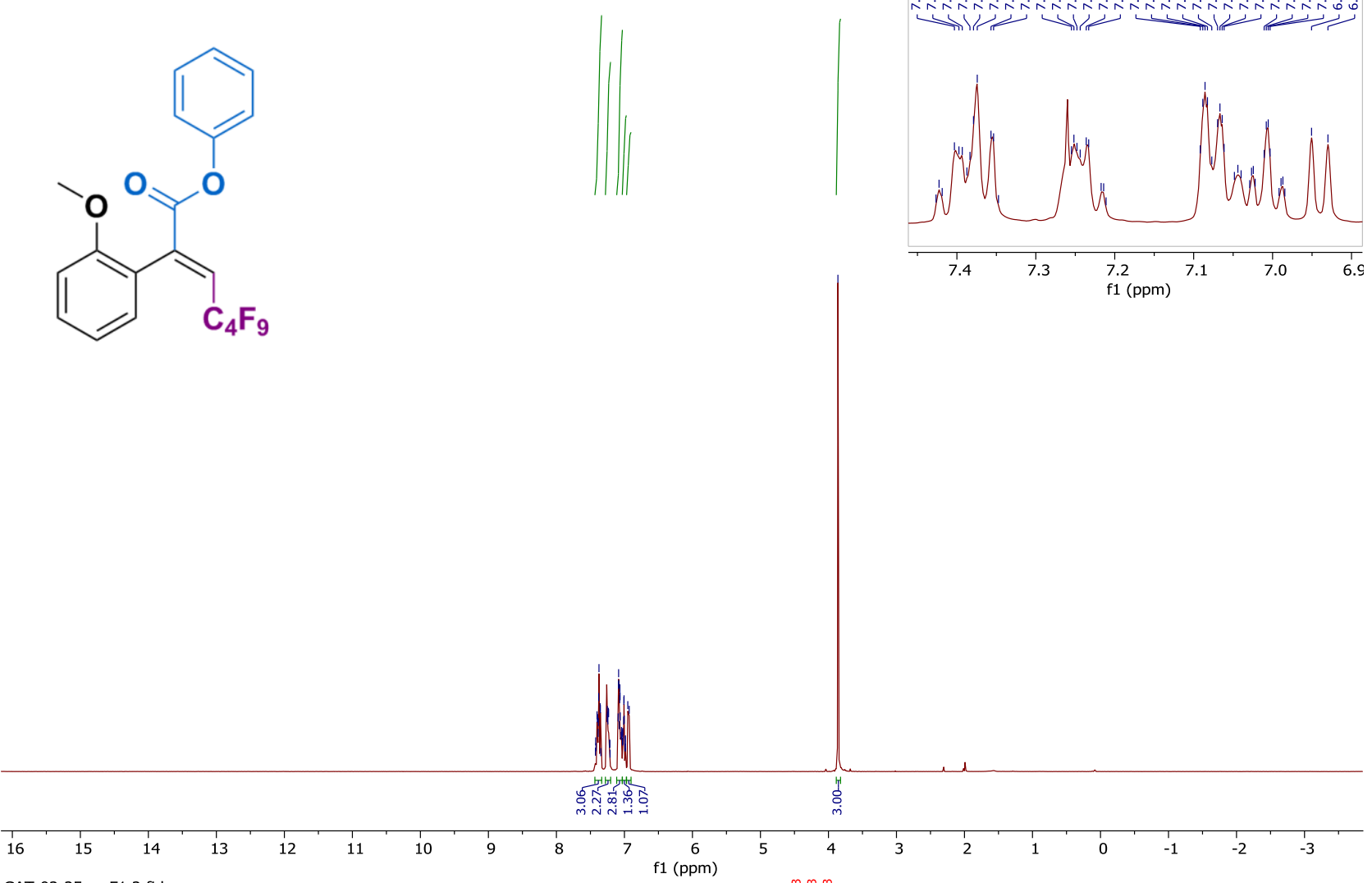

GAT-02-25-rp-F1.2.fid

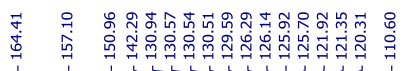

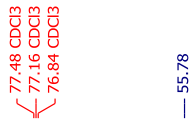

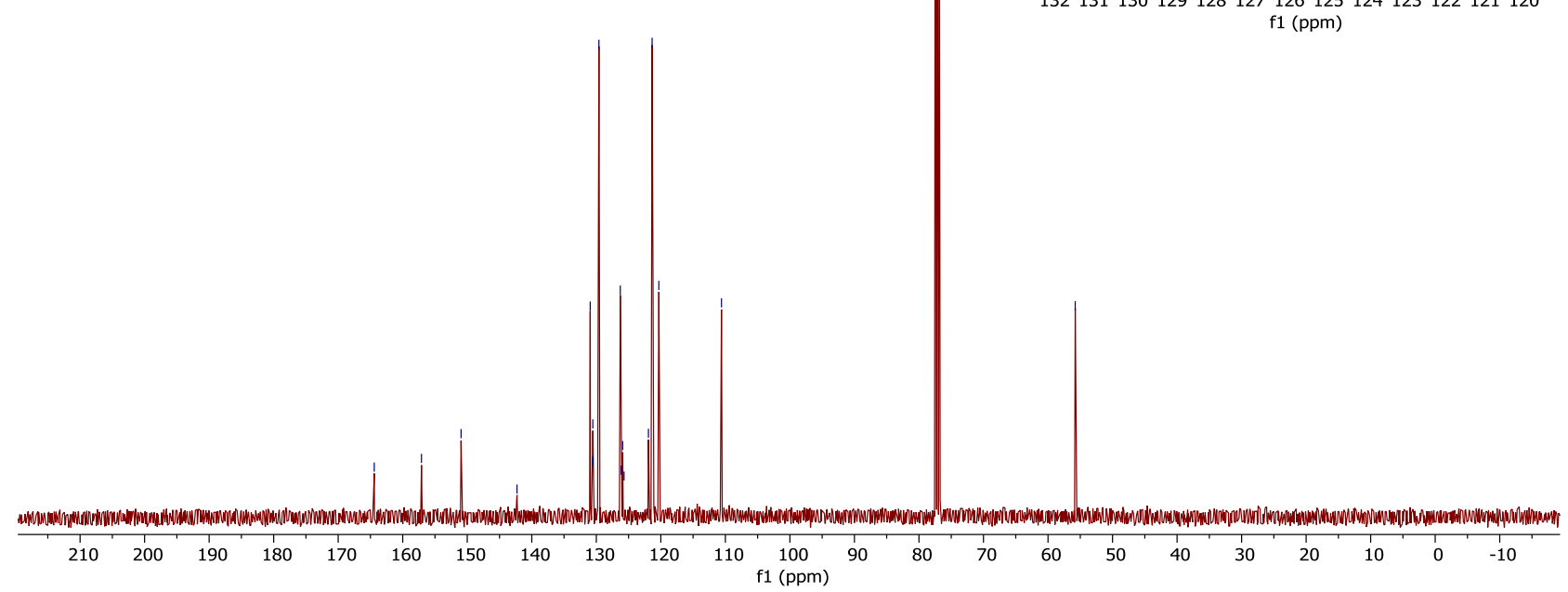




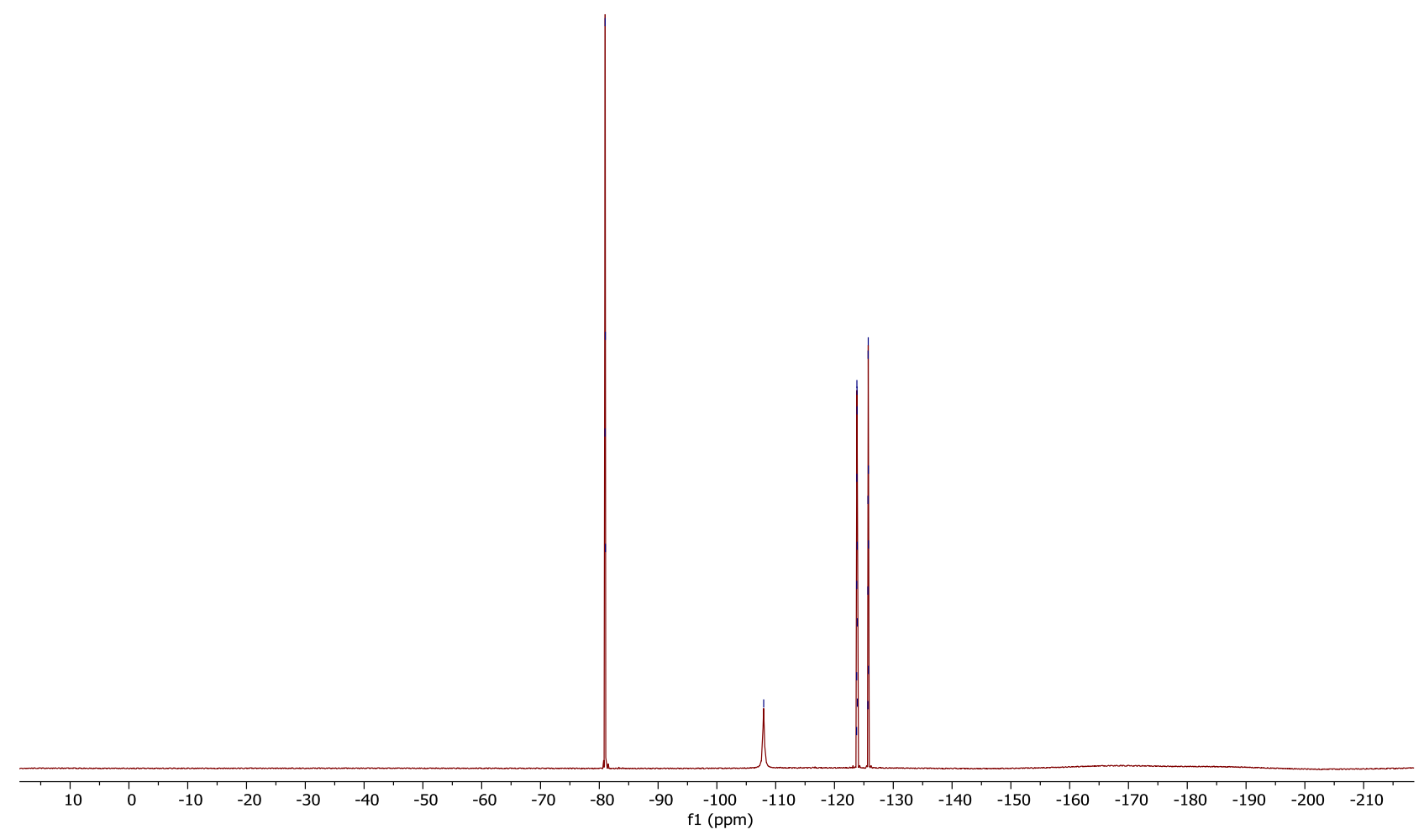


Phenyl (2E)-4,4,5,5,6,6,7,7,7-nonafluoro-2-(4-(4,4,5,5-tetramethyl-1,3,2-dioxaborolan-2-yl)phenyl)hept-2-enoate (10) GAT-02-75-rp1.1.fid
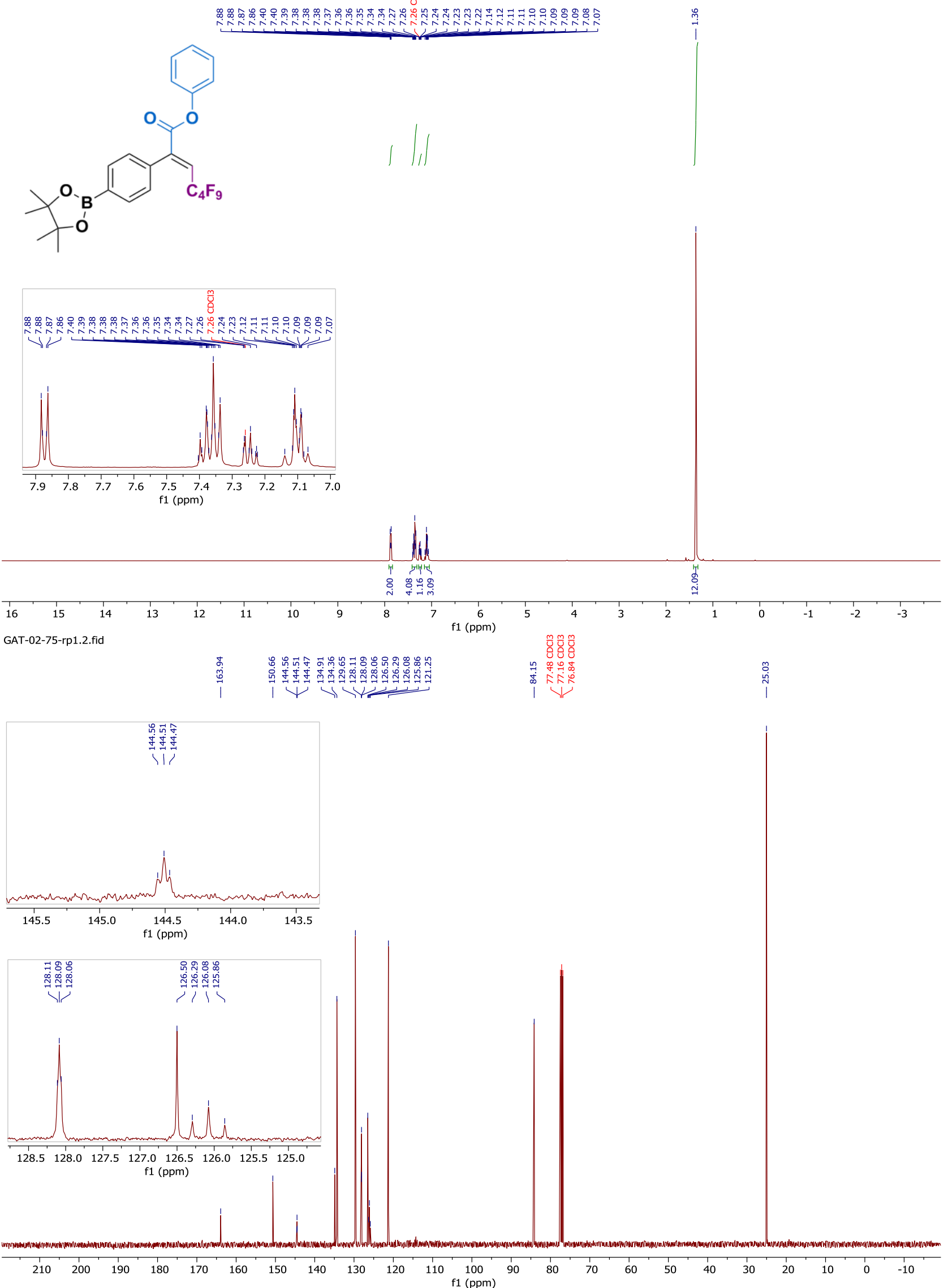


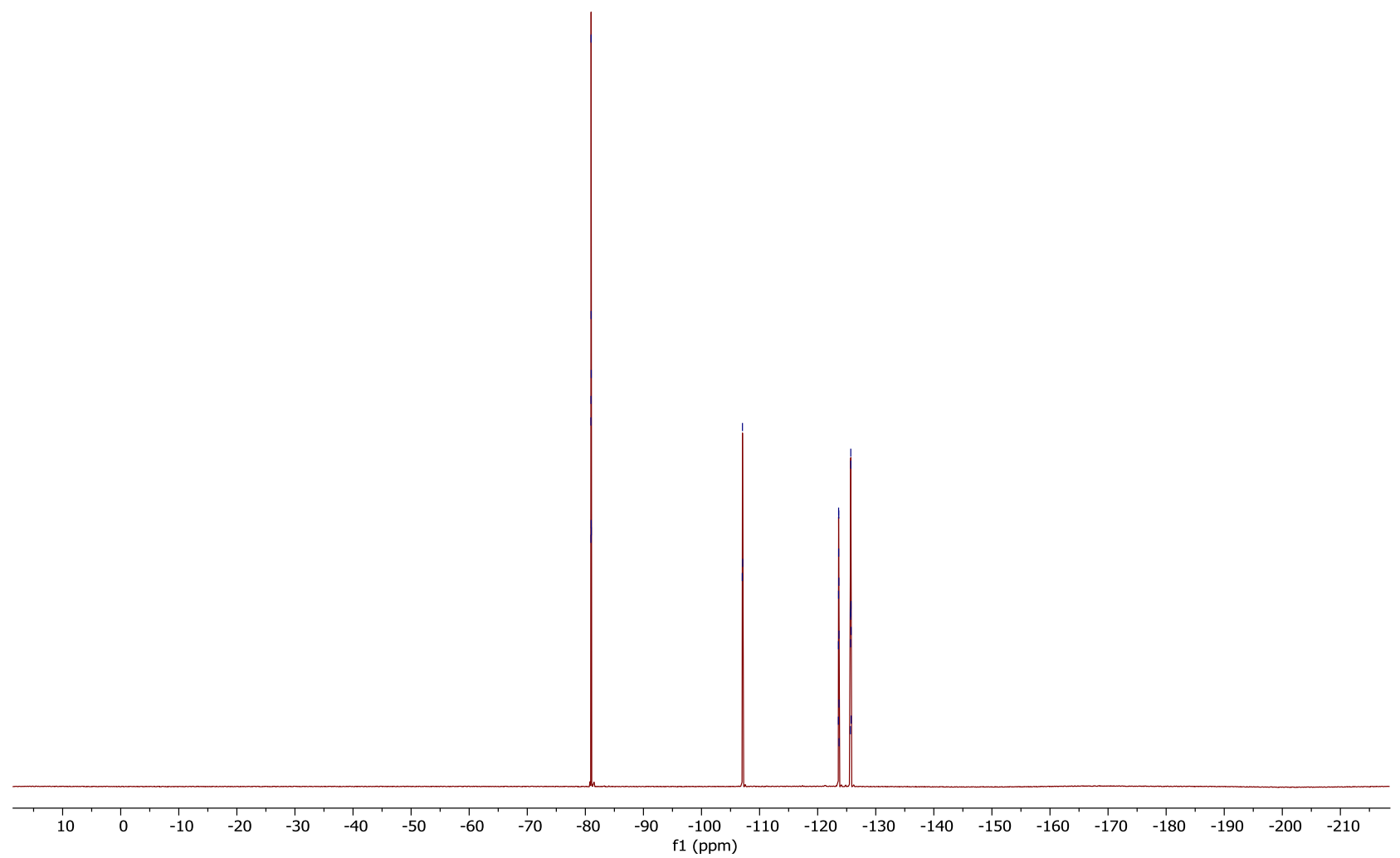


Phenyl (2E)-4,4,5,5,6,6,7,7,7-nonafluoro-2-(4-(trifluoromethyl)phenyl)hept-2-enoate (11a) GAT-02-34-rp-F1.1.fid<smiles>O=C(Oc1ccccc1)/C(=C\C(F)(F)F)c1ccc(C(F)(F)F)cc1</smiles>
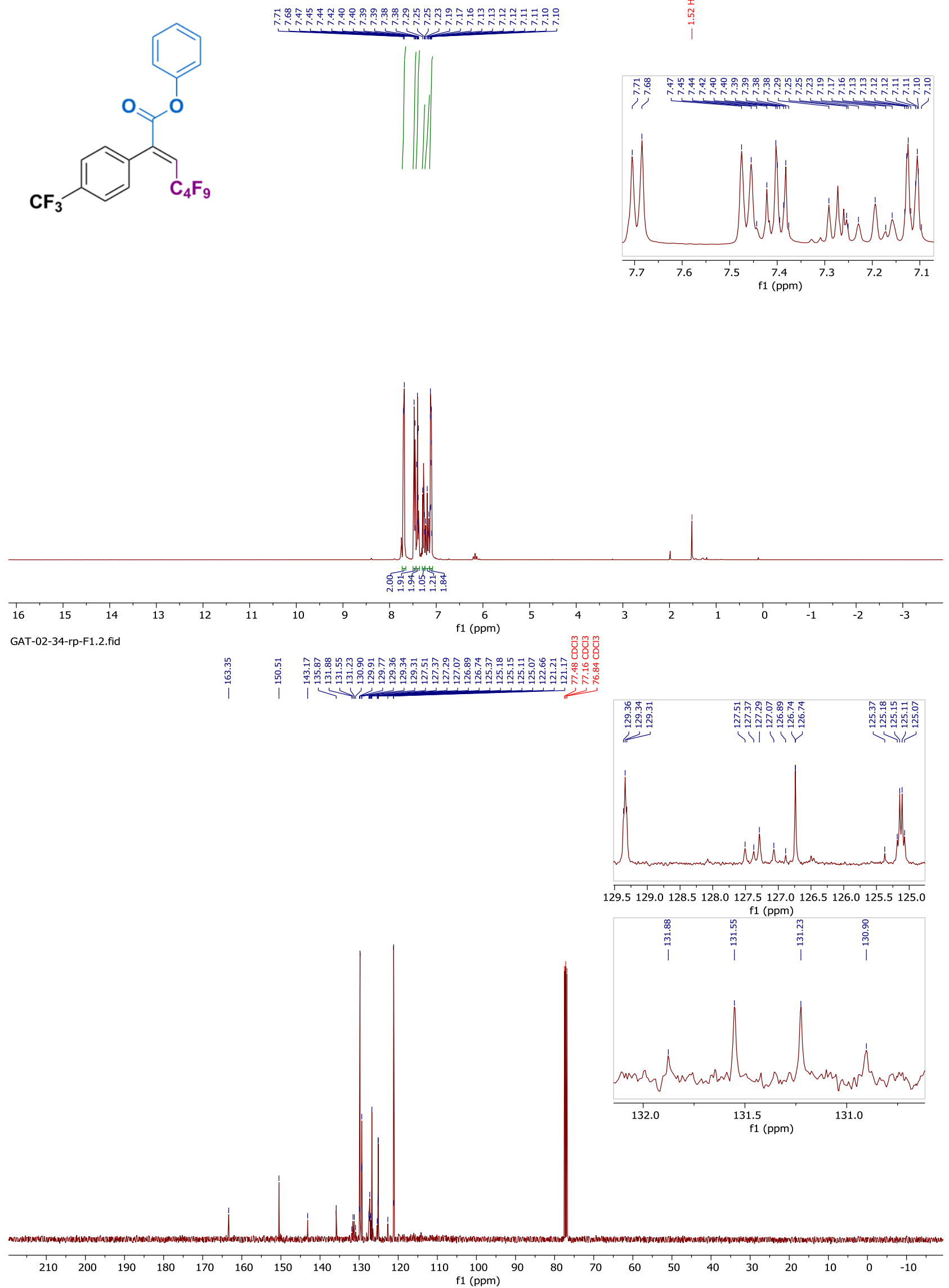


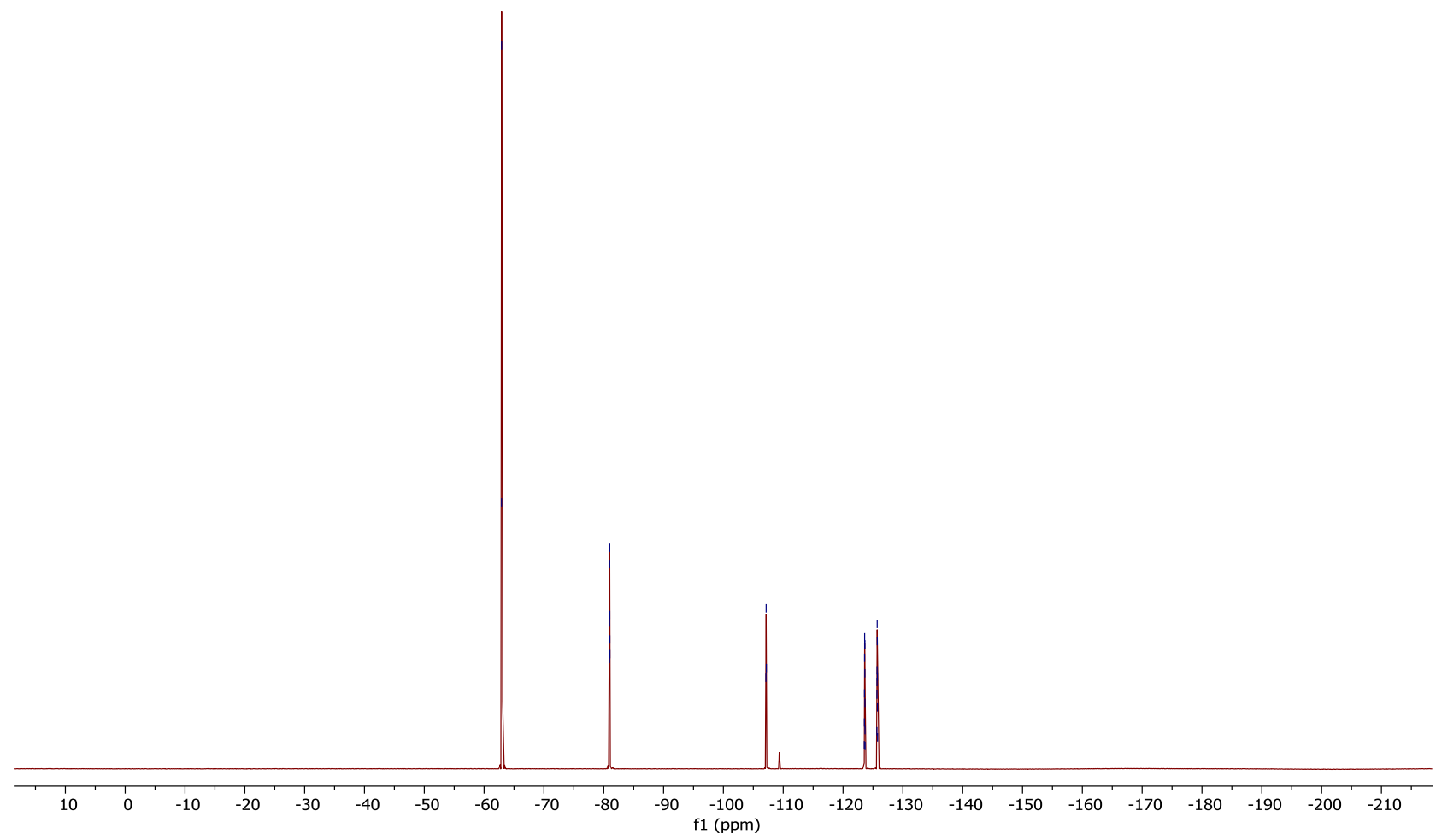


Phenyl (2E)-4,4,5,5,6,6,7,7,7-nonafluoro-2-(4-fluorophenyl)hept-2-enoate (12)

GAT-02-26-F2-rp.1.fid<smiles>O=C(Oc1ccccc1)/C(=C/C(F)(F)F)c1ccc(F)cc1</smiles>

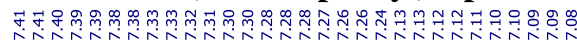

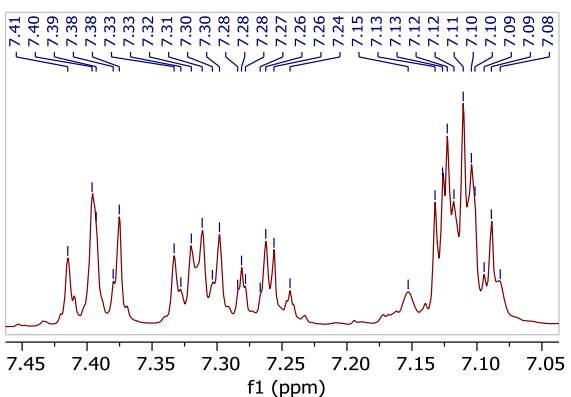

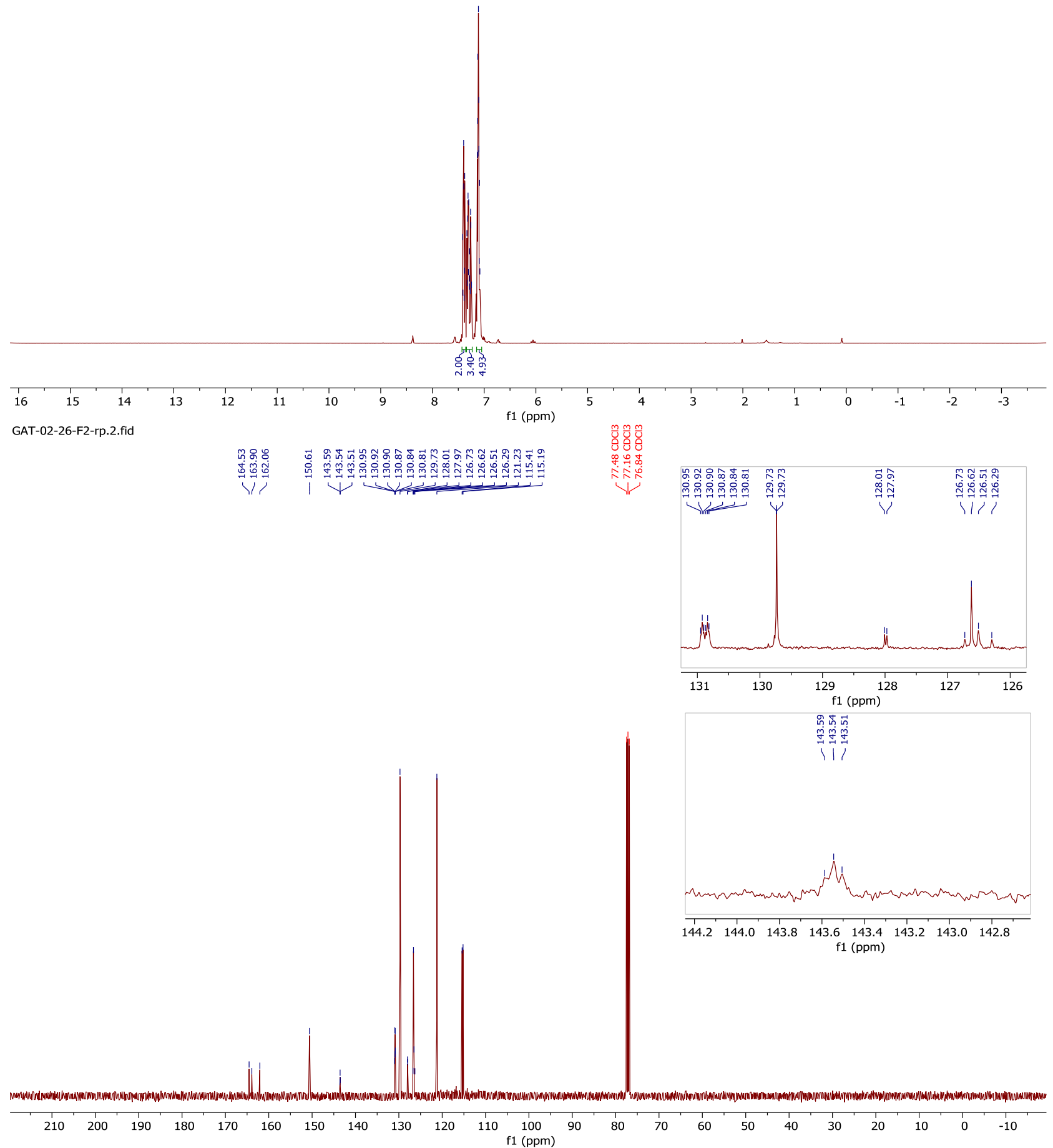




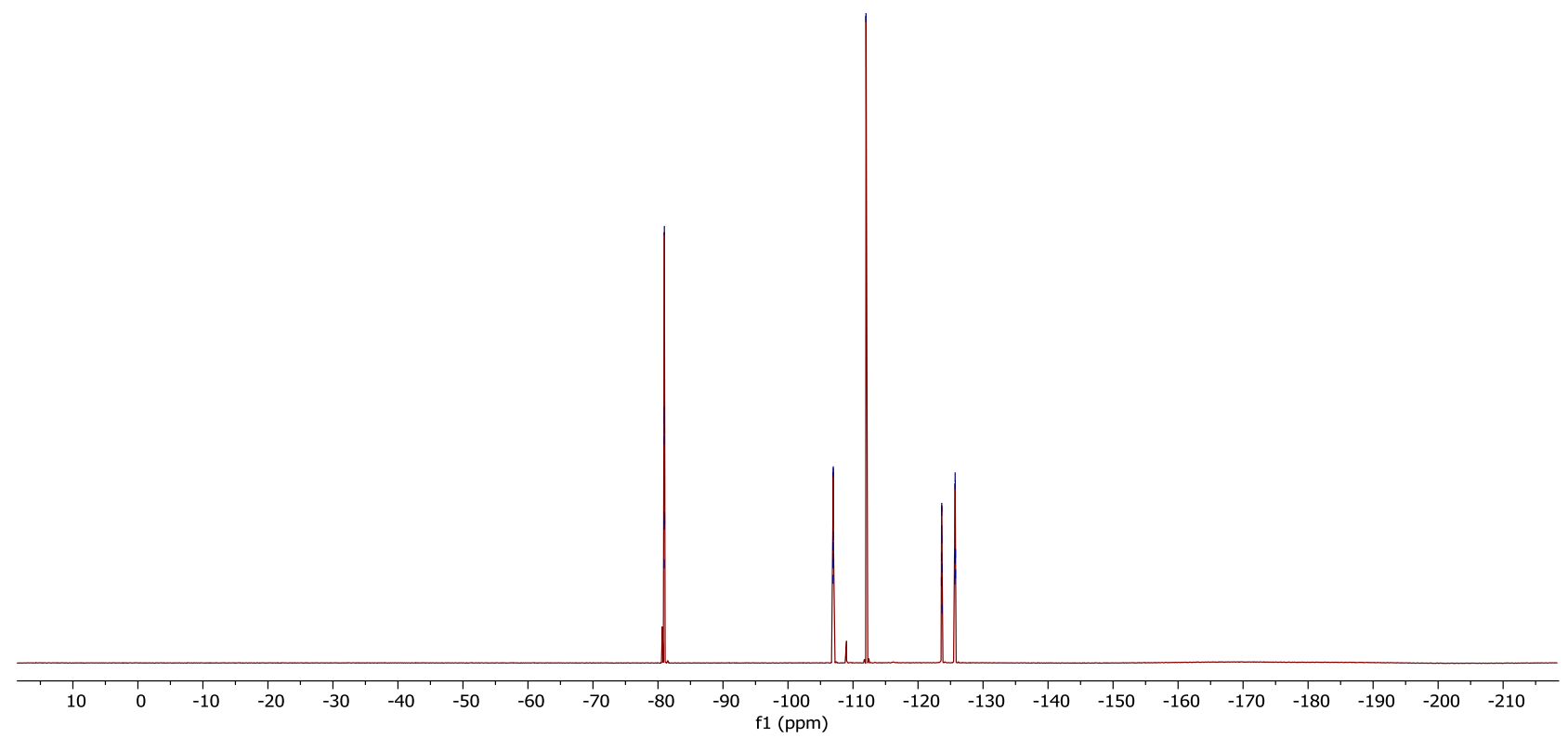


Phenyl (2E)-2-(4-chlorophenyl)-4,4,5,5,6,6,7,7,7-nonafluorohept-2-enoate (13)

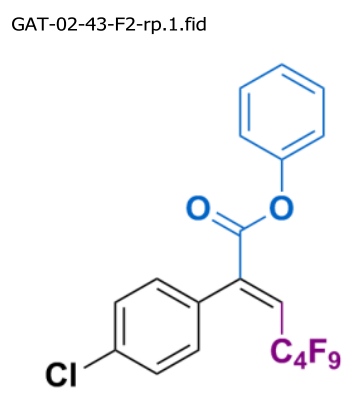

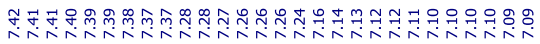
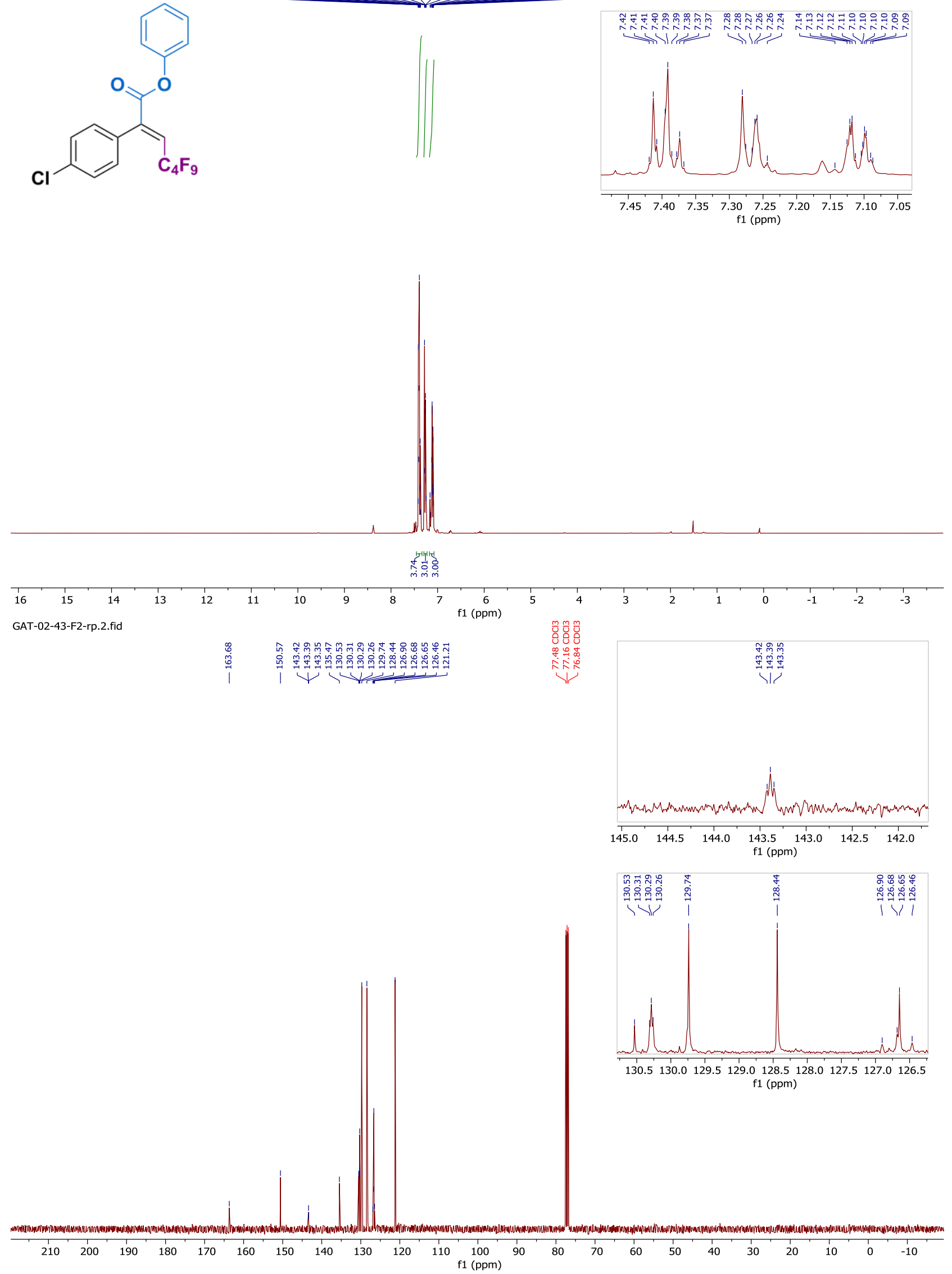


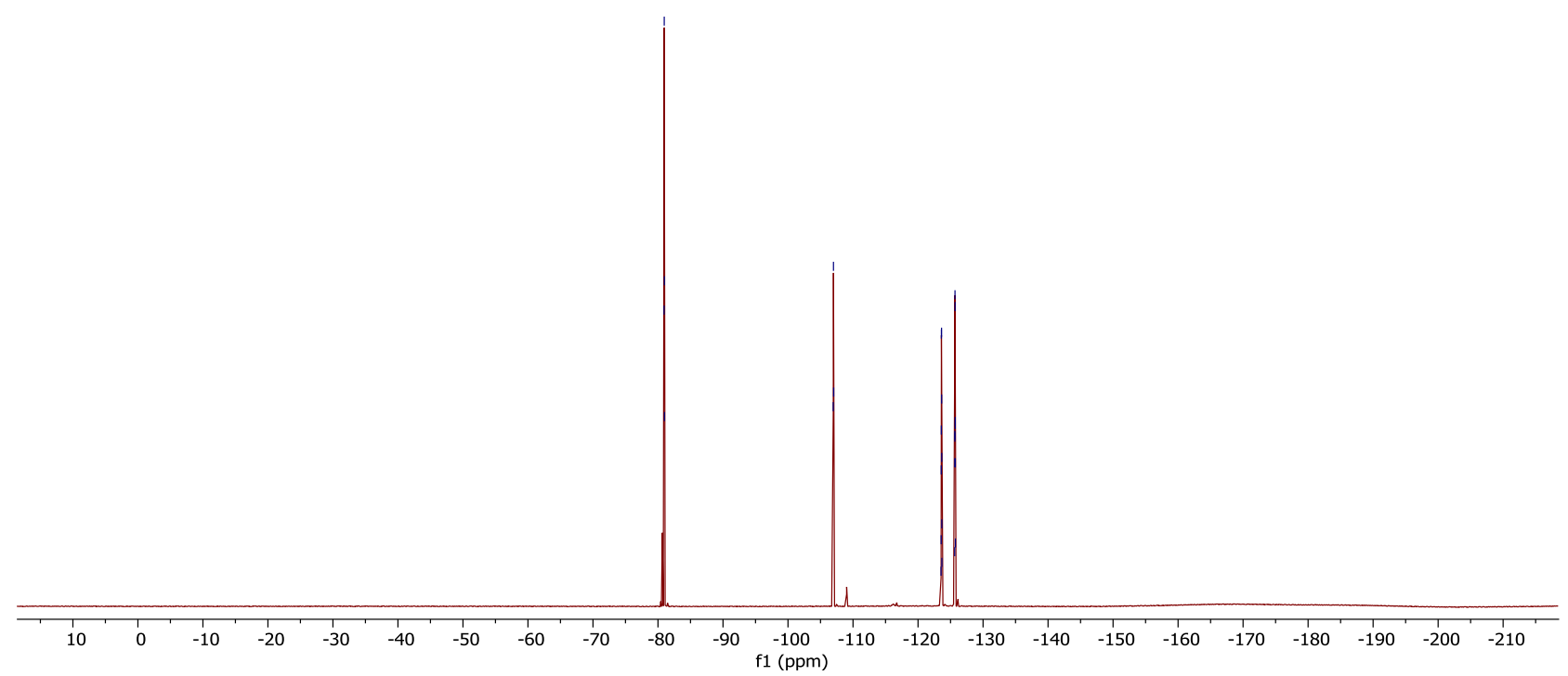


Phenyl (2E)-2-(4-bromophenyl)-4,4,5,5,6,6,7,7,7-nonafluorohept-2-enoate (14) GAT-02-56-F2-rp.1.fid

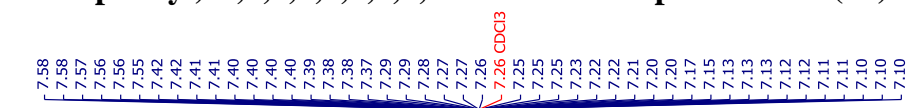<smiles>O=C(Oc1ccccc1)/C(=C/C(F)(F)F)c1ccc(Br)cc1</smiles>
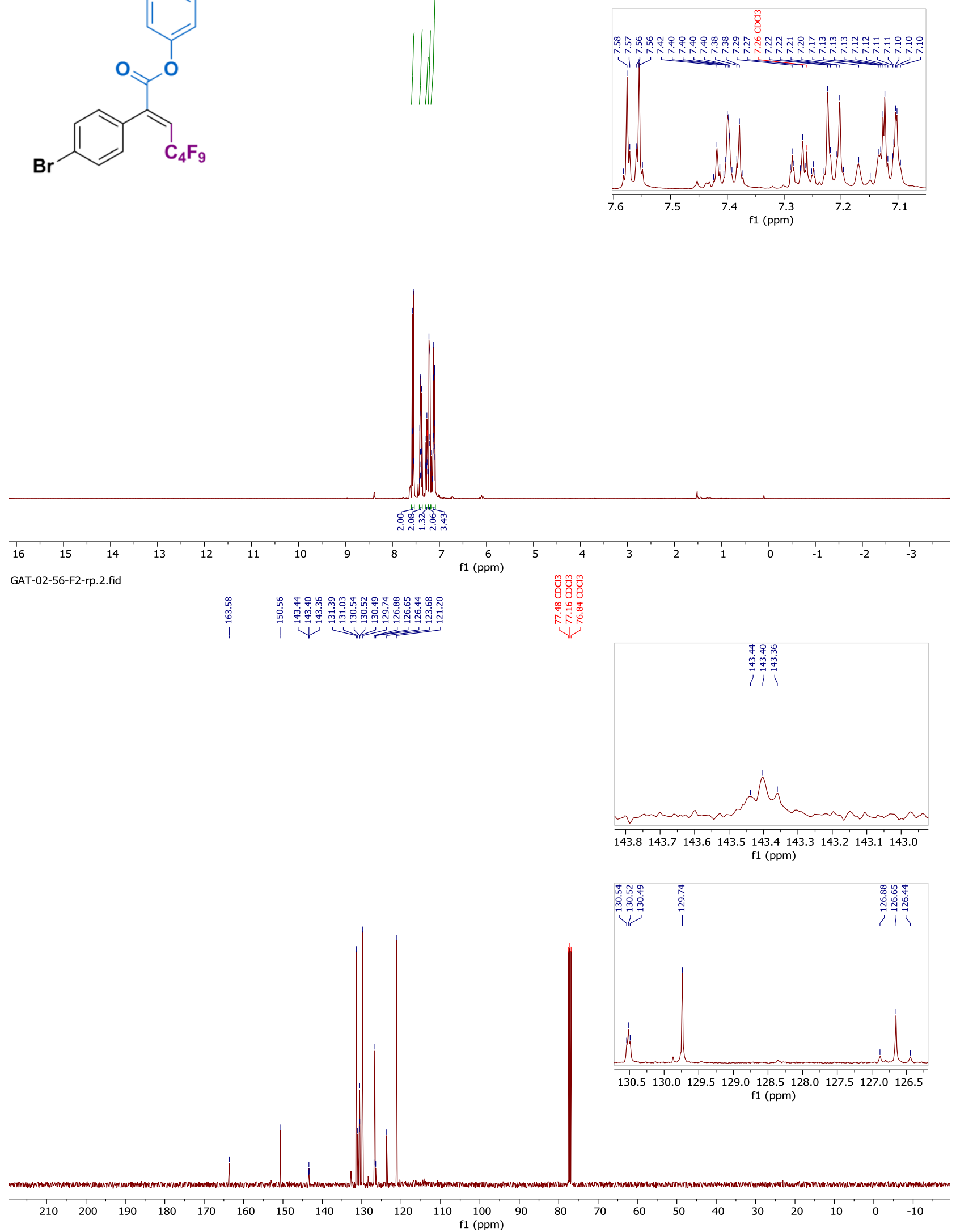


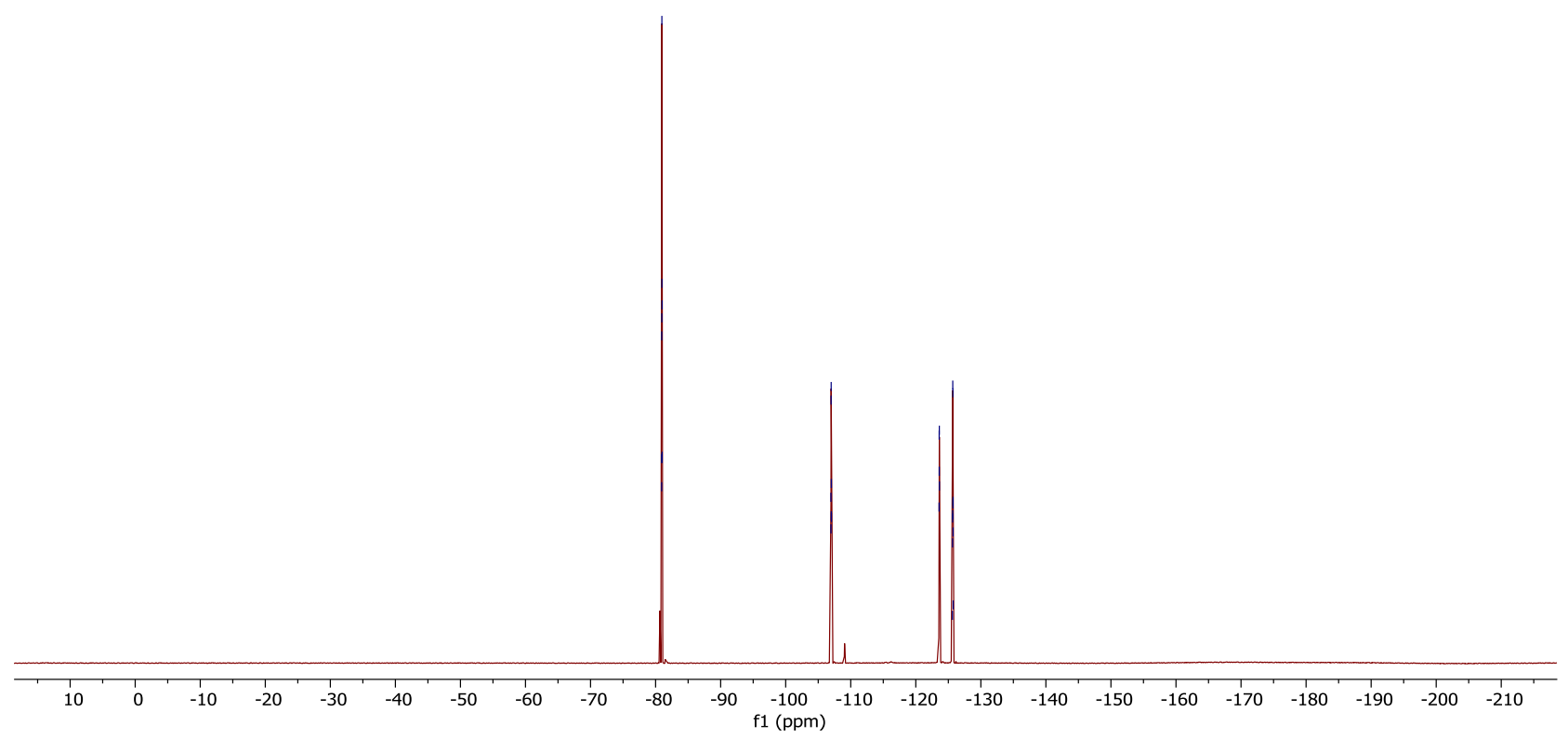


Phenyl (2E)-2-(2-bromophenyl)-4,4,5,5,6,6,7,7,7-nonafluorohept-2-enoate (15) GAT-02-57-F4-rp1.1.fid<smiles>O=C(Oc1ccccc1)/C(=C/C(F)(F)F)c1ccccc1Br</smiles>
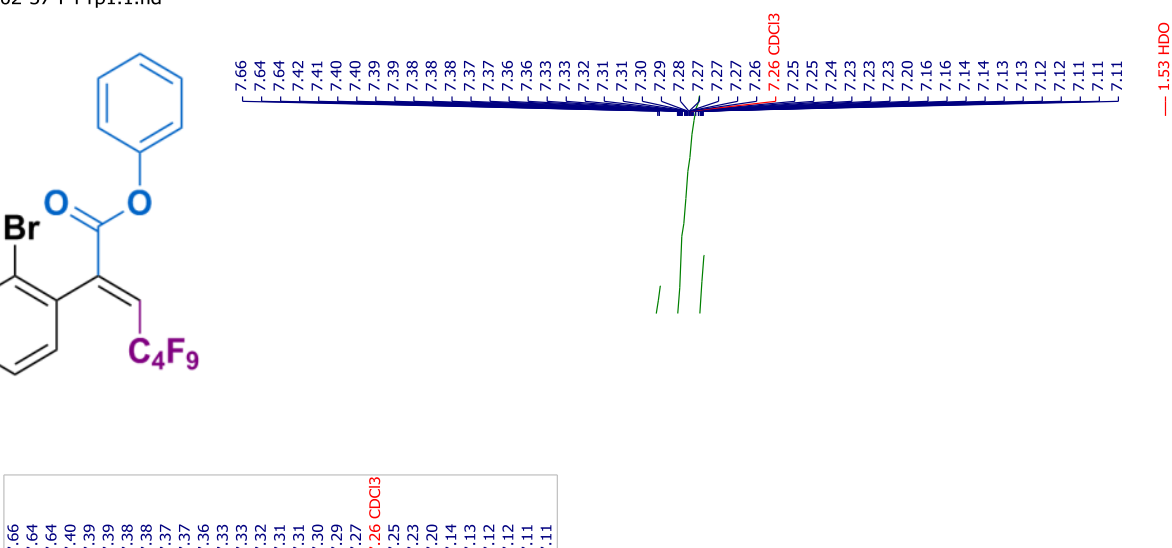

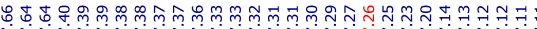
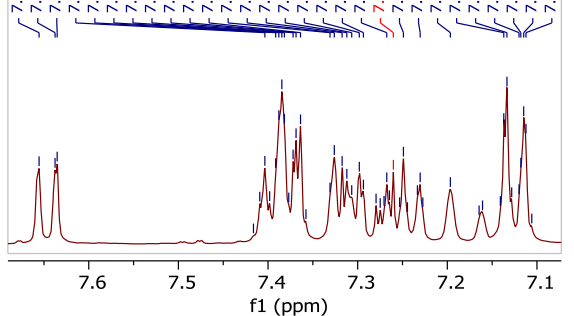

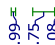
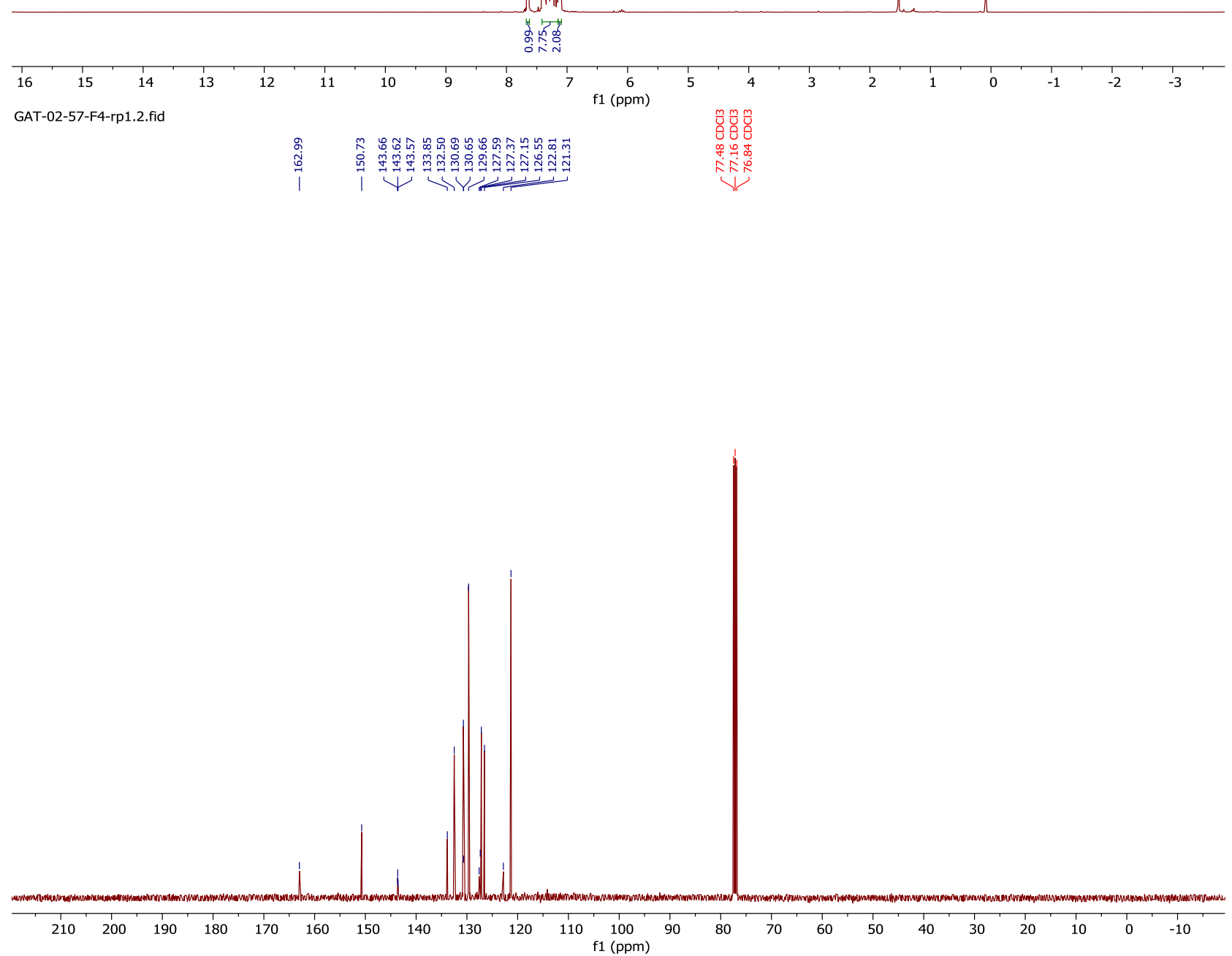


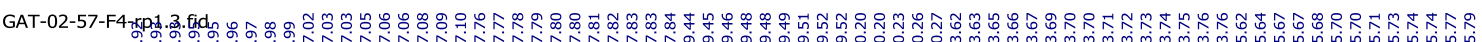

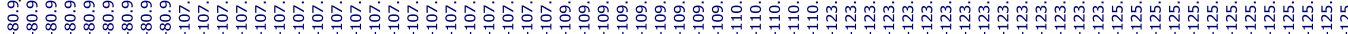

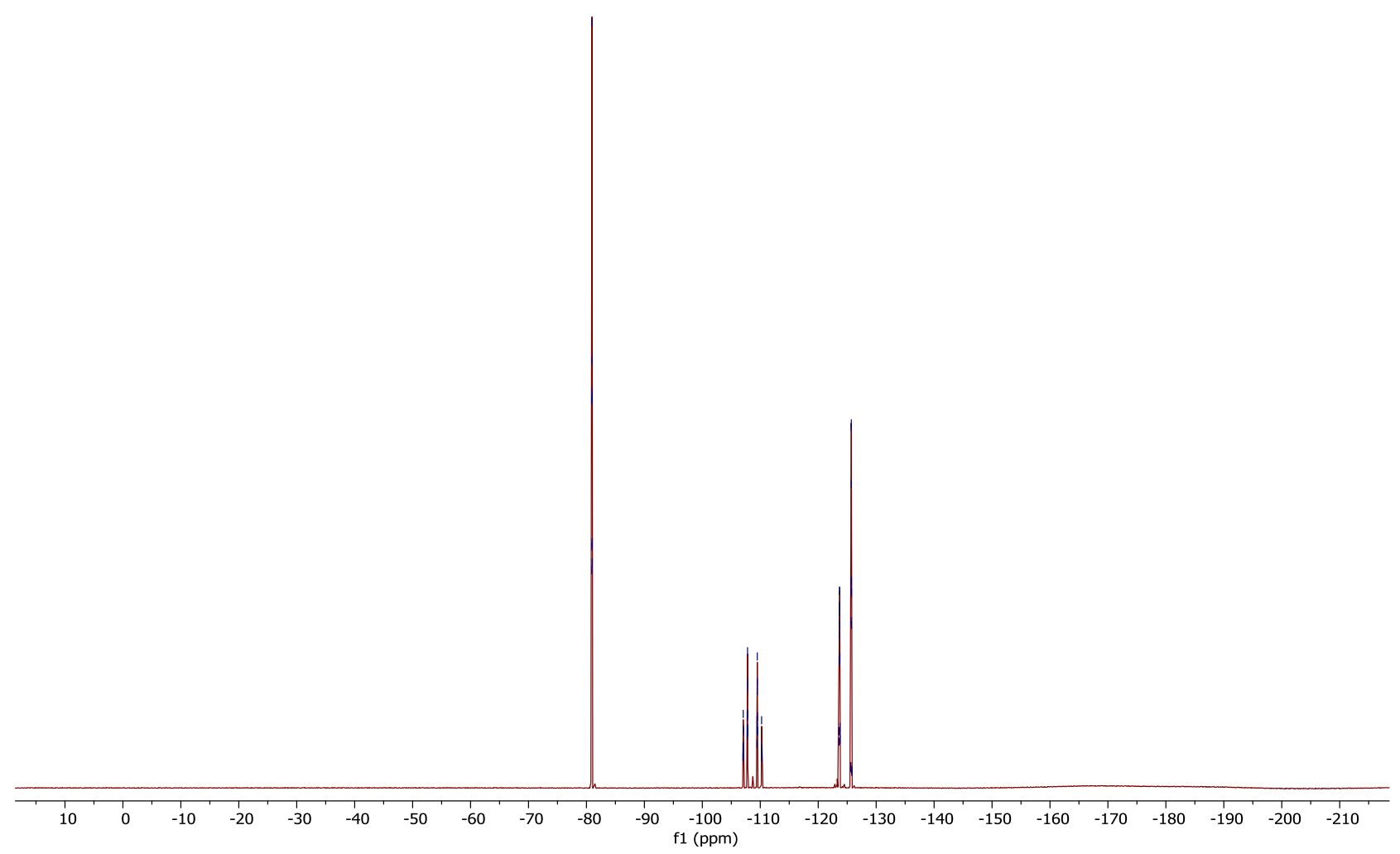


Phenyl (2E)-4,4,5,5,6,6,7,7,7-nonafluoro-2-(thiophen-3-yl)hept-2-enoate (16a)<smiles>O=C(Oc1ccccc1)C(=CC(F)(F)F)c1ccsc1</smiles>
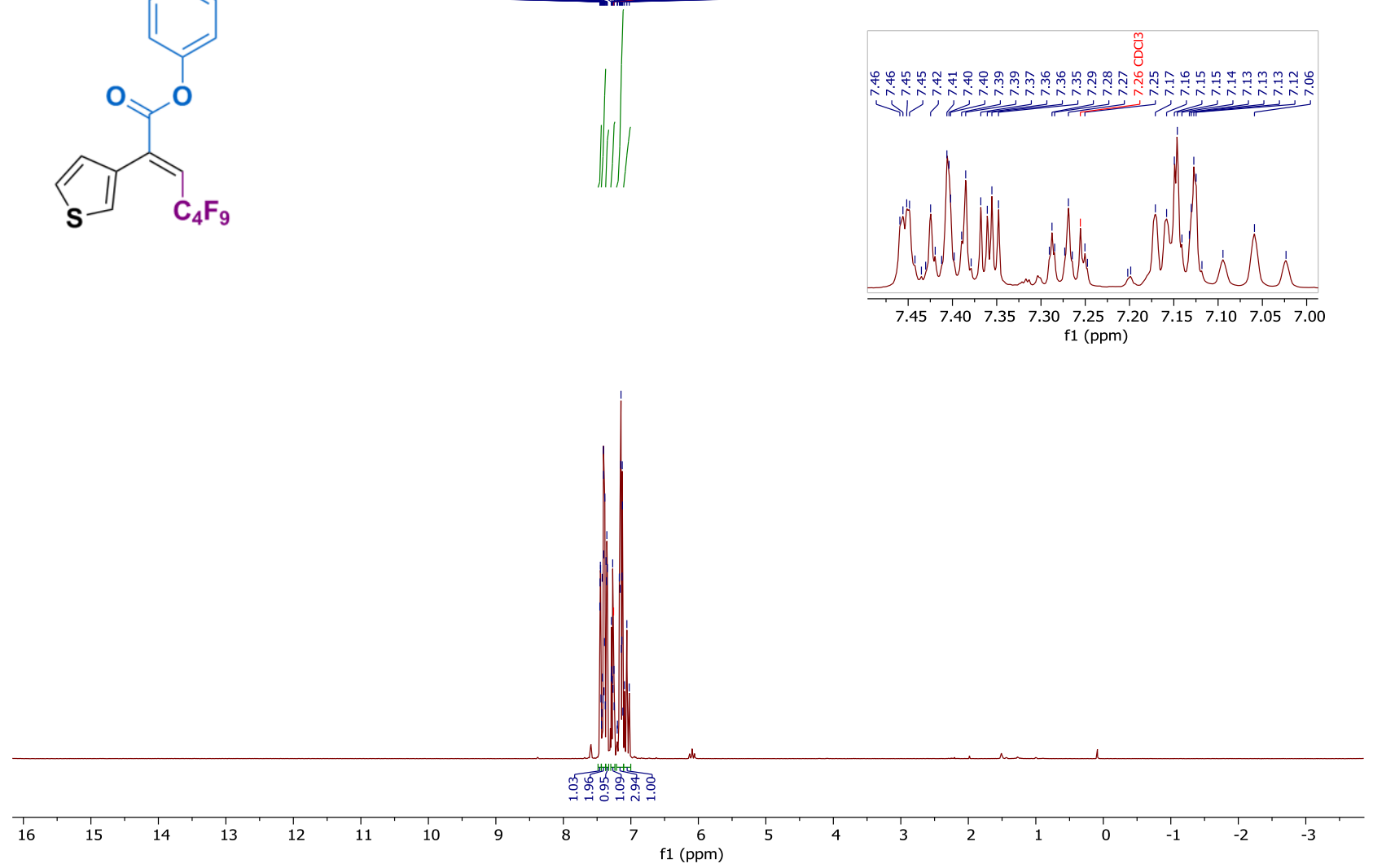

GAT-02-114-F3-rp.2.fid
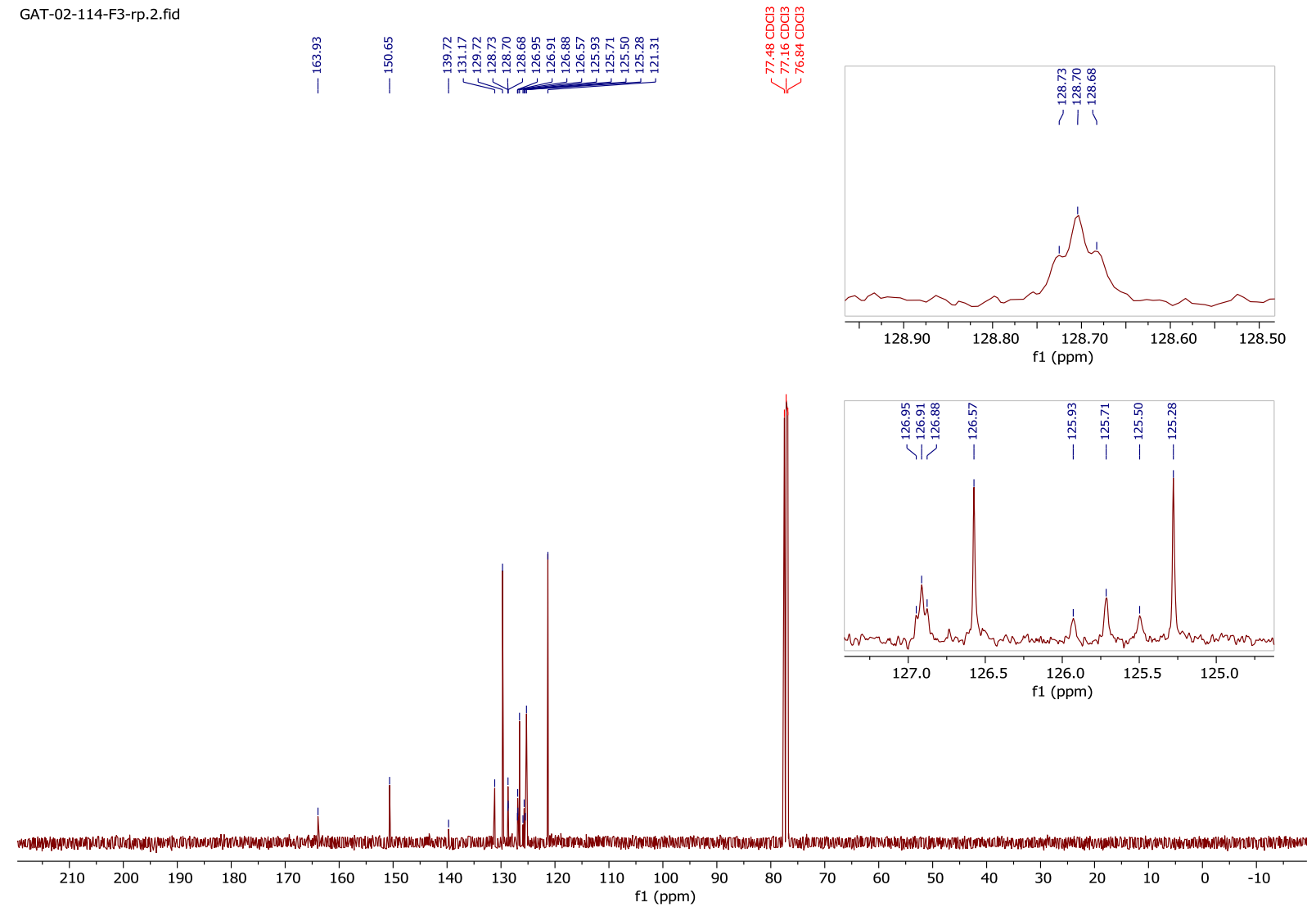


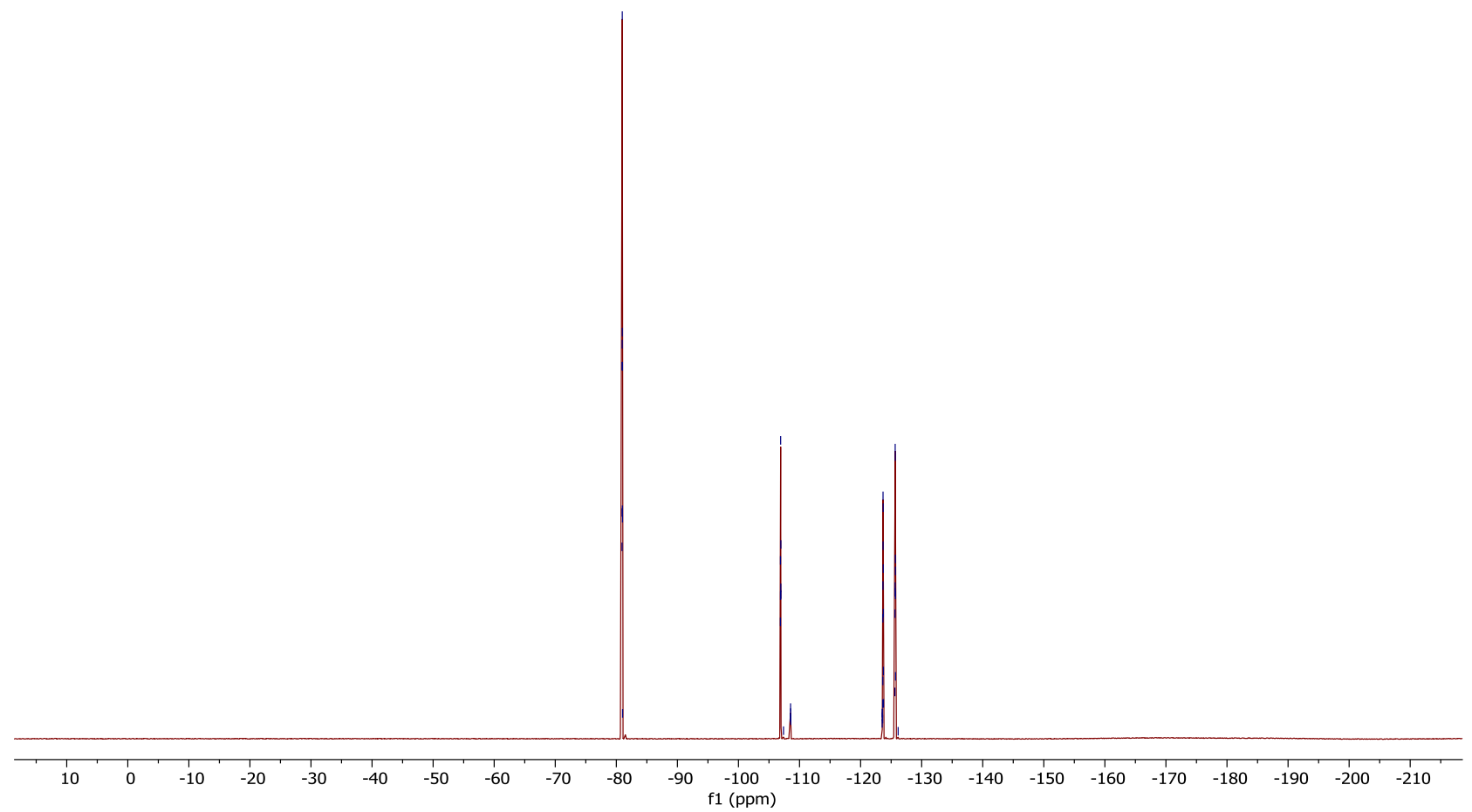


(E)-4-fluorophenyl 4,4,5,5,6,6,7,7,7-nonafluoro-2-(thiophen-3-yl)hept-2-enoate (16b)<smiles>O=C(Oc1ccc(F)cc1)C(=CC(F)(F)F)c1ccsc1</smiles>

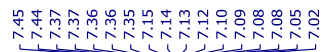
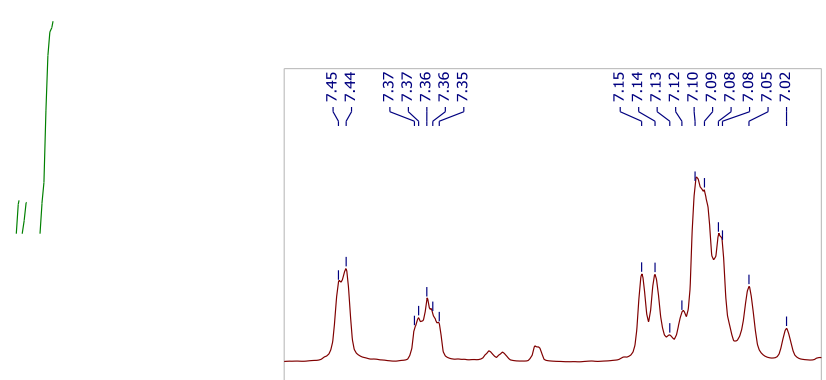

$\begin{array}{llllllllll}7.45 & 7.40 & 7.35 & 7.30 & 7.25 & 7.20 & 7.15 & 7.10 & 7.05 & 7.00\end{array}$

f1 (ppm)

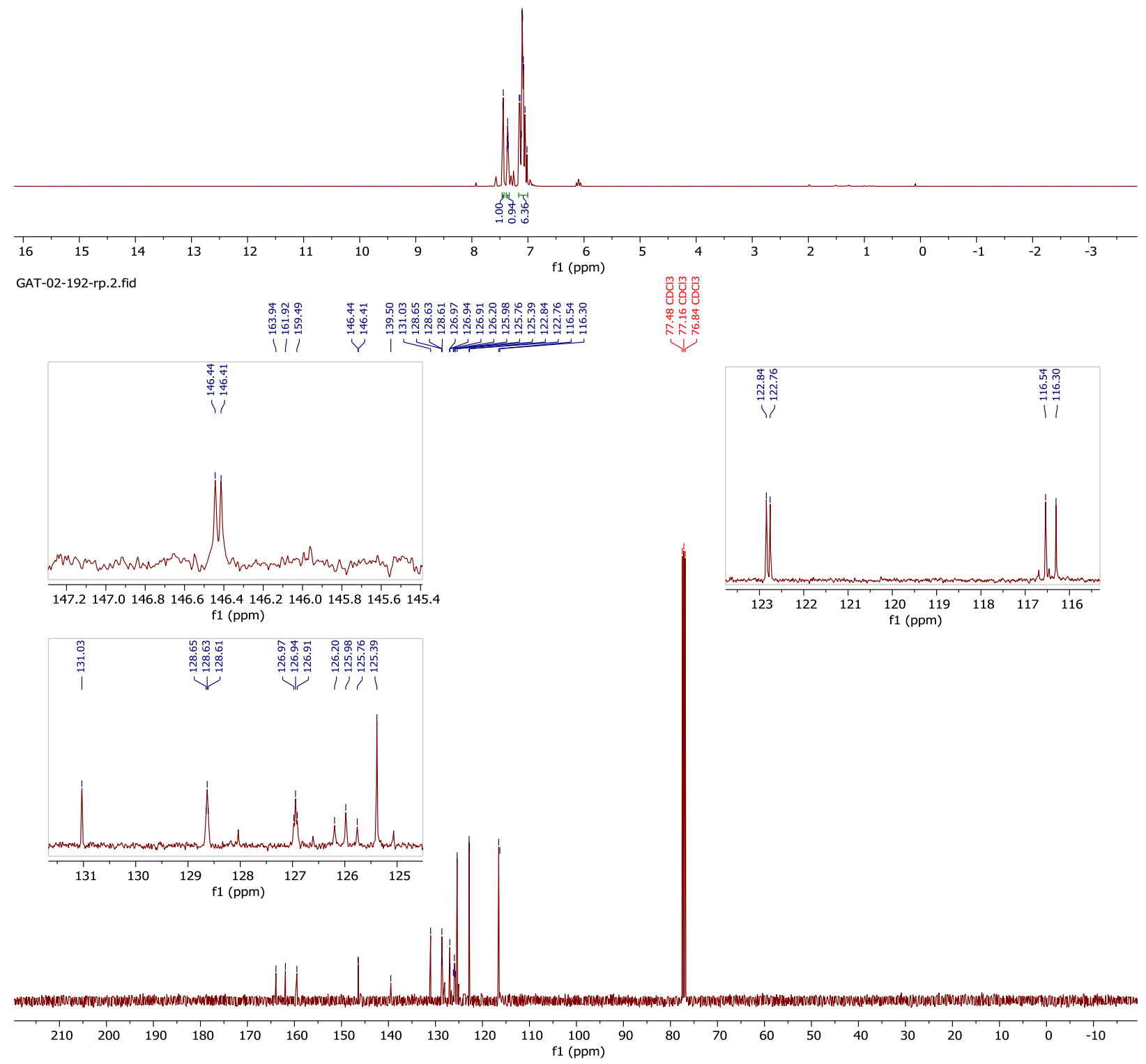




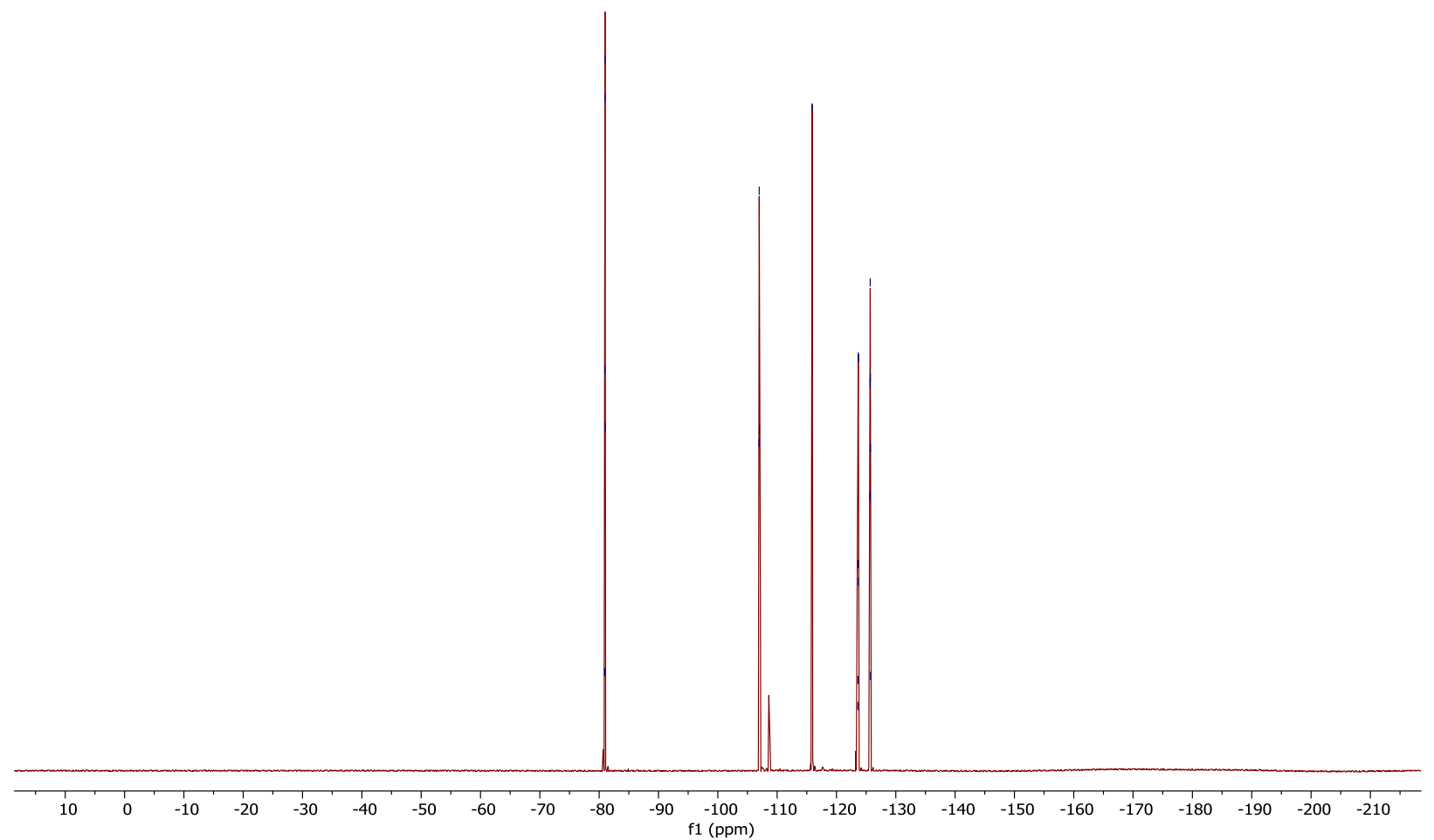


Phenyl (2E)-2-ferrocenyl-4,4,5,5,6,6,7,7,7-nonafluorohept-2-enoate $(E-17)$ GAT-02-55-F3-rp-F1_2.1.fid
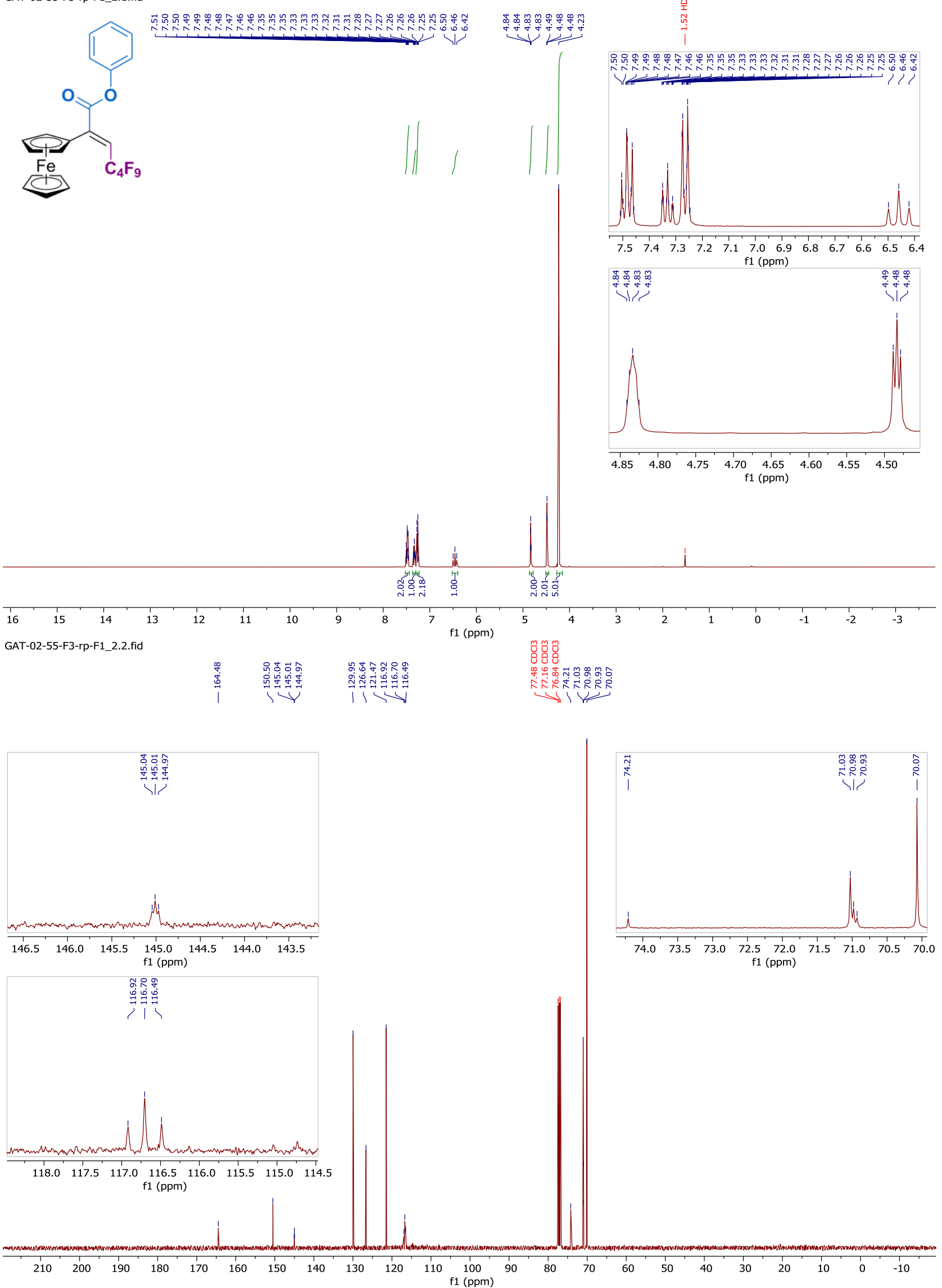


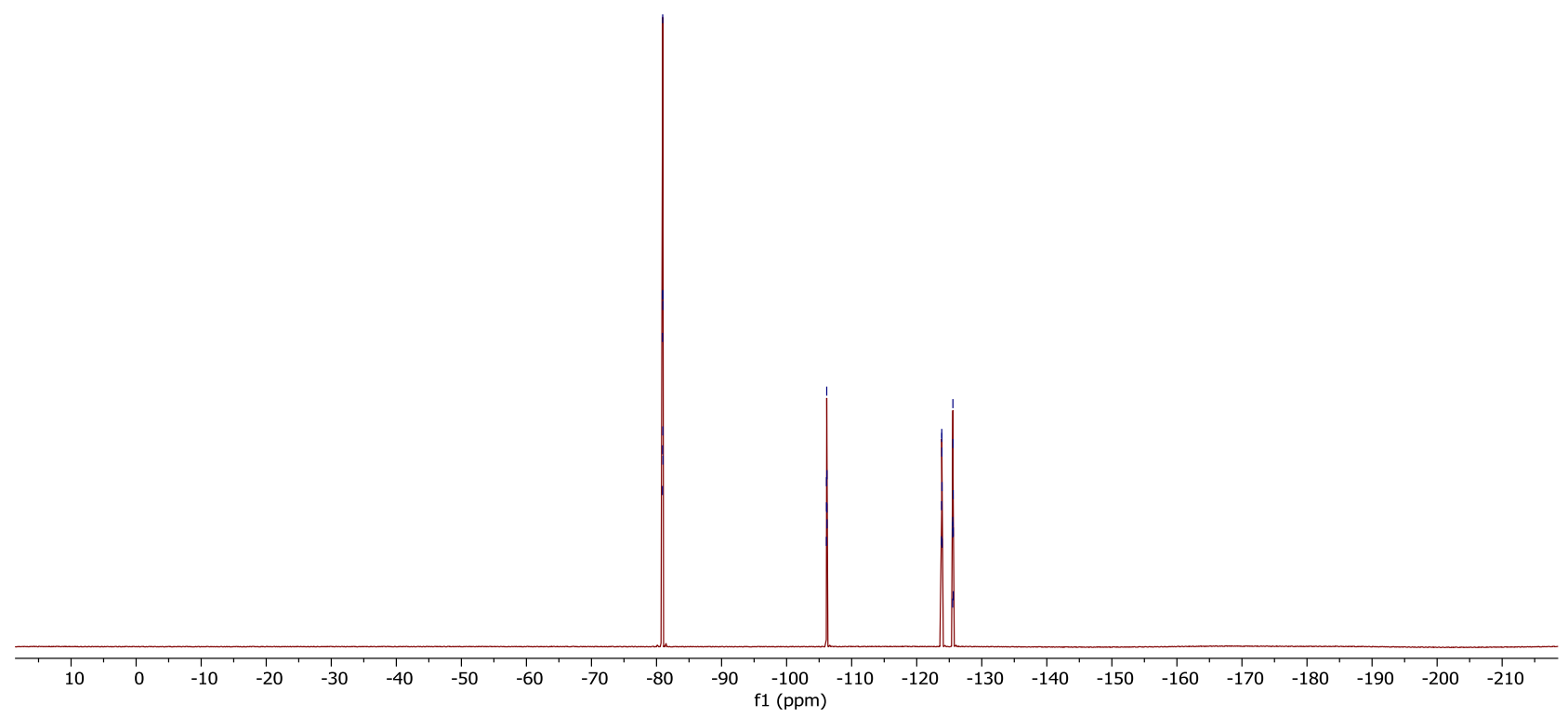


Phenyl (2Z)-2-ferrocenyl-4,4,5,5,6,6,7,7,7-nonafluorohept-2-enoate ( $Z$-17)
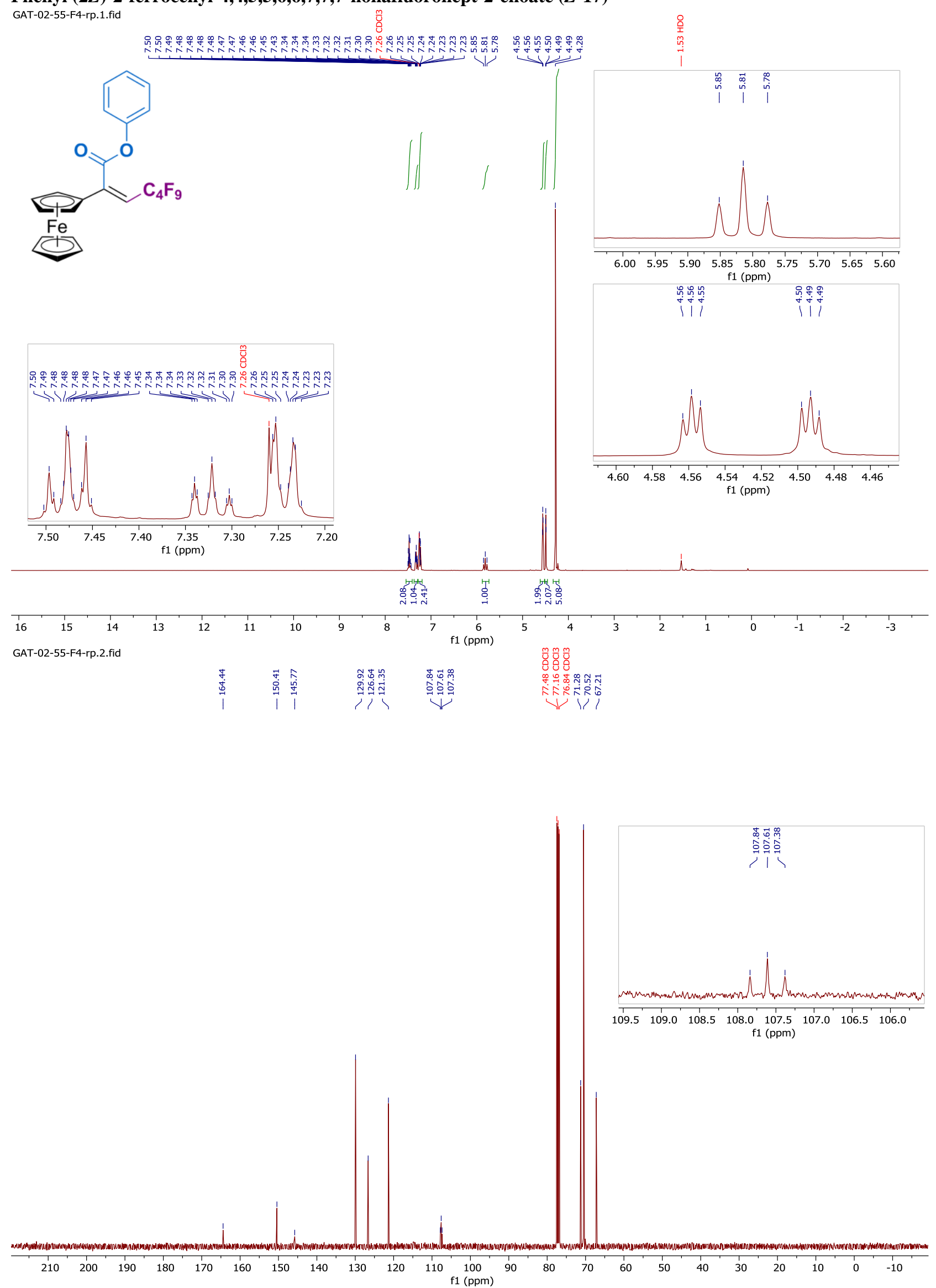


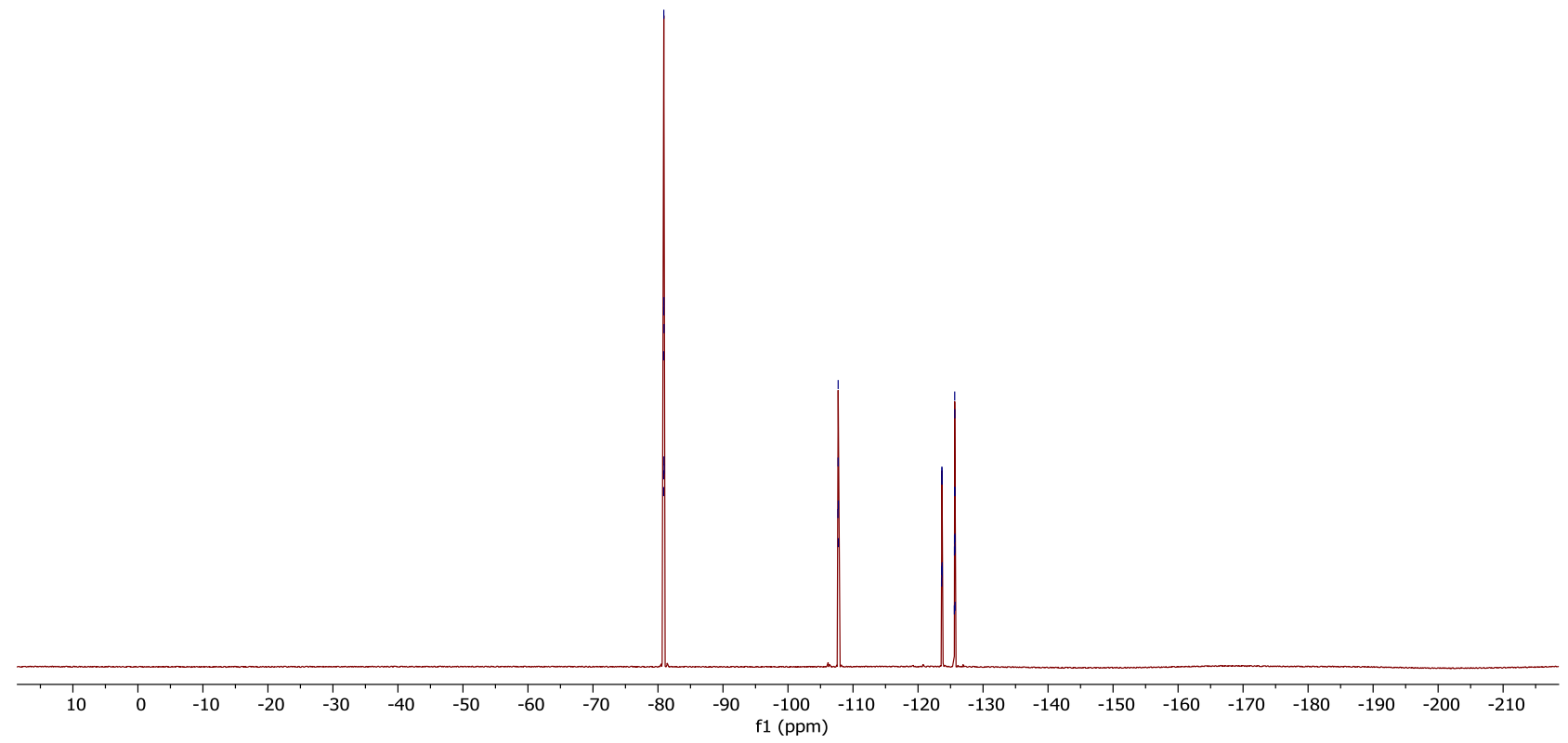


Phenyl (2E)-2-(2,2,3,3,4,4,5,5,5-nonafluoropentylidene)octanoate (18) GAT-02-40-F2-rp.1.fid<smiles>CCCCCC/C(=C\C(F)(F)F)C(=O)Oc1ccccc1</smiles>

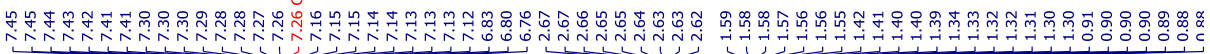
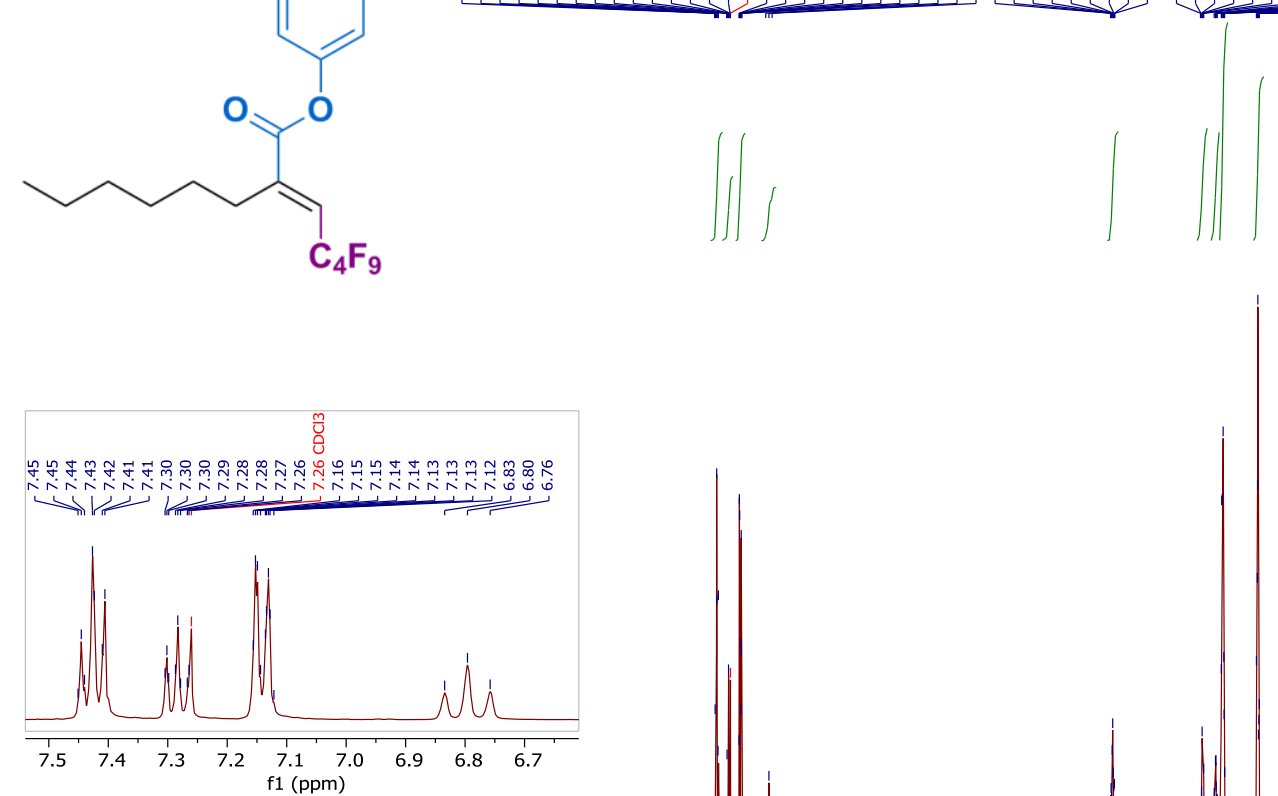

(at)

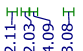

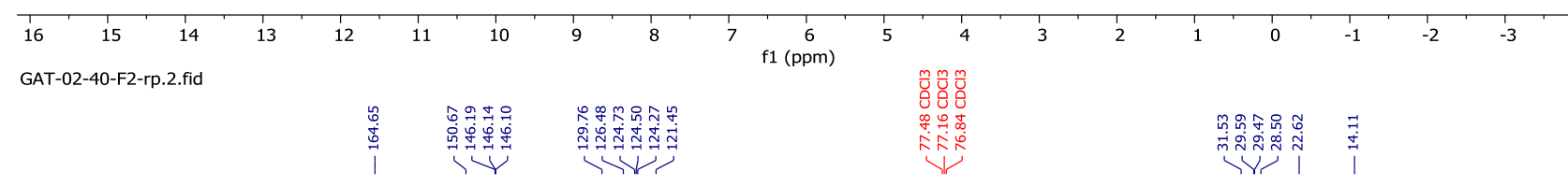
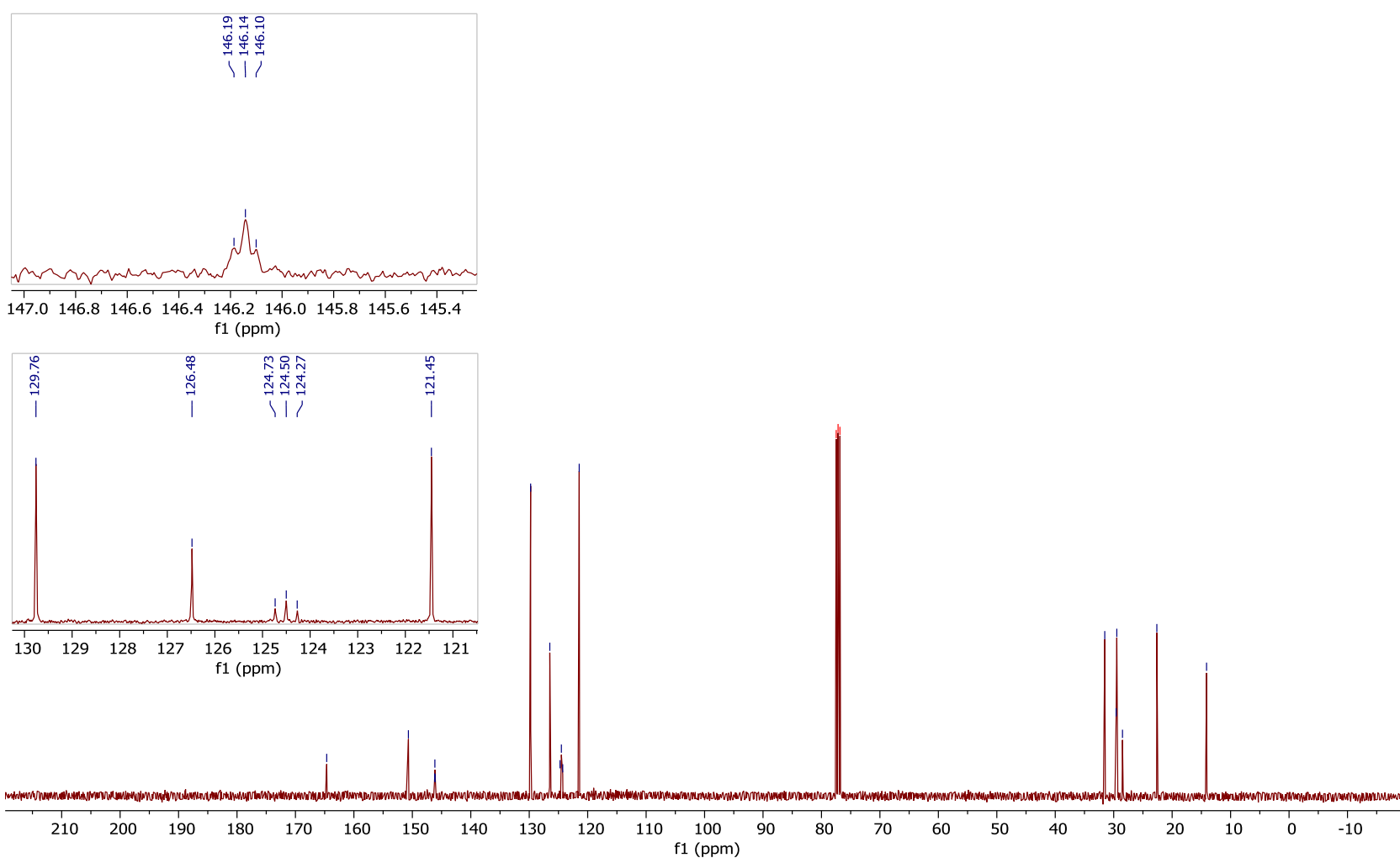


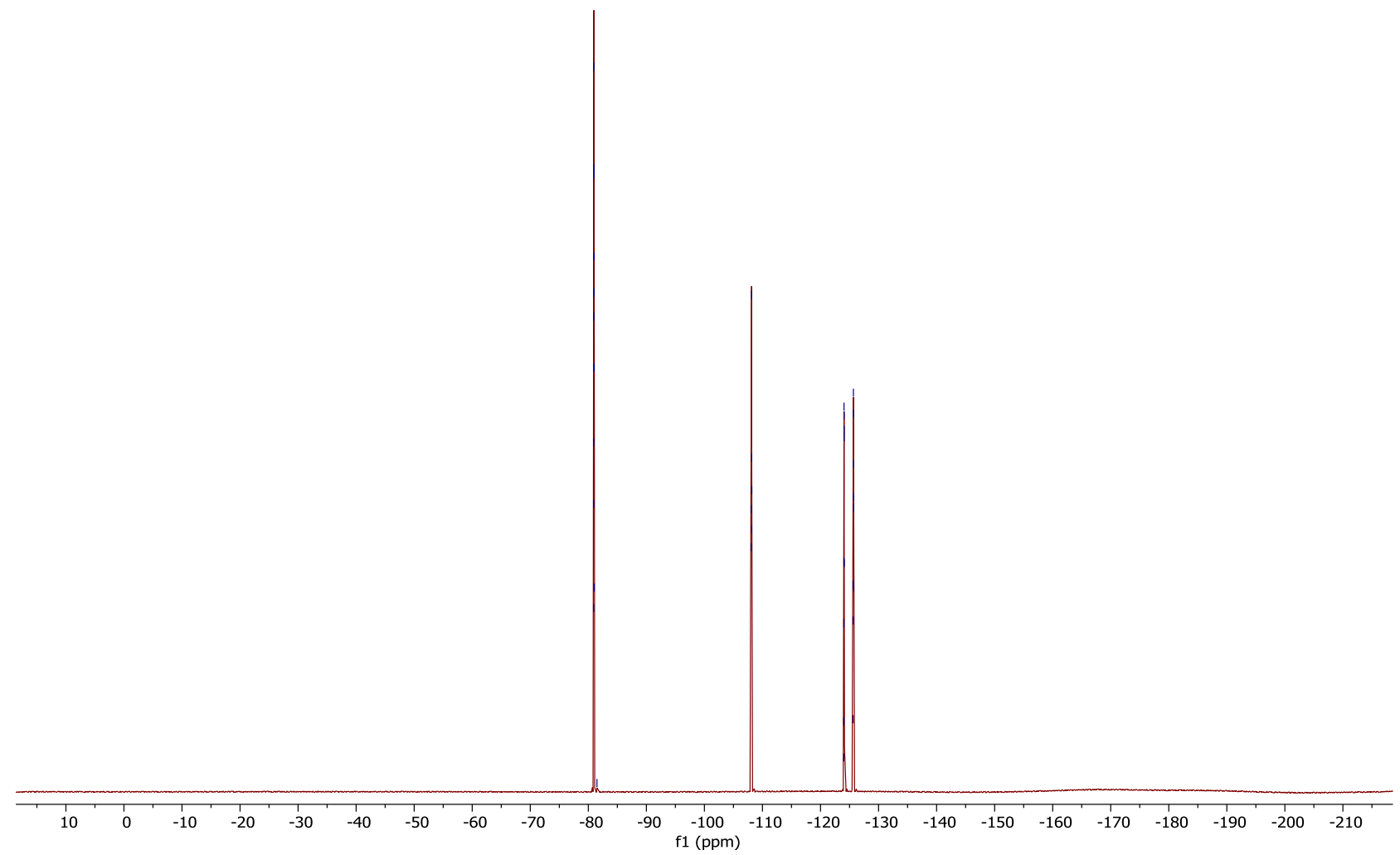


Phenyl (2E)-2-cyclopropyl-4,4,5,5,6,6,7,7,7-nonafluorohept-2-enoate (19a)

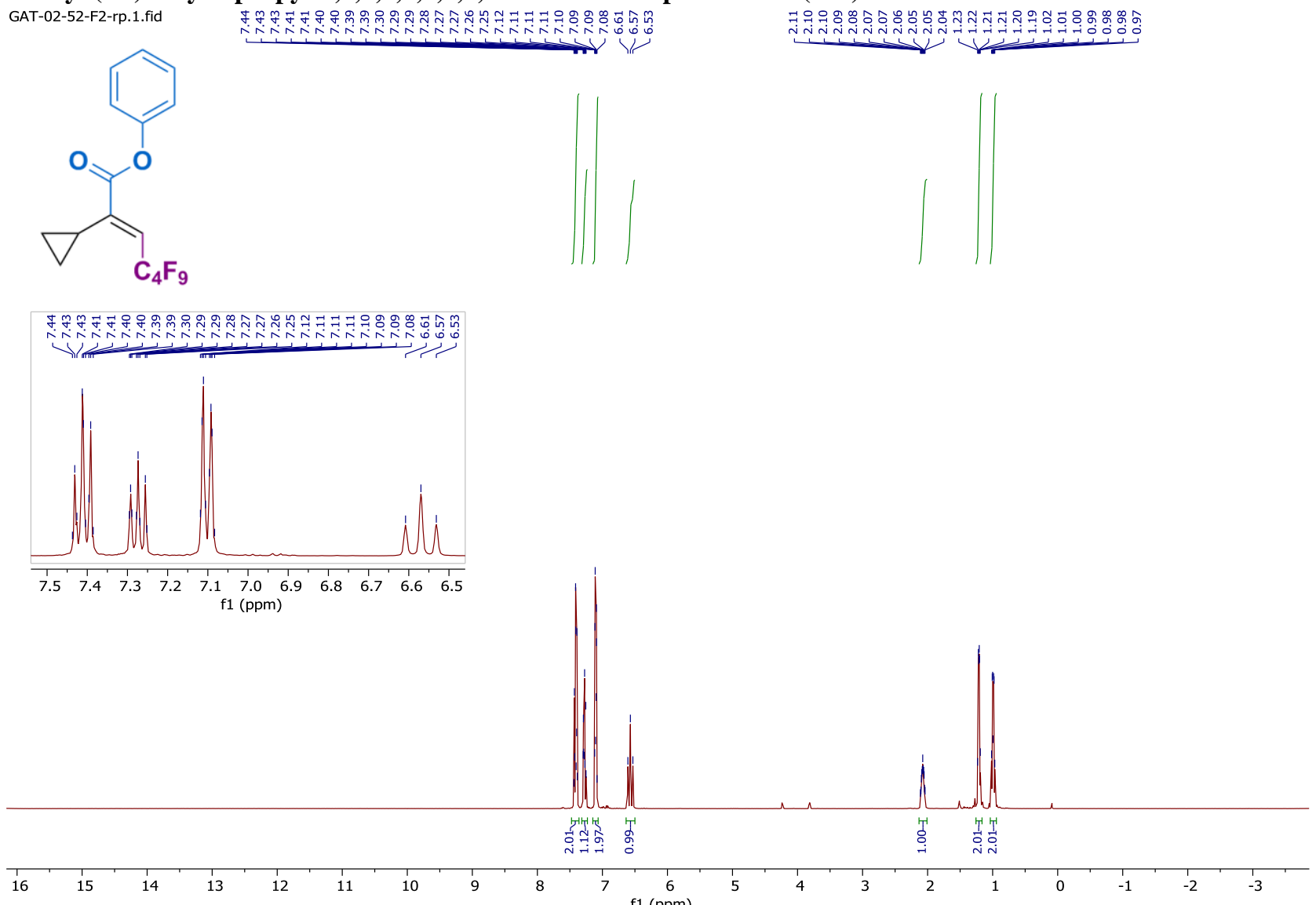

GAT-02-52-F2-rp.2.fid

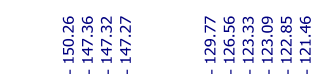

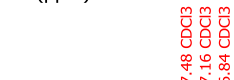

र्य

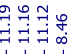
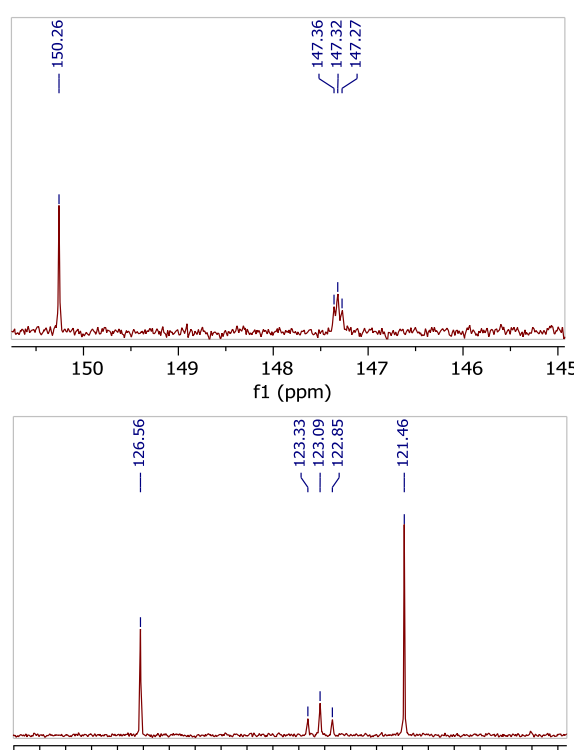

$\begin{array}{lllllllllll}129 & 128 & 127 & 126 & 125 & 124 & 123 & 122 & 121 & 120 & 119\end{array}$ f1 (ppm)
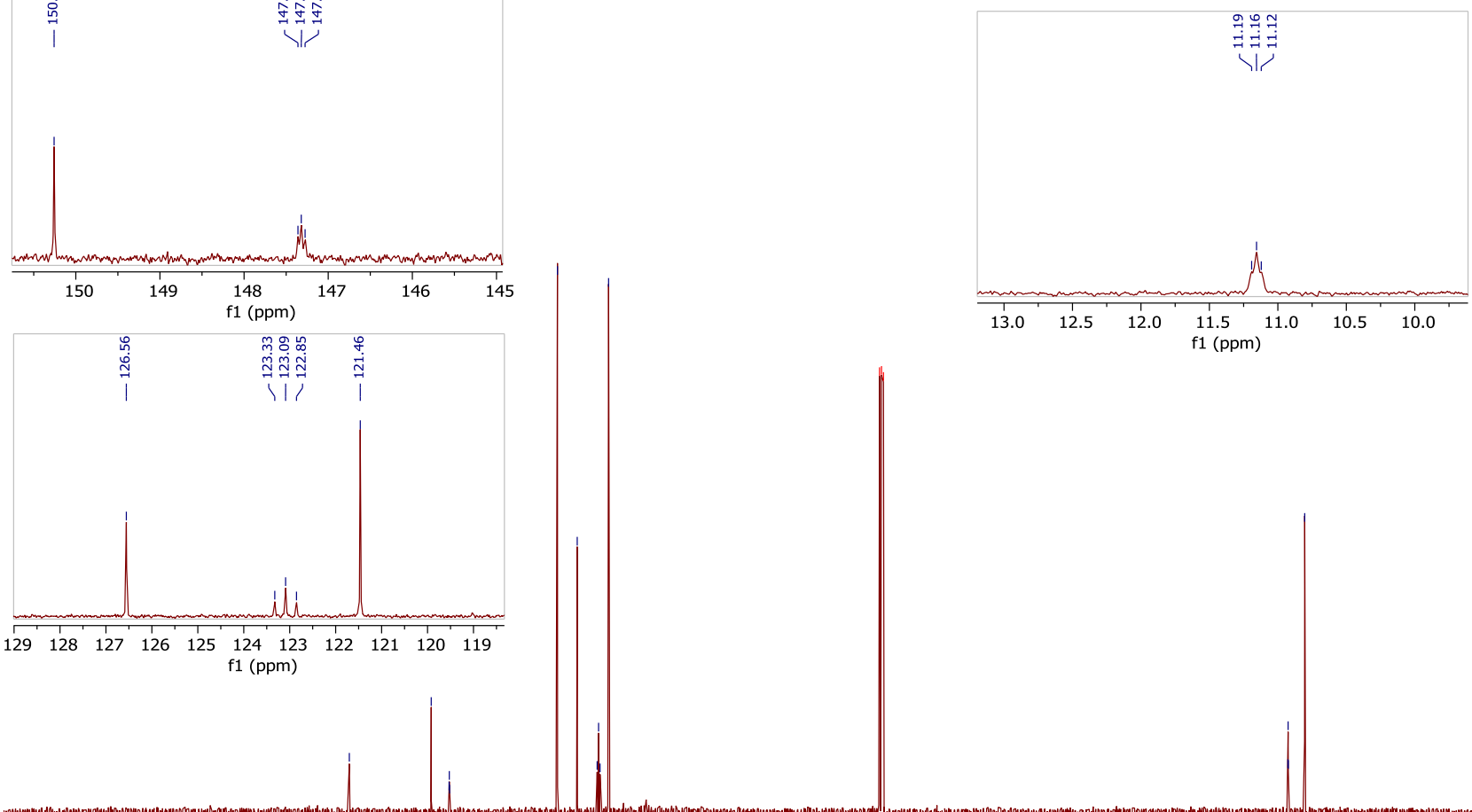

$\begin{array}{llllllllllll}210 & 200 & 190 & 180 & 170 & 160 & 150 & 140 & 130 & 120 & 110 & 100\end{array}$ 


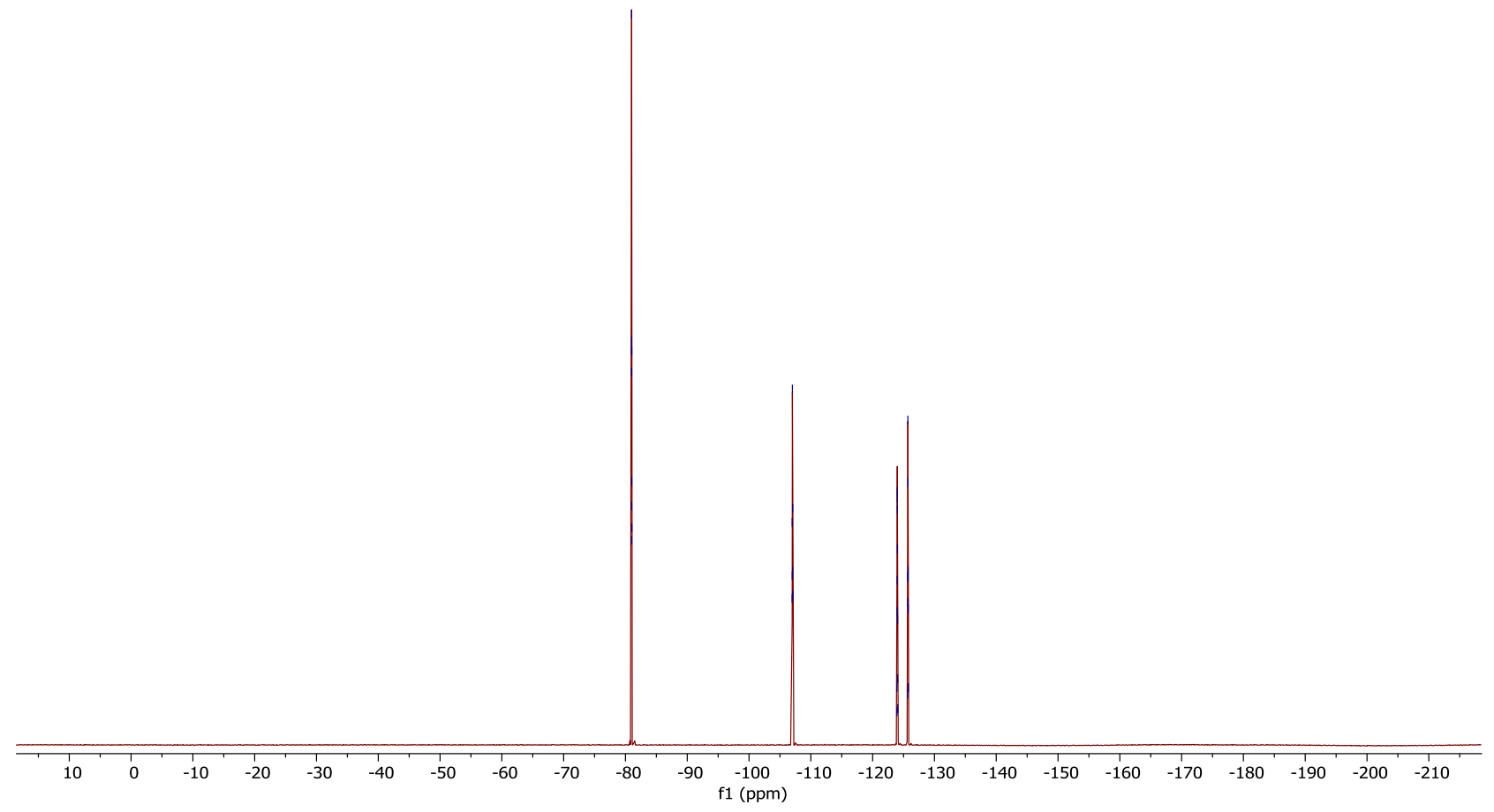


Phenyl (2E)-2-(cyclohex-1-en-1-yl)-4,4,5,5,6,6,7,7,7-nonafluorohept-2-enoate (20a)

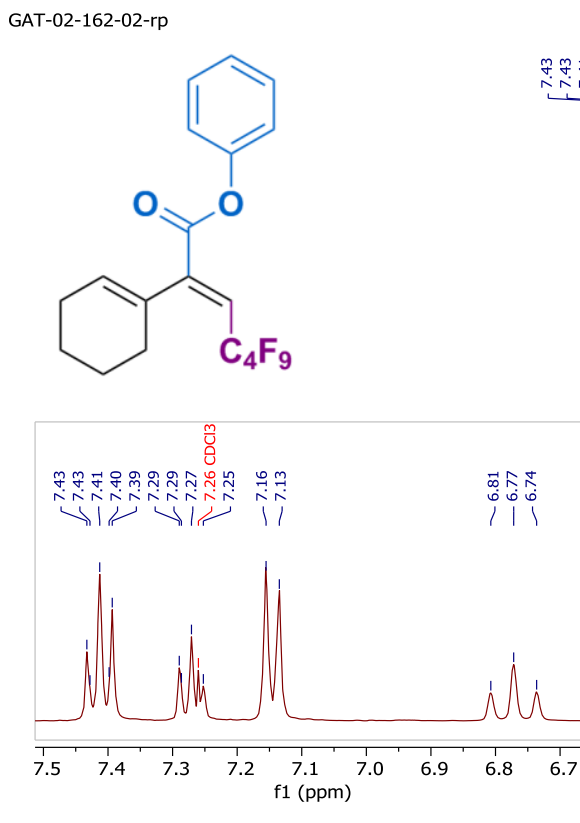

学

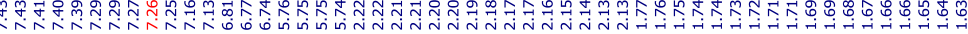
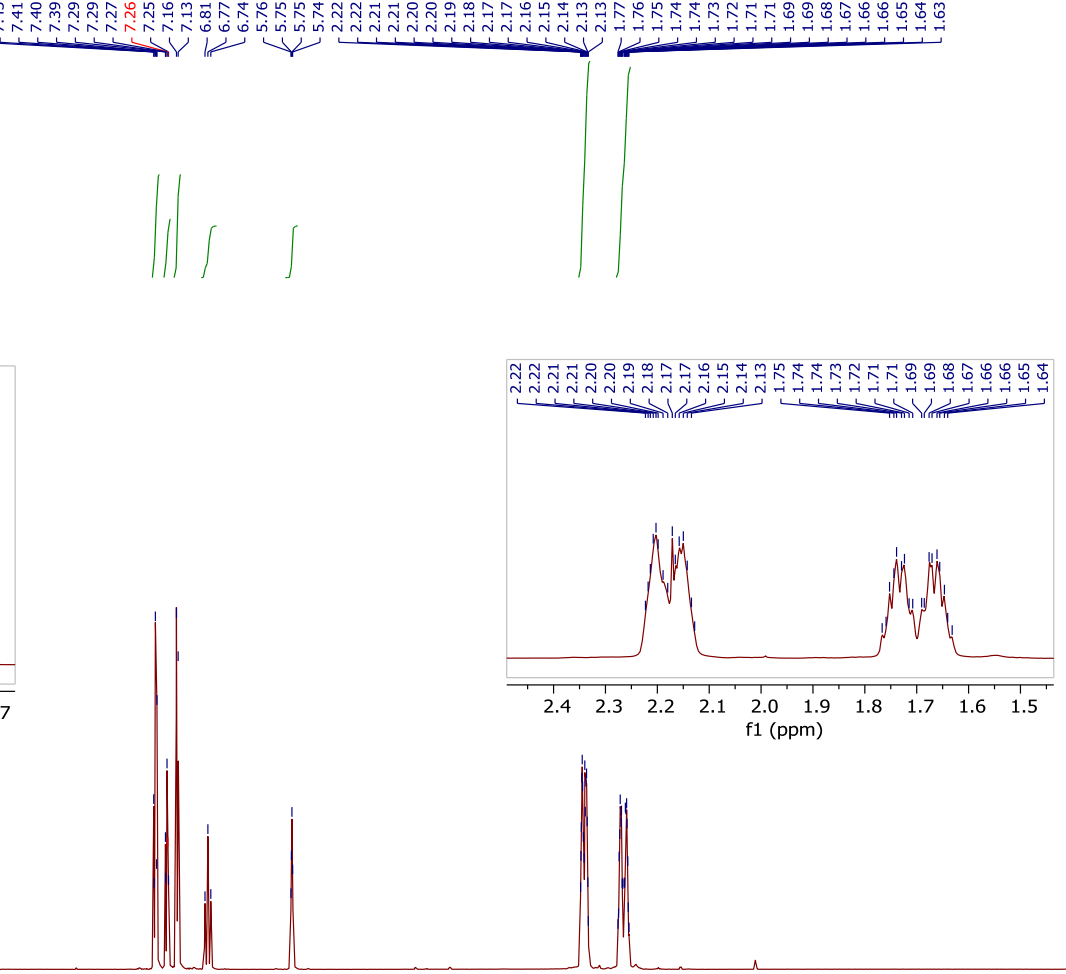

bith

管管

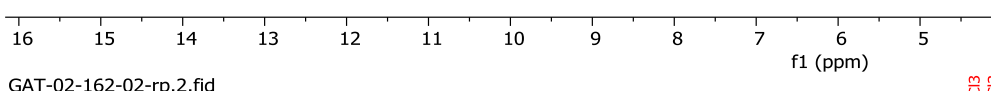

GAT-02-162-02-rp.2.fid

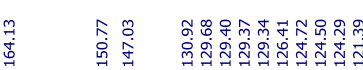

$$
\begin{aligned}
& \text { । } 1 \text { }
\end{aligned}
$$
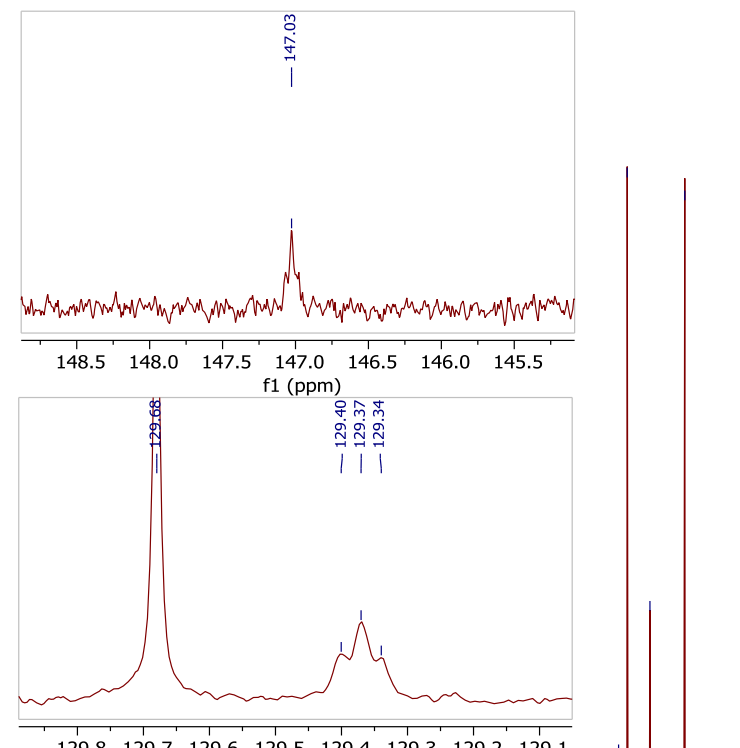

$\begin{array}{llllllll}129.8 & 129.7 & 129.6 & 129.5 & 129.4 & 129.3 & 129.2 & 129.1\end{array}$ $\mathrm{f} 1(\mathrm{ppm})$
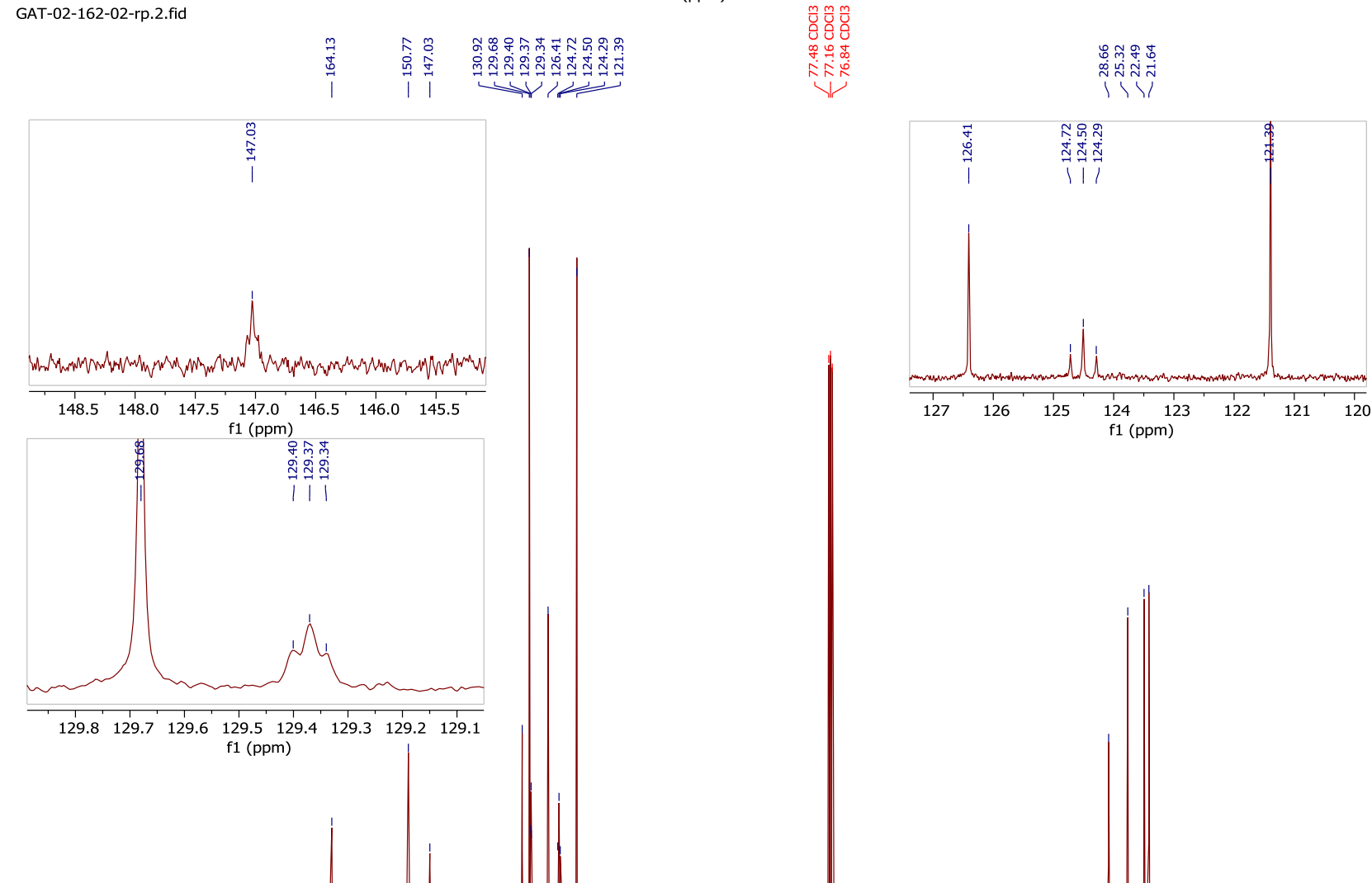

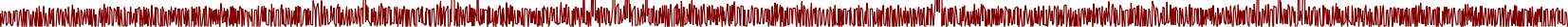

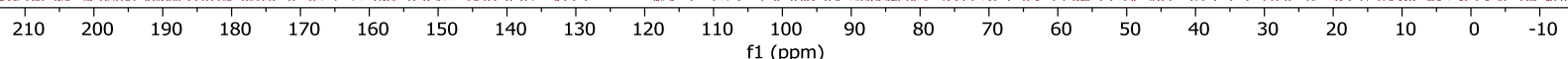




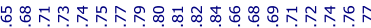

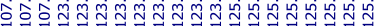

$\psi$

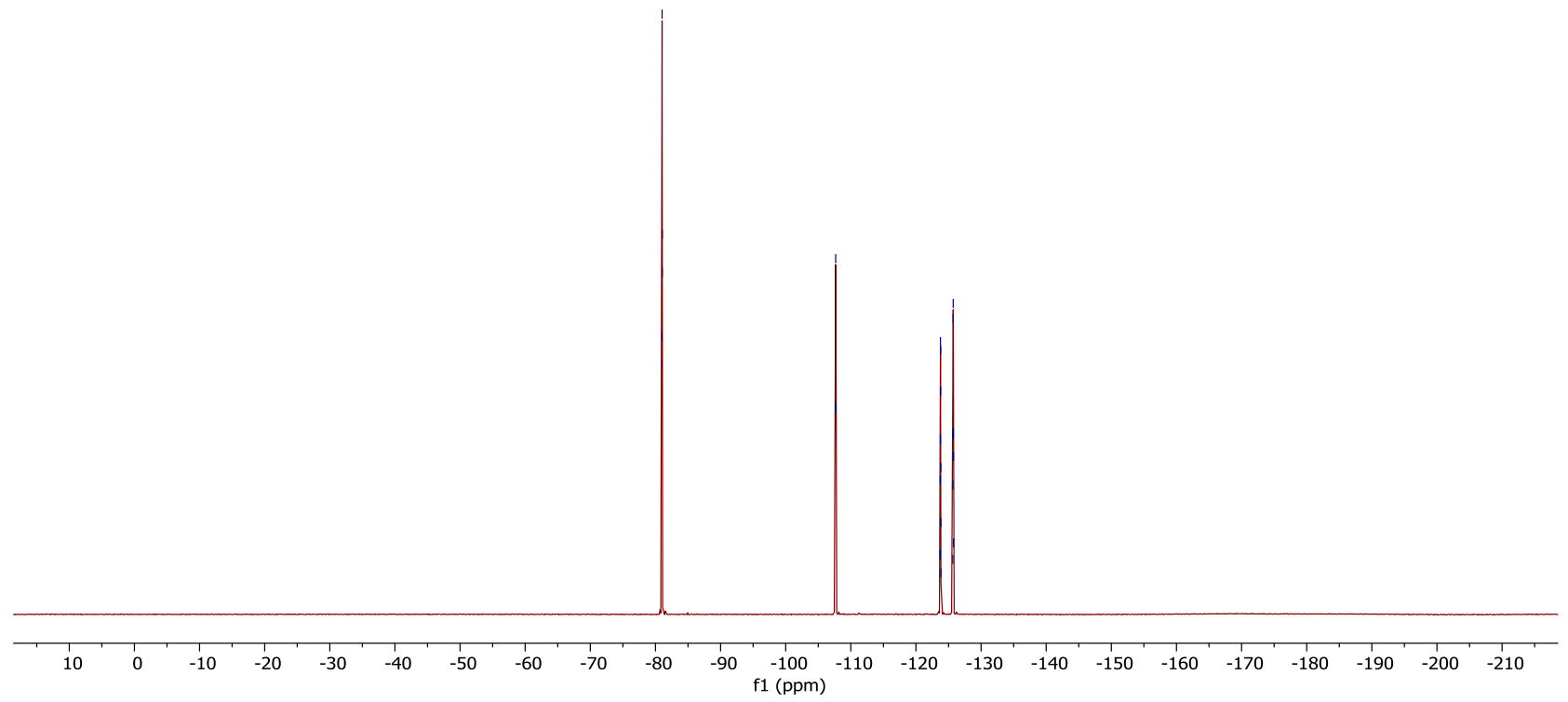


Phenyl (2E)-2-(3-chloropropyl)-4,4,5,5,6,6,7,7,7-nonafluorohept-2-enoate (21) GAT-02-51-F2-rp1.1.fid<smiles>O=C(Oc1ccccc1)C(=CC(F)(F)F)CCCCl</smiles>

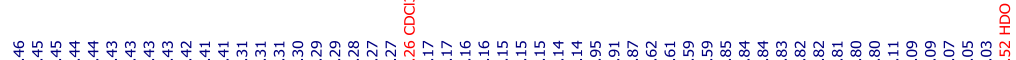

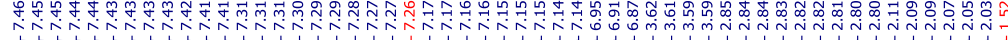
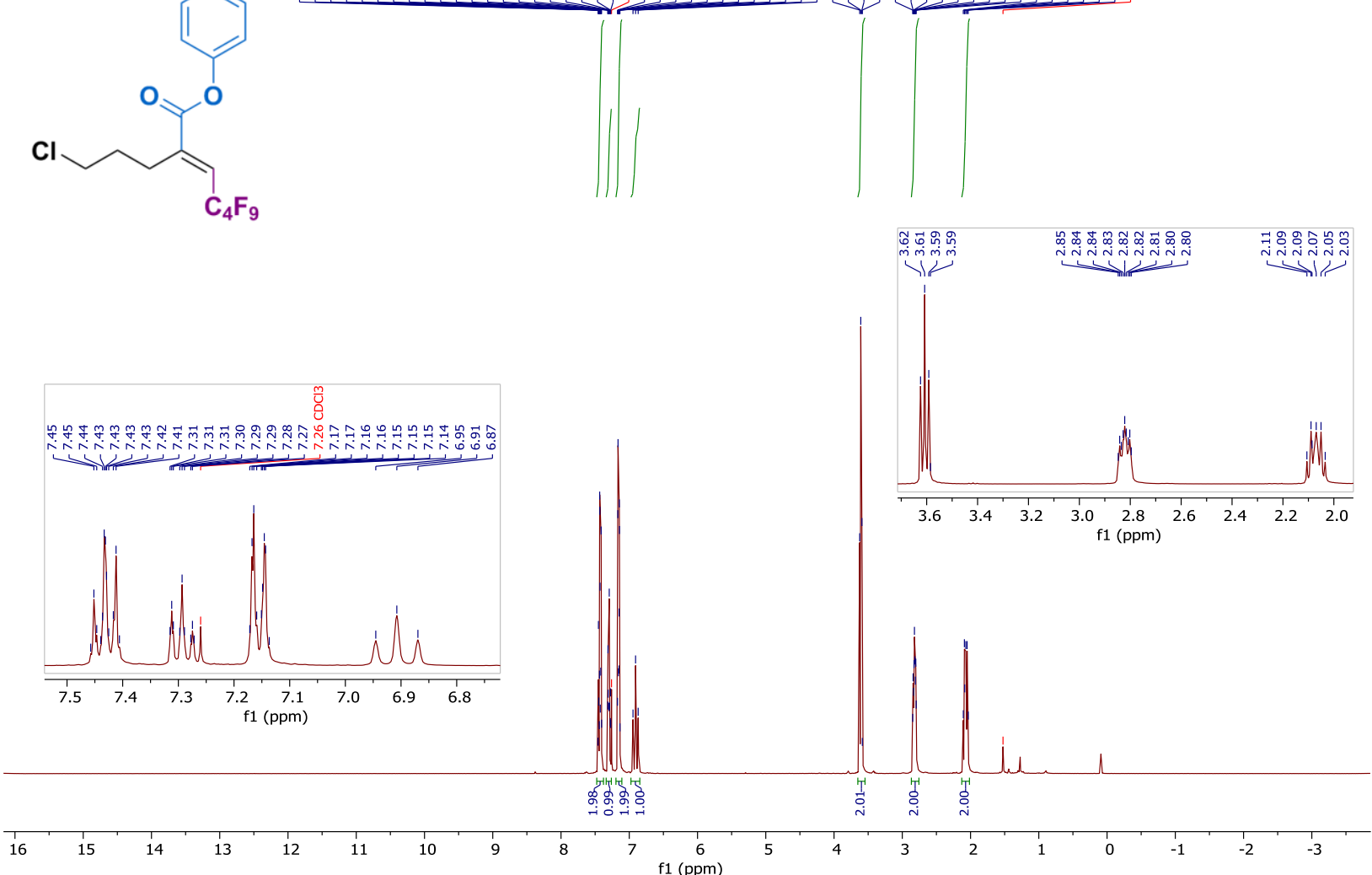

GAT-02-51-F2-rp1.2.fid
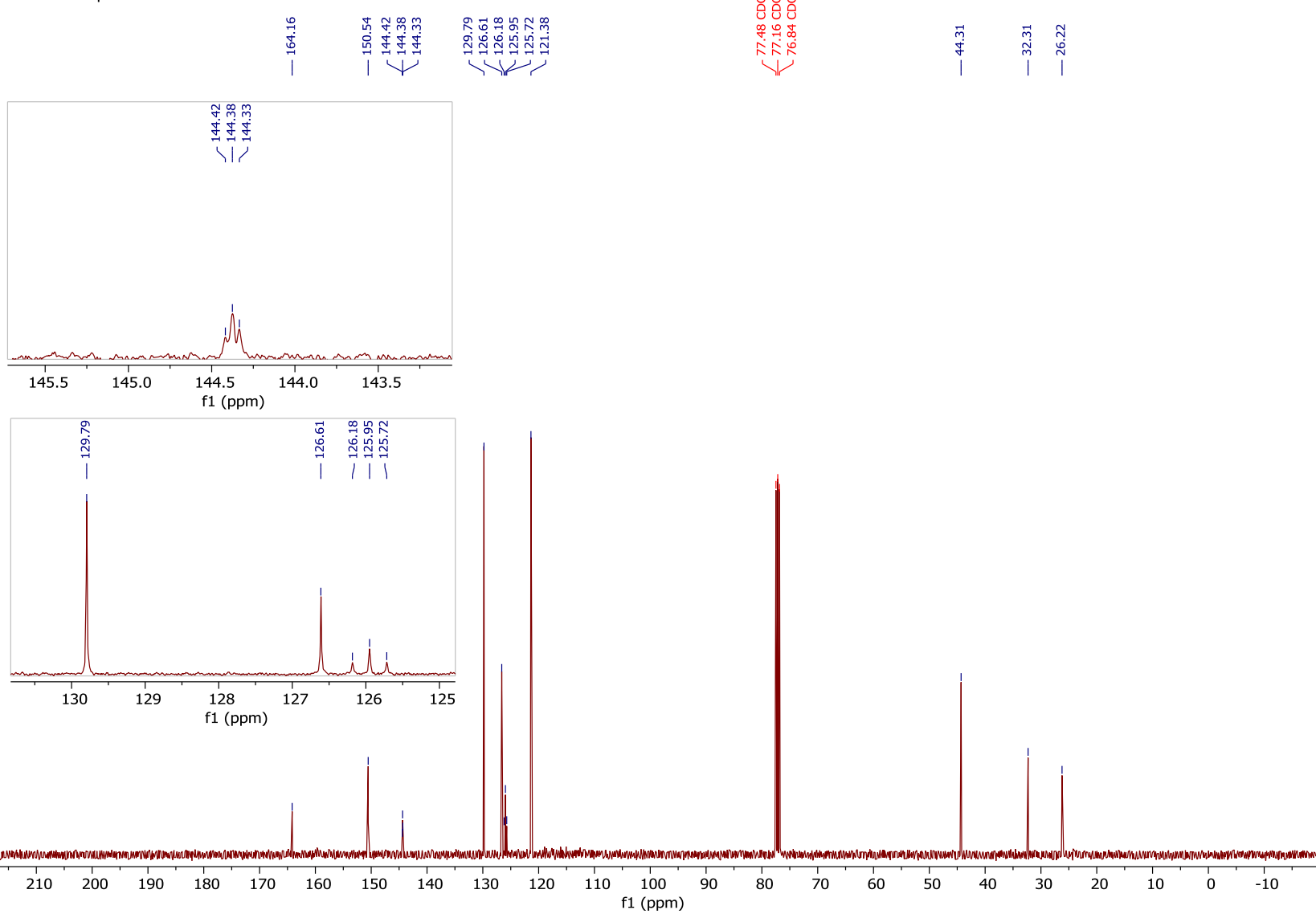

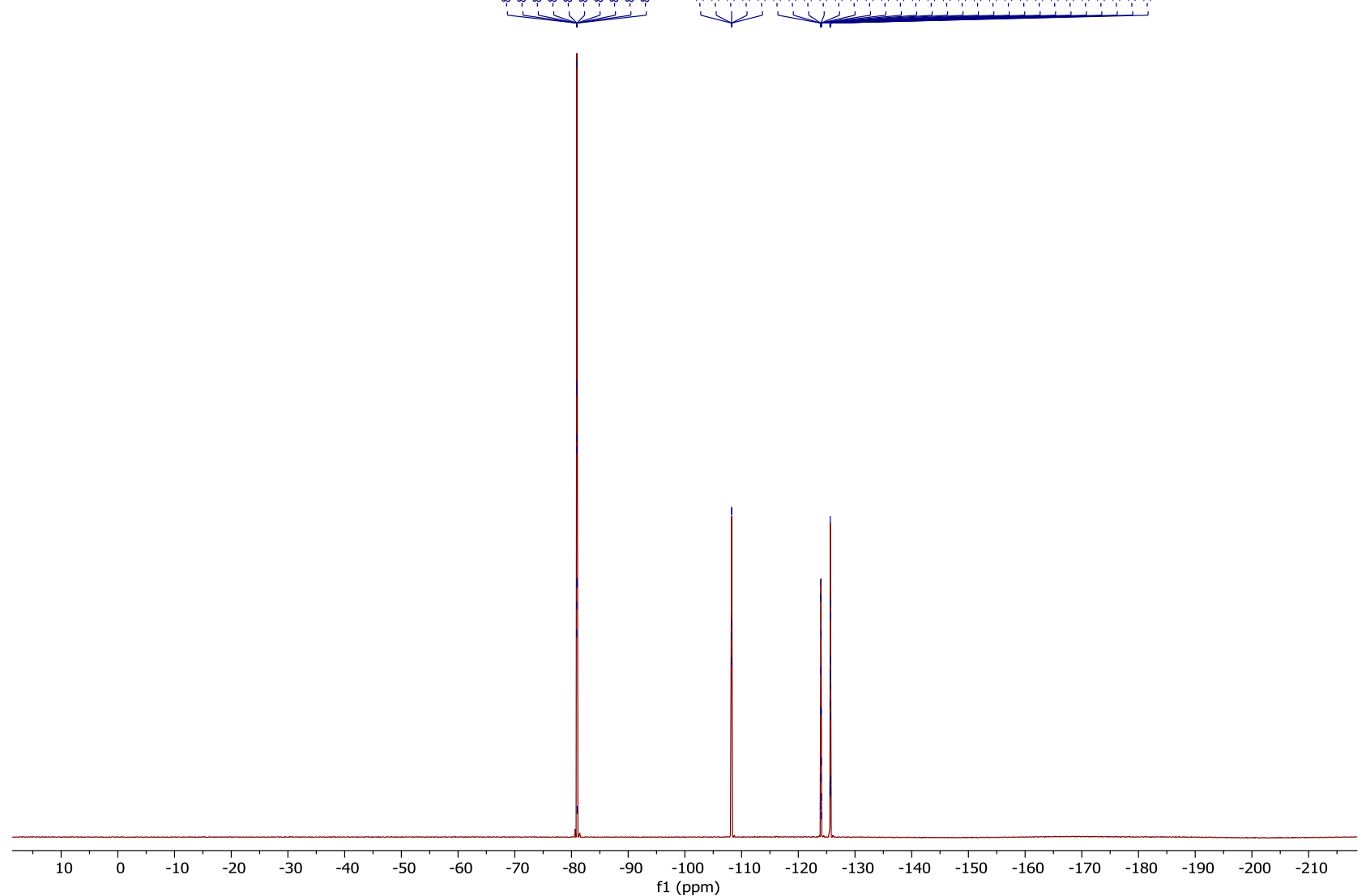
Phenyl (2E)-4,4,5,5,6,6,7,7,7-nonafluoro-2-(4-(formyloxy)butyl)hept-2-enoate (22)

GAT-02-47-F3-rp1.1.fid

若

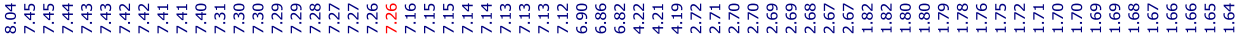<smiles>O=COCCCC/C(=C\C(F)(F)F)C(=O)Oc1ccccc1</smiles>

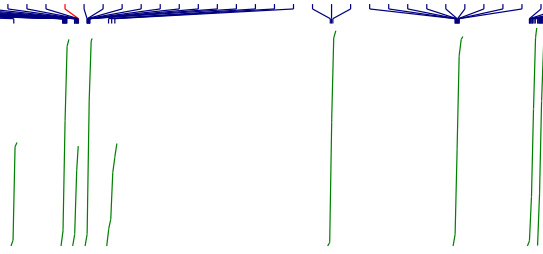

$\operatorname{mon}$

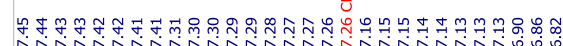
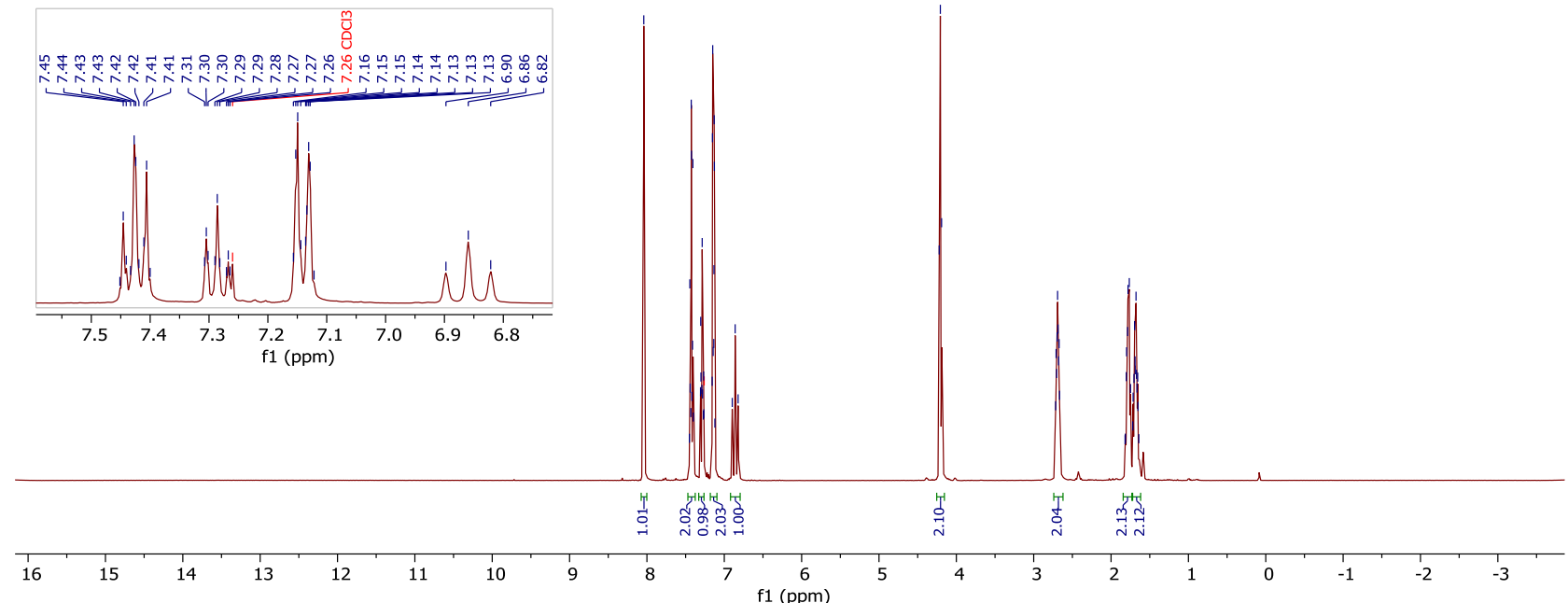

GAT-02-47-F3-rp1.2.fid

|

f1 (ppm)
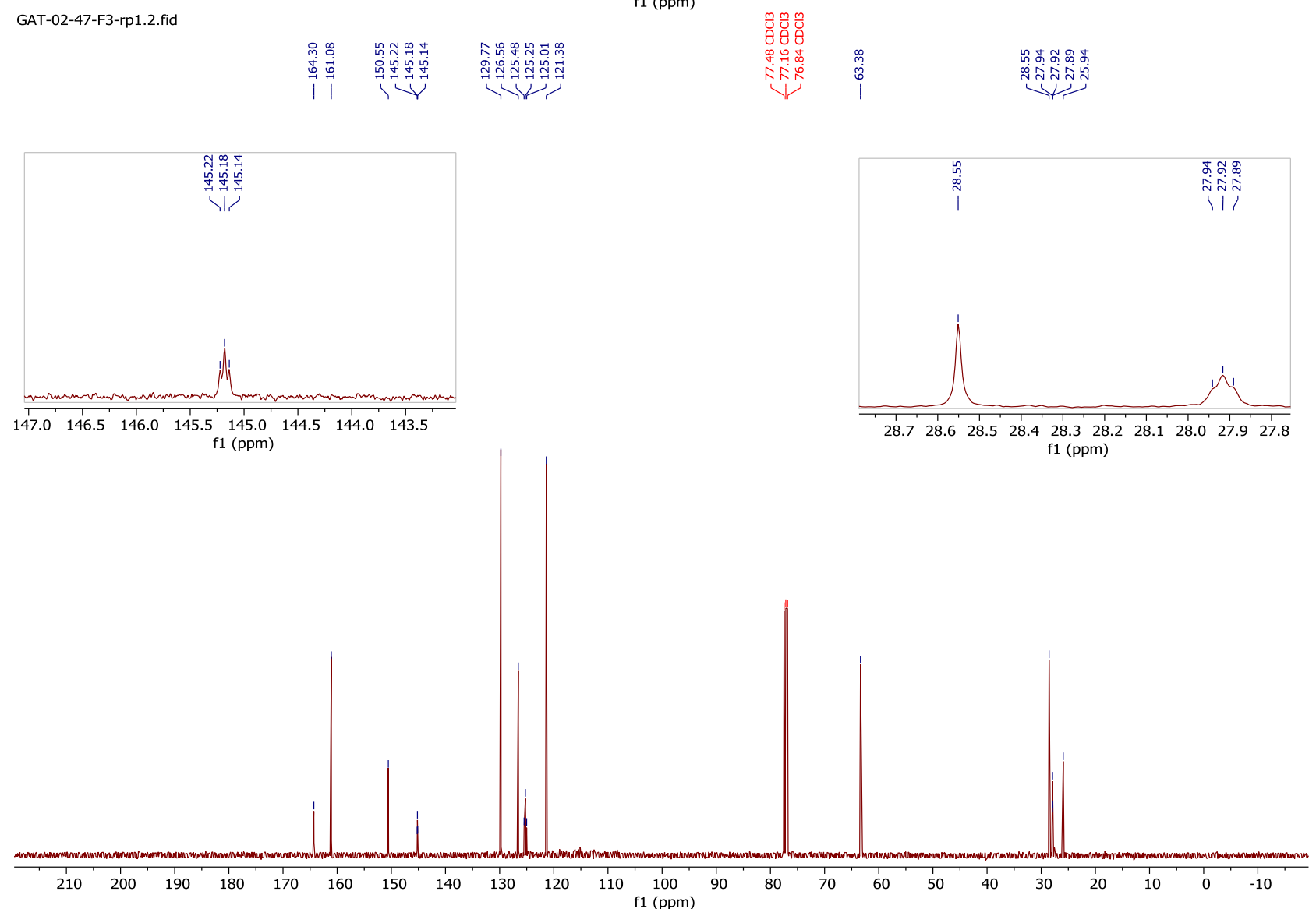


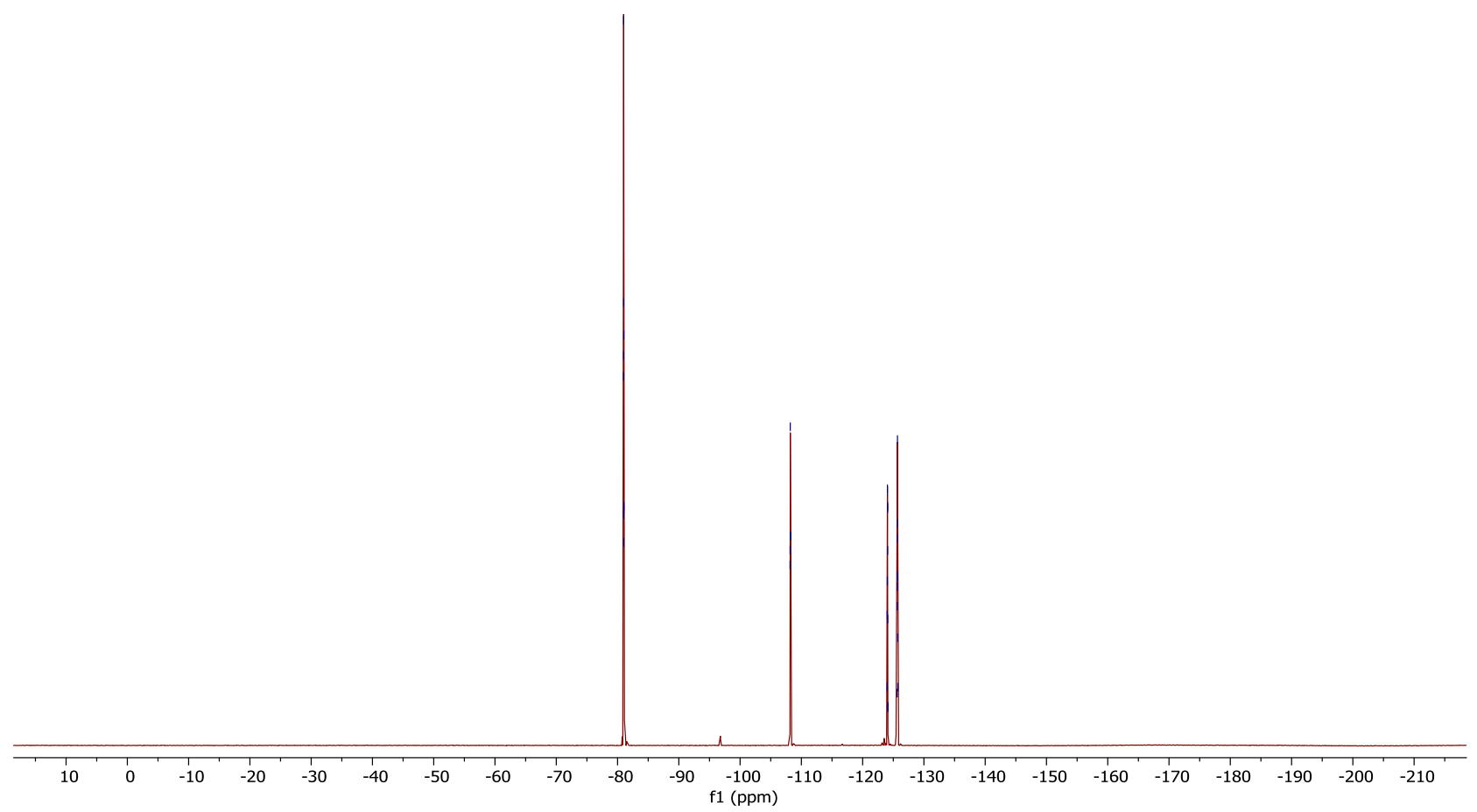


2-Methoxyphenyl (2E)-4,4,5,5,6,6,7,7,7-nonafluoro-2-(4-methoxyphenyl)hept-2-enoate (23) GAT-02-96-rp.1.fid

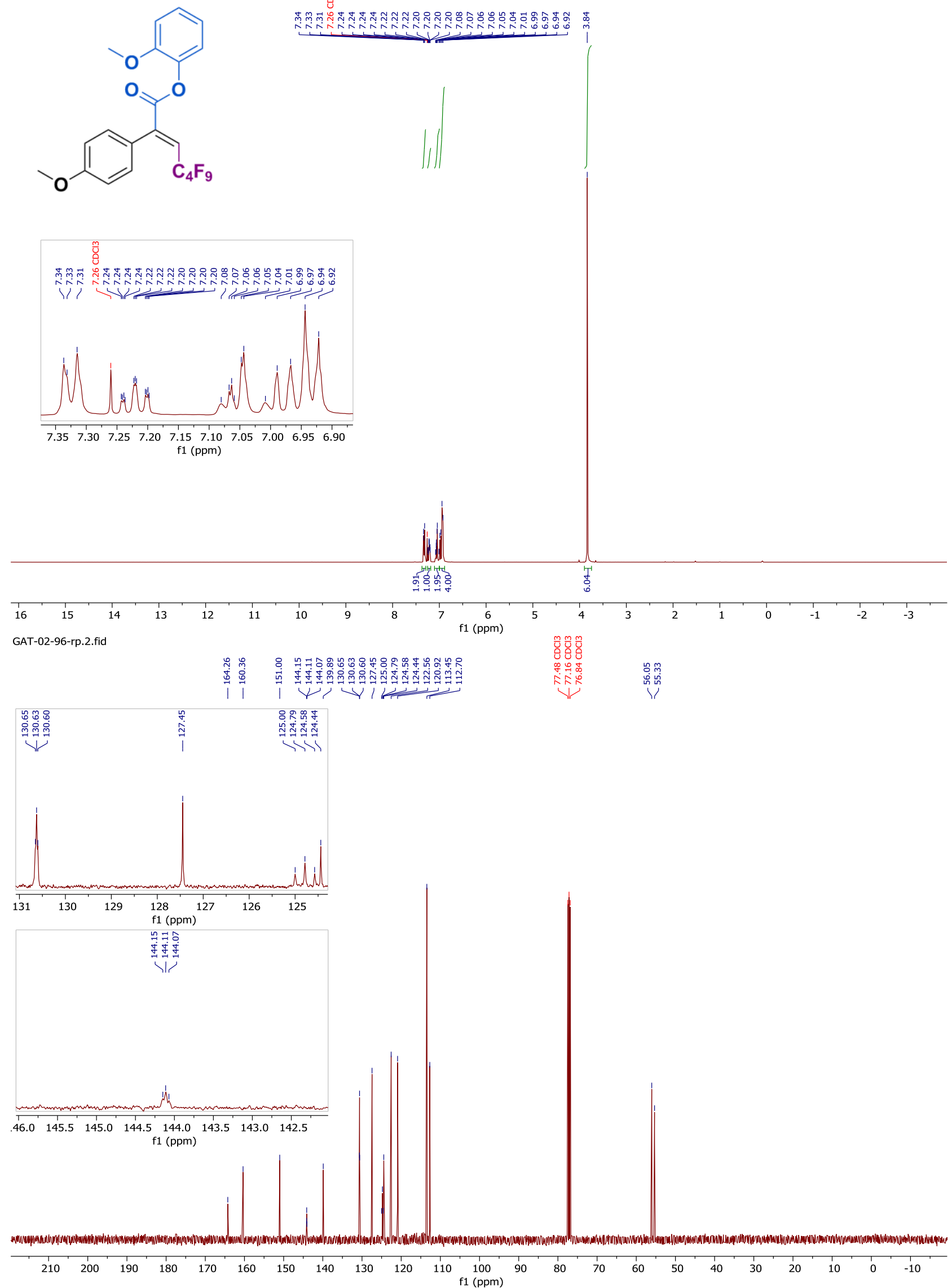
雚 


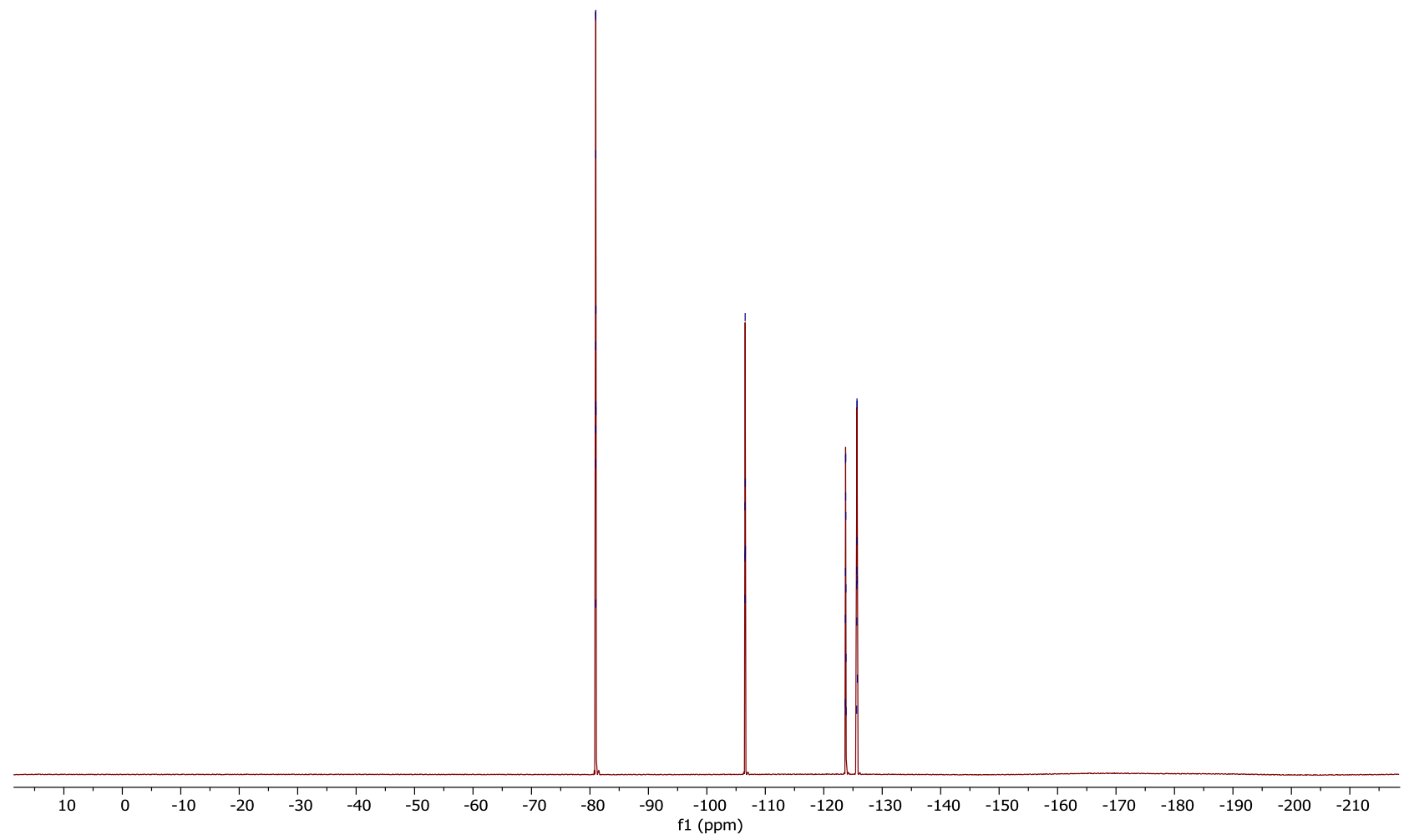


4-Methoxyphenyl (2E)-4,4,5,5,6,6,7,7,7-nonafluoro-2-(4-methoxyphenyl)hept-2-enoate (24) GAT-02-95-F3-rp-1.1.fid
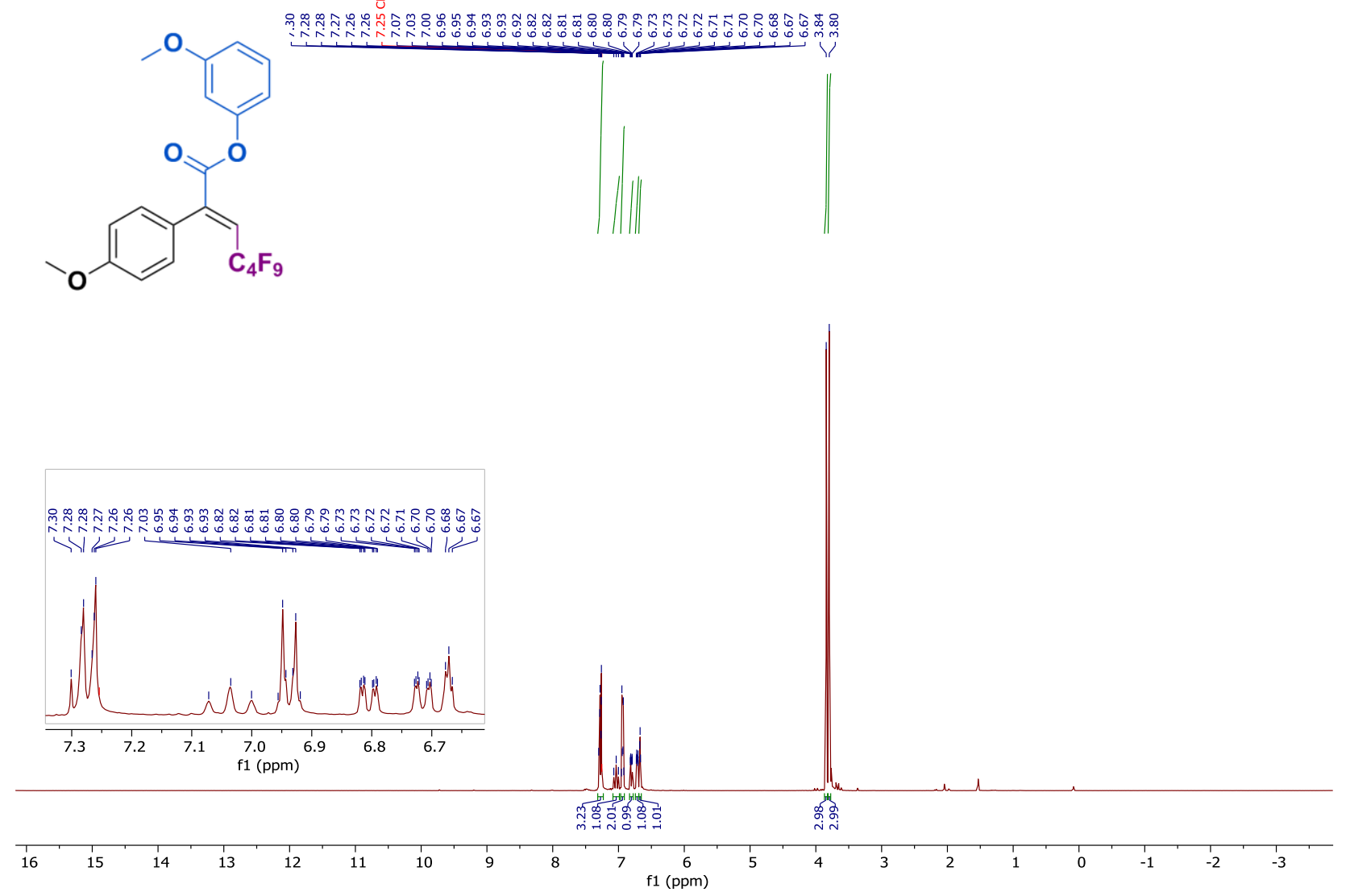

GAT-02-95-F3-rp.2.fid
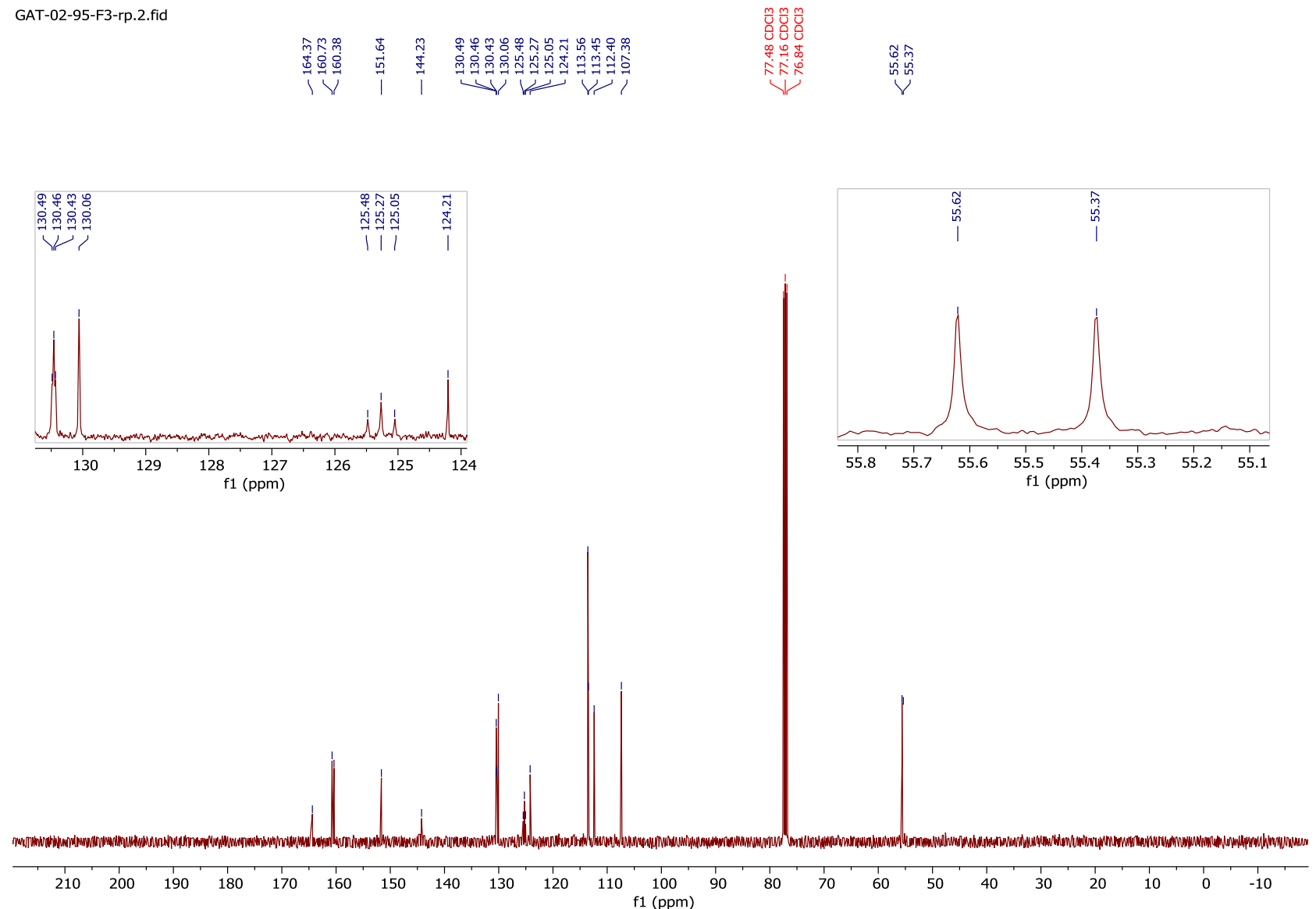


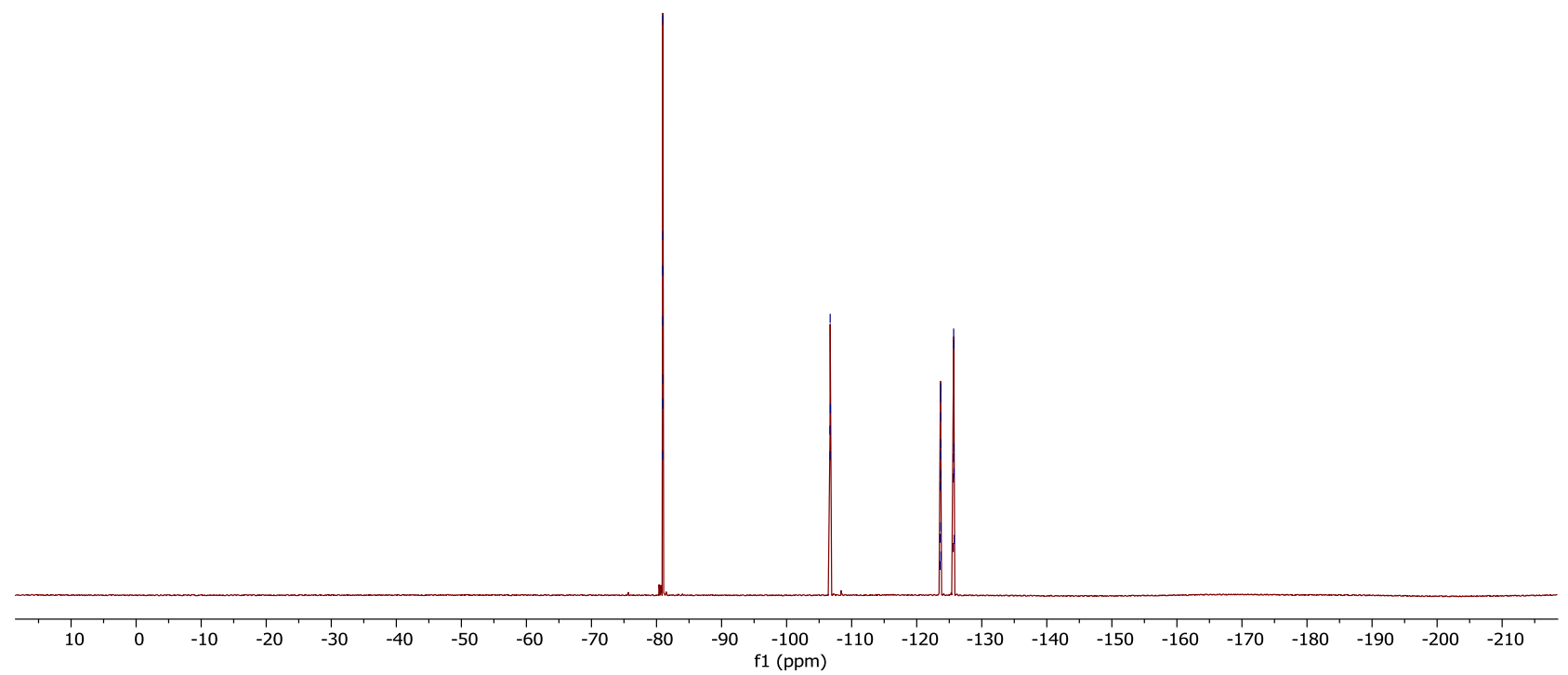


4-Methoxyphenyl (2E)-4,4,5,5,6,6,7,7,7-nonafluoro-2-(4-methoxyphenyl)hept-2-enoate (25) GAT-02-94-F5-rp.1.fid

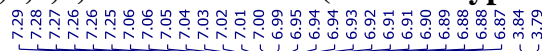<smiles>COc1ccc(OC(=O)/C(=C\C(F)(F)F)c2ccc(OC)cc2)cc1</smiles>
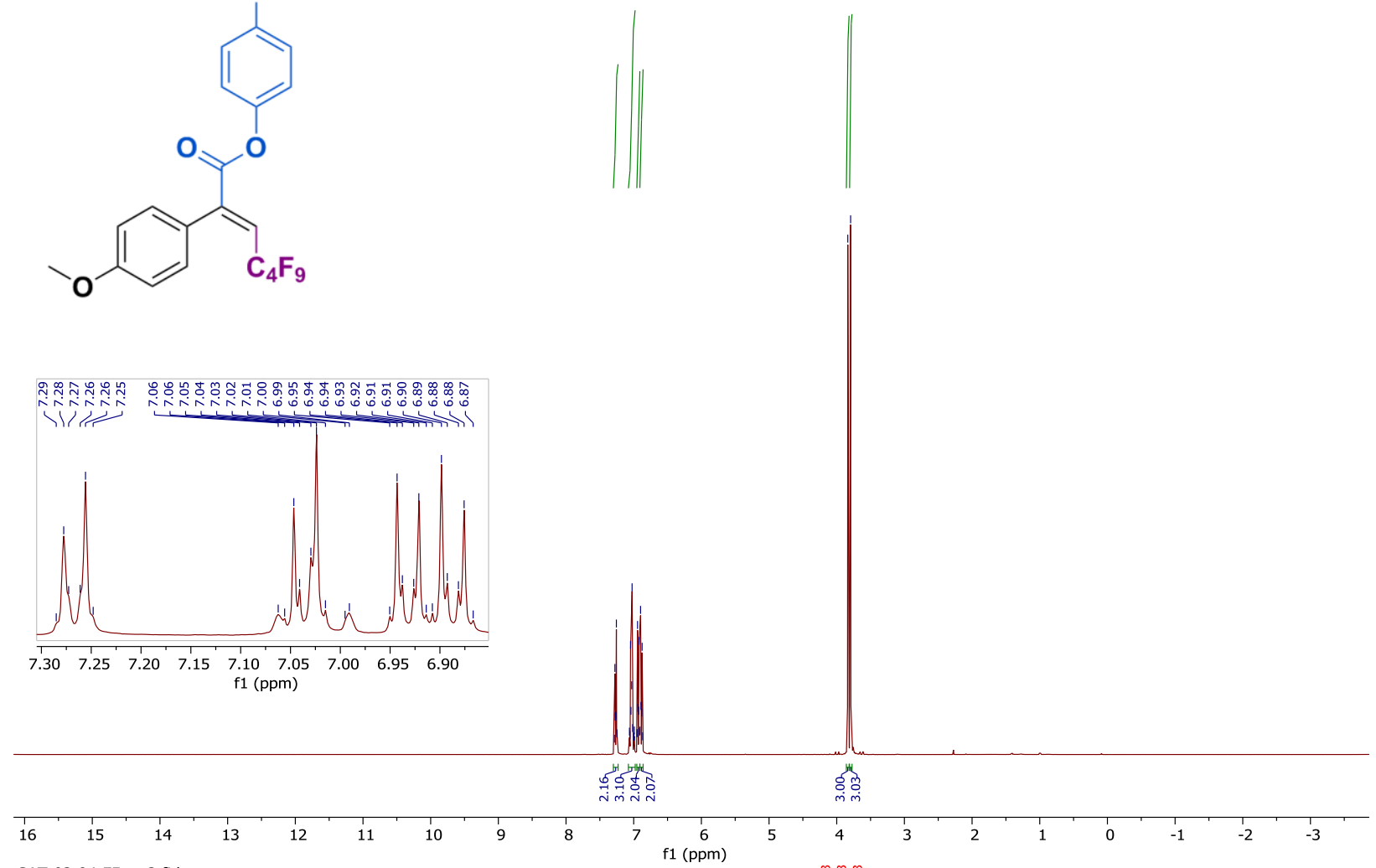

GAT-02-94-F5-rp.2.fid
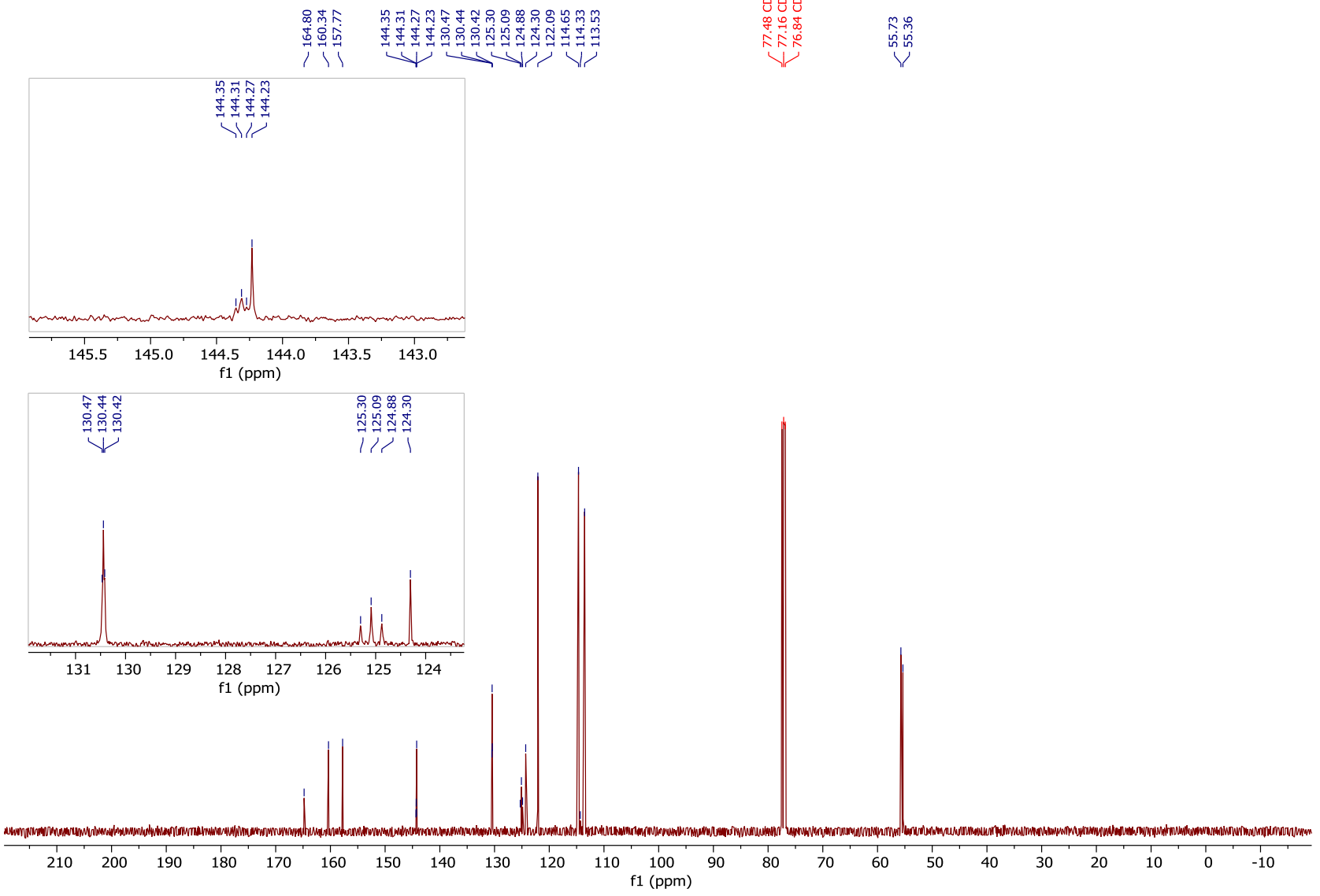


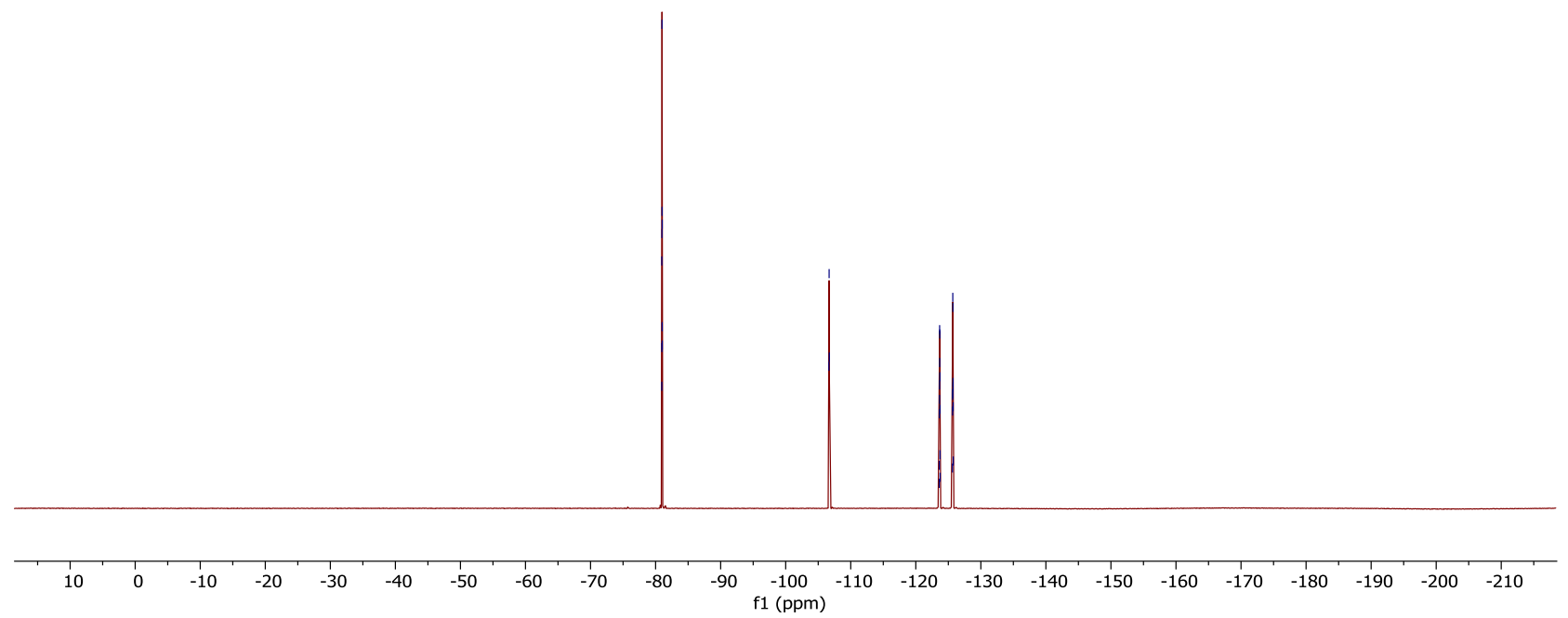


4-Methylphenyl (2E)-4,4,5,5,6,6,7,7,7-nonafluoro-2-(4-methoxyphenyl)hept-2-enoate (26) GAT-02-107-F3-rp.1.fid<smiles>COc1ccc(C(=CC(F)(F)F)C(=O)Oc2ccc(C)cc2)cc1</smiles>
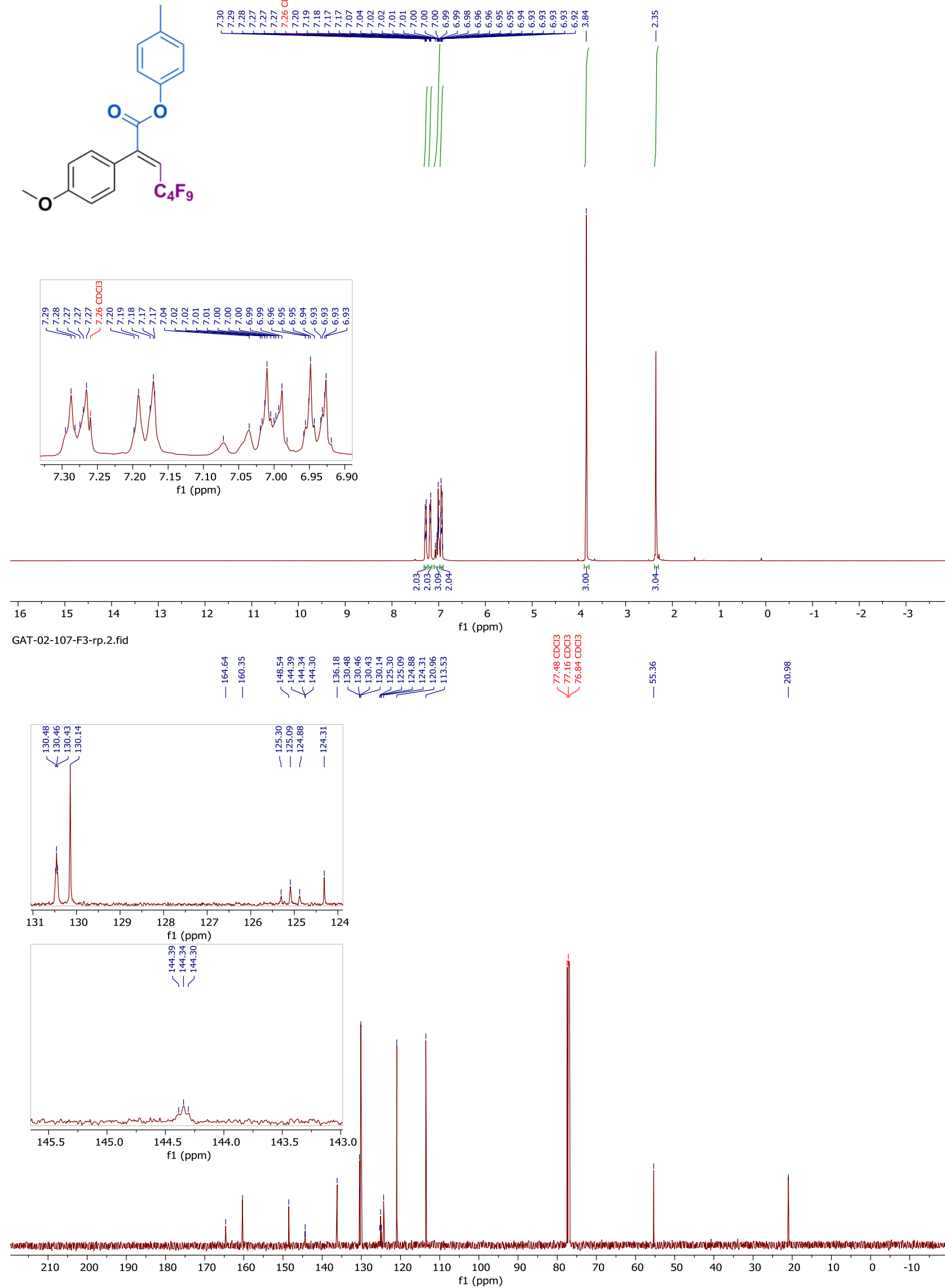


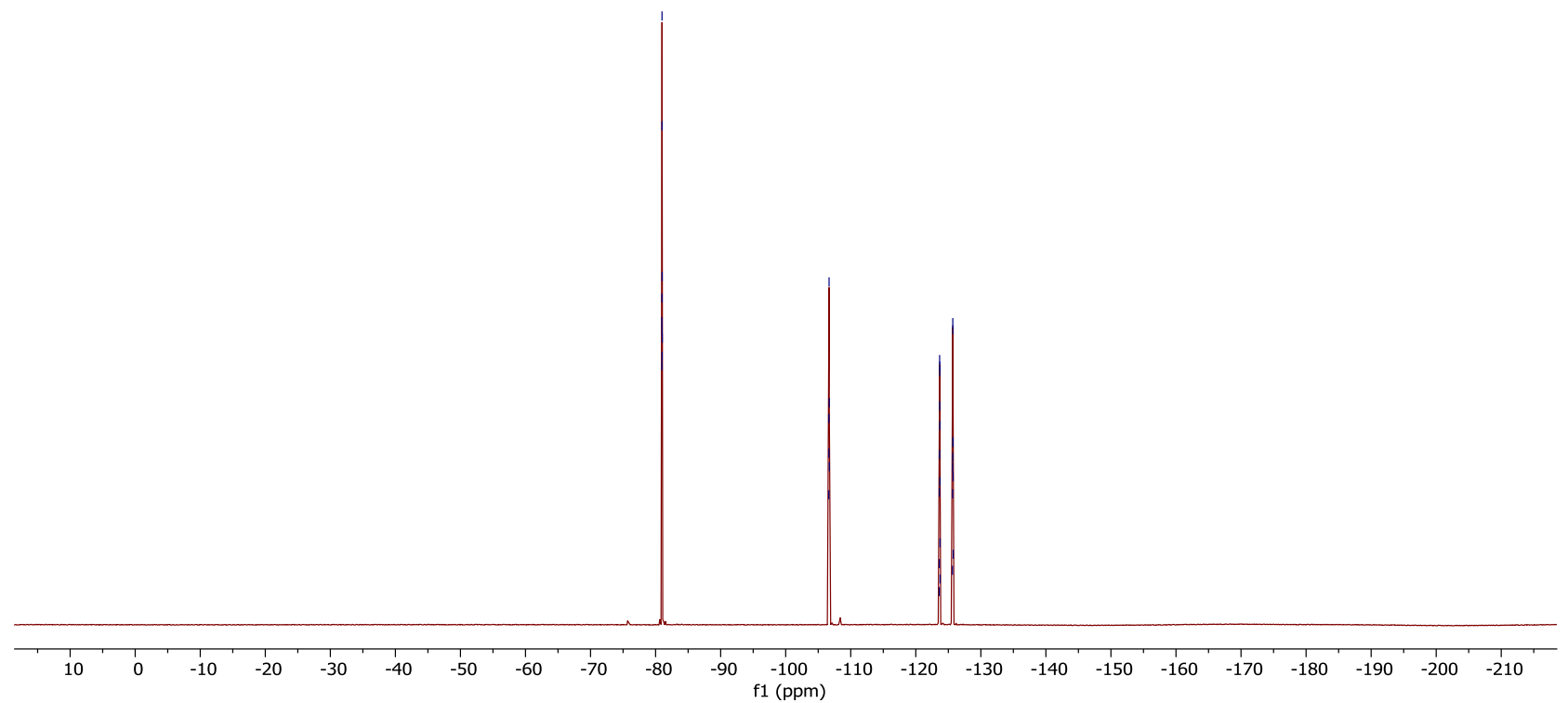


4-Nitrophenyl (2E)-4,4,5,5,6,6,7,7,7-nonafluoro-2-(4-methoxyphenyl)hept-2-enoate (27) GAT-02-80-F3-rpp.1.fid

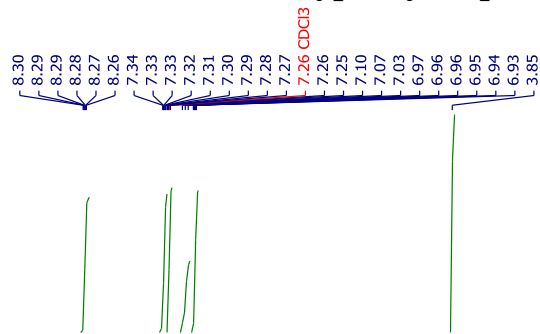<smiles>COc1ccc(/C(=C\C(F)(F)F)C(=O)Oc2ccc([N+](=O)[O-])cc2)cc1</smiles>
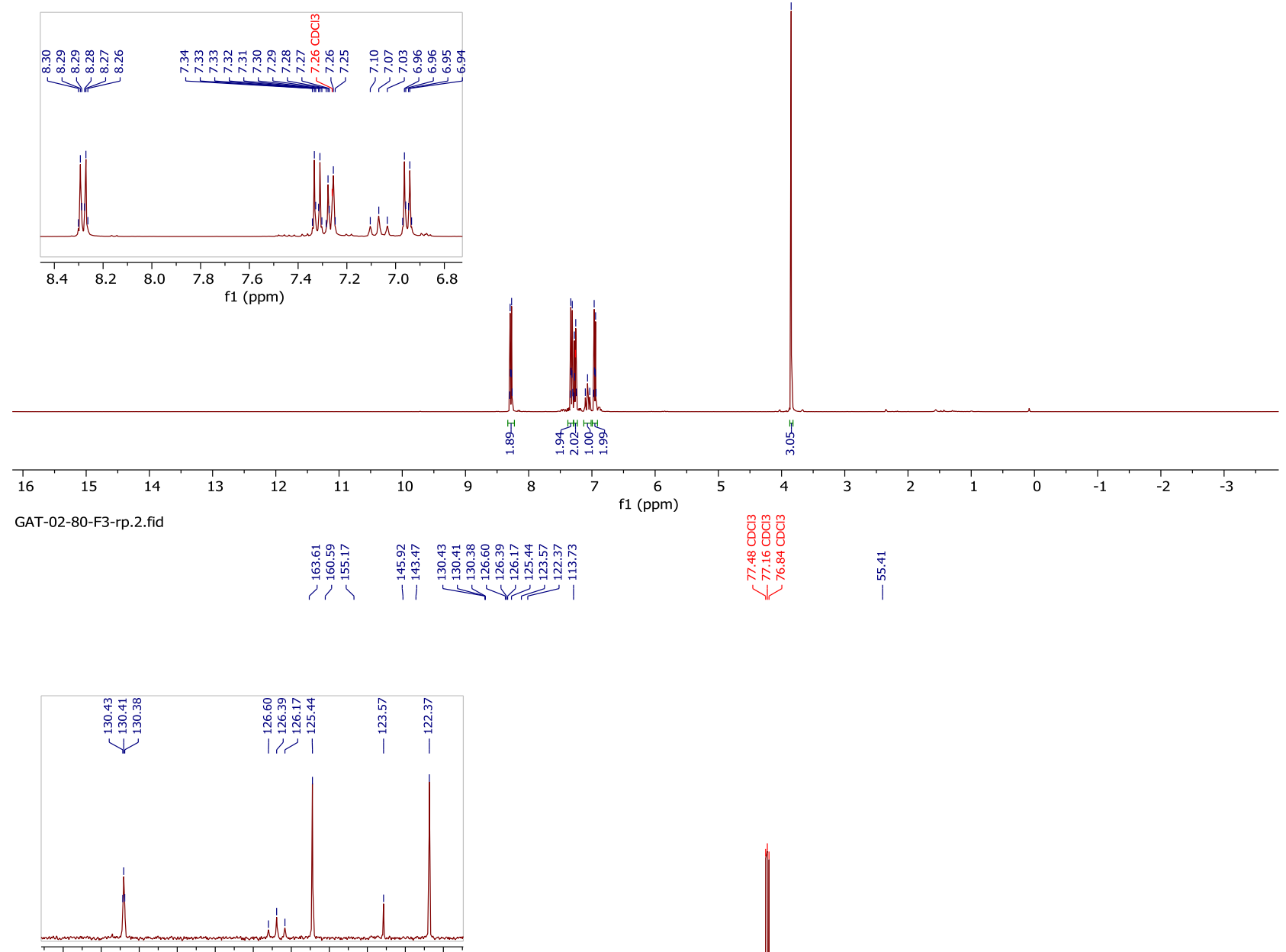

$\begin{array}{lllllllllll}132 & 131 & 130 & 129 & 128 & 127 & 126 & 125 & 124 & 123 & 122\end{array}$ f1 (ppm)

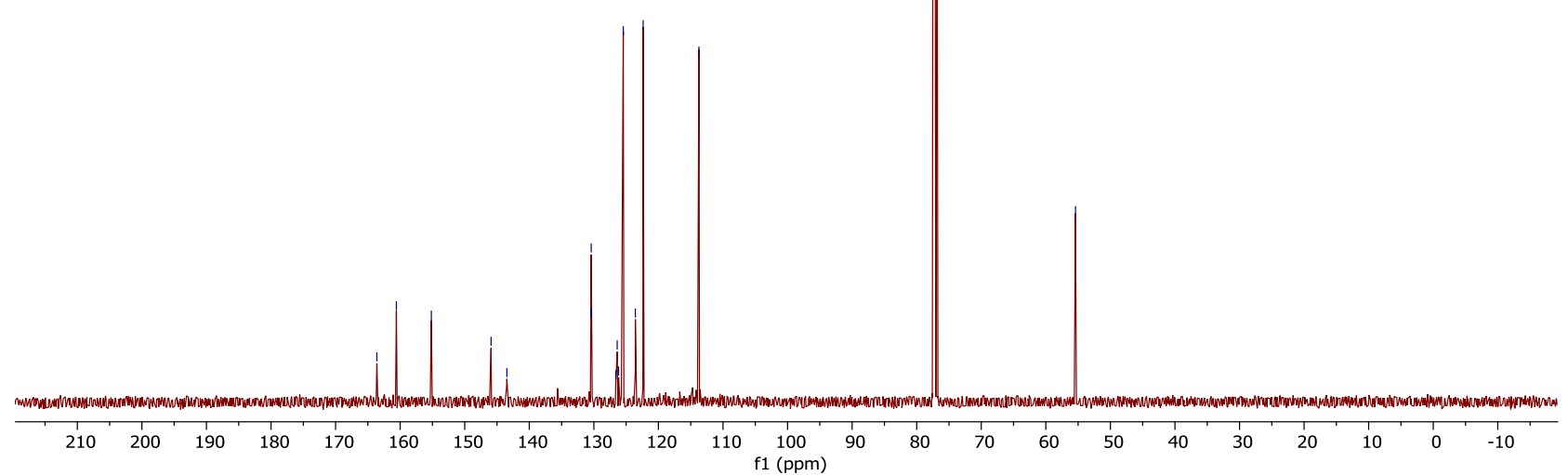




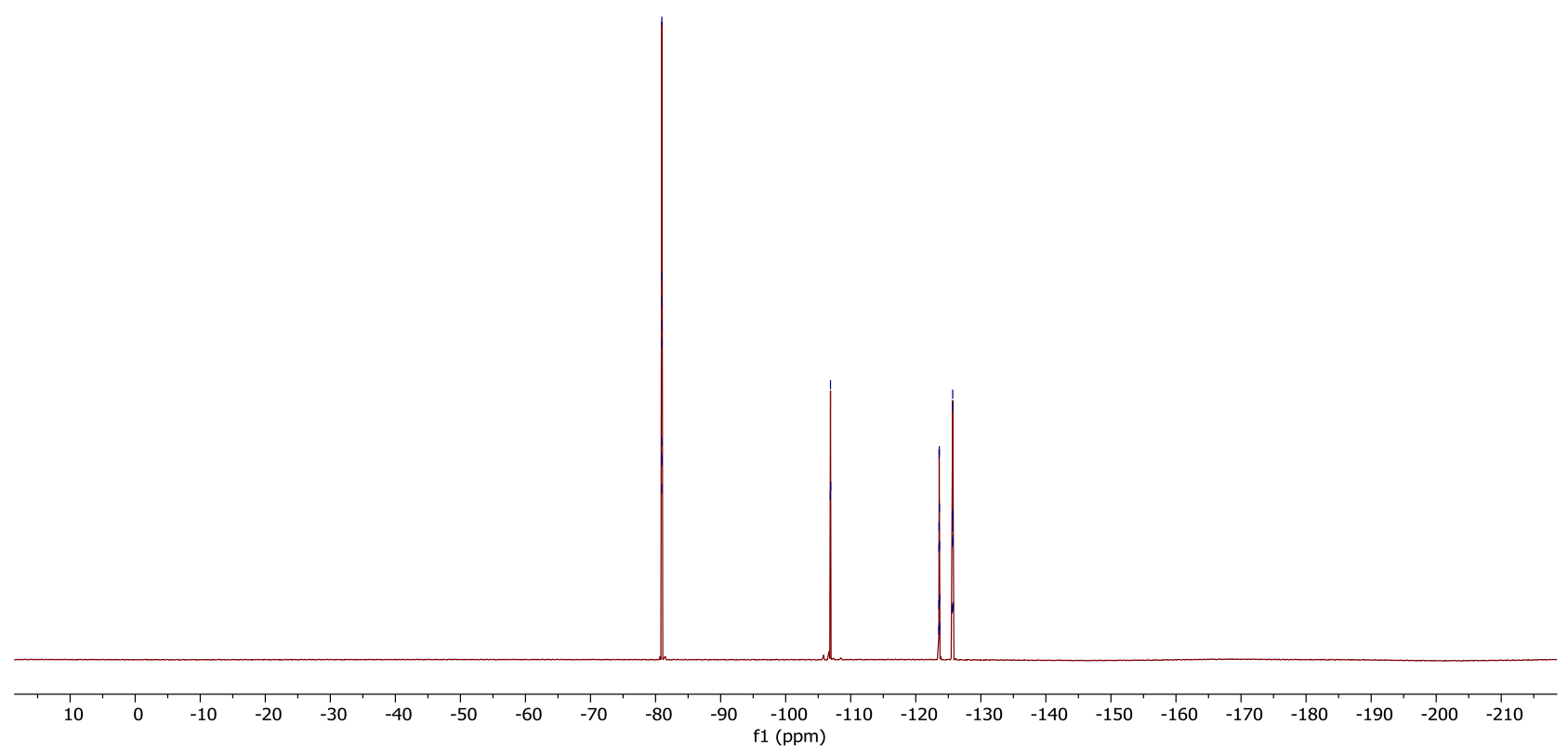


2,4,6-Trichlorophenyl (2E)-4,4,5,5,6,6,7,7,7-nonafluoro-2-(4-methoxyphenyl)hept-2-enoate (28) GAT-02-100-rp.1.fid<smiles>COc1ccc(/C(=C\C(F)(F)F)C(=O)Oc2c(Cl)cc(Cl)cc2Cl)cc1</smiles>
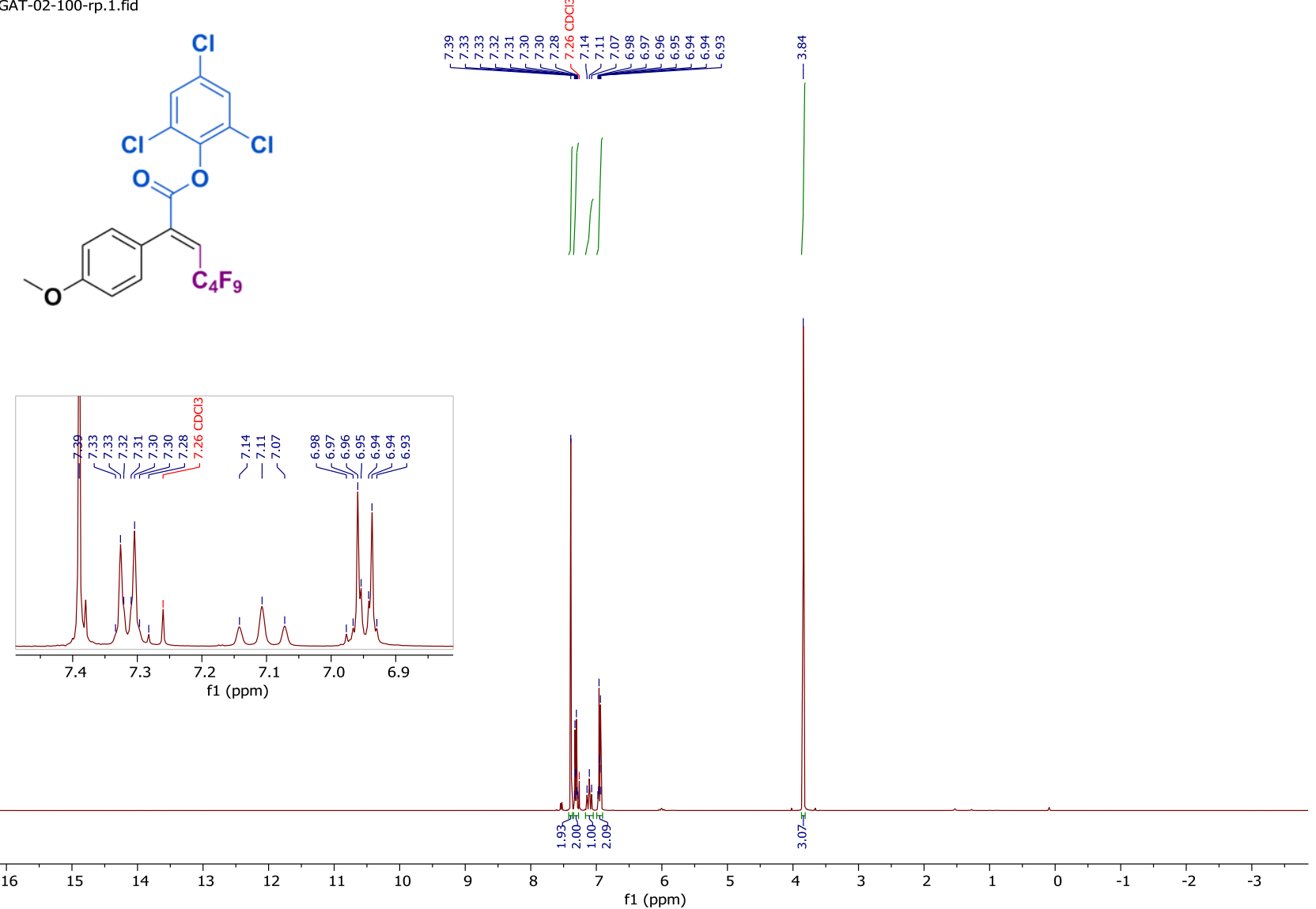

GAT-02-100-rp.2.fid
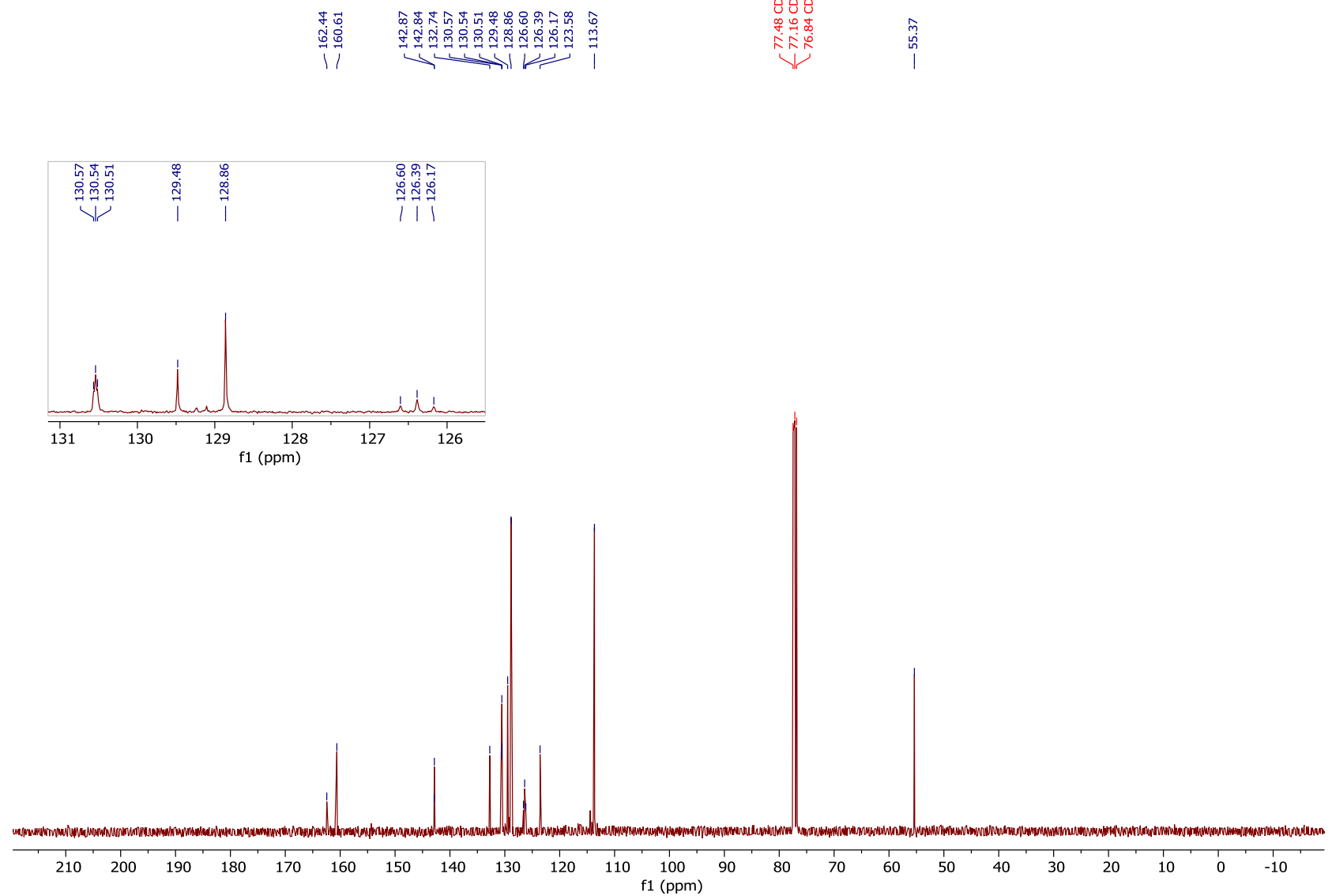


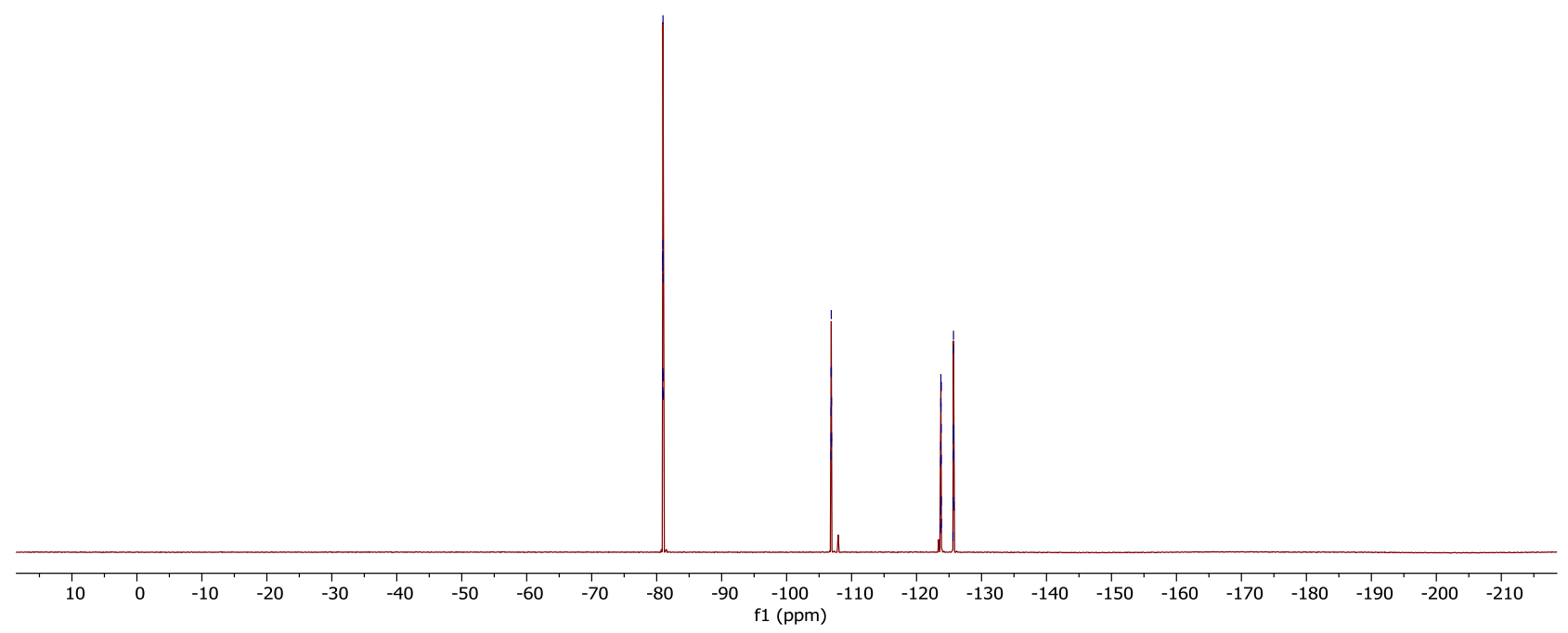


4-Bromophenyl (2E)-4,4,5,5,6,6,7,7,7-nonafluoro-2-(4-methoxyphenyl)hept-2-enoate (29) GAT-02-99-F2-rpp.1.fid
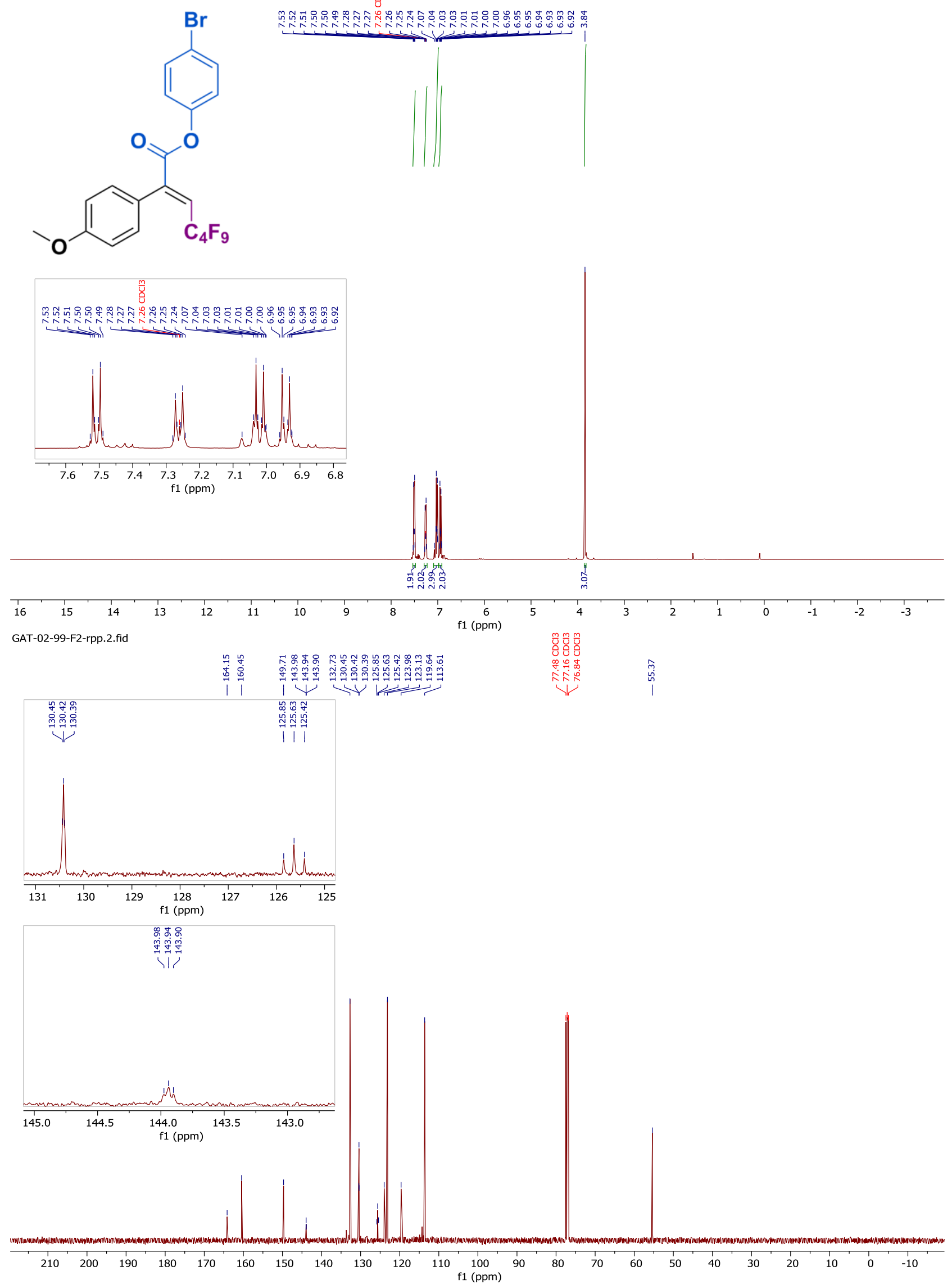


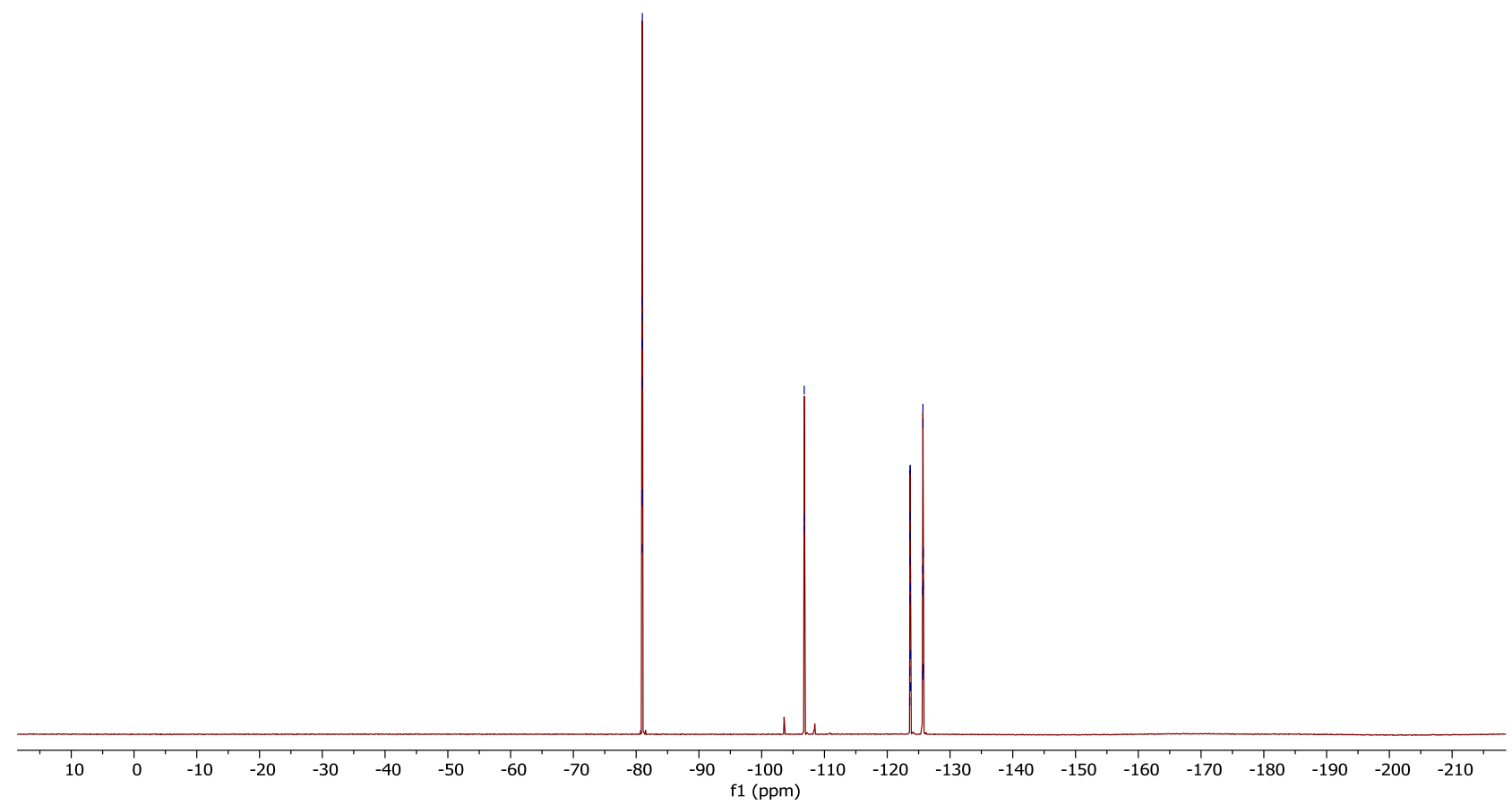


4-Fluorophenyl ( $2 E$ )-4,4,5,5,6,6,7,7,7-nonafluoro-2-(4-methoxyphenyl)hept-2-enoate (30) GAT-02-11.1.fid

等<smiles>COc1ccc(/C(=C\C(F)(F)F)C(=O)Oc2ccc(F)cc2)cc1</smiles>
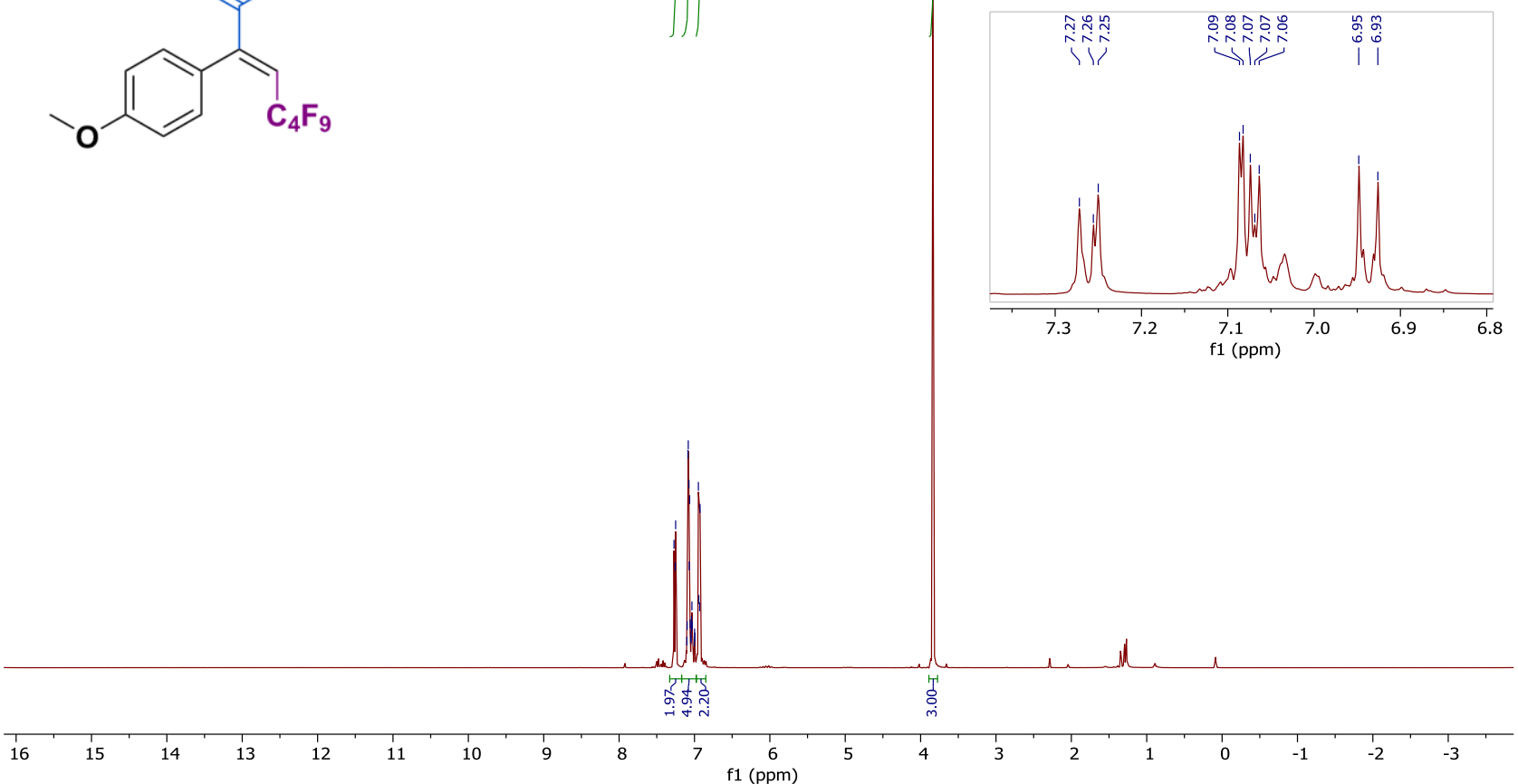

GAT-02-11.2.fid

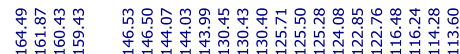
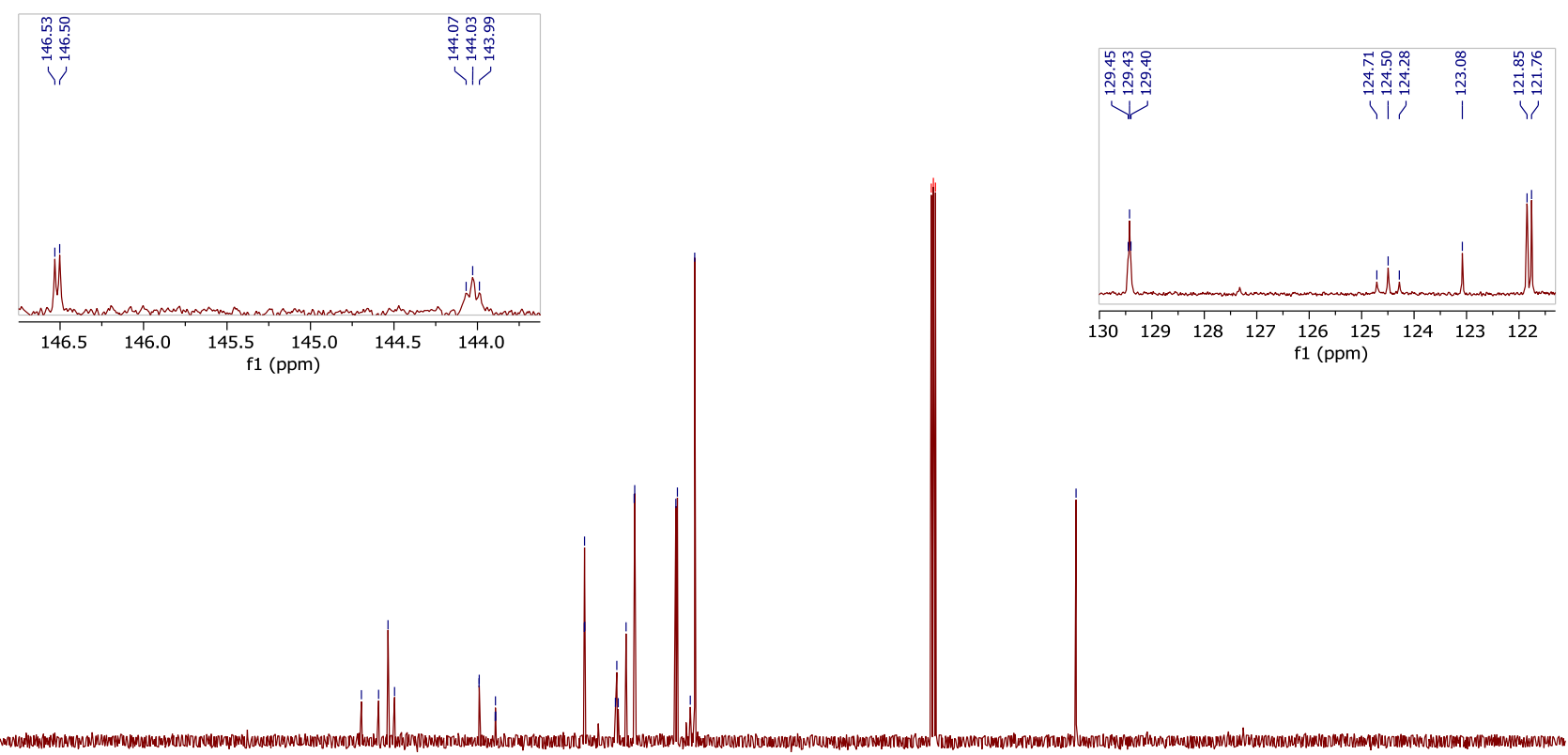

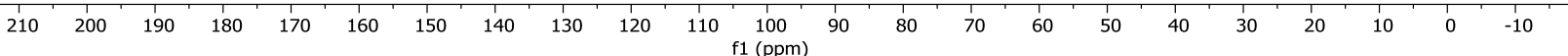




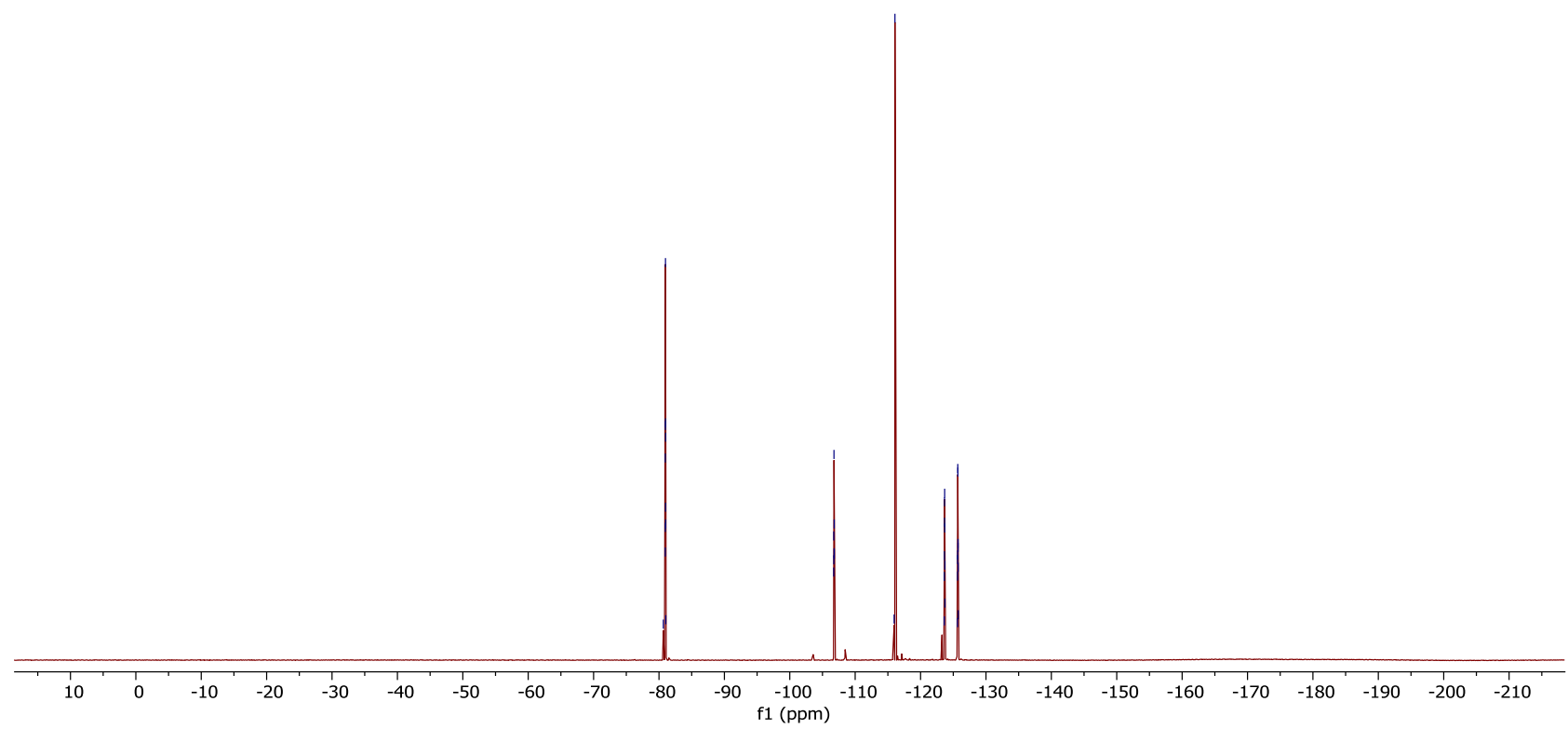


Phenyl (2E)-4,4,5,5,6,6,7,7,8,8,9,9,9-tridecafluoro-2-(4-methoxyphenyl)non-2-enoate (31) GAT-02-65-F3-rp.1.fid<smiles>COc1ccc(/C(=C\C(F)(F)F)C(=O)Oc2ccccc2)cc1</smiles>
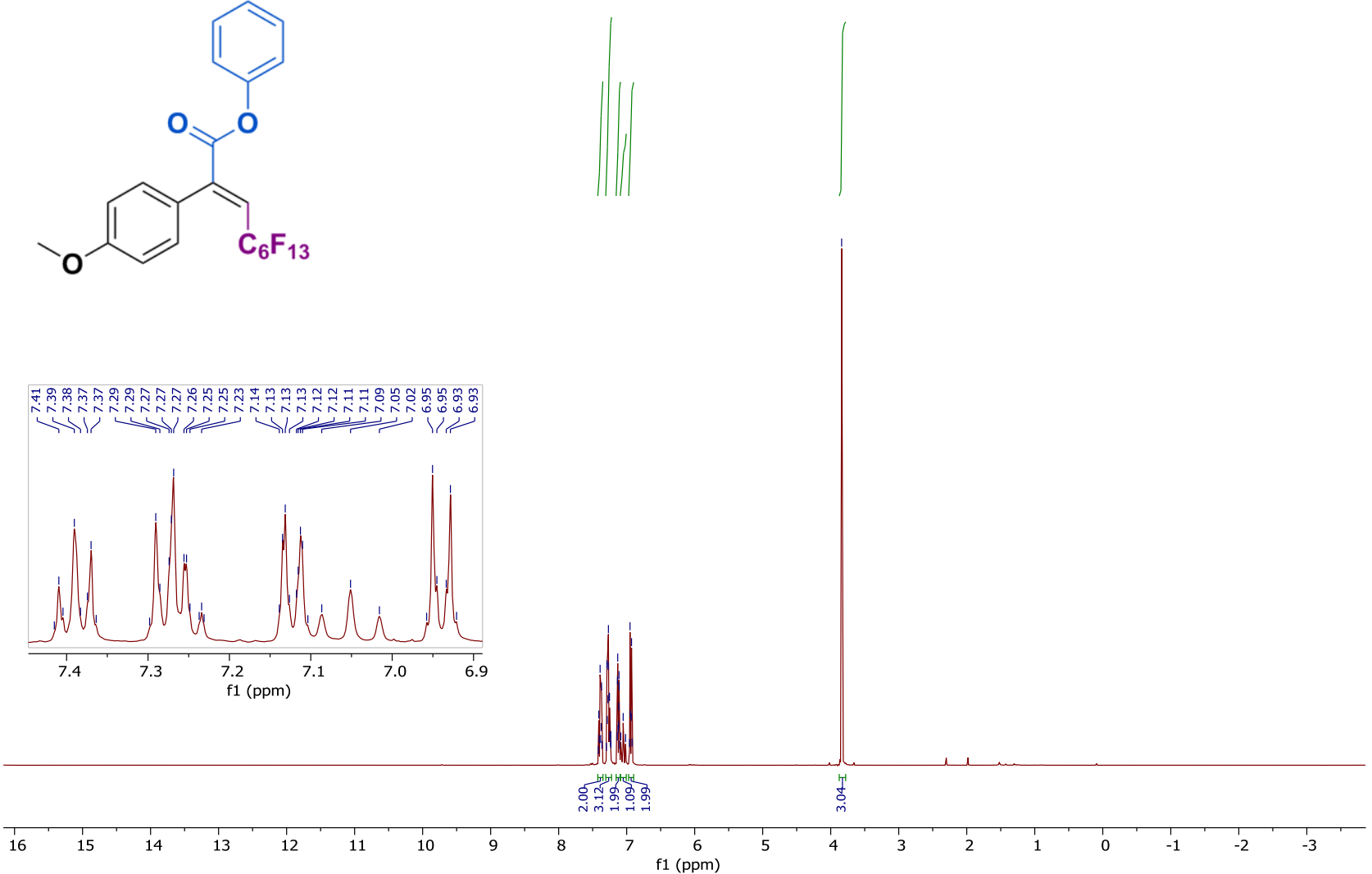

GAT-02-65-F3-rp.2.fid
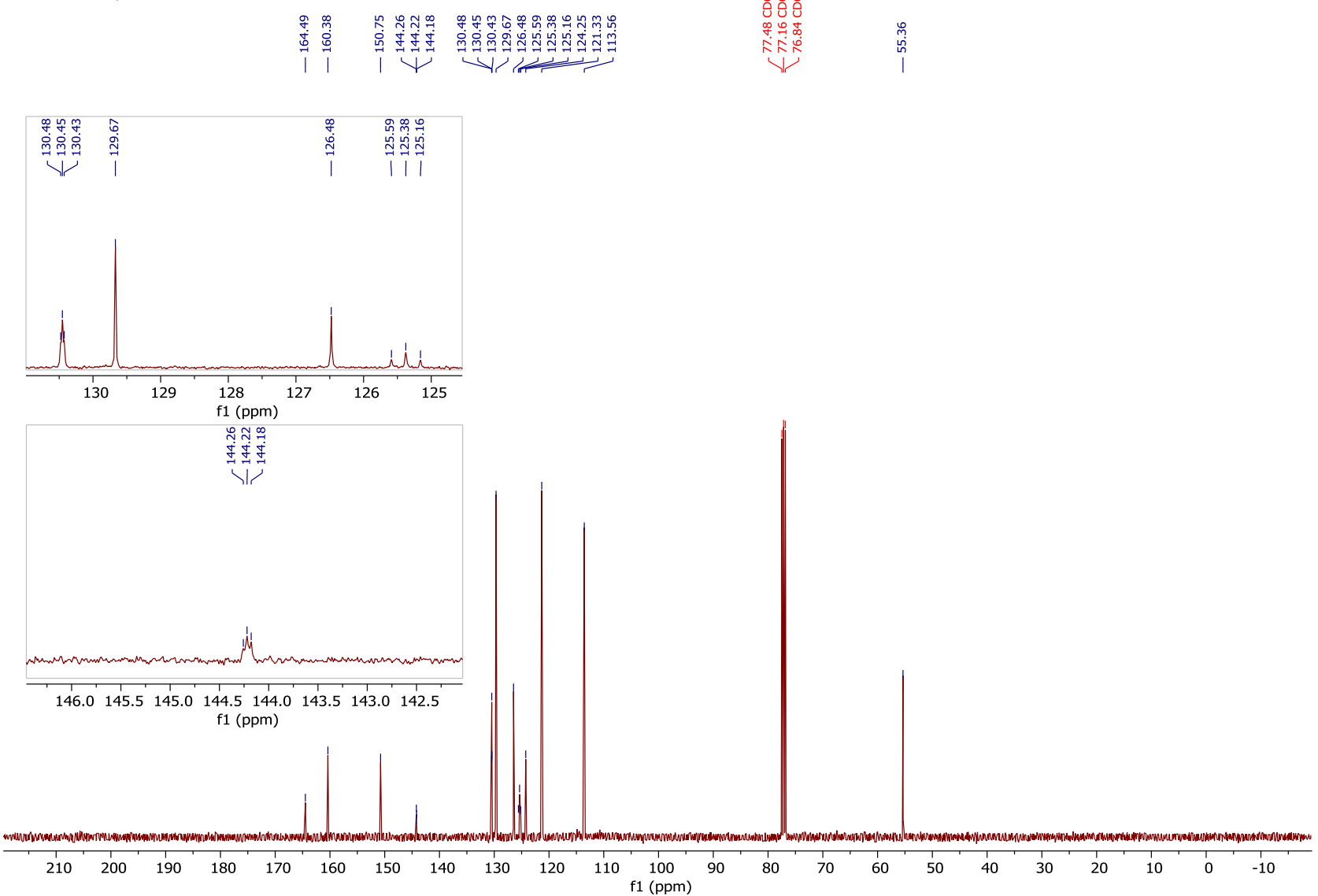


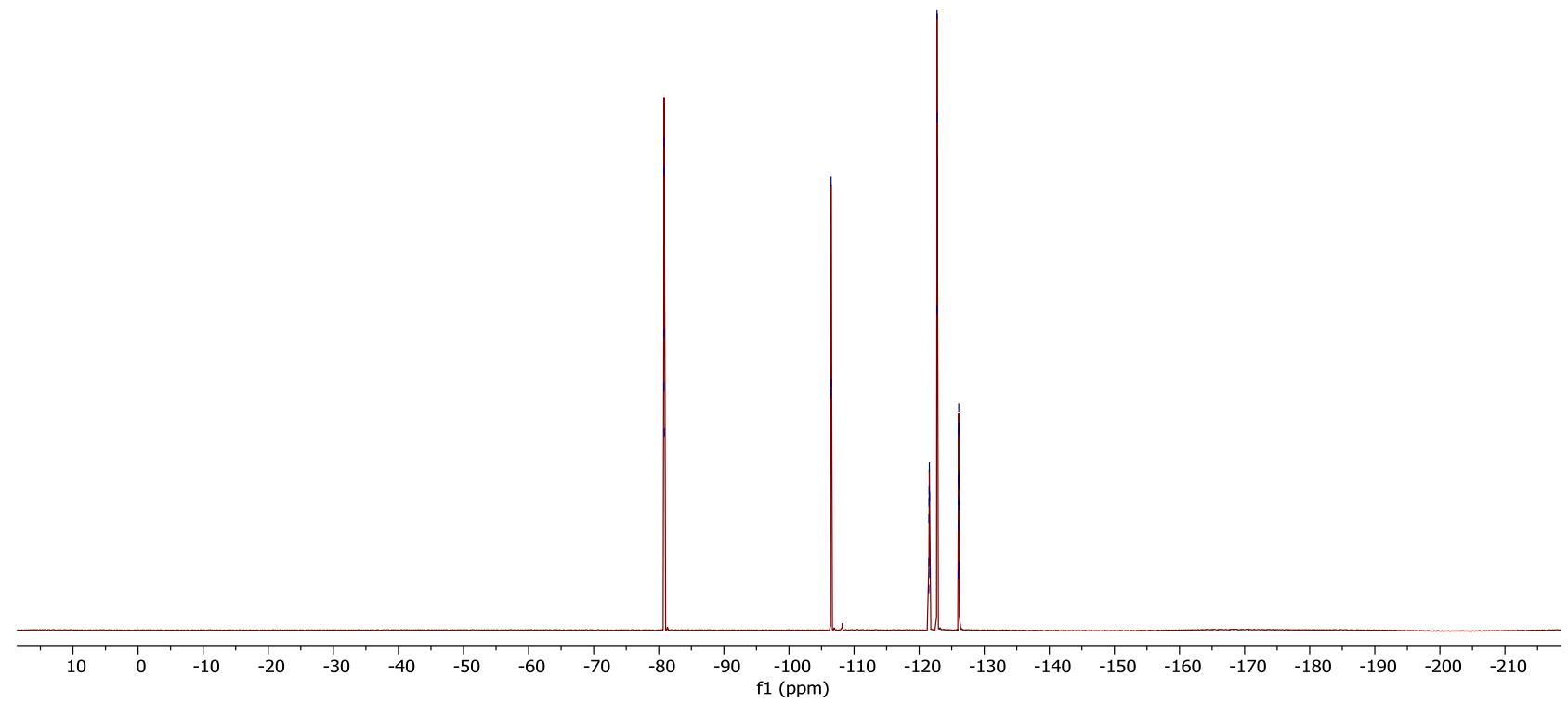


Phenyl (2E)-4,4,5,5,6,6,7,7,8,8,9,9,10,10,11,11,12,12,13,13,13-henicosafluoro-2-(4-methoxyphenyl)tridec-2-enoate (32) GAT-02-66-F5-rp.1.fid

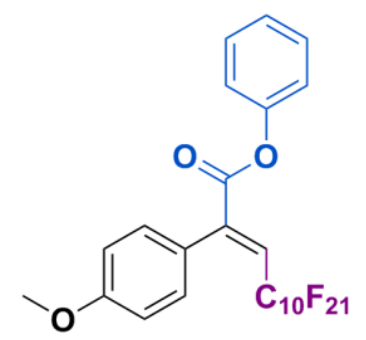
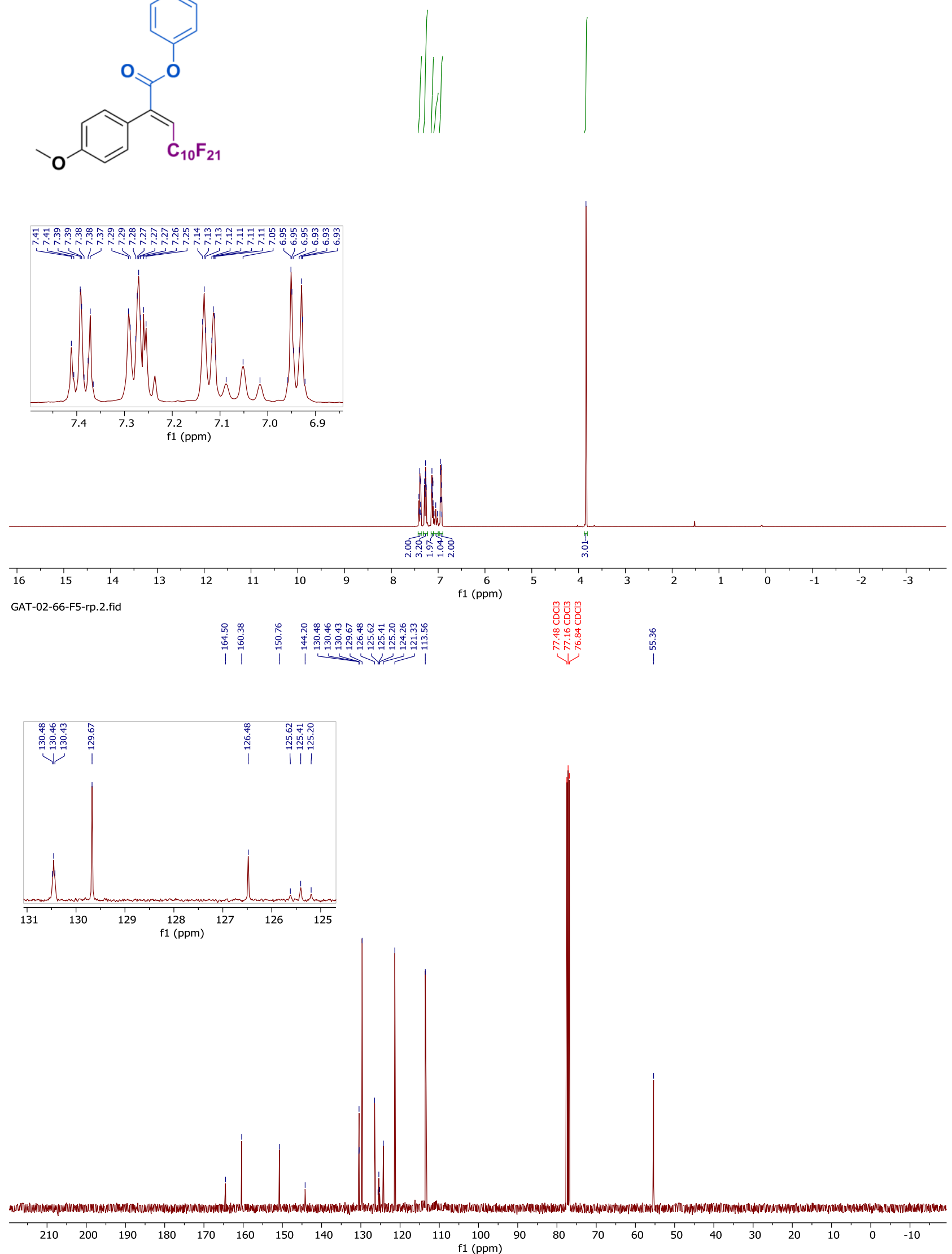


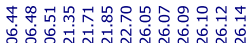

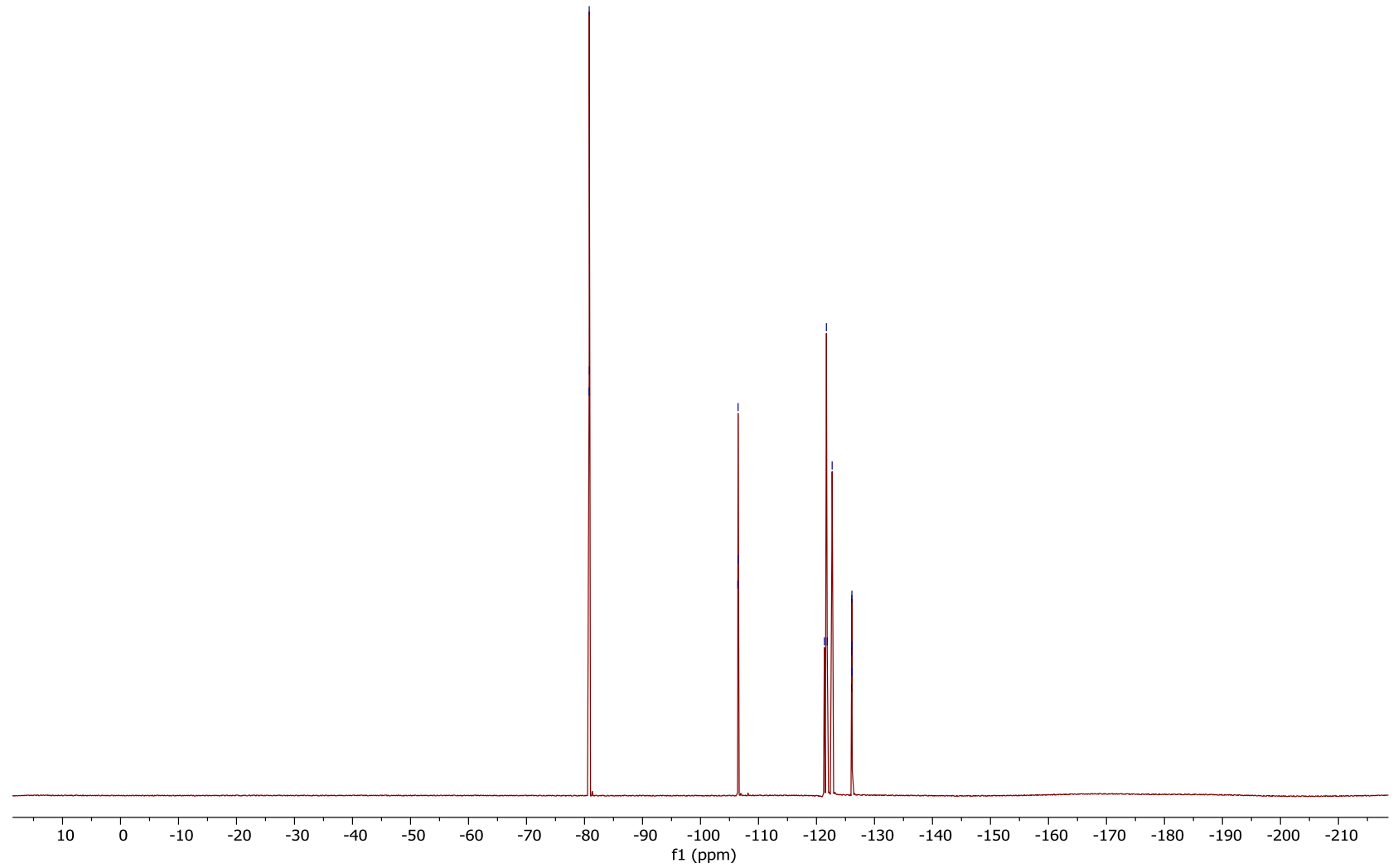


Phenyl (2E)-4,4,5,5,6,6,7,7,8,9,9,9-dodecafluoro-2-(4-methoxyphenyl)-8-(trifluoromethyl)non-2-enoate (33)

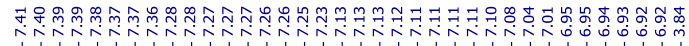<smiles>COc1ccc(/C(=C/C(F)(F)C(F)(F)C(F)(F)C(F)(F)C(F)(F)C(F)(F)F)C(=O)Oc2ccccc2)cc1</smiles>
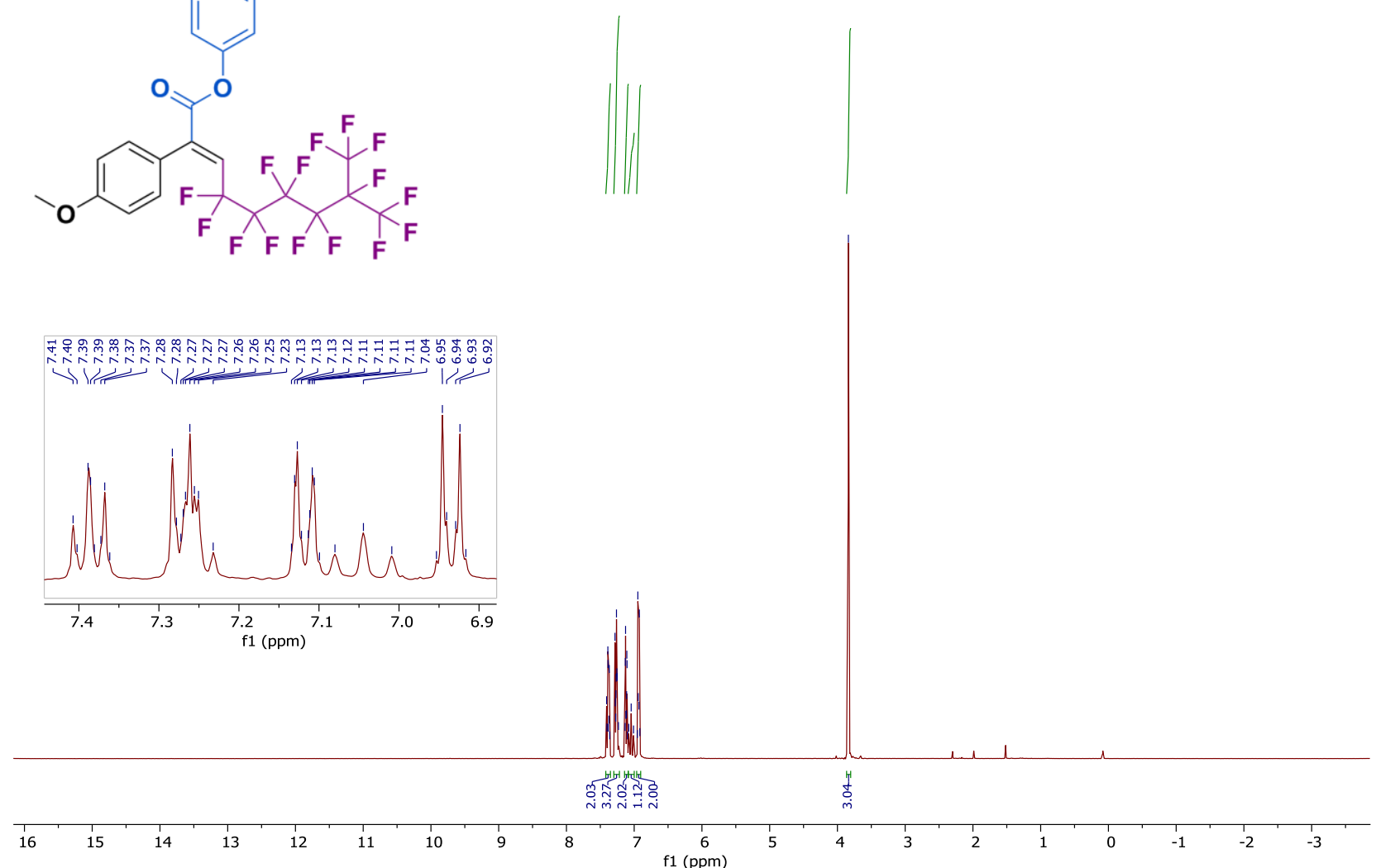

GAT-02-67-F2-rp.2.fid

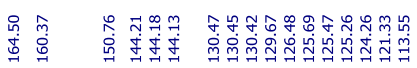

|
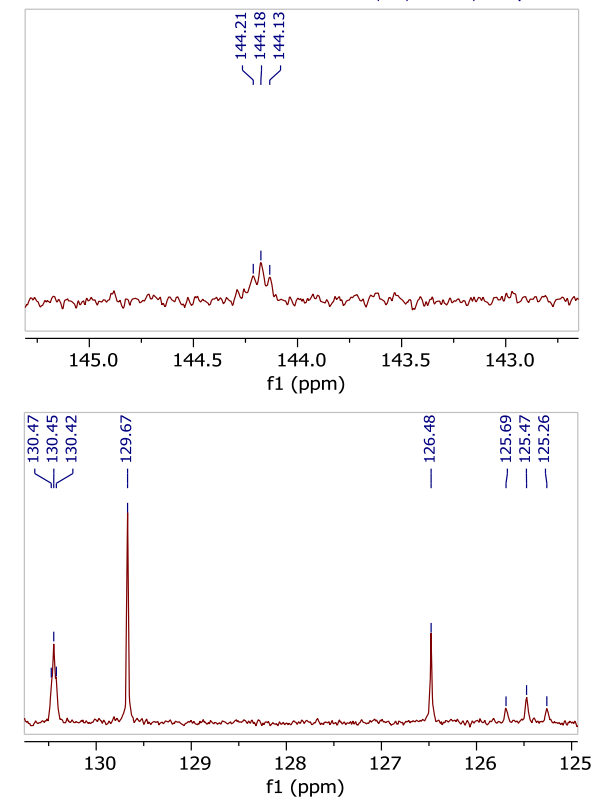

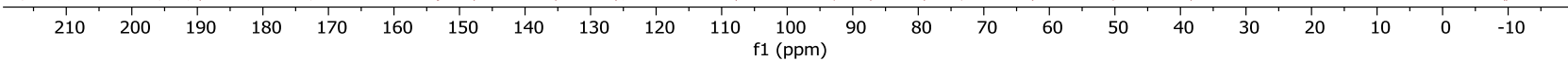




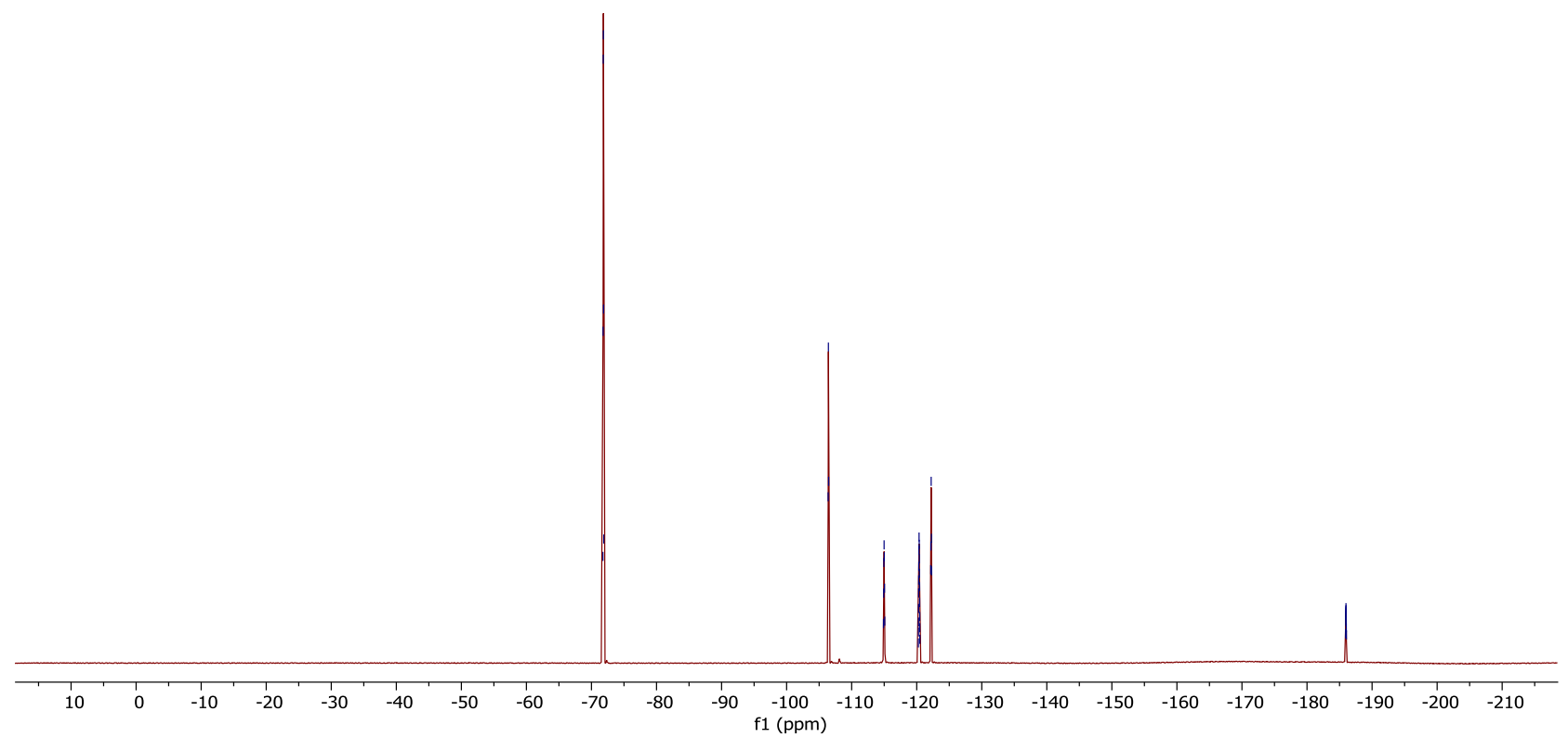


Phenyl (2E)-4,4,5,5,6,6,7,7-octafluoro-7-iodo-2-(4-methoxyphenyl)hept-2-enoate (34)

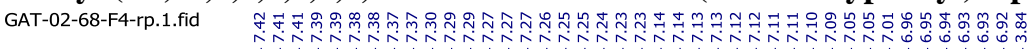<smiles>COc1ccc(/C(=C\C(F)(F)C(F)(F)C(F)(F)C(F)(F)I)C(=O)Oc2ccccc2)cc1</smiles>
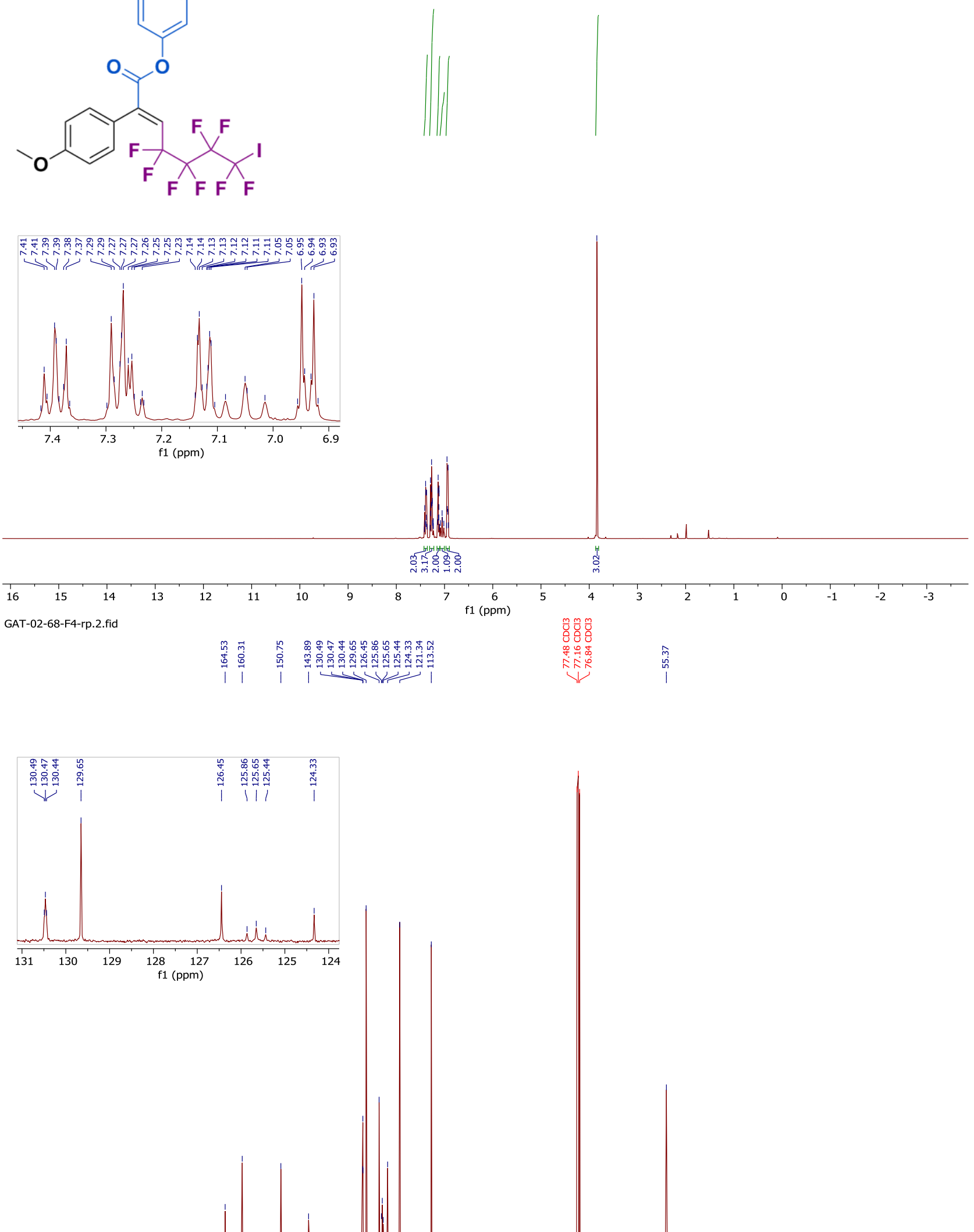

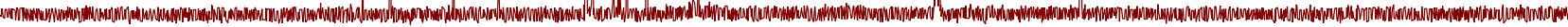

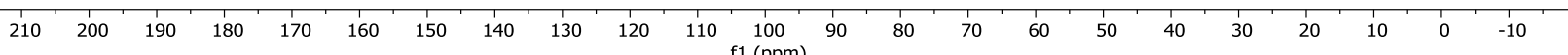




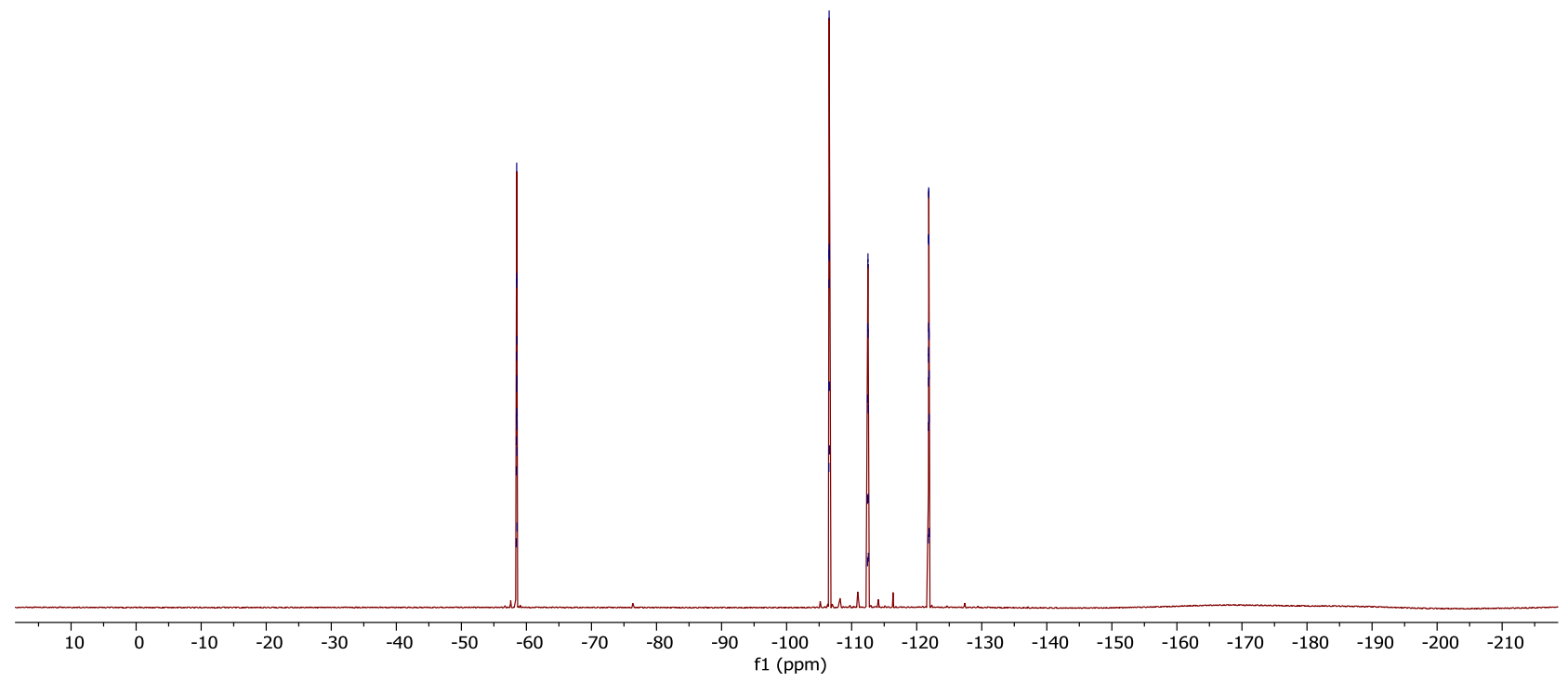


Phenyl (2E)-4,4,5,5,6,6,7,7,7-nonafluoro-2-phenylhept-2-enoate-d (35)

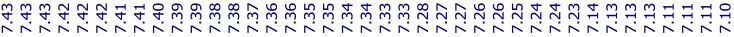<smiles>[2H]/C(=C(\C(=O)Oc1ccccc1)c1ccccc1)C(F)(F)F</smiles>

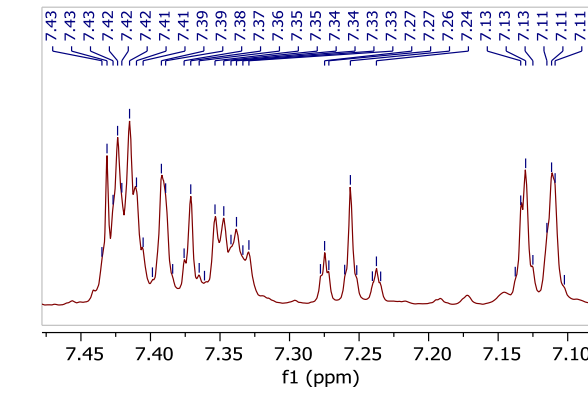

Nats

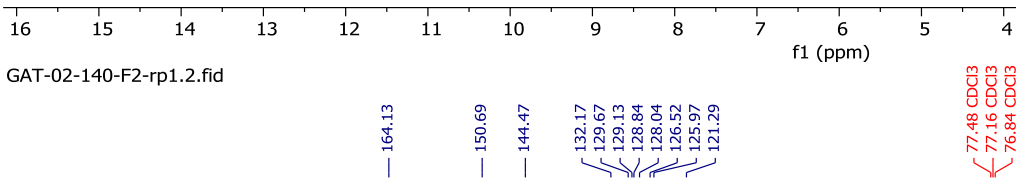
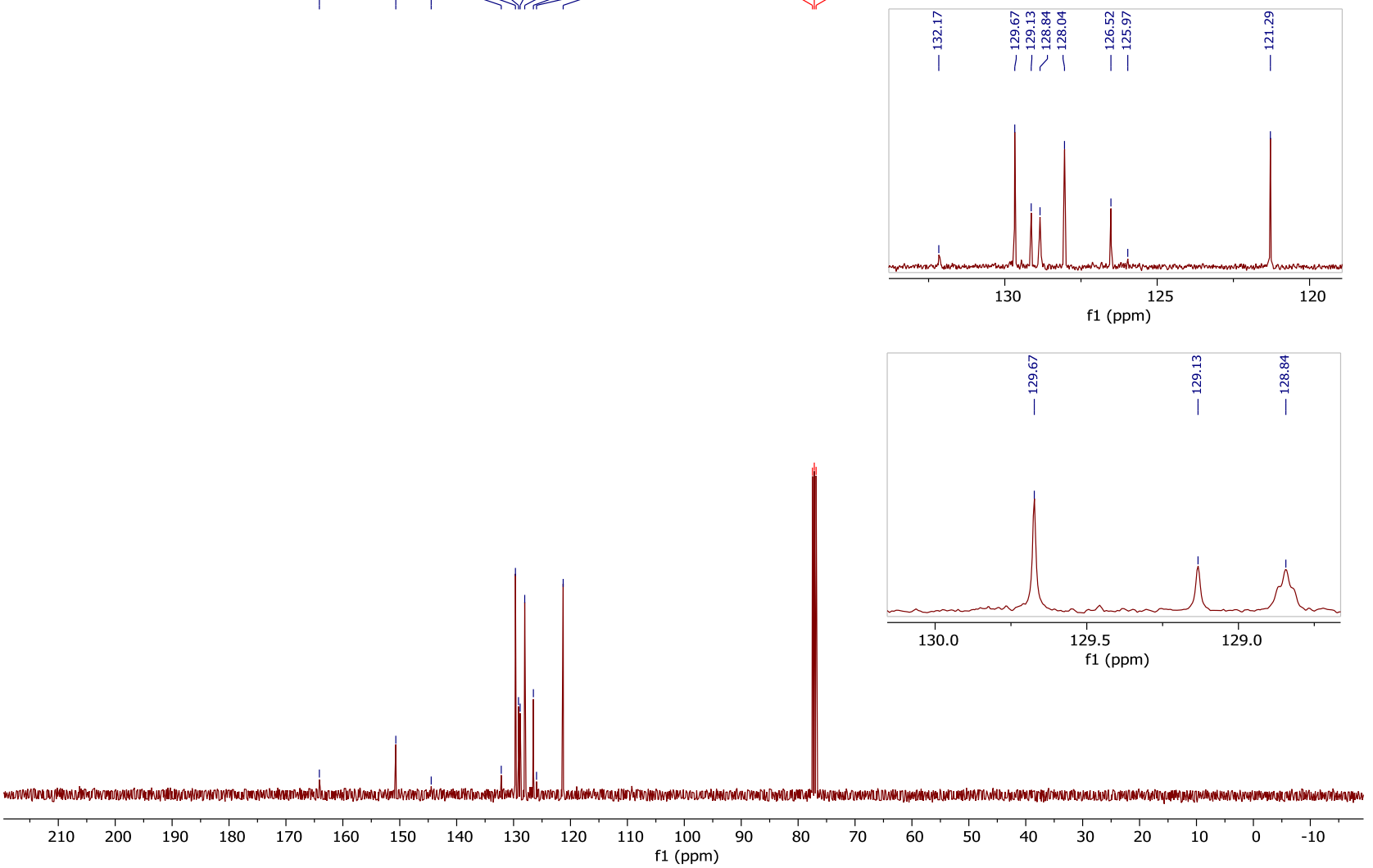


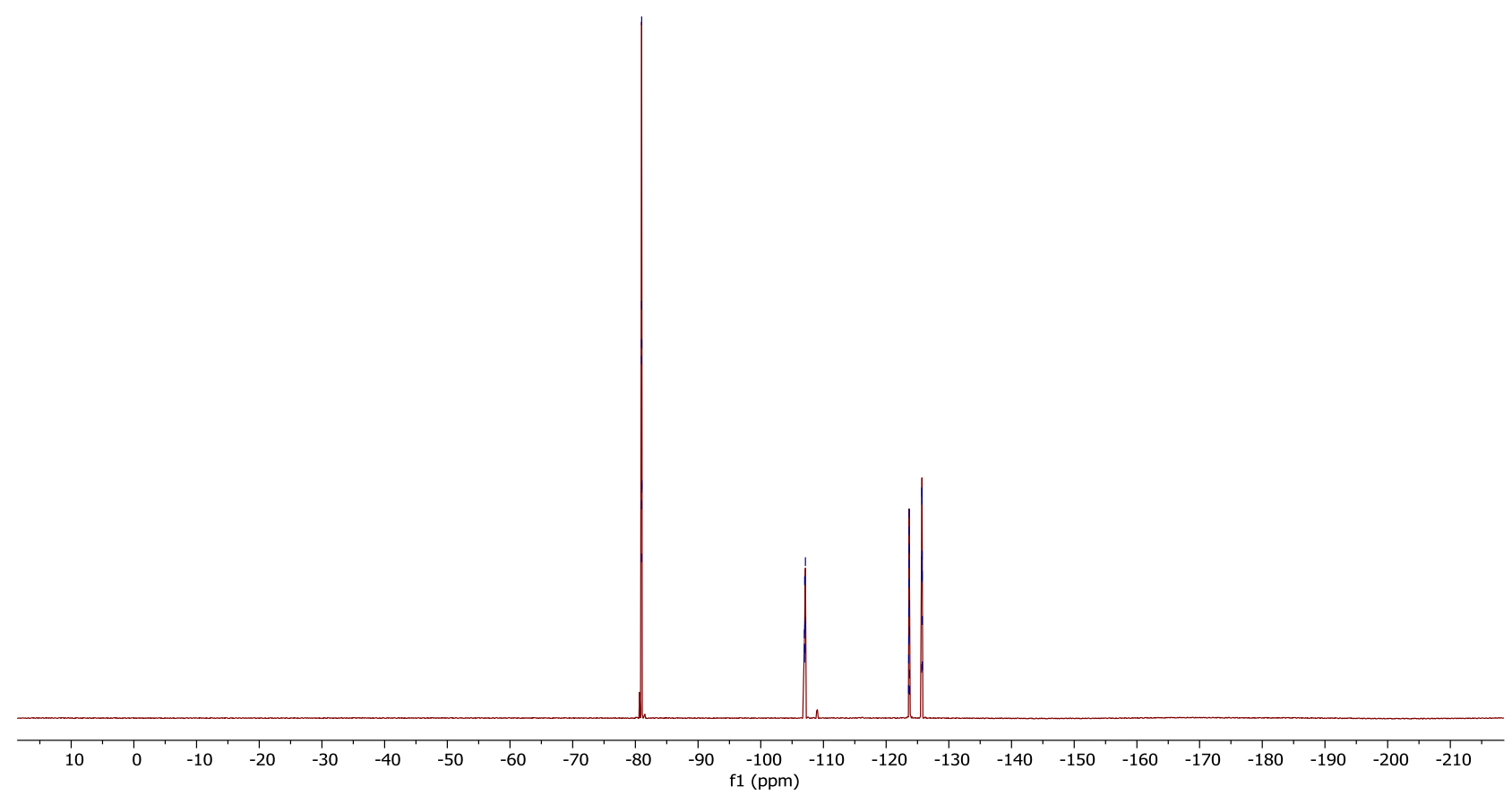


3-Methoxyphenyl (2E)-4,4,5,5,6,6,7,7,7-nonafluoro-2-(4-methoxyphenyl)hept-2-enoate - ${ }^{13} \mathrm{C}$ (36)

GAT-02-151-F3.1.fid

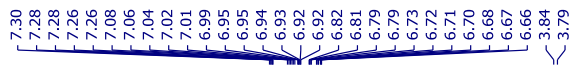<smiles>COc1ccc(C(=CC(F)(F)F)[C@H](O)Oc2cccc(OC)c2)cc1</smiles>
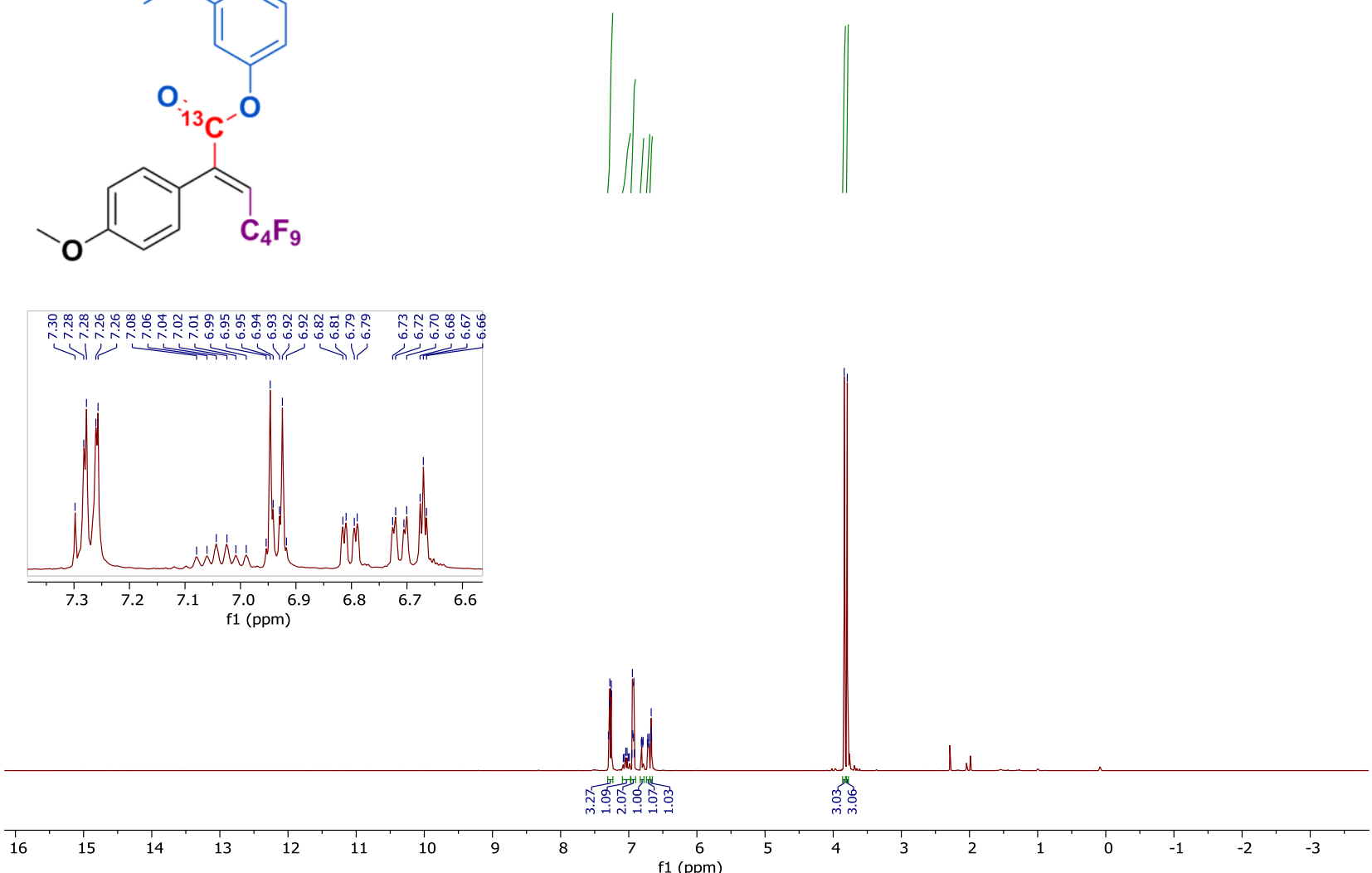

GAT-02-151-F3.2.fid

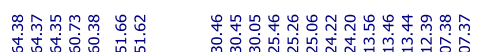

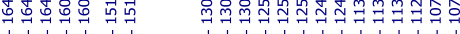

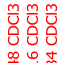

年
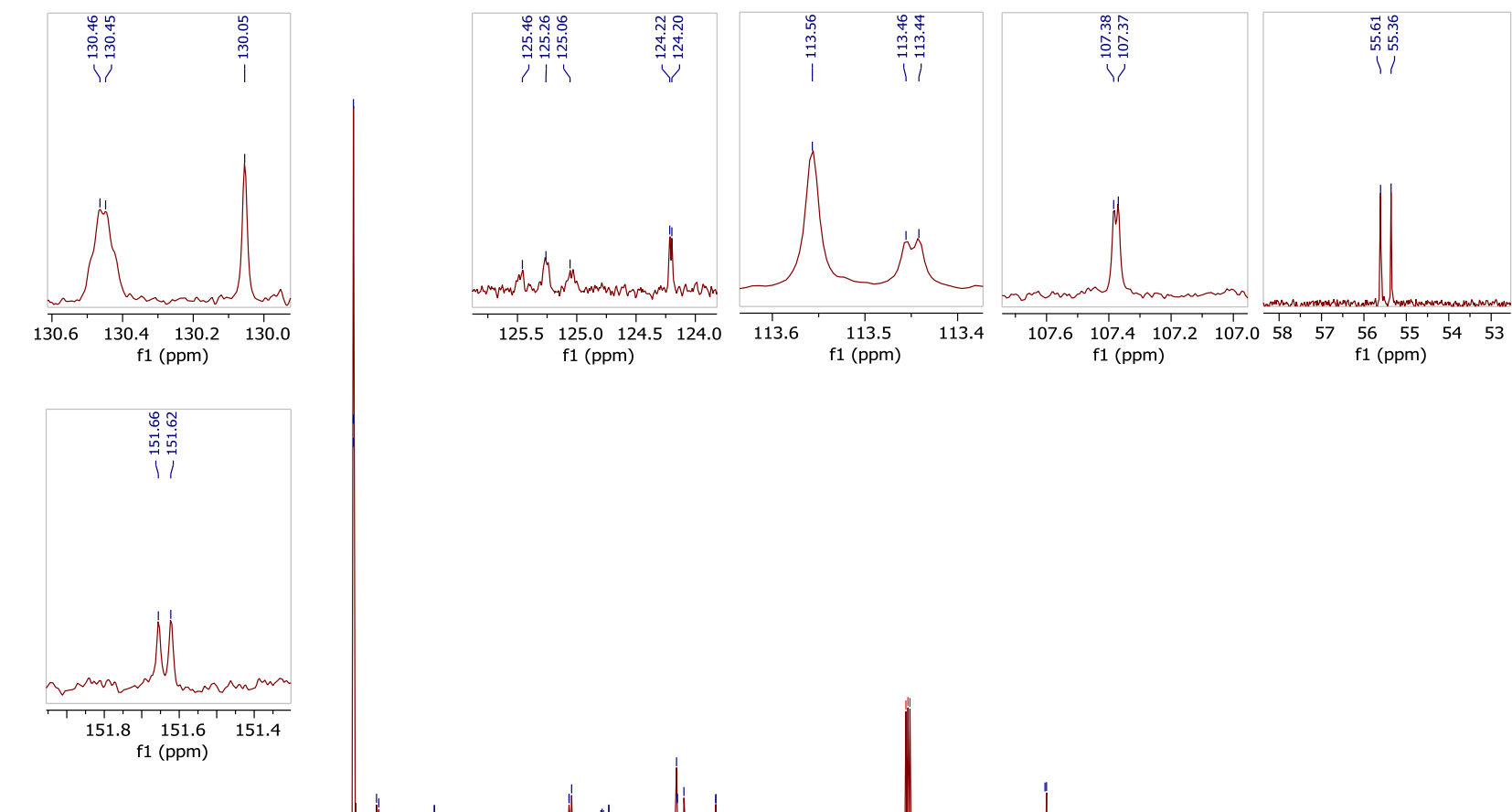

$\begin{array}{lllllll}210 & 200 & 190 & 180 & 170 & 160 & 150\end{array}$
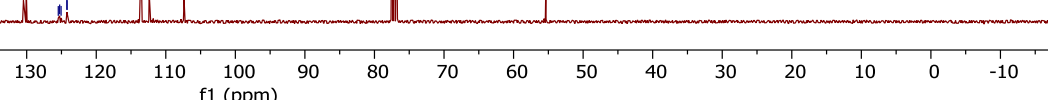
GAT-02-151-F3.31.fid

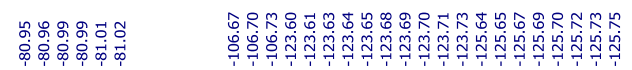

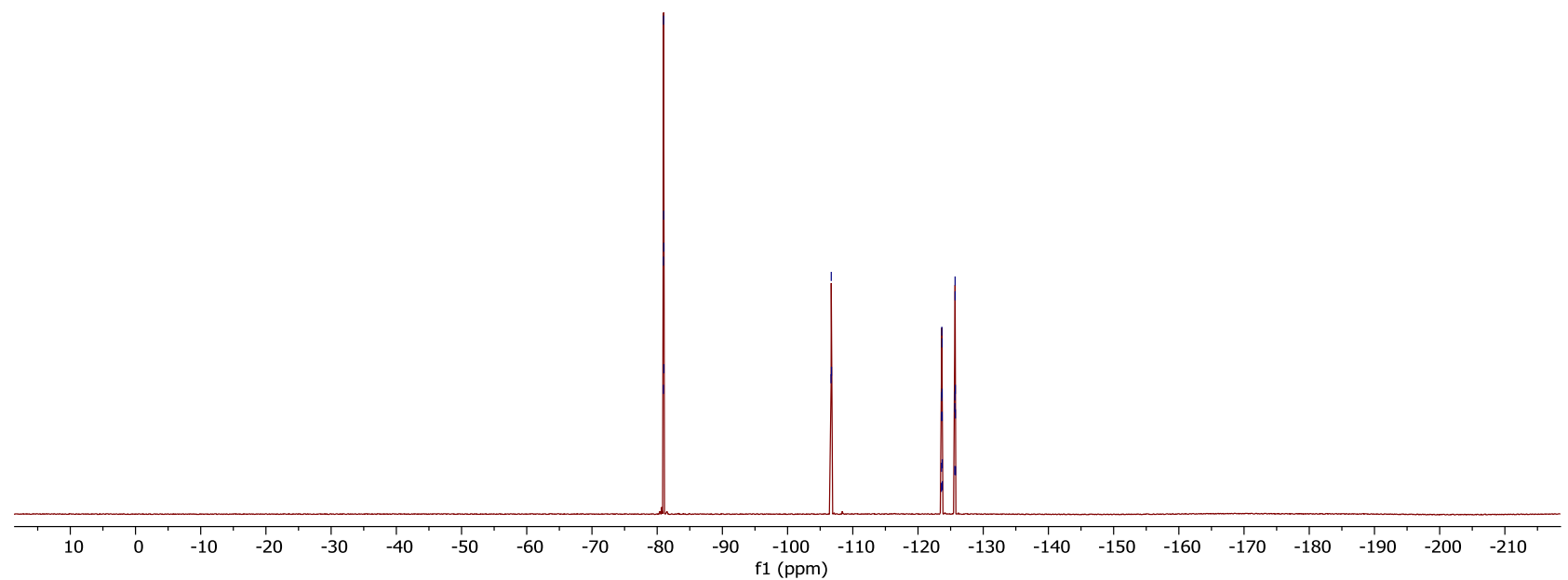




\subsection{Synthetic utility of perfluorosubstituted $\alpha, \beta$-unsaturated esters}

\section{1,1-dimethyl 3-phenyl 3-(4-fluorophenyl)-2-(perfluorobutyl)propane-1,1,3-tricarboxylate}

GAT-02-188-F1.1.fid<smiles>COC(=O)C(C(C(=O)Oc1ccccc1)c1ccc(F)cc1)C(C(C)(F)F)C(F)(F)F</smiles>

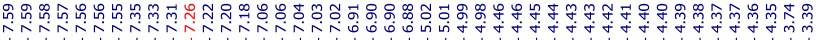
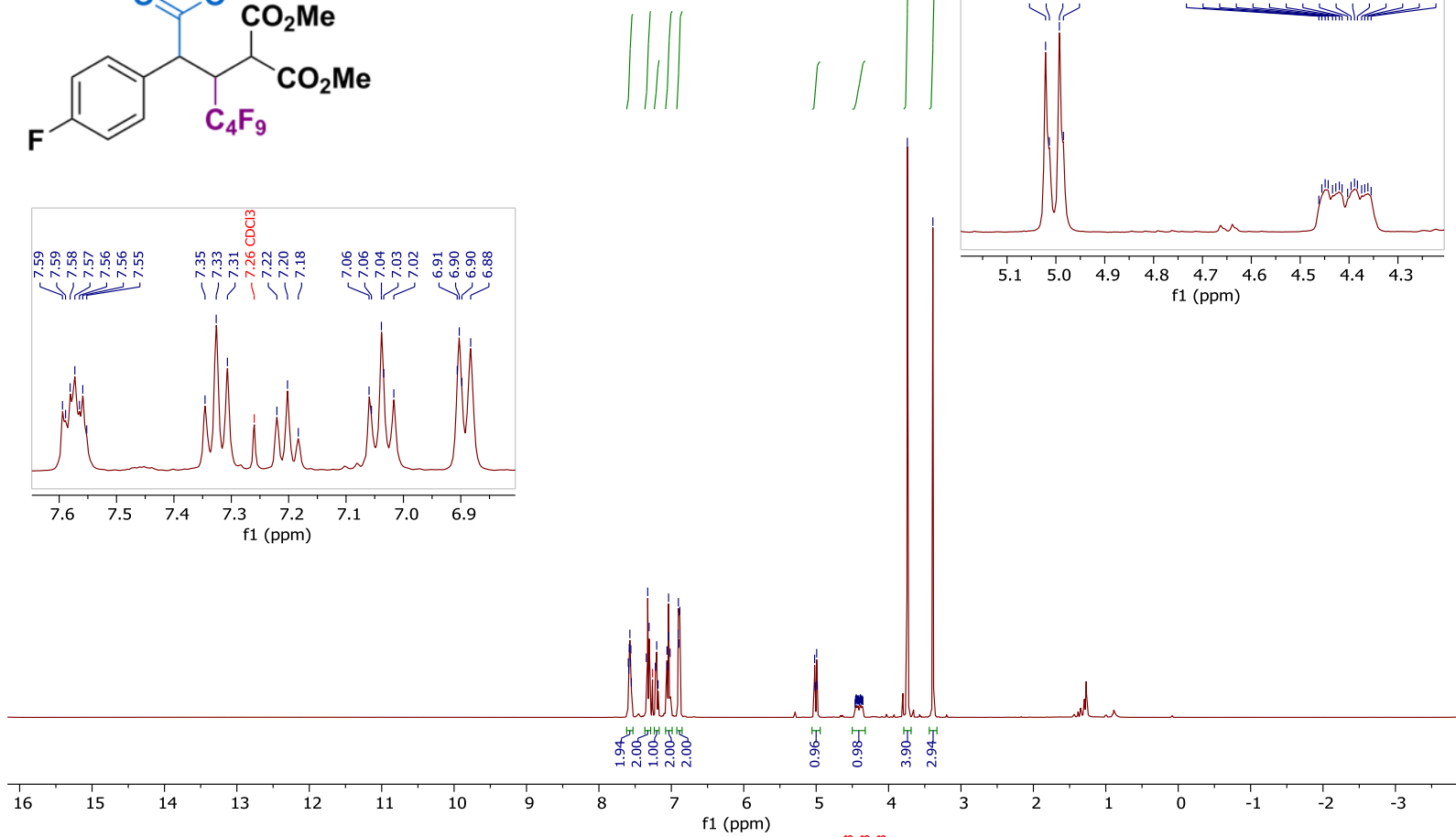

GAT-02-188-F1.2.fid

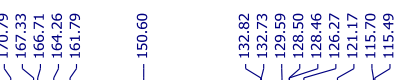

Vil

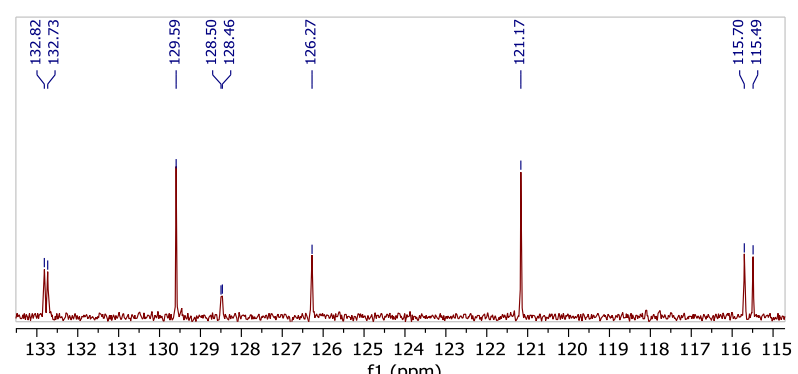
f1 (ppm)

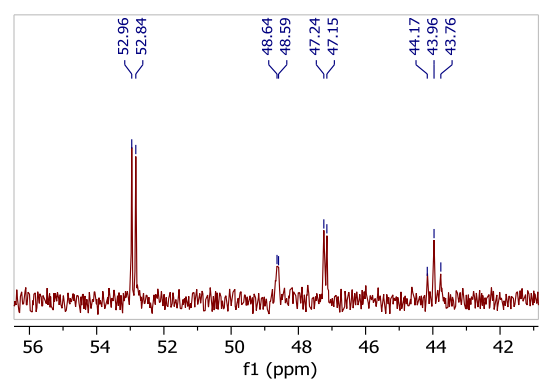

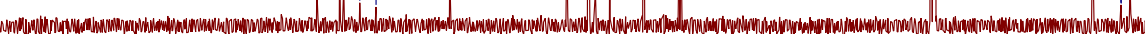

$\begin{array}{lllllllllll}210 & 200 & 190 & 180 & 170 & 160 & 150 & 140 & 130 & 120 & 110 \\ \mathrm{f} 1(\mathrm{ppm})\end{array}$ 


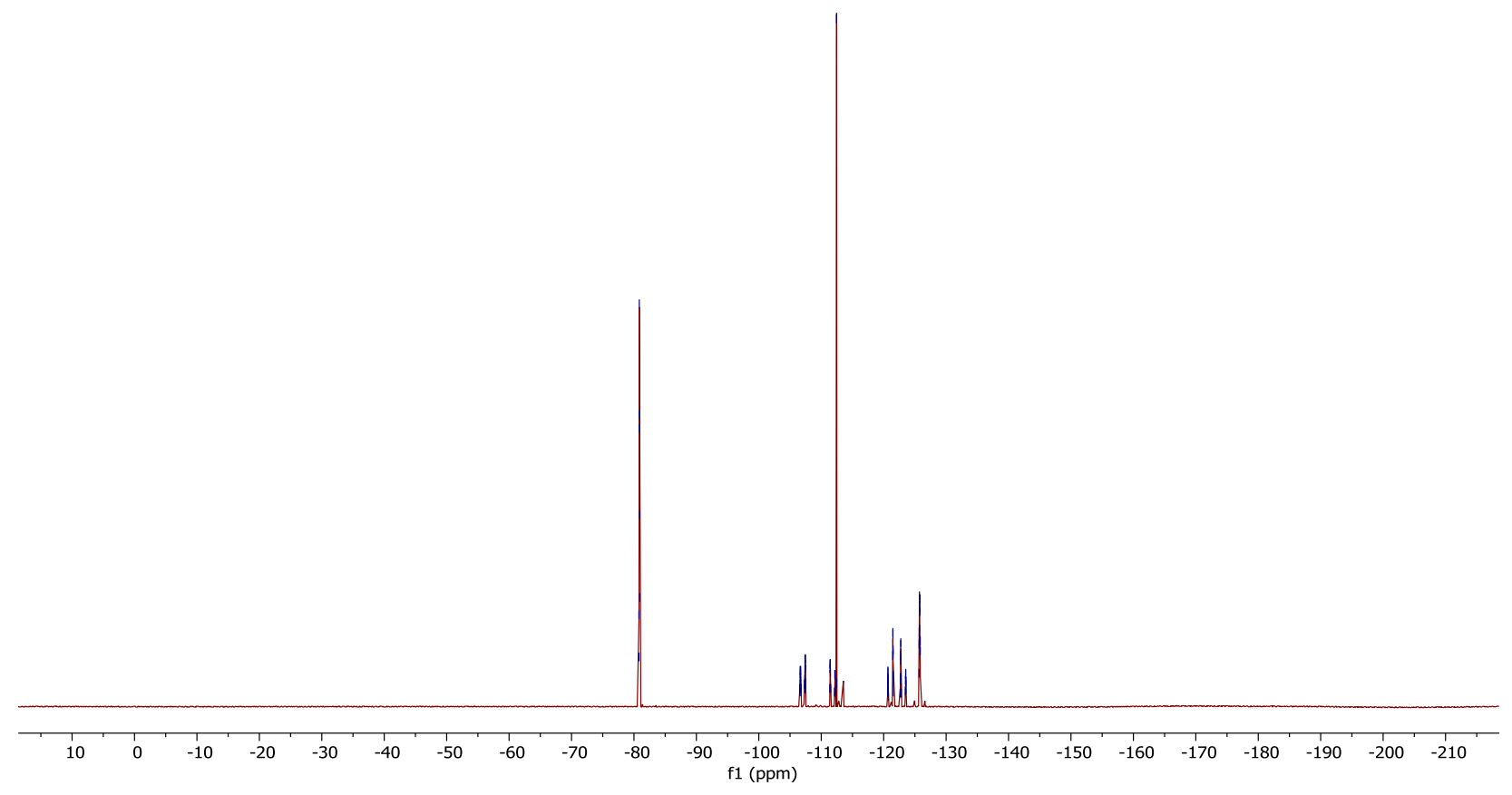


(E)-4,4,5,5,6,6,7,7,7-nonafluoro-2-(4-fluorophenyl)-1-morpholinohept-2-en-1-one GAT-02-194-F3p.1.fid<smiles>O=C(C(=CC(F)(F)F)c1ccc(F)cc1)N1CCOCC1</smiles>
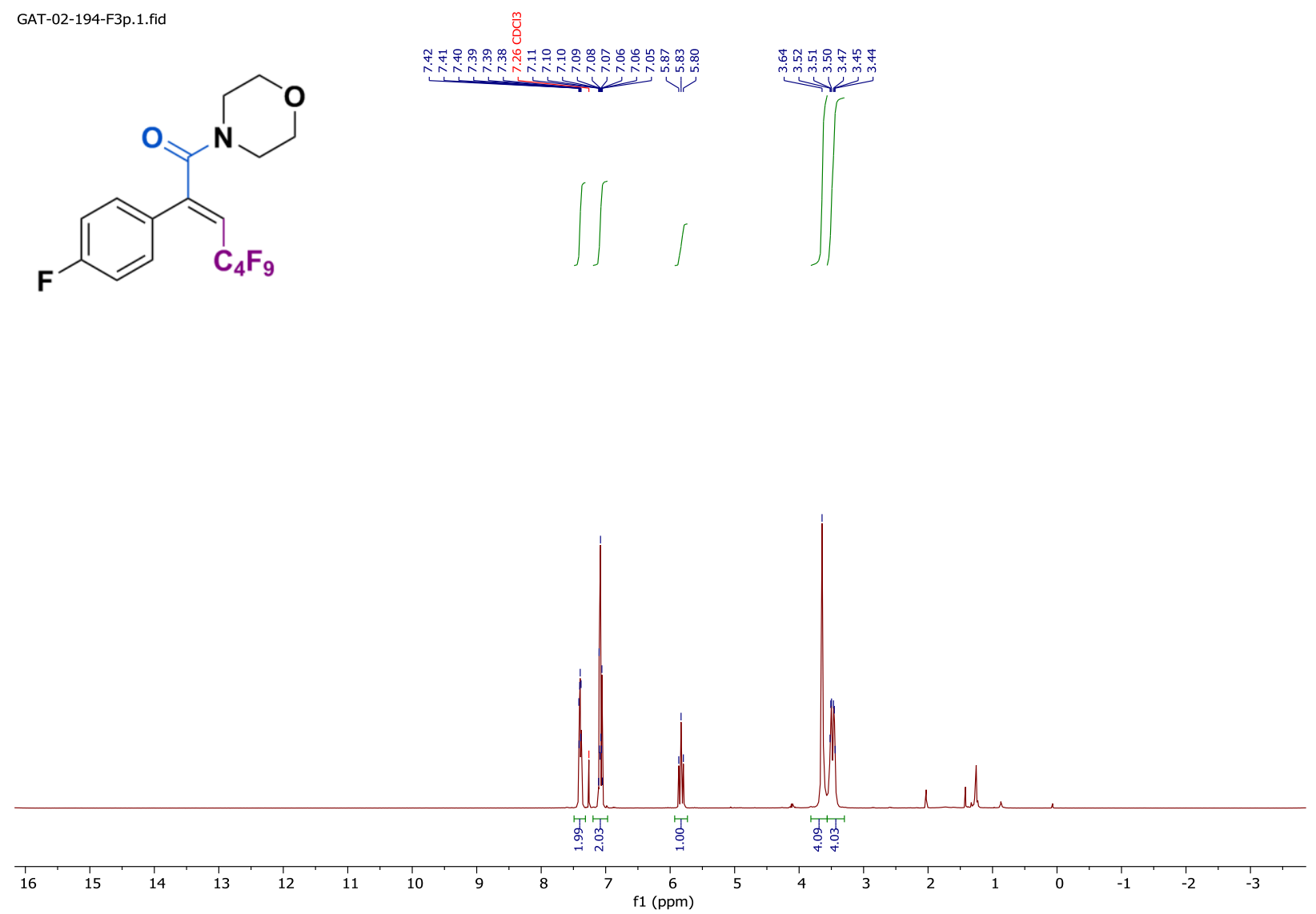

GAT-02-194-F3p.2.fid
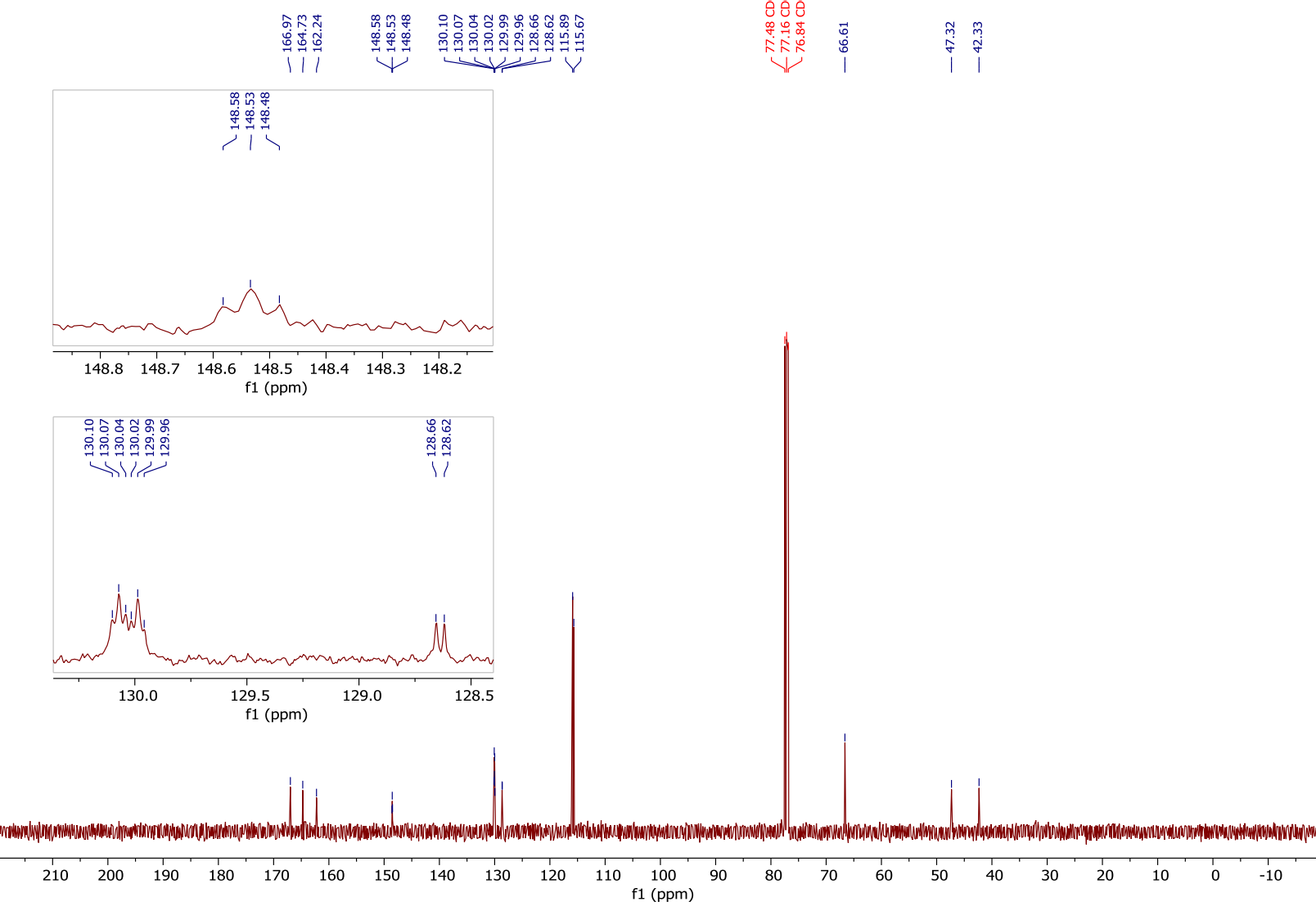


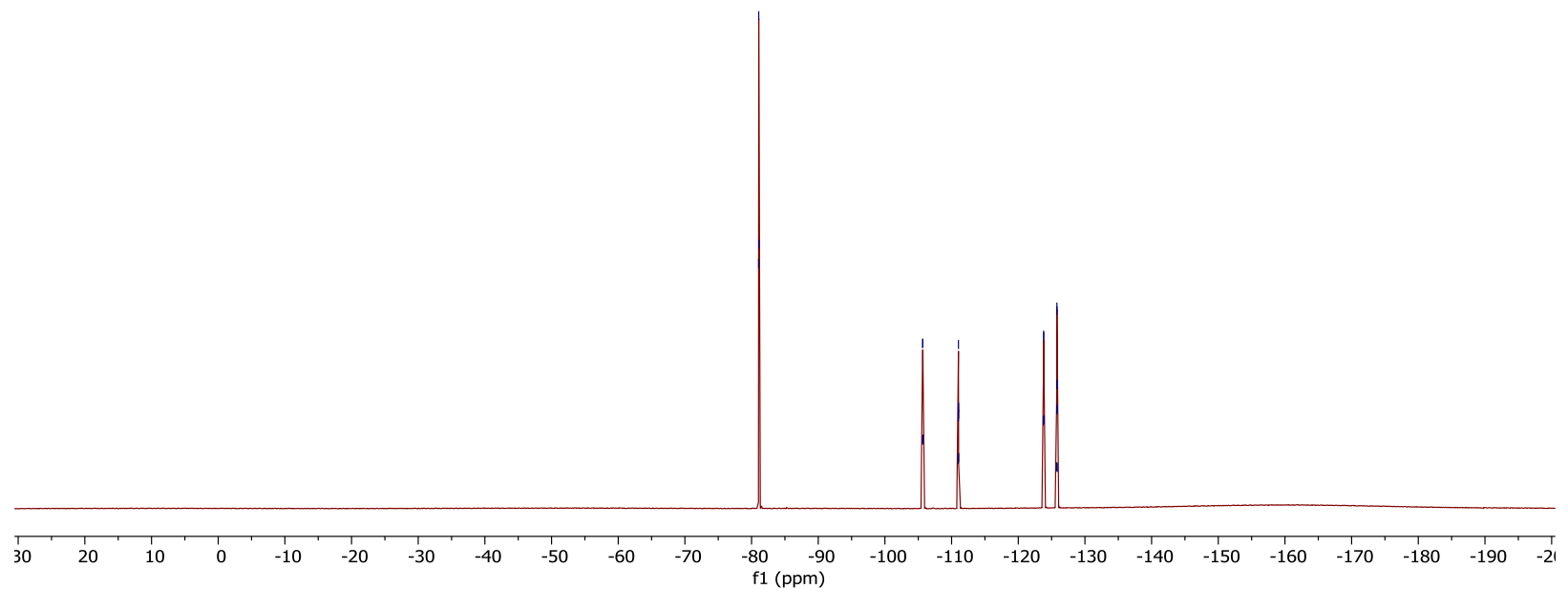




\section{(E)-butyl 4,4,5,5,6,6,7,7,7-nonafluoro-2-phenylhept-2-enoate}
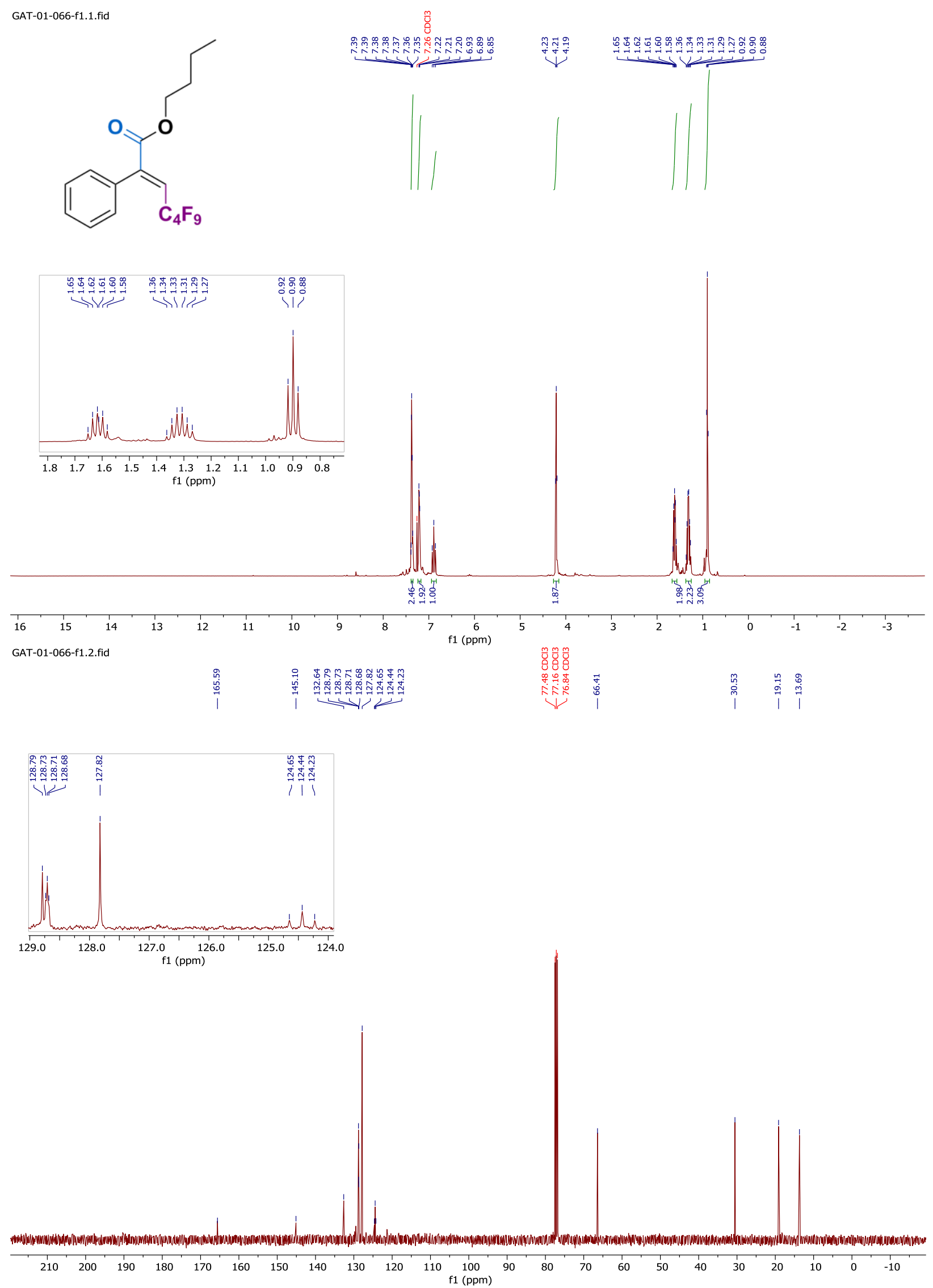


\section{X-Ray Diffraction Data of Compound 10}

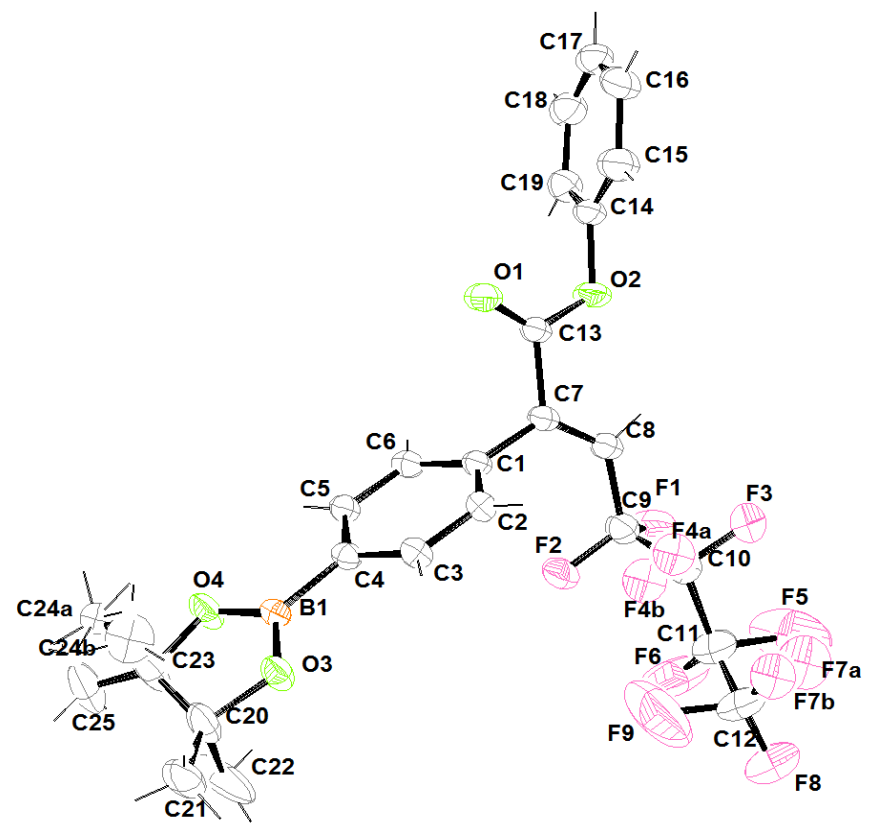

Comment on B alerts: An attempt was made to resolve the problem by introduction of ca. $0.5 / 0.5$ disorder during refinement of F4, F7 and C24 atoms. This resulted in disappearence of several B-alerts. However, further refinement of $\mathrm{C} 22$ and some of the carbon and fluorine atom positions with geometric restrains lead to poor convergence of the whole refinement and an increased R-factor. Fluctuation in geometry of the side chain is probably derivative of additional disorder in this part. Our experience with low-T data for other compounds of this series was not good.

Crystal colour

Crystal description

Crystal dimensions $[\mathrm{mm}]$

Temperature [K]

Radiation type

Crystal system

Space group

Unit cell parameters

$\mathrm{a}[\AA]$

$\mathrm{b}[\AA]$

$\mathrm{c}[\AA]$

$\left.\alpha{ }^{\circ}\right]$

$\beta\left[^{\circ}\right]$

$\gamma\left[{ }^{\circ}\right]$

$\mathrm{V}[\AA 3]$

$\mathrm{F}(000)$

Dx [g cm-3]

Reflections used in refinement

Parameters refined

Restraints

Goodness of fit colourless

plate

$0.506 \times 0.321 \times 0.176$

296(2)

CuKla

monoclinic

P 21/c

18.977(3)

11.6497(18)

12.004(2)

90

97.393(10)

90

2631.7(8)

1160

1.434

4571

368

29

1.101 


\section{Optimized geometries, energies and corrections to thermodynamic functions.}

\section{General}

All calculations were performed using Gaussian 09 package. Structures of minima and transition states were optimized employing B3LYP functional and LANL2DZ basis set for Pd and I, and the 6-31G(d) basis set for the other atoms (later on denoted as BS1). Frequency calculations were then performed at the same level of theory to confirm the nature of stationary points and provide corrections to thermodynamic functions. Single point energies and solvation (dichloroethane, PCM model) were calculated at M06 level of theory using SDD basis set for Pd and I, and the 6-311++g(d,p) basis set for the other atoms (later on denoted as BS2). In all cases, wavefunctions were tested for stability; if necessary, the unrestricted wavefunctions were obtained using "stable $=$ opt" keyword. Structures were visualized using: CYLview, 1.0b; Legault, C. Y., Université de Sherbrooke, 2009 (http://www.cylview.org)

\section{RuPhosPd}

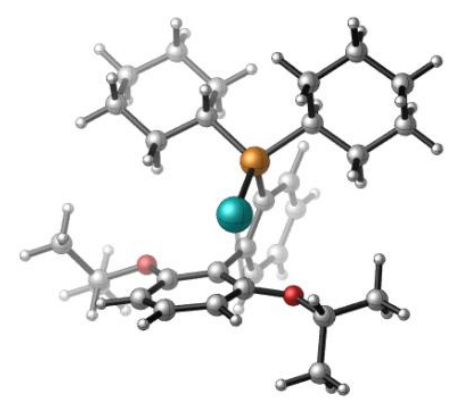

$\mathrm{E}(\mathrm{B} 3 \mathrm{LYP} / \mathrm{BS} 1)=-1787.65799$

$\mathrm{E}(\mathrm{PCM}($ dichloroethane $) / \mathrm{M} 06 / \mathrm{BS} 2 / / \mathrm{B} 3 \mathrm{LYP} / \mathrm{BS} 1)=-1788.200818$

Zero-point correction $=$

Thermal correction to Energy=

Thermal correction to Enthalpy=

Thermal correction to Gibbs Free Energy=
0.674363

0.710193

0.711137

0.606275 


\begin{tabular}{|c|c|c|c|}
\hline $\mathrm{C}$ & 4.76843500 & 0.01067400 & 0.25452400 \\
\hline $\mathrm{H}$ & 5.17578800 & -0.96352500 & -0.04510500 \\
\hline $\mathrm{C}$ & 5.01838900 & 1.04425100 & -0.84527600 \\
\hline $\mathrm{H}$ & 6.09226800 & 1.13508600 & -1.04679100 \\
\hline $\mathrm{H}$ & 4.64296800 & 2.02477300 & -0.53271300 \\
\hline $\mathrm{H}$ & 4.51554700 & 0.77026400 & -1.77727800 \\
\hline $\mathrm{C}$ & 5.39084300 & 0.42094400 & 1.58652300 \\
\hline $\mathrm{H}$ & 6.47272200 & 0.55664500 & 1.47860300 \\
\hline $\mathrm{H}$ & 5.20832100 & -0.34244900 & 2.34900700 \\
\hline $\mathrm{H}$ & 4.95552100 & 1.36396100 & 1.93451400 \\
\hline $\mathrm{C}$ & -1.25214500 & -3.96771300 & 0.09956500 \\
\hline $\mathrm{H}$ & -1.50577900 & -3.81017900 & -0.95705600 \\
\hline $\mathrm{C}$ & -2.52994300 & -3.90420000 & 0.92949600 \\
\hline $\mathrm{H}$ & -3.20585600 & -4.71964300 & 0.64888600 \\
\hline $\mathrm{H}$ & -3.04350700 & -2.95226000 & 0.77281600 \\
\hline $\mathrm{H}$ & -2.29536500 & -3.99937500 & 1.99545200 \\
\hline $\mathrm{C}$ & -0.51400900 & -5.29607100 & 0.28523100 \\
\hline $\mathrm{H}$ & -1.13350700 & -6.12815500 & -0.06934800 \\
\hline $\mathrm{H}$ & -0.29652900 & -5.45677500 & 1.34706900 \\
\hline $\mathrm{H}$ & 0.43195500 & -5.31578000 & -0.26303800 \\
\hline $\mathrm{C}$ & -2.75177800 & 1.26638600 & 0.21415200 \\
\hline $\mathrm{H}$ & -2.76884900 & 2.02411000 & 1.00991300 \\
\hline $\mathrm{C}$ & -3.48166000 & 1.85689500 & -1.01190000 \\
\hline $\mathrm{H}$ & -2.99191200 & 2.77863300 & -1.34818600 \\
\hline $\mathrm{H}$ & -3.41057200 & 1.14213700 & -1.84424300 \\
\hline $\mathrm{C}$ & -4.96168300 & 2.14993200 & -0.70796800 \\
\hline $\mathrm{H}$ & -5.02575100 & 2.94976000 & 0.04503400 \\
\hline $\mathrm{H}$ & -5.45425500 & 2.53320600 & -1.61109400 \\
\hline $\mathrm{C}$ & -5.69430600 & 0.90577400 & -0.18668800 \\
\hline $\mathrm{H}$ & -5.73672600 & 0.15063000 & -0.98554400 \\
\hline $\mathrm{H}$ & -6.73338300 & 1.15330600 & 0.06641200 \\
\hline $\mathrm{C}$ & -4.97248400 & 0.31115700 & 1.03062200 \\
\hline $\mathrm{H}$ & -5.47388700 & -0.60819000 & 1.36056500 \\
\hline $\mathrm{H}$ & -5.03481500 & 1.01824500 & 1.87149900 \\
\hline $\mathrm{C}$ & -3.49555700 & 0.01473000 & 0.72184100 \\
\hline $\mathrm{H}$ & -3.43294400 & -0.76248200 & -0.05320600 \\
\hline $\mathrm{H}$ & -2.99784800 & -0.38875000 & 1.61085100 \\
\hline $\mathrm{C}$ & -0.20955100 & 2.54407800 & -0.60176400 \\
\hline $\mathrm{H}$ & -0.77180200 & 2.86190000 & -1.49280900 \\
\hline $\mathrm{C}$ & -0.35822800 & 3.63759600 & 0.47375700 \\
\hline $\mathrm{H}$ & 0.16249300 & 3.32128700 & 1.38693800 \\
\hline $\mathrm{H}$ & -1.41231800 & 3.78060700 & 0.74325800 \\
\hline $\mathrm{C}$ & 0.23142000 & 4.97868200 & -0.00163400 \\
\hline $\mathrm{H}$ & -0.35748400 & 5.35214500 & -0.85274900 \\
\hline $\mathrm{H}$ & 0.13725900 & 5.72813800 & 0.79534400 \\
\hline $\mathrm{C}$ & 1.69868900 & 4.83183500 & -0.42797200 \\
\hline $\mathrm{H}$ & 2.30522100 & 4.57052600 & 0.45203700 \\
\hline $\mathrm{H}$ & 2.08450700 & 5.78928500 & -0.80178600 \\
\hline $\mathrm{C}$ & 1.85548200 & 3.73696800 & -1.49304400 \\
\hline $\mathrm{H}$ & 2.91424800 & 3.60713100 & -1.75359500 \\
\hline $\mathrm{H}$ & 1.34521200 & 4.05380500 & -2.41514700 \\
\hline $\mathrm{C}$ & 1.26645800 & 2.39620900 & -1.02320300 \\
\hline $\mathrm{H}$ & 1.85243600 & 2.01571500 & -0.17604300 \\
\hline $\mathrm{H}$ & 1.34726900 & 1.64532100 & -1.81878900 \\
\hline $\mathrm{O}$ & 3.35907700 & -0.13609600 & 0.50710100 \\
\hline $\mathrm{O}$ & -0.43427700 & -2.87089300 & 0.54921000 \\
\hline P & -0.96429400 & 0.84857700 & -0.22145600 \\
\hline
\end{tabular}




\section{CF3I}

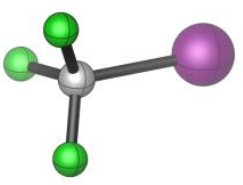

$\mathrm{E}(\mathrm{B} 3 \mathrm{LYP} / \mathrm{BS} 1)=-348.988781$

$\mathrm{E}(\mathrm{PCM}$ (dichloroethane)/M06/BS2// B3LYP/BS1) = -348.998922

Zero-point correction $=$ 0.013999

Thermal correction to Energy=

0.018810

Thermal correction to Enthalpy= 0.019754

Thermal correction to Gibbs Free Energy= $-0.015302$

Charge $=0$ Multiplicity $=1$

C $\quad 0.00000000 \quad 0.00000000 \quad-1.22390200$

I $\quad 0.00000000 \quad 0.00000000 \quad 0.99292000$

F $\quad 0.00000000 \quad 1.25055800 \quad-1.67708700$

F $\quad 1.08301500 \quad-0.62527900-1.67708700$

F $\quad-1.08301500 \quad-0.62527900 \quad-1.67708700$

\section{CO}

$\mathrm{E}(\mathrm{B} 3 \mathrm{LYP} / \mathrm{BS} 1)=-113.309453$

$\mathrm{E}(\mathrm{PCM}($ dichloroethane $) / \mathrm{M} 06 / \mathrm{BS} 2 / / \mathrm{B} 3 \mathrm{LYP} / \mathrm{BS} 1)=-113.286339$

Zero-point correction=

Thermal correction to Energy=

Thermal correction to Enthalpy=

Thermal correction to Gibbs Free Energy=
0.005033

0.007393

0.008337

$-0.014106$

Charge $=0$ Multiplicity $=1$

C $\quad 0.00000000 \quad 0.00000000 \quad-0.65025200$

$\begin{array}{llll}\mathrm{O} & 0.00000000 & 0.00000000 & 0.48768900\end{array}$ 


\section{IM1}

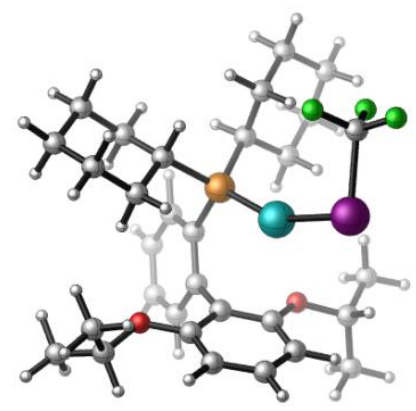

$\mathrm{E}(\mathrm{B} 3 \mathrm{LYP} / \mathrm{BS} 1)=-2136.666226$

$\mathrm{E}(\mathrm{PCM}($ dichloroethane $) / \mathrm{M} 06 / \mathrm{BS} 2 / / \mathrm{B} 3 \mathrm{LYP} / \mathrm{BS} 1)=-2137.226092$

Zero-point correction=

Thermal correction to Energy=

Thermal correction to Enthalpy=

Thermal correction to Gibbs Free Energy=
0.689620

0.732773

0.733717

0.605435

$\begin{array}{lrrr}\text { Charge }= & \text { Multiplicity }=1 & \\ \mathrm{C} & 2.00202600 & 0.95420700 & 1.26704400 \\ \mathrm{C} & 2.66056500 & 1.96685700 & 1.99113200 \\ \mathrm{H} & 2.25256600 & 2.97157200 & 1.99951200 \\ \mathrm{C} & 3.82972000 & 1.72702900 & 2.70969800 \\ \mathrm{H} & 4.30590200 & 2.53693200 & 3.25646500 \\ \mathrm{C} & 4.37710200 & 0.44522000 & 2.71966300 \\ \mathrm{H} & 5.28818700 & 0.23670300 & 3.27464100 \\ \mathrm{C} & 3.74058500 & -0.57222200 & 2.01550000 \\ \mathrm{H} & 4.15674900 & -1.57586500 & 2.02494700 \\ \mathrm{C} & 2.55769100 & -0.34830200 & 1.29045300 \\ \mathrm{C} & 1.97926200 & -1.55055600 & 0.60232400 \\ \mathrm{C} & 1.00044100 & -2.34134600 & 1.24572000 \\ \mathrm{C} & 0.55332600 & -3.53969900 & 0.66247900 \\ \mathrm{H} & -0.19657100 & -4.15128700 & 1.14776700 \\ \mathrm{C} & 1.10810000 & -3.95831700 & -0.54456600 \\ \mathrm{H} & 0.76609900 & -4.88808600 & -0.99189400 \\ \mathrm{C} & 2.09855400 & -3.21863500 & -1.18449200 \\ \mathrm{H} & 2.51354100 & -3.57375900 & -2.11901300 \\ \mathrm{C} & 2.53971100 & -2.02053500 & -0.60313600 \\ \mathrm{C} & 4.39871100 & -1.75483700 & -2.15975900 \\ \mathrm{H} & 4.52440500 & -2.83405600 & -2.00199400 \\ \mathrm{C} & 3.81401200 & -1.49314000 & -3.54897100 \\ \mathrm{H} & 4.46686200 & -1.91687400 & -4.32112800 \\ \mathrm{H} & 3.72670400 & -0.41598400 & -3.72576600 \\ \mathrm{H} & 2.81952000 & -1.93487200 & -3.65987400 \\ \mathrm{C} & 5.74349100 & -1.06246500 & -1.95644500 \\ \mathrm{H} & 6.46962900 & -1.41270400 & -2.69840800 \\ \mathrm{H} & 6.13594800 & -1.26931600 & -0.95626400 \\ \mathrm{H} & 5.63418700 & 0.02217000 & -2.06394900 \\ \mathrm{C} & -0.28033900 & -2.67403400 & 3.29255100 \\ \mathrm{H} & -1.09012100 & -3.08381700 & 2.67502000 \\ \mathrm{C} & -0.88260200 & -1.70938800 & 4.30758300 \\ \mathrm{H} & -1.55799300 & -2.24550400 & 4.98342400 \\ \mathrm{H} & -1.44538400 & -0.92145900 & 3.80032000\end{array}$




$\begin{array}{lrrr}\mathrm{H} & -0.09232600 & -1.24150600 & 4.90474900 \\ \mathrm{C} & 0.50494100 & -3.80401900 & 3.96221500 \\ \mathrm{H} & -0.15990500 & -4.41044700 & 4.58802800 \\ \mathrm{H} & 1.29152700 & -3.38464100 & 4.59928200 \\ \mathrm{H} & 0.97559500 & -4.46188000 & 3.22587900 \\ \mathrm{C} & -0.42607500 & 2.59704200 & 1.44805900 \\ \mathrm{H} & 0.27279700 & 3.41366100 & 1.67642400 \\ \mathrm{C} & -1.65600600 & 3.21643500 & 0.74932400 \\ \mathrm{H} & -1.35930300 & 3.73660900 & -0.16939200 \\ \mathrm{H} & -2.33823000 & 2.41009700 & 0.44734100 \\ \mathrm{C} & -2.39501600 & 4.20248000 & 1.67174400 \\ \mathrm{H} & -1.74441000 & 5.06619000 & 1.87650900 \\ \mathrm{H} & -3.28042900 & 4.59605800 & 1.15553600 \\ \mathrm{C} & -2.79833000 & 3.54855600 & 3.00065900 \\ \mathrm{H} & -3.54380200 & 2.76365600 & 2.80508400 \\ \mathrm{H} & -3.28179000 & 4.28426300 & 3.65661200 \\ \mathrm{C} & -1.58162800 & 2.92471100 & 3.69854500 \\ \mathrm{H} & -1.89043500 & 2.41231000 & 4.61926500 \\ \mathrm{H} & -0.88897300 & 3.72336500 & 4.00447600 \\ \mathrm{C} & -0.84751100 & 1.93739200 & 2.77639200 \\ \mathrm{H} & -1.50859300 & 1.09085300 & 2.54401200 \\ \mathrm{H} & 0.02641400 & 1.52034600 & 3.28937700 \\ \mathrm{C} & 1.00015600 & 2.40143800 & -1.13448100 \\ \mathrm{H} & 0.04289200 & 2.75047400 & -1.54920700 \\ \mathrm{C} & 1.86652400 & 3.63744700 & -0.82523400 \\ \mathrm{H} & 2.81585200 & 3.31419400 & -0.37869200 \\ \mathrm{H} & 1.37456000 & 4.28698300 & -0.09022700 \\ \mathrm{C} & 2.16492500 & 4.44756000 & -2.10061800 \\ \mathrm{H} & 1.22523800 & 4.86240000 & -2.49492100 \\ \mathrm{H} & 2.80515200 & 5.30495000 & -1.85410800 \\ \mathrm{C} & 2.82600100 & 3.57839700 & -3.17925800 \\ \mathrm{H} & 3.81789100 & 3.25863900 & -2.82607900 \\ \mathrm{H} & 2.99236500 & 4.16478400 & -4.09240800 \\ \mathrm{C} & 1.97390600 & 2.33817900 & -3.48480200 \\ \mathrm{H} & 2.47999300 & 1.70077900 & -4.22243900 \\ \mathrm{H} & 1.02734200 & 2.65513500 & -3.94794200 \\ \mathrm{C} & 1.66793900 & 1.52729500 & -2.21415000 \\ \mathrm{H} & 2.59700300 & 1.10030300 & -1.81508800 \\ \mathrm{H} & 1.01315100 & 0.67999300 & -2.45167500 \\ \mathrm{~F} & 3.53277800 & -1.24120400 & -1.12975700 \\ \mathrm{O} & -3.82226600 & 0.89054600 & -2.54384200 \\ \mathrm{O} & & & \\ \mathrm{P} & 0.58487600 & -1.87931200 & 2.46003700 \\ \mathrm{Pd} & -0.87473900 & 1.34191000 & 0.32921700 \\ \mathrm{I} & -3.07834900 & -1.6 .43671200 & -0.28339000 \\ \mathrm{C} & -4.35226700 & 0.13365400 & -1.22979600 \\ & -5.57033200 & -0.25826800 & -1.58745700 \\ \mathrm{~F} & -4.45358000 & 0.8521600 & -0.47059200 \\ \mathrm{H} & & & \\ \mathrm{H} & & & \end{array}$




\section{IM2}

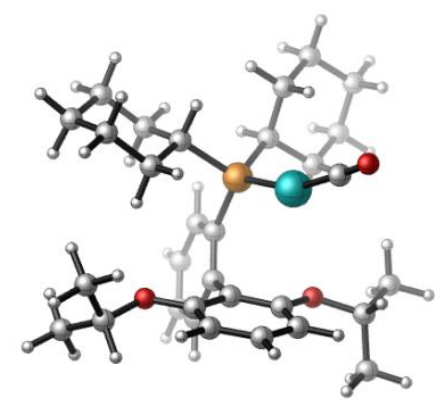

$\mathrm{E}(\mathrm{B} 3 \mathrm{LYP} / \mathrm{BS} 1)=-1901.026486$

$\mathrm{E}(\mathrm{PCM}$ (dichloroethane $) / \mathrm{M} 06 / \mathrm{BS} 2 / / \mathrm{B} 3 \mathrm{LYP} / \mathrm{BS} 1)=-1901.544449$

Zero-point correction=

0.682915

Thermal correction to Energy=

0.721766

Thermal correction to Enthalpy=

0.722710

Thermal correction to Gibbs Free Energy=

0.608160

Charge $=0$ Multiplicity $=1$

$\begin{array}{lrrr}\mathrm{C} & 0.11293300 & 0.49664900 & 1.63209500 \\ \mathrm{C} & -0.13791200 & 1.22065900 & 2.81386700 \\ \mathrm{H} & -0.83325300 & 2.05261400 & 2.79727600 \\ \mathrm{C} & 0.47948700 & 0.90450600 & 4.02158700 \\ \mathrm{H} & 0.25776100 & 1.48665500 & 4.91220000 \\ \mathrm{C} & 1.37730900 & -0.16057900 & 4.07385700 \\ \mathrm{H} & 1.86734700 & -0.42527900 & 5.00721900 \\ \mathrm{C} & 1.63953500 & -0.88847500 & 2.91752100 \\ \mathrm{H} & 2.33514600 & -1.72222200 & 2.95120900 \\ \mathrm{C} & 1.02339700 & -0.58576100 & 1.69113200 \\ \mathrm{C} & 1.40103700 & -1.46231100 & 0.53421600 \\ \mathrm{C} & 0.60682800 & -2.57642600 & 0.18676700 \\ \mathrm{C} & 1.03085100 & -3.47338400 & -0.80644300 \\ \mathrm{H} & 0.42353000 & -4.32374800 & -1.08797200 \\ \mathrm{C} & 2.25287100 & -3.26248100 & -1.43762600 \\ \mathrm{H} & 2.57675600 & -3.95419100 & -2.21063300 \\ \mathrm{C} & 3.07078700 & -2.18735600 & -1.09969900 \\ \mathrm{H} & 4.01679000 & -2.05192300 & -1.60814200 \\ \mathrm{C} & 2.64747500 & -1.29543300 & -0.10468100 \\ \mathrm{C} & 4.79756600 & -0.14882200 & 0.02401500 \\ \mathrm{H} & 5.19967800 & -1.16828000 & -0.03854200 \\ \mathrm{C} & 5.02831700 & 0.58701900 & -1.29698300 \\ \mathrm{H} & 6.09831400 & 0.61382700 & -1.53410300 \\ \mathrm{H} & 4.66749400 & 1.61852400 & -1.22383400 \\ \mathrm{H} & 4.50501800 & 0.10376200 & -2.12707500 \\ \mathrm{C} & 5.43949600 & 0.56925300 & 1.20775400 \\ \mathrm{H} & 6.52063100 & 0.66318400 & 1.05718700 \\ \mathrm{H} & 5.26108800 & 0.01812900 & 2.13598000 \\ \mathrm{H} & 5.01681300 & 1.57372900 & 1.31906300 \\ \mathrm{C} & -1.34191500 & -3.91128500 & 0.78937500 \\ \mathrm{H} & -1.45873200 & -4.16567300 & -0.27147900 \\ \mathrm{C} & -2.71094700 & -3.56001500 & 1.35926300 \\ \mathrm{H} & -3.37309800 & -4.43153600 & 1.31360000 \\ \mathrm{H} & -3.16545100 & -2.74520400 & 0.78957500\end{array}$




\begin{tabular}{lrrr}
$\mathrm{H}$ & -2.62096500 & -3.24599000 & 2.40496100 \\
$\mathrm{C}$ & -0.67627000 & -5.06296600 & 1.54519700 \\
$\mathrm{H}$ & -1.27734100 & -5.97484200 & 1.45346900 \\
$\mathrm{H}$ & -0.58911800 & -4.81187100 & 2.60817400 \\
$\mathrm{H}$ & 0.32531800 & -5.27423200 & 1.15952100 \\
$\mathrm{C}$ & -2.45550500 & 1.54721300 & 0.65018700 \\
$\mathrm{H}$ & -2.32080600 & 2.31243400 & 1.42678400 \\
$\mathrm{C}$ & -3.26240600 & 2.18688300 & -0.50114600 \\
$\mathrm{H}$ & -2.73643600 & 3.05935000 & -0.90636500 \\
$\mathrm{H}$ & -3.34649700 & 1.46156500 & -1.32231600 \\
$\mathrm{C}$ & -4.66638700 & 2.61356300 & -0.03674700 \\
$\mathrm{H}$ & -4.57387700 & 3.42335400 & 0.70276200 \\
$\mathrm{H}$ & -5.22196000 & 3.03054000 & -0.88651800 \\
$\mathrm{C}$ & -5.44199300 & 1.44543500 & 0.58763700 \\
$\mathrm{H}$ & -5.64074800 & 0.68934600 & -0.18597800 \\
$\mathrm{H}$ & -6.41955100 & 1.78794900 & 0.95076000 \\
$\mathrm{C}$ & -4.64213700 & 0.80087100 & 1.72838000 \\
$\mathrm{H}$ & -5.18075200 & -0.06699300 & 2.13052700 \\
$\mathrm{H}$ & -4.54559800 & 1.51877600 & 2.55688600 \\
$\mathrm{C}$ & -3.24226900 & 0.36929500 & 1.26133300 \\
$\mathrm{H}$ & -3.33869700 & -0.41513700 & 0.49792500 \\
$\mathrm{H}$ & -2.68482600 & -0.07151800 & 2.09535400 \\
$\mathrm{C}$ & 0.07280900 & 2.56813800 & -0.51380500 \\
$\mathrm{H}$ & -0.59400100 & 2.90314100 & -1.32173300 \\
$\mathrm{C}$ & 0.18521300 & 3.70319200 & 0.52158900 \\
$\mathrm{H}$ & 0.81335300 & 3.37063600 & 1.35830200 \\
$\mathrm{H}$ & -0.79882600 & 3.94798600 & 0.94130700 \\
$\mathrm{C}$ & 0.80424400 & 4.96883800 & -0.09988300 \\
$\mathrm{H}$ & 0.12291200 & 5.36659500 & -0.86660600 \\
$\mathrm{H}$ & 0.89997800 & 5.74848000 & 0.66713300 \\
$\mathrm{C}$ & 2.16860400 & 4.67504800 & -0.73912500 \\
$\mathrm{H}$ & 2.88138200 & 4.38781000 & 0.04841800 \\
$\mathrm{H}$ & 2.57074500 & 5.58104600 & -1.21070900 \\
$\mathrm{C}$ & 2.06241100 & 3.53796500 & -1.76543200 \\
$\mathrm{H}$ & 3.05158700 & 3.30552400 & -2.18150100 \\
$\mathrm{H}$ & 1.44145200 & 3.87001600 & -2.61081900 \\
$\mathrm{C}$ & 1.44480200 & 2.27021000 & -1.15140600 \\
$\mathrm{H}$ & 2.12127200 & 1.86106200 & -0.39051000 \\
$\mathrm{H}$ & 1.33200200 & 1.49512400 & -1.91960300 \\
$\mathrm{O}$ & 3.39162000 & -0.23214000 & 0.32989200 \\
$\mathrm{O}$ & -0.55129000 & -2.71038800 & 0.89672600 \\
$\mathrm{P}$ & -0.76074300 & 0.97344300 & 0.05803500 \\
$\mathrm{Pd}$ & -0.98362000 & -0.57537100 & -1.78153100 \\
$\mathrm{C}$ & -1.51447800 & -1.35516100 & -3.42588400 \\
$\mathrm{O}$ & -1.87166500 & -1.77532200 & -4.43945700 \\
& & & \\
\hline
\end{tabular}




\section{IM3}

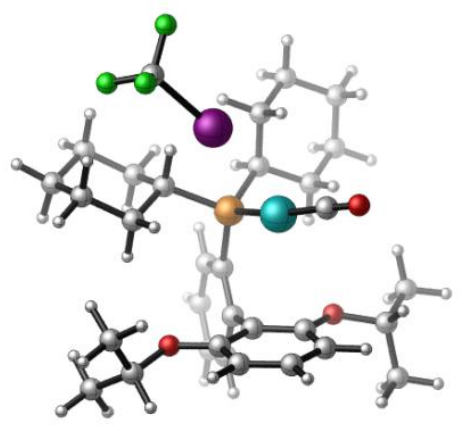

$\mathrm{E}(\mathrm{B} 3 \mathrm{LYP} / \mathrm{BS} 1)=-2250.022151$

$\mathrm{E}(\mathrm{PCM}$ (dichloroethane $) / \mathrm{M} 06 / \mathrm{BS} 2 / / \mathrm{B} 3 \mathrm{LYP} / \mathrm{BS} 1)=-2250.559567$

Zero-point correction=

Thermal correction to Energy=

Thermal correction to Enthalpy=

0.697334

0.743408

Thermal correction to Gibbs Free Energy=
0.744352

0.607927

$\begin{array}{crrr}\text { Charge }=0 \text { Multiplicity }= & & \\ \mathrm{C} & 2.02399200 & 0.19227100 & 1.70422800 \\ \mathrm{C} & 2.43537200 & -0.00295200 & 3.03754100 \\ \mathrm{H} & 1.83419600 & -0.61440900 & 3.70116000 \\ \mathrm{C} & 3.60369600 & 0.56286300 & 3.54123200 \\ \mathrm{H} & 3.88801700 & 0.38895900 & 4.57563900 \\ \mathrm{C} & 4.39856900 & 1.34843400 & 2.70773700 \\ \mathrm{H} & 5.31366000 & 1.80003200 & 3.08183100 \\ \mathrm{C} & 4.01558500 & 1.54303300 & 1.38449400 \\ \mathrm{H} & 4.63714400 & 2.14316400 & 0.72599300 \\ \mathrm{C} & 2.84311600 & 0.97487000 & 0.85672500 \\ \mathrm{C} & 2.58772500 & 1.24274000 & -0.59712900 \\ \mathrm{C} & 3.09667600 & 0.36368500 & -1.57770800 \\ \mathrm{C} & 3.00772900 & 0.68528500 & -2.94102000 \\ \mathrm{H} & 3.38866000 & 0.01470700 & -3.70054700 \\ \mathrm{C} & 2.41803800 & 1.88825700 & -3.31805200 \\ \mathrm{H} & 2.34352700 & 2.13428300 & -4.37390600 \\ \mathrm{C} & 1.92277400 & 2.78460600 & -2.37447300 \\ \mathrm{H} & 1.47130300 & 3.71203100 & -2.70228800 \\ \mathrm{C} & 2.02071900 & 2.46598100 & -1.01219700 \\ \mathrm{C} & 1.30132600 & 4.67530200 & -0.27176700 \\ \mathrm{H} & 1.95090300 & 5.02700500 & -1.08383100 \\ \mathrm{C} & -0.16720500 & 4.83621300 & -0.66767700 \\ \mathrm{H} & -0.38317900 & 5.88520500 & -0.90159000 \\ \mathrm{H} & -0.81816600 & 4.52721200 & 0.15678200 \\ \mathrm{H} & -0.42047000 & 4.23095500 & -1.54285400 \\ \mathrm{C} & 1.64684000 & 5.43579400 & 1.00516400 \\ \mathrm{H} & 1.45216100 & 6.50610100 & 0.87567100 \\ \mathrm{H} & 2.70187600 & 5.29980300 & 1.26144500 \\ \mathrm{H} & 1.04059300 & 5.07310900 & 1.84233700 \\ \mathrm{C} & 4.45119900 & -1.62692500 & -1.96260100 \\ \mathrm{H} & 3.88201400 & -1.81081400 & -2.88282900 \\ \mathrm{C} & 4.60891900 & -2.94608000 & -1.21534400 \\ \mathrm{H} & 5.19054100 & -3.65283100 & -1.81709700 \\ \mathrm{H} & 3.63181300 & -3.38897900 & -1.00398500\end{array}$




\begin{tabular}{|c|c|c|c|}
\hline & 5.13074300 & -2.78675300 & -0.26539700 \\
\hline & 5.79865700 & -0.98157700 & -2.29160200 \\
\hline $\mathrm{H}$ & 6.37952800 & -1.63252900 & -2.95485700 \\
\hline $\mathrm{H}$ & 6.37242800 & -0.82463400 & -1.37146300 \\
\hline $\mathrm{H}$ & 5.67297800 & -0.01452800 & -2.78713600 \\
\hline $\mathrm{C}$ & 0.48104500 & -2.29971900 & 1.90060000 \\
\hline $\mathrm{H}$ & 0.65451800 & -2.19174500 & 2.98001900 \\
\hline $\mathrm{C}$ & -0.86263200 & -3.03516700 & 1.70664000 \\
\hline $\mathrm{H}$ & -1.68367500 & -2.47498000 & 2.16929900 \\
\hline $\mathrm{H}$ & -1.08741400 & -3.08984500 & 0.63235300 \\
\hline $\mathrm{C}$ & -0.81671000 & -4.45525300 & 2.29808500 \\
\hline $\mathrm{H}$ & -0.70576700 & -4.38990600 & 3.39098500 \\
\hline $\mathrm{H}$ & -1.77299700 & -4.96033400 & 2.11191800 \\
\hline $\mathrm{C}$ & 0.34402500 & -5.27691900 & 1.72011300 \\
\hline $\mathrm{H}$ & 0.16830900 & -5.44540400 & 0.64750500 \\
\hline $\mathrm{H}$ & 0.38111300 & -6.26738300 & 2.19150900 \\
\hline $\mathrm{C}$ & 1.68181600 & -4.54636900 & 1.90341900 \\
\hline $\mathrm{H}$ & 2.49621400 & -5.11717700 & 1.43861300 \\
\hline $\mathrm{H}$ & 1.91873600 & -4.48357900 & 2.97621200 \\
\hline $\mathrm{C}$ & 1.63817300 & -3.12962700 & 1.30749800 \\
\hline $\mathrm{H}$ & 1.49810000 & -3.19423400 & 0.21912000 \\
\hline $\mathrm{H}$ & 2.59447700 & -2.62019400 & 1.46950100 \\
\hline $\mathrm{C}$ & -0.93612100 & 0.31190700 & 2.06247300 \\
\hline $\mathrm{H}$ & -1.83019900 & -0.23933200 & 1.73865000 \\
\hline $\mathrm{C}$ & -0.88938600 & 0.28108300 & 3.60225700 \\
\hline $\mathrm{H}$ & 0.00089400 & 0.81896400 & 3.95285000 \\
\hline $\mathrm{H}$ & -0.80912000 & -0.74829100 & 3.97342200 \\
\hline $\mathrm{C}$ & -2.14226200 & 0.94517400 & 4.20346300 \\
\hline $\mathrm{H}$ & -3.02971800 & 0.35422700 & 3.93357000 \\
\hline $\mathrm{H}$ & -2.07799200 & 0.93030600 & 5.29931300 \\
\hline $\mathrm{C}$ & -2.31635500 & 2.38374600 & 3.69670900 \\
\hline $\mathrm{H}$ & -1.48516400 & 3.00003400 & 4.07100500 \\
\hline $\mathrm{H}$ & -3.23780400 & 2.82042400 & 4.10267000 \\
\hline $\mathrm{C}$ & -2.33222800 & 2.43260200 & 2.16200900 \\
\hline $\mathrm{H}$ & -2.39615400 & 3.47356400 & 1.81767500 \\
\hline $\mathrm{H}$ & -3.23364800 & 1.92704300 & 1.78703700 \\
\hline $\mathrm{C}$ & -1.08785300 & 1.76057800 & 1.55598500 \\
\hline $\mathrm{H}$ & -0.18893600 & 2.33386200 & 1.81686200 \\
\hline $\mathrm{H}$ & -1.15497700 & 1.76812400 & 0.46234000 \\
\hline $\mathrm{O}$ & 1.61614200 & 3.29389600 & -0.00370700 \\
\hline $\mathrm{O}$ & 3.67680800 & -0.77451100 & -1.09533300 \\
\hline$P$ & 0.43648900 & -0.58421500 & 1.12855200 \\
\hline $\mathrm{Pc}$ & 0.01582100 & -0.76342400 & -1.25905800 \\
\hline $\mathrm{C}$ & -0.30642600 & -1.26396100 & -3.06688500 \\
\hline $\mathrm{O}$ & -0.42707900 & -1.60585400 & -4.15946400 \\
\hline I & -3.26450600 & -0.39463600 & -1.19409900 \\
\hline $\mathrm{C}$ & -5.38179200 & 0.14487000 & -0.75016000 \\
\hline $\mathrm{F}$ & -5.44370200 & 0.89851700 & 0.35915900 \\
\hline & -5.92034600 & 0.84049700 & $-1.7567970 \mathrm{C}$ \\
\hline & -6.12311200 & -0.95029200 & -0.5501830 \\
\hline
\end{tabular}




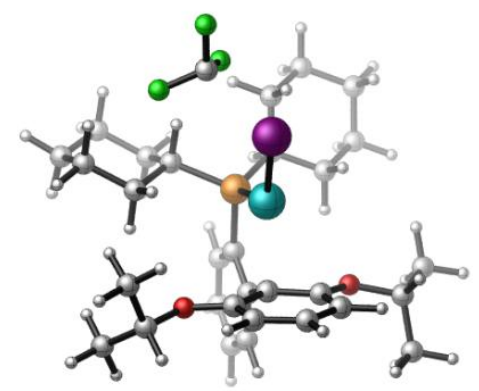

$\mathrm{E}(\mathrm{B} 3 \mathrm{LYP} / \mathrm{BS} 1)=-2136.657617$

$\mathrm{E}(\mathrm{PCM}($ dichloroethane $) / \mathrm{M} 06 / \mathrm{BS} 2 / / \mathrm{B} 3 \mathrm{LYP} / \mathrm{BS} 1)=-2137.212978$

Zero-point correction $=$

0.688446

Thermal correction to Energy=

0.731300

Thermal correction to Enthalpy=

0.732245

Thermal correction to Gibbs Free Energy=

0.606731

Charge $=0$ Multiplicity $=1$

$\begin{array}{lrrr}\mathrm{C} & -1.45866100 & 0.62253400 & 1.86718200 \\ \mathrm{C} & -1.82191500 & 1.29460900 & 3.04815700 \\ \mathrm{H} & -1.18286400 & 2.07970700 & 3.43899400 \\ \mathrm{C} & -2.99663800 & 0.98334000 & 3.72902100 \\ \mathrm{H} & -3.25421700 & 1.51549500 & 4.64093000 \\ \mathrm{C} & -3.83703000 & -0.01224000 & 3.22896600 \\ \mathrm{H} & -4.75609400 & -0.26811500 & 3.74967000 \\ \mathrm{C} & -3.50039200 & -0.67135000 & 2.04965200 \\ \mathrm{H} & -4.16249000 & -1.43557600 & 1.65149300 \\ \mathrm{C} & -2.32188400 & -0.36556400 & 1.34902300 \\ \mathrm{C} & -2.09514900 & -1.12112400 & 0.06064300 \\ \mathrm{C} & -2.80045700 & -0.71791600 & -1.11482300 \\ \mathrm{C} & -2.93306100 & -1.58916600 & -2.20826700 \\ \mathrm{H} & -3.44865700 & -1.27926700 & -3.10777400 \\ \mathrm{C} & -2.41546400 & -2.87854000 & -2.12200500 \\ \mathrm{H} & -2.52255600 & -3.54928200 & -2.96983900 \\ \mathrm{C} & -1.75112600 & -3.32681700 & -0.98243500 \\ \mathrm{H} & -1.35787000 & -4.33429600 & -0.95550600 \\ \mathrm{C} & -1.60676800 & -2.46417700 & 0.11371900 \\ \mathrm{C} & -0.66760300 & -4.19271400 & 1.55315400 \\ \mathrm{H} & -1.39597300 & -4.86047200 & 1.07508300 \\ \mathrm{C} & 0.73230800 & -4.45252100 & 0.99641100 \\ \mathrm{H} & 1.00494100 & -5.50415100 & 1.14237200 \\ \mathrm{H} & 1.46855900 & -3.83082800 & 1.51571000 \\ \mathrm{H} & 0.79511800 & -4.22590000 & -0.07169700 \\ \mathrm{C} & -0.74405200 & -4.38062700 & 3.06466200 \\ \mathrm{H} & -0.47126200 & -5.40732200 & 3.33182100 \\ \mathrm{H} & -1.75666100 & -4.18129200 & 3.42839900 \\ \mathrm{H} & -0.05471000 & -3.69676300 & 3.57118900 \\ \mathrm{C} & -4.29541300 & 0.95843500 & -2.06533300 \\ \mathrm{H} & -3.88513400 & 0.72297400 & -3.05520300 \\ \mathrm{C} & -4.38827600 & 2.47299400 & -1.92335300 \\ \mathrm{H} & -5.08847000 & 2.87615500 & -2.66290800 \\ \mathrm{H} & -3.41110100 & 2.93792200 & -2.07925300\end{array}$




\begin{tabular}{lrrr}
$\mathrm{H}$ & -4.74609000 & 2.74244700 & -0.92376800 \\
$\mathrm{C}$ & -5.64883500 & 0.27289200 & -1.87169700 \\
$\mathrm{H}$ & -6.35536500 & 0.60558700 & -2.64042500 \\
$\mathrm{H}$ & -6.06020200 & 0.52986200 & -0.88933800 \\
$\mathrm{H}$ & -5.56356000 & -0.81576500 & -1.93535600 \\
$\mathrm{C}$ & 0.03239500 & 2.88595500 & 0.76077000 \\
$\mathrm{H}$ & -0.12032200 & 3.30788000 & 1.76561800 \\
$\mathrm{C}$ & 1.3498500 & 3.45282000 & 0.19160800 \\
$\mathrm{H}$ & 2.19544800 & 3.20179900 & 0.84173300 \\
$\mathrm{H}$ & 1.55455200 & 2.98169600 & -0.77837800 \\
$\mathrm{C}$ & 1.27564400 & 4.98015000 & 0.01901600 \\
$\mathrm{H}$ & 1.18898800 & 5.45355200 & 1.00875500 \\
$\mathrm{H}$ & 2.21307500 & 5.34478000 & -0.41971300 \\
$\mathrm{C}$ & 0.08047400 & 5.39989000 & -0.84778800 \\
$\mathrm{H}$ & 0.22812900 & 5.02632300 & -1.87166900 \\
$\mathrm{H}$ & 0.02557100 & 6.49390200 & -0.91731400 \\
$\mathrm{C}$ & -1.23135000 & 4.83224200 & -0.28792900 \\
$\mathrm{H}$ & -2.07213500 & 5.09441700 & -0.94365000 \\
$\mathrm{H}$ & -1.44211300 & 5.29569000 & 0.68767300 \\
$\mathrm{C}$ & -1.16051600 & 3.30530800 & -0.12165100 \\
$\mathrm{H}$ & -1.04853800 & 2.83345000 & -1.10796200 \\
$\mathrm{H}$ & -2.09713700 & 2.92505700 & 0.29962000 \\
$\mathrm{C}$ & 1.55233500 & 0.68936000 & 2.07405500 \\
$\mathrm{H}$ & 2.39789800 & 1.00600600 & 1.44728900 \\
$\mathrm{C}$ & 1.61405000 & 1.47299400 & 3.39995100 \\
$\mathrm{H}$ & 0.77529800 & 1.17152300 & 4.04091500 \\
$\mathrm{H}$ & 1.51479400 & 2.55174500 & 3.22631900 \\
$\mathrm{C}$ & 2.93174100 & 1.18987500 & 4.14523000 \\
$\mathrm{H}$ & 3.77331000 & 1.56989700 & 3.54783800 \\
$\mathrm{H}$ & 2.94617100 & 1.74225200 & 5.09391000 \\
$\mathrm{C}$ & 3.12341500 & -0.31255100 & 4.39658300 \\
$\mathrm{H}$ & 2.34527000 & -0.66329500 & 5.09113400 \\
$\mathrm{H}$ & 4.08734600 & -0.49517800 & 4.88865300 \\
$\mathrm{C}$ & 3.03228000 & -1.10944600 & 3.08725000 \\
$\mathrm{H}$ & 3.11396400 & -2.18542500 & 3.29143700 \\
$\mathrm{H}$ & 3.88003100 & -0.85022700 & 2.43812800 \\
$\mathrm{C}$ & 1.72578000 & -0.82184900 & 2.32774600 \\
$\mathrm{H}$ & 0.86726200 & -1.19751000 & 2.90153800 \\
$\mathrm{H}$ & 1.72407200 & -1.35440600 & 1.37064600 \\
$\mathrm{O}$ & -1.07631800 & -2.82837400 & 1.31004500 \\
$\mathrm{O}$ & -3.35855300 & 0.52271800 & -1.05557300 \\
$\mathrm{P}$ & 0.08867200 & 1.01527900 & 0.93640100 \\
$\mathrm{Pd}$ & 0.04504700 & -0.32939300 & -0.94915000 \\
$\mathrm{I}$ & 1.94918200 & -0.89396200 & -2.77561400 \\
$\mathrm{C}$ & 3.91643000 & 0.09110500 & -1.28184700 \\
$\mathrm{~F}$ & 5.04165900 & 0.20089800 & -1.99058600 \\
$\mathrm{~F}$ & 3.58176000 & 1.30176600 & -0.79956700 \\
$\mathrm{~F}$ & 4.12890500 & -0.73092600 & -0.24145600 \\
& & & \\
\hline
\end{tabular}




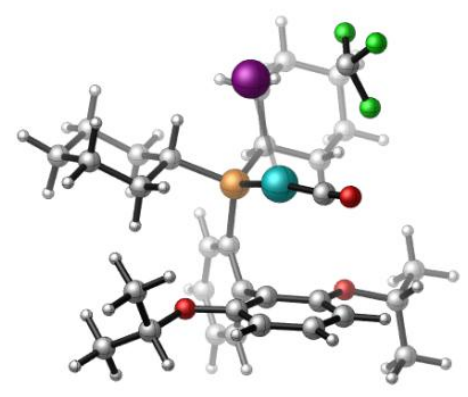

$\mathrm{E}(\mathrm{B} 3 \mathrm{LYP} / \mathrm{BS} 1)=-2249.98659$

$\mathrm{E}(\mathrm{PCM}($ dichloroethane $) / \mathrm{M} 06 / \mathrm{BS} 2 / / \mathrm{B} 3 \mathrm{LYP} / \mathrm{BS} 1)=-2250.517479$

Zero-point correction=

0.696294

Thermal correction to Energy=

0.742009

Thermal correction to Enthalpy=

0.742953

Thermal correction to Gibbs Free Energy=

0.610122

Charge $=0$ Multiplicity $=1$

$\begin{array}{lrrr}\mathrm{C} & 1.73332900 & 0.12949900 & 1.81980100 \\ \mathrm{C} & 2.04287800 & 0.41874700 & 3.16383300 \\ \mathrm{H} & 1.28447600 & 0.84598200 & 3.80980600 \\ \mathrm{C} & 3.30205500 & 0.17085500 & 3.70313400 \\ \mathrm{H} & 3.50237800 & 0.40934900 & 4.74424800 \\ \mathrm{C} & 4.29444800 & -0.38426000 & 2.89667400 \\ \mathrm{H} & 5.28471400 & -0.58454100 & 3.29718100 \\ \mathrm{C} & 4.00347700 & -0.69193900 & 1.57174200 \\ \mathrm{H} & 4.76818300 & -1.13904000 & 0.94284600 \\ \mathrm{C} & 2.73605000 & -0.45281600 & 1.01109200 \\ \mathrm{C} & 2.58053300 & -0.88852500 & -0.41785200 \\ \mathrm{C} & 2.27858000 & -2.24059000 & -0.70598100 \\ \mathrm{C} & 2.36153000 & -2.72690800 & -2.01944700 \\ \mathrm{H} & 2.12855700 & -3.75813900 & -2.25134600 \\ \mathrm{C} & 2.75572000 & -1.86502800 & -3.03945000 \\ \mathrm{H} & 2.81521600 & -2.24119800 & -4.05714500 \\ \mathrm{C} & 3.07411200 & -0.53286600 & -2.78845800 \\ \mathrm{H} & 3.37797500 & 0.10749100 & -3.60619900 \\ \mathrm{C} & 3.00402900 & -0.04887200 & -1.47340600 \\ \mathrm{C} & 4.05145100 & 2.08148600 & -2.02876400 \\ \mathrm{H} & 4.70862700 & 1.46527500 & -2.65587700 \\ \mathrm{C} & 3.05869200 & 2.84906300 & -2.90278000 \\ \mathrm{H} & 3.59674400 & 3.47218700 & -3.62650500 \\ \mathrm{H} & 2.43401300 & 3.50190900 & -2.28466100 \\ \mathrm{H} & 2.39787600 & 2.17519000 & -3.45575700 \\ \mathrm{C} & 4.91017500 & 3.00490500 & -1.17013000 \\ \mathrm{H} & 5.48750100 & 3.68543400 & -1.80545300 \\ \mathrm{H} & 5.60525800 & 2.42473800 & -0.55572600 \\ \mathrm{H} & 4.28070800 & 3.60497400 & -0.50422000 \\ \mathrm{C} & 1.81843800 & -4.43444200 & 0.25578600 \\ \mathrm{H} & 1.23758100 & -4.67563800 & -0.64343300 \\ \mathrm{C} & 1.03317600 & -4.89070200 & 1.47983300 \\ \mathrm{H} & 0.90927100 & -5.97887900 & 1.46305300 \\ \mathrm{H} & 0.04258900 & -4.42771000 & 1.49832100\end{array}$




\begin{tabular}{|c|c|c|c|}
\hline & 1.56307200 & -4.61580200 & 2.39831800 \\
\hline $\mathrm{C}$ & 3.20533300 & -5.07478200 & 0.18043800 \\
\hline $\mathrm{H}$ & 3.11656200 & -6.16322100 & 0.08950800 \\
\hline $\mathrm{H}$ & 3.77125000 & -4.84867800 & 1.09091000 \\
\hline $\mathrm{H}$ & 3.77409400 & -4.70616600 & -0.67830000 \\
\hline $\mathrm{C}$ & -1.08657500 & -0.17569800 & 2.53453300 \\
\hline $\mathrm{H}$ & -0.64007900 & 0.08723400 & 3.50329700 \\
\hline $\mathrm{C}$ & -2.51482800 & 0.40712000 & 2.49853400 \\
\hline $\mathrm{H}$ & -2.49444500 & 1.49427200 & 2.63503000 \\
\hline $\mathrm{H}$ & -2.96681900 & 0.22408300 & 1.51791800 \\
\hline $\mathrm{C}$ & -3.40089800 & -0.22682200 & 3.58620700 \\
\hline $\mathrm{H}$ & -3.02000300 & 0.05617400 & 4.57939300 \\
\hline $\mathrm{H}$ & -4.41557500 & 0.18375400 & 3.51133500 \\
\hline $\mathrm{C}$ & -3.43378900 & -1.75719900 & 3.47080400 \\
\hline $\mathrm{H}$ & -3.91214200 & -2.03213600 & 2.52054600 \\
\hline $\mathrm{H}$ & -4.04759300 & -2.18702300 & 4.27275800 \\
\hline $\mathrm{C}$ & -2.01390900 & -2.33954300 & 3.51231400 \\
\hline $\mathrm{H}$ & -2.04269100 & -3.42949400 & 3.38356700 \\
\hline $\mathrm{H}$ & -1.57050700 & -2.15386900 & 4.50229400 \\
\hline $\mathrm{C}$ & -1.12270400 & -1.71570500 & 2.42569600 \\
\hline $\mathrm{H}$ & -1.51588900 & -1.98771300 & 1.43682200 \\
\hline $\mathrm{H}$ & -0.10645500 & -2.12156400 & 2.48383400 \\
\hline $\mathrm{C}$ & -0.16381300 & 2.38788900 & 1.27982300 \\
\hline $\mathrm{H}$ & -1.22237400 & 2.51476300 & 1.01053100 \\
\hline $\mathrm{C}$ & 0.07592900 & 3.07037900 & 2.64025600 \\
\hline $\mathrm{H}$ & 1.12784900 & 2.95049300 & 2.92913700 \\
\hline $\mathrm{H}$ & -0.52806200 & 2.60254200 & 3.42802700 \\
\hline $\mathrm{C}$ & -0.24635200 & 4.57423200 & 2.56484400 \\
\hline $\mathrm{H}$ & -1.31856100 & 4.70628200 & 2.35918200 \\
\hline $\mathrm{H}$ & -0.05402500 & 5.04227200 & 3.53901700 \\
\hline $\mathrm{C}$ & 0.57280100 & 5.26740900 & 1.46682000 \\
\hline $\mathrm{H}$ & 1.63885800 & 5.23306000 & 1.73738400 \\
\hline $\mathrm{H}$ & 0.30031500 & 6.32850600 & 1.40143100 \\
\hline $\mathrm{C}$ & 0.37062200 & 4.57984400 & 0.10879400 \\
\hline $\mathrm{H}$ & 1.00566300 & 5.05055300 & -0.65364400 \\
\hline $\mathrm{H}$ & -0.66842600 & 4.72309800 & -0.22062300 \\
\hline $\mathrm{C}$ & 0.66901700 & 3.07224400 & 0.17643800 \\
\hline $\mathrm{H}$ & 1.73710700 & 2.90955700 & 0.37200800 \\
\hline $\mathrm{H}$ & 0.45241400 & 2.60573900 & -0.79184100 \\
\hline $\mathrm{O}$ & 3.36266900 & 1.21306800 & -1.10394000 \\
\hline $\mathrm{O}$ & 1.92849800 & -3.00003500 & 0.37062300 \\
\hline $\mathrm{P}$ & 0.03009000 & 0.51873600 & 1.18723900 \\
\hline $\mathrm{Pd}$ & -0.46342700 & -0.28638000 & -1.04635700 \\
\hline $\mathrm{C}$ & -0.66182300 & -1.20040000 & -2.76986000 \\
\hline $\mathrm{O}$ & -0.83902300 & -1.70143100 & -3.78494800 \\
\hline I & -2.94807000 & 1.29411100 & -1.59165000 \\
\hline $\mathrm{C}$ & -3.96914900 & -1.15321200 & -0.99711200 \\
\hline $\mathrm{F}$ & -4.70763500 & -1.03043100 & 0.11433300 \\
\hline $\mathrm{F}$ & -4.74360100 & -1.54769700 & -2.00496400 \\
\hline & -3.01982800 & -2.07993300 & -0.78098 \\
\hline
\end{tabular}




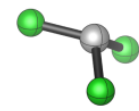

$\mathrm{E}(\mathrm{B} 3 \mathrm{LYP} / \mathrm{BS} 1)=-337.551022$

$\mathrm{E}(\mathrm{PCM}($ dichloroethane $) / \mathrm{M} 06 / \mathrm{BS} 2 / / \mathrm{B} 3 \mathrm{LYP} / \mathrm{BS} 1)=-337.541907$

\begin{tabular}{|c|c|c|c|}
\hline \multicolumn{4}{|c|}{ rrection $=$} \\
\hline & orrection to En & $\operatorname{rgy}=$ & \\
\hline & orrection to En & halpy $=$ & \\
\hline & orrection to $\mathrm{Gi}$ & bs Free Energy & \\
\hline \multicolumn{4}{|c|}{ Charge $=0$ Multiplicity $=2$} \\
\hline $\mathrm{C}$ & 0.00001200 & -0.00004300 & 0.328900 \\
\hline $\mathrm{F}$ & 0.97804500 & -0.80154800 & -0.073094 \\
\hline $\mathrm{F}$ & -1.18324800 & -0.44616200 & -0.073090 \\
\hline & 0.20519500 & 1.24773900 & -0.0730830 \\
\hline
\end{tabular}

\section{RuPhosPdI}

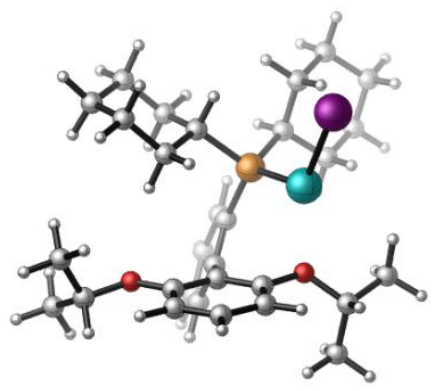

$\mathrm{E}(\mathrm{B} 3 \mathrm{LYP} / \mathrm{BS} 1)=-1799.099914$

$\mathrm{E}(\mathrm{PCM}$ (dichloroethane)/M06/BS2// B3LYP/BS1) $=-1799.653467$

Zero-point correction= 0.676286

Thermal correction to Energy= 0.714548

Thermal correction to Enthalpy=

0.715492

Thermal correction to Gibbs Free Energy=

0.600708

$\begin{array}{lrrr}\text { Charge }=0 \text { Multiplicity }=2 & \\ \mathrm{C} & 0.95891500 & 0.70829100 & 1.72118900 \\ \mathrm{C} & 1.02376300 & 1.53074300 & 2.86295500 \\ \mathrm{H} & 0.27687200 & 2.30118600 & 3.01355900 \\ \mathrm{C} & 2.02226500 & 1.39400900 & 3.82406800 \\ \mathrm{H} & 2.03216800 & 2.04958300 & 4.69066600 \\ \mathrm{C} & 2.99694900 & 0.41186200 & 3.66311300 \\ \mathrm{H} & 3.77971000 & 0.28023400 & 4.40532500 \\ \mathrm{C} & 2.96166200 & -0.40173700 & 2.53545700 \\ \mathrm{H} & 3.72565700 & -1.16129500 & 2.39876000 \\ \mathrm{C} & 1.96412900 & -0.27858000 & 1.55177600 \\ \mathrm{C} & 2.09880900 & -1.21833300 & 0.38871000 \\ \mathrm{C} & 1.24034800 & -2.31491900 & 0.20574200\end{array}$




\begin{tabular}{|c|c|c|c|}
\hline $\mathrm{C}$ & 1.46498700 & -3.27197700 & -0.79005700 \\
\hline $\mathrm{H}$ & 0.77717500 & -4.09759400 & -0.92876500 \\
\hline $\mathrm{C}$ & 2.56935100 & -3.13019100 & -1.62252800 \\
\hline $\mathrm{H}$ & 2.74731900 & -3.86196900 & -2.40532500 \\
\hline $\mathrm{C}$ & 3.44159700 & -2.05174400 & -1.48339200 \\
\hline $\mathrm{H}$ & 4.28319100 & -1.95758700 & -2.15758100 \\
\hline $\mathrm{C}$ & 3.21098000 & -1.10048400 & -0.48150000 \\
\hline $\mathrm{C}$ & 5.28109800 & 0.11989900 & -0.93363100 \\
\hline $\mathrm{H}$ & 5.72634700 & -0.87695000 & -1.04769600 \\
\hline $\mathrm{C}$ & 5.10019700 & 0.77729700 & -2.30236500 \\
\hline $\mathrm{H}$ & 6.06592800 & 0.85692300 & -2.81455600 \\
\hline $\mathrm{H}$ & 4.68964000 & 1.78597200 & -2.18460900 \\
\hline $\mathrm{H}$ & 4.42026800 & 0.20626200 & -2.94121400 \\
\hline $\mathrm{C}$ & 6.15469400 & 0.95203300 & -0.00000500 \\
\hline $\mathrm{H}$ & 7.14759500 & 1.09887700 & -0.43862500 \\
\hline $\mathrm{H}$ & 6.26989200 & 0.45486000 & 0.96786400 \\
\hline $\mathrm{H}$ & 5.70158300 & 1.93451300 & 0.17017000 \\
\hline $\mathrm{C}$ & -0.31188600 & -3.64943200 & 1.56653800 \\
\hline $\mathrm{H}$ & -0.49054600 & -4.33443200 & 0.72930700 \\
\hline $\mathrm{C}$ & -1.63235200 & -3.37366400 & 2.27100700 \\
\hline $\mathrm{H}$ & -2.04441700 & -4.30734100 & 2.66855200 \\
\hline $\mathrm{H}$ & -2.35910500 & -2.94220400 & 1.57446600 \\
\hline $\mathrm{H}$ & -1.48710900 & -2.67419300 & 3.10106400 \\
\hline $\mathrm{C}$ & 0.75646700 & -4.22183500 & 2.49389100 \\
\hline $\mathrm{H}$ & 0.42216200 & -5.18268700 & 2.90066700 \\
\hline $\mathrm{H}$ & 0.94314900 & -3.53620300 & 3.32746200 \\
\hline $\mathrm{H}$ & 1.69817400 & -4.38838300 & 1.96163700 \\
\hline $\mathrm{C}$ & -1.81762500 & 1.74753700 & 1.54149500 \\
\hline $\mathrm{H}$ & -1.39863600 & 2.63302000 & 2.03875300 \\
\hline $\mathrm{C}$ & -2.97430600 & 2.23315700 & 0.63803300 \\
\hline $\mathrm{H}$ & -2.61238700 & 2.96478500 & -0.09336200 \\
\hline $\mathrm{H}$ & -3.37048400 & 1.38642700 & 0.06355900 \\
\hline $\mathrm{C}$ & -4.10144500 & 2.86927600 & 1.47103100 \\
\hline $\mathrm{H}$ & -3.72969600 & 3.78943300 & 1.94728600 \\
\hline $\mathrm{H}$ & -4.91792500 & 3.17124000 & 0.80340200 \\
\hline $\mathrm{C}$ & -4.62245900 & 1.91459200 & 2.55383700 \\
\hline $\mathrm{H}$ & -5.10221900 & 1.05020800 & 2.07279800 \\
\hline $\mathrm{H}$ & -5.39471900 & 2.40810700 & 3.15767600 \\
\hline $\mathrm{C}$ & -3.47749300 & 1.42275600 & 3.44989100 \\
\hline $\mathrm{H}$ & -3.84991300 & 0.69864900 & 4.18593900 \\
\hline $\mathrm{H}$ & -3.07266600 & 2.27036100 & 4.02354500 \\
\hline $\mathrm{C}$ & -2.35074600 & 0.78187300 & 2.62253000 \\
\hline $\mathrm{H}$ & -2.73299300 & -0.11947500 & 2.12389900 \\
\hline $\mathrm{H}$ & -1.53824400 & 0.45696600 & 3.28307400 \\
\hline $\mathrm{C}$ & 0.07863600 & 2.24292300 & -0.69642600 \\
\hline $\mathrm{H}$ & -0.83708000 & 2.40916000 & -1.28118300 \\
\hline $\mathrm{C}$ & 0.51851800 & 3.58086600 & -0.06922800 \\
\hline $\mathrm{H}$ & 1.40320000 & 3.41202800 & 0.55984300 \\
\hline $\mathrm{H}$ & -0.26445400 & 3.98201400 & 0.58597100 \\
\hline $\mathrm{C}$ & 0.85503500 & 4.61986500 & -1.15490800 \\
\hline $\mathrm{H}$ & -0.06184400 & 4.87275700 & -1.70712600 \\
\hline $\mathrm{H}$ & 1.20092100 & 5.54824500 & -0.68201000 \\
\hline $\mathrm{C}$ & 1.90871600 & 4.09390300 & -2.13984100 \\
\hline $\mathrm{H}$ & 2.86271600 & 3.95759400 & -1.60792900 \\
\hline $\mathrm{H}$ & 2.09295900 & 4.83458800 & -2.92853500 \\
\hline $\mathrm{C}$ & 1.47391000 & 2.75465800 & -2.75252900 \\
\hline $\mathrm{H}$ & 2.25718700 & 2.36622400 & -3.41613000 \\
\hline
\end{tabular}




$\begin{array}{lccc}\mathrm{H} & 0.58440200 & 2.91118900 & -3.37961200 \\ \mathrm{C} & 1.15134800 & 1.71243600 & -1.66827900 \\ \mathrm{H} & 2.06650200 & 1.47768100 & -1.10985800 \\ \mathrm{H} & 0.80471200 & 0.78128100 & -2.13075900 \\ \mathrm{O} & 4.01325200 & -0.01891800 & -0.25919100 \\ \mathrm{O} & 0.12764600 & -2.36662800 & 1.03137500 \\ \mathrm{P} & -0.45845800 & 0.92567700 & 0.53073500 \\ \mathrm{Pd} & -1.30035400 & -1.00989200 & -0.47433600 \\ \mathrm{I} & -3.07195000 & -0.79974100 & -2.47960000\end{array}$

\section{$\operatorname{RuPhosPd}(\mathbf{C O}) \mathbf{I}$}

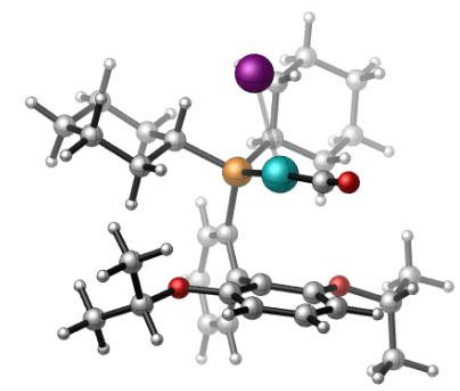

$\mathrm{E}(\mathrm{B} 3 \mathrm{LYP} / \mathrm{BS} 1)=-1912.438672$

$\mathrm{E}(\mathrm{PCM}$ (dichloroethane $) / \mathrm{M} 06 / \mathrm{BS} 2 / / \mathrm{B} 3 \mathrm{LYP} / \mathrm{BS} 1)=-1912.972716$

Zero-point correction $=\quad 0.683794$

Thermal correction to Energy= $\quad 0.724959$

Thermal correction to Enthalpy $=\quad 0.725903$

Thermal correction to Gibbs Free Energy $=\quad 0.604453$

$\begin{array}{lrrr}\text { Charge }= & \text { Multiplicity }=2 & \\ \text { C } & -0.79824600 & 0.03473500 & 2.03700400 \\ \mathrm{C} & -0.71661500 & 0.29173700 & 3.41966900 \\ \mathrm{H} & 0.17086500 & 0.76295900 & 3.82679100 \\ \mathrm{C} & -1.75147200 & -0.03482400 & 4.29181800 \\ \mathrm{H} & -1.65323700 & 0.17609100 & 5.35329300 \\ \mathrm{C} & -2.90792400 & -0.63027900 & 3.78946300 \\ \mathrm{H} & -3.72567800 & -0.89584800 & 4.45418700 \\ \mathrm{C} & -3.01504300 & -0.87124800 & 2.42343100 \\ \mathrm{H} & -3.92198800 & -1.31748300 & 2.02517700 \\ \mathrm{C} & -1.98285500 & -0.54246100 & 1.52702200 \\ \mathrm{C} & -2.27990000 & -0.81339100 & 0.07876200 \\ \mathrm{C} & -3.04072700 & 0.12387000 & -0.66204100 \\ \mathrm{C} & -3.54602600 & -0.20688100 & -1.92902800 \\ \mathrm{H} & -4.12384000 & 0.50447900 & -2.50496500 \\ \mathrm{C} & -3.30611900 & -1.47759800 & -2.44515800 \\ \mathrm{H} & -3.69596400 & -1.73253300 & -3.42686000 \\ \mathrm{C} & -2.57735600 & -2.42962500 & -1.73654300 \\ \mathrm{H} & -2.41081100 & -3.40691600 & -2.17019600 \\ \mathrm{C} & -2.07757200 & -2.10418200 & -0.46641600 \\ \mathrm{C} & -1.36086300 & -4.38394900 & 0.01709800 \\ \mathrm{H} & -2.30815500 & -4.66459800 & -0.46119600 \\ \mathrm{C} & -0.18901500 & -4.68289700 & -0.91823100 \\ \mathrm{H} & -0.18470200 & -5.74533900 & -1.18787600 \\ \mathrm{H} & 0.76022200 & -4.44943600 & -0.42568400\end{array}$




\begin{tabular}{|c|c|c|c|}
\hline $\mathrm{H}$ & -0.24259300 & -4.09621200 & -1.83982400 \\
\hline $\mathrm{C}$ & -1.24561100 & -5.11270300 & 1.35214800 \\
\hline $\mathrm{H}$ & -1.22201300 & -6.19601400 & 1.19136800 \\
\hline $\mathrm{H}$ & -2.09584900 & -4.87285400 & 1.99783000 \\
\hline $\mathrm{H}$ & -0.32602900 & -4.82036900 & 1.87022400 \\
\hline $\mathrm{C}$ & -4.20340800 & 2.26718900 & -0.56992800 \\
\hline $\mathrm{H}$ & -4.04324200 & 2.37499500 & -1.65027600 \\
\hline $\mathrm{C}$ & -3.88748500 & 3.59652200 & 0.10550500 \\
\hline $\mathrm{H}$ & -4.57756700 & 4.37062700 & -0.24689600 \\
\hline $\mathrm{H}$ & -2.86510200 & 3.91244500 & -0.12016900 \\
\hline $\mathrm{H}$ & -3.99158900 & 3.50720200 & 1.19225200 \\
\hline $\mathrm{C}$ & -5.63079800 & 1.79559100 & -0.28889400 \\
\hline $\mathrm{H}$ & -6.35364200 & 2.51538500 & -0.68907300 \\
\hline $\mathrm{H}$ & -5.78993400 & 1.70983200 & 0.79166300 \\
\hline $\mathrm{H}$ & -5.83179000 & 0.82161500 & -0.74461500 \\
\hline $\mathrm{C}$ & 1.02888800 & 2.24192400 & 1.45252900 \\
\hline $\mathrm{H}$ & 1.09682400 & 2.25910300 & 2.54984100 \\
\hline $\mathrm{C}$ & 2.37404900 & 2.73605800 & 0.88018500 \\
\hline $\mathrm{H}$ & 3.19850500 & 2.09809900 & 1.21814800 \\
\hline $\mathrm{H}$ & 2.35640000 & 2.65858600 & -0.21393900 \\
\hline $\mathrm{C}$ & 2.65039100 & 4.19239700 & 1.29407500 \\
\hline $\mathrm{H}$ & 2.78801800 & 4.24234100 & 2.38500200 \\
\hline $\mathrm{H}$ & 3.59441800 & 4.52503300 & 0.84487000 \\
\hline $\mathrm{C}$ & 1.50505400 & 5.12961200 & 0.88596300 \\
\hline $\mathrm{H}$ & 1.44710300 & 5.17195100 & -0.21140600 \\
\hline $\mathrm{H}$ & 1.70808100 & 6.15199200 & 1.22967400 \\
\hline $\mathrm{C}$ & 0.16277700 & 4.63552700 & 1.44372000 \\
\hline $\mathrm{H}$ & -0.65572900 & 5.28260600 & 1.10188800 \\
\hline $\mathrm{H}$ & 0.17593500 & 4.70467900 & 2.54188600 \\
\hline $\mathrm{C}$ & -0.11977000 & 3.18179900 & 1.02989600 \\
\hline $\mathrm{H}$ & -0.23292000 & 3.12847500 & -0.06257500 \\
\hline $\mathrm{H}$ & -1.06844200 & 2.84107600 & 1.45874300 \\
\hline $\mathrm{C}$ & 2.09987800 & -0.55586800 & 1.51524200 \\
\hline $\mathrm{H}$ & 2.89366200 & -0.19395200 & 0.84645100 \\
\hline $\mathrm{C}$ & 2.57290200 & -0.38807600 & 2.97200900 \\
\hline $\mathrm{H}$ & 1.79316000 & -0.74574300 & 3.65659700 \\
\hline $\mathrm{H}$ & 2.75156400 & 0.66805600 & 3.21007200 \\
\hline $\mathrm{C}$ & 3.85701700 & -1.19987500 & 3.22448800 \\
\hline $\mathrm{H}$ & 4.66943500 & -0.79550400 & 2.60375300 \\
\hline $\mathrm{H}$ & 4.17004800 & -1.07910300 & 4.26975400 \\
\hline $\mathrm{C}$ & 3.65318400 & -2.68541800 & 2.89432000 \\
\hline $\mathrm{H}$ & 2.92129200 & -3.11274500 & 3.59632400 \\
\hline $\mathrm{H}$ & 4.58911000 & -3.23915300 & 3.04228000 \\
\hline $\mathrm{C}$ & 3.14660100 & -2.86849200 & 1.45639100 \\
\hline $\mathrm{H}$ & 2.95044100 & -3.93024100 & 1.25546600 \\
\hline $\mathrm{H}$ & 3.92877900 & -2.55679700 & 0.75022000 \\
\hline $\mathrm{C}$ & 1.87608700 & -2.04524600 & 1.18349500 \\
\hline $\mathrm{H}$ & 1.04145000 & -2.43329100 & 1.78314500 \\
\hline $\mathrm{H}$ & 1.58738200 & -2.14678800 & 0.13185400 \\
\hline $\mathrm{O}$ & -1.41807300 & -2.97726200 & 0.34250200 \\
\hline $\mathrm{O}$ & -3.24466000 & 1.32310800 & -0.04673400 \\
\hline $\mathrm{P}$ & 0.63605100 & 0.47566300 & 0.94716400 \\
\hline $\mathrm{Pd}$ & 0.20564100 & 0.20849400 & -1.41040000 \\
\hline $\mathrm{C}$ & -0.35360300 & 0.13815600 & -3.30404000 \\
\hline $\mathrm{O}$ & -0.58281400 & 0.11511600 & -4.42475700 \\
\hline I & 2.90705100 & 0.18183600 & -2.32536000 \\
\hline
\end{tabular}




\section{Phenylacetylene}

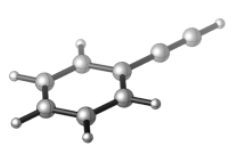

$\mathrm{E}(\mathrm{B} 3 \mathrm{LYP} / \mathrm{BS} 1)=-308.39359$

$\mathrm{E}(\mathrm{PCM}($ dichloroethane $) / \mathrm{M} 06 / \mathrm{BS} 2 / / \mathrm{B} 3 \mathrm{LYP} / \mathrm{BS} 1)=-308.226078$

$\begin{array}{ll}\text { Zero-point correction }= & 0.109497 \\ \text { Thermal correction to Energy= } & 0.115978 \\ \text { Thermal correction to Enthalpy= } & 0.116923 \\ \text { Thermal correction to Gibbs Free Energy= } & 0.079062\end{array}$

Charge $=0$ Multiplicity $=1$

$\begin{array}{lrrr}\mathrm{C} & -1.51254700 & -1.20860500 & -0.00001300 \\ \mathrm{C} & -0.11992700 & -1.21313000 & 0.00001100 \\ \mathrm{C} & 0.59417800 & -0.00002100 & 0.00003200 \\ \mathrm{C} & -0.11990800 & 1.21312000 & 0.00001300 \\ \mathrm{C} & -1.51251400 & 1.20862600 & -0.00001500 \\ \mathrm{C} & -2.21296400 & 0.00001200 & -0.00002400 \\ \mathrm{H} & -2.05301100 & -2.15130300 & -0.00002300 \\ \mathrm{H} & 0.42860100 & -2.14998200 & 0.00002200 \\ \mathrm{H} & 0.42866300 & 2.14994700 & 0.00002400 \\ \mathrm{H} & -2.05297500 & 2.15132600 & -0.00002500 \\ \mathrm{H} & -3.29955900 & 0.00003500 & -0.00004400 \\ \mathrm{C} & 2.02414400 & -0.00001300 & 0.00007200 \\ \mathrm{C} & 3.23418700 & 0.00000400 & 0.00001400 \\ \mathrm{H} & 4.30038500 & 0.00001800 & -0.00048300\end{array}$

TS3

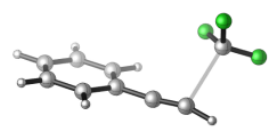

$\mathrm{E}(\mathrm{B} 3 \mathrm{LYP} / \mathrm{BS} 1)=-645.94489$

$\mathrm{E}(\mathrm{PCM}($ dichloroethane $) / \mathrm{M} 06 / \mathrm{BS} 2 / / \mathrm{B} 3 \mathrm{LYP} / \mathrm{BS} 1)=-645.768727$

Zero-point correction $=$

Thermal correction to Energy=

0.121867

Thermal correction to Enthalpy=

0.133107

Thermal correction to Gibbs Free Energy=

$\begin{array}{lrrr}\text { Charge }=0 \text { Multiplicity }=2 & \\ \mathrm{C} & 2.69549300 & -1.43169000 & -0.31646000 \\ \mathrm{C} & 1.47163200 & -0.84571000 & -0.62534700 \\ \mathrm{C} & 1.23917600 & 0.51116700 & -0.32112800 \\ \mathrm{C} & 2.25779500 & 1.26044000 & 0.30013600 \\ \mathrm{C} & 3.47838600 & 0.66429100 & 0.60417700 \\ \mathrm{C} & 3.70182600 & -0.68033000 & 0.29665700 \\ \mathrm{H} & 2.86440600 & -2.47884500 & -0.55192100 \\ \mathrm{H} & 0.67893700 & -1.42489200 & -1.08790100\end{array}$




$\begin{array}{lrrr}\mathrm{H} & 2.07933100 & 2.30476900 & 0.53688500 \\ \mathrm{H} & 4.25815000 & 1.25009700 & 1.08303300 \\ \mathrm{H} & 4.65606500 & -1.14155400 & 0.53580200 \\ \mathrm{C} & -0.01389000 & 1.10819800 & -0.64002700 \\ \mathrm{C} & -1.14777800 & 1.50457400 & -0.85912400 \\ \mathrm{H} & -1.98166000 & 2.05624600 & -1.23437400 \\ \mathrm{C} & -2.65940100 & -0.14287500 & 0.18114200 \\ \mathrm{~F} & -3.78707700 & -0.16840200 & -0.53484400 \\ \mathrm{~F} & -2.02286600 & -1.30936200 & 0.07655000 \\ \mathrm{~F} & -2.93390800 & 0.11618500 & 1.45810700\end{array}$

\section{IM4}

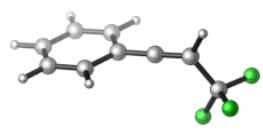

$\mathrm{E}(\mathrm{B} 3 \mathrm{LYP} / \mathrm{BS} 1)=-646.014847$

$\mathrm{E}(\mathrm{PCM}($ dichloroethane $) / \mathrm{M} 06 / \mathrm{BS} 2 / / \mathrm{B} 3 \mathrm{LYP} / \mathrm{BS} 1)=-645.839033$

$\begin{array}{ll}\text { Zero-point correction }= & 0.124940 \\ \text { Thermal correction to Energy= } & 0.135315 \\ \text { Thermal correction to Enthalpy= } & 0.136259 \\ \text { Thermal correction to Gibbs Free Energy= } & 0.085533\end{array}$

Charge $=0$ Multiplicity $=2$

$\begin{array}{llll}\text { C } & 3.21678600 & 1.21332500 & -0.14012200\end{array}$

$\begin{array}{llll}\mathrm{C} & 1.86709100 & 1.22866700 & 0.16546500\end{array}$

$\begin{array}{llll}\text { C } & 1.14805700 & 0.00000700 & 0.32908000\end{array}$

$\begin{array}{llll}\mathrm{C} & 1.86708700 & -1.22866300 & 0.16547000\end{array}$

$\begin{array}{llll}\text { C } & 3.21678000 & -1.21333100 & -0.14011600\end{array}$

$\begin{array}{llll}\text { C } & 3.90434500 & -0.00000300 & -0.29553800\end{array}$

$\begin{array}{llll}\mathrm{H} & 3.74671600 & 2.15429500 & -0.26127400\end{array}$

$\begin{array}{llll}\mathrm{H} & 1.33462700 & 2.16693000 & 0.28381200\end{array}$

$\begin{array}{llll}\mathrm{H} & 1.33461500 & -2.16692000 & 0.28382800\end{array}$

$\mathrm{H} \quad 3.74670800 \quad-2.15430200 \quad-0.26126100$

$\begin{array}{llll}\mathrm{H} & 4.96323100 & -0.00000800 & -0.53614700\end{array}$

$\begin{array}{llll}\text { C } & -0.19039700 & -0.00000500 & 0.63271500\end{array}$

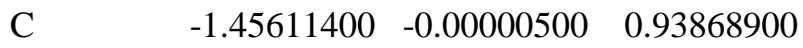

$\begin{array}{llll}\mathrm{H} & -1.81806800 & -0.00000700 & 1.97051900\end{array}$

$\begin{array}{llll}\text { C } & -2.56365100 & -0.00000100 & -0.08377700\end{array}$

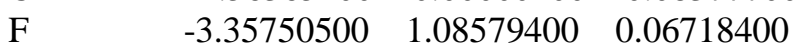

F $\quad \begin{array}{llll}\text { F } & -2.10366000 & 0.00004300 & -1.34659100\end{array}$

$\begin{array}{lllll}\mathrm{F} & & -3.35747100 & -1.08583000 & 0.06711100\end{array}$

TS4

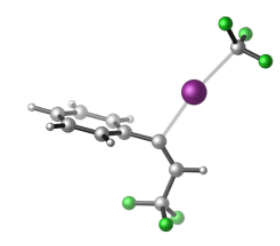

$\mathrm{E}(\mathrm{B} 3 \mathrm{LYP} / \mathrm{BS} 1)=-994.98506$ 
$\mathrm{E}(\mathrm{PCM}($ dichloroethane $) / \mathrm{M} 06 / \mathrm{BS} 2 / / \mathrm{B} 3 \mathrm{LYP} / \mathrm{BS} 1)=-994.827607$

$\begin{array}{ll}\text { Zero-point correction }= & 0.140130 \\ \text { Thermal correction to Energy= } & 0.156913 \\ \text { Thermal correction to Enthalpy= } & 0.157857 \\ \text { Thermal correction to Gibbs Free Energy= } & 0.086317\end{array}$

Charge $=0$ Multiplicity $=2$

$\begin{array}{llll}\text { C } & 2.83932500 & 2.69438300 & -1.21430100\end{array}$

C $\quad 2.16174700 \quad 1.47957900 \quad-1.20935100$

$\begin{array}{llll}\text { C } & 1.70733800 & 0.92632400 & 0.00647300\end{array}$

$\begin{array}{llll}\mathrm{C} & 1.92098600 & 1.63399200 & 1.20803500\end{array}$

$\begin{array}{llll}\mathrm{C} & 2.60051200 & 2.84712700 & 1.19238800\end{array}$

C $\quad 3.06034000 \quad 3.38125200 \quad-0.01626500$

$\mathrm{H} \quad 3.19657100 \quad 3.10757800 \quad-2.15322200$

$\mathrm{H} \quad \begin{array}{llll}1.98447600 & 0.94270800 & -2.13608900\end{array}$

$\begin{array}{llll}\mathrm{H} & 1.55630500 & 1.21806400 & 2.14216900\end{array}$

$\begin{array}{llll}\mathrm{H} & 2.77231400 & 3.37998000 & 2.12322300\end{array}$

$\begin{array}{llll}\mathrm{H} & 3.58670100 & 4.33152400 & -0.02435200\end{array}$

$\begin{array}{llll}\mathrm{C} & 1.00097900 & -0.33366400 & 0.00944900\end{array}$

$\begin{array}{llll}\mathrm{C} & 1.34095600 & -1.61214700 & 0.00381000\end{array}$

$\begin{array}{llll}\mathrm{H} & 0.60214100 & -2.40696900 & -0.02075600\end{array}$

$\begin{array}{llll}\text { C } & 2.75807700 & -2.12216800 & 0.02976500\end{array}$

F $\quad 3.02209500 \quad-2.82977900 \quad-1.09257500$

F $\quad 3.68271500-1.15297100 \quad 0.12788100$

F $\quad 2.93030100 \quad-2.96352600 \quad 1.07426900$

$\begin{array}{llll}\text { I } & -1.41961000 & 0.00313400 & -0.00988300\end{array}$

$\begin{array}{llll}\mathrm{C} & -3.93690400 & -0.04454000 & -0.01308000\end{array}$

$\begin{array}{llll}\text { F } & -4.37137700 & 0.14893100 & 1.22758900\end{array}$

F $\quad-4.37608600 \quad 0.92721900 \quad-0.80649700$

F $\quad-4.35201600 \quad-1.22318900 \quad-0.46274900$

TS5

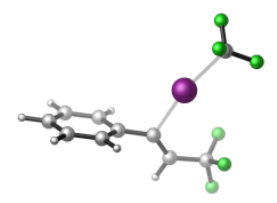

$\mathrm{E}(\mathrm{B} 3 \mathrm{LYP} / \mathrm{BS} 1)=-994.980336$

$\mathrm{E}(\mathrm{PCM}($ dichloroethane $) / \mathrm{M} 06 / \mathrm{BS} 2 / / \mathrm{B} 3 \mathrm{LYP} / \mathrm{BS} 1)=-994.825694$

Zero-point correction=

0.139808

Thermal correction to Energy=

0.156536

Thermal correction to Enthalpy=

0.157480

Thermal correction to Gibbs Free Energy $=\quad 0.087756$

Charge $=0$ Multiplicity $=2$

$\begin{array}{llll}\mathrm{C} & -3.74899200 & -2.01332200 & -1.14392200 \\ \mathrm{C} & -2.70643600 & -1.09378000 & -1.18465100 \\ \mathrm{C} & -2.40425900 & -0.31310000 & -0.04940300 \\ \mathrm{C} & -3.15191500 & -0.50066900 & 1.13207700 \\ \mathrm{C} & -4.19138200 & -1.42642900 & 1.16419500 \\ \mathrm{C} & -4.49288300 & -2.18412700 & 0.02895100 \\ \mathrm{H} & -3.98272100 & -2.60135900 & -2.02686700 \\ \mathrm{H} & -2.12098200 & -0.96446400 & -2.08978100\end{array}$




$\begin{array}{cccc}\mathrm{H} & -2.90846000 & 0.08745300 & 2.01159500 \\ \mathrm{H} & -4.76683200 & -1.55793500 & 2.07618300 \\ \mathrm{H} & -5.30198300 & -2.90847200 & 0.05810900 \\ \mathrm{C} & -1.33762200 & 0.66707900 & -0.07763000 \\ \mathrm{C} & -1.38104600 & 1.98985100 & -0.11664700 \\ \mathrm{H} & -2.35600800 & 2.47227900 & -0.19757700 \\ \mathrm{C} & -0.23246000 & 2.96428100 & -0.06896000 \\ \mathrm{~F} & -0.71942800 & 4.22357500 & -0.15116400 \\ \mathrm{~F} & 0.47031400 & 2.88102700 & 1.07830600 \\ \mathrm{~F} & 0.63510800 & 2.80310300 & -1.08691800 \\ \mathrm{I} & 0.83040400 & -0.50351000 & 0.02988000 \\ \mathrm{C} & 3.17461500 & -1.26474100 & 0.05457600 \\ \mathrm{~F} & 3.42184600 & -1.84971800 & 1.22327500 \\ \mathrm{~F} & 3.34641400 & -2.14010700 & -0.93274900 \\ \mathrm{~F} & 3.98573100 & -0.22807600 & -0.11373200\end{array}$

\section{Z-1-iodo-1-phenyl-2-trifluoromethylethane}<smiles>Cc1c(C)c(C)c(C(O)C(C)C(C)C)c(C)c1C</smiles>

$\mathrm{E}(\mathrm{B} 3 \mathrm{LYP} / \mathrm{BS} 1)=-657.444446$

$\mathrm{E}(\mathrm{PCM}($ dichloroethane $) / \mathrm{M} 06 / \mathrm{BS} 2 / / \mathrm{B} 3 \mathrm{LYP} / \mathrm{BS} 1)=-657.291113$

Zero-point correction=

Thermal correction to Energy=

Thermal correction to Enthalpy=

Thermal correction to Gibbs Free Energy= 0.087451

$\begin{array}{lrrr}\text { Charge }= & \text { Multiplicity }=1 & \\ \text { C } & 3.79076500 & -0.30183700 & 0.79907300 \\ \text { C } & 2.40634800 & -0.45457300 & 0.79090300 \\ \text { C } & 1.59775800 & 0.41770600 & 0.04400900 \\ \text { C } & 2.21425900 & 1.42861900 & -0.71495100 \\ \text { C } & 3.60101300 & 1.57307200 & -0.71068800 \\ \text { C } & 4.39357600 & 0.71096400 & 0.04853800 \\ \text { H } & 4.40062300 & -0.97776800 & 1.39186400 \\ \text { H } & 1.94600000 & -1.24630800 & 1.37337200 \\ \text { H } & 1.60109400 & 2.08513300 & -1.32473700 \\ \text { H } & 4.06095200 & 2.35360700 & -1.31034800 \\ \text { H } & 5.47442900 & 0.82072200 & 0.04881300 \\ \text { C } & 0.12124100 & 0.33233800 & 0.06048100 \\ \text { C } & -0.65829500 & 1.41265800 & 0.18547100 \\ \text { H } & -0.16882300 & 2.36978500 & 0.34603200 \\ \text { C } & -2.15797700 & 1.50672000 & 0.16131700 \\ \text { F } & -2.51953900 & 2.80388200 & 0.28892200 \\ \text { F } & -2.69572800 & 1.05407300 & -0.99011900 \\ \text { F } & -2.74114400 & 0.82845900 & 1.17110900 \\ \text { I } & -0.70865500 & -1.64786600 & -0.16488900\end{array}$




\section{$E$-1-iodo-1-phenyl-2-trifluoromethylethane}<smiles>CB1C(C)C(C)=C(C)C(=O)C1B(O)OC(C)C(O)(O)C(C)(O)O</smiles>

$\mathrm{E}(\mathrm{B} 3 \mathrm{LYP} / \mathrm{BS} 1)=-657.446477$

$\mathrm{E}(\mathrm{PCM}$ (dichloroethane)/M06/BS2// B3LYP/BS1 $)=-657.29052$

$\begin{array}{ll}\text { Zero-point correction= } & 0.128637 \\ \text { Thermal correction to Energy= } & 0.140272 \\ \text { Thermal correction to Enthalpy= } & 0.141217 \\ \text { Thermal correction to Gibbs Free Energy= } & 0.087879\end{array}$

Charge $=0$ Multiplicity $=1$

$\begin{array}{lrrr}\mathrm{C} & 1.15767500 & 3.10012900 & -0.90065500 \\ \mathrm{C} & 0.36649900 & 1.95471700 & -0.91386100 \\ \mathrm{C} & 0.60611400 & 0.91512800 & -0.00008500 \\ \mathrm{C} & 1.63659400 & 1.06079900 & 0.94494900 \\ \mathrm{C} & 2.41839800 & 2.21491400 & 0.96109400 \\ \mathrm{C} & 2.18386400 & 3.23550800 & 0.03866100 \\ \mathrm{H} & 0.96924800 & 3.89135900 & -1.62076500 \\ \mathrm{H} & -0.43811400 & 1.85881000 & -1.63637700 \\ \mathrm{H} & 1.82116800 & 0.26734700 & 1.66087500 \\ \mathrm{H} & 3.20910200 & 2.31581400 & 1.69916100 \\ \mathrm{H} & 2.79267300 & 4.13525100 & 0.05422300 \\ \mathrm{C} & -0.20361700 & -0.31742300 & -0.00416900 \\ \mathrm{C} & 0.21401200 & -1.58773000 & -0.02931300 \\ \mathrm{H} & -0.48443800 & -2.41256000 & 0.04994900 \\ \mathrm{C} & 1.64358500 & -2.03936100 & -0.15655900 \\ \mathrm{~F} & 1.67430300 & -3.26862700 & -0.71625600 \\ \mathrm{~F} & 2.40874300 & -1.23052300 & -0.91030400 \\ \mathrm{~F} & 2.24752600 & -2.14767300 & 1.05590400 \\ \mathrm{I} & -2.35818000 & -0.02744700 & 0.09978200\end{array}$

\section{IM5}

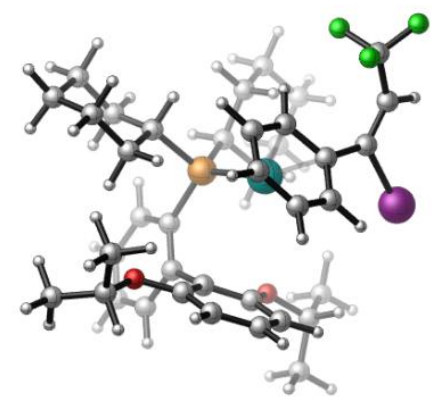

$\mathrm{E}(\mathrm{B} 3 \mathrm{LYP} / \mathrm{BS} 1)=-2445.123518$

$\mathrm{E}(\mathrm{PCM}$ (dichloroethane)/M06/BS2// B3LYP/BS1 $)=-2445.528652$

Zero-point correction $=$ 0.804675

Thermal correction to Energy=

Thermal correction to Enthalpy=
0.854073

0.855017 


\begin{tabular}{|c|c|c|c|}
\hline \multicolumn{4}{|c|}{ Charge $=0$ Multiplicity $=1$} \\
\hline C & -2.60168200 & 0.31843400 & 1.49034100 \\
\hline $\mathrm{C}$ & -3.39397600 & 0.14268400 & 2.64268000 \\
\hline $\mathrm{H}$ & -3.11363200 & -0.60119600 & 3.37970400 \\
\hline $\mathrm{C}$ & -4.53834000 & 0.89967100 & 2.88107400 \\
\hline $\mathrm{H}$ & -5.12060400 & 0.73243900 & 3.78357000 \\
\hline $\mathrm{C}$ & -4.92473100 & 1.86699400 & 1.95476300 \\
\hline $\mathrm{H}$ & -5.81594400 & 2.46700400 & 2.11983900 \\
\hline $\mathrm{C}$ & -4.14896900 & 2.06769200 & 0.81774300 \\
\hline $\mathrm{H}$ & -4.43373200 & 2.83094400 & 0.09884200 \\
\hline $\mathrm{C}$ & -2.98562400 & 1.31887700 & 0.56671100 \\
\hline $\mathrm{C}$ & -2.23087500 & 1.69885300 & -0.67280600 \\
\hline $\mathrm{C}$ & -1.21519400 & 2.67413800 & -0.6039830 \\
\hline $\mathrm{C}$ & -0.61629600 & 3.16819100 & -1.77190800 \\
\hline $\mathrm{H}$ & 0.17216400 & 3.90875900 & -1.72545300 \\
\hline $\mathrm{C}$ & -1.04512700 & 2.69191000 & -3.00731800 \\
\hline $\mathrm{H}$ & -0.58189300 & 3.07366300 & $-3.9134080 c$ \\
\hline $\mathrm{C}$ & -2.05828900 & 1.74169400 & -3.11012900 \\
\hline $\mathrm{H}$ & -2.36791200 & 1.39316000 & -4.0869540 \\
\hline $\mathrm{C}$ & -2.65653700 & 1.25268300 & $-1.9402530 c$ \\
\hline $\mathrm{C}$ & -4.37934600 & 0.01787300 & -3.14388200 \\
\hline $\mathrm{H}$ & -4.41333600 & 0.90906700 & -3.78436700 \\
\hline $\mathrm{C}$ & -3.67297700 & -1.12579800 & -3.8747110( \\
\hline $\mathrm{H}$ & -4.17816100 & -1.33926000 & -4.8240890 \\
\hline $\mathrm{H}$ & -3.69214600 & -2.03383300 & -3.2628510 \\
\hline $\mathrm{H}$ & -2.62721800 & -0.88418300 & -4.0862200 \\
\hline $\mathrm{C}$ & -5.80179900 & -0.34677900 & -2.7289540 \\
\hline $\mathrm{H}$ & -6.40229900 & -0.60269700 & -3.6088980 \\
\hline $\mathrm{H}$ & -6.27822500 & 0.49086400 & -2.21063700 \\
\hline $\mathrm{H}$ & -5.79382600 & -1.20853700 & -2.0528030 \\
\hline $\mathrm{C}$ & -0.13949200 & 4.30069300 & 0.85906500 \\
\hline $\mathrm{H}$ & 0.74784500 & 4.28887100 & 0.21320700 \\
\hline $\mathrm{C}$ & 0.31813800 & 4.26819600 & 2.31267000 \\
\hline $\mathrm{H}$ & 0.90475800 & 5.16413600 & 2.54387600 \\
\hline $\mathrm{H}$ & 0.93658800 & 3.38619900 & 2.50076800 \\
\hline $\mathrm{H}$ & -0.54619100 & 4.23610800 & 2.98493800 \\
\hline $\mathrm{C}$ & -1.00221200 & 5.52450600 & 0.54611200 \\
\hline $\mathrm{H}$ & -0.42711200 & 6.44457800 & 0.70113100 \\
\hline $\mathrm{H}$ & -1.87584400 & 5.54648300 & 1.20710800 \\
\hline $\mathrm{H}$ & -1.35468700 & 5.51293700 & -0.4895120 \\
\hline $\mathrm{C}$ & -0.36930000 & -0.86712300 & 2.9665120 \\
\hline $\mathrm{H}$ & -1.15742800 & -1.16896900 & 3.66962200 \\
\hline $\mathrm{C}$ & 0.75287200 & -1.92462100 & 3.03684100 \\
\hline $\mathrm{H}$ & 0.36458900 & -2.91832500 & 2.78251900 \\
\hline $\mathrm{H}$ & 1.51583900 & -1.68412100 & 2.28306600 \\
\hline $\mathrm{C}$ & 1.40026700 & -1.97437200 & 4.43220200 \\
\hline $\mathrm{H}$ & 0.65966700 & -2.33235800 & 5.16330400 \\
\hline $\mathrm{H}$ & 2.21714900 & -2.70756000 & 4.43245400 \\
\hline $\mathrm{C}$ & 1.91690400 & -0.59714900 & 4.87086800 \\
\hline $\mathrm{H}$ & 2.74108100 & -0.29238700 & 4.20907200 \\
\hline $\mathrm{H}$ & 2.33263200 & -0.65172500 & 5.88536800 \\
\hline $\mathrm{C}$ & 0.80219300 & 0.45617200 & 4.80383200 \\
\hline $\mathrm{H}$ & 1.19537800 & 1.44589000 & 5.07076200 \\
\hline $\mathrm{H}$ & 0.02958300 & 0.21643800 & 5.55008600 \\
\hline & 0.16264800 & 0.51225300 & 3.40667000 \\
\hline
\end{tabular}




$\begin{array}{lrrr}\mathrm{H} & 0.91109000 & 0.84864600 & 2.67563100 \\ \mathrm{H} & -0.64408200 & 1.25348200 & 3.38917300 \\ \mathrm{C} & -1.81803200 & -2.49710600 & 0.95059400 \\ \mathrm{H} & -0.90546900 & -3.11041700 & 0.90807800 \\ \mathrm{C} & -2.73678900 & -3.08492800 & 2.03924700 \\ \mathrm{H} & -3.64580600 & -2.47506100 & 2.11741800 \\ \mathrm{H} & -2.24825800 & -3.05790900 & 3.02137400 \\ \mathrm{C} & -3.13924200 & -4.53395900 & 1.70788500 \\ \mathrm{H} & -2.24292300 & -5.17189000 & 1.72056400 \\ \mathrm{H} & -3.80824800 & -4.92025800 & 2.48803800 \\ \mathrm{C} & -3.81083200 & -4.63019600 & 0.33114600 \\ \mathrm{H} & -4.76517100 & -4.08322000 & 0.35665500 \\ \mathrm{H} & -4.05413800 & -5.67474800 & 0.09715600 \\ \mathrm{C} & -2.91336400 & -4.03062200 & -0.76061000 \\ \mathrm{H} & -3.42582600 & -4.05867600 & -1.73184200 \\ \mathrm{H} & -2.01196400 & -4.65313500 & -0.86756800 \\ \mathrm{C} & -2.49408900 & -2.58748800 & -0.43172400 \\ \mathrm{H} & -3.37504600 & -1.93246500 & -0.44956500 \\ \mathrm{H} & -1.81074300 & -2.20900000 & -1.20028200 \\ \mathrm{O} & -3.68870500 & 0.35533600 & -1.92686900 \\ \mathrm{O} & -0.88533700 & 3.08505800 & 0.65693000 \\ \mathrm{P} & -1.10394100 & -0.76401700 & 1.23209600 \\ \mathrm{Pd} & 0.56308700 & -0.35517900 & -0.36266600 \\ \mathrm{I} & 3.48647400 & 1.97030100 & -0.18265200 \\ \mathrm{C} & 3.45630000 & -0.13281500 & -0.78395400 \\ \mathrm{C} & 4.44879800 & -0.86395600 & -0.25109700 \\ \mathrm{H} & 5.11802900 & -0.43788900 & 0.48654500 \\ \mathrm{C} & 4.78100400 & -2.29179400 & -0.56906900 \\ \mathrm{C} & 2.39074800 & -0.52607300 & -1.72163500 \\ \mathrm{C} & 2.11399300 & 0.24352600 & -2.89258800 \\ \mathrm{C} & 1.77111500 & -1.81753400 & -1.60178000 \\ \mathrm{C} & 1.34442100 & -0.27128100 & -3.91915100 \\ \mathrm{H} & 2.55364000 & 1.23125900 & -2.98614100 \\ \mathrm{C} & 0.99548100 & -2.31776200 & -2.67730500 \\ \mathrm{H} & 2.08304000 & -2.49845700 & -0.82064800 \\ \mathrm{C} & 0.79178300 & -1.56516500 & -3.82067200 \\ \mathrm{H} & 1.17269600 & 0.32630600 & -4.80975600 \\ \mathrm{H} & 0.57979200 & -3.31842600 & -2.59527600 \\ \mathrm{H} & 0.20486400 & -1.96797100 & -4.64183300 \\ \mathrm{~F} & 4.58775000 & -2.62936400 & -1.85912600 \\ \mathrm{~F} & 4.06531300 & -3.17718000 & 0.18130800 \\ \mathrm{~F} & 6.08276400 & -2.51949200 & -0.28341200\end{array}$

\section{TS6}

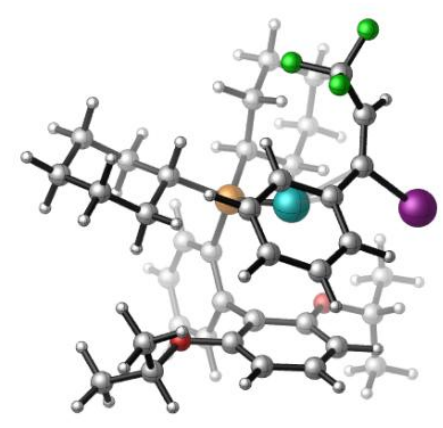


$\mathrm{E}(\mathrm{B} 3 \mathrm{LYP} / \mathrm{BS} 1)=-2445.119744$

$\mathrm{E}(\mathrm{PCM}($ dichloroethane $) / \mathrm{M} 06 / \mathrm{BS} 2 / / \mathrm{B} 3 \mathrm{LYP} / \mathrm{BS} 1)=-2445.526311$

Zero-point correction=

0.803511

Thermal correction to Energy=

0.852512

Thermal correction to Enthalpy=

0.853456

Thermal correction to Gibbs Free Energy=

0.714424

Charge $=0$ Multiplicity $=1$

C

C

$\mathrm{H}$ $\begin{array}{lll}2.42783000 & 0.04368700 & 1.62045100\end{array}$

$\begin{array}{llll}3.17791400 & 0.48280100 & 2.73015100\end{array}$

$\begin{array}{llll}\mathrm{C} & 4.34791300 & -0.15349200 & 3.13707800\end{array}$

$\begin{array}{llll}\mathrm{H} & 4.89484400 & 0.21846200 & 3.99961300\end{array}$

$\begin{array}{llll}\text { C } & 4.80509500 & -1.26402000 & 2.42972100\end{array}$

$\begin{array}{llll}\mathrm{H} & 5.71725200 & -1.77412600 & 2.72847500\end{array}$

$\begin{array}{llll}\text { C } & 4.07341500 & -1.72273900 & 1.33947100\end{array}$

$\begin{array}{llll}\mathrm{H} & 4.41332200 & -2.59722000 & 0.79162600\end{array}$

$\begin{array}{llll}\mathrm{C} & 2.88534700 & -1.09774600 & 0.92100100\end{array}$

$\begin{array}{llll}\text { C } & 2.18891400 & -1.75325500 & -0.23390400\end{array}$

$\begin{array}{llll}\mathrm{C} & 1.22215300 & -2.75031900 & 0.00785000\end{array}$

$\begin{array}{llll}\mathrm{C} & 0.68077800 & -3.49373200 & -1.05067300\end{array}$

$\begin{array}{llll}\mathrm{H} & -0.06959700 & -4.25358300 & -0.87392500\end{array}$

$\begin{array}{llll}\mathrm{C} & 1.11711100 & -3.24311800 & -2.34833000\end{array}$

$\begin{array}{llll}\mathrm{H} & 0.69588400 & -3.81641500 & -3.16997000\end{array}$

$\begin{array}{llll}\mathrm{C} & 2.08524200 & -2.27897100 & -2.61789700\end{array}$

$\mathrm{H} \quad 2.40271900 \quad-2.10972500 \quad-3.63868600$

C $\quad 2.62931400 \quad-1.54296500 \quad-1.55589600$

$\begin{array}{llll}\mathrm{C} & 4.34702100 & -0.50632700 & -2.93981900\end{array}$

$\mathrm{H} \quad 4.42795800 \quad-1.50776700 \quad-3.38244700$

$\begin{array}{lllll}\text { C } & & 3.63751800 & 0.43950600 & -3.91084500\end{array}$

$\mathrm{H} \quad 4.17143500 \quad 0.47164100 \quad-4.86799200$

$\mathrm{H} \quad 3.61052400 \quad 1.45355500 \quad-3.49810100$

$\begin{array}{llll}\mathrm{H} & 2.60675500 & 0.12564000 & -4.09979500\end{array}$

$\begin{array}{lllll}\text { C } & 5.74293300 & -0.01620600 & -2.56568500\end{array}$

$\mathrm{H} \quad \begin{array}{lllll}\mathrm{H} & 6.36786700 & 0.07620600 & -3.46088800\end{array}$

$\begin{array}{lllll}\mathrm{H} & 6.22332100 & -0.71396200 & -1.87313900\end{array}$

$\begin{array}{lllll}\mathrm{H} & 5.68659400 & 0.96428000 & -2.08046600\end{array}$

$\begin{array}{llll}\mathrm{C} & 0.16777400 & -4.10298900 & 1.74148300\end{array}$

$\begin{array}{llll}\mathrm{H} & -0.70043400 & -4.24864600 & 1.08622100\end{array}$

$\begin{array}{llll}\text { C } & -0.33102500 & -3.79907100 & 3.14955900\end{array}$

$\mathrm{H} \quad-0.89887600 \quad-4.64998000 \quad 3.54166600$

$\mathrm{H} \quad-0.97830400 \quad-2.91760000 \quad 3.14548700$

$\begin{array}{llll}\mathrm{H} & 0.51363300 & -3.60644000 & 3.82006200\end{array}$

$\begin{array}{llll}\mathrm{C} & 1.07574700 & -5.33350200 & 1.69708800\end{array}$

$\mathrm{H} \quad 0.52520400 \quad-6.22462200 \quad 2.01974100$

$\begin{array}{llll}\mathrm{H} & 1.93022600 & -5.19222600 & 2.36823900\end{array}$

$\begin{array}{llll}\mathrm{H} & 1.45751100 & -5.51482600 & 0.68797100\end{array}$

$\begin{array}{llll}\mathrm{C} & 0.12637100 & 1.43165700 & 2.79159400\end{array}$

$\begin{array}{llll}\mathrm{H} & 0.89801500 & 1.87979200 & 3.43162000\end{array}$

$\begin{array}{llll}\mathrm{C} & -1.00652100 & 2.46830000 & 2.63523100\end{array}$

$\begin{array}{llll}\mathrm{H} & -0.62240600 & 3.39793600 & 2.19844600\end{array}$

$\begin{array}{llll}\mathrm{H} & -1.75580400 & 2.08069500 & 1.93138100\end{array}$

$\begin{array}{llll}\text { C } & -1.67699000 & 2.78325800 & 3.98448200\end{array}$

$\mathrm{H} \quad-0.95246500 \quad 3.28893000 \quad 4.64061400$

$\begin{array}{llll}\mathrm{H} & -2.50129400 & 3.49096300 & 3.82795400\end{array}$

$\begin{array}{llll}\text { C } & -2.18608300 & 1.51363400 & 4.68103100\end{array}$ 


\begin{tabular}{lccc}
$\mathrm{H}$ & -2.99557600 & 1.07168100 & 4.08130300 \\
$\mathrm{H}$ & -2.62008500 & 1.76306100 & 5.65794500 \\
$\mathrm{C}$ & -1.05869000 & 0.48417200 & 4.84137700 \\
$\mathrm{H}$ & -1.44470800 & -0.43820800 & 5.29474900 \\
$\mathrm{H}$ & -0.30166500 & 0.87919900 & 5.53561400 \\
$\mathrm{C}$ & -0.39612300 & 0.16033100 & 3.49222600 \\
$\mathrm{H}$ & -1.12903900 & -0.32668000 & 2.83363300 \\
$\mathrm{H}$ & 0.42038000 & -0.55671500 & 3.63373600 \\
$\mathrm{C}$ & 1.56007000 & 2.65324400 & 0.49924900 \\
$\mathrm{H}$ & 0.62993500 & 3.21254700 & 0.31807400 \\
$\mathrm{C}$ & 2.43680300 & 3.48525500 & 1.45507900 \\
$\mathrm{H}$ & 3.36548000 & 2.93961100 & 1.66506700 \\
$\mathrm{H}$ & 1.93259900 & 3.64015000 & 2.41706800 \\
$\mathrm{C}$ & 2.79226300 & 4.85132900 & 0.83910000 \\
$\mathrm{H}$ & 1.87332100 & 5.44291200 & 0.71291100 \\
$\mathrm{H}$ & 3.43293800 & 5.41396500 & 1.53075700 \\
$\mathrm{C}$ & 3.48458800 & 4.69228000 & -0.52157300 \\
$\mathrm{H}$ & 4.45903600 & 4.20293600 & -0.37499800 \\
$\mathrm{H}$ & 3.69086500 & 5.67631800 & -0.96218200 \\
$\mathrm{C}$ & 2.63242300 & 3.84446200 & -1.47668300 \\
$\mathrm{H}$ & 3.16313600 & 3.69403900 & -2.42668900 \\
$\mathrm{H}$ & 1.70978400 & 4.39304400 & -1.72008400 \\
$\mathrm{C}$ & 2.26347200 & 2.48339200 & -0.86185500 \\
$\mathrm{H}$ & 3.17003200 & 1.87877000 & -0.73004300 \\
$\mathrm{H}$ & 1.61530800 & 1.92320300 & -1.54495500 \\
$\mathrm{O}$ & 3.62096400 & -0.61181900 & -1.70128700 \\
$\mathrm{O}$ & 0.88328900 & -2.92674700 & 1.31964700 \\
$\mathrm{P}$ & 0.89644300 & 0.99129400 & 1.12462600 \\
$\mathrm{Pd}$ & -0.75458800 & 0.18949100 & -0.33515300 \\
$\mathrm{I}$ & -3.23208500 & -2.03671000 & -0.06367600 \\
$\mathrm{C}$ & -3.06930700 & -0.00884000 & -0.95357600 \\
$\mathrm{C}$ & -3.85655100 & 0.89907600 & -0.32572500 \\
$\mathrm{H}$ & -4.30993300 & 0.66507700 & 0.62908100 \\
$\mathrm{C}$ & -4.22679800 & 2.25651200 & -0.82765400 \\
$\mathrm{C}$ & -2.24687700 & 0.11868900 & -2.16213800 \\
$\mathrm{C}$ & -2.08027300 & -0.93109700 & -3.10465700 \\
$\mathrm{C}$ & -1.56002900 & 1.34925500 & -2.40483700 \\
$\mathrm{C}$ & -1.31772500 & -0.75011100 & -4.24562500 \\
$\mathrm{~F}$ & -2.57739100 & -1.87922200 & -2.93288200 \\
$\mathrm{~F}$ & -3.32609300 & 3.22493800 & -0.47524300 \\
$\mathrm{C}$ & -5.40624900 & 2.63405900 & -0.28413800 \\
$\mathrm{H}$ & -1.73937400 & 2.21031300 & -1.77661000 \\
$\mathrm{C}$ & -0.66476400 & 0.47101800 & -4.48524500 \\
$\mathrm{H}$ & -1.22851300 & -1.56299500 & -4.96094100 \\
$\mathrm{H}$ & -0.29007300 & 2.45569200 & -3.74172400 \\
$\mathrm{H}$ & -0.07529100 & 0.60571500 & -5.38814500 \\
& & & \\
$\mathrm{~F}$ & -3.35748800 & 2.32954100 & -2.16874900 \\
\hline
\end{tabular}




\section{IM5}

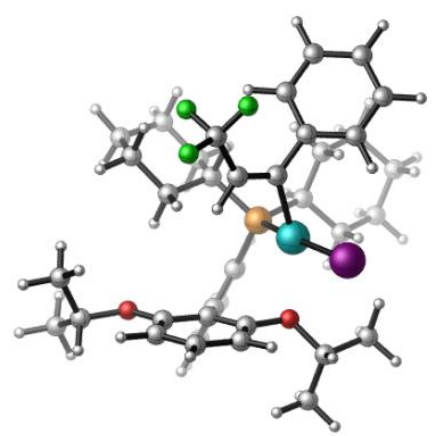

$\mathrm{E}(\mathrm{B} 3 \mathrm{LYP} / \mathrm{BS} 1)=-2445.172123$

$\mathrm{E}(\mathrm{PCM}$ (dichloroethane $) / \mathrm{M} 06 / \mathrm{BS} 2 / / \mathrm{B} 3 \mathrm{LYP} / \mathrm{BS} 1)=-2445.574281$

Zero-point correction=

0.806406

Thermal correction to Energy=

0.855363

Thermal correction to Enthalpy=

0.856307

Thermal correction to Gibbs Free Energy=

0.720915

Charge $=0$ Multiplicity $=1$

$\begin{array}{lrrr}\mathrm{C} & 1.94060600 & 0.13069100 & 1.96661900 \\ \mathrm{C} & 2.31336300 & 0.52304800 & 3.26829100 \\ \mathrm{H} & 1.62762300 & 1.09743900 & 3.87828600 \\ \mathrm{C} & 3.54966700 & 0.19837200 & 3.82017300 \\ \mathrm{H} & 3.79131700 & 0.52041800 & 4.82938200 \\ \mathrm{C} & 4.46107300 & -0.54145800 & 3.07030300 \\ \mathrm{H} & 5.42805000 & -0.81483800 & 3.48377100 \\ \mathrm{C} & 4.11990000 & -0.92811900 & 1.77952600 \\ \mathrm{H} & 4.82691700 & -1.49964100 & 1.18633600 \\ \mathrm{C} & 2.87747600 & -0.60677200 & 1.20224800 \\ \mathrm{C} & 2.67985500 & -1.10105800 & -0.19629700 \\ \mathrm{C} & 1.77989900 & -2.13706900 & -0.48818600 \\ \mathrm{C} & 1.72182600 & -2.73057300 & -1.75330000 \\ \mathrm{H} & 0.99255900 & -3.50217300 & -1.96382800 \\ \mathrm{C} & 2.57026100 & -2.26420200 & -2.75054800 \\ \mathrm{H} & 2.52298100 & -2.70637300 & -3.74157000 \\ \mathrm{C} & 3.46107500 & -1.21708000 & -2.51115500 \\ \mathrm{H} & 4.09302100 & -0.86264000 & -3.31552000 \\ \mathrm{C} & 3.52180100 & -0.63777400 & -1.23816100 \\ \mathrm{C} & 5.39892800 & 0.82037800 & -1.80765700 \\ \mathrm{H} & 5.78801400 & -0.05111600 & -2.35005300 \\ \mathrm{C} & 4.84064100 & 1.84881000 & -2.79194100 \\ \mathrm{H} & 5.62117500 & 2.16506400 & -3.49331600 \\ \mathrm{H} & 4.48564100 & 2.73284100 & -2.25137700 \\ \mathrm{H} & 4.00441600 & 1.44614000 & -3.37071400 \\ \mathrm{C} & 6.50684000 & 1.39590200 & -0.93110900 \\ \mathrm{H} & 7.33501300 & 1.75429600 & -1.55191000 \\ \mathrm{H} & 6.88980100 & 0.63667900 & -0.24244000 \\ \mathrm{H} & 6.12752000 & 2.23641700 & -0.34006100 \\ \mathrm{C} & 0.71800000 & -3.93745500 & 0.79622900 \\ \mathrm{H} & 0.29264300 & -4.38801200 & -0.10563000 \\ \mathrm{C} & -0.30056600 & -4.02690200 & 1.92077500 \\ \mathrm{H} & -0.50856600 & -5.07939000 & 2.14185300 \\ \mathrm{H} & -1.23932400 & -3.54929800 & 1.62657800 \\ & & & \end{array}$




\begin{tabular}{|c|c|c|c|}
\hline 4 & 0.08035200 & -3.54937600 & 2.82986100 \\
\hline & 2.05238400 & -4.58769500 & 1.15027000 \\
\hline $\mathrm{H}$ & 1.89745200 & -5.65280800 & 1.35513300 \\
\hline $\mathrm{H}$ & 2.48343700 & -4.12107300 & 2.04289800 \\
\hline $\mathrm{H}$ & 2.77232000 & -4.50416600 & 0.33053800 \\
\hline $\mathrm{C}$ & -0.77041300 & 0.76491500 & 2.91317800 \\
\hline $\mathrm{H}$ & -0.22324000 & 1.41494700 & 3.60754100 \\
\hline $\mathrm{C}$ & -2.14634600 & 1.41943800 & 2.67674600 \\
\hline $\mathrm{H}$ & -2.03914700 & 2.40879000 & 2.21923700 \\
\hline $\mathrm{H}$ & -2.72464400 & 0.81258300 & 1.97321000 \\
\hline $\mathrm{C}$ & -2.92842200 & 1.54367300 & 3.99604200 \\
\hline $\mathrm{H}$ & -2.40095400 & 2.23260300 & 4.67336300 \\
\hline $\mathrm{H}$ & -3.90824800 & 1.99261000 & 3.79232000 \\
\hline $\mathrm{C}$ & -3.09629900 & 0.18172800 & 4.68394300 \\
\hline $\mathrm{H}$ & -3.73046100 & -0.46182600 & 4.05725300 \\
\hline $\mathrm{H}$ & -3.61753900 & 0.29887100 & 5.64254100 \\
\hline $\mathrm{C}$ & -1.73805900 & -0.50171300 & 4.89623100 \\
\hline $\mathrm{H}$ & -1.87526000 & -1.50099200 & 5.32862900 \\
\hline $\mathrm{H}$ & -1.14942400 & 0.07537100 & 5.62532800 \\
\hline $\mathrm{C}$ & -0.94642700 & -0.61489200 & 3.58197200 \\
\hline $\mathrm{H}$ & -1.48281900 & -1.27620100 & 2.88850500 \\
\hline $\mathrm{H}$ & 0.02777700 & -1.07955500 & 3.77157600 \\
\hline $\mathrm{C}$ & 0.48972900 & 2.40770700 & 0.74839200 \\
\hline $\mathrm{H}$ & -0.49922100 & 2.66877700 & 0.34845200 \\
\hline $\mathrm{C}$ & 0.85803900 & 3.43456800 & 1.83862000 \\
\hline $\mathrm{H}$ & 1.83148500 & 3.17119600 & 2.27427800 \\
\hline $\mathrm{H}$ & 0.12750400 & 3.42101900 & 2.65563800 \\
\hline $\mathrm{C}$ & 0.93214100 & 4.85582000 & 1.24933800 \\
\hline $\mathrm{H}$ & -0.07016900 & 5.15563100 & 0.91004300 \\
\hline $\mathrm{H}$ & 1.22018400 & 5.56485300 & 2.03621000 \\
\hline $\mathrm{C}$ & 1.91189200 & 4.93558400 & 0.07043400 \\
\hline $\mathrm{H}$ & 2.93347900 & 4.75944600 & 0.43935800 \\
\hline $\mathrm{H}$ & 1.90410000 & 5.94486100 & -0.36035400 \\
\hline $\mathrm{C}$ & 1.57579300 & 3.89195400 & -1.00386400 \\
\hline $\mathrm{H}$ & 2.32596200 & 3.91717500 & -1.80483400 \\
\hline $\mathrm{H}$ & 0.61381600 & 4.13243200 & -1.47609900 \\
\hline $\mathrm{C}$ & 1.50286700 & 2.47320500 & -0.41427700 \\
\hline $\mathrm{H}$ & 2.49472400 & 2.17371300 & -0.05245500 \\
\hline $\mathrm{H}$ & 1.22392700 & 1.76212800 & -1.19750600 \\
\hline $\mathrm{O}$ & 4.37375900 & 0.37344600 & -0.89671900 \\
\hline $\mathrm{O}$ & 0.91140300 & -2.50661400 & 0.53484600 \\
\hline $\mathrm{P}$ & 0.25681100 & 0.63684000 & 1.33740300 \\
\hline $\mathrm{Pc}$ & -0.80613200 & -0.89075500 & $-0.1904520 c$ \\
\hline I & -2.18176900 & -2.82118200 & -1.57084600 \\
\hline $\mathrm{C}$ & -1.78784800 & 0.53835000 & -1.21485400 \\
\hline $\mathrm{C}$ & -1.16142100 & 0.90329000 & -2.34529900 \\
\hline $\mathrm{H}$ & -0.20965300 & 0.45943600 & -2.61928000 \\
\hline $\mathrm{C}$ & -1.64112800 & 1.88496500 & -3.37188300 \\
\hline $\mathrm{C}$ & -3.06157800 & 1.08253000 & -0.69978800 \\
\hline $\mathrm{C}$ & -4.10534700 & 0.23987000 & -0.27316700 \\
\hline $\mathrm{C}$ & -3.24986400 & 2.47653900 & -0.60535900 \\
\hline $\mathrm{C}$ & -5.29574900 & 0.77433500 & 0.21705800 \\
\hline $\mathrm{H}$ & -3.97623200 & -0.83438100 & -0.34343600 \\
\hline $\mathrm{C}$ & -4.43756400 & 3.00604400 & -0.10306200 \\
\hline $\mathrm{H}$ & -2.46705300 & 3.14532300 & $-0.9463340 c$ \\
\hline & -5.46587300 & 2.15796400 & $0.3129340 \mathrm{C}$ \\
\hline 1 & -6.09359500 & 0.10449800 & 0.5270540 \\
\hline
\end{tabular}




$$
\begin{array}{lrrr}
\mathrm{H} & -4.56020300 & 4.08440800 & -0.04220600 \\
\mathrm{H} & -6.39235100 & 2.57101200 & 0.70314800 \\
\mathrm{~F} & -1.27304300 & 3.17334400 & -3.07021100 \\
\mathrm{~F} & -2.97493100 & 1.90684800 & -3.54958900 \\
\mathrm{~F} & -1.07643200 & 1.61330500 & -4.57090200 \\
& & & \\
\mathbf{I} & & &
\end{array}
$$

$\mathrm{E}(\mathrm{B} 3 \mathrm{LYP} / \mathrm{BS} 1)=-11.47211$

$\mathrm{E}(\mathrm{PCM}$ (dichloroethane $) / \mathrm{M} 06 / \mathrm{BS} 2 / / \mathrm{B} 3 \mathrm{LYP} / \mathrm{BS} 1)=-11.5701$

$\begin{array}{ll}\text { Zero-point correction }= & 0.000000 \\ \text { Thermal correction to Energy= } & 0.001416 \\ \text { Thermal correction to Enthalpy= } & 0.002360 \\ \text { Thermal correction to Gibbs Free Energy= } & -0.016848\end{array}$

Charge $=-1$ Multiplicity $=1$

$\begin{array}{llll}\text { I } & 0.00000000 & 0.00000000 & 0.00000000\end{array}$

PhO $^{-}$

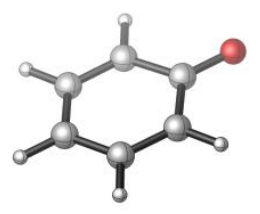

$\mathrm{E}(\mathrm{B} 3 \mathrm{LYP} / \mathrm{BS} 1)=-306.884159$

$\mathrm{E}(\mathrm{PCM}($ dichloroethane $) / \mathrm{M} 06 / \mathrm{BS} 2 / / \mathrm{B} 3 \mathrm{LYP} / \mathrm{BS} 1)=-306.850558$

Zero-point correction= 0.090715

Thermal correction to Energy= 0.095960

Thermal correction to Enthalpy= 0.096904

Thermal correction to Gibbs Free Energy= $\quad 0.061884$

$\begin{array}{lrrr}\text { Charge }= & -1 \text { Multiplicity }=1 \\ \text { C } & -1.10206200 & 1.20101300 & 0.00003500 \\ \text { C } & -1.83108700 & -0.00000400 & -0.00006900 \\ \text { C } & -1.10206500 & -1.20100300 & 0.00004200 \\ \text { C } & 0.28636200 & -1.21262400 & 0.00001900 \\ \text { C } & 1.08472800 & -0.00001300 & -0.00020600 \\ \text { C } & 0.28635300 & 1.21262200 & 0.00002400 \\ \text { H } & -1.64167700 & 2.15244500 & 0.00006500 \\ \text { H } & -2.92013100 & 0.00000700 & 0.00006000 \\ \text { H } & -1.64166200 & -2.15244500 & 0.00006100 \\ \text { H } & 0.83173500 & -2.15716600 & 0.00011900 \\ \text { H } & 0.83177000 & 2.15713900 & 0.00012200 \\ \text { O } & 2.35082400 & 0.00000900 & 0.00006300\end{array}$

CO

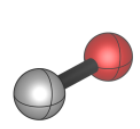

$\mathrm{E}(\mathrm{B} 3 \mathrm{LYP} / \mathrm{BS} 1)=-113.309453$

$\mathrm{E}(\mathrm{PCM}$ (dichloroethane)/M06/BS2// B3LYP/BS1 $)=-113.286339$

Zero-point correction $=$

0.005033 
Charge $=0$ Multiplicity $=1$

$\begin{array}{lllr}\text { C } & 0.00000000 & 0.00000000 & -0.65025200 \\ \mathrm{O} & 0.00000000 & 0.00000000 & 0.48768900\end{array}$

\section{IM6}

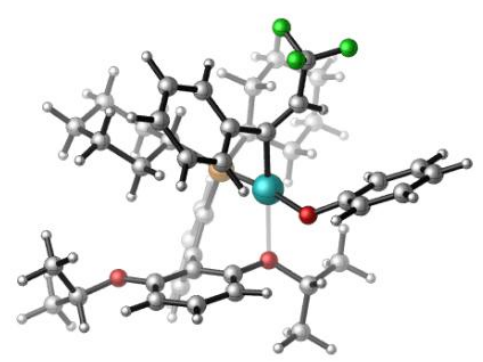

$\mathrm{E}(\mathrm{B} 3 \mathrm{LYP} / \mathrm{BS} 1)=-2740.605819$

$\mathrm{E}(\mathrm{PCM}$ (dichloroethane $) / \mathrm{M} 06 / \mathrm{BS} 2 / / \mathrm{B} 3 \mathrm{LYP} / \mathrm{BS} 1)=-2740.870649$

Zero-point correction=

0.899908

Thermal correction to Energy=

0.953135

Thermal correction to Enthalpy=

0.954080

Thermal correction to Gibbs Free Energy=

0.808862

$\begin{array}{lrrr}\text { Charge }= & \text { Multiplicity }=1 & \\ \mathrm{C} & 2.15885200 & 0.10951000 & 1.90823200 \\ \mathrm{C} & 2.63236500 & 0.60556500 & 3.13882000 \\ \mathrm{H} & 2.12602900 & 1.43080000 & 3.62202400 \\ \mathrm{C} & 3.74810400 & 0.07505200 & 3.78233600 \\ \mathrm{H} & 4.07038700 & 0.49196800 & 4.73258500 \\ \mathrm{C} & 4.43539300 & -0.98547800 & 3.19910800 \\ \mathrm{H} & 5.30459000 & -1.41989000 & 3.68546000 \\ \mathrm{C} & 3.99236200 & -1.48866300 & 1.98113500 \\ \mathrm{H} & 4.52140900 & -2.31639400 & 1.51937300 \\ \mathrm{C} & 2.86704300 & -0.97002300 & 1.31607100 \\ \mathrm{C} & 2.51841900 & -1.65621800 & 0.03125600 \\ \mathrm{C} & 1.36579900 & -2.44820600 & -0.07018900 \\ \mathrm{C} & 1.07382400 & -3.19926200 & -1.21272800 \\ \mathrm{H} & 0.13810000 & -3.73844600 & -1.28408200 \\ \mathrm{C} & 1.97600900 & -3.17409600 & -2.26987500 \\ \mathrm{H} & 1.76264200 & -3.74581400 & -3.16880100 \\ \mathrm{C} & 3.14388300 & -2.41222300 & -2.20795900 \\ \mathrm{H} & 3.82053800 & -2.40646000 & -3.05302300 \\ \mathrm{C} & 3.41744300 & -1.65488200 & -1.06250800 \\ \mathrm{C} & 5.62507600 & -0.95420600 & -1.84381500 \\ \mathrm{H} & 5.71541400 & -1.98945700 & -2.19730100 \\ \mathrm{C} & 5.38373100 & -0.01240200 & -3.02403300 \\ \mathrm{H} & 6.20414400 & -0.09237000 & -3.74651300 \\ \mathrm{H} & 5.33393700 & 1.02457400 & -2.67456200 \\ \mathrm{H} & 4.44790500 & -0.24139500 & -3.54203900 \\ \mathrm{C} & 6.87702400 & -0.58618900 & -1.05366300\end{array}$




\begin{tabular}{|c|c|c|c|}
\hline $\mathrm{H}$ & 7.75978000 & -0.62152300 & -1.70115000 \\
\hline $\mathrm{H}$ & 7.02675800 & -1.27984300 & -0.22075700 \\
\hline $\mathrm{H}$ & 6.78588400 & 0.42626700 & -0.64590400 \\
\hline $\mathrm{C}$ & -0.15405300 & -3.63014800 & 1.46859400 \\
\hline $\mathrm{H}$ & -0.85446400 & -3.92364300 & 0.68271000 \\
\hline $\mathrm{C}$ & -0.92909000 & -3.23875500 & 2.71754300 \\
\hline $\mathrm{H}$ & -1.50289500 & -4.09833100 & 3.07966300 \\
\hline $\mathrm{H}$ & -1.63013300 & -2.43045500 & 2.49625500 \\
\hline $\mathrm{H}$ & -0.24734500 & -2.91240500 & 3.51090300 \\
\hline $\mathrm{C}$ & 0.87399100 & -4.72349200 & 1.74451800 \\
\hline $\mathrm{H}$ & 0.35862700 & -5.62100200 & 2.10399100 \\
\hline $\mathrm{H}$ & 1.58167900 & -4.39912800 & 2.51586200 \\
\hline $\mathrm{H}$ & 1.43641100 & -4.99590800 & 0.84712500 \\
\hline $\mathrm{C}$ & -0.16635700 & 1.84280200 & 2.56471400 \\
\hline $\mathrm{H}$ & 0.61789100 & 2.37336800 & 3.11850300 \\
\hline $\mathrm{C}$ & -1.16324400 & 2.92309400 & 2.08937900 \\
\hline $\mathrm{H}$ & -0.65508000 & 3.66714200 & 1.46593600 \\
\hline $\mathrm{H}$ & -1.93806200 & 2.47139600 & 1.46377400 \\
\hline $\mathrm{C}$ & -1.81760500 & 3.63598100 & 3.28683700 \\
\hline $\mathrm{H}$ & -1.05149900 & 4.20297600 & 3.83733500 \\
\hline $\mathrm{H}$ & -2.54374500 & 4.37035800 & 2.91694900 \\
\hline $\mathrm{C}$ & -2.49687900 & 2.64557900 & 4.24237400 \\
\hline $\mathrm{H}$ & -3.33878200 & 2.16236100 & 3.72543800 \\
\hline $\mathrm{H}$ & -2.92196600 & 3.17756700 & 5.10274900 \\
\hline $\mathrm{C}$ & -1.50876200 & 1.57063000 & 4.71450100 \\
\hline $\mathrm{H}$ & -2.01531300 & 0.83749300 & 5.35488000 \\
\hline $\mathrm{H}$ & -0.72890600 & 2.03948500 & 5.33349600 \\
\hline $\mathrm{C}$ & -0.85033600 & 0.84844700 & 3.52736600 \\
\hline $\mathrm{H}$ & -1.61311100 & 0.28858500 & 2.96901900 \\
\hline $\mathrm{H}$ & -0.12709600 & 0.10929800 & 3.89197500 \\
\hline $\mathrm{C}$ & 1.35921700 & 2.28712600 & 0.05067900 \\
\hline $\mathrm{H}$ & 0.46253500 & 2.80669500 & -0.31567600 \\
\hline $\mathrm{C}$ & 2.24894900 & 3.30031200 & 0.80139800 \\
\hline $\mathrm{H}$ & 3.12505800 & 2.77730100 & 1.20715700 \\
\hline $\mathrm{H}$ & 1.71685700 & 3.73517800 & 1.65546000 \\
\hline $\mathrm{C}$ & 2.71930400 & 4.42820500 & -0.13458000 \\
\hline $\mathrm{H}$ & 1.84744400 & 5.01551800 & -0.45817800 \\
\hline $\mathrm{H}$ & 3.37165400 & 5.11608600 & 0.41875700 \\
\hline $\mathrm{C}$ & 3.44352900 & 3.87557800 & -1.37002400 \\
\hline $\mathrm{H}$ & 4.37942700 & 3.39127100 & -1.05251900 \\
\hline $\mathrm{H}$ & 3.72755400 & 4.69624400 & -2.04129000 \\
\hline $\mathrm{C}$ & 2.56760200 & 2.85519500 & -2.10982300 \\
\hline $\mathrm{H}$ & 3.11414900 & 2.42640100 & -2.95934900 \\
\hline $\mathrm{H}$ & 1.68810400 & 3.36028800 & -2.53135200 \\
\hline $\mathrm{C}$ & 2.10118700 & 1.72298300 & -1.17853400 \\
\hline $\mathrm{H}$ & 2.97645900 & 1.15242100 & -0.84551900 \\
\hline $\mathrm{H}$ & 1.45149400 & 1.03388300 & -1.72761300 \\
\hline $\mathrm{O}$ & 4.53512500 & -0.88601500 & -0.90156000 \\
\hline $\mathrm{O}$ & 0.51168500 & -2.39930800 & 1.02341400 \\
\hline $\mathrm{P}$ & 0.65381300 & 0.92198100 & 1.13645700 \\
\hline $\mathrm{Pc}$ & -0.83511900 & -0.63656800 & 0.14503400 \\
\hline $\mathrm{C}$ & -1.97282000 & 0.69383800 & -0.83198000 \\
\hline $\mathrm{C}$ & -3.12248400 & 1.08904700 & -0.26775700 \\
\hline $\mathrm{H}$ & -3.39123500 & 0.77404200 & 0.73490000 \\
\hline C & -4.17136300 & 1.96647600 & -0.88273100 \\
\hline$c$ & -1.49275600 & 1.04501300 & -2.18544300 \\
\hline & -1.12567500 & 0.02862400 & -3.0890230 \\
\hline
\end{tabular}




$\begin{array}{llrr}\text { C } & -1.38589600 & 2.38614300 & -2.59932900 \\ \text { C } & -0.67737200 & 0.34927700 & -4.36885600 \\ \text { H } & -1.21868000 & -1.00532900 & -2.77090100 \\ \text { C } & -0.92894200 & 2.70033600 & -3.87978400 \\ \text { H } & -1.68686500 & 3.17898200 & -1.92198800 \\ \text { C } & -0.57141700 & 1.68472000 & -4.76877000 \\ \text { H } & -0.41178200 & -0.44746500 & -5.05910000 \\ \text { H } & -0.86074800 & 3.74159100 & -4.18496700 \\ \text { H } & -0.21794600 & 1.93128700 & -5.76658100 \\ \text { F } & -4.35856200 & 1.77655400 & -2.20259300 \\ \text { F } & -3.88022500 & 3.29617500 & -0.72114600 \\ \text { F } & -5.36205200 & 1.77028900 & -0.27216500 \\ \text { O } & -1.95466200 & -2.17146000 & -0.68986900 \\ \text { C } & -3.26795200 & -2.37706400 & -0.59708700 \\ \text { C } & -4.02187700 & -2.09659500 & 0.56601600 \\ \text { C } & -3.95254200 & -2.95759000 & -1.69089900 \\ \text { C } & -5.38321900 & -2.39132600 & 0.63104400 \\ \text { H } & -3.51712300 & -1.64066600 & 1.41460900 \\ \text { C } & -5.30994700 & -3.25701200 & -1.61301500 \\ \text { H } & -3.38510100 & -3.17090300 & -2.59319900 \\ \text { C } & -6.04067800 & -2.97616800 & -0.45375600 \\ \text { H } & -5.93553400 & -2.16056200 & 1.53994200 \\ \text { H } & -5.80614300 & -3.70738200 & -2.47035100 \\ \text { H } & -7.10169000 & -3.20375200 & -0.39983500\end{array}$

\section{IM7}

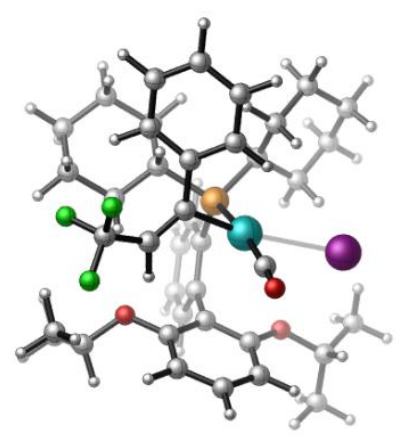

$\mathrm{E}(\mathrm{B} 3 \mathrm{LYP} / \mathrm{BS} 1)=-2558.491782$

$\mathrm{E}(\mathrm{PCM}$ (dichloroethane)/M06/BS2// B3LYP/BS1 $)=-2558.491782$

Zero-point correction=

Thermal correction to Energy=

Thermal correction to Enthalpy=

Thermal correction to Gibbs Free Energy=
0.813842

0.865736

0.866681

0.723376

$\begin{array}{lrrr}\text { Charge }=0 \text { Multiplicity }=1 & \\ \mathrm{C} & 1.00386000 & 0.89547800 & 2.10826600 \\ \mathrm{C} & 1.18645900 & 0.80805300 & 3.50445100 \\ \mathrm{H} & 0.84987500 & -0.07495900 & 4.03591000 \\ \mathrm{C} & 1.79401500 & 1.82212800 & 4.23786100 \\ \mathrm{H} & 1.91635700 & 1.71446000 & 5.31211500 \\ \mathrm{C} & 2.24153800 & 2.96691000 & 3.57984200 \\ \mathrm{H} & 2.71859800 & 3.77201200 & 4.13223100 \\ \mathrm{C} & 2.08305700 & 3.06416700 & 2.20219900 \\ \mathrm{H} & 2.44973500 & 3.94259500 & 1.67891500\end{array}$




\begin{tabular}{|c|c|c|c|}
\hline $\mathrm{C}$ & 1.47293800 & 2.04968800 & 1.44118900 \\
\hline $\mathrm{C}$ & 1.43928100 & 2.29265100 & -0.03736900 \\
\hline $\mathrm{C}$ & 2.58328900 & 1.97249800 & -0.80501200 \\
\hline $\mathrm{C}$ & 2.63785300 & 2.30005600 & -2.16800500 \\
\hline $\mathrm{H}$ & 3.49484700 & 2.02684700 & -2.76990900 \\
\hline $\mathrm{C}$ & 1.57575900 & 2.98993300 & -2.74835200 \\
\hline $\mathrm{H}$ & 1.62087000 & 3.24170400 & -3.80460000 \\
\hline $\mathrm{C}$ & 0.46341700 & 3.37210400 & -2.00117600 \\
\hline $\mathrm{H}$ & -0.34733000 & 3.92264300 & -2.46462600 \\
\hline $\mathrm{C}$ & 0.40790400 & 3.03547400 & -0.64113500 \\
\hline $\mathrm{C}$ & -1.23399100 & 4.71481400 & 0.01326200 \\
\hline $\mathrm{H}$ & -0.55799200 & 5.32256400 & -0.60071200 \\
\hline $\mathrm{C}$ & -2.59813400 & 4.60804000 & -0.66388800 \\
\hline $\mathrm{H}$ & -3.06615400 & 5.59741300 & -0.72598500 \\
\hline $\mathrm{H}$ & -3.26239200 & 3.95039400 & -0.09302100 \\
\hline $\mathrm{H}$ & -2.52365600 & 4.20449800 & -1.67706500 \\
\hline $\mathrm{C}$ & -1.32319800 & 5.31372200 & 1.41481700 \\
\hline $\mathrm{H}$ & -1.73950600 & 6.32655800 & 1.37246500 \\
\hline $\mathrm{H}$ & -0.33361700 & 5.36009300 & 1.87934800 \\
\hline $\mathrm{H}$ & -1.97148700 & 4.70114400 & 2.05099100 \\
\hline $\mathrm{C}$ & 4.88911500 & 1.17411100 & -0.70340500 \\
\hline $\mathrm{H}$ & 4.75698100 & 0.72075900 & -1.69247400 \\
\hline $\mathrm{C}$ & 5.59395300 & 0.17437800 & 0.20522300 \\
\hline $\mathrm{H}$ & 6.59634800 & -0.04249500 & -0.17993900 \\
\hline $\mathrm{H}$ & 5.02666100 & -0.75863900 & 0.25160900 \\
\hline $\mathrm{H}$ & 5.69078200 & 0.58153100 & 1.21799800 \\
\hline $\mathrm{C}$ & 5.64594600 & 2.49989200 & -0.80680400 \\
\hline $\mathrm{H}$ & 6.63185900 & 2.33611200 & -1.25650800 \\
\hline $\mathrm{H}$ & 5.78997400 & 2.92933500 & 0.19107500 \\
\hline $\mathrm{H}$ & 5.10984800 & 3.23008600 & -1.42027000 \\
\hline $\mathrm{C}$ & 0.77827800 & -2.05940600 & 2.13937100 \\
\hline $\mathrm{H}$ & 0.44010700 & -2.02917400 & 3.18322500 \\
\hline $\mathrm{C}$ & 0.17268000 & -3.32186200 & 1.48607200 \\
\hline $\mathrm{H}$ & -0.92410900 & -3.28028100 & 1.49426500 \\
\hline $\mathrm{H}$ & 0.47805900 & -3.35567100 & 0.43287700 \\
\hline $\mathrm{C}$ & 0.65337500 & -4.59797300 & 2.19839300 \\
\hline $\mathrm{H}$ & 0.25800400 & -4.61429600 & 3.22561600 \\
\hline $\mathrm{H}$ & 0.23878100 & -5.47796700 & 1.69055400 \\
\hline $\mathrm{C}$ & 2.18619500 & -4.67497900 & 2.24031500 \\
\hline $\mathrm{H}$ & 2.56982200 & -4.78006000 & 1.21603300 \\
\hline $\mathrm{H}$ & 2.50603900 & -5.56819300 & 2.79230700 \\
\hline C & 2.78658400 & -3.41230500 & 2.87564500 \\
\hline $\mathrm{H}$ & 3.88265700 & -3.46004200 & 2.85444200 \\
\hline $\mathrm{H}$ & 2.49653000 & -3.36621400 & 3.93696000 \\
\hline $\mathrm{C}$ & 2.31887200 & -2.13288700 & 2.15870200 \\
\hline $\mathrm{H}$ & 2.69972100 & -2.12874900 & 1.13320500 \\
\hline $\mathrm{H}$ & 2.73738900 & -1.25287400 & 2.65792400 \\
\hline $\mathrm{C}$ & -1.67490800 & -0.34866900 & 2.08572700 \\
\hline $\mathrm{H}$ & -2.30314000 & -0.87809400 & 1.36199900 \\
\hline $\mathrm{C}$ & -1.91503800 & -1.01536900 & 3.45801300 \\
\hline $\mathrm{H}$ & -1.29049000 & -0.54033900 & 4.22642700 \\
\hline $\mathrm{H}$ & -1.64794700 & -2.07686700 & 3.43422400 \\
\hline $\mathrm{C}$ & -3.39330800 & -0.88766800 & 3.87398500 \\
\hline $\mathrm{H}$ & -4.01380900 & -1.45008500 & 3.16124100 \\
\hline $\mathrm{H}$ & -3.53695500 & -1.36059200 & 4.85411300 \\
\hline C & -3.85469800 & 0.57478400 & 3.90948200 \\
\hline $\mathrm{H}$ & -3.30697800 & 1.10702000 & 4.70147900 \\
\hline
\end{tabular}




$\begin{array}{lccc}\mathrm{H} & -4.91875500 & 0.63160300 & 4.17243100 \\ \mathrm{C} & -3.59582200 & 1.26324300 & 2.56341600 \\ \mathrm{H} & -3.85711100 & 2.32797700 & 2.62415400 \\ \mathrm{H} & -4.25059200 & 0.83237400 & 1.79462800 \\ \mathrm{C} & -2.12741900 & 1.12723000 & 2.12525700 \\ \mathrm{H} & -1.49279400 & 1.67664800 & 2.83182600 \\ \mathrm{H} & -1.98309700 & 1.60118100 & 1.15210900 \\ \mathrm{O} & -0.64053600 & 3.39894900 & 0.16408700 \\ \mathrm{O} & 3.58708000 & 1.37753300 & -0.11136900 \\ \mathrm{P} & 0.06822200 & -0.51261600 & 1.32835600 \\ \mathrm{Pd} & 0.01985000 & -0.73258900 & -1.11848500 \\ \mathrm{C} & -2.03492400 & -0.44462200 & -1.24663600 \\ \mathrm{C} & -2.49051000 & 0.78433100 & -1.53201900 \\ \mathrm{H} & -1.81156300 & 1.62982600 & -1.53702300 \\ \mathrm{C} & -3.89469300 & 1.18946600 & -1.86930500 \\ \mathrm{C} & -2.81839000 & -1.69463600 & -1.13428800 \\ \mathrm{C} & -2.35619700 & -2.90026600 & -1.69902600 \\ \mathrm{C} & -4.03616700 & -1.72837600 & -0.42216600 \\ \mathrm{C} & -3.08932800 & -4.07967000 & -1.57963600 \\ \mathrm{H} & -1.41326600 & -2.91410200 & -2.23607200 \\ \mathrm{C} & -4.76148500 & -2.91120100 & -0.29427200 \\ \mathrm{H} & -4.42010400 & -0.81678800 & 0.02153900 \\ \mathrm{C} & -4.29351200 & -4.09281000 & -0.87301800 \\ \mathrm{H} & -2.71286400 & -4.99136700 & -2.03542000 \\ \mathrm{H} & -5.69772900 & -2.90710100 & 0.25767200 \\ \mathrm{H} & -4.85987700 & -5.01453500 & -0.77202700 \\ \mathrm{~F} & -4.61899300 & 0.24321000 & -2.49635200 \\ \mathrm{~F} & -3.87748400 & 2.27631600 & -2.68229000 \\ \mathrm{~F} & -4.60851500 & 1.56036200 & -0.76237100 \\ \mathrm{I} & 2.55896900 & -1.87710100 & -1.75206900 \\ \mathrm{C} & -0.01024100 & -0.68504500 & -3.08030000 \\ \mathrm{O} & -0.04752400 & -0.57955900 & -4.21628900\end{array}$

\section{IM8}

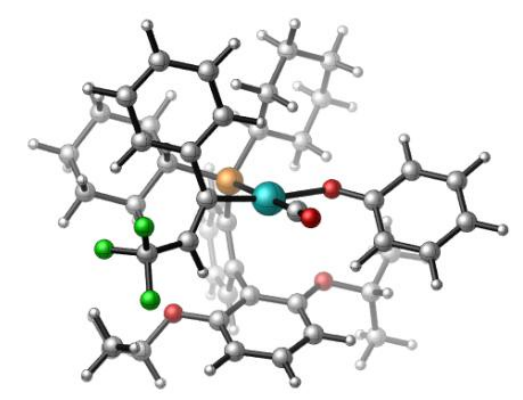

$\mathrm{E}(\mathrm{B} 3 \mathrm{LYP} / \mathrm{BS} 1)=-2853.923801$

$\mathrm{E}(\mathrm{PCM}($ dichloroethane $) / \mathrm{M} 06 / \mathrm{BS} 2 / / \mathrm{B} 3 \mathrm{LYP} / \mathrm{BS} 1)=-2854.166604$

Zero-point correction $=$

Thermal correction to Energy=

Thermal correction to Enthalpy=

Thermal correction to Gibbs Free Energy=
0.907717

0.963748

0.964693

0.813259 


\begin{tabular}{|c|c|c|c|}
\hline \multicolumn{4}{|c|}{ Charge $=0$ Multiplicity $=1$} \\
\hline $\mathrm{C}$ & 0.31559400 & 1.29566900 & 2.18281100 \\
\hline $\mathrm{C}$ & 0.27967600 & 1.37616900 & 3.59119900 \\
\hline $\mathrm{H}$ & 0.06099200 & 0.48952300 & 4.17477100 \\
\hline $\mathrm{C}$ & 0.52633900 & 2.56322800 & 4.27324200 \\
\hline $\mathrm{H}$ & 0.48984900 & 2.58089900 & 5.35904600 \\
\hline $\mathrm{C}$ & 0.82269300 & 3.71757600 & 3.55000200 \\
\hline $\mathrm{H}$ & 1.01934500 & 4.65624900 & 4.06106400 \\
\hline $\mathrm{C}$ & 0.87772600 & 3.65333700 & 2.16264500 \\
\hline $\mathrm{H}$ & 1.12903000 & 4.54297500 & 1.59229500 \\
\hline $\mathrm{C}$ & 0.63275100 & 2.46239800 & 1.45337200 \\
\hline $\mathrm{C}$ & 0.78821300 & 2.56842700 & -0.03301100 \\
\hline $\mathrm{C}$ & 2.07410100 & 2.40877100 & -0.59819600 \\
\hline $\mathrm{C}$ & 2.28939400 & 2.68612300 & -1.95772500 \\
\hline $\mathrm{H}$ & 3.26941900 & 2.56151800 & -2.39971500 \\
\hline $\mathrm{C}$ & 1.22896400 & 3.13906100 & -2.73957000 \\
\hline $\mathrm{H}$ & 1.39820000 & 3.34925900 & -3.79241800 \\
\hline $\mathrm{C}$ & -0.03978700 & 3.34002500 & -2.19952200 \\
\hline $\mathrm{H}$ & -0.84796600 & 3.70389500 & -2.82279500 \\
\hline $\mathrm{C}$ & -0.25002800 & 3.07401200 & -0.83811500 \\
\hline $\mathrm{C}$ & -2.29232600 & 4.38936500 & -0.63428700 \\
\hline $\mathrm{H}$ & -1.67179400 & 5.09675200 & -1.19865400 \\
\hline $\mathrm{C}$ & -3.43885200 & 3.88671900 & -1.50834600 \\
\hline $\mathrm{H}$ & -4.09184800 & 4.72192800 & -1.78765000 \\
\hline $\mathrm{H}$ & -4.03863400 & 3.14756200 & -0.96773300 \\
\hline $\mathrm{H}$ & -3.08179800 & 3.41300600 & -2.42617600 \\
\hline $\mathrm{C}$ & -2.78641600 & 5.05970000 & 0.64522900 \\
\hline $\mathrm{H}$ & -3.41300900 & 5.92614400 & 0.40569800 \\
\hline $\mathrm{H}$ & -1.94311500 & 5.39414800 & 1.25699600 \\
\hline $\mathrm{H}$ & -3.38217400 & 4.35697900 & 1.23810500 \\
\hline $\mathrm{C}$ & 4.43806000 & 2.05213700 & -0.09022600 \\
\hline $\mathrm{H}$ & 4.55951900 & 1.57209900 & -1.06722300 \\
\hline $\mathrm{C}$ & 5.14736900 & 1.20628400 & 0.95979200 \\
\hline $\mathrm{H}$ & 6.22290600 & 1.17849800 & 0.75418300 \\
\hline $\mathrm{H}$ & 4.76408600 & 0.18335300 & 0.94457400 \\
\hline $\mathrm{H}$ & 4.99330100 & 1.62934800 & 1.95890600 \\
\hline $\mathrm{C}$ & 4.94884000 & 3.49363900 & -0.12326800 \\
\hline $\mathrm{H}$ & 6.00951800 & 3.51123600 & -0.39832000 \\
\hline $\mathrm{H}$ & 4.84122000 & 3.95370200 & 0.86553800 \\
\hline $\mathrm{H}$ & 4.40012600 & 4.10375800 & -0.84734900 \\
\hline $\mathrm{C}$ & 0.73442200 & -1.60262300 & 2.56363700 \\
\hline $\mathrm{H}$ & 0.22706400 & -1.55495300 & 3.53634300 \\
\hline $\mathrm{C}$ & 0.55256300 & -3.03256900 & 2.00797500 \\
\hline $\mathrm{H}$ & -0.50807400 & -3.26100200 & 1.84106800 \\
\hline $\mathrm{H}$ & 1.05178500 & -3.08773300 & 1.03319100 \\
\hline $\mathrm{C}$ & 1.16942200 & -4.07378700 & 2.95787500 \\
\hline $\mathrm{H}$ & 0.61724200 & -4.07361000 & 3.91007200 \\
\hline $\mathrm{H}$ & 1.05111500 & -5.07743300 & 2.52959500 \\
\hline $\mathrm{C}$ & 2.65159200 & -3.77735000 & 3.22733300 \\
\hline $\mathrm{H}$ & 3.21784500 & -3.90393900 & 2.29353800 \\
\hline $\mathrm{H}$ & 3.05999700 & -4.50143900 & 3.94421300 \\
\hline $\mathrm{C}$ & 2.84635600 & -2.34452900 & 3.74492100 \\
\hline $\mathrm{H}$ & 3.91473400 & -2.13077000 & 3.87725100 \\
\hline $\mathrm{H}$ & 2.38581200 & -2.25512700 & 4.74133000 \\
\hline $\mathrm{C}$ & 2.23000100 & -1.29957300 & 2.79777100 \\
\hline $\mathrm{H}$ & 2.74030300 & -1.30732000 & 1.83090400 \\
\hline $\mathrm{H}$ & 2.35626500 & -0.29859700 & 3.22274300 \\
\hline
\end{tabular}




\begin{tabular}{|c|c|c|c|}
\hline & -1.99049100 & -0.52725500 & 1.90636800 \\
\hline $\mathrm{H}$ & -2.34195800 & -1.29332500 & 1.20673500 \\
\hline $\mathrm{C}$ & -2.32322900 & -1.02791400 & 3.32893100 \\
\hline $\mathrm{H}$ & -1.95970200 & -0.31227000 & 4.07800000 \\
\hline $\mathrm{H}$ & -1.83407100 & -1.98644100 & 3.53199900 \\
\hline $\mathrm{C}$ & -3.84368100 & -1.19840800 & 3.50901500 \\
\hline $\mathrm{H}$ & -4.19873300 & -1.98729000 & 2.83019500 \\
\hline $\mathrm{H}$ & -4.05197900 & -1.54546900 & 4.52938700 \\
\hline $\mathrm{C}$ & -4.60128400 & 0.10323000 & 3.21760500 \\
\hline $\mathrm{H}$ & -4.31884800 & 0.86166300 & 3.96295200 \\
\hline $\mathrm{H}$ & -5.68238400 & -0.05346200 & 3.32397500 \\
\hline $\mathrm{C}$ & -4.26953000 & 0.62147100 & 1.81252400 \\
\hline $\mathrm{H}$ & -4.76353400 & 1.58538100 & 1.63318800 \\
\hline $\mathrm{H}$ & -4.67185100 & -0.07555000 & 1.06545300 \\
\hline $\mathrm{C}$ & -2.75363000 & 0.78166600 & 1.60437100 \\
\hline $\mathrm{H}$ & -2.38428900 & 1.57374400 & 2.26740400 \\
\hline $\mathrm{H}$ & -2.55211900 & 1.11706000 & 0.58422700 \\
\hline $\mathrm{O}$ & -1.44855400 & 3.28861900 & -0.21411500 \\
\hline $\mathrm{O}$ & 3.03849500 & 2.02247400 & 0.27353300 \\
\hline $\mathrm{P}$ & -0.14905400 & -0.35484700 & 1.45963900 \\
\hline $\mathrm{Pc}$ & 0.33246000 & -0.96825300 & -0.88197800 \\
\hline $\mathrm{C}$ & -1.67036700 & -1.01477000 & -1.43114800 \\
\hline $\mathrm{C}$ & -2.13733700 & 0.07246400 & -2.07048300 \\
\hline $\mathrm{H}$ & -1.51600100 & 0.95960500 & -2.15097200 \\
\hline $\mathrm{C}$ & -3.45970400 & 0.25696100 & -2.75302900 \\
\hline $\mathrm{C}$ & -2.38810000 & -2.29070600 & -1.19583900 \\
\hline $\mathrm{C}$ & -1.71033900 & -3.52501100 & -1.25863700 \\
\hline $\mathrm{C}$ & -3.75871300 & -2.32004900 & -0.86015700 \\
\hline $\mathrm{C}$ & -2.37281300 & -4.73046800 & -1.03120000 \\
\hline $\mathrm{H}$ & -0.64914300 & -3.54174600 & -1.48469400 \\
\hline $\mathrm{C}$ & -4.41723900 & -3.52429100 & -0.62010200 \\
\hline $\mathrm{H}$ & -4.31147200 & -1.39170900 & -0.78880200 \\
\hline $\mathrm{C}$ & -3.73055700 & -4.73709600 & -0.70821500 \\
\hline $\mathrm{H}$ & -1.82226900 & -5.66484000 & -1.10010400 \\
\hline $\mathrm{H}$ & -5.47345100 & -3.51270200 & -0.36404700 \\
\hline $\mathrm{H}$ & -4.24612500 & -5.67512600 & -0.52142900 \\
\hline $\mathrm{F}$ & -3.92137700 & -0.84062900 & -3.38540900 \\
\hline $\mathrm{F}$ & -3.36096100 & 1.23747500 & -3.68717700 \\
\hline $\mathrm{F}$ & -4.45024000 & 0.65619800 & -1.89606200 \\
\hline $\mathrm{C}$ & 0.58156300 & -1.51138300 & -2.75792800 \\
\hline $\mathrm{O}$ & 0.61933100 & -1.83882100 & -3.84927800 \\
\hline $\mathrm{O}$ & 2.34629200 & -1.23757200 & -0.33492100 \\
\hline $\mathrm{C}$ & 3.36554500 & -1.39081000 & -1.17254500 \\
\hline $\mathrm{C}$ & 3.50958600 & -0.67763100 & -2.38703700 \\
\hline $\mathrm{C}$ & 4.40282200 & -2.29730200 & -0.83195900 \\
\hline $\mathrm{C}$ & 4.61686800 & -0.87517800 & -3.21597500 \\
\hline $\mathrm{H}$ & 2.75394700 & 0.05672000 & -2.65165200 \\
\hline $\mathrm{C}$ & 5.50447500 & -2.47965200 & -1.66251500 \\
\hline $\mathrm{H}$ & 4.30932600 & -2.85563000 & 0.09593800 \\
\hline $\mathrm{C}$ & 5.62501700 & -1.77449800 & -2.86585800 \\
\hline $\mathrm{H}$ & 4.69037500 & -0.31215600 & -4.14470300 \\
\hline $\mathrm{H}$ & 6.27699400 & -3.18854000 & -1.37096600 \\
\hline $\mathrm{H}$ & 6.48420300 & -1.92495700 & -3.5133170 \\
\hline
\end{tabular}


TS7

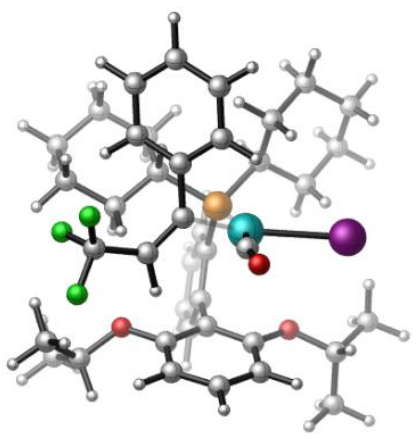

$\mathrm{E}(\mathrm{B} 3 \mathrm{LYP} / \mathrm{BS} 1)=-2558.471324$

$\mathrm{E}(\mathrm{PCM}$ (dichloroethane)/M06/BS2// B3LYP/BS1 $)=-2558.851778$

Zero-point correction=

0.813212

Thermal correction to Energy=

0.864467

Thermal correction to Enthalpy=

0.865411

Thermal correction to Gibbs Free Energy=

0.723246

Charge $=0$ Multiplicity $=1$

$\begin{array}{lrrr}\mathrm{C} & 1.05227200 & 1.51505900 & 1.91430000 \\ \mathrm{C} & 1.44823300 & 1.73276600 & 3.24961000 \\ \mathrm{H} & 1.45656800 & 0.90535400 & 3.94972500 \\ \mathrm{C} & 1.84656500 & 2.98486000 & 3.70978700 \\ \mathrm{H} & 2.14659800 & 3.10991000 & 4.74669400 \\ \mathrm{C} & 1.85989400 & 4.06566700 & 2.82958200 \\ \mathrm{H} & 2.16829400 & 5.05121600 & 3.16821800 \\ \mathrm{C} & 1.48768700 & 3.86787200 & 1.50416300 \\ \mathrm{H} & 1.51744200 & 4.69956400 & 0.80591200 \\ \mathrm{C} & 1.08612000 & 2.61027900 & 1.02035300 \\ \mathrm{C} & 0.77490500 & 2.54502900 & -0.44374500 \\ \mathrm{C} & 1.80752700 & 2.22100800 & -1.35320900 \\ \mathrm{C} & 1.57581500 & 2.26622400 & -2.73753700 \\ \mathrm{H} & 2.35013800 & 1.98243300 & -3.43850100 \\ \mathrm{C} & 0.33588500 & 2.69085300 & -3.20746200 \\ \mathrm{H} & 0.16032100 & 2.73001500 & -4.27924800 \\ \mathrm{C} & -0.68132500 & 3.07597600 & -2.33408200 \\ \mathrm{H} & -1.63082800 & 3.42565400 & -2.72275200 \\ \mathrm{C} & -0.45347900 & 3.01186100 & -0.95075700 \\ \mathrm{C} & -2.30556300 & 4.46737100 & -0.31951400 \\ \mathrm{H} & -1.89166400 & 5.05302400 & -1.15002700 \\ \mathrm{C} & -3.67564200 & 3.91200400 & -0.70277300 \\ \mathrm{H} & -4.38162800 & 4.73354300 & -0.87126800 \\ \mathrm{H} & -4.06909500 & 3.28018000 & 0.10047600 \\ \mathrm{H} & -3.63764400 & 3.30789100 & -1.61266400 \\ \mathrm{C} & -2.36235300 & 5.33756700 & 0.93362100 \\ \mathrm{H} & -3.02701400 & 6.19415700 & 0.77518200 \\ \mathrm{H} & -1.36612800 & 5.70920800 & 1.19147100 \\ \mathrm{H} & -2.74175900 & 4.75866700 & 1.78278800 \\ \mathrm{C} & 4.21076000 & 1.84297100 & -1.56738900 \\ \mathrm{H} & 4.03251500 & 1.18533700 & -2.42582800 \\ \mathrm{C} & 5.24557000 & 1.19349400 & -0.65732700 \\ \mathrm{H} & 6.20049600 & 1.09611700 & -1.18529800 \\ \mathrm{H} & 4.91161900 & 0.19845800 & -0.35374300\end{array}$




\begin{tabular}{|c|c|c|c|}
\hline $\mathrm{H}$ & 5.40339500 & 1.80494600 & 0.23826700 \\
\hline $\mathrm{C}$ & 4.63892300 & 3.23834300 & -2.02634300 \\
\hline $\mathrm{H}$ & 5.55896700 & 3.17183600 & -2.61801400 \\
\hline $\mathrm{H}$ & 4.83381900 & 3.87662800 & -1.15714500 \\
\hline $\mathrm{H}$ & 3.87390900 & 3.72059800 & -2.64190100 \\
\hline $\mathrm{C}$ & 1.59213400 & -1.34066100 & 2.42196100 \\
\hline $\mathrm{H}$ & 1.41955300 & -1.13958200 & 3.48749900 \\
\hline $\mathrm{C}$ & 1.20594000 & -2.81324100 & 2.15738500 \\
\hline $\mathrm{H}$ & 0.14835900 & -2.98692100 & 2.39542100 \\
\hline $\mathrm{H}$ & 1.33021700 & -3.02878900 & 1.08868300 \\
\hline $\mathrm{C}$ & 2.08549900 & -3.77416500 & 2.97657900 \\
\hline $\mathrm{H}$ & 1.87883100 & -3.63400900 & 4.04874500 \\
\hline $\mathrm{H}$ & 1.81401100 & -4.81071400 & 2.73926900 \\
\hline $\mathrm{C}$ & 3.57891400 & -3.53702800 & 2.71040600 \\
\hline $\mathrm{H}$ & 3.80319900 & -3.79140200 & 1.66524600 \\
\hline $\mathrm{H}$ & 4.18609300 & -4.20326400 & 3.33688600 \\
\hline $\mathrm{C}$ & 3.95963100 & -2.07136500 & 2.96484400 \\
\hline $\mathrm{H}$ & 5.01750700 & -1.90570400 & 2.72442800 \\
\hline $\mathrm{H}$ & 3.84394700 & -1.84625300 & 4.03653000 \\
\hline $\mathrm{C}$ & 3.09060600 & -1.10547400 & 2.14037900 \\
\hline $\mathrm{H}$ & 3.28744400 & -1.25760900 & 1.07412700 \\
\hline $\mathrm{H}$ & 3.36288100 & -0.06981000 & 2.37053600 \\
\hline $\mathrm{C}$ & -1.20880300 & -0.33754700 & 2.35597500 \\
\hline $\mathrm{H}$ & -1.64406000 & -1.24998400 & 1.92364000 \\
\hline $\mathrm{C}$ & -1.15397400 & -0.52080500 & 3.88756800 \\
\hline $\mathrm{H}$ & -0.67075200 & 0.35177700 & 4.34596300 \\
\hline $\mathrm{H}$ & -0.55695400 & -1.39874000 & 4.15653400 \\
\hline $\mathrm{C}$ & -2.56297000 & -0.67192000 & 4.49017600 \\
\hline $\mathrm{H}$ & -3.02110400 & -1.59773000 & 4.11176300 \\
\hline $\mathrm{H}$ & -2.48356100 & -0.78551100 & 5.57911300 \\
\hline $\mathrm{C}$ & -3.46000000 & 0.52053700 & 4.13613400 \\
\hline $\mathrm{H}$ & -3.05338100 & 1.43095200 & 4.60092900 \\
\hline $\mathrm{H}$ & -4.46637000 & 0.37881000 & 4.55067800 \\
\hline $\mathrm{C}$ & -3.52751800 & 0.70975900 & 2.61590000 \\
\hline $\mathrm{H}$ & -4.12795900 & 1.59308100 & 2.36318200 \\
\hline $\mathrm{H}$ & -4.04571800 & -0.15170900 & 2.17272600 \\
\hline $\mathrm{C}$ & -2.12768300 & 0.84872800 & 1.99253800 \\
\hline $\mathrm{H}$ & -1.67009600 & 1.78032000 & 2.34667800 \\
\hline $\mathrm{H}$ & -2.21542300 & 0.94832100 & 0.90720100 \\
\hline $\mathrm{O}$ & -1.38421200 & 3.39108000 & -0.02235800 \\
\hline $\mathrm{O}$ & 2.99921100 & 1.91425300 & -0.78035400 \\
\hline $\mathrm{P}$ & 0.46139000 & -0.18968500 & 1.45099100 \\
\hline $\mathrm{Pd}$ & 0.21251800 & -0.91733100 & -0.94763100 \\
\hline $\mathrm{C}$ & -2.00623700 & -1.17483000 & -1.45266000 \\
\hline $\mathrm{C}$ & -2.67387700 & -0.04953800 & -1.76421800 \\
\hline $\mathrm{H}$ & -2.13013900 & 0.81995600 & -2.12271300 \\
\hline $\mathrm{C}$ & -4.16149800 & 0.16827600 & -1.73724100 \\
\hline $\mathrm{C}$ & -2.50426800 & -2.45420100 & -0.91436500 \\
\hline $\mathrm{C}$ & -1.95231800 & -3.68190000 & -1.33056500 \\
\hline $\mathrm{C}$ & -3.51834100 & -2.47992100 & 0.06485500 \\
\hline $\mathrm{C}$ & -2.41537400 & -4.88754900 & -0.80773900 \\
\hline $\mathrm{H}$ & -1.15893600 & -3.69999600 & -2.06997000 \\
\hline $\mathrm{C}$ & -3.97047100 & -3.68702400 & 0.59210700 \\
\hline $\mathrm{H}$ & -3.95329400 & -1.55053500 & 0.41115300 \\
\hline C & -3.42411000 & -4.89660300 & 0.15725900 \\
\hline $\mathrm{H}$ & -1.97977200 & -5.82082000 & -1.1529590 \\
\hline त & -4.75270800 & -3.68068800 & 1.3462980 \\
\hline
\end{tabular}




$\begin{array}{llll}\mathrm{H} & -3.77796200 & -5.83698200 & 0.57051800 \\ \mathrm{~F} & -4.87660400 & -0.92225300 & -2.06505500 \\ \mathrm{~F} & -4.49277800 & 1.14583300 & -2.61387300 \\ \mathrm{~F} & -4.60154100 & 0.58205800 & -0.51532000 \\ \mathrm{I} & 2.67121000 & -1.86566000 & -1.76264800 \\ \mathrm{C} & -0.50716600 & -1.35233300 & -2.63859400 \\ \mathrm{O} & -0.63551600 & -1.57056300 & -3.77172400\end{array}$

\section{IM9}

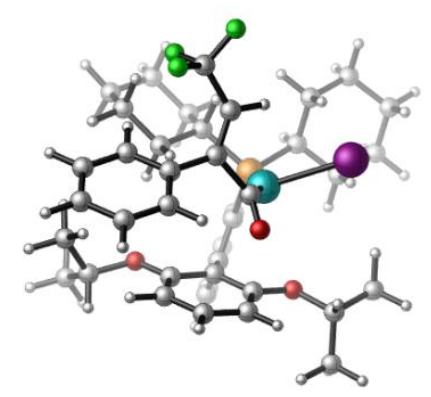

$\mathrm{E}(\mathrm{B} 3 \mathrm{LYP} / \mathrm{BS} 1)=-2558.492643$

$\mathrm{E}(\mathrm{PCM}$ (dichloroethane $) / \mathrm{M} 06 / \mathrm{BS} 2 / / \mathrm{B} 3 \mathrm{LYP} / \mathrm{BS} 1)=-2558.867017$

Zero-point correction=

0.815116

Thermal correction to Energy=

0.866603

Thermal correction to Enthalpy=

0.867547

Thermal correction to Gibbs Free Energy=

0.723907

$\begin{array}{lrrr}\text { Charge }= & \text { Multiplicity }=1 & \\ \mathrm{C} & 2.19090300 & 2.01371900 & 0.74506300 \\ \mathrm{C} & 3.20353300 & 2.58093600 & 1.54270000 \\ \mathrm{H} & 3.58427100 & 2.03509600 & 2.39883400 \\ \mathrm{C} & 3.74546600 & 3.83356600 & 1.26697800 \\ \mathrm{H} & 4.52743800 & 4.23789700 & 1.90418900 \\ \mathrm{C} & 3.27875000 & 4.55571100 & 0.16943700 \\ \mathrm{H} & 3.69267800 & 5.53275800 & -0.06557400 \\ \mathrm{C} & 2.27767300 & 4.01266400 & -0.62945700 \\ \mathrm{H} & 1.91238600 & 4.56867300 & -1.48827100 \\ \mathrm{C} & 1.71949400 & 2.74962500 & -0.36608700 \\ \mathrm{C} & 0.65027400 & 2.30127700 & -1.31908000 \\ \mathrm{C} & 0.98072400 & 1.51294600 & -2.44841600 \\ \mathrm{C} & 0.03123700 & 1.28494000 & -3.45694700 \\ \mathrm{H} & 0.26329800 & 0.66665300 & -4.31333500 \\ \mathrm{C} & -1.23702400 & 1.84405900 & -3.33969900 \\ \mathrm{H} & -1.97342800 & 1.64804300 & -4.11357700 \\ \mathrm{C} & -1.58984800 & 2.63636000 & -2.24948300 \\ \mathrm{H} & -2.58788700 & 3.05084000 & -2.18605700 \\ \mathrm{C} & -0.63858900 & 2.87862100 & -1.24948500 \\ \mathrm{C} & -1.95503100 & 4.62710400 & -0.16852800 \\ \mathrm{H} & -2.15582500 & 4.92628000 & -1.20502300 \\ \mathrm{C} & -3.20573600 & 4.00062300 & 0.44853100 \\ \mathrm{H} & -4.03717000 & 4.71482600 & 0.42410800 \\ \mathrm{H} & -3.01648400 & 3.73235000 & 1.49382000 \\ \mathrm{H} & -3.51598000 & 3.09611400 & -0.08248300 \\ \mathrm{C} & -1.46422900 & 5.84034900 & 0.61631300\end{array}$




\begin{tabular}{|c|c|c|c|}
\hline $\mathrm{H}_{\mathrm{H}}$ & -2.24105300 & 6.61211700 & 0.65100900 \\
\hline $\mathrm{H}$ & -0.56909400 & 6.26432500 & 0.15152400 \\
\hline $\mathrm{H}$ & -1.21410400 & 5.55491700 & 1.64394900 \\
\hline $\mathrm{C}$ & 2.76817100 & 0.36906000 & -3.65058100 \\
\hline $\mathrm{H}$ & 2.01709000 & -0.35377000 & -3.98965000 \\
\hline $\mathrm{C}$ & 4.00156400 & -0.39519500 & -3.18754300 \\
\hline $\mathrm{H}$ & 4.45380100 & -0.92157000 & -4.03513900 \\
\hline $\mathrm{H}$ & 3.73131400 & -1.13206000 & -2.42713200 \\
\hline $\mathrm{H}$ & 4.74474200 & 0.29259200 & -2.76898300 \\
\hline C & 3.08452700 & 1.37852800 & -4.75515000 \\
\hline $\mathrm{H}$ & 3.45883000 & 0.85996700 & -5.64503900 \\
\hline $\mathrm{H}$ & 3.85519100 & 2.07860800 & -4.41370900 \\
\hline $\mathrm{H}$ & 2.20061100 & 1.95519200 & -5.04371300 \\
\hline $\mathrm{C}$ & 3.01837400 & -0.59349200 & 1.82038300 \\
\hline $\mathrm{H}$ & 3.48345600 & 0.02770900 & 2.59834100 \\
\hline $\mathrm{C}$ & 2.62128600 & -1.93444300 & 2.47651200 \\
\hline $\mathrm{H}$ & 1.92742500 & -1.76849700 & 3.30949200 \\
\hline $\mathrm{H}$ & 2.09841600 & -2.55872000 & 1.74141300 \\
\hline $\mathrm{C}$ & 3.86104900 & -2.68517900 & 2.99610000 \\
\hline $\mathrm{H}$ & 4.31636800 & -2.11049500 & 3.81721300 \\
\hline $\mathrm{H}$ & 3.55121400 & -3.64741600 & 3.42304600 \\
\hline $\mathrm{C}$ & 4.90328000 & -2.90199200 & 1.89012400 \\
\hline $\mathrm{H}$ & 4.48526800 & -3.57351900 & 1.12726500 \\
\hline $\mathrm{H}$ & 5.79176100 & -3.40048500 & 2.29876200 \\
\hline $\mathrm{C}$ & 5.29257300 & -1.57111400 & 1.23128600 \\
\hline $\mathrm{H}$ & 5.99705200 & -1.74403500 & 0.40767000 \\
\hline $\mathrm{H}$ & 5.81767400 & -0.93977000 & 1.96440500 \\
\hline $\mathrm{C}$ & 4.05751800 & -0.82276000 & 0.70295300 \\
\hline $\mathrm{H}$ & 3.59082500 & -1.41263800 & -0.09546900 \\
\hline $\mathrm{H}$ & 4.35722900 & 0.13385800 & 0.25977100 \\
\hline $\mathrm{C}$ & 0.43404000 & 0.57521500 & 2.67864500 \\
\hline $\mathrm{H}$ & 0.18871000 & -0.45936000 & 2.96162300 \\
\hline $\mathrm{C}$ & 1.08038100 & 1.26277800 & 3.89590800 \\
\hline $\mathrm{H}$ & 1.36322300 & 2.28861400 & 3.62506400 \\
\hline $\mathrm{H}$ & 2.00186500 & 0.74638000 & 4.19193800 \\
\hline $\mathrm{C}$ & 0.11069200 & 1.30348000 & 5.09169200 \\
\hline $\mathrm{H}$ & -0.08782000 & 0.27618400 & 5.43099700 \\
\hline $\mathrm{H}$ & 0.58391800 & 1.82428700 & 5.93413000 \\
\hline $\mathrm{C}$ & -1.21677000 & 1.98059900 & 4.72267500 \\
\hline $\mathrm{H}$ & -1.03073200 & 3.04205500 & 4.50083200 \\
\hline $\mathrm{H}$ & -1.90680100 & 1.95479400 & 5.57568500 \\
\hline $\mathrm{C}$ & -1.85773200 & 1.31405400 & 3.49648500 \\
\hline $\mathrm{H}$ & -2.77499900 & 1.84659000 & 3.21131200 \\
\hline $\mathrm{H}$ & -2.15974800 & 0.28747200 & 3.75069300 \\
\hline $\mathrm{C}$ & -0.88872500 & 1.27224200 & 2.30298000 \\
\hline $\mathrm{H}$ & -0.68184400 & 2.29110100 & 1.95357500 \\
\hline $\mathrm{H}$ & -1.36174300 & 0.74916600 & 1.46037100 \\
\hline $\mathrm{O}$ & -0.85875900 & 3.68880200 & -0.17068500 \\
\hline $\mathrm{O}$ & 2.26062500 & 1.05129600 & -2.47978600 \\
\hline $\mathrm{P}$ & 1.52072200 & 0.33179800 & 1.15322200 \\
\hline $\mathrm{Pc}$ & 0.06354200 & -0.87308700 & -0.53547600 \\
\hline $\mathrm{C}$ & -2.65902000 & -1.63870000 & -0.51692400 \\
\hline $\mathrm{C}$ & -2.42344800 & -2.51632400 & 0.47788400 \\
\hline $\mathrm{H}$ & -1.45679700 & -3.00769100 & 0.5364040 \\
\hline C & -3.37756900 & -2.93908700 & 1.5567490 \\
\hline & -3.89237800 & -0.83460100 & -0.73048300 \\
\hline & -4.50408600 & -0.78166600 & $-1.994 \supset \supset$ \\
\hline
\end{tabular}




$\begin{array}{llcc}\text { C } & -4.45009500 & -0.09559800 & 0.32725200 \\ \text { C } & -5.66000400 & -0.02573400 & -2.18525100 \\ \text { H } & -4.07445300 & -1.33576300 & -2.82076800 \\ \text { C } & -5.60060000 & 0.66600200 & 0.12764000 \\ \text { H } & -3.98489100 & -0.12425300 & 1.30663900 \\ \text { C } & -6.21073700 & 0.70175900 & -1.12822800 \\ \text { H } & -6.13068400 & -0.00615100 & -3.16436900 \\ \text { H } & -6.02124500 & 1.22892900 & 0.95637000 \\ \text { H } & -7.10961300 & 1.29279000 & -1.28169500 \\ \text { F } & -4.66663800 & -2.97013500 & 1.17525100 \\ \text { F } & -3.29675100 & -2.10384200 & 2.63204700 \\ \text { F } & -3.05354800 & -4.17053800 & 1.99796900 \\ \text { I } & 1.02029600 & -3.35573100 & -1.16753500 \\ \text { C } & -1.53667900 & -1.43953300 & -1.53294900 \\ \text { O } & -1.67935700 & -1.36589500 & -2.71926400\end{array}$

TS9

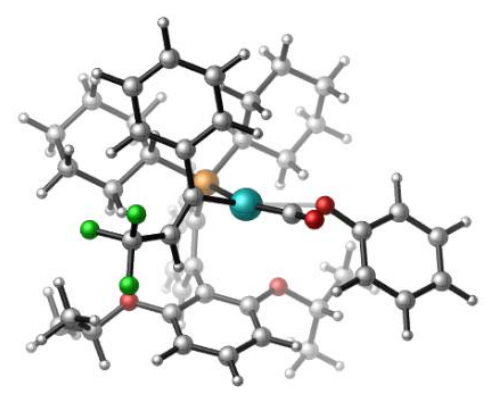

$\mathrm{E}(\mathrm{B} 3 \mathrm{LYP} / \mathrm{BS} 1)=-2853.910305$

$\mathrm{E}(\mathrm{PCM}$ (dichloroethane $) / \mathrm{M} 06 / \mathrm{BS} 2 / / \mathrm{B} 3 \mathrm{LYP} / \mathrm{BS} 1)=-2854.149631$

Zero-point correction=

0.906686

Thermal correction to Energy=

0.962296

Thermal correction to Enthalpy=

0.963241

Thermal correction to Gibbs Free Energy=

0.811892

$\begin{array}{lccc}\text { Charge }= & \text { Multiplicity }=1 & \\ \mathrm{C} & 0.02644100 & 1.52827400 & 2.13857900 \\ \mathrm{C} & 0.00510200 & 1.73296300 & 3.53331800 \\ \mathrm{H} & -0.13549000 & 0.89072800 & 4.20043600 \\ \mathrm{C} & 0.16819200 & 2.99509300 & 4.09738200 \\ \mathrm{H} & 0.14777800 & 3.11150500 & 5.17752100 \\ \mathrm{C} & 0.35622800 & 4.09778800 & 3.26551800 \\ \mathrm{H} & 0.47902400 & 5.09263000 & 3.68529800 \\ \mathrm{C} & 0.40619400 & 3.91068300 & 1.88830000 \\ \mathrm{H} & 0.58314300 & 4.75999600 & 1.23468500 \\ \mathrm{C} & 0.25899600 & 2.64165100 & 1.30055100 \\ \mathrm{C} & 0.45234400 & 2.59053500 & -0.18338900 \\ \mathrm{C} & 1.74734400 & 2.34380900 & -0.68732300 \\ \mathrm{C} & 2.01538400 & 2.47171600 & -2.05870100 \\ \mathrm{H} & 3.00679500 & 2.27569000 & -2.44675500 \\ \mathrm{C} & 0.99083000 & 2.86072100 & -2.91726500 \\ \mathrm{H} & 1.19497100 & 2.95571800 & -3.98041700 \\ \mathrm{C} & -0.29232900 & 3.13546000 & -2.44470300 \\ \mathrm{H} & -1.06832600 & 3.43921600 & -3.13617800\end{array}$




\begin{tabular}{|c|c|c|c|}
\hline $\mathrm{C}$ & -0.55654300 & 3.01389000 & -1.07232900 \\
\hline $\mathrm{C}$ & -2.69298800 & 4.18952500 & -1.16842600 \\
\hline $\mathrm{H}$ & -2.11734400 & 4.86841600 & -1.81067000 \\
\hline $\mathrm{C}$ & -3.69916900 & 3.40148100 & -2.00531200 \\
\hline $\mathrm{H}$ & -4.41372300 & 4.08775200 & -2.47481000 \\
\hline $\mathrm{H}$ & -4.25817900 & 2.70329000 & -1.37408300 \\
\hline $\mathrm{H}$ & -3.21924000 & 2.82033700 & -2.79645100 \\
\hline $\mathrm{C}$ & -3.36424800 & 4.99645500 & -0.06039400 \\
\hline $\mathrm{H}$ & -4.07015000 & 5.71721300 & -0.48748700 \\
\hline $\mathrm{H}$ & -2.61893700 & 5.54141300 & 0.52691000 \\
\hline $\mathrm{H}$ & -3.91530600 & 4.33281900 & 0.61493100 \\
\hline $\mathrm{C}$ & 4.08544900 & 2.28558200 & 0.02126000 \\
\hline $\mathrm{H}$ & 4.39760400 & 1.81910600 & -0.91862900 \\
\hline $\mathrm{C}$ & 4.82139700 & 1.60607600 & 1.16856300 \\
\hline $\mathrm{H}$ & 5.89971300 & 1.76890600 & 1.06845700 \\
\hline $\mathrm{H}$ & 4.63590000 & 0.52917000 & 1.15670000 \\
\hline $\mathrm{H}$ & 4.49284900 & 2.01617800 & 2.13032100 \\
\hline $\mathrm{C}$ & 4.32844800 & 3.79441200 & -0.01856900 \\
\hline $\mathrm{H}$ & 5.39347200 & 3.99838600 & -0.17631100 \\
\hline $\mathrm{H}$ & 4.02496500 & 4.25153400 & 0.93013200 \\
\hline $\mathrm{H}$ & 3.76880600 & 4.27332600 & -0.82807800 \\
\hline $\mathrm{C}$ & 0.58729700 & -1.31000200 & 2.70550900 \\
\hline $\mathrm{H}$ & 0.24574900 & -1.08315600 & 3.72434500 \\
\hline $\mathrm{C}$ & 0.26972500 & -2.79543400 & 2.42267100 \\
\hline $\mathrm{H}$ & -0.80722200 & -2.98820800 & 2.50083200 \\
\hline $\mathrm{H}$ & 0.55517700 & -3.03384700 & 1.38937800 \\
\hline $\mathrm{C}$ & 1.03533300 & -3.71837600 & 3.38671600 \\
\hline $\mathrm{H}$ & 0.67226100 & -3.55384200 & 4.41258300 \\
\hline $\mathrm{H}$ & 0.81560400 & -4.76540400 & 3.14239800 \\
\hline $\mathrm{C}$ & 2.54798500 & -3.46062900 & 3.33423700 \\
\hline $\mathrm{H}$ & 2.92548900 & -3.73656700 & 2.33923900 \\
\hline $\mathrm{H}$ & 3.06726100 & -4.10091700 & 4.05877000 \\
\hline $\mathrm{C}$ & 2.86709500 & -1.98251800 & 3.60126600 \\
\hline $\mathrm{H}$ & 3.94555200 & -1.80262100 & 3.50829200 \\
\hline $\mathrm{H}$ & 2.59676400 & -1.73314600 & 4.63884400 \\
\hline $\mathrm{C}$ & 2.10907200 & -1.05509800 & 2.63607900 \\
\hline $\mathrm{H}$ & 2.45897600 & -1.23313700 & 1.61126800 \\
\hline $\mathrm{H}$ & 2.32915300 & -0.00773500 & 2.86982300 \\
\hline $\mathrm{C}$ & -2.17240700 & -0.42749500 & 1.91836100 \\
\hline $\mathrm{H}$ & -2.41316300 & -1.37466300 & 1.41788000 \\
\hline $\mathrm{C}$ & -2.52756600 & -0.57690400 & 3.41317100 \\
\hline $\mathrm{H}$ & -2.26615100 & 0.34437700 & 3.94859800 \\
\hline $\mathrm{H}$ & -1.95718300 & -1.39131700 & 3.87381900 \\
\hline $\mathrm{C}$ & -4.03273000 & -0.84005600 & 3.60259500 \\
\hline $\mathrm{H}$ & -4.29397400 & -1.80354700 & 3.14113500 \\
\hline $\mathrm{H}$ & -4.25474500 & -0.93484100 & 4.67339500 \\
\hline $\mathrm{C}$ & -4.87976200 & 0.27383200 & 2.97370200 \\
\hline $\mathrm{H}$ & -4.69085600 & 1.21705300 & 3.50752000 \\
\hline $\mathrm{H}$ & -5.94847200 & 0.05439000 & 3.09278000 \\
\hline $\mathrm{C}$ & -4.53164700 & 0.44800800 & 1.48981800 \\
\hline $\mathrm{H}$ & -5.09810800 & 1.28333700 & 1.05808600 \\
\hline $\mathrm{H}$ & -4.84155300 & -0.45275700 & 0.94233400 \\
\hline $\mathrm{C}$ & -3.02663400 & 0.68613500 & 1.27207700 \\
\hline $\mathrm{H}$ & -2.74708100 & 1.65401600 & 1.70623400 \\
\hline $\mathrm{H}$ & -2.81618700 & 0.75553000 & 0.20141900 \\
\hline $\mathrm{O}$ & -1.76398500 & 3.30242500 & -0.50311100 \\
\hline $\mathrm{O}$ & 2.68088500 & 2.00291300 & 0.24464200 \\
\hline
\end{tabular}




$\begin{array}{lrrr}\mathrm{P} & -0.33884000 & -0.18535800 & 1.51707800 \\ \mathrm{Pd} & 0.39226100 & -1.03209700 & -0.67427200 \\ \mathrm{C} & -1.45859800 & -1.29888200 & -1.44378100 \\ \mathrm{C} & -1.90222000 & -0.31623900 & -2.24360100 \\ \mathrm{H} & -1.33263400 & 0.60103300 & -2.35488100 \\ \mathrm{C} & -3.15936300 & -0.30471000 & -3.05893800 \\ \mathrm{C} & -2.12961700 & -2.58201800 & -1.13564900 \\ \mathrm{C} & -1.42139600 & -3.79933800 & -1.12779900 \\ \mathrm{C} & -3.50313000 & -2.61894900 & -0.81790000 \\ \mathrm{C} & -2.06136200 & -5.00275600 & -0.83870000 \\ \mathrm{H} & -0.36216500 & -3.80094300 & -1.35945400 \\ \mathrm{C} & -4.13811800 & -3.82257500 & -0.51532000 \\ \mathrm{H} & -4.07604600 & -1.69955200 & -0.82270600 \\ \mathrm{C} & -3.42211400 & -5.02080200 & -0.52470800 \\ \mathrm{H} & -1.49207800 & -5.92825600 & -0.85471500 \\ \mathrm{H} & -5.19800000 & -3.82147100 & -0.27456200 \\ \mathrm{H} & -3.91819500 & -5.95834100 & -0.28885500 \\ \mathrm{~F} & -3.49041700 & -1.49322000 & -3.59862100 \\ \mathrm{~F} & -3.03590400 & 0.58014700 & -4.08137400 \\ \mathrm{~F} & -4.24871300 & 0.11120300 & -2.33948200 \\ \mathrm{C} & 1.31625100 & -1.94628500 & -2.16089700 \\ \mathrm{O} & 1.67881000 & -2.47764000 & -3.12073200 \\ \mathrm{O} & 2.64676800 & -1.71001300 & -0.76627100 \\ \mathrm{C} & 3.88420100 & -1.40278300 & -1.18925700 \\ \mathrm{C} & 4.14421200 & -0.58594000 & -2.30942200 \\ \mathrm{C} & 4.98725600 & -1.95079300 & -0.49835900 \\ \mathrm{C} & 5.45543800 & -0.33270600 & -2.71607700 \\ \mathrm{H} & 3.31136900 & -0.15741200 & -2.85785100 \\ \mathrm{C} & 6.29183300 & -1.68018900 & -0.90640500 \\ \mathrm{H} & 4.79157800 & -2.59629200 & 0.35344800 \\ \mathrm{C} & 6.53980600 & -0.86970300 & -2.01836900 \\ \mathrm{H} & 5.62850800 & 0.29128700 & -3.59057200 \\ \mathrm{H} & 7.12274700 & -2.11394900 & -0.35423700 \\ \mathrm{H} & 7.55789200 & -0.66771300 & -2.33917500\end{array}$

\section{TS8}

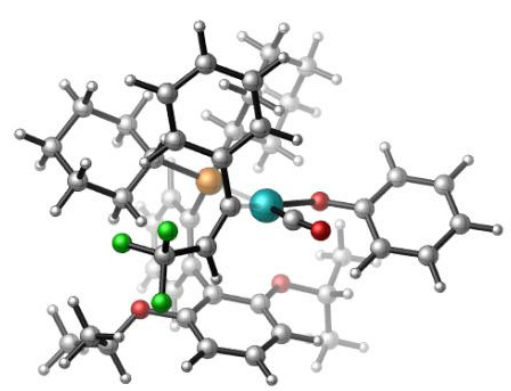

$\mathrm{E}(\mathrm{B} 3 \mathrm{LYP} / \mathrm{BS} 1)=-2853.900676$

$\mathrm{E}(\mathrm{PCM}($ dichloroethane $) / \mathrm{M} 06 / \mathrm{BS} 2 / / \mathrm{B} 3 \mathrm{LYP} / \mathrm{BS} 1)=-2854.139566$

Zero-point correction=

Thermal correction to Energy=

Thermal correction to Enthalpy=

Thermal correction to Gibbs Free Energy=
0.906833

0.962329

0.963273

0.812469 


\begin{tabular}{|c|c|c|c|}
\hline \multicolumn{4}{|c|}{ Charge $=0$ Multiplicity $=1$} \\
\hline $\mathrm{C}$ & 0.01746300 & 2.08059000 & 1.80442200 \\
\hline $\mathrm{C}$ & 0.03347100 & 2.59523300 & 3.11652100 \\
\hline $\mathrm{H}$ & 0.09659500 & 1.91689200 & 3.95978700 \\
\hline $\mathrm{C}$ & -0.01837300 & 3.96217000 & 3.37663800 \\
\hline $\mathrm{H}$ & -0.00124900 & 4.31870500 & 4.40305200 \\
\hline $\mathrm{C}$ & -0.08833000 & 4.85977400 & 2.31255300 \\
\hline $\mathrm{H}$ & -0.13022000 & 5.93052100 & 2.49394300 \\
\hline $\mathrm{C}$ & -0.08737200 & 4.37221600 & 1.00981600 \\
\hline $\mathrm{H}$ & -0.11633700 & 5.06596400 & 0.17437500 \\
\hline $\mathrm{C}$ & -0.02876700 & 2.99642300 & 0.72724900 \\
\hline $\mathrm{C}$ & 0.03705700 & 2.62941900 & -0.72405000 \\
\hline $\mathrm{C}$ & 1.30293700 & 2.47324400 & -1.33492200 \\
\hline $\mathrm{C}$ & 1.39967000 & 2.24789400 & -2.71842400 \\
\hline $\mathrm{H}$ & 2.36311000 & 2.11204100 & -3.19214300 \\
\hline $\mathrm{C}$ & 0.23936700 & 2.21990400 & -3.48816000 \\
\hline $\mathrm{H}$ & 0.31751400 & 2.04908300 & -4.55881000 \\
\hline $\mathrm{C}$ & -1.01782300 & 2.42189000 & -2.91982400 \\
\hline $\mathrm{H}$ & -1.90431800 & 2.41510300 & -3.54279600 \\
\hline $\mathrm{C}$ & -1.11244300 & 2.64553000 & -1.53687500 \\
\hline $\mathrm{C}$ & -3.37921300 & 3.54112500 & -1.60605700 \\
\hline $\mathrm{H}$ & -2.95564400 & 4.07807800 & -2.46415900 \\
\hline $\mathrm{C}$ & -4.40353800 & 2.51425500 & -2.08550200 \\
\hline $\mathrm{H}$ & -5.23440700 & 3.01977600 & -2.59147400 \\
\hline $\mathrm{H}$ & -4.80841300 & 1.95542800 & -1.23516700 \\
\hline $\mathrm{H}$ & -3.97073300 & 1.79252600 & -2.78251000 \\
\hline $\mathrm{C}$ & -3.98120900 & 4.54827600 & -0.62939000 \\
\hline $\mathrm{H}$ & -4.80116900 & 5.09862300 & -1.10412800 \\
\hline $\mathrm{H}$ & -3.22376100 & 5.26469200 & -0.29747800 \\
\hline $\mathrm{H}$ & -4.37624100 & 4.03418800 & 0.25367400 \\
\hline $\mathrm{C}$ & 3.71575500 & 2.63364200 & -1.00100800 \\
\hline $\mathrm{H}$ & 3.84325100 & 1.82096000 & -1.72385100 \\
\hline $\mathrm{C}$ & 4.60477100 & 2.35688100 & 0.20416200 \\
\hline $\mathrm{H}$ & 5.65732500 & 2.35740700 & -0.09898800 \\
\hline $\mathrm{H}$ & 4.36283700 & 1.38133500 & 0.63013400 \\
\hline $\mathrm{H}$ & 4.46133500 & 3.12715100 & 0.97058700 \\
\hline $\mathrm{C}$ & 4.01226500 & 3.98953100 & -1.64488700 \\
\hline $\mathrm{H}$ & 5.04000600 & 4.00846900 & -2.02491600 \\
\hline $\mathrm{H}$ & 3.90402100 & 4.78938700 & -0.90362500 \\
\hline $\mathrm{H}$ & 3.33749300 & 4.20287500 & -2.47951700 \\
\hline $\mathrm{C}$ & 1.10223000 & -0.40824600 & 2.97491200 \\
\hline $\mathrm{H}$ & 0.64417700 & -0.11766700 & 3.92980700 \\
\hline $\mathrm{C}$ & 1.15743800 & -1.95202400 & 2.93191600 \\
\hline $\mathrm{H}$ & 0.14935800 & -2.38326100 & 2.98503300 \\
\hline $\mathrm{H}$ & 1.58679400 & -2.26103200 & 1.96972900 \\
\hline $\mathrm{C}$ & 2.01970700 & -2.50915100 & 4.07809400 \\
\hline $\mathrm{H}$ & 1.53767600 & -2.27968800 & 5.04061900 \\
\hline $\mathrm{H}$ & 2.06572000 & -3.60342600 & 4.00558300 \\
\hline $\mathrm{C}$ & 3.43351100 & -1.91088100 & 4.06156400 \\
\hline $\mathrm{H}$ & 3.95505100 & -2.24842200 & 3.15438600 \\
\hline $\mathrm{H}$ & 4.01394500 & -2.28403100 & 4.91534500 \\
\hline $\mathrm{C}$ & 3.38719300 & -0.37585000 & 4.07509000 \\
\hline $\mathrm{H}$ & 4.40258000 & 0.03494100 & 4.00460800 \\
\hline $\mathrm{H}$ & 2.97993200 & -0.03499200 & 5.03973300 \\
\hline $\mathrm{C}$ & 2.52698300 & 0.18154000 & 2.92754500 \\
\hline $\mathrm{H}$ & 2.97324600 & -0.07094400 & 1.96042300 \\
\hline $\mathrm{H}$ & 2.48947900 & 1.27461700 & 2.98933100 \\
\hline
\end{tabular}




\begin{tabular}{|c|c|c|c|}
\hline $\mathrm{C}$ & -1.75335900 & -0.25550700 & 2.15846000 \\
\hline $\mathrm{H}$ & -1.81149300 & -1.32240600 & 1.89801700 \\
\hline $\mathrm{C}$ & -2.05556300 & -0.11879100 & 3.66545600 \\
\hline $\mathrm{H}$ & -1.94428300 & 0.93052300 & 3.96760500 \\
\hline $\mathrm{H}$ & -1.34575300 & -0.70271000 & 4.26161000 \\
\hline $\mathrm{C}$ & -3.48669500 & -0.57680200 & 4.00123600 \\
\hline $\mathrm{H}$ & -3.58133500 & -1.65301500 & 3.79349000 \\
\hline $\mathrm{H}$ & -3.66823400 & -0.44938200 & 5.07635100 \\
\hline $\mathrm{C}$ & -4.53178600 & 0.19203900 & 3.18330800 \\
\hline $\mathrm{H}$ & -4.50364800 & 1.25472000 & 3.46625400 \\
\hline $\mathrm{H}$ & -5.54182100 & -0.16875600 & 3.41664300 \\
\hline $\mathrm{C}$ & -4.24671000 & 0.05592200 & 1.68259200 \\
\hline $\mathrm{H}$ & -4.96506000 & 0.64559400 & 1.09857400 \\
\hline $\mathrm{H}$ & -4.39478200 & -0.99212000 & 1.38676700 \\
\hline $\mathrm{C}$ & -2.81561600 & 0.49603600 & 1.32832800 \\
\hline $\mathrm{H}$ & -2.71803300 & 1.57341700 & 1.50673000 \\
\hline $\mathrm{H}$ & -2.63977700 & 0.34965800 & 0.25872900 \\
\hline $\mathrm{O}$ & -2.29679200 & 2.89325100 & -0.89866900 \\
\hline $\mathrm{O}$ & 2.35990600 & 2.60337300 & -0.49838000 \\
\hline $\mathrm{P}$ & -0.00368400 & 0.23086000 & 1.59225200 \\
\hline $\mathrm{Pd}$ & 0.64032700 & -0.97569500 & -0.56489900 \\
\hline $\mathrm{C}$ & -1.19222100 & -2.01813000 & -1.44919600 \\
\hline $\mathrm{C}$ & -1.95128500 & -1.17625900 & -2.17829100 \\
\hline $\mathrm{H}$ & -1.50035100 & -0.29534100 & -2.62790400 \\
\hline $\mathrm{C}$ & -3.40824300 & -1.31755300 & -2.52429000 \\
\hline $\mathrm{C}$ & -1.57962300 & -3.23848500 & -0.71184300 \\
\hline $\mathrm{C}$ & -2.80459600 & -3.31038100 & -0.01847200 \\
\hline $\mathrm{C}$ & -0.71393900 & -4.34800400 & -0.64112100 \\
\hline $\mathrm{C}$ & -3.15198900 & -4.44992600 & 0.70334300 \\
\hline $\mathrm{H}$ & -3.48572200 & -2.46934500 & -0.04250900 \\
\hline $\mathrm{C}$ & -1.06976800 & -5.48977200 & 0.07387000 \\
\hline $\mathrm{H}$ & 0.23942700 & -4.32943000 & -1.15735800 \\
\hline $\mathrm{C}$ & -2.28861800 & -5.54631700 & 0.75186200 \\
\hline $\mathrm{H}$ & -4.10187000 & -4.47878700 & 1.23027500 \\
\hline $\mathrm{H}$ & -0.38820700 & -6.33522400 & 0.10217000 \\
\hline $\mathrm{H}$ & -2.56162500 & -6.43425000 & 1.31521000 \\
\hline $\mathrm{F}$ & -3.78682300 & -2.58759000 & -2.75244500 \\
\hline $\mathrm{F}$ & -3.67939500 & -0.60621400 & -3.64390700 \\
\hline $\mathrm{F}$ & -4.22551400 & -0.82585900 & -1.55073100 \\
\hline $\mathrm{C}$ & 0.54596600 & -2.04077400 & -2.11186500 \\
\hline $\mathrm{O}$ & 0.83820800 & -2.61912100 & -3.08104700 \\
\hline $\mathrm{O}$ & 2.65039400 & -0.62912500 & -0.19522600 \\
\hline $\mathrm{C}$ & 3.64266900 & -1.07474500 & -0.97143200 \\
\hline $\mathrm{C}$ & 3.68026100 & -0.89596800 & -2.37333500 \\
\hline $\mathrm{C}$ & 4.75971800 & -1.69553500 & -0.36347000 \\
\hline $\mathrm{C}$ & 4.78290500 & -1.30911200 & -3.12078500 \\
\hline $\mathrm{H}$ & 2.82914900 & -0.42896000 & -2.86083300 \\
\hline $\mathrm{C}$ & 5.85982900 & -2.09560100 & -1.11866200 \\
\hline $\mathrm{H}$ & 4.74226500 & -1.84225600 & 0.71328100 \\
\hline $\mathrm{C}$ & 5.88409600 & -1.90874500 & -2.50413600 \\
\hline $\mathrm{H}$ & 4.77963200 & -1.15988100 & -4.19870400 \\
\hline $\mathrm{H}$ & 6.70531900 & -2.56485000 & -0.61959500 \\
\hline $\mathrm{H}$ & 6.74023400 & -2.22934600 & -3.09116400 \\
\hline
\end{tabular}




\section{IM10}

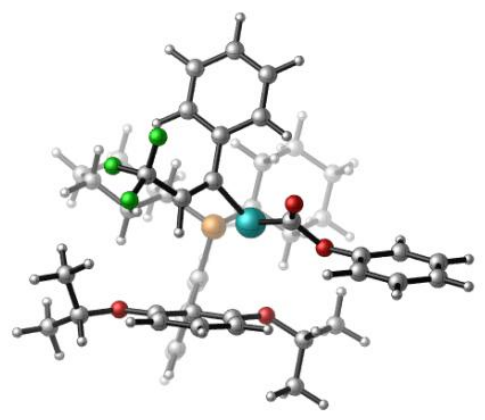

$\mathrm{E}(\mathrm{B} 3 \mathrm{LYP} / \mathrm{BS} 1)=-2853.924833$

$\mathrm{E}(\mathrm{PCM}$ (dichloroethane $) / \mathrm{M} 06 / \mathrm{BS} 2 / / \mathrm{B} 3 \mathrm{LYP} / \mathrm{BS} 1)=-2854.165500$

Zero-point correction=

0.908099

Thermal correction to Energy=

0.963972

Thermal correction to Enthalpy=

0.964916

Thermal correction to Gibbs Free Energy=

0.810891

$\begin{array}{lrrr}\text { Charge }= & \text { Multiplicity }=1 & \\ \mathrm{C} & 1.41447100 & -1.82227000 & 1.79333900 \\ \mathrm{C} & 1.95162600 & -2.22407100 & 3.03177600 \\ \mathrm{H} & 2.05844300 & -1.50042400 & 3.83224500 \\ \mathrm{C} & 2.35468000 & -3.53534500 & 3.27195800 \\ \mathrm{H} & 2.76276500 & -3.80798200 & 4.24152400 \\ \mathrm{C} & 2.23089600 & -4.48562200 & 2.25964900 \\ \mathrm{H} & 2.54293800 & -5.51337500 & 2.42529800 \\ \mathrm{C} & 1.69166400 & -4.11064800 & 1.03307200 \\ \mathrm{H} & 1.57983800 & -4.85014300 & 0.24549900 \\ \mathrm{C} & 1.26633500 & -2.79541400 & 0.77606700 \\ \mathrm{C} & 0.64339500 & -2.56697900 & -0.56933300 \\ \mathrm{C} & -0.75845200 & -2.52912400 & -0.70363100 \\ \mathrm{C} & -1.36509500 & -2.50914600 & -1.96773800 \\ \mathrm{H} & -2.44270500 & -2.46536400 & -2.06343200 \\ \mathrm{C} & -0.56295100 & -2.52007400 & -3.10307100 \\ \mathrm{H} & -1.02717100 & -2.49439600 & -4.08501200 \\ \mathrm{C} & 0.82801200 & -2.54562400 & -3.00848600 \\ \mathrm{H} & 1.42278200 & -2.53950300 & -3.91242700 \\ \mathrm{C} & 1.43182400 & -2.57469400 & -1.74339700 \\ \mathrm{C} & 3.68287500 & -2.79691600 & -2.65959100 \\ \mathrm{H} & 3.22399900 & -3.48756500 & -3.37915500 \\ \mathrm{C} & 3.98103000 & -1.45545600 & -3.33183100 \\ \mathrm{H} & 4.65240700 & -1.60693900 & -4.18507800 \\ \mathrm{H} & 4.47353700 & -0.77907800 & -2.62577100 \\ \mathrm{H} & 3.07875500 & -0.95735600 & -3.69706600 \\ \mathrm{C} & 4.93522900 & -3.44784700 & -2.08060100 \\ \mathrm{H} & 5.67027100 & -3.62775200 & -2.87250400 \\ \mathrm{H} & 4.69047600 & -4.40330300 & -1.60661000 \\ \mathrm{H} & 5.39125700 & -2.79671400 & -1.32703300 \\ \mathrm{C} & -2.79400100 & -3.08815500 & 0.52313700 \\ \mathrm{H} & -3.41999800 & -2.63487000 & -0.25177800 \\ \mathrm{C} & -3.36500600 & -2.71397500 & 1.88353300 \\ \mathrm{H} & -4.37267900 & -3.12946900 & 1.99049700 \\ \mathrm{H} & -3.42770400 & -1.62757400 & 1.98448300\end{array}$




\begin{tabular}{lrcc}
$\mathrm{H}$ & -2.73702800 & -3.11053700 & 2.68889200 \\
$\mathrm{C}$ & -2.70166100 & -4.59974900 & 0.31973200 \\
$\mathrm{H}$ & -3.70108400 & -5.04633500 & 0.36880600 \\
$\mathrm{H}$ & -2.08063200 & -5.05035500 & 1.10198100 \\
$\mathrm{H}$ & -2.26742000 & -4.84814800 & -0.65351600 \\
$\mathrm{C}$ & 0.29563500 & 0.52233100 & 3.20255600 \\
$\mathrm{H}$ & 1.02698200 & 0.27836700 & 3.98442100 \\
$\mathrm{C}$ & 0.07616700 & 2.05202700 & 3.22113000 \\
$\mathrm{H}$ & 1.01664500 & 2.58326100 & 3.03461200 \\
$\mathrm{H}$ & -0.60362400 & 2.32956100 & 2.40394900 \\
$\mathrm{C}$ & -0.51736100 & 2.51575600 & 4.56313200 \\
$\mathrm{H}$ & 0.21629100 & 2.34137400 & 5.36463000 \\
$\mathrm{H}$ & -0.69189300 & 3.59847700 & 4.53034800 \\
$\mathrm{C}$ & -1.81885400 & 1.77368500 & 4.89797100 \\
$\mathrm{H}$ & -2.58918100 & 2.04553500 & 4.16129900 \\
$\mathrm{H}$ & -2.19665700 & 2.09044400 & 5.87842300 \\
$\mathrm{C}$ & -1.61048300 & 0.25285800 & 4.87151600 \\
$\mathrm{H}$ & -2.55873800 & -0.26754900 & 5.05752200 \\
$\mathrm{H}$ & -0.93049400 & -0.03666400 & 5.68676400 \\
$\mathrm{C}$ & -1.02065100 & -0.21237900 & 3.53003500 \\
$\mathrm{H}$ & -1.74794600 & -0.01059500 & 2.72977000 \\
$\mathrm{H}$ & -0.86157600 & -1.29622500 & 3.53979900 \\
$\mathrm{C}$ & 2.62600900 & 0.84878400 & 1.37997100 \\
$\mathrm{H}$ & 2.32936900 & 1.89178300 & 1.20072900 \\
$\mathrm{C}$ & 3.53070800 & 0.81023500 & 2.62875700 \\
$\mathrm{H}$ & 3.80287300 & -0.23028400 & 2.84887300 \\
$\mathrm{H}$ & 3.00338900 & 1.19672200 & 3.50885000 \\
$\mathrm{C}$ & 4.81971900 & 1.62394200 & 2.41160600 \\
$\mathrm{H}$ & 4.56110200 & 2.68552500 & 2.28503000 \\
$\mathrm{H}$ & 2.31218900 & 5.18752300 & -0.11226900 \\
$\mathrm{H}$ & 0.29155700 & 5.87997700 & -0.85806500 \\
$\mathrm{H}$ & -1.86423100 & 5.78776600 & 0.20025400 \\
$\mathrm{H}$ & 2.42985000 & 5.66643400 & 0.01135900 \\
$\mathrm{C}$ & 5.44911700 & 1.56047800 & 3.30879500 \\
$\mathrm{H}$ & 5.59117100 & 1.13767800 & 1.17769200 \\
$\mathrm{C}$ & 5.94498700 & 0.11099100 & 1.35457300 \\
$\mathrm{H}$ & 6.48495900 & 1.75468200 & 1.01945700 \\
$\mathrm{C}$ & 4.69721100 & 1.15983300 & -0.06893300 \\
$\mathrm{H}$ & 5.24048700 & 0.76276700 & -0.93598100 \\
$\mathrm{H}$ & 4.44574800 & 2.20099700 & -0.31652900 \\
$\mathrm{C}$ & 3.39959000 & 0.35870400 & 0.13740200 \\
$\mathrm{H}$ & 3.64019800 & -0.70559900 & 0.25353400 \\
$\mathrm{H}$ & 2.77333000 & 0.44519300 & -0.75456200 \\
$\mathrm{O}$ & 2.78028100 & -2.63539100 & -1.54427700 \\
$\mathrm{O}$ & -1.47261800 & -2.48878800 & 0.46919700 \\
$\mathrm{P}$ & 0.96544800 & -0.03768200 & 1.53548200 \\
$\mathrm{Pd}$ & -0.86590100 & 0.50488600 & -0.08362100 \\
$\mathrm{C}$ & 0.10234500 & 1.83395800 & -1.24040800 \\
$\mathrm{H}$ & 0.69077200 & 1.30876400 & -2.32500200 \\
$\mathrm{C}$ & 0.60151400 & 0.25005200 & -2.54519100 \\
$\mathrm{H}$ & 1.54916000 & 2.02287700 & -3.32152700 \\
\hline
\end{tabular}




$\begin{array}{lccc}\text { H } & 0.34090300 & 6.89949700 & 0.57350600 \\ \mathrm{~F} & 1.16308700 & 3.28000600 & -3.60606400 \\ \mathrm{~F} & 1.57732500 & 1.33045900 & -4.48952800 \\ \mathrm{~F} & 2.85758800 & 2.10316500 & -2.91414600 \\ \mathrm{C} & -2.56367800 & 0.89843700 & -1.11666200 \\ \mathrm{O} & -2.91719800 & 1.56612400 & -2.05103400 \\ \mathrm{O} & -3.49915300 & 0.08737100 & -0.40116100 \\ \mathrm{C} & -4.83791600 & 0.03385700 & -0.78194700 \\ \mathrm{C} & -5.23007000 & -0.23881100 & -2.09556100 \\ \mathrm{C} & -5.79097200 & 0.19303900 & 0.22613600 \\ \mathrm{C} & -6.59009600 & -0.35155100 & -2.38931800 \\ \mathrm{H} & -4.48334400 & -0.34724700 & -2.87275600 \\ \mathrm{C} & -7.14721700 & 0.07119800 & -0.07910100 \\ \mathrm{H} & -5.45775900 & 0.40739100 & 1.23712500 \\ \mathrm{C} & -7.55199200 & -0.19972900 & -1.38792300 \\ \mathrm{H} & -6.89695800 & -0.55913200 & -3.41110600 \\ \mathrm{H} & -7.88682600 & 0.19244200 & 0.70806800 \\ \mathrm{H} & -8.60807000 & -0.29027800 & -1.62630000\end{array}$

\section{IM11}

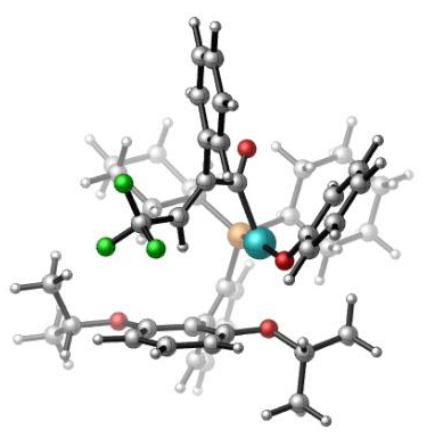

$\mathrm{E}(\mathrm{B} 3 \mathrm{LYP} / \mathrm{BS} 1)=-2853.945864$

$\mathrm{E}(\mathrm{PCM}$ (dichloroethane) $/ \mathrm{M} 06 / \mathrm{BS} 2 / / \mathrm{B} 3 \mathrm{LYP} / \mathrm{BS} 1)=-2854.181973$

Zero-point correction $=$

Thermal correction to Energy=

Thermal correction to Enthalpy=

Thermal correction to Gibbs Free Energy=
0.908896

0.964444

0.965388

0.813669

$\begin{array}{lrrr}\text { Charge }= & \text { Multiplicity }=1 \\ \mathrm{C} & 1.51128000 & 0.24433500 & 1.12772800 \\ \mathrm{C} & 2.32372000 & -1.01662900 & 0.84778100 \\ \mathrm{C} & 1.77374800 & -1.92475700 & 0.02586900 \\ \mathrm{H} & 0.84804700 & -1.68889500 & -0.49045100 \\ \mathrm{C} & -3.13440700 & 0.83166400 & -0.04646900 \\ \mathrm{C} & -4.29022000 & 1.53168500 & 0.35426100 \\ \mathrm{H} & -4.23933800 & 2.22566100 & 1.18497300 \\ \mathrm{C} & -5.51676400 & 1.36787800 & -0.28326700 \\ \mathrm{H} & -6.38258100 & 1.92943800 & 0.05682400 \\ \mathrm{C} & -5.61631300 & 0.48442300 & -1.35611200 \\ \mathrm{H} & -6.56193100 & 0.34474800 & -1.87299600 \\ \mathrm{C} & -4.48909400 & -0.22004000 & -1.76390400 \\ \mathrm{H} & -4.56106800 & -0.91137200 & -2.59806600 \\ \mathrm{C} & -3.23964000 & -0.06952500 & -1.13466300 \\ \mathrm{C} & -2.13808000 & -0.90783600 & -1.71248800\end{array}$




\begin{tabular}{|c|c|c|c|}
\hline $\mathrm{C}$ & -1.13504300 & -0.34759800 & -2.52621300 \\
\hline $\mathrm{C}$ & -0.22827400 & -1.15381800 & -3.22533100 \\
\hline $\mathrm{H}$ & 0.56511600 & -0.71098200 & -3.81083300 \\
\hline $\mathrm{C}$ & -0.31796300 & -2.53505400 & -3.09955200 \\
\hline $\mathrm{H}$ & 0.39748600 & -3.16509000 & -3.62001000 \\
\hline $\mathrm{C}$ & -1.28173600 & -3.12794400 & -2.28561100 \\
\hline $\mathrm{H}$ & -1.30304000 & -4.20491200 & -2.18746100 \\
\hline $\mathrm{C}$ & -2.19116100 & -2.31780500 & -1.59407500 \\
\hline $\mathrm{C}$ & -3.40213100 & -4.22190300 & -0.66506700 \\
\hline $\mathrm{H}$ & -3.27443700 & -4.67902200 & -1.65485600 \\
\hline $\mathrm{C}$ & -2.42659700 & -4.84733600 & 0.33403400 \\
\hline $\mathrm{H}$ & -2.57115300 & -5.93325700 & 0.37161400 \\
\hline $\mathrm{H}$ & -2.60571200 & -4.44471700 & 1.33683500 \\
\hline $\mathrm{H}$ & -1.38351200 & -4.65046800 & 0.07058500 \\
\hline $\mathrm{C}$ & -4.85693400 & -4.37593800 & -0.23369200 \\
\hline $\mathrm{H}$ & -5.11234100 & -5.43622000 & -0.13213200 \\
\hline $\mathrm{H}$ & -5.52885400 & -3.92322000 & -0.96919600 \\
\hline $\mathrm{H}$ & -5.02335200 & -3.88574200 & 0.73168500 \\
\hline $\mathrm{C}$ & -0.50055200 & 1.71025100 & -3.70263100 \\
\hline $\mathrm{H}$ & 0.51516700 & 1.33207600 & -3.84030300 \\
\hline $\mathrm{C}$ & -0.41556100 & 3.18298400 & -3.33050900 \\
\hline $\mathrm{H}$ & -0.01115300 & 3.75034100 & -4.17580300 \\
\hline $\mathrm{H}$ & 0.25346500 & 3.32455100 & -2.47741100 \\
\hline $\mathrm{H}$ & -1.40513800 & 3.58402500 & -3.08522800 \\
\hline $\mathrm{C}$ & -1.36728100 & 1.46372000 & -4.93521800 \\
\hline $\mathrm{H}$ & -0.93119700 & 1.97219000 & -5.80230000 \\
\hline $\mathrm{H}$ & -2.37804200 & 1.85630200 & -4.77634300 \\
\hline $\mathrm{H}$ & -1.44267800 & 0.39826300 & -5.17187800 \\
\hline $\mathrm{C}$ & -1.67970600 & 2.92623200 & 1.45217500 \\
\hline $\mathrm{H}$ & -2.63630200 & 3.05174200 & 1.97625800 \\
\hline $\mathrm{C}$ & -0.55888600 & 3.29697500 & 2.44969700 \\
\hline $\mathrm{H}$ & -0.59073000 & 2.64726900 & 3.33135800 \\
\hline $\mathrm{H}$ & 0.41818000 & 3.12903500 & 1.98309900 \\
\hline $\mathrm{C}$ & -0.67933800 & 4.76588300 & 2.89106000 \\
\hline $\mathrm{H}$ & -1.61177700 & 4.90164100 & 3.45994100 \\
\hline $\mathrm{H}$ & 0.14203800 & 5.00751500 & 3.57715300 \\
\hline $\mathrm{C}$ & -0.67343000 & 5.72349400 & 1.69182900 \\
\hline $\mathrm{H}$ & 0.30530600 & 5.67133100 & 1.19316600 \\
\hline $\mathrm{H}$ & -0.79906300 & 6.75994800 & 2.02984400 \\
\hline $\mathrm{C}$ & -1.77284600 & 5.35336100 & 0.68610000 \\
\hline $\mathrm{H}$ & -1.72318400 & 6.00652300 & -0.19438900 \\
\hline $\mathrm{H}$ & -2.75871800 & 5.52262300 & 1.14490800 \\
\hline $\mathrm{C}$ & -1.66357800 & 3.88484600 & 0.24287000 \\
\hline $\mathrm{H}$ & -0.72442000 & 3.73914900 & -0.30688100 \\
\hline $\mathrm{H}$ & -2.47475200 & 3.63984100 & -0.45229700 \\
\hline $\mathrm{C}$ & -1.70680800 & 0.12826400 & 2.47209900 \\
\hline $\mathrm{H}$ & -0.78303000 & 0.38523000 & 3.00714000 \\
\hline $\mathrm{C}$ & -2.90946600 & 0.48336700 & 3.37056700 \\
\hline $\mathrm{H}$ & -3.84296300 & 0.28207400 & 2.82818300 \\
\hline $\mathrm{H}$ & -2.90937500 & 1.55094000 & 3.62100000 \\
\hline $\mathrm{C}$ & -2.89327000 & -0.34032400 & 4.67137700 \\
\hline $\mathrm{H}$ & -2.01279900 & -0.05691800 & 5.26637600 \\
\hline $\mathrm{H}$ & -3.77373200 & -0.08787500 & 5.27618800 \\
\hline $\mathrm{C}$ & -2.84991800 & -1.84812100 & 4.38926300 \\
\hline $\mathrm{H}$ & -3.78640900 & -2.15197500 & 3.89797400 \\
\hline $\mathrm{H}$ & -2.79337000 & -2.40909400 & 5.33073100 \\
\hline $\mathrm{C}$ & -1.66301200 & -2.20236300 & 3.4829990 \\
\hline
\end{tabular}




$\begin{array}{lrrr}\mathrm{H} & -1.66719600 & -3.27422600 & 3.24749400 \\ \mathrm{H} & -0.72390800 & -2.00707100 & 4.02131000 \\ \mathrm{C} & -1.67655800 & -1.38595300 & 2.17934400 \\ \mathrm{H} & -2.55313700 & -1.66278500 & 1.57927700 \\ \mathrm{H} & -0.79571800 & -1.63661900 & 1.57846600 \\ \mathrm{O} & 1.57765400 & 0.80978700 & 2.19267000 \\ \mathrm{O} & -3.18092500 & -2.80136200 & -0.78559300 \\ \mathrm{O} & -1.07724400 & 1.03257100 & -2.54261900 \\ \mathrm{P} & -1.55641100 & 1.13267000 & 0.89147500 \\ \mathrm{Pd} & 0.40232100 & 0.88378300 & -0.39468300 \\ \mathrm{C} & 3.64088900 & -1.13520300 & 1.52910400 \\ \mathrm{C} & 3.75350400 & -1.08526300 & 2.92703000 \\ \mathrm{C} & 4.80263800 & -1.26350200 & 0.75134500 \\ \mathrm{C} & 5.00467400 & -1.19038800 & 3.53347000 \\ \mathrm{H} & 2.86332000 & -0.97049200 & 3.53518400 \\ \mathrm{C} & 6.05168300 & -1.35765300 & 1.36301300 \\ \mathrm{H} & 4.72318600 & -1.26369700 & -0.33141400 \\ \mathrm{C} & 6.15617100 & -1.32602400 & 2.75476400 \\ \mathrm{H} & 5.07959900 & -1.16267400 & 4.61738200 \\ \mathrm{H} & 6.94321500 & -1.44758600 & 0.74869200 \\ \mathrm{H} & 7.13048300 & -1.39963400 & 3.23084300 \\ \mathrm{C} & 2.29621400 & -3.28665800 & -0.31272600 \\ \mathrm{~F} & 2.94187700 & -3.28134600 & -1.50410800 \\ \mathrm{~F} & 1.25587100 & -4.15499000 & -0.44192600 \\ \mathrm{~F} & 3.13194900 & -3.81279500 & 0.59803300 \\ \mathrm{O} & 1.91117400 & 0.88673700 & -1.79605200 \\ \mathrm{C} & 3.17025500 & 1.29499700 & -1.67685700 \\ \mathrm{C} & 4.13964600 & 0.79881100 & -2.58331300 \\ \mathrm{C} & 3.60586800 & 2.22130600 & -0.70094900 \\ \mathrm{C} & 5.46711100 & 1.21507800 & -2.51536200 \\ \mathrm{H} & 3.81589100 & 0.08651000 & -3.33812300 \\ \mathrm{C} & 4.93945700 & 2.62295900 & -0.63675200 \\ \mathrm{H} & 2.87672200 & 2.61437300 & 0.00385900 \\ \mathrm{C} & 5.88349600 & 2.12716900 & -1.53991800 \\ \mathrm{H} & 6.18657200 & 0.81785100 & -3.22892100 \\ \mathrm{H} & 5.24337100 & 3.33234600 & 0.13031600 \\ \mathrm{H} & 6.92108100 & 2.44497600 & -1.48590100\end{array}$

\section{TS10}

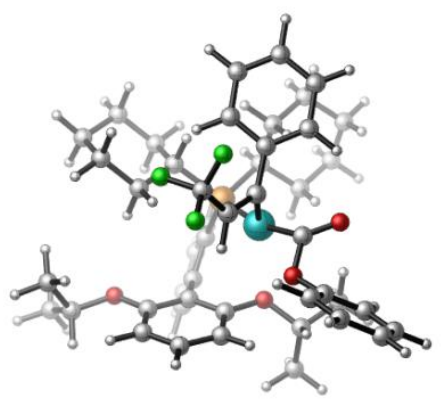

$\mathrm{E}(\mathrm{B} 3 \mathrm{LYP} / \mathrm{BS} 1)=-2853.912876$

$\mathrm{E}(\mathrm{PCM}$ (dichloroethane)/M06/BS2// B3LYP/BS1 $)=-2854.153289$

Zero-point correction=

Thermal correction to Energy=
0.907297

0.962623 
Thermal correction to Enthalpy= $\quad 0.963567$

Thermal correction to Gibbs Free Energy $=\quad 0.810133$

Charge $=0$ Multiplicity $=1$

C $\quad-2.89777300 \quad-0.14358200 \quad-1.37427800$

$\begin{array}{lllll}\mathrm{C} & & -3.93749100 & 0.38121200 & -2.16647300\end{array}$

$\mathrm{H} \quad-3.97370100 \quad 1.44550200 \quad-2.36883000$

$\begin{array}{llll}\mathrm{C} & -4.93548700 & -0.42241400 & -2.71215500\end{array}$

$\begin{array}{lllll}\mathrm{H} & -5.71773600 & 0.02380100 & -3.32053400\end{array}$

C $\quad-4.91628700 \quad-1.79517100 \quad-2.47288100$

$\mathrm{H} \quad-5.68055100 \quad-2.44209100 \quad-2.89549500$

C $\quad-3.90597100 \quad-2.33323200 \quad-1.68274100$

$\mathrm{H} \quad-3.88924000 \quad-3.40114200 \quad-1.48519400$

C $\quad \begin{array}{llll}\text { C } & -2.89046500 & -1.53741300 & -1.12309500\end{array}$

$\begin{array}{llll}\text { C } & -1.89117000 & -2.27053900 & -0.27813900\end{array}$

$\begin{array}{llll}\text { C } & -0.59626800 & -2.56130500 & -0.74418500\end{array}$

$\begin{array}{llll}\text { C } & 0.26939300 & -3.39030400 & -0.01790800\end{array}$

$\begin{array}{llll}\mathrm{H} & 1.27281200 & -3.58698400 & -0.37158600\end{array}$

$\begin{array}{llll}\mathrm{C} & -0.16206000 & -3.92706600 & 1.18964800\end{array}$

$\begin{array}{llll}\mathrm{H} & 0.50858100 & -4.56374600 & 1.76030300\end{array}$

$\begin{array}{lllll}\text { C } & & -1.43401900 & -3.65285300 & 1.69069300\end{array}$

$\begin{array}{llll}\mathrm{H} & -1.73639000 & -4.07453600 & 2.64077100\end{array}$

$\begin{array}{llll}\text { C } & -2.29808400 & -2.83206300 & 0.95558200\end{array}$

$\begin{array}{llll}\text { C } & -4.19716700 & -3.21538000 & 2.44486600\end{array}$

$\begin{array}{llll}\mathrm{H} & -3.84641900 & -4.25560000 & 2.44705900\end{array}$

C $\quad \begin{array}{llll}\text { C } & -3.85832600 & -2.54297000 & 3.77616400\end{array}$

$\begin{array}{lllll}\mathrm{H} & -4.31462100 & -3.09417600 & 4.60653300\end{array}$

$\begin{array}{llll}\mathrm{H} & -4.24555600 & -1.51841400 & 3.79053700\end{array}$

$\mathrm{H} \quad-2.77860300 \quad-2.49989400 \quad 3.94595300$

$\begin{array}{llll}\text { C } & -5.69373300 & -3.19458400 & 2.14846700\end{array}$

$\mathrm{H} \quad-6.24641800 \quad-3.71186900 \quad 2.94030900$

$\mathrm{H} \quad \begin{array}{llll}\mathrm{H} & -5.90567600 & -3.68659100 & 1.19442700\end{array}$

$\mathrm{H} \quad \begin{array}{llll}-6.05571700 & -2.16245800 & 2.08998100\end{array}$

C $\quad 0.67457400 \quad-2.64265800 \quad-2.83536300$

$\begin{array}{llll}\mathrm{H} & 1.58932300 & -2.90382800 & -2.29136800\end{array}$

C $\quad 1.02152100 \quad-1.62800400 \quad-3.91705700$

$\mathrm{H} \quad \begin{array}{llll}\mathrm{H} & 1.70351200 & -2.07889400 & -4.64646800\end{array}$

$\mathrm{H} \quad \begin{array}{llll}1.51036500 & -0.75041600 & -3.48289800\end{array}$

$\mathrm{H} \quad \begin{array}{llll}0.11739100 & -1.30257900 & -4.44281500\end{array}$

C $\quad \begin{array}{llll}0.01357300 & -3.90230700 & -3.39145100\end{array}$

$\mathrm{H} \quad 0.69775000 \quad-4.41487900 \quad-4.07700900$

$\mathrm{H} \quad-0.89807000 \quad-3.64123400 \quad-3.94017600$

$\mathrm{H} \quad-0.25057100 \quad-4.60044400 \quad-2.59111100$

C $\quad \begin{array}{llll}-1.52183900 & 2.38889400 & -1.99322800\end{array}$

$\begin{array}{llll}\mathrm{H} & -2.53471000 & 2.79132300 & -2.12905700\end{array}$

C $\quad \begin{array}{llll}-0.62515200 & 3.54988700 & -1.50909900\end{array}$

$\mathrm{H} \quad \begin{array}{llll}\mathrm{H} & -0.99992300 & 3.96535400 & -0.56696700\end{array}$

$\begin{array}{llll}\mathrm{H} & 0.38308200 & 3.16929200 & -1.30184600\end{array}$

C $\quad \begin{array}{lllll}-0.54107300 & 4.67140300 & -2.55922400\end{array}$

$\mathrm{H} \quad \begin{array}{llll}\mathrm{H} & -1.53247300 & 5.13396700 & -2.68072300\end{array}$

$\begin{array}{llll}\mathrm{H} & 0.12946500 & 5.45895100 & -2.19323500\end{array}$

$\begin{array}{lllll}\text { C } & & -0.05773200 & 4.14538500 & -3.91772200\end{array}$

$\mathrm{H} \quad 0.98010500 \quad 3.79511200 \quad-3.82041100$

$\begin{array}{lllll}\mathrm{H} & -0.04874500 & 4.95456700 & -4.65938700\end{array}$

$\begin{array}{lllll}\mathrm{C} & & -0.93772000 & 2.98521800 & -4.40339600\end{array}$

$\mathrm{H} \quad-\begin{array}{llll}\mathrm{H} & -5.55061400 & 2.57979600 & -5.34719100\end{array}$

$\begin{array}{llll}\mathrm{H} & -1.95025200 & 3.36002800 & -4.61701600\end{array}$ 


\begin{tabular}{|c|c|c|c|}
\hline $\mathrm{C}$ & -1.02106200 & 1.86258200 & -3.35564500 \\
\hline $\mathrm{H}$ & -0.02327100 & 1.42319400 & -3.21549300 \\
\hline $\mathrm{H}$ & -1.67067700 & 1.05785600 & -3.71876800 \\
\hline C & -2.32834000 & 1.80211100 & 0.80393100 \\
\hline $\mathrm{H}$ & -1.58167400 & 2.56661800 & 1.06372600 \\
\hline $\mathrm{C}$ & -3.68864200 & 2.49682200 & 0.60265500 \\
\hline $\mathrm{H}$ & -4.43255400 & 1.75243400 & 0.28850000 \\
\hline $\mathrm{H}$ & -3.62973800 & 3.24355200 & -0.19886600 \\
\hline C & -4.16878500 & 3.17648300 & 1.89839300 \\
\hline $\mathrm{H}$ & -3.48296100 & 3.99883100 & 2.15068700 \\
\hline $\mathrm{H}$ & -5.15408400 & 3.63184900 & 1.73375200 \\
\hline $\mathrm{C}$ & -4.22433300 & 2.18722400 & 3.07090100 \\
\hline $\mathrm{H}$ & -4.99666200 & 1.43015400 & 2.86741800 \\
\hline $\mathrm{H}$ & -4.52683500 & 2.70422200 & 3.99070000 \\
\hline $\mathrm{C}$ & -2.87105500 & 1.48924700 & 3.26826800 \\
\hline $\mathrm{H}$ & -2.93530000 & 0.75106400 & 4.07810400 \\
\hline $\mathrm{H}$ & -2.12328500 & 2.23154200 & 3.58505900 \\
\hline $\mathrm{C}$ & -2.39132900 & 0.80339600 & 1.97755200 \\
\hline $\mathrm{H}$ & -3.07766500 & -0.01508100 & 1.72500700 \\
\hline $\mathrm{H}$ & -1.40500200 & 0.35133700 & 2.13575400 \\
\hline $\mathrm{O}$ & -3.57166400 & -2.52374600 & 1.34579200 \\
\hline $\mathrm{O}$ & -0.22910800 & -1.95784800 & -1.92873100 \\
\hline$P$ & -1.57273000 & 0.99532800 & -0.72354300 \\
\hline $\mathrm{Pd}$ & 0.72497200 & 0.16313200 & -0.45724900 \\
\hline $\mathrm{C}$ & 2.10659900 & 0.85990300 & 1.00007100 \\
\hline $\mathrm{C}$ & 2.23286300 & 0.07550500 & 2.09289300 \\
\hline $\mathrm{H}$ & 2.10517000 & -0.99642600 & 2.00230300 \\
\hline $\mathrm{C}$ & 2.59487500 & 0.48982400 & 3.48491600 \\
\hline $\mathrm{C}$ & 2.20274700 & 2.34133600 & 1.00016300 \\
\hline $\mathrm{C}$ & 3.07578600 & 3.05526200 & 0.16008400 \\
\hline C & 1.36487700 & 3.07149500 & 1.86837300 \\
\hline $\mathrm{C}$ & 3.10575900 & 4.45037900 & 0.19303400 \\
\hline $\mathrm{H}$ & 3.72401900 & 2.51363800 & -0.51649700 \\
\hline $\mathrm{C}$ & 1.39862900 & 4.46349700 & 1.89499500 \\
\hline $\mathrm{H}$ & 0.69288600 & 2.53442300 & 2.53056900 \\
\hline $\mathrm{C}$ & 2.26901700 & 5.16215700 & 1.05421000 \\
\hline $\mathrm{H}$ & 3.79410900 & 4.98221600 & -0.45898000 \\
\hline $\mathrm{H}$ & 0.74723300 & 5.00318600 & 2.57789400 \\
\hline $\mathrm{H}$ & 2.29783600 & 6.24842800 & 1.07518700 \\
\hline $\mathrm{F}$ & 3.38449900 & 1.57733100 & 3.55757500 \\
\hline $\mathrm{F}$ & 3.25863100 & -0.52628600 & 4.09835200 \\
\hline $\mathrm{F}$ & 1.49793600 & 0.74350300 & 4.25210200 \\
\hline $\mathrm{C}$ & 2.74683400 & -0.12577600 & -0.52988900 \\
\hline $\mathrm{O}$ & 3.41065500 & 0.46937100 & -1.35473400 \\
\hline $\mathrm{O}$ & 3.06262300 & -1.42044700 & -0.12247900 \\
\hline $\mathrm{C}$ & 4.36212700 & -1.90250300 & -0.31272500 \\
\hline C & 5.10572500 & -2.18271500 & 0.83368400 \\
\hline $\mathrm{C}$ & 4.87108500 & -2.15795700 & -1.58576400 \\
\hline $\mathrm{C}$ & 6.38072900 & -2.73481900 & 0.70079800 \\
\hline $\mathrm{H}$ & 4.68348300 & -1.95941000 & 1.80904000 \\
\hline $\mathrm{C}$ & 6.14915800 & -2.70729700 & -1.70497200 \\
\hline $\mathrm{H}$ & 4.27850700 & -1.92144300 & -2.46189700 \\
\hline C & 6.90479600 & -2.99860000 & -0.56686100 \\
\hline $\mathrm{H}$ & 6.96449900 & -2.95556200 & 1.59031300 \\
\hline $\mathrm{H}$ & 6.55449800 & -2.90678200 & -2.69337700 \\
\hline $\mathrm{H}$ & 7.89787100 & -3.42757500 & -0.66766400 \\
\hline
\end{tabular}




\section{TS11}

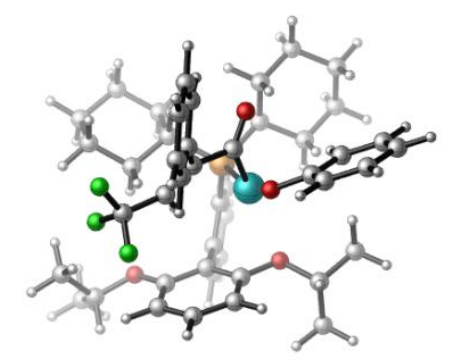

$\mathrm{E}(\mathrm{B} 3 \mathrm{LYP} / \mathrm{BS} 1)=-2853.929766$

$\mathrm{E}(\mathrm{PCM}$ (dichloroethane $) / \mathrm{M} 06 / \mathrm{BS} 2 / / \mathrm{B} 3 \mathrm{LYP} / \mathrm{BS} 1)=-2854.162301$

Zero-point correction=

0.908134

Thermal correction to Energy=

0.963149

Thermal correction to Enthalpy=

0.964094

Thermal correction to Gibbs Free Energy=

0.812008

$\begin{array}{lrrr}\text { Charge }= & \text { Multiplicity }=1 & & \\ \mathrm{C} & -2.24086400 & 0.17171400 & 0.63634700 \\ \mathrm{C} & -2.30909100 & 1.68473000 & 0.45843400 \\ \mathrm{C} & -1.21627500 & 2.34153100 & 0.04142200 \\ \mathrm{H} & -0.33075500 & 1.77091400 & -0.22301300 \\ \mathrm{C} & 2.87035100 & -1.35556000 & 0.24959900 \\ \mathrm{C} & 3.82320500 & -2.19754500 & 0.85556200 \\ \mathrm{H} & 3.59860200 & -2.66656300 & 1.80668200 \\ \mathrm{C} & 5.06007700 & -2.46108400 & 0.27193700 \\ \mathrm{H} & 5.76563300 & -3.11882900 & 0.77250600 \\ \mathrm{C} & 5.37688300 & -1.87843600 & -0.95352700 \\ \mathrm{H} & 6.33412600 & -2.07499400 & -1.42909800 \\ \mathrm{C} & 4.45270100 & -1.03892900 & -1.56715700 \\ \mathrm{H} & 4.69437900 & -0.57809000 & -2.52056500 \\ \mathrm{C} & 3.19941100 & -0.76123700 & -0.99442200 \\ \mathrm{C} & 2.32380800 & 0.17439900 & -1.77471400 \\ \mathrm{C} & 1.26357600 & -0.30419800 & -2.56938300 \\ \mathrm{C} & 0.52817800 & 0.55899300 & -3.39310400 \\ \mathrm{H} & -0.29802100 & 0.19091000 & -3.98829500 \\ \mathrm{C} & 0.85194500 & 1.91228200 & -3.41611500 \\ \mathrm{H} & 0.26999000 & 2.58897900 & -4.03523400 \\ \mathrm{C} & 1.90052900 & 2.41955700 & -2.65205400 \\ \mathrm{H} & 2.12106800 & 3.47865900 & -2.68296100 \\ \mathrm{C} & 2.64758700 & 1.54842900 & -1.84636600 \\ \mathrm{C} & 4.34730400 & 3.22006500 & -1.32345300 \\ \mathrm{H} & 4.23889300 & 3.47518600 & -2.38536800 \\ \mathrm{C} & 3.70522500 & 4.30117700 & -0.45410700 \\ \mathrm{H} & 4.15442200 & 5.27711400 & -0.67237300 \\ \mathrm{H} & 3.87008000 & 4.07896100 & 0.60590800 \\ \mathrm{H} & 2.62722200 & 4.37267100 & -0.61857900 \\ \mathrm{C} & 5.82661900 & 3.02057200 & -1.00735700 \\ \mathrm{H} & 6.37768000 & 3.95497300 & -1.16039000 \\ \mathrm{H} & 6.26028300 & 2.24913700 & -1.65098600 \\ \mathrm{H} & 5.95485600 & 2.71000100 & 0.03520300 \\ \mathrm{C} & 0.36421200 & -2.35969300 & -3.55011000\end{array}$




\begin{tabular}{|c|c|c|c|}
\hline $\mathrm{H}$ & -0.56151600 & -1.84110400 & -3.82662100 \\
\hline $\mathrm{C}$ & 0.00516900 & -3.73416100 & -3.00105400 \\
\hline $\mathrm{H}$ & -0.46152300 & -4.33880100 & -3.78610400 \\
\hline $\mathrm{H}$ & -0.70134400 & -3.64647500 & -2.17075000 \\
\hline $\mathrm{H}$ & 0.90281700 & -4.25303700 & -2.64779500 \\
\hline $\mathrm{C}$ & 1.30930500 & -2.43009600 & -4.74866500 \\
\hline $\mathrm{H}$ & 0.82974900 & -2.96641100 & -5.57515400 \\
\hline $\mathrm{H}$ & 2.22698400 & -2.96252800 & -4.47509700 \\
\hline $\mathrm{H}$ & 1.58082800 & -1.43136200 & -5.10376900 \\
\hline $\mathrm{C}$ & 0.92695200 & -2.69747400 & 2.02567300 \\
\hline $\mathrm{H}$ & 1.80231100 & -2.91785500 & 2.65080800 \\
\hline $\mathrm{C}$ & -0.29549800 & -2.56909600 & 2.96357900 \\
\hline $\mathrm{H}$ & -0.13208700 & -1.77531100 & 3.70235400 \\
\hline $\mathrm{H}$ & -1.17666200 & -2.27149200 & 2.38267000 \\
\hline $\mathrm{C}$ & -0.57761700 & -3.89225000 & 3.69707400 \\
\hline $\mathrm{H}$ & 0.25743000 & -4.11941800 & 4.37737600 \\
\hline $\mathrm{H}$ & -1.46980100 & -3.77683400 & 4.32512800 \\
\hline $\mathrm{C}$ & -0.76159100 & -5.05936500 & 2.71740700 \\
\hline $\mathrm{H}$ & -1.66639200 & -4.88612700 & 2.11657700 \\
\hline $\mathrm{H}$ & -0.92320500 & -5.99638000 & 3.26564600 \\
\hline $\mathrm{C}$ & 0.44937900 & -5.18946900 & 1.78296200 \\
\hline $\mathrm{H}$ & 0.28692100 & -5.99310700 & 1.05302400 \\
\hline $\mathrm{H}$ & 1.33379600 & -5.47738200 & 2.37121400 \\
\hline $\mathrm{C}$ & 0.73657600 & -3.87213700 & 1.04337700 \\
\hline $\mathrm{H}$ & -0.10449200 & -3.63917100 & 0.37594000 \\
\hline $\mathrm{H}$ & 1.61997100 & -3.98381900 & 0.40414500 \\
\hline $\mathrm{C}$ & 1.59644400 & 0.15653400 & 2.48087800 \\
\hline $\mathrm{H}$ & 0.61084600 & 0.28728600 & 2.95134500 \\
\hline $\mathrm{C}$ & 2.59240900 & -0.31984000 & 3.55879700 \\
\hline $\mathrm{H}$ & 3.57147100 & -0.49552100 & 3.09292900 \\
\hline $\mathrm{H}$ & 2.27217700 & -1.27148700 & 3.99783900 \\
\hline $\mathrm{C}$ & 2.74842100 & 0.72378600 & 4.68064300 \\
\hline $\mathrm{H}$ & 1.79281900 & 0.82132300 & 5.21660300 \\
\hline $\mathrm{H}$ & 3.48253900 & 0.36650400 & 5.41452700 \\
\hline $\mathrm{C}$ & 3.16216200 & 2.09478500 & 4.12958700 \\
\hline $\mathrm{H}$ & 4.17306200 & 2.02238100 & 3.70122000 \\
\hline $\mathrm{H}$ & 3.21758600 & 2.83043300 & 4.94222900 \\
\hline $\mathrm{C}$ & 2.18460800 & 2.56509700 & 3.04460300 \\
\hline $\mathrm{H}$ & 2.51310100 & 3.52116500 & 2.61788100 \\
\hline $\mathrm{H}$ & 1.19960600 & 2.75118400 & 3.49744100 \\
\hline $\mathrm{C}$ & 2.03579100 & 1.52446100 & 1.92152800 \\
\hline $\mathrm{H}$ & 2.99152300 & 1.41467600 & 1.39380000 \\
\hline $\mathrm{H}$ & 1.31482500 & 1.88974800 & 1.18616000 \\
\hline $\mathrm{O}$ & -2.74247500 & -0.43167000 & 1.55948900 \\
\hline $\mathrm{O}$ & 3.72074600 & 1.93912900 & -1.09769200 \\
\hline $\mathrm{O}$ & 0.99255800 & -1.64874100 & -2.45363600 \\
\hline $\mathrm{P}$ & 1.23366200 & -1.08315800 & 1.10144000 \\
\hline $\mathrm{Pd}$ & -0.59278300 & -0.72114400 & -0.30438900 \\
\hline $\mathrm{C}$ & -3.64252600 & 2.29080600 & 0.72662900 \\
\hline $\mathrm{C}$ & -4.35262400 & 2.01354200 & 1.90612000 \\
\hline $\mathrm{C}$ & -4.23048900 & 3.12730900 & -0.23814800 \\
\hline $\mathrm{C}$ & -5.60771800 & 2.58165300 & 2.12246100 \\
\hline $\mathrm{H}$ & -3.92003500 & 1.34986100 & 2.64504300 \\
\hline C & -5.48924900 & 3.68486100 & -0.02125000 \\
\hline $\mathrm{H}$ & -3.69643600 & 3.33623500 & -1.15946800 \\
\hline $\mathrm{C}$ & -6.18112200 & 3.41666800 & 1.16167100 \\
\hline $\mathrm{H}$ & -6.14107700 & 2.36510400 & 3.04423 \\
\hline
\end{tabular}




$\begin{array}{lrrr}\text { H } & -5.93007100 & 4.32696900 & -0.77922200 \\ \mathrm{H} & -7.16274700 & 3.85143800 & 1.33098800 \\ \mathrm{C} & -1.03692200 & 3.81291900 & -0.14121700 \\ \mathrm{~F} & -1.28629300 & 4.20591200 & -1.41968800 \\ \mathrm{~F} & 0.26116100 & 4.14513200 & 0.10908600 \\ \mathrm{~F} & -1.79340200 & 4.57953900 & 0.66440900 \\ \mathrm{O} & -2.64705300 & -0.32809300 & -0.97248200 \\ \mathrm{C} & -3.59449600 & -1.31302300 & -1.07020400 \\ \mathrm{C} & -4.84157400 & -0.96456700 & -1.60942100 \\ \mathrm{C} & -3.35814900 & -2.63404700 & -0.67033200 \\ \mathrm{C} & -5.82736100 & -1.93660000 & -1.76956200 \\ \mathrm{H} & -5.01492400 & 0.06833500 & -1.89572600 \\ \mathrm{C} & -4.35421500 & -3.59957000 & -0.82810500 \\ \mathrm{H} & -2.39567700 & -2.88623200 & -0.23455900 \\ \mathrm{C} & -5.58978800 & -3.25865500 & -1.38132000 \\ \mathrm{H} & -6.78798400 & -1.65895700 & -2.19633300 \\ \mathrm{H} & -4.16149900 & -4.62284600 & -0.51482400 \\ \mathrm{H} & -6.36159200 & -4.01333000 & -1.50542700\end{array}$

\section{IM12}

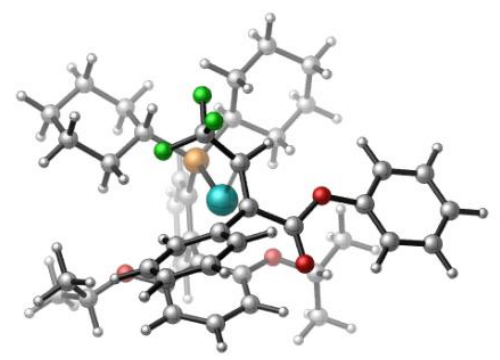

$\mathrm{E}(\mathrm{B} 3 \mathrm{LYP} / \mathrm{BS} 1)=-2853.982018$

$\mathrm{E}(\mathrm{PCM}$ (dichloroethane) $/ \mathrm{M} 06 / \mathrm{BS} 2 / / \mathrm{B} 3 \mathrm{LYP} / \mathrm{BS} 1)=-2854.224333$

Zero-point correction $=$

Thermal correction to Energy=

Thermal correction to Enthalpy=

Thermal correction to Gibbs Free Energy=
0.910381

0.965500

0.966444

0.813645

$\begin{array}{crrr}\text { Charge }= & \text { Multiplicity }=1 & \\ \mathrm{C} & 2.81575500 & -1.71235500 & 0.30034300 \\ \mathrm{C} & 1.53046600 & -2.01012800 & 0.98033200 \\ \mathrm{C} & 1.06552100 & -1.12014000 & 1.99848200 \\ \mathrm{H} & 1.75659000 & -0.35298900 & 2.33394600 \\ \mathrm{C} & -1.87235900 & 2.13548900 & -1.02208600 \\ \mathrm{C} & -2.52844000 & 3.37645100 & -1.12264100 \\ \mathrm{H} & -2.70140700 & 3.96814800 & -0.22980600 \\ \mathrm{C} & -2.96139100 & 3.87760000 & -2.34777900 \\ \mathrm{H} & -3.46582700 & 4.83914300 & -2.39378200 \\ \mathrm{C} & -2.73835500 & 3.13775000 & -3.50933600 \\ \mathrm{H} & -3.06699700 & 3.51527900 & -4.47409000 \\ \mathrm{C} & -2.08324400 & 1.91245300 & -3.42848000 \\ \mathrm{H} & -1.90091900 & 1.33755600 & -4.33225700 \\ \mathrm{C} & -1.63847600 & 1.39381400 & -2.20056100 \\ \mathrm{C} & -0.93504400 & 0.06522900 & -2.25928600\end{array}$




\begin{tabular}{|c|c|c|c|}
\hline $\mathrm{C}$ & 0.46266300 & 0.00197900 & -2.50377200 \\
\hline $\mathrm{C}$ & 1.07588900 & -1.22411200 & -2.82604500 \\
\hline $\mathrm{H}$ & 2.14675500 & -1.29469800 & -2.95830800 \\
\hline $\mathrm{C}$ & 0.29223600 & -2.36293700 & -2.96114300 \\
\hline $\mathrm{H}$ & 0.77176200 & -3.30735600 & -3.19954500 \\
\hline $\mathrm{C}$ & -1.09035700 & -2.32704600 & -2.77730200 \\
\hline $\mathrm{H}$ & -1.66668700 & -3.23639400 & -2.88749700 \\
\hline $\mathrm{C}$ & -1.70183400 & -1.11889400 & -2.42789600 \\
\hline $\mathrm{C}$ & -3.96765800 & -1.99140800 & -2.66924300 \\
\hline $\mathrm{H}$ & -3.55778400 & -2.49224400 & -3.55581700 \\
\hline $\mathrm{C}$ & -4.18051500 & -3.00495500 & -1.54409900 \\
\hline $\mathrm{H}$ & -4.87047000 & -3.79219800 & -1.86925600 \\
\hline $\mathrm{H}$ & -4.61021700 & -2.51143400 & -0.66598200 \\
\hline $\mathrm{H}$ & -3.24152200 & -3.47760000 & -1.24155100 \\
\hline C & -5.25544200 & -1.27014200 & -3.05561000 \\
\hline $\mathrm{H}$ & -6.00523600 & -1.98952300 & -3.40257900 \\
\hline $\mathrm{H}$ & -5.06668100 & -0.54701200 & -3.85472400 \\
\hline $\mathrm{H}$ & -5.66557700 & -0.73110700 & -2.19471900 \\
\hline $\mathrm{C}$ & 2.46773600 & 1.31249900 & -2.97746400 \\
\hline $\mathrm{H}$ & 3.07846700 & 0.50906700 & -2.54958900 \\
\hline $\mathrm{C}$ & 2.99078700 & 2.64796100 & -2.46391000 \\
\hline $\mathrm{H}$ & 4.01737300 & 2.80429000 & -2.81137100 \\
\hline $\mathrm{H}$ & 2.99349500 & 2.66765500 & -1.37132700 \\
\hline $\mathrm{H}$ & 2.36701000 & 3.47049900 & -2.83113700 \\
\hline $\mathrm{C}$ & 2.47381800 & 1.23662700 & -4.50490000 \\
\hline $\mathrm{H}$ & 3.49908700 & 1.32444200 & -4.88151100 \\
\hline $\mathrm{H}$ & 1.88010200 & 2.05712600 & -4.92327600 \\
\hline $\mathrm{H}$ & 2.05895800 & 0.29128600 & -4.86641900 \\
\hline $\mathrm{C}$ & -0.49282500 & 3.00868400 & 1.39597500 \\
\hline $\mathrm{H}$ & -1.21225300 & 3.83571500 & 1.31265000 \\
\hline $\mathrm{C}$ & -0.18465400 & 2.80223800 & 2.89391300 \\
\hline $\mathrm{H}$ & -1.10233600 & 2.58316400 & 3.45173500 \\
\hline $\mathrm{H}$ & 0.46616200 & 1.92791300 & 3.01768600 \\
\hline $\mathrm{C}$ & 0.49504000 & 4.04168700 & 3.50307900 \\
\hline $\mathrm{H}$ & -0.21222900 & 4.88464900 & 3.49200900 \\
\hline $\mathrm{H}$ & 0.73250300 & 3.84862200 & 4.55700400 \\
\hline $\mathrm{C}$ & 1.76154200 & 4.43796600 & 2.73204600 \\
\hline $\mathrm{H}$ & 2.51396100 & 3.64221400 & 2.84106200 \\
\hline $\mathrm{H}$ & 2.20210600 & 5.34586400 & 3.16362500 \\
\hline $\mathrm{C}$ & 1.45695000 & 4.64462600 & 1.24176900 \\
\hline $\mathrm{H}$ & 2.37770100 & 4.87613600 & 0.69071600 \\
\hline $\mathrm{H}$ & 0.79602300 & 5.51640400 & 1.12364500 \\
\hline $\mathrm{C}$ & 0.78429500 & 3.40637000 & 0.62738400 \\
\hline $\mathrm{H}$ & 1.48393300 & 2.55925500 & 0.64967100 \\
\hline $\mathrm{H}$ & 0.55138500 & 3.58549300 & -0.42797600 \\
\hline $\mathrm{C}$ & -2.78504500 & 1.12684700 & 1.65748100 \\
\hline $\mathrm{H}$ & -2.33588900 & 0.86393800 & 2.62650400 \\
\hline $\mathrm{C}$ & -3.77651900 & 2.28564400 & 1.87687500 \\
\hline $\mathrm{H}$ & -4.21174700 & 2.57792100 & 0.91222800 \\
\hline $\mathrm{H}$ & -3.26469400 & 3.16987100 & 2.27735200 \\
\hline $\mathrm{C}$ & -4.91357900 & 1.87308900 & 2.82977900 \\
\hline $\mathrm{H}$ & -4.49525300 & 1.66881700 & 3.82634300 \\
\hline $\mathrm{H}$ & -5.61634600 & 2.70782600 & 2.95042000 \\
\hline C & -5.64679200 & 0.62415800 & 2.32083400 \\
\hline $\mathrm{H}$ & -6.15886100 & 0.86650500 & 1.37750400 \\
\hline $\mathrm{H}$ & -6.42656200 & 0.32427700 & 3.03264800 \\
\hline $\mathrm{C}$ & -4.66579900 & -0.53265000 & 2.083089 \\
\hline
\end{tabular}




$\begin{array}{lrrc}\mathrm{H} & -5.19520000 & -1.40223000 & 1.67109500 \\ \mathrm{H} & -4.24276300 & -0.85504400 & 3.04562200 \\ \mathrm{C} & -3.51762700 & -0.12903100 & 1.14239700 \\ \mathrm{H} & -3.91011900 & 0.06382300 & 0.13531700 \\ \mathrm{H} & -2.80398100 & -0.95490100 & 1.05024400 \\ \mathrm{O} & 3.30595100 & -2.38169400 & -0.58993300 \\ \mathrm{O} & -3.04685700 & -0.95819100 & -2.26361500 \\ \mathrm{O} & 1.11681600 & 1.19791500 & -2.46976100 \\ \mathrm{P} & -1.26610700 & 1.48658200 & 0.60352200 \\ \mathrm{Pd} & 0.14003700 & -0.37640300 & 0.28386800 \\ \mathrm{C} & 1.05779000 & -3.42949900 & 0.83316000 \\ \mathrm{C} & 1.93782900 & -4.48282800 & 1.13603900 \\ \mathrm{C} & -0.24401800 & -3.75482500 & 0.42864500 \\ \mathrm{C} & 1.52701200 & -5.81156700 & 1.04743000 \\ \mathrm{H} & 2.95524600 & -4.25722000 & 1.44108700 \\ \mathrm{C} & -0.65966800 & -5.08478600 & 0.34306900 \\ \mathrm{H} & -0.93053200 & -2.95345100 & 0.17558300 \\ \mathrm{C} & 0.22328100 & -6.11946800 & 0.65219800 \\ \mathrm{H} & 2.22647800 & -6.60736900 & 1.29038800 \\ \mathrm{H} & -1.67717700 & -5.31108000 & 0.03280800 \\ \mathrm{H} & -0.09907600 & -7.15536700 & 0.58459500 \\ \mathrm{C} & 0.16231600 & -1.52886200 & 3.12497200 \\ \mathrm{~F} & -0.98538100 & -2.13779000 & 2.75117700 \\ \mathrm{~F} & -0.20304700 & -0.43233300 & 3.84781000 \\ \mathrm{~F} & 0.78397300 & -2.36657800 & 3.99056800 \\ \mathrm{O} & 3.40874600 & -0.56497200 & 0.79321000 \\ \mathrm{C} & 4.56801600 & 0.01465000 & 0.30328000 \\ \mathrm{C} & 5.46718300 & -0.56404200 & -0.60153100 \\ \mathrm{C} & 4.81932500 & 1.28751100 & 0.83308400 \\ \mathrm{C} & 6.60668700 & 0.15862000 & -0.97040400 \\ \mathrm{H} & 5.27907700 & -1.54922300 & -0.99929200 \\ \mathrm{C} & 5.96112400 & 1.98921800 & 0.45559400 \\ \mathrm{H} & 4.11158900 & 1.70456200 & 1.54308100 \\ \mathrm{C} & 6.86239000 & 1.42835900 & -0.45353100 \\ \mathrm{H} & 7.30443500 & -0.29186800 & -1.67174200 \\ \mathrm{H} & 6.14623500 & 2.97452000 & 0.87504600 \\ \mathrm{H} & 7.75462100 & 1.97290900 & -0.74938600\end{array}$

\section{Propyne}

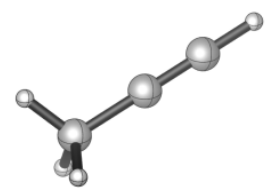

$\mathrm{E}(\mathrm{B} 3 \mathrm{LYP} / \mathrm{BS} 1)=-116.653269$

$\mathrm{E}(\mathrm{PCM}($ dichloroethane $) / \mathrm{M} 06 / \mathrm{BS} 2 / / \mathrm{B} 3 \mathrm{LYP} / \mathrm{BS} 1)=-116.593689$

Zero-point correction=

0.055717

Thermal correction to Energy=

0.059714

Thermal correction to Enthalpy=

0.060659

Thermal correction to Gibbs Free Energy=

0.031488

Charge $=0$ Multiplicity $=1$

$\begin{array}{llll}\text { C } & -0.21903000 & -0.00050600 & -0.00000400\end{array}$ 


$\begin{array}{lrrr}\mathrm{C} & -1.42599400 & 0.00022500 & -0.00002500 \\ \mathrm{H} & -2.49206800 & 0.00023800 & -0.00004300 \\ \mathrm{C} & 1.24134500 & 0.00019300 & 0.00002100 \\ \mathrm{H} & 1.63801200 & 1.02239300 & 0.00002600 \\ \mathrm{H} & 1.63804900 & -0.51105000 & 0.88532000 \\ \mathrm{H} & 1.63808000 & -0.51105400 & -0.88526000\end{array}$

\section{$\mathrm{C}_{4} \mathrm{~F}_{9} \mathrm{I}$}

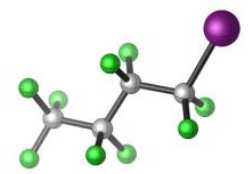

$\mathrm{E}(\mathrm{B} 3 \mathrm{LYP} / \mathrm{BS} 1)=-1062.325661$

$\mathrm{E}(\mathrm{PCM}$ (dichloroethane)/M06/BS2// B3LYP/BS1 $)=-1062.311753$

Zero-point correction $=$ 0.050979

Thermal correction to Energy=

0.064357

Thermal correction to Enthalpy=

0.065301

Thermal correction to Gibbs Free Energy=

0.008176

$\begin{array}{lrrr}\text { Charge }= & \text { Multiplicity }=1 & \\ \text { C } & 0.78494800 & 0.76073100 & 0.09663600 \\ \text { F } & 0.71494400 & 1.39847900 & 1.27076400 \\ \text { F } & 0.71702200 & 1.65976000 & -0.89576100 \\ \text { C } & -0.41082100 & -0.22202000 & -0.02386200 \\ \text { C } & -1.78960800 & 0.49432800 & -0.13637300 \\ \text { C } & -3.00946300 & -0.44455300 & 0.06496500 \\ \text { F } & -0.25735600 & -0.98208800 & -1.12725900 \\ \text { F } & -0.42121800 & -1.01391000 & 1.07161400 \\ \text { F } & -1.88626100 & 1.04184300 & -1.36680900 \\ \text { F } & -1.87316300 & 1.47317500 & 0.79357700 \\ \text { F } & -2.90432800 & -1.52660900 & -0.71696700 \\ \text { F } & -4.12326300 & 0.22057600 & -0.26266600 \\ \text { F } & -3.09667400 & -0.83104600 & 1.34148000 \\ \text { I } & 2.73061000 & -0.31118000 & -0.01849000\end{array}$

\section{$\mathbf{C}_{4} \mathbf{F}_{9}$}

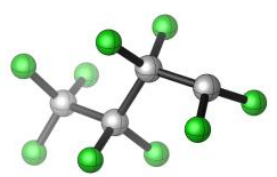

$\mathrm{E}(\mathrm{B} 3 \mathrm{LYP} / \mathrm{BS} 1)=-1050.89967$

$\mathrm{E}(\mathrm{PCM}$ (dichloroethane)/M06/BS2// B3LYP/BS1 $)=-1050.859248$

Zero-point correction $=$

Thermal correction to Energy=

Thermal correction to Enthalpy=

Thermal correction to Gibbs Free Energy=
0.049135

0.061002

0.061946

0.008863 


$\begin{array}{lrrr}\text { Charge }= & \text { Multiplicity }=2 \\ \text { C } & 2.19597400 & 0.16521500 & 0.02587400 \\ \text { F } & 2.54810300 & 0.05417800 & -1.24747000 \\ \text { F } & 2.57084000 & -0.88659200 & 0.74403200 \\ \text { C } & 0.76269400 & 0.60656800 & 0.25753900 \\ \text { C } & -0.30381500 & -0.47615000 & -0.06763100 \\ \text { C } & -1.77548500 & 0.00973200 & -0.06247500 \\ \text { F } & 0.62902400 & 0.94753500 & 1.56234400 \\ \text { F } & 0.52628600 & 1.69026900 & -0.52274700 \\ \text { F } & -0.18537800 & -1.46854000 & 0.84426700 \\ \text { F } & -0.03665300 & -0.97602000 & -1.30006600 \\ \text { F } & -2.05492600 & 0.63834400 & 1.08694300 \\ \text { F } & -2.58734800 & -1.04787200 & -0.18615100 \\ \text { F } & -1.99619500 & 0.84512200 & -1.08335600\end{array}$

TS12

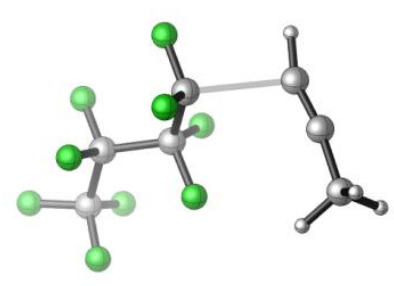

$\mathrm{E}(\mathrm{B} 3 \mathrm{LYP} / \mathrm{BS} 1)=-1167.552346$

$\mathrm{E}(\mathrm{PCM}$ (dichloroethane)/M06/BS2// B3LYP/BS1 $)=-1167.453613$

Zero-point correction=

0.105275

Thermal correction to Energy=

0.122142

Thermal correction to Enthalpy=

0.123086

Thermal correction to Gibbs Free Energy= 0.057025

$\begin{array}{lrrr}\text { Charge }= & \text { Multiplicity }=2 & & \\ \mathrm{C} & 3.64890000 & -0.69371200 & -0.29822500 \\ \mathrm{C} & 3.30253000 & 0.37811500 & -0.77461900 \\ \mathrm{H} & 3.34650400 & 1.26058700 & -1.37728400 \\ \mathrm{C} & 1.26595300 & 1.09116700 & 0.15068000 \\ \mathrm{~F} & 1.43597000 & 1.19776300 & 1.46962200 \\ \mathrm{~F} & 1.04510000 & 2.29278300 & -0.40184600 \\ \mathrm{C} & 3.85984100 & -1.96766900 & 0.37602100 \\ \mathrm{H} & 2.92683300 & -2.31042500 & 0.84134300 \\ \mathrm{H} & 4.18669400 & -2.74059800 & -0.32987900 \\ \mathrm{H} & 4.62107000 & -1.88177900 & 1.15992600 \\ \mathrm{C} & 0.21251600 & 0.06717400 & -0.23371200 \\ \mathrm{C} & -1.24734700 & 0.43912900 & 0.14730900 \\ \mathrm{C} & -2.29971600 & -0.68259900 & -0.04480800 \\ \mathrm{~F} & 0.25271500 & -0.10984900 & -1.57726300 \\ \mathrm{~F} & 0.51977600 & -1.10672800 & 0.38495600 \\ \mathrm{~F} & -1.62508700 & 1.49547200 & -0.61069100 \\ \mathrm{~F} & -1.27411400 & 0.80259400 & 1.45383700 \\ \mathrm{~F} & -2.24325500 & -1.17395600 & -1.28994900 \\ \mathrm{~F} & -3.52345500 & -0.17814800 & 0.16399900 \\ \mathrm{~F} & -2.09178000 & -1.67742000 & 0.82622600\end{array}$




\section{IM13}

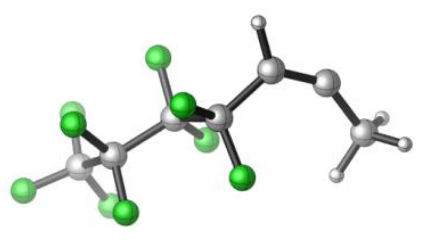

$\mathrm{E}(\mathrm{B} 3 \mathrm{LYP} / \mathrm{BS} 1)=-1167.607088$

$\mathrm{E}(\mathrm{PCM}($ dichloroethane $) / \mathrm{M} 06 / \mathrm{BS} 2 / / \mathrm{B} 3 \mathrm{LYP} / \mathrm{BS} 1)=-1167.507185$

Zero-point correction=

0.109210

Thermal correction to Energy=

0.124971

Thermal correction to Enthalpy=

0.125915

Thermal correction to Gibbs Free Energy=

0.063807

Charge $=0$ Multiplicity $=2$

$\begin{array}{llll}\text { C } & -3.55675900 & -0.47166300 & 0.52020500\end{array}$

$\begin{array}{llll}\mathrm{C} & -2.67982100 & 0.47241900 & 0.78097700\end{array}$

$\begin{array}{llll}\mathrm{H} & -2.75580300 & 1.10580700 & 1.66342000\end{array}$

$\begin{array}{lllll}\text { C } & & -1.46814600 & 0.75472800 & -0.07774000\end{array}$

F $\quad \begin{array}{llll}\text { C } & -1.68103600 & 0.38671400 & -1.37440100\end{array}$

F $\quad-1.18514000 \quad 2.09149700 \quad-0.05700300$

C $\quad-3.89102600 \quad-1.54361900 \quad-0.42410700$

$\mathrm{H} \quad-\quad-3.09398100 \quad-1.68209100 \quad-1.16902500$

$\mathrm{H} \quad-\quad-4.03702800 \quad-2.49454100 \quad 0.10290600$

$\begin{array}{llll}\mathrm{H} & -4.82294500 & -1.31687600 & -0.95642800\end{array}$

$\begin{array}{lllll}\text { C } & & -0.19932000 & 0.00302300 & 0.41136600\end{array}$

$\begin{array}{lllll}\text { C } & & 1.14019000 & 0.43634400 & -0.24392700\end{array}$

$\begin{array}{llll}\mathrm{C} & 2.32429600 & -0.53622400 & 0.00550000\end{array}$

$\begin{array}{lllll}\text { F } & & -0.07946600 & 0.19279600 & 1.75016000\end{array}$

$\begin{array}{llll}\text { F } & -0.38771300 & -1.32324600 & 0.17555600\end{array}$

$\begin{array}{llll}\mathrm{F} & 1.49301100 & 1.64304600 & 0.25476900\end{array}$

$\begin{array}{lllll}\text { F } & 0.98085700 & 0.53918900 & -1.58497500\end{array}$

F $\quad \begin{array}{llll}2.43958900 & -0.81489000 & 1.31159600\end{array}$

F $\quad 3.45850900 \quad 0.04020100 \quad-0.41338700$

$\begin{array}{llll}\mathrm{F} & 2.14953200 & -1.67779100 & -0.67059500\end{array}$

\section{IM14}

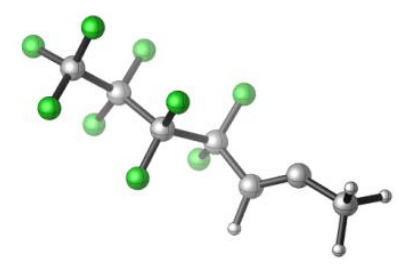

$\mathrm{E}(\mathrm{B} 3 \mathrm{LYP} / \mathrm{BS} 1)=-1167.606597$ 
$\mathrm{E}(\mathrm{PCM}($ dichloroethane $) / \mathrm{M} 06 / \mathrm{BS} 2 / / \mathrm{B} 3 \mathrm{LYP} / \mathrm{BS} 1)=-1167.50751$

$\begin{array}{ll}\text { Zero-point correction }= & 0.108847 \\ \text { Thermal correction to Energy= } & 0.124733 \\ \text { Thermal correction to Enthalpy= } & 0.125677 \\ \text { Thermal correction to Gibbs Free Energy= } & 0.063516\end{array}$

Charge $=0$ Multiplicity $=2$

C $\quad-3.57456900 \quad-0.43389400 \quad-0.21996000$

$\begin{array}{llll}\text { C } & -2.65241900 & 0.12448500 & 0.53185900\end{array}$

$\begin{array}{llll}\mathrm{H} & -2.74265100 & 0.19175200 & 1.61995000\end{array}$

$\begin{array}{lllll}\mathrm{C} & & -1.38524300 & 0.70536800 & -0.03107500\end{array}$

F $\quad \begin{array}{llll}\text { C } & -1.47062500 & 0.87109900 & -1.37701100\end{array}$

F $\quad \begin{array}{llll}-1.14322700 & 1.92873900 & 0.53755400\end{array}$

C $\quad-4.88709900 \quad-1.08168500 \quad-0.12382600$

$\mathrm{H} \quad-5.23641900 \quad-1.13152100 \quad 0.92066300$

$\begin{array}{llll}\mathrm{H} & -5.63896800 & -0.53451200 & -0.70568000\end{array}$

$\mathrm{H} \quad-4.84965500 \quad-2.10511600 \quad-0.51680200$

$\begin{array}{llll}\text { C } & -0.14090600 & -0.18106100 & 0.25469500\end{array}$

$\begin{array}{lllll}\mathrm{C} & & 1.23655900 & 0.47295300 & -0.04038000\end{array}$

$\begin{array}{llll}\text { C } & 2.42593500 & -0.52404300 & -0.08002000\end{array}$

F $\quad-0.15565400 \quad-0.52341300 \quad 1.57090400$

F $\quad \begin{array}{llll}-0.25118800 & -1.31261400 & -0.48723700\end{array}$

$\begin{array}{llll}\mathrm{F} & 1.50081200 & 1.38079600 & 0.92702000\end{array}$

F $\quad \begin{array}{llll}1.19791200 & 1.10023400 & -1.23979200\end{array}$

F $\quad \begin{array}{llll}2.42685100 & -1.30123100 & 1.01267900\end{array}$

F $\quad 3.57360500 \quad 0.16619800 \quad-0.11713400$

F $\quad 2.35864200 \quad-1.30017800 \quad-1.16763800$

TS13

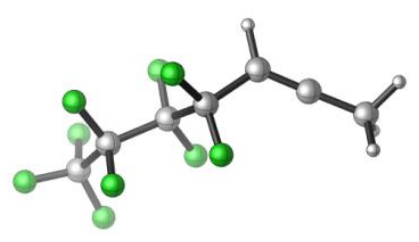

$\mathrm{E}(\mathrm{B} 3 \mathrm{LYP} / \mathrm{BS} 1)=-1167.600302$

$\mathrm{E}(\mathrm{PCM}$ (dichloroethane) $/ \mathrm{M} 06 / \mathrm{BS} 2 / / \mathrm{B} 3 \mathrm{LYP} / \mathrm{BS} 1)=-1167.502419$

Zero-point correction $=\quad 0.107666$

Thermal correction to Energy= $\quad 0.123211$

Thermal correction to Enthalpy $=\quad 0.124155$

Thermal correction to Gibbs Free Energy $=\quad 0.061869$

$\begin{array}{lrrr}\text { Charge }= & \text { Multiplicity }=2 & \\ \text { C } & -3.58683800 & -0.42589100 & 0.22623100 \\ \text { C } & -2.63611000 & 0.29768500 & 0.74489700 \\ \text { H } & -2.63893300 & 0.66016900 & 1.77856000 \\ \text { C } & -1.40385400 & 0.71179100 & -0.03540000 \\ \text { F } & -1.58932800 & 0.54382800 & -1.37148300 \\ \text { F } & -1.13255000 & 2.03357400 & 0.19805400 \\ \text { C } & -4.66721400 & -1.21339800 & -0.33485700\end{array}$




$\begin{array}{lrrr}\text { H } & -4.77720000 & -1.04951100 & -1.41790300 \\ \mathrm{H} & -4.47261600 & -2.28288800 & -0.18402500 \\ \mathrm{H} & -5.63897500 & -0.98579500 & 0.13094700 \\ \mathrm{C} & -0.13876700 & -0.09198800 & 0.36951600 \\ \mathrm{C} & 1.21084500 & 0.44879400 & -0.17682600 \\ \mathrm{C} & 2.40007200 & -0.54092000 & -0.04981800 \\ \mathrm{~F} & -0.05486100 & -0.09988400 & 1.72647100 \\ \mathrm{~F} & -0.29766100 & -1.37061500 & -0.06124500 \\ \mathrm{~F} & 1.53895800 & 1.56707200 & 0.50988400 \\ \mathrm{~F} & 1.08246300 & 0.75549600 & -1.48978000 \\ \mathrm{~F} & 2.48532400 & -1.01904100 & 1.19988600 \\ \mathrm{~F} & 3.53803400 & 0.10317000 & -0.34191400 \\ \mathrm{~F} & 2.25839100 & -1.56453300 & -0.89987600\end{array}$

\section{TS14}

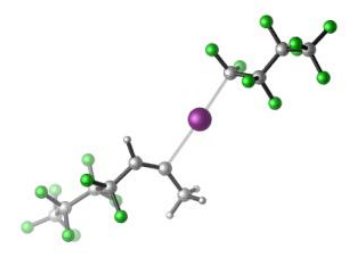

$\mathrm{E}(\mathrm{B} 3 \mathrm{LYP} / \mathrm{BS} 1)=-2229.921111$

$\mathrm{E}(\mathrm{PCM}($ dichloroethane $) / \mathrm{M} 06 / \mathrm{BS} 2 / / \mathrm{B} 3 \mathrm{LYP} / \mathrm{BS} 1)=-2229.820516$

Zero-point correction=

Thermal correction to Energy=

Thermal correction to Enthalpy=

Thermal correction to Gibbs Free Energy=
0.160687

0.191485

0.192429

0.088486

$\begin{array}{lrcc}\text { Charge }= & \text { Multiplicity }=2 & & \\ \text { C } & -1.33176100 & -0.76322000 & -0.54715600 \\ \text { C } & -2.09444700 & -0.59645300 & 0.51593900 \\ \text { H } & -1.73657700 & -0.10582900 & 1.41603900 \\ \text { C } & -3.54254900 & -1.03206000 & 0.58930200 \\ \text { F } & -3.85442300 & -1.33547800 & 1.88131500 \\ \text { F } & -3.77885000 & -2.13589500 & -0.17371100 \\ \text { I } & 1.08046100 & 0.13135300 & -0.17031700 \\ \text { C } & -1.43152200 & -1.31536300 & -1.91209200 \\ \text { H } & -2.45904200 & -1.64499900 & -2.10877600 \\ \text { H } & -1.15625100 & -0.56090400 & -2.65713900 \\ \text { H } & -0.75693800 & -2.16955100 & -2.03826600 \\ \text { C } & 3.26149600 & 1.01979400 & 0.20625500 \\ \text { F } & 3.44235000 & 2.04734100 & -0.63007600 \\ \text { F } & 3.30756300 & 1.45027700 & 1.46874100 \\ \text { C } & -4.51945900 & 0.07992300 & 0.11726200 \\ \text { C } & -6.02065500 & -0.17299100 & 0.42035700 \\ \text { C } & -6.99422700 & 0.76027600 & -0.34708900 \\ \text { F } & -6.22834000 & 0.01574600 & 1.74206900 \\ \text { F } & -4.16688700 & 1.24097800 & 0.72080300 \\ \text { F } & -4.36283900 & 0.22152300 & -1.22606800 \\ \text { F } & -6.34323100 & -1.44672500 & 0.09576600 \\ \text { F } & -7.01329300 & 0.45191900 & -1.64862600\end{array}$




$\begin{array}{lrrr}\text { F } & -6.62820500 & 2.04138900 & -0.20502500 \\ \text { F } & -8.22739400 & 0.60540000 & 0.14969100 \\ \text { C } & 4.33436200 & -0.06256700 & -0.03573200 \\ \text { C } & 5.79231500 & 0.47945400 & 0.03631800 \\ \text { C } & 6.88573400 & -0.62059900 & 0.08201800 \\ \text { F } & 8.08520400 & -0.04369100 & -0.05962800 \\ \text { F } & 6.85257400 & -1.26577600 & 1.25230000 \\ \text { F } & 6.70862100 & -1.50132500 & -0.91118400 \\ \text { F } & 5.93444100 & 1.23988900 & 1.14679800 \\ \text { F } & 6.01704800 & 1.24293400 & -1.05519000 \\ \text { F } & 4.15862900 & -0.59485100 & -1.26427500 \\ \text { F } & 4.18688200 & -1.02672400 & 0.90103700\end{array}$

\section{TS15}

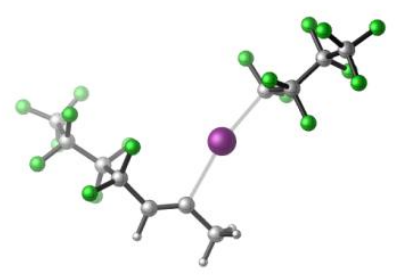

$\mathrm{E}(\mathrm{B} 3 \mathrm{LYP} / \mathrm{BS} 1)=-2229.915659$

$\mathrm{E}(\mathrm{PCM}$ (dichloroethane $) / \mathrm{M} 06 / \mathrm{BS} 2 / / \mathrm{B} 3 \mathrm{LYP} / \mathrm{BS} 1)=-2229.817107$

Zero-point correction=

0.160460

Thermal correction to Energy=

0.191246

Thermal correction to Enthalpy=

0.192191

Thermal correction to Gibbs Free Energy=

0.087384

$\begin{array}{lrrr}\text { Charge }= & \text { Multiplicity }= & \\ \text { C } & 1.34923100 & 2.62778200 & -0.25661100 \\ \text { C } & 2.54391500 & 2.42108100 & 0.26726600 \\ \text { H } & 3.26676400 & 3.23963800 & 0.21648400 \\ \text { C } & 3.10837000 & 1.19209600 & 0.92637000 \\ \text { F } & 2.15355100 & 0.41239100 & 1.48538900 \\ \text { F } & 3.96522000 & 1.59114300 & 1.91681800 \\ \text { I } & -0.49039100 & 0.82094800 & -0.39890800 \\ \text { C } & 0.76307800 & 3.82936600 & -0.89719100 \\ \text { H } & 1.49963200 & 4.64602100 & -0.90122900 \\ \text { H } & 0.46561900 & 3.62132300 & -1.93057500 \\ \text { H } & -0.12908500 & 4.16814700 & -0.35938600 \\ \text { C } & -2.43084500 & -0.52314800 & -0.80876400 \\ \text { F } & -2.75137700 & -0.40834400 & -2.10110700 \\ \text { F } & -2.10124800 & -1.78541200 & -0.53221400 \\ \text { C } & 3.93218000 & 0.31650100 & -0.05976900 \\ \text { C } & 4.80249300 & -0.78923100 & 0.59691800 \\ \text { C } & 5.32605200 & -1.86065400 & -0.39591700 \\ \text { F } & 4.08516800 & -1.43413900 & 1.54549800 \\ \text { F } & 3.07032400 & -0.26380900 & -0.93006400 \\ \text { F } & 4.76547300 & 1.13470000 & -0.75400500 \\ \text { F } & 5.87795500 & -0.20631600 & 1.17221800 \\ \text { F } & 5.90193100 & -1.27692200 & -1.45658400 \\ \text { F } & 4.32745800 & -2.64416900 & -0.81551600\end{array}$




$\begin{array}{lrrr}\text { F } & 6.23721300 & -2.62066500 & 0.22480000 \\ \text { C } & -3.60296400 & -0.05824300 & 0.07977900 \\ \text { C } & -4.94410500 & -0.78560800 & -0.23177700 \\ \text { C } & -6.06175100 & -0.55209100 & 0.81891100 \\ \text { F } & -7.20977600 & -1.05322900 & 0.34714300 \\ \text { F } & -5.76147500 & -1.16718200 & 1.96687800 \\ \text { F } & -6.22336500 & 0.75719500 & 1.05013200 \\ \text { F } & -4.72538300 & -2.11911800 & -0.30330900 \\ \text { F } & -5.39843700 & -0.34661200 & -1.42599300 \\ \text { F } & -3.80452600 & 1.26399400 & -0.10820800 \\ \text { F } & -3.27827800 & -0.28822900 & 1.37162800\end{array}$

\section{E-1-nonafluorobutyl-2-iodo-propene}

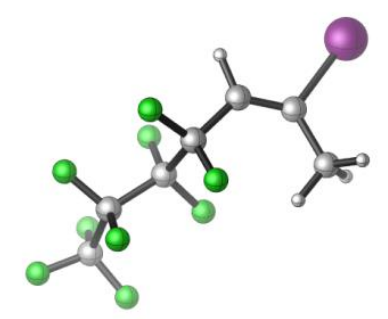

$\mathrm{E}(\mathrm{B} 3 \mathrm{LYP} / \mathrm{BS} 1)=-1179.049575$

$\mathrm{E}(\mathrm{PCM}$ (dichloroethane)/M06/BS2// B3LYP/BS1 $)=-1178.970747$

$\begin{array}{ll}\text { Zero-point correction }= & 0.112712 \\ \text { Thermal correction to Energy= } & 0.129798 \\ \text { Thermal correction to Enthalpy= } & 0.130743 \\ \text { Thermal correction to Gibbs Free Energy= } & 0.064860\end{array}$

Charge $=0$ Multiplicity $=1$

$\begin{array}{lrrr}\text { C } & 1.98382600 & 0.11702900 & 0.53321100 \\ \mathrm{C} & 1.11413300 & 0.72455200 & -0.27713800 \\ \mathrm{H} & 1.37247900 & 0.97183000 & -1.30008500 \\ \mathrm{C} & -0.29828500 & 1.11500000 & 0.07773700 \\ \mathrm{~F} & -0.42750600 & 1.50622400 & 1.37736600 \\ \mathrm{~F} & -0.66578600 & 2.16753700 & -0.71200000 \\ \mathrm{C} & 1.81623600 & -0.33922000 & 1.95028400 \\ \mathrm{H} & 0.80201900 & -0.15550100 & 2.30431300 \\ \mathrm{H} & 2.03198400 & -1.41001500 & 2.02924800 \\ \mathrm{H} & 2.52202700 & 0.18447400 & 2.60461400 \\ \mathrm{C} & -1.32478500 & -0.02511400 & -0.17499400 \\ \mathrm{C} & -2.81926100 & 0.39287300 & -0.12697600 \\ \mathrm{C} & -3.81252700 & -0.79614300 & -0.03740000 \\ \mathrm{~F} & -1.08348600 & -0.54843300 & -1.40178400 \\ \mathrm{~F} & -1.11394700 & -0.99187300 & 0.75844200 \\ \mathrm{~F} & -3.10866400 & 1.08307500 & -1.25194800 \\ \mathrm{~F} & -3.03909200 & 1.18276500 & 0.94977400 \\ \mathrm{~F} & -3.53983300 & -1.70964800 & -0.97913800 \\ \mathrm{~F} & -5.05633700 & -0.33895500 & -0.22659400 \\ \mathrm{~F} & -3.74642600 & -1.37526000 & 1.16662100 \\ \mathrm{I} & 3.94990900 & -0.29251900 & -0.27232300\end{array}$




\section{Z-1-nonafluorobutyl-2-iodo-propene}

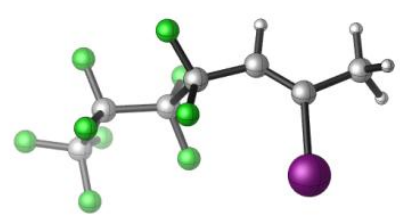

$\mathrm{E}(\mathrm{B} 3 \mathrm{LYP} / \mathrm{BS} 1)=-1179.045066$

$\mathrm{E}(\mathrm{PCM}($ dichloroethane $) / \mathrm{M} 06 / \mathrm{BS} 2 / / \mathrm{B} 3 \mathrm{LYP} / \mathrm{BS} 1)=-1178.969424$

Zero-point correction=

Thermal correction to Energy=

0.112475

Thermal correction to Enthalpy=

Thermal correction to Gibbs Free Energy=
0.129548

0.130492

0.064490 


\section{$\mathrm{pNO}_{2}-\mathrm{PhO}^{-}$}

$\mathrm{E}(\mathrm{B} 3 \mathrm{LYP} / \mathrm{BS} 1)=-511.43122$

$\mathrm{E}(\mathrm{PCM}$ (dichloroethane)/M06/BS2// B3LYP/BS1 $)=-511.349837$

$\begin{array}{ll}\text { Zero-point correction }= & 0.094613 \\ \text { Thermal correction to Energy= } & 0.102188 \\ \text { Thermal correction to Enthalpy= } & 0.103132 \\ \text { Thermal correction to Gibbs Free Energy= } & 0.061837\end{array}$

$\begin{array}{lrrr}\text { Charge }= & -1 \text { Multiplicity }=1 \\ \mathrm{C} & -0.05019200 & -1.22247600 & -0.00001400 \\ \mathrm{C} & 0.66636100 & 0.00000000 & 0.00003000 \\ \mathrm{C} & -0.05019200 & 1.22247600 & -0.00000300 \\ \mathrm{C} & -1.42154700 & 1.22609000 & -0.00009900 \\ \mathrm{C} & -2.21395600 & 0.00000000 & -0.00022300 \\ \mathrm{C} & -1.42154700 & -1.22609000 & -0.00010500 \\ \mathrm{H} & 0.51917400 & -2.14630200 & 0.00003700 \\ \mathrm{H} & 0.51917400 & 2.14630200 & 0.00005400 \\ \mathrm{H} & -1.97481900 & 2.16324400 & -0.00010400 \\ \mathrm{H} & -1.97481900 & -2.16324400 & -0.00010900 \\ \mathrm{O} & -3.46736900 & 0.00000000 & -0.00017100 \\ \mathrm{~N} & 2.07744900 & 0.00000000 & 0.00013400 \\ \mathrm{O} & 2.69090800 & -1.09479400 & 0.00034500 \\ \mathrm{O} & 2.69090900 & 1.09479400 & 0.00003500\end{array}$

\section{IM6-NO2}

$\mathrm{E}(\mathrm{B} 3 \mathrm{LYP} / \mathrm{BS} 1)=-2945.114993$

$\mathrm{E}(\mathrm{PCM}($ dichloroethane $) / \mathrm{M} 06 / \mathrm{BS} 2 / / \mathrm{B} 3 \mathrm{LYP} / \mathrm{BS} 1)=-2945.357979$

Zero-point correction=

0.902815

Thermal correction to Energy=

0.958625

Thermal correction to Enthalpy=

0.959569

Thermal correction to Gibbs Free Energy=
0.807534

\begin{tabular}{|c|c|c|c|}
\hline \multicolumn{4}{|c|}{ Charge $=0$ Multiplicity $=1$} \\
\hline $\mathrm{C}$ & 2.42677500 & -0.28732500 & 1.95382500 \\
\hline $\mathrm{C}$ & 2.93506200 & 0.06711300 & 3.21891400 \\
\hline $\mathrm{H}$ & 2.60528200 & 0.97645200 & 3.70303300 \\
\hline $\mathrm{C}$ & 3.86688100 & -0.71376900 & 3.89889900 \\
\hline $\mathrm{H}$ & 4.22463800 & -0.39659200 & 4.87449900 \\
\hline $\mathrm{C}$ & 4.32697600 & -1.89222700 & 3.31914600 \\
\hline $\mathrm{H}$ & 5.05060100 & -2.51947400 & 3.83239800 \\
\hline $\mathrm{C}$ & 3.84207100 & -2.26390200 & 2.07017900 \\
\hline $\mathrm{H}$ & 4.19105300 & -3.18413800 & 1.61267500 \\
\hline $\mathrm{C}$ & 2.89692500 & -1.49426700 & 1.36896500 \\
\hline $\mathrm{C}$ & 2.45164300 & -2.07662300 & 0.06310500 \\
\hline $\mathrm{C}$ & 1.14801500 & -2.56702100 & -0.09220900 \\
\hline $\mathrm{C}$ & 0.73034500 & -3.23512500 & -1.24620500 \\
\hline $\mathrm{H}$ & -0.30237400 & -3.54152700 & -1.35359600 \\
\hline $\mathrm{C}$ & 1.65532400 & -3.43615500 & -2.26463900 \\
\hline $\mathrm{H}$ & 1.34949400 & -3.94811800 & -3.17267900 \\
\hline $\mathrm{C}$ & 2.96777800 & -2.97509700 & -2.15113100 \\
\hline $\mathrm{H}$ & 3.66001100 & -3.13778700 & -2.96743100 \\
\hline $\mathrm{C}$ & 3.36954600 & -2.29856400 & -0.99270100 \\
\hline $\mathrm{C}$ & 5.71122100 & -2.15073200 & -1.68208700 \\
\hline
\end{tabular}




\begin{tabular}{|c|c|c|c|}
\hline $\mathrm{H}$ & 5.56453300 & -3.17845200 & -2.03822400 \\
\hline $\mathrm{C}$ & 5.75072100 & -1.18222100 & -2.86441300 \\
\hline $\mathrm{H}$ & 6.55928400 & -1.45676200 & -3.55140700 \\
\hline $\mathrm{H}$ & 5.93309900 & -0.16199000 & -2.50989100 \\
\hline $\mathrm{H}$ & 4.81088400 & -1.18513500 & -3.42416200 \\
\hline $\mathrm{C}$ & 6.98099300 & -2.09133900 & -0.83891000 \\
\hline $\mathrm{H}$ & 7.85539400 & -2.33620500 & -1.45133000 \\
\hline $\mathrm{H}$ & 6.92811500 & -2.80065800 & -0.00743200 \\
\hline $\mathrm{H}$ & 7.11743300 & -1.08618600 & -0.42580000 \\
\hline $\mathrm{C}$ & -0.61154600 & -3.37451200 & 1.43375200 \\
\hline $\mathrm{H}$ & -1.33695000 & -3.57114400 & 0.63934000 \\
\hline $\mathrm{C}$ & -1.32560400 & -2.80466600 & 2.64985700 \\
\hline $\mathrm{H}$ & -2.04571600 & -3.53680300 & 3.02992100 \\
\hline $\mathrm{H}$ & -1.87372100 & -1.89575000 & 2.39037300 \\
\hline $\mathrm{H}$ & -0.60814800 & -2.57401700 & 3.44510700 \\
\hline $\mathrm{C}$ & 0.19238800 & -4.62729900 & 1.76869200 \\
\hline $\mathrm{H}$ & -0.48495500 & -5.39926900 & 2.14973400 \\
\hline $\mathrm{H}$ & 0.93803700 & -4.40880600 & 2.54123300 \\
\hline $\mathrm{H}$ & 0.70508800 & -5.03275100 & 0.89207700 \\
\hline $\mathrm{C}$ & 0.59945100 & 1.99748800 & 2.50982300 \\
\hline $\mathrm{H}$ & 1.47451200 & 2.28583300 & 3.10401600 \\
\hline $\mathrm{C}$ & -0.02025300 & 3.31898000 & 1.99986600 \\
\hline $\mathrm{H}$ & 0.71092600 & 3.88340600 & 1.41074700 \\
\hline $\mathrm{H}$ & -0.86287300 & 3.11400400 & 1.33435200 \\
\hline C & -0.49478400 & 4.19051400 & 3.17723200 \\
\hline $\mathrm{H}$ & 0.37682200 & 4.51061400 & 3.76806100 \\
\hline $\mathrm{H}$ & -0.95864600 & 5.10383300 & 2.78512600 \\
\hline $\mathrm{C}$ & -1.47556300 & 3.44096600 & 4.08931900 \\
\hline $\mathrm{H}$ & -2.39653900 & 3.22103900 & 3.53005200 \\
\hline $\mathrm{H}$ & -1.76827200 & 4.07524700 & 4.93517200 \\
\hline $\mathrm{C}$ & -0.86303400 & 2.12778300 & 4.59492400 \\
\hline $\mathrm{H}$ & -1.58916500 & 1.57294800 & 5.20207400 \\
\hline $\mathrm{H}$ & -0.01189500 & 2.35313100 & 5.25497700 \\
\hline $\mathrm{C}$ & -0.38406100 & 1.24358600 & 3.43164400 \\
\hline $\mathrm{H}$ & -1.24890700 & 0.92166200 & 2.83540800 \\
\hline $\mathrm{H}$ & 0.08063000 & 0.32970900 & 3.82104800 \\
\hline C & 2.26301400 & 1.99114400 & 0.04928600 \\
\hline $\mathrm{H}$ & 1.53869000 & 2.72102900 & -0.33822700 \\
\hline C & 3.36454600 & 2.74505800 & 0.82510500 \\
\hline $\mathrm{H}$ & 4.06889900 & 2.01634500 & 1.24782900 \\
\hline $\mathrm{H}$ & 2.94344100 & 3.30290300 & 1.66955200 \\
\hline $\mathrm{C}$ & 4.12793300 & 3.71553800 & -0.09471900 \\
\hline $\mathrm{H}$ & 3.44317500 & 4.50762900 & -0.43158800 \\
\hline $\mathrm{H}$ & 4.92252300 & 4.21231800 & 0.47663100 \\
\hline $\mathrm{C}$ & 4.71281500 & 2.99665900 & -1.31797500 \\
\hline $\mathrm{H}$ & 5.48901500 & 2.29164200 & -0.98384900 \\
\hline $\mathrm{H}$ & 5.20838200 & 3.71790400 & -1.98022900 \\
\hline $\mathrm{C}$ & 3.62272800 & 2.22998800 & -2.07915500 \\
\hline $\mathrm{H}$ & 4.06026900 & 1.67630400 & -2.91939200 \\
\hline $\mathrm{H}$ & 2.90739700 & 2.94006400 & -2.51574200 \\
\hline C & 2.86742700 & 1.25226600 & -1.16153200 \\
\hline $\mathrm{H}$ & 3.56582200 & 0.48471300 & -0.80809900 \\
\hline $\mathrm{H}$ & 2.08039400 & 0.74291500 & -1.72688100 \\
\hline $\mathrm{O}$ & 4.63034800 & -1.81948500 & -0.78383100 \\
\hline $\mathrm{O}$ & 0.28026700 & -2.30554100 & 0.96299300 \\
\hline $\mathrm{P}$ & 1.20503400 & 0.86924800 & 1.12507800 \\
\hline $\mathrm{da}$ & $-0.5 / 568500$ & -0.29439100 & 0.0901940 \\
\hline
\end{tabular}




$\begin{array}{lrrr}\text { C } & -1.33489300 & 1.28398400 & -0.88345100 \\ \mathrm{C} & -2.34622300 & 1.95964700 & -0.32045800 \\ \mathrm{H} & -2.67145500 & 1.74414200 & 0.69152700 \\ \mathrm{C} & -3.14590600 & 3.06639300 & -0.94253600 \\ \mathrm{C} & -0.77651000 & 1.50185600 & -2.23442300 \\ \mathrm{C} & -0.67017200 & 0.43081400 & -3.14292300 \\ \mathrm{C} & -0.33402000 & 2.77618100 & -2.63751200 \\ \mathrm{C} & -0.14662400 & 0.63669800 & -4.41797200 \\ \mathrm{H} & -1.02406100 & -0.54854700 & -2.83656100 \\ \mathrm{C} & 0.19473000 & 2.97349800 & -3.91329900 \\ \mathrm{H} & -0.43250800 & 3.61516900 & -1.95606700 \\ \mathrm{C} & 0.29286700 & 1.90525800 & -4.80726500 \\ \mathrm{H} & -0.08562900 & -0.19663100 & -5.11319300 \\ \mathrm{H} & 0.52190500 & 3.96637800 & -4.21127300 \\ \mathrm{H} & 0.70206900 & 2.06135900 & -5.80188400 \\ \mathrm{~F} & -3.35296700 & 2.93089200 & -2.26536000 \\ \mathrm{~F} & -2.54567900 & 4.28433200 & -0.76414300 \\ \mathrm{~F} & -4.35649500 & 3.15501400 & -0.34779600 \\ \mathrm{O} & -2.01216200 & -1.50201500 & -0.84618400 \\ \mathrm{C} & -3.30801500 & -1.55579400 & -0.64270300 \\ \mathrm{C} & -3.95601900 & -1.01132700 & 0.50043300 \\ \mathrm{C} & -4.12479500 & -2.23327000 & -1.59337600 \\ \mathrm{C} & -5.32089900 & -1.14776300 & 0.69072400 \\ \mathrm{H} & -3.35439500 & -0.48219200 & 1.23337100 \\ \mathrm{C} & -5.48671800 & -2.37438300 & -1.40406200 \\ \mathrm{H} & -3.64275900 & -2.64341600 & -2.47600600 \\ \mathrm{C} & -6.08693000 & -1.83242600 & -0.25893700 \\ \mathrm{H} & -5.81265000 & -0.73632600 & 1.56440800 \\ \mathrm{H} & -6.10564500 & -2.89382600 & -2.12600500 \\ \mathrm{~N} & -7.51187300 & -1.98485200 & -0.05439800 \\ \mathrm{O} & -8.01091800 & -1.49875300 & 0.96860100 \\ \mathrm{O} & -8.16239000 & -2.59729900 & -0.91045200\end{array}$

\section{IM8-NO2}

$\mathrm{E}(\mathrm{B} 3 \mathrm{LYP} / \mathrm{BS} 1)=-3058.432328$

$\mathrm{E}(\mathrm{PCM}$ (dichloroethane)/M06/BS2// B3LYP/BS1 $)=-3058.652986$

\begin{tabular}{|c|c|c|c|}
\hline \multicolumn{4}{|c|}{ Zero-point correction } \\
\hline \multicolumn{4}{|c|}{ Thermal correction to Energy= } \\
\hline \multicolumn{4}{|c|}{ Thermal correction to Enthalpy= } \\
\hline \multicolumn{4}{|c|}{ Thermal correction to Gibbs Free Energy= } \\
\hline \multicolumn{4}{|c|}{ Charge $=0$ Multiplicity $=1$} \\
\hline $\mathrm{C}$ & -0.71948800 & 1.62937300 & 2.0451850 \\
\hline $\mathrm{C}$ & -1.08447200 & 1.88368500 & 3.38448 \\
\hline $\mathrm{H}$ & -1.33646700 & 1.05752200 & \\
\hline $\mathrm{C}$ & -1.12659100 & 3.17105100 & 3.90 \\
\hline $\mathrm{H}$ & -1.411 & 3.32335500 & 4.946 \\
\hline $\mathrm{C}$ & -0.79638200 & 4.25227500 & 3.093 \\
\hline $\mathrm{H}$ & -0.82139300 & 5.26683000 & 3.481956 \\
\hline $\mathrm{C}$ & -0.42115500 & 4.01877300 & 1.775780 \\
\hline $\mathrm{H}$ & -0.14342900 & 4.85420000 & 1.139570 \\
\hline $\mathrm{C}$ & -0.37117200 & 2.72430300 & 1.2247830 \\
\hline $\mathrm{C}$ & 0.10152500 & 2.66144200 & -0.1960590 \\
\hline $\mathrm{C}$ & 1.49167600 & 2.64362400 & -0.453158 \\
\hline & 1.97253400 & 2.78890600 & -1.764164 \\
\hline
\end{tabular}




\begin{tabular}{|c|c|c|c|}
\hline $\mathrm{H}$ & 3.03557200 & 2.77828000 & -1.96832300 \\
\hline $\mathrm{C}$ & 1.06806500 & 2.96668700 & -2.80918900 \\
\hline $\mathrm{H}$ & 1.44359000 & 3.07633100 & -3.82311400 \\
\hline C & -0.30531600 & 3.01945000 & -2.58015300 \\
\hline $\mathrm{H}$ & -0.99212200 & 3.17060100 & -3.40464100 \\
\hline $\mathrm{C}$ & -0.78332300 & 2.88989700 & -1.26751900 \\
\hline $\mathrm{C}$ & -2.96190400 & 3.90539000 & -1.67615200 \\
\hline $\mathrm{H}$ & -2.31549200 & 4.62330200 & -2.19603200 \\
\hline $\mathrm{C}$ & -3.82832600 & 3.15613500 & -2.68514300 \\
\hline $\mathrm{H}$ & -4.49615400 & 3.85677100 & -3.19973000 \\
\hline $\mathrm{H}$ & -4.44297300 & 2.40345900 & -2.18089500 \\
\hline $\mathrm{H}$ & -3.22964200 & 2.64075900 & -3.44040600 \\
\hline $\mathrm{C}$ & -3.79142900 & 4.63930000 & -0.62572400 \\
\hline $\mathrm{H}$ & -4.44093600 & 5.38196400 & -1.10239800 \\
\hline $\mathrm{H}$ & -3.14248800 & 5.14958500 & 0.09239200 \\
\hline $\mathrm{H}$ & -4.42241400 & 3.93263800 & -0.07551200 \\
\hline C & 3.69343400 & 2.81581800 & 0.59632900 \\
\hline $\mathrm{H}$ & 4.13890100 & 2.28250900 & -0.25086400 \\
\hline $\mathrm{C}$ & 4.27125500 & 2.25241500 & 1.88866700 \\
\hline $\mathrm{H}$ & 5.34968700 & 2.43882600 & 1.92964700 \\
\hline $\mathrm{H}$ & 4.10335700 & 1.17393200 & 1.94547800 \\
\hline $\mathrm{H}$ & 3.80120100 & 2.72913900 & 2.75600300 \\
\hline $\mathrm{C}$ & 3.91718000 & 4.32267100 & 0.46385800 \\
\hline $\mathrm{H}$ & 4.99016300 & 4.54167300 & 0.42858100 \\
\hline $\mathrm{H}$ & 3.48632300 & 4.84410100 & 1.32599200 \\
\hline $\mathrm{H}$ & 3.45940600 & 4.72366900 & -0.44558100 \\
\hline C & -0.06544600 & -1.10764400 & 2.94772000 \\
\hline $\mathrm{H}$ & -0.78150700 & -1.01892100 & 3.77535900 \\
\hline $\mathrm{C}$ & 0.05348600 & -2.60527000 & 2.58727500 \\
\hline $\mathrm{H}$ & -0.90785800 & -3.00646200 & 2.24134800 \\
\hline $\mathrm{H}$ & 0.76140700 & -2.70528900 & 1.75552400 \\
\hline $\mathrm{C}$ & 0.56031200 & -3.42122900 & 3.78909500 \\
\hline $\mathrm{H}$ & -0.18863800 & -3.38930200 & 4.59488300 \\
\hline $\mathrm{H}$ & 0.66244100 & -4.47510900 & 3.50053100 \\
\hline $\mathrm{C}$ & 1.89680200 & -2.87634600 & 4.31247700 \\
\hline $\mathrm{H}$ & 2.67068100 & -3.02835200 & 3.54632900 \\
\hline $\mathrm{H}$ & 2.21658800 & -3.44023200 & 5.19802700 \\
\hline C & 1.79667700 & -1.37922400 & 4.64010500 \\
\hline $\mathrm{H}$ & 2.77353100 & -0.99376100 & 4.95866400 \\
\hline $\mathrm{H}$ & 1.11507300 & -1.24154300 & 5.49383700 \\
\hline $\mathrm{C}$ & 1.28993400 & -0.55737300 & 3.44117400 \\
\hline $\mathrm{H}$ & 2.00654700 & -0.60095800 & 2.61690800 \\
\hline $\mathrm{H}$ & 1.19414700 & 0.49402500 & 3.73038500 \\
\hline $\mathrm{C}$ & -2.68422900 & -0.53321700 & 1.59245500 \\
\hline $\mathrm{H}$ & -2.78083100 & -1.41533600 & 0.95054700 \\
\hline $\mathrm{C}$ & -3.26076700 & -0.91670100 & 2.97296900 \\
\hline $\mathrm{H}$ & -3.16144600 & -0.07659200 & 3.67280800 \\
\hline $\mathrm{H}$ & -2.71773200 & -1.76429200 & 3.40405300 \\
\hline $\mathrm{C}$ & -4.75138600 & -1.28898600 & 2.85835300 \\
\hline $\mathrm{H}$ & -4.84794100 & -2.19265900 & 2.23928900 \\
\hline $\mathrm{H}$ & -5.13814000 & -1.54751900 & 3.85246000 \\
\hline $\mathrm{C}$ & -5.57675600 & -0.15469500 & 2.23760800 \\
\hline $\mathrm{H}$ & -5.56359300 & 0.71402900 & 2.91253100 \\
\hline $\mathrm{H}$ & -6.62663800 & -0.45841300 & 2.13846200 \\
\hline $\mathrm{C}$ & -5.00446500 & 0.25208400 & 0.87384700 \\
\hline $\mathrm{H}$ & -5.55774900 & 1.10842000 & 0.46679400 \\
\hline $\mathrm{H}$ & -5.14325700 & -0.57100900 & 0.16031600 \\
\hline
\end{tabular}




$\begin{array}{lrrr}\mathrm{C} & -3.51104600 & 0.61034200 & 0.96378500 \\ \mathrm{H} & -3.39709300 & 1.51337700 & 1.57619500 \\ \mathrm{H} & -3.12808800 & 0.86259900 & -0.02773200 \\ \mathrm{O} & -2.11091800 & 2.98116800 & -0.94985100 \\ \mathrm{O} & 2.27839900 & 2.51529300 & 0.64584100 \\ \mathrm{P} & -0.82301100 & -0.14850900 & 1.51197600 \\ \mathrm{Pd} & 0.24365500 & -0.94852900 & -0.56340900 \\ \mathrm{C} & -1.56264700 & -1.38786700 & -1.47295100 \\ \mathrm{C} & -2.04225000 & -0.46929600 & -2.32851900 \\ \mathrm{H} & -1.55892300 & 0.49940100 & -2.40912200 \\ \mathrm{C} & -3.20993900 & -0.58999700 & -3.26263600 \\ \mathrm{C} & -2.11034400 & -2.73735000 & -1.19860200 \\ \mathrm{C} & -1.25778100 & -3.83847000 & -0.98196200 \\ \mathrm{C} & -3.50051000 & -2.96301100 & -1.10639500 \\ \mathrm{C} & -1.76660300 & -5.10925700 & -0.71810400 \\ \mathrm{H} & -0.18206300 & -3.69981400 & -1.01797500 \\ \mathrm{C} & -4.00775700 & -4.23103000 & -0.83090700 \\ \mathrm{H} & -4.18732000 & -2.13924700 & -1.25658100 \\ \mathrm{C} & -3.14515400 & -5.31233200 & -0.63879700 \\ \mathrm{H} & -1.08178100 & -5.93945700 & -0.56849500 \\ \mathrm{H} & -5.08331800 & -4.37312100 & -0.76786900 \\ \mathrm{H} & -3.54279600 & -6.30046000 & -0.42471100 \\ \mathrm{~F} & -3.37873900 & -1.81804600 & -3.79062500 \\ \mathrm{~F} & -3.05521100 & 0.26867300 & -4.30158900 \\ \mathrm{~F} & -4.39600800 & -0.25349100 & -2.66906400 \\ \mathrm{C} & 0.95919100 & -1.61133600 & -2.28078700 \\ \mathrm{O} & 1.28135600 & -2.00266200 & -3.30105600 \\ \mathrm{O} & 2.13050900 & -0.88530200 & 0.41746800 \\ \mathrm{C} & 3.32653600 & -0.92102800 & -0.11267700 \\ \mathrm{C} & 3.66171600 & -0.33939700 & -1.36887200 \\ \mathrm{C} & 4.38212800 & -1.55930500 & 0.60675900 \\ \mathrm{C} & 4.95065000 & -0.40329000 & -1.87574400 \\ \mathrm{H} & 2.89172300 & 0.19513300 & -1.91581800 \\ \mathrm{C} & 5.66795300 & -1.62017100 & 0.10380400 \\ \mathrm{H} & 4.14357700 & -2.01119700 & 1.56496100 \\ \mathrm{C} & 5.95480200 & -1.04258300 & -1.14157300 \\ \mathrm{H} & 5.20176000 & 0.03996200 & -2.83257900 \\ \mathrm{H} & 6.46397900 & -2.11225100 & 0.65025500 \\ \mathrm{~N} & 7.30131200 & -1.10230800 & -1.66936100 \\ \mathrm{O} & 7.52141800 & -0.57774800 & -2.76850900 \\ \mathrm{O} & 8.16727800 & -1.67281500 & -0.99470600 \\ & & & \\ & & & \end{array}$

\section{TS9-NO2}

$\mathrm{E}(\mathrm{B} 3 \mathrm{LYP} / \mathrm{BS} 1)=-3058.416478$

$\mathrm{E}(\mathrm{PCM}($ dichloroethane $) / \mathrm{M} 06 / \mathrm{BS} 2 / / \mathrm{B} 3 \mathrm{LYP} / \mathrm{BS} 1)=-3058.632347$

Zero-point correction=

Thermal correction to Energy=

Thermal correction to Enthalpy=

Thermal correction to Gibbs Free Energy=
0.909318

0.967618

0.968562

0.809724

Charge $=0$ Multiplicity $=1$

$\begin{array}{llll}\text { C } & -0.94979100 & 1.64685900 & 2.11112400\end{array}$

$\begin{array}{llll}\mathrm{C} & -1.24416600 & 1.91349800 & 3.46351400\end{array}$

$\begin{array}{llll}\mathrm{H} & -1.46058400 & 1.09564700 & 4.14109900\end{array}$

$\begin{array}{llll}\text { C } & -1.26105300 & 3.20942500 & 3.97172300\end{array}$ 


\begin{tabular}{lrrr}
$\mathrm{H}$ & -1.49004600 & 3.37631700 & 5.02062200 \\
$\mathrm{C}$ & -0.98305600 & 4.28111600 & 3.12448300 \\
$\mathrm{H}$ & -0.99700500 & 5.30048900 & 3.50063800 \\
$\mathrm{C}$ & -0.66697600 & 4.03396800 & 1.79257500 \\
$\mathrm{H}$ & -0.42114200 & 4.86187200 & 1.13392800 \\
$\mathrm{C}$ & -0.62969600 & 2.73181400 & 1.26401500 \\
$\mathrm{C}$ & -0.16680900 & 2.61825300 & -0.15594200 \\
$\mathrm{C}$ & 1.20760000 & 2.41836900 & -0.40799200 \\
$\mathrm{C}$ & 1.71055500 & 2.48238000 & -1.71661700 \\
$\mathrm{H}$ & 2.76350000 & 2.32379000 & -1.91244900 \\
$\mathrm{C}$ & 0.83898000 & 2.76420100 & -2.76499900 \\
$\mathrm{H}$ & 1.22561700 & 2.81290600 & -3.77935500 \\
$\mathrm{C}$ & -0.51948800 & 2.99031200 & -2.54429300 \\
$\mathrm{H}$ & -1.17085100 & 3.21200900 & -3.38032300 \\
$\mathrm{C}$ & -1.02008900 & 2.92971000 & -1.23507800 \\
$\mathrm{C}$ & -3.18180900 & 3.91380700 & -1.80321200 \\
$\mathrm{H}$ & -2.55583600 & 4.59819800 & -2.39000600 \\
$\mathrm{C}$ & -3.94640400 & 2.97232000 & -2.73261500 \\
$\mathrm{H}$ & -4.61755900 & 3.54960400 & -3.37911100 \\
$\mathrm{H}$ & -4.55209400 & 2.26926900 & -2.15149000 \\
$\mathrm{H}$ & -3.28266100 & 2.38752800 & -3.37425300 \\
$\mathrm{C}$ & -4.11133700 & 4.72851100 & -0.90826100 \\
$\mathrm{H}$ & -4.78329100 & 5.34351800 & -1.51667400 \\
$\mathrm{H}$ & -3.53573800 & 5.38552800 & -0.24924600 \\
$\mathrm{H}$ & -4.72071100 & 4.06477600 & -0.28517800 \\
$\mathrm{C}$ & 3.38981400 & 2.49438800 & 0.69055900 \\
$\mathrm{H}$ & 3.86691600 & 1.97837300 & -0.14956900 \\
$\mathrm{H}$ & -2.93031700 & -0.48552500 & 1.58466000 \\
$\mathrm{C}$ & -3.55943700 & -0.57831900 & 2.99120500 \\
$\mathrm{H}$ & -3.47966400 & 0.39346200 & 3.49422400 \\
$\mathrm{H}$ & -3.02934300 & -1.30856800 & 3.61315800 \\
$\mathrm{H}$ & 3.93571900 & 1.91967900 & 1.99107400 \\
$\mathrm{H}$ & 5.01239700 & 2.10676600 & 2.05938400 \\
$\mathrm{H}$ & 3.77209100 & 0.83976200 & 2.03301600 \\
$\mathrm{C}$ & 3.44437600 & 2.38714100 & 2.85176600 \\
$\mathrm{H}$ & 3.60469200 & 4.00382700 & 0.58349200 \\
$\mathrm{H}$ & 4.67646900 & 4.23071700 & 0.59108800 \\
$\mathrm{H}$ & 3.13639800 & 4.51307800 & 1.43334200 \\
$\mathrm{C}$ & 3.18002800 & 4.40911400 & -0.33999300 \\
$\mathrm{H}$ & -0.31225600 & -1.12213900 & 2.91272800 \\
$\mathrm{C}$ & -0.82617000 & -0.86633400 & 3.84894000 \\
$\mathrm{H}$ & -0.50071600 & -2.63572400 & 2.66712900 \\
$\mathrm{H}$ & -1.56413800 & -2.89025700 & 2.58416100 \\
$\mathrm{C}$ & -0.04098500 & -2.90549900 & 1.70659200 \\
$\mathrm{H}$ & 0.14502500 & -3.46390500 & 3.79181900 \\
$\mathrm{H}$ & -0.39059600 & -3.27515600 & 4.73434900 \\
$\mathrm{H}$ & 0.02453200 & -4.53244500 & 3.57367600 \\
\hline & 1.62974800 & -3.11683900 & 3.97185200 \\
$\mathrm{H}$ & 2.18251600 & -3.41895600 & 3.07049400 \\
$\mathrm{H}$ & 1.05401800 & -3.68754700 & 4.80745600 \\
$\mathrm{H}$ & 1.82304000 & -1.61085500 & 4.20069900 \\
\hline
\end{tabular}




\begin{tabular}{lrrr}
$\mathrm{C}$ & -5.04751800 & -0.96482800 & 2.90688400 \\
$\mathrm{H}$ & -5.13670300 & -1.97443000 & 2.47991700 \\
$\mathrm{H}$ & -5.46954600 & -1.01454400 & 3.91879100 \\
$\mathrm{C}$ & -5.83715000 & 0.02744300 & 2.04301000 \\
$\mathrm{H}$ & -5.83538800 & 1.01299800 & 2.53167500 \\
$\mathrm{H}$ & -6.88681600 & -0.28350800 & 1.96796300 \\
$\mathrm{C}$ & -5.21468400 & 0.15013400 & 0.64605800 \\
$\mathrm{H}$ & -5.74755500 & 0.90505200 & 0.05373800 \\
$\mathrm{H}$ & -5.33715300 & -0.80331700 & 0.11332700 \\
$\mathrm{C}$ & -3.72024800 & 0.51239800 & 0.70903200 \\
$\mathrm{H}$ & -3.60811400 & 1.52260400 & 1.12177500 \\
$\mathrm{H}$ & -3.30643700 & 0.54359900 & -0.30222200 \\
$\mathrm{O}$ & -2.32194100 & 3.17316200 & -0.90556200 \\
$\mathrm{O}$ & 1.97384600 & 2.17917000 & 0.69380000 \\
$\mathrm{P}$ & -1.08002400 & -0.11040600 & 1.52831100 \\
$\mathrm{Pd}$ & 0.15246400 & -0.88609900 & -0.44476500 \\
$\mathrm{C}$ & -1.45447600 & -1.45995400 & -1.52014600 \\
$\mathrm{C}$ & -1.88327200 & -0.57270000 & -2.42905900 \\
$\mathrm{H}$ & -1.42992200 & 0.41110000 & -2.49491200 \\
$\mathrm{C}$ & -2.98942100 & -0.76042400 & -3.42332600 \\
$\mathrm{C}$ & -1.97795700 & -2.81445800 & -1.24553600 \\
$\mathrm{C}$ & -1.11800700 & -3.91930600 & -1.09490400 \\
$\mathrm{C}$ & -3.36472600 & -3.03164200 & -1.11240400 \\
$\mathrm{C}$ & -1.62425600 & -5.19220500 & -0.84184000 \\
$\mathrm{H}$ & -0.04676300 & -3.77786200 & -1.18705800 \\
$\mathrm{C}$ & -3.86704600 & -4.30464000 & -0.84657600 \\
$\mathrm{H}$ & -4.04889100 & -2.20041100 & -1.23683600 \\
$\mathrm{C}$ & -3.00076500 & -5.39063900 & -0.70982100 \\
$\mathrm{H}$ & -0.93985200 & -6.03047000 & -0.74434500 \\
$\mathrm{H}$ & -4.94018300 & -4.44640900 & -0.75016200 \\
$\mathrm{H}$ & -3.39367000 & -6.38220200 & -0.50266800 \\
$\mathrm{~F}$ & -3.09159100 & -2.00654500 & -3.92069600 \\
$\mathrm{~F}$ & -2.81076900 & 0.08018300 & -4.47281800 \\
$\mathrm{~F}$ & -4.21425100 & -0.44728800 & -2.89805100 \\
$\mathrm{C}$ & 1.42096900 & -1.64061700 & -1.77631900 \\
$\mathrm{O}$ & 1.92191000 & -2.08636600 & -2.71891200 \\
$\mathrm{O}$ & 2.58897700 & -1.34188400 & -0.29078000 \\
$\mathrm{C}$ & 3.86745600 & -1.07978400 & -0.51777200 \\
$\mathrm{C}$ & 4.32719800 & -0.35958900 & -1.65118300 \\
$\mathrm{C}$ & 4.83582700 & -1.54548800 & 0.41072400 \\
$\mathrm{C}$ & 5.67924400 & -0.11227900 & -1.83794000 \\
$\mathrm{H}$ & 3.60846900 & -0.00214900 & -2.37982400 \\
$\mathrm{C}$ & 6.18464600 & -1.29141700 & 0.22975500 \\
$\mathrm{H}$ & 4.48934600 & -2.11103400 & 1.27027100 \\
$\mathrm{C}$ & 6.60400000 & -0.57232000 & -0.89574500 \\
$\mathrm{H}$ & 6.03650900 & 0.43552200 & -2.70193900 \\
$\mathrm{H}$ & 6.92544900 & -1.64142700 & 0.93877500 \\
& 8.01764500 & -0.29831200 & -1.08619400 \\
& 8.35595700 & 0.34340200 & -2.08687200 \\
\hline & 8.81017100 & -0.71752700 & -0.23562500 \\
\hline
\end{tabular}

\section{IM10-NO2}

$\mathrm{E}(\mathrm{B} 3 \mathrm{LYP} / \mathrm{BS} 1)=-3058.431624$

$\mathrm{E}(\mathrm{PCM}$ (dichloroethane)/M06/BS2// B3LYP/BS1 $)=-3058.644437$ 
Zero-point correction=

Thermal correction to Energy=

Thermal correction to Enthalpy=

Thermal correction to Gibbs Free Energy=

Charge $=0$ Multiplicity $=1$

\begin{tabular}{|c|c|c|c|}
\hline $\mathrm{C}$ & 2.91232000 & -0.59313700 & 1.72929600 \\
\hline $\mathrm{C}$ & 3.74025900 & -0.26703900 & 2.82222600 \\
\hline $\mathrm{H}$ & 3.72087300 & 0.73397300 & 3.23453900 \\
\hline $\mathrm{C}$ & 4.59934000 & -1.19043400 & 3.41215900 \\
\hline $\mathrm{H}$ & 5.21643800 & -0.89174200 & 4.25519900 \\
\hline $\mathrm{C}$ & 4.65300000 & -2.49059600 & 2.91523800 \\
\hline $\mathrm{H}$ & 5.30691300 & -3.23168500 & 3.36684600 \\
\hline $\mathrm{C}$ & 3.86055300 & -2.83091600 & 1.82509300 \\
\hline $\mathrm{H}$ & 3.90695800 & -3.83820300 & 1.42269500 \\
\hline $\mathrm{C}$ & 2.99130300 & -1.90986900 & 1.21155400 \\
\hline $\mathrm{C}$ & 2.23990900 & -2.44050400 & 0.02956500 \\
\hline $\mathrm{C}$ & 0.85802000 & -2.67353900 & 0.06010300 \\
\hline $\mathrm{C}$ & 0.18905700 & -3.32492700 & -0.98000300 \\
\hline $\mathrm{H}$ & -0.88587000 & -3.44429300 & -0.95400600 \\
\hline $\mathrm{C}$ & 0.91979700 & -3.74410700 & -2.08508100 \\
\hline $\mathrm{H}$ & 0.40958400 & -4.23666800 & -2.90767700 \\
\hline $\mathrm{C}$ & 2.29294900 & -3.51268200 & -2.17081200 \\
\hline $\mathrm{H}$ & 2.83080700 & -3.83002000 & -3.05512600 \\
\hline $\mathrm{C}$ & 2.95384400 & -2.86461000 & -1.12101300 \\
\hline $\mathrm{C}$ & 5.16441900 & -3.11627500 & -2.13560200 \\
\hline $\mathrm{H}$ & 4.81948100 & -4.11985200 & -2.41652000 \\
\hline $\mathrm{C}$ & 5.16411900 & -2.19603800 & -3.35678300 \\
\hline $\mathrm{H}$ & 5.81292600 & -2.60543200 & -4.13945900 \\
\hline $\mathrm{H}$ & 5.54104400 & -1.20513400 & -3.08136600 \\
\hline $\mathrm{H}$ & 4.16056500 & -2.07402900 & -3.77453800 \\
\hline $\mathrm{C}$ & 6.54218700 & -3.22629500 & -1.49021200 \\
\hline $\mathrm{H}$ & 7.26841800 & -3.61931500 & -2.20977700 \\
\hline $\mathrm{H}$ & 6.51203500 & -3.89431000 & -0.62411200 \\
\hline $\mathrm{H}$ & 6.88481900 & -2.24198800 & -1.15361400 \\
\hline $\mathrm{C}$ & -0.74485400 & -3.07434300 & 1.87385600 \\
\hline $\mathrm{H}$ & -1.48720300 & -3.44462000 & 1.15753500 \\
\hline $\mathrm{C}$ & -1.43959800 & -2.23141600 & 2.93212000 \\
\hline $\mathrm{H}$ & -2.15476000 & -2.85137000 & 3.48382600 \\
\hline $\mathrm{H}$ & -1.98390000 & -1.40157400 & 2.47144900 \\
\hline $\mathrm{H}$ & -0.71118600 & -1.82751700 & 3.64297200 \\
\hline $\mathrm{C}$ & 0.04449900 & -4.24020700 & 2.46088300 \\
\hline $\mathrm{H}$ & -0.63360700 & -4.91108500 & 2.99975900 \\
\hline $\mathrm{H}$ & 0.80421300 & -3.87501900 & 3.16007600 \\
\hline $\mathrm{H}$ & 0.54119000 & -4.82063200 & 1.67744500 \\
\hline $\mathrm{C}$ & 1.47767800 & 1.84190300 & 2.52794600 \\
\hline $\mathrm{H}$ & 2.45632700 & 2.10671700 & 2.94763700 \\
\hline $\mathrm{C}$ & 0.78758400 & 3.16500600 & 2.13853800 \\
\hline $\mathrm{H}$ & 1.37363200 & 3.70501400 & 1.38697100 \\
\hline $\mathrm{H}$ & -0.18487700 & 2.95373200 & 1.68332600 \\
\hline $\mathrm{C}$ & 0.58119500 & 4.06404200 & 3.37016400 \\
\hline $\mathrm{H}$ & 1.55952900 & 4.36818400 & 3.77254300 \\
\hline $\mathrm{H}$ & 0.06819000 & 4.98328300 & 3.06221700 \\
\hline $\mathrm{C}$ & -0.21891600 & 3.34682400 & 4.46621000 \\
\hline $\mathrm{H}$ & -1.23730600 & 3.15353700 & 4.09896100 \\
\hline $\mathrm{H}$ & -0.31996700 & 3.99008600 & 5.34961900 \\
\hline $\mathrm{C}$ & 0.44113700 & 2.01493500 & 4.84965500 \\
\hline
\end{tabular}




\begin{tabular}{|c|c|c|c|}
\hline $\mathrm{H}$ & -0.17039300 & 1.48248800 & 5.58946100 \\
\hline $\mathrm{H}$ & 1.41011600 & 2.21386100 & 5.33176200 \\
\hline $\mathrm{C}$ & 0.66206300 & 1.11668400 & 3.62055000 \\
\hline $\mathrm{H}$ & -0.31158200 & 0.83003900 & 3.19908100 \\
\hline $\mathrm{H}$ & 1.16188500 & 0.18855600 & 3.92188900 \\
\hline $\mathrm{C}$ & 2.80631900 & 1.71824500 & -0.14590300 \\
\hline $\mathrm{H}$ & 2.10407900 & 2.49104900 & -0.49042600 \\
\hline $\mathrm{C}$ & 4.04148200 & 2.41583300 & 0.45530400 \\
\hline $\mathrm{H}$ & 4.73861200 & 1.65653500 & 0.83559600 \\
\hline $\mathrm{H}$ & 3.76078500 & 3.04533700 & 1.30831600 \\
\hline $\mathrm{C}$ & 4.75399200 & 3.27931800 & -0.60265900 \\
\hline $\mathrm{H}$ & 4.08891500 & 4.10346600 & -0.89970100 \\
\hline $\mathrm{H}$ & 5.64620700 & 3.74214300 & -0.16140100 \\
\hline $\mathrm{C}$ & 5.13375400 & 2.46186500 & -1.84567300 \\
\hline $\mathrm{H}$ & 5.89745300 & 1.71982100 & -1.56761400 \\
\hline $\mathrm{H}$ & 5.59100400 & 3.11304800 & -2.60148600 \\
\hline $\mathrm{C}$ & 3.91441700 & 1.73542600 & -2.43150700 \\
\hline $\mathrm{H}$ & 4.21902800 & 1.10381900 & -3.27607900 \\
\hline $\mathrm{H}$ & 3.19880500 & 2.46483200 & -2.83546700 \\
\hline $\mathrm{C}$ & 3.20541000 & 0.87273600 & -1.37383000 \\
\hline $\mathrm{H}$ & 3.87234900 & 0.06053300 & -1.05625900 \\
\hline $\mathrm{H}$ & 2.31740600 & 0.40562800 & -1.81196600 \\
\hline $\mathrm{O}$ & 4.29309900 & -2.60280500 & -1.10652500 \\
\hline $\mathrm{O}$ & 0.15887800 & -2.16922100 & 1.16034700 \\
\hline$P$ & 1.75936900 & 0.71011900 & 1.04610500 \\
\hline $\mathrm{Pd}$ & -0.42302100 & -0.08550800 & 0.12778700 \\
\hline $\mathrm{C}$ & -0.74171800 & 1.39369800 & -1.23468000 \\
\hline $\mathrm{C}$ & -0.42723900 & 1.05355500 & -2.49817100 \\
\hline $\mathrm{H}$ & -0.10153100 & 0.04416000 & -2.73013400 \\
\hline $\mathrm{C}$ & -0.47724500 & 1.91906900 & -3.72082900 \\
\hline $\mathrm{C}$ & -1.21144500 & 2.72290800 & -0.78317700 \\
\hline $\mathrm{C}$ & -2.33133800 & 2.83973800 & 0.06210300 \\
\hline $\mathrm{C}$ & -0.53895000 & 3.90193400 & -1.16041100 \\
\hline $\mathrm{C}$ & -2.77400100 & 4.08934300 & 0.49373300 \\
\hline $\mathrm{H}$ & -2.84790800 & 1.93980200 & 0.37708500 \\
\hline $\mathrm{C}$ & -0.97491700 & 5.14886600 & -0.71325500 \\
\hline $\mathrm{H}$ & 0.32281900 & 3.83916300 & -1.81562000 \\
\hline $\mathrm{C}$ & -2.09520400 & 5.25033400 & 0.11388400 \\
\hline $\mathrm{H}$ & -3.64975900 & 4.15562200 & 1.13439100 \\
\hline $\mathrm{H}$ & -0.43856200 & 6.04420700 & -1.01731700 \\
\hline $\mathrm{H}$ & -2.43644900 & 6.22308400 & 0.45800300 \\
\hline $\mathrm{F}$ & -1.50965300 & 2.78654600 & -3.75263400 \\
\hline $\mathrm{F}$ & -0.56688600 & 1.14960100 & -4.83077000 \\
\hline F & 0.65982300 & 2.67101700 & -3.87954400 \\
\hline $\mathrm{C}$ & -2.22768800 & -0.76548200 & -0.53208900 \\
\hline $\mathrm{O}$ & -2.45816200 & -1.50813400 & -1.45218400 \\
\hline $\mathrm{O}$ & -3.28899600 & -0.36822700 & 0.34069300 \\
\hline $\mathrm{C}$ & -4.59561500 & -0.75026200 & 0.10445100 \\
\hline $\mathrm{C}$ & -5.19456800 & -0.69096200 & -1.16202200 \\
\hline $\mathrm{C}$ & -5.34196000 & -1.13048300 & 1.22741600 \\
\hline $\mathrm{C}$ & -6.53844600 & -1.02171600 & -1.29743200 \\
\hline $\mathrm{H}$ & -4.61560600 & -0.39178600 & -2.02541100 \\
\hline $\mathrm{C}$ & -6.68590100 & -1.45738800 & 1.09529400 \\
\hline $\mathrm{H}$ & -4.85538500 & -1.15824700 & 2.19692200 \\
\hline $\mathrm{C}$ & -7.26877900 & -1.40282800 & -0.17098900 \\
\hline $\mathrm{H}$ & -7.02981500 & -0.98444300 & -2.26180300 \\
\hline $\mathrm{H}$ & -7.28531100 & -1.75266500 & 1.94745000 \\
\hline
\end{tabular}




$\begin{array}{llrr}\mathrm{N} & -8.68409000 & -1.75036100 & -0.31919900 \\ \mathrm{O} & -9.17419700 & -1.69706400 & -1.44816200 \\ \mathrm{O} & -9.30415400 & -2.07676200 & 0.69498800\end{array}$

\section{TS10-NO2}

$\mathrm{E}(\mathrm{B} 3 \mathrm{LYP} / \mathrm{BS} 1)=-3058.411876$

$\mathrm{E}(\mathrm{PCM}($ dichloroethane $) / \mathrm{M} 06 / \mathrm{BS} 2 / / \mathrm{B} 3 \mathrm{LYP} / \mathrm{BS} 1)=-3058.626197$

Zero-point correction $=$ 0.910074

Thermal correction to Energy= 0.967802

Thermal correction to Enthalpy= 0.968746

Thermal correction to Gibbs Free Energy= 0.809588

$\begin{array}{lrrr}\text { Charge }= & \text { Multiplicity }=1 & & \\ \mathrm{C} & -3.24787400 & -0.62134500 & -1.35059000 \\ \mathrm{C} & -4.38617500 & -0.28693700 & -2.11006400 \\ \mathrm{H} & -4.64720600 & 0.75444500 & -2.25752100 \\ \mathrm{C} & -5.20347300 & -1.25247300 & -2.69247600 \\ \mathrm{H} & -6.07028100 & -0.94878900 & -3.27333900 \\ \mathrm{C} & -4.89603400 & -2.60127300 & -2.52543200 \\ \mathrm{H} & -5.51465200 & -3.37132400 & -2.97857500 \\ \mathrm{C} & -3.78552400 & -2.95546700 & -1.76690000 \\ \mathrm{H} & -3.54558200 & -4.00496700 & -1.62388000 \\ \mathrm{C} & -2.94979000 & -1.99426100 & -1.17010100 \\ \mathrm{C} & -1.81094200 & -2.54697800 & -0.36630900 \\ \mathrm{C} & -0.48365800 & -2.50222100 & -0.82300800 \\ \mathrm{C} & 0.54752500 & -3.16792800 & -0.14873700 \\ \mathrm{H} & 1.56921400 & -3.09513100 & -0.49829100 \\ \mathrm{C} & 0.24795400 & -3.88431400 & 1.00386700 \\ \mathrm{H} & 1.04321400 & -4.39791500 & 1.53705700 \\ \mathrm{C} & -1.05424900 & -3.93992700 & 1.50071300 \\ \mathrm{H} & -1.25435400 & -4.49143400 & 2.41050300 \\ \mathrm{C} & -2.08104800 & -3.27707800 & 0.81788400 \\ \mathrm{C} & -3.84055400 & -4.15489800 & 2.27052000 \\ \mathrm{H} & -3.27692900 & -5.09521400 & 2.21425300 \\ \mathrm{C} & -3.63744200 & -3.49902600 & 3.63700700 \\ \mathrm{H} & -3.96098500 & -4.17739400 & 4.43477600 \\ \mathrm{H} & -4.23036100 & -2.58056900 & 3.70590100 \\ \mathrm{H} & -2.58909400 & -3.24052100 & 3.81219300 \\ \mathrm{C} & -5.31028500 & -4.43633700 & 1.97381100 \\ \mathrm{H} & -5.73133800 & -5.10237900 & 2.73469400 \\ \mathrm{H} & -5.42346400 & -4.90915000 & 0.99361200 \\ \mathrm{H} & -5.88399000 & -3.50332800 & 1.97334900 \\ \mathrm{C} & 0.66165500 & -2.22978900 & -2.97024100 \\ \mathrm{H} & 1.62721700 & -2.45810000 & -2.50364400 \\ \mathrm{C} & 0.84760700 & -1.08772100 & -3.95959000 \\ \mathrm{H} & 1.53019200 & -1.39583300 & -4.75919800 \\ \mathrm{H} & 1.26978200 & -0.20795800 & -3.46395900 \\ \mathrm{H} & -0.11113600 & -0.81041100 & -4.41050200 \\ \mathrm{C} & 0.08064900 & -3.48786600 & -3.61063600 \\ \mathrm{H} & 0.75800500 & -3.86071000 & -4.38702500 \\ \mathrm{H} & -0.88910600 & -3.26734100 & -4.06980800 \\ \mathrm{H} & -0.05729500 & -4.28304800 & -2.87130500 \\ \mathrm{C} & -2.46458400 & 2.17598800 & -1.83748600 \\ \mathrm{H} & -3.54445800 & 2.34606000 & -1.94067500 \\ \mathrm{C} & -1.84539800 & 3.48267400 & -1.29578100\end{array}$




\begin{tabular}{lrrr}
$\mathrm{H}$ & -2.28390800 & 3.74915400 & -0.32772900 \\
$\mathrm{H}$ & -0.77266000 & 3.33455500 & -1.12190500 \\
$\mathrm{C}$ & -2.04367100 & 4.64857200 & -2.28019000 \\
$\mathrm{H}$ & -3.11690700 & 4.87733000 & -2.36571200 \\
$\mathrm{H}$ & -1.56242800 & 5.54738700 & -1.87517600 \\
$\mathrm{C}$ & -1.48262500 & 4.32134100 & -3.67079900 \\
$\mathrm{H}$ & -0.39035400 & 4.21367700 & -3.60259700 \\
$\mathrm{H}$ & -1.67452200 & 5.14966000 & -4.36486100 \\
$\mathrm{C}$ & -2.08493200 & 3.01848900 & -4.21477100 \\
$\mathrm{H}$ & -1.63606200 & 2.76385000 & -5.18353500 \\
$\mathrm{H}$ & -3.16055900 & 3.16240400 & -4.39773700 \\
$\mathrm{C}$ & -1.88782300 & 1.85150900 & -3.23262900 \\
$\mathrm{H}$ & -0.81346800 & 1.64368100 & -3.12765300 \\
$\mathrm{H}$ & -2.34670100 & 0.94117200 & -3.63561600 \\
$\mathrm{C}$ & -3.04141700 & 1.27212900 & 0.93544600 \\
$\mathrm{H}$ & -2.46014300 & 2.15518000 & 1.23911600 \\
$\mathrm{C}$ & -4.51935300 & 1.68459600 & 0.79687700 \\
$\mathrm{H}$ & -5.10307100 & 0.82546800 & 0.43980000 \\
$\mathrm{H}$ & -4.63346000 & 2.47822700 & 0.04821900 \\
$\mathrm{C}$ & -5.09602000 & 2.16473300 & 2.14160300 \\
$\mathrm{H}$ & -4.58631500 & 3.09186000 & 2.44296000 \\
$\mathrm{H}$ & -6.15705500 & 2.41819300 & 2.01909400 \\
$\mathrm{C}$ & -4.92020100 & 1.11031500 & 3.24332500 \\
$\mathrm{H}$ & -5.52708100 & 0.22618700 & 2.99625700 \\
$\mathrm{H}$ & -5.29891200 & 1.49301800 & 4.19972100 \\
$\mathrm{C}$ & -3.44884400 & 0.69280000 & 3.37818000 \\
$\mathrm{H}$ & -3.34238500 & -0.09562200 & 4.13440700 \\
$\mathrm{~F}$ & -2.85981100 & 1.54895100 & 3.73908800 \\
$\mathrm{H}$ & -2.87053700 & 0.20685400 & 2.03801900 \\
$\mathrm{O}$ & 2.28789400 & 0.49378400 & -0.52383900 \\
$\mathrm{C}$ & 2.85863400 & 1.18555000 & -1.34221000 \\
$\mathrm{C}$ & 2.88289300 & -0.69720700 & -0.04841000 \\
$\mathrm{H}$ & 4.25218000 & -0.86826500 & -0.16005000 \\
$\mathrm{H}$ & -3.38202300 & -0.71649000 & 1.73657300 \\
$\mathrm{O}$ & -1.80913200 & -0.04174000 & 2.15510700 \\
$\mathrm{O}$ & -3.38866100 & -3.28591900 & 1.21179200 \\
$\mathrm{P}$ & -0.24136500 & -1.72687200 & -1.94527700 \\
$\mathrm{H}$ & -2.17742700 & 0.73763200 & -0.65204000 \\
$\mathrm{C}$ & 0.26290400 & 0.36554100 & -0.48906800 \\
$\mathrm{C}$ & 1.42956200 & 1.37154200 & 0.96446500 \\
$\mathrm{C}$ & 1.69567600 & 0.65134500 & 2.07762400 \\
$\mathrm{H}$ & 1.75686500 & -0.42834400 & 2.01587300 \\
$\mathrm{C}$ & 2.00026400 & 1.15256800 & 3.45538000 \\
$\mathrm{C}$ & 1.25319000 & 2.84654700 & 0.93941200 \\
$\mathrm{C}$ & 1.94788800 & 3.69346500 & 0.05724400 \\
$\mathrm{C}$ & 0.32464600 & 3.42678000 & 1.82832500 \\
$\mathrm{C}$ & 1.72111900 & 5.07060100 & 0.07230300 \\
$\mathrm{H}$ & 2.66200400 & 3.26911300 & -0.63626900 \\
$\mathrm{H}$ & 0.10195400 & 4.80144500 & 1.83751500 \\
$\mathrm{H}$ & -0.21594800 & 2.78845300 & 2.52005900 \\
$\mathrm{H}$ & 0.79896200 & 5.63254300 & 0.95685800 \\
\hline
\end{tabular}




$\begin{array}{lrrr}\mathrm{C} & 4.97420000 & -0.99637300 & 1.03175700 \\ \mathrm{C} & 4.88558300 & -0.97472400 & -1.40307400 \\ \mathrm{C} & 6.34163300 & -1.24552600 & 0.98524300 \\ \mathrm{H} & 4.45759500 & -0.88593300 & 1.97988100 \\ \mathrm{C} & 6.25398600 & -1.22039700 & -1.45276000 \\ \mathrm{H} & 4.31030300 & -0.86157100 & -2.31336200 \\ \mathrm{C} & 6.96300300 & -1.35629100 & -0.25899000 \\ \mathrm{H} & 6.93013600 & -1.35045100 & 1.88815400 \\ \mathrm{H} & 6.77818500 & -1.31037700 & -2.39604700 \\ \mathrm{~N} & 8.40478600 & -1.62499800 & -0.31407000 \\ \mathrm{O} & 8.92898100 & -1.71563600 & -1.42499700 \\ \mathrm{O} & 9.00621100 & -1.74632200 & 0.75371800\end{array}$

\section{pF-PhO'}

$\mathrm{E}(\mathrm{B} 3 \mathrm{LYP} / \mathrm{BS} 1)=-406.120504$

$\mathrm{E}(\mathrm{PCM}($ dichloroethane $) / \mathrm{M} 06 / \mathrm{BS} 2 / / \mathrm{B} 3 \mathrm{LYP} / \mathrm{BS} 1)=-406.091988$

$\begin{array}{ll}\text { Zero-point correction }= & 0.082742 \\ \text { Thermal correction to Energy= } & 0.088826 \\ \text { Thermal correction to Enthalpy= } & 0.089770 \\ \text { Thermal correction to Gibbs Free Energy= } & 0.052533\end{array}$

$\begin{array}{lrrr}\text { Charge }=-1 \text { Multiplicity }=1 \\ \text { C } & 0.65640400 & -1.20944900 & 0.00000100 \\ \text { C } & 1.34658400 & 0.00000000 & -0.00001700 \\ \text { C } & 0.65640400 & 1.20944800 & -0.00000100 \\ \text { C } & -0.73559300 & 1.21258700 & -0.00003400 \\ \text { C } & -1.53360900 & 0.00000000 & -0.00021600 \\ \text { C } & -0.73559300 & -1.21258700 & -0.00003300 \\ \text { H } & 1.22333800 & -2.14075600 & 0.00006500 \\ \text { H } & 1.22333800 & 2.14075600 & 0.00006300 \\ \text { H } & -1.27838000 & 2.15729600 & 0.00004000 \\ \text { H } & -1.27838000 & -2.15729500 & 0.00004100 \\ \text { O } & -2.80038700 & 0.00000000 & 0.00013800 \\ \text { F } & 2.73173200 & 0.00000000 & 0.00005400\end{array}$

\section{IM6-F}

$\mathrm{E}(\mathrm{B} 3 \mathrm{LYP} / \mathrm{BS} 1)=-2839.837957$

$\mathrm{E}(\mathrm{PCM}($ dichloroethane $) / \mathrm{M} 06 / \mathrm{BS} 2 / / \mathrm{B} 3 \mathrm{LYP} / \mathrm{BS} 1)=-2840.112225$

Zero-point correction $=$

Thermal correction to Energy=

Thermal correction to Enthalpy=

Thermal correction to Gibbs Free Energy=
0.891470

0.945661

0.946606

0.798708 


\begin{tabular}{|c|c|c|c|}
\hline & 4.50503600 & -2.48049700 & 1.66120000 \\
\hline $\mathrm{C}$ & 2.94964900 & -1.03728300 & 1.36484400 \\
\hline $\mathrm{C}$ & 2.52816100 & -1.80295900 & 0.14851200 \\
\hline $\mathrm{C}$ & 1.31462700 & -2.50505600 & 0.12893100 \\
\hline $\mathrm{C}$ & 0.94462300 & -3.32956500 & -0.93765300 \\
\hline $\mathrm{H}$ & -0.03173500 & -3.79672700 & -0.94888000 \\
\hline $\mathrm{C}$ & 1.82838700 & -3.47197900 & -2.00120100 \\
\hline $\mathrm{H}$ & 1.55597600 & -4.10331200 & -2.84240900 \\
\hline $\mathrm{C}$ & 3.05336900 & -2.80304900 & -2.01976800 \\
\hline $\mathrm{H}$ & 3.71369100 & -2.92697200 & -2.86869700 \\
\hline $\mathrm{C}$ & 3.40561400 & -1.97081600 & -0.95021200 \\
\hline $\mathrm{C}$ & 5.64645900 & -1.51716000 & -1.81665000 \\
\hline $\mathrm{H}$ & 5.64994300 & -2.58301500 & -2.07885900 \\
\hline $\mathrm{C}$ & 5.45709400 & -0.66539200 & -3.07228300 \\
\hline $\mathrm{H}$ & 6.25725400 & -0.86988000 & -3.79286400 \\
\hline $\mathrm{H}$ & 5.49073700 & 0.39859600 & -2.81423200 \\
\hline $\mathrm{H}$ & 4.49847500 & -0.86705000 & -3.55901400 \\
\hline $\mathrm{C}$ & 6.93749500 & -1.18173900 & -1.07632100 \\
\hline $\mathrm{H}$ & 7.80243700 & -1.33879600 & -1.72975000 \\
\hline $\mathrm{H}$ & 7.05101400 & -1.81268000 & -0.18955400 \\
\hline $\mathrm{H}$ & 6.93107300 & -0.13469200 & -0.75490100 \\
\hline $\mathrm{C}$ & -0.26284100 & -3.41718400 & 1.79163000 \\
\hline $\mathrm{H}$ & -1.00988300 & -3.71752700 & 1.05243000 \\
\hline $\mathrm{C}$ & -0.96138300 & -2.85413000 & 3.02036700 \\
\hline $\mathrm{H}$ & -1.59497100 & -3.62562400 & 3.47058400 \\
\hline $\mathrm{H}$ & -1.59626200 & -2.00716300 & 2.74814900 \\
\hline $\mathrm{H}$ & -0.22904700 & -2.52292200 & 3.76517100 \\
\hline $\mathrm{C}$ & 0.68094600 & -4.56462000 & 2.13924300 \\
\hline $\mathrm{H}$ & 0.10650200 & -5.37821600 & 2.59576500 \\
\hline $\mathrm{H}$ & 1.44044800 & -4.23335000 & 2.85650300 \\
\hline $\mathrm{H}$ & 1.18610100 & -4.96444900 & 1.25576900 \\
\hline $\mathrm{C}$ & 0.17866200 & 2.12546900 & 2.37932100 \\
\hline $\mathrm{H}$ & 1.02060200 & 2.62213000 & 2.87633300 \\
\hline $\mathrm{C}$ & -0.71150000 & 3.25664100 & 1.81550900 \\
\hline $\mathrm{H}$ & -0.14117600 & 3.88454200 & 1.12176500 \\
\hline $\mathrm{H}$ & -1.54309600 & 2.83732200 & 1.24229100 \\
\hline $\mathrm{C}$ & -1.26281900 & 4.13727200 & 2.95191300 \\
\hline $\mathrm{H}$ & -0.42998200 & 4.66504100 & 3.44106000 \\
\hline $\mathrm{H}$ & -1.91492400 & 4.90969600 & 2.52594900 \\
\hline $\mathrm{C}$ & -2.02357100 & 3.31346100 & 4.00008300 \\
\hline $\mathrm{H}$ & -2.92166900 & 2.87861500 & 3.53779700 \\
\hline $\mathrm{H}$ & -2.37342400 & 3.96228800 & 4.81283000 \\
\hline $\mathrm{C}$ & -1.14455600 & 2.18598500 & 4.55831300 \\
\hline $\mathrm{H}$ & -1.71325200 & 1.56938800 & 5.26583100 \\
\hline $\mathrm{H}$ & -0.30942100 & 2.62263500 & 5.12660500 \\
\hline $\mathrm{C}$ & -0.58612900 & 1.29725700 & 3.43467100 \\
\hline $\mathrm{H}$ & -1.41285500 & 0.77206000 & 2.93705300 \\
\hline $\mathrm{H}$ & 0.06411400 & 0.52163900 & 3.85686900 \\
\hline $\mathrm{C}$ & 1.68481300 & 2.18929400 & -0.17706200 \\
\hline $\mathrm{H}$ & 0.82846400 & 2.75103000 & -0.57558400 \\
\hline $\mathrm{C}$ & 2.67566100 & 3.18297000 & 0.46592700 \\
\hline $\mathrm{H}$ & 3.51141100 & 2.62325300 & 0.90666500 \\
\hline $\mathrm{H}$ & 2.20286100 & 3.73930300 & 1.28374700 \\
\hline $\mathrm{C}$ & 3.22300600 & 4.17793900 & -0.57385600 \\
\hline H & 2.40021400 & 4.81287500 & -0.93408700 \\
\hline & 3.94718400 & 4.84852000 & -0.09341800 \\
\hline & 3.86547900 & 3.45675100 & -1.7664500 \\
\hline
\end{tabular}




$\begin{array}{llcc}\mathrm{H} & 4.76266700 & 2.92042500 & -1.42188300 \\ \mathrm{H} & 4.20386600 & 4.18651300 & -2.51320000 \\ \mathrm{C} & 2.88608800 & 2.45762400 & -2.39716500 \\ \mathrm{H} & 3.37188500 & 1.91011100 & -3.21477700 \\ \mathrm{H} & 2.04291200 & 2.99978000 & -2.84626300 \\ \mathrm{C} & 2.34730400 & 1.45592500 & -1.36067200 \\ \mathrm{H} & 3.17851400 & 0.84388500 & -0.99068000 \\ \mathrm{H} & 1.62974500 & 0.77929000 & -1.83570400 \\ \mathrm{O} & 4.58225900 & -1.28157200 & -0.87152300 \\ \mathrm{O} & 0.48477700 & -2.28915600 & 1.22108800 \\ \mathrm{P} & 0.88660100 & 0.99893200 & 1.04235000 \\ \mathrm{Pd} & -0.73797600 & -0.51574100 & 0.21870300 \\ \mathrm{C} & -1.80319600 & 0.82054900 & -0.82719700 \\ \mathrm{C} & -2.90427400 & 1.33112400 & -0.25794900 \\ \mathrm{H} & -3.15343400 & 1.10716800 & 0.77420900 \\ \mathrm{C} & -3.91700300 & 2.23207900 & -0.89832500 \\ \mathrm{C} & -1.34123500 & 1.04750000 & -2.21270300 \\ \mathrm{C} & -1.04815600 & -0.04869100 & -3.04712800 \\ \mathrm{C} & -1.17540400 & 2.34796000 & -2.72531500 \\ \mathrm{C} & -0.61710300 & 0.15464300 & -4.35660900 \\ \mathrm{H} & -1.18358400 & -1.05105900 & -2.65248900 \\ \mathrm{C} & -0.73487900 & 2.54486000 & -4.03462300 \\ \mathrm{H} & -1.41800500 & 3.20205200 & -2.10111500 \\ \mathrm{C} & -0.45289300 & 1.45053900 & -4.85481000 \\ \mathrm{H} & -0.40968100 & -0.70248200 & -4.99225800 \\ \mathrm{H} & -0.62060100 & 3.55642000 & -4.41618100 \\ \mathrm{H} & -0.11270900 & 1.60585500 & -5.87531900 \\ \mathrm{~F} & -4.14716500 & 1.98074400 & -2.20100900 \\ \mathrm{~F} & -3.54678200 & 3.54963100 & -0.81784700 \\ \mathrm{~F} & -5.10105800 & 2.13879900 & -0.25165100 \\ \mathrm{O} & -1.98990800 & -2.02918400 & -0.45579900 \\ \mathrm{C} & -3.31848700 & -2.10904900 & -0.38426800 \\ \mathrm{C} & -4.05990000 & -1.73673000 & 0.76111200 \\ \mathrm{C} & -4.04020900 & -2.64754200 & -1.47583600 \\ \mathrm{C} & -5.44339700 & -1.90486800 & 0.82072200 \\ \mathrm{H} & -3.53096700 & -1.31126600 & 1.60984100 \\ \mathrm{C} & -5.42019400 & -2.82810900 & -1.41901700 \\ \mathrm{H} & -3.48622400 & -2.93073200 & -2.36645500 \\ \mathrm{C} & -6.10733600 & -2.45253000 & -0.26965200 \\ \mathrm{H} & -6.00970000 & -1.61650700 & 1.70147600 \\ \mathrm{H} & -5.96946200 & -3.24783000 & -2.25658500 \\ \mathrm{~F} & -7.45384700 & -2.62279900 & -0.21293100\end{array}$

\section{IM8- F}

$\mathrm{E}(\mathrm{B} 3 \mathrm{LYP} / \mathrm{BS} 1)=-2953.155466$

$\mathrm{E}(\mathrm{PCM}$ (dichloroethane)/M06/BS2// B3LYP/BS1 $)=-2953.407576$

Zero-point correction $=$

Thermal correction to Energy=

Thermal correction to Enthalpy=

Thermal correction to Gibbs Free Energy=
0.899361

0.956341

0.957286

0.802648

\footnotetext{
Charge $=0$ Multiplicity $=1$

$\begin{array}{llll}\text { C } & -0.23126700 & 1.65496600 & 2.04341400\end{array}$

$\begin{array}{llll}\text { C } & -0.44751700 & 1.90730300 & 3.41465400\end{array}$

$\begin{array}{llll}\mathrm{H} & -0.63345500 & 1.08069200 & 4.09065800\end{array}$
} 


\begin{tabular}{|c|c|c|c|}
\hline $\mathrm{C}$ & -0.42371100 & 3.19333100 & 3.94465700 \\
\hline $\mathrm{H}$ & -0.59399500 & 3.34388800 & 5.00716600 \\
\hline $\mathrm{C}$ & -0.17658500 & 4.27537500 & 3.10106100 \\
\hline $\mathrm{H}$ & -0.15319400 & 5.28877700 & 3.49271300 \\
\hline $\mathrm{C}$ & 0.05449800 & 4.04383300 & 1.75005800 \\
\hline $\mathrm{H}$ & 0.27036900 & 4.87911300 & 1.09005300 \\
\hline $\mathrm{C}$ & 0.03658900 & 2.75098100 & 1.19406000 \\
\hline $\mathrm{C}$ & 0.36397800 & 2.68357300 & -0.26647000 \\
\hline $\mathrm{C}$ & 1.72207400 & 2.63251200 & -0.65709100 \\
\hline $\mathrm{C}$ & 2.07346200 & 2.74872700 & -2.01154300 \\
\hline $\mathrm{H}$ & 3.11000300 & 2.71213300 & -2.32087100 \\
\hline $\mathrm{C}$ & 1.07366700 & 2.93398400 & -2.96447500 \\
\hline $\mathrm{H}$ & 1.34942500 & 3.02138600 & -4.01216900 \\
\hline $\mathrm{C}$ & -0.26862600 & 3.02433400 & -2.60272000 \\
\hline $\mathrm{H}$ & -1.02969600 & 3.18145900 & -3.35787600 \\
\hline $\mathrm{C}$ & -0.61679000 & 2.92077400 & -1.24733500 \\
\hline $\mathrm{C}$ & -2.81161500 & 3.96074200 & -1.46045800 \\
\hline $\mathrm{H}$ & -2.21153400 & 4.66403500 & -2.05129500 \\
\hline $\mathrm{C}$ & -3.77563800 & 3.20362500 & -2.37066400 \\
\hline $\mathrm{H}$ & -4.48417000 & 3.90201200 & -2.83125900 \\
\hline $\mathrm{H}$ & -4.34530300 & 2.46418100 & -1.79850100 \\
\hline $\mathrm{H}$ & -3.25543700 & 2.67108300 & -3.17077000 \\
\hline $\mathrm{C}$ & -3.53329500 & 4.72006600 & -0.34998000 \\
\hline $\mathrm{H}$ & -4.21949300 & 5.46043600 & -0.77624900 \\
\hline $\mathrm{H}$ & -2.81557200 & 5.23629800 & 0.29472200 \\
\hline $\mathrm{H}$ & -4.11444600 & 4.02825800 & 0.26963000 \\
\hline $\mathrm{C}$ & 4.03029400 & 2.61068800 & 0.14277400 \\
\hline $\mathrm{H}$ & 4.29269900 & 2.00724400 & -0.73292800 \\
\hline $\mathrm{C}$ & 4.67548300 & 1.98771000 & 1.37434000 \\
\hline $\mathrm{H}$ & 5.76659500 & 2.01667600 & 1.28220400 \\
\hline $\mathrm{H}$ & 4.36002200 & 0.94705700 & 1.47884600 \\
\hline $\mathrm{H}$ & 4.386688800 & 2.53820800 & 2.27683800 \\
\hline $\mathrm{C}$ & 4.44032500 & 4.07046800 & -0.05948500 \\
\hline $\mathrm{H}$ & 5.52102300 & 4.13923000 & -0.22792500 \\
\hline $\mathrm{H}$ & 4.19218500 & 4.65761800 & 0.83188300 \\
\hline $\mathrm{H}$ & 3.93307200 & 4.52062300 & -0.91838500 \\
\hline $\mathrm{C}$ & 0.46766600 & -1.09841500 & 2.85723200 \\
\hline $\mathrm{H}$ & -0.14826500 & -0.99362900 & 3.76022500 \\
\hline $\mathrm{C}$ & 0.50826700 & -2.59725500 & 2.48628900 \\
\hline $\mathrm{H}$ & -0.49453100 & -2.97472900 & 2.24855800 \\
\hline $\mathrm{H}$ & 1.11567500 & -2.71097000 & 1.57986500 \\
\hline $\mathrm{C}$ & 1.12509600 & -3.42866300 & 3.62439900 \\
\hline $\mathrm{H}$ & 0.47118400 & -3.37936900 & 4.50833300 \\
\hline $\mathrm{H}$ & 1.16763600 & -4.48435400 & 3.32724800 \\
\hline $\mathrm{C}$ & 2.52489100 & -2.91945700 & 3.99669300 \\
\hline $\mathrm{H}$ & 3.20595700 & -3.09235500 & 3.15101200 \\
\hline $\mathrm{H}$ & 2.92469500 & -3.49176300 & 4.84373200 \\
\hline $\mathrm{C}$ & 2.50209500 & -1.41980500 & 4.32769000 \\
\hline $\mathrm{H}$ & 3.51894400 & -1.06101000 & 4.53224400 \\
\hline $\mathrm{H}$ & 1.92554400 & -1.26340300 & 5.25280200 \\
\hline $\mathrm{C}$ & 1.88482100 & -0.58634500 & 3.19047500 \\
\hline $\mathrm{H}$ & 2.49718800 & -0.65107000 & 2.28674300 \\
\hline $\mathrm{H}$ & 1.85223900 & 0.46829300 & 3.48267000 \\
\hline $\mathrm{C}$ & -2.26444600 & -0.47064800 & 1.78723300 \\
\hline $\mathrm{H}$ & -2.44168400 & -1.35028000 & 1.15927800 \\
\hline $\mathrm{C}$ & -2.70110400 & -0.84516800 & 3.22044100 \\
\hline $\mathrm{H}$ & -2.51566600 & -0.00704200 & 3.90507300 \\
\hline
\end{tabular}




$\begin{array}{lrrr}\mathrm{H} & -2.12996000 & -1.70148000 & 3.59442600 \\ \mathrm{C} & -4.20134700 & -1.19305800 & 3.26181600 \\ \mathrm{H} & -4.37633700 & -2.09452500 & 2.65686700 \\ \mathrm{H} & -4.48749400 & -1.44597400 & 4.29094500 \\ \mathrm{C} & -5.06816800 & -0.04503000 & 2.72889400 \\ \mathrm{H} & -4.97080000 & 0.82270900 & 3.39825800 \\ \mathrm{H} & -6.12761600 & -0.33146900 & 2.73949000 \\ \mathrm{C} & -4.63382600 & 0.35394400 & 1.31282300 \\ \mathrm{H} & -5.21206200 & 1.22018400 & 0.96551500 \\ \mathrm{H} & -4.86008400 & -0.46522200 & 0.61740400 \\ \mathrm{C} & -3.13328800 & 0.68670900 & 1.24683900 \\ \mathrm{H} & -2.94102100 & 1.58751800 & 1.84281000 \\ \mathrm{H} & -2.85147100 & 0.93289700 & 0.22046100 \\ \mathrm{O} & -1.90471000 & 3.04218400 & -0.80039200 \\ \mathrm{O} & 2.60523600 & 2.51622300 & 0.36424200 \\ \mathrm{P} & -0.41559900 & -0.11794600 & 1.51152400 \\ \mathrm{Pd} & 0.41505800 & -0.92494500 & -0.66970100 \\ \mathrm{C} & -1.49004700 & -1.36255800 & -1.37548000 \\ \mathrm{C} & -2.05961200 & -0.44556000 & -2.17676300 \\ \mathrm{H} & -1.58333900 & 0.52037100 & -2.31573800 \\ \mathrm{C} & -3.32577100 & -0.56089000 & -2.97181800 \\ \mathrm{C} & -2.01663200 & -2.70681300 & -1.03916700 \\ \mathrm{C} & -1.15498300 & -3.81620400 & -0.92458800 \\ \mathrm{C} & -3.38748700 & -2.91981400 & -0.77985000 \\ \mathrm{C} & -1.64036100 & -5.08185800 & -0.59870300 \\ \mathrm{H} & -0.09032200 & -3.68673000 & -1.09010200 \\ \mathrm{C} & -3.86997400 & -4.18231300 & -0.44112200 \\ \mathrm{H} & -4.07950400 & -2.08949700 & -0.84942600 \\ \mathrm{C} & -3.00093300 & -5.27179900 & -0.35203400 \\ \mathrm{H} & -0.95023700 & -5.91852600 & -0.53110700 \\ \mathrm{H} & -4.93142100 & -4.31376000 & -0.24799800 \\ \mathrm{H} & -3.37901300 & -6.25575400 & -0.08852800 \\ \mathrm{~F} & -3.56706700 & -1.79076300 & -3.46752700 \\ \mathrm{~F} & -3.28298300 & 0.28842700 & -4.03003800 \\ \mathrm{~F} & -4.43539000 & -0.20677200 & -2.25202000 \\ \mathrm{C} & 0.93476900 & -1.59067500 & -2.44407200 \\ \mathrm{O} & 1.14594500 & -1.97486100 & -3.49633100 \\ \mathrm{O} & 2.37758200 & -0.76363000 & 0.08143200 \\ \mathrm{C} & 3.48649400 & -1.04107300 & -0.60156500 \\ \mathrm{C} & 3.78196700 & -0.49711800 & -1.87478300 \\ \mathrm{C} & 4.46612600 & -1.88703200 & -0.02255100 \\ \mathrm{C} & 4.98008700 & -0.78435500 & -2.53395900 \\ \mathrm{H} & 3.06295000 & 0.17548400 & -2.33318800 \\ \mathrm{C} & 5.66616200 & -2.16865900 & -0.67167000 \\ \mathrm{C} & 4.26058900 & -2.31787500 & 0.95330300 \\ \mathrm{H} & 5.91026800 & -1.61542700 & -1.92451800 \\ & 5.19758100 & -0.36551900 & -3.51240300 \\ & 6.41061500 & -2.81850000 & -0.22081700 \\ & 7.07730600 & -1.89440800 & -2.56158300\end{array}$

\section{TS9- F}

$\mathrm{E}(\mathrm{B} 3 \mathrm{LYP} / \mathrm{BS} 1)=-2953.145805$

$\mathrm{E}(\mathrm{PCM}$ (dichloroethane $) / \mathrm{M} 06 / \mathrm{BS} 2 / / \mathrm{B} 3 \mathrm{LYP} / \mathrm{BS} 1)=-2953.395171$

$\begin{array}{ll}\text { Zero-point correction }= & 0.898573 \\ \text { Thermal correction to Energy= } & 0.954993\end{array}$ 
Thermal correction to Enthalpy= $\quad 0.955937$

Thermal correction to Gibbs Free Energy $=\quad 0.802662$

\begin{tabular}{|c|c|c|c|}
\hline \multicolumn{4}{|c|}{ Charge $=0$ Multiplicity $=1$} \\
\hline $\mathrm{C}$ & 1.01698600 & -1.76902000 & 1.91297500 \\
\hline $\mathrm{C}$ & 1.15298800 & -2.12357400 & 3.27046300 \\
\hline $\mathrm{H}$ & 0.85120600 & -1.42682300 & 4.04381700 \\
\hline $\mathrm{C}$ & 1.66306000 & -3.35753500 & 3.66469500 \\
\hline $\mathrm{H}$ & 1.75017400 & -3.59397400 & 4.72167500 \\
\hline $\mathrm{C}$ & 2.05656900 & -4.27742800 & 2.69428200 \\
\hline $\mathrm{H}$ & 2.46026500 & -5.24482900 & 2.98086600 \\
\hline $\mathrm{C}$ & 1.91317000 & -3.95213100 & 1.34968300 \\
\hline $\mathrm{H}$ & 2.19617900 & -4.67229700 & 0.58751100 \\
\hline $\mathrm{C}$ & 1.39028800 & -2.71612600 & 0.93083500 \\
\hline $\mathrm{C}$ & 1.22302800 & -2.54850000 & -0.54829800 \\
\hline $\mathrm{C}$ & -0.01772700 & -2.87296000 & -1.14027500 \\
\hline $\mathrm{C}$ & -0.16547500 & -2.86005500 & -2.53649100 \\
\hline $\mathrm{H}$ & -1.12003700 & -3.07973900 & -2.99634400 \\
\hline $\mathrm{C}$ & 0.93664500 & -2.56342600 & -3.33285800 \\
\hline $\mathrm{H}$ & 0.82217600 & -2.55188800 & -4.41337600 \\
\hline $\mathrm{C}$ & 2.18508200 & -2.28338900 & -2.77879200 \\
\hline $\mathrm{H}$ & 3.02381900 & -2.06201000 & -3.42655700 \\
\hline $\mathrm{C}$ & 2.32868800 & -2.28444600 & -1.38333300 \\
\hline $\mathrm{C}$ & 4.76676100 & -2.15824800 & -1.44650600 \\
\hline $\mathrm{H}$ & 4.64894900 & -2.90267700 & -2.24446800 \\
\hline $\mathrm{C}$ & 5.18494700 & -0.81310000 & -2.04010600 \\
\hline $\mathrm{H}$ & 6.15379900 & -0.91221400 & -2.54348600 \\
\hline $\mathrm{H}$ & 5.28556700 & -0.06144300 & -1.25013400 \\
\hline $\mathrm{H}$ & 4.46452900 & -0.43433100 & -2.76920400 \\
\hline $\mathrm{C}$ & 5.77233800 & -2.67844300 & -0.42304200 \\
\hline $\mathrm{H}$ & 6.75437400 & -2.81484200 & -0.88891800 \\
\hline $\mathrm{H}$ & 5.44355600 & -3.63743700 & -0.01122000 \\
\hline $\mathrm{H}$ & 5.87834400 & -1.96773500 & 0.40383800 \\
\hline $\mathrm{C}$ & -2.22076300 & -3.83693400 & -0.73122800 \\
\hline $\mathrm{H}$ & -2.62054800 & -3.24312700 & -1.55867700 \\
\hline $\mathrm{C}$ & -3.19491500 & -3.76121300 & 0.43710100 \\
\hline $\mathrm{H}$ & -4.14678000 & -4.22863000 & 0.16257200 \\
\hline $\mathrm{H}$ & -3.38598300 & -2.71985800 & 0.70521600 \\
\hline $\mathrm{H}$ & -2.78941300 & -4.28608600 & 1.30951700 \\
\hline $\mathrm{C}$ & -1.94636100 & -5.27803900 & -1.16455300 \\
\hline $\mathrm{H}$ & -2.87145200 & -5.74691600 & -1.51885100 \\
\hline $\mathrm{H}$ & -1.56759400 & -5.86073400 & -0.31724900 \\
\hline $\mathrm{H}$ & -1.20998400 & -5.32803400 & -1.97214900 \\
\hline $\mathrm{C}$ & -0.90038800 & 0.28057400 & 2.81884700 \\
\hline $\mathrm{H}$ & -0.44612100 & 0.13714000 & 3.80784400 \\
\hline $\mathrm{C}$ & -1.40116100 & 1.73952300 & 2.74418300 \\
\hline $\mathrm{H}$ & -0.57186500 & 2.44624100 & 2.86802900 \\
\hline $\mathrm{H}$ & -1.81753800 & 1.92808000 & 1.74537500 \\
\hline $\mathrm{C}$ & -2.47614800 & 2.01049000 & 3.81206300 \\
\hline $\mathrm{H}$ & -2.01777400 & 1.93788100 & 4.80982500 \\
\hline $\mathrm{H}$ & -2.84008000 & 3.04091700 & 3.71192200 \\
\hline $\mathrm{C}$ & -3.64361300 & 1.01723100 & 3.71580300 \\
\hline $\mathrm{H}$ & -4.18384000 & 1.17832900 & 2.77265600 \\
\hline $\mathrm{H}$ & -4.36272500 & 1.20023900 & 4.52425800 \\
\hline $\mathrm{C}$ & -3.14656800 & -0.43489400 & 3.76321100 \\
\hline $\mathrm{H}$ & -3.98607200 & -1.12834300 & 3.62932400 \\
\hline $\mathrm{H}$ & -2.72254400 & -0.64446900 & 4.75694300 \\
\hline
\end{tabular}




\begin{tabular}{|c|c|c|c|}
\hline $\mathrm{C}$ & -2.07932300 & -0.70660600 & 2.68914700 \\
\hline $\mathrm{H}$ & -2.52963500 & -0.60630200 & 1.69300900 \\
\hline $\mathrm{H}$ & -1.72144200 & -1.73890500 & 2.76636100 \\
\hline $\mathrm{C}$ & 1.87100600 & 1.06132500 & 2.01539000 \\
\hline $\mathrm{H}$ & 1.56202100 & 2.04895900 & 1.64705300 \\
\hline $\mathrm{C}$ & 2.15281000 & 1.18212100 & 3.52822300 \\
\hline $\mathrm{H}$ & 2.43260500 & 0.19953700 & 3.92937600 \\
\hline $\mathrm{H}$ & 1.25998500 & 1.51022200 & 4.07221600 \\
\hline $\mathrm{C}$ & 3.30253500 & 2.16958800 & 3.80103600 \\
\hline $\mathrm{H}$ & 2.99795200 & 3.17700900 & 3.48155600 \\
\hline $\mathrm{H}$ & 3.48634300 & 2.22651700 & 4.88169600 \\
\hline $\mathrm{C}$ & 4.58233400 & 1.76379500 & 3.05839900 \\
\hline $\mathrm{H}$ & 4.94417000 & 0.80546400 & 3.45958000 \\
\hline $\mathrm{H}$ & 5.37687200 & 2.49919000 & 3.23814200 \\
\hline $\mathrm{C}$ & 4.31696600 & 1.61814200 & 1.55446200 \\
\hline $\mathrm{H}$ & 5.22038600 & 1.26833900 & 1.03843100 \\
\hline $\mathrm{H}$ & 4.08420900 & 2.60525800 & 1.13114800 \\
\hline $\mathrm{C}$ & 3.15672200 & 0.64983300 & 1.26512700 \\
\hline $\mathrm{H}$ & 3.44440500 & -0.36372800 & 1.57010400 \\
\hline $\mathrm{H}$ & 2.97268500 & 0.60950200 & 0.18904100 \\
\hline $\mathrm{O}$ & 3.51048100 & -2.04893500 & -0.74023800 \\
\hline $\mathrm{O}$ & -1.00380400 & -3.20847100 & -0.26496700 \\
\hline $\mathrm{P}$ & 0.42109200 & -0.04963500 & 1.52344400 \\
\hline $\mathrm{Pd}$ & -0.64424800 & 0.40624100 & -0.65678200 \\
\hline $\mathrm{C}$ & 0.68157400 & 1.79649600 & -1.28255200 \\
\hline $\mathrm{C}$ & 1.67509900 & 1.37845900 & -2.08044300 \\
\hline $\mathrm{H}$ & 1.78958000 & 0.32422900 & -2.30956200 \\
\hline $\mathrm{C}$ & 2.73385200 & 2.22074900 & -2.72318300 \\
\hline $\mathrm{C}$ & 0.40332300 & 3.17990700 & -0.83751700 \\
\hline $\mathrm{C}$ & -0.89076300 & 3.72812100 & -0.92878400 \\
\hline $\mathrm{C}$ & 1.42457900 & 3.98211100 & -0.29017600 \\
\hline $\mathrm{C}$ & -1.14956300 & 5.02944700 & -0.50362300 \\
\hline $\mathrm{H}$ & -1.69238900 & 3.12997100 & -1.34900700 \\
\hline $\mathrm{C}$ & 1.15914800 & 5.27919700 & 0.14775200 \\
\hline $\mathrm{H}$ & 2.43356900 & 3.59039500 & -0.22902400 \\
\hline $\mathrm{C}$ & -0.12800100 & 5.80919200 & 0.04464500 \\
\hline $\mathrm{H}$ & -2.15352100 & 5.43429200 & -0.59965400 \\
\hline $\mathrm{H}$ & 1.96425200 & 5.87794800 & 0.56568700 \\
\hline $\mathrm{H}$ & -0.33334600 & 6.82037100 & 0.38531300 \\
\hline $\mathrm{F}$ & 2.32299700 & 3.44053300 & -3.11784400 \\
\hline $\mathrm{F}$ & 3.22564100 & 1.58775300 & -3.81941100 \\
\hline $\mathrm{F}$ & 3.80860200 & 2.42055600 & -1.89747800 \\
\hline $\mathrm{C}$ & -1.77646000 & 0.52782800 & -2.26782400 \\
\hline $\mathrm{O}$ & -2.23260000 & 0.78956800 & -3.30195500 \\
\hline $\mathrm{O}$ & -2.74516000 & -0.65046200 & -1.22251100 \\
\hline $\mathrm{C}$ & -4.02814500 & -0.23926200 & -1.08639600 \\
\hline $\mathrm{C}$ & -5.04874100 & -0.93302200 & -1.76493900 \\
\hline $\mathrm{C}$ & -4.38751500 & 0.86712800 & -0.29322700 \\
\hline $\mathrm{C}$ & -6.38441100 & -0.55761700 & -1.63029800 \\
\hline $\mathrm{H}$ & -4.77521000 & -1.77258400 & -2.39661900 \\
\hline $\mathrm{C}$ & -5.72167900 & 1.25561300 & -0.16226100 \\
\hline $\mathrm{H}$ & -3.60709200 & 1.42201700 & 0.21891400 \\
\hline $\mathrm{C}$ & -6.70372900 & 0.53291600 & -0.82869900 \\
\hline $\mathrm{H}$ & -7.17637400 & -1.09300700 & -2.14480900 \\
\hline $\mathrm{H}$ & -6.00509700 & 2.10820000 & 0.44733500 \\
\hline $\mathrm{F}$ & -8.00102800 & 0.90200600 & -0.69822900 \\
\hline
\end{tabular}




\section{IM10- F}

$\mathrm{E}(\mathrm{B} 3 \mathrm{LYP} / \mathrm{BS} 1)=-2953.156831$

$\mathrm{E}(\mathrm{PCM}$ (dichloroethane)/M06/BS2// B3LYP/BS1 $)=-2953.40674$

$\begin{array}{ll}\text { Zero-point correction= } & 0.899995 \\ \text { Thermal correction to Energy= } & 0.956648 \\ \text { Thermal correction to Enthalpy= } & 0.957593 \\ \text { Thermal correction to Gibbs Free Energy= } & 0.801386\end{array}$

$\begin{array}{crrr}\text { Charge }= & \text { Multiplicity } & 1 & \\ \mathrm{C} & 1.74568400 & -1.66798100 & 1.82278700 \\ \mathrm{C} & 2.36808800 & -1.97463500 & 3.04818300 \\ \mathrm{H} & 2.49769100 & -1.19908100 & 3.79488100 \\ \mathrm{C} & 2.82875600 & -3.25560300 & 3.34281600 \\ \mathrm{H} & 3.30186900 & -3.45444300 & 4.30067800 \\ \mathrm{C} & 2.67879500 & -4.27071400 & 2.39937900 \\ \mathrm{H} & 3.03465900 & -5.27611700 & 2.60804100 \\ \mathrm{C} & 2.05756800 & -3.98977000 & 1.18645100 \\ \mathrm{H} & 1.92682400 & -4.78008100 & 0.45311900 \\ \mathrm{C} & 1.57297300 & -2.70675500 & 0.87659400 \\ \mathrm{C} & 0.86519900 & -2.58079100 & -0.43965800 \\ \mathrm{C} & -0.54065200 & -2.53504200 & -0.48980200 \\ \mathrm{C} & -1.22945200 & -2.61252200 & -1.70807500 \\ \mathrm{H} & -2.31073000 & -2.55923300 & -1.73316500 \\ \mathrm{C} & -0.50376400 & -2.72309500 & -2.88806500 \\ \mathrm{H} & -1.02968800 & -2.77190700 & -3.83742400 \\ \mathrm{C} & 0.89082500 & -2.74966100 & -2.88011300 \\ \mathrm{H} & 1.42549900 & -2.81822300 & -3.81836500 \\ \mathrm{C} & 1.57632200 & -2.68353800 & -1.65917500 \\ \mathrm{C} & 3.76369400 & -2.94905200 & -2.70703900 \\ \mathrm{H} & 3.27138400 & -3.68846900 & -3.35215500 \\ \mathrm{C} & 3.98986400 & -1.64517000 & -3.47476000 \\ \mathrm{H} & 4.59185300 & -1.83829900 & -4.37023300 \\ \mathrm{H} & 4.52952200 & -0.92699000 & -2.84900400 \\ \mathrm{H} & 3.05393100 & -1.17607100 & -3.79144800 \\ \mathrm{C} & 5.06402200 & -3.54506600 & -2.17725000 \\ \mathrm{H} & 5.74867200 & -3.75815200 & -3.00523200 \\ \mathrm{H} & 4.87039500 & -4.47573800 & -1.63519000 \\ \mathrm{H} & 5.55532000 & -2.84429000 & -1.49368900 \\ \mathrm{C} & -2.44722200 & -3.06581800 & 0.93843200 \\ \mathrm{H} & -3.15686800 & -2.76175000 & 0.16270100 \\ \mathrm{C} & -2.96161000 & -2.57537500 & 2.28377200 \\ \mathrm{H} & -3.92471500 & -3.04689600 & 2.50682600 \\ \mathrm{H} & -3.10391400 & -1.49200800 & 2.26388600 \\ \mathrm{H} & -2.25702600 & -2.82809700 & 3.08349400 \\ \mathrm{C} & -2.23475500 & -4.57820200 & 0.90356500 \\ \mathrm{H} & -3.18453100 & -5.09366800 & 1.08493400 \\ \mathrm{H} & -1.52119700 & -4.87979000 & 1.67839000 \\ \mathrm{H} & -1.85200900 & -4.90692700 & -0.06759200 \\ \mathrm{C} & 0.68371200 & 0.75269900 & 3.15331700 \\ \mathrm{H} & 1.49252400 & 0.59557600 & 3.87909900 \\ \mathrm{C} & 0.41307800 & 2.27215200 & 3.07039600 \\ \mathrm{H} & 1.31119900 & 2.81178000 & 2.74863300 \\ \mathrm{H} & -0.35204600 & 2.46158400 & 2.30539400 \\ \mathrm{H} & -0.06291800 & 2.82986100 & 4.42306600 \\ \mathrm{H} & 0.75069400 & 2.74279300 & 5.15893800 \\ & -0.27759500 & 3.90093900 & 4.32084800\end{array}$




\begin{tabular}{|c|c|c|c|}
\hline $\mathrm{C}$ & -1.29913500 & 2.08354900 & 4.94423900 \\
\hline $\mathrm{H}$ & -2.14669100 & 2.27374100 & 4.26933700 \\
\hline $\mathrm{H}$ & -1.59008800 & 2.46926500 & 5.92956700 \\
\hline $\mathrm{C}$ & -1.04217400 & 0.57164400 & 5.01713600 \\
\hline $\mathrm{H}$ & -1.94953900 & 0.04350400 & 5.33715400 \\
\hline $\mathrm{H}$ & -0.27702300 & 0.36805600 & 5.78129100 \\
\hline $\mathrm{C}$ & -0.56930800 & 0.01281400 & 3.66491800 \\
\hline $\mathrm{H}$ & -1.37593100 & 0.12750200 & 2.92647000 \\
\hline $\mathrm{H}$ & -0.37209200 & -1.06180400 & 3.74672900 \\
\hline $\mathrm{C}$ & 2.84294300 & 0.98761800 & 1.12352100 \\
\hline $\mathrm{H}$ & 2.50727800 & 2.00821200 & 0.89268800 \\
\hline $\mathrm{C}$ & 3.84344000 & 1.06166600 & 2.29534700 \\
\hline $\mathrm{H}$ & 4.15900600 & 0.04628500 & 2.56915200 \\
\hline $\mathrm{H}$ & 3.37688100 & 1.50281200 & 3.18387500 \\
\hline $\mathrm{C}$ & 5.08830700 & 1.88459200 & 1.91657400 \\
\hline $\mathrm{H}$ & 4.79097800 & 2.92812800 & 1.73636200 \\
\hline $\mathrm{H}$ & 5.78851100 & 1.90085600 & 2.76187500 \\
\hline $\mathrm{C}$ & 5.77292100 & 1.32717600 & 0.66157700 \\
\hline $\mathrm{H}$ & 6.17058700 & 0.32527700 & 0.88213600 \\
\hline $\mathrm{H}$ & 6.63221000 & 1.95232100 & 0.38706500 \\
\hline $\mathrm{C}$ & 4.78236700 & 1.23510200 & -0.50638500 \\
\hline $\mathrm{H}$ & 5.26686900 & 0.78741000 & -1.38349300 \\
\hline $\mathrm{H}$ & 4.48025100 & 2.24770500 & -0.80884100 \\
\hline $\mathrm{C}$ & 3.53005000 & 0.42083900 & -0.13718500 \\
\hline $\mathrm{H}$ & 3.81090900 & -0.62532300 & 0.03851400 \\
\hline $\mathrm{H}$ & 2.83288600 & 0.42451200 & -0.97927200 \\
\hline $\mathrm{O}$ & 2.93476100 & -2.73442100 & -1.54453000 \\
\hline $\mathrm{O}$ & -1.18757800 & -2.37367500 & 0.71596100 \\
\hline $\mathrm{P}$ & 1.22397800 & 0.08200500 & 1.48019600 \\
\hline $\mathrm{Pd}$ & -0.74390900 & 0.44162700 & -0.02417600 \\
\hline $\mathrm{C}$ & 0.09117100 & 1.76243500 & -1.29010500 \\
\hline $\mathrm{C}$ & 0.63260100 & 1.20813100 & -2.38517200 \\
\hline $\mathrm{H}$ & 0.57647800 & 0.13654400 & -2.54779800 \\
\hline $\mathrm{C}$ & 1.38875900 & 1.90386600 & -3.47358500 \\
\hline $\mathrm{C}$ & 0.11597700 & 3.18329500 & -0.88483700 \\
\hline $\mathrm{C}$ & -1.06107700 & 3.85572200 & -0.50422400 \\
\hline $\mathrm{C}$ & 1.33119200 & 3.89485400 & -0.84453800 \\
\hline $\mathrm{C}$ & -1.02193900 & 5.18977200 & -0.10598900 \\
\hline $\mathrm{H}$ & -2.00575800 & 3.32670000 & -0.54609600 \\
\hline $\mathrm{C}$ & 1.36740100 & 5.22714400 & -0.43151500 \\
\hline $\mathrm{H}$ & 2.24625800 & 3.40434500 & -1.15725200 \\
\hline $\mathrm{C}$ & 0.19259200 & 5.88059800 & -0.05820100 \\
\hline $\mathrm{H}$ & -1.94546400 & 5.69278400 & 0.16898200 \\
\hline $\mathrm{H}$ & 2.31751300 & 5.75491200 & -0.40818700 \\
\hline $\mathrm{H}$ & 0.22067500 & 6.91863100 & 0.26248500 \\
\hline $\mathrm{F}$ & 0.92206900 & 3.12357500 & -3.79764800 \\
\hline $\mathrm{F}$ & 1.37604400 & 1.14856600 & -4.60154100 \\
\hline F & 2.71501900 & 2.06855500 & -3.15987900 \\
\hline $\mathrm{C}$ & -2.51641600 & 0.73326700 & -0.97148800 \\
\hline $\mathrm{O}$ & -2.93340100 & 1.35626700 & -1.91095500 \\
\hline $\mathrm{O}$ & -3.40372200 & -0.07439600 & -0.18761300 \\
\hline $\mathrm{C}$ & -4.76046500 & -0.13011600 & -0.49406100 \\
\hline $\mathrm{C}$ & -5.22565100 & -0.45178400 & -1.77312600 \\
\hline $\mathrm{C}$ & -5.66123100 & 0.08229200 & 0.55188600 \\
\hline $\mathrm{C}$ & -6.59760300 & -0.56364100 & -2.00046600 \\
\hline $\mathrm{H}$ & -4.52363500 & -0.60122000 & -2.58405900 \\
\hline $\mathrm{C}$ & -7.03375600 & -0.03600100 & 0.33066500 \\
\hline
\end{tabular}




\begin{tabular}{|c|c|c|c|}
\hline & -5.27842900 & 0.33590500 & 1.53543400 \\
\hline & -7.48005900 & -0.35573800 & -0.94622300 \\
\hline & -6.98640600 & -0.80784200 & -2.98363700 \\
\hline & -7.75227100 & 0.12054700 & 1.12852300 \\
\hline & -8.80876500 & -0.46974400 & -1.16878100 \\
\hline
\end{tabular}

\section{TS10- F}

$\mathrm{E}(\mathrm{B} 3 \mathrm{LYP} / \mathrm{BS} 1)=-2953.144887$

$\mathrm{E}(\mathrm{PCM}$ (dichloroethane)/M06/BS2// B3LYP/BS1 $)=-2953.394287$

$\begin{array}{ll}\text { Zero-point correction= } & 0.899161 \\ \text { Thermal correction to Energy= } & 0.955275 \\ \text { Thermal correction to Enthalpy= } & 0.956219 \\ \text { Thermal correction to Gibbs Free Energy= } & 0.801031\end{array}$

$\begin{array}{lrrr}\text { Charge }= & \text { Multiplicity }=1 & \\ \mathrm{C} & -3.00308200 & -0.40775500 & -1.39393500 \\ \mathrm{C} & -4.08118600 & 0.00578900 & -2.20056900 \\ \mathrm{H} & -4.22598900 & 1.06045000 & -2.40421000 \\ \mathrm{C} & -4.98229100 & -0.89728200 & -2.75916300 \\ \mathrm{H} & -5.79849700 & -0.53470100 & -3.37839900 \\ \mathrm{C} & -4.82325000 & -2.26064900 & -2.51857400 \\ \mathrm{H} & -5.50990700 & -2.98359600 & -2.95102500 \\ \mathrm{C} & -3.77324200 & -2.69063200 & -1.71415300 \\ \mathrm{H} & -3.64798000 & -3.75104500 & -1.51531100 \\ \mathrm{C} & -2.85407000 & -1.79342100 & -1.14142200 \\ \mathrm{C} & -1.79564400 & -2.41958400 & -0.28262500 \\ \mathrm{C} & -0.47024700 & -2.56893500 & -0.72780500 \\ \mathrm{C} & 0.46736000 & -3.30536200 & 0.00854500 \\ \mathrm{H} & 1.49148500 & -3.39111200 & -0.32997500 \\ \mathrm{C} & 0.07644700 & -3.89211200 & 1.20659000 \\ \mathrm{H} & 0.80151500 & -4.45764700 & 1.78553800 \\ \mathrm{C} & -1.22509500 & -3.75638200 & 1.68815100 \\ \mathrm{H} & -1.49585800 & -4.21304700 & 2.63157100 \\ \mathrm{C} & -2.15979200 & -3.02735000 & 0.94287800 \\ \mathrm{C} & -4.02973800 & -3.61336000 & 2.40320900 \\ \mathrm{H} & -3.57274200 & -4.61145700 & 2.40848200 \\ \mathrm{C} & -3.78139500 & -2.91359400 & 3.74031400 \\ \mathrm{H} & -4.19155200 & -3.51087600 & 4.56282300 \\ \mathrm{H} & -4.27149100 & -1.93401600 & 3.74997300 \\ \mathrm{H} & -2.71444300 & -2.76101800 & 3.92697200 \\ \mathrm{C} & -5.51600700 & -3.74733500 & 2.08544600 \\ \mathrm{H} & -6.02259000 & -4.32279300 & 2.86790200 \\ \mathrm{H} & -5.66217300 & -4.25482100 & 1.12718900 \\ \mathrm{H} & -5.98297300 & -2.75841000 & 2.02466700 \\ \mathrm{C} & 0.81549500 & -2.51839900 & -2.81137600 \\ \mathrm{H} & 1.74786100 & -2.70056200 & -2.26481500 \\ \mathrm{C} & 1.07399700 & -1.47119000 & -3.88669200 \\ \mathrm{H} & 1.79647600 & -1.85549000 & -4.61526300 \\ \mathrm{H} & 1.48011100 & -0.55516800 & -3.44676700 \\ \mathrm{H} & 0.14626600 & -1.22624400 & -4.41490400 \\ \mathrm{C} & 0.27051100 & -3.82871600 & -3.37574000 \\ \mathrm{H} & 0.99913100 & -4.27433900 & -4.06226700 \\ \mathrm{H} & -0.65957800 & -3.64685900 & -3.92542500 \\ \mathrm{H} & 0.06809700 & -4.55211800 & -2.57987200 \\ \mathrm{C} & -1.89921600 & 2.25976500 & -1.99087700 \\ \mathrm{H} & -2.94846700 & 2.54579400 & -2.14403200 \\ & & & \\ & & \end{array}$




\begin{tabular}{|c|c|c|c|}
\hline $\mathrm{C}$ & -1.14894700 & 3.51304100 & -1.48887400 \\
\hline $\mathrm{H}$ & -1.58737900 & 3.88090200 & -0.55449900 \\
\hline $\mathrm{H}$ & -0.10855400 & 3.24840900 & -1.26192400 \\
\hline $\mathrm{C}$ & -1.17143900 & 4.63971000 & -2.53656200 \\
\hline $\mathrm{H}$ & -2.20607400 & 4.98802900 & -2.67719700 \\
\hline $\mathrm{H}$ & -0.60096900 & 5.49650900 & -2.15680700 \\
\hline $\mathrm{C}$ & -0.60567700 & 4.17545700 & -3.88559400 \\
\hline $\mathrm{H}$ & 0.46266200 & 3.94296600 & -3.76795700 \\
\hline $\mathrm{H}$ & -0.67265700 & 4.98292200 & -4.62611300 \\
\hline $\mathrm{C}$ & -1.34090800 & 2.92578800 & -4.38970300 \\
\hline $\mathrm{H}$ & -0.89265800 & 2.56902900 & -5.32602800 \\
\hline $\mathrm{H}$ & -2.38462800 & 3.18578300 & -4.62283200 \\
\hline $\mathrm{C}$ & -1.31834800 & 1.79761100 & -3.34484300 \\
\hline $\mathrm{H}$ & -0.28063900 & 1.47113400 & -3.18676800 \\
\hline $\mathrm{H}$ & -1.86792900 & 0.92692900 & -3.72102700 \\
\hline $\mathrm{C}$ & -2.66481200 & 1.57800800 & 0.79696400 \\
\hline $\mathrm{H}$ & -2.00841800 & 2.41880300 & 1.06517100 \\
\hline $\mathrm{C}$ & -4.09078000 & 2.12102000 & 0.58384000 \\
\hline $\mathrm{H}$ & -4.74617000 & 1.30082100 & 0.26142200 \\
\hline $\mathrm{H}$ & -4.10610700 & 2.87160900 & -0.21610200 \\
\hline $\mathrm{C}$ & -4.65422700 & 2.74108500 & 1.87588400 \\
\hline $\mathrm{H}$ & -4.06451600 & 3.63261700 & 2.13602000 \\
\hline $\mathrm{H}$ & -5.68167200 & 3.08678600 & 1.70247500 \\
\hline $\mathrm{C}$ & -4.61257400 & 1.74869900 & 3.04632400 \\
\hline $\mathrm{H}$ & -5.29585300 & 0.91247200 & 2.83437500 \\
\hline $\mathrm{H}$ & -4.97816400 & 2.22731300 & 3.96395400 \\
\hline C & -3.19322700 & 1.20184400 & 3.25563000 \\
\hline $\mathrm{H}$ & -3.18436900 & 0.45883200 & 4.06354700 \\
\hline $\mathrm{H}$ & -2.53374300 & 2.02023800 & 3.58093800 \\
\hline $\mathrm{C}$ & -2.62934500 & 0.57558000 & 1.96859900 \\
\hline $\mathrm{H}$ & -3.22010400 & -0.31214200 & 1.70848900 \\
\hline $\mathrm{H}$ & -1.60102700 & 0.23387900 & 2.13615700 \\
\hline $\mathrm{O}$ & -3.46418200 & -2.85672800 & 1.31421800 \\
\hline $\mathrm{O}$ & -0.14803600 & -1.92143700 & -1.90340900 \\
\hline$P$ & -1.81314700 & 0.86335300 & -0.72613100 \\
\hline $\mathrm{Pd}$ & 0.55784300 & 0.26639700 & -0.45103600 \\
\hline $\mathrm{C}$ & 1.85230000 & 1.12970800 & 0.99985800 \\
\hline $\mathrm{C}$ & 2.04363400 & 0.38189800 & 2.10930700 \\
\hline $\mathrm{H}$ & 2.02099000 & -0.69882100 & 2.03831700 \\
\hline $\mathrm{C}$ & 2.35531900 & 0.85307100 & 3.49543500 \\
\hline $\mathrm{C}$ & 1.80182100 & 2.61337300 & 0.97422400 \\
\hline $\mathrm{C}$ & 2.60231900 & 3.39593000 & 0.12310800 \\
\hline $\mathrm{C}$ & 0.89269100 & 3.27139700 & 1.82825200 \\
\hline $\mathrm{C}$ & 2.49433600 & 4.78754400 & 0.13266600 \\
\hline $\mathrm{H}$ & 3.30264000 & 2.90991700 & -0.54362400 \\
\hline $\mathrm{C}$ & 0.78891000 & 4.66010100 & 1.83178900 \\
\hline $\mathrm{H}$ & 0.27345400 & 2.68132800 & 2.49674100 \\
\hline $\mathrm{C}$ & 1.58903700 & 5.42726600 & 0.98092000 \\
\hline $\mathrm{H}$ & 3.12858600 & 5.37389200 & -0.52737600 \\
\hline $\mathrm{H}$ & 0.08487900 & 5.14406300 & 2.50408300 \\
\hline $\mathrm{H}$ & 1.51065900 & 6.51127100 & 0.98397300 \\
\hline $\mathrm{F}$ & 3.02888400 & 2.01650100 & 3.55331500 \\
\hline $\mathrm{F}$ & 3.11886200 & -0.08073300 & 4.12493800 \\
\hline $\mathrm{F}$ & 1.23584100 & 1.00287200 & 4.25648300 \\
\hline $\mathrm{C}$ & 2.59834700 & 0.18721700 & -0.49952300 \\
\hline $\mathrm{O}$ & 3.20633200 & 0.82827800 & -1.33347800 \\
\hline $\mathrm{O}$ & 3.04685700 & -1.06234100 & -0.0647310 \\
\hline
\end{tabular}




$\begin{array}{lrrr}\mathrm{C} & 4.39853700 & -1.38581400 & -0.21091300 \\ \mathrm{C} & 5.14858000 & -1.53247500 & 0.95679000 \\ \mathrm{C} & 4.96655400 & -1.61719100 & -1.46417100 \\ \mathrm{C} & 6.48493200 & -1.92446600 & 0.87494000 \\ \mathrm{H} & 4.68432700 & -1.33143200 & 1.91743400 \\ \mathrm{C} & 6.30392300 & -2.00581600 & -1.54988700 \\ \mathrm{H} & 4.37099300 & -1.48653600 & -2.35988700 \\ \mathrm{C} & 7.03983300 & -2.15474100 & -0.37921900 \\ \mathrm{H} & 7.09457800 & -2.04807300 & 1.76378900 \\ \mathrm{H} & 6.77828400 & -2.19158800 & -2.50783100 \\ \mathrm{~F} & 8.33302100 & -2.53592700 & -0.46358000\end{array}$

\section{pOMe-PhO-}

$\mathrm{E}(\mathrm{B} 3 \mathrm{LYP} / \mathrm{BS} 1)=-421.401807$

$\mathrm{E}(\mathrm{PCM}($ dichloroethane $) / \mathrm{M} 06 / \mathrm{BS} 2 / / \mathrm{B} 3 \mathrm{LYP} / \mathrm{BS} 1)=-421.338549$

$\begin{array}{ll}\text { Zero-point correction }= & 0.123397 \\ \text { Thermal correction to Energy= } & 0.131257 \\ \text { Thermal correction to Enthalpy= } & 0.132202 \\ \text { Thermal correction to Gibbs Free Energy= } & 0.090403\end{array}$

$\begin{array}{lrrr}\text { Charge }= & -1 \text { Multiplicity }=1 \\ \text { C } & -0.00466100 & 1.36792100 & 0.00006400 \\ \text { C } & 0.88146400 & 0.28213900 & 0.00022600 \\ \text { C } & 0.35214700 & -1.01368500 & 0.00032000 \\ \text { C } & -1.02876200 & -1.21728100 & 0.00016300 \\ \text { C } & -1.99631900 & -0.14204900 & -0.00006200 \\ \text { C } & -1.37869000 & 1.17191900 & -0.00010500 \\ \text { H } & 0.41662600 & 2.37414800 & -0.00001500 \\ \text { H } & 1.01142900 & -1.88089900 & 0.00045700 \\ \text { H } & -1.42251900 & -2.23364000 & 0.00017300 \\ \text { H } & -2.05045700 & 2.03037400 & -0.00033900 \\ \text { O } & -3.25132900 & -0.32244400 & -0.00022600 \\ \text { O } & 2.25472900 & 0.59819900 & 0.00039000 \\ \text { C } & 3.14276500 & -0.48050400 & -0.00050100 \\ \text { H } & 4.15554600 & -0.05959500 & -0.00056800 \\ \text { H } & 3.02755500 & -1.12410300 & 0.88933500 \\ \text { H } & 3.02696100 & -1.12309300 & -0.89098800\end{array}$

\section{IM6-OMe}

$\mathrm{E}(\mathrm{B} 3 \mathrm{LYP} / \mathrm{BS} 1)=-2855.123695$

$\mathrm{E}(\mathrm{PCM}($ dichloroethane $) / \mathrm{M} 06 / \mathrm{BS} 2 / / \mathrm{B} 3 \mathrm{LYP} / \mathrm{BS} 1)=-2855.361852$

Zero-point correction $=$

Thermal correction to Energy=

Thermal correction to Enthalpy=

Thermal correction to Gibbs Free Energy=
0.932273

0.988254

0.989198

0.837165 


\begin{tabular}{|c|c|c|c|}
\hline & 5.44629900 & -1.79024500 & 3.73183600 \\
\hline $\mathrm{C}$ & 4.11565000 & -1.79587900 & 2.04031000 \\
\hline $\mathrm{H}$ & 4.53707900 & -2.70507100 & 1.62319100 \\
\hline $\mathrm{C}$ & 3.05223900 & -1.18453100 & 1.35264500 \\
\hline $\mathrm{C}$ & 2.60672800 & -1.89763100 & 0.11298100 \\
\hline $\mathrm{C}$ & 1.36298500 & -2.54364800 & 0.06508300 \\
\hline $\mathrm{C}$ & 0.96469700 & -3.31846200 & -1.02848900 \\
\hline $\mathrm{H}$ & -0.03189800 & -3.73974700 & -1.06031900 \\
\hline $\mathrm{C}$ & 1.84953900 & -3.46963700 & -2.08981200 \\
\hline $\mathrm{H}$ & 1.55535100 & -4.06265900 & -2.95135000 \\
\hline $\mathrm{C}$ & 3.10393700 & -2.85729200 & -2.08017900 \\
\hline $\mathrm{H}$ & 3.76489000 & -2.98639300 & -2.92786000 \\
\hline $\mathrm{C}$ & 3.48447800 & -2.07342000 & -0.98416700 \\
\hline $\mathrm{C}$ & 5.75033900 & -1.69695800 & -1.82161100 \\
\hline $\mathrm{H}$ & 5.70791200 & -2.75388300 & -2.11481800 \\
\hline $\mathrm{C}$ & 5.60990300 & -0.80170400 & -3.05328700 \\
\hline $\mathrm{H}$ & 6.40550300 & -1.02182200 & -3.77435000 \\
\hline $\mathrm{H}$ & 5.68986000 & 0.25180700 & -2.76426000 \\
\hline $\mathrm{H}$ & 4.64684500 & -0.94521000 & -3.55167000 \\
\hline $\mathrm{C}$ & 7.04935600 & -1.44180700 & -1.06336300 \\
\hline $\mathrm{H}$ & 7.91157500 & -1.61910200 & -1.71524900 \\
\hline $\mathrm{H}$ & 7.12709700 & -2.10232200 & -0.19445100 \\
\hline $\mathrm{H}$ & 7.08749300 & -0.40518500 & -0.71178300 \\
\hline $\mathrm{C}$ & -0.27642500 & -3.42834800 & 1.68226000 \\
\hline $\mathrm{H}$ & -1.03163200 & -3.66045500 & 0.92712100 \\
\hline $\mathrm{C}$ & -0.95635200 & -2.86888200 & 2.92309400 \\
\hline $\mathrm{H}$ & -1.63555900 & -3.61914000 & 3.34172900 \\
\hline $\mathrm{H}$ & -1.54104000 & -1.98013000 & 2.67217500 \\
\hline $\mathrm{H}$ & -0.21536800 & -2.60214700 & 3.68511500 \\
\hline $\mathrm{C}$ & 0.59959900 & -4.63693500 & 1.99863400 \\
\hline $\mathrm{H}$ & -0.02407000 & -5.43393600 & 2.41850900 \\
\hline $\mathrm{H}$ & 1.36642300 & -4.37410400 & 2.73622200 \\
\hline $\mathrm{H}$ & 1.09408200 & -5.03191200 & 1.10695000 \\
\hline $\mathrm{C}$ & 0.41789300 & 2.07148800 & 2.43007000 \\
\hline $\mathrm{H}$ & 1.27656200 & 2.52147900 & 2.94282200 \\
\hline $\mathrm{C}$ & -0.42241800 & 3.25032800 & 1.88767900 \\
\hline $\mathrm{H}$ & 0.17681000 & 3.86994400 & 1.21105800 \\
\hline $\mathrm{H}$ & -1.26708500 & 2.87764100 & 1.30117900 \\
\hline $\mathrm{C}$ & -0.94452600 & 4.12734100 & 3.04044600 \\
\hline $\mathrm{H}$ & -0.09416700 & 4.60939600 & 3.54643100 \\
\hline $\mathrm{H}$ & -1.56180600 & 4.93521100 & 2.62845600 \\
\hline $\mathrm{C}$ & -1.74527900 & 3.31198600 & 4.06505600 \\
\hline $\mathrm{H}$ & -2.65694700 & 2.92464500 & 3.58734700 \\
\hline $\mathrm{H}$ & -2.07403500 & 3.95598100 & 4.89047000 \\
\hline $\mathrm{C}$ & -0.91664300 & 2.13711800 & 4.60228500 \\
\hline $\mathrm{H}$ & -1.51465000 & 1.52847000 & 5.29241600 \\
\hline $\mathrm{H}$ & -0.06826800 & 2.52651600 & 5.18518200 \\
\hline $\mathrm{C}$ & -0.38731400 & 1.25188100 & 3.46180800 \\
\hline $\mathrm{H}$ & -1.23114600 & 0.77241500 & 2.94713600 \\
\hline $\mathrm{H}$ & 0.22777200 & 0.44103000 & 3.87026100 \\
\hline $\mathrm{C}$ & 1.94091700 & 2.13445400 & -0.11468100 \\
\hline $\mathrm{H}$ & 1.11055600 & 2.73919900 & -0.50536600 \\
\hline $\mathrm{C}$ & 2.96710200 & 3.07133700 & 0.55716600 \\
\hline $\mathrm{H}$ & 3.77666100 & 2.46786700 & 0.98914000 \\
\hline $\mathrm{H}$ & 2.51212300 & 3.62715100 & 1.38540700 \\
\hline $\mathrm{C}$ & 3.56109000 & 4.06749400 & -0.45554300 \\
\hline & 2.76689600 & 4.74354300 & -0.8049740 \\
\hline
\end{tabular}




$\begin{array}{lrcc}\mathrm{H} & 4.30888100 & 4.69664300 & 0.04447500 \\ \mathrm{C} & 4.18116100 & 3.34922500 & -1.66169300 \\ \mathrm{H} & 5.05352700 & 2.76857100 & -1.32525500 \\ \mathrm{H} & 4.55380800 & 4.08209600 & -2.38889500 \\ \mathrm{C} & 3.16590500 & 2.40614000 & -2.32144600 \\ \mathrm{H} & 3.63442300 & 1.85896500 & -3.14936100 \\ \mathrm{H} & 2.34822900 & 2.99267200 & -2.76186000 \\ \mathrm{C} & 2.58053900 & 1.40303000 & -1.31189200 \\ \mathrm{H} & 3.38379700 & 0.74884400 & -0.95237500 \\ \mathrm{H} & 1.83866500 & 0.76774300 & -1.80627600 \\ \mathrm{O} & 4.69062200 & -1.44061400 & -0.87737500 \\ \mathrm{O} & 0.53647400 & -2.32352400 & 1.15784400 \\ \mathrm{P} & 1.08419900 & 0.94839800 & 1.06884700 \\ \mathrm{Pd} & -0.60565200 & -0.46618600 & 0.19549300 \\ \mathrm{C} & -1.61404700 & 0.94580300 & -0.80917300 \\ \mathrm{C} & -2.70365500 & 1.46729800 & -0.22767900 \\ \mathrm{H} & -2.97169100 & 1.20306700 & 0.79035900 \\ \mathrm{C} & -3.68009800 & 2.42838700 & -0.83486600 \\ \mathrm{C} & -1.13137600 & 1.21602900 & -2.17972400 \\ \mathrm{C} & -0.86133100 & 0.14575000 & -3.05464000 \\ \mathrm{C} & -0.92219500 & 2.53006400 & -2.63898400 \\ \mathrm{C} & -0.41133400 & 0.38736800 & -4.35111500 \\ \mathrm{H} & -1.02878100 & -0.86717600 & -2.70121100 \\ \mathrm{C} & -0.46197100 & 2.76534800 & -3.93524800 \\ \mathrm{H} & -1.14624600 & 3.36574300 & -1.98367900 \\ \mathrm{C} & -0.20386700 & 1.69652300 & -4.79587200 \\ \mathrm{H} & -0.22246000 & -0.44987100 & -5.01829700 \\ \mathrm{H} & -0.31364100 & 3.78739200 & -4.27498500 \\ \mathrm{H} & 0.15158600 & 1.88182100 & -5.80614400 \\ \mathrm{~F} & -3.90806300 & 2.24314800 & -2.14893800 \\ \mathrm{~F} & -3.26778900 & 3.72884900 & -0.69395600 \\ \mathrm{~F} & -4.87288600 & 2.34771000 & -0.20123100 \\ \mathrm{O} & -1.92034400 & -1.90761300 & -0.50357700 \\ \mathrm{C} & -3.25623100 & -1.90139400 & -0.49041500 \\ \mathrm{C} & -4.02097900 & -1.58070200 & 0.64841300 \\ \mathrm{C} & -3.96876600 & -2.29170000 & -1.64998600 \\ \mathrm{C} & -5.41792700 & -1.65220100 & 0.64473900 \\ \mathrm{H} & -3.50492100 & -1.26867200 & 1.55320700 \\ \mathrm{C} & -5.35397900 & -2.37604400 & -1.65711200 \\ \mathrm{H} & -3.40032000 & -2.53690300 & -2.54322600 \\ \mathrm{C} & -6.09440500 & -2.05438000 & -0.51023000 \\ \mathrm{H} & -5.96093500 & -1.39132800 & 1.54720300 \\ \mathrm{H} & -5.89164400 & -2.68232600 & -2.55013300 \\ \mathrm{O} & -7.46104000 & -2.16525300 & -0.62564700 \\ \mathrm{C} & -8.24541600 & -1.80902200 & 0.49317200 \\ \mathrm{H} & -8.09090600 & -0.75968900 & 0.78293800 \\ \mathrm{H} & -9.28658600 & -1.94780000 & 0.19223300 \\ \mathrm{H} & -8.03482500 & -2.44959400 & 1.36220400 \\ & & & \end{array}$

\section{IM8- OMe}

$\mathrm{E}(\mathrm{B} 3 \mathrm{LYP} / \mathrm{BS} 1)=-2968.438445$

$\mathrm{E}(\mathrm{PCM}$ (dichloroethane)/M06/BS2// B3LYP/BS1 $)=-2968.654687$

Zero-point correction $=$

Thermal correction to Energy=

Thermal correction to Enthalpy=
0.939603

0.998669

0.999614 
Thermal correction to Gibbs Free Energy= $\quad 0.837895$

\begin{tabular}{|c|c|c|c|}
\hline \multicolumn{4}{|c|}{ Charge $=0$ Multiplicity $=1$} \\
\hline C & -0.60394700 & 1.73739600 & 1.98697100 \\
\hline $\mathrm{C}$ & -0.91428400 & 2.02377000 & 3.33316400 \\
\hline $\mathrm{H}$ & -1.08375700 & 1.21131100 & 4.03027600 \\
\hline $\mathrm{C}$ & -1.00565200 & 3.32679700 & 3.81194200 \\
\hline $\mathrm{H}$ & -1.24579500 & 3.50374100 & 4.85676500 \\
\hline $\mathrm{C}$ & -0.78342300 & 4.39196100 & 2.94044600 \\
\hline $\mathrm{H}$ & -0.85014500 & 5.41809300 & 3.29196200 \\
\hline $\mathrm{C}$ & -0.45996400 & 4.12808300 & 1.61453600 \\
\hline $\mathrm{H}$ & -0.26209600 & 4.95169300 & 0.93453100 \\
\hline $\mathrm{C}$ & -0.35948900 & 2.81786000 & 1.11072600 \\
\hline $\mathrm{C}$ & 0.06050800 & 2.71874800 & -0.32407700 \\
\hline $\mathrm{C}$ & 1.44074400 & 2.73620700 & -0.63041800 \\
\hline $\mathrm{C}$ & 1.86820400 & 2.81897300 & -1.96531400 \\
\hline $\mathrm{H}$ & 2.92221600 & 2.83220000 & -2.21077700 \\
\hline $\mathrm{C}$ & 0.92022100 & 2.90417700 & -2.98295500 \\
\hline $\mathrm{H}$ & 1.25468000 & 2.96528200 & -4.01531200 \\
\hline $\mathrm{C}$ & -0.44502900 & 2.92805100 & -2.70616700 \\
\hline $\mathrm{H}$ & -1.16485100 & 3.00891800 & $-3.5119720 c$ \\
\hline $\mathrm{C}$ & -0.86994200 & 2.85790700 & -1.37073600 \\
\hline $\mathrm{C}$ & -3.10996800 & 3.73484000 & -1.77021300 \\
\hline $\mathrm{H}$ & -2.52321300 & 4.45196200 & -2.35795100 \\
\hline $\mathrm{C}$ & -3.95455800 & 2.86531700 & -2.69872800 \\
\hline $\mathrm{H}$ & -4.67925700 & 3.48634700 & -3.23823600 \\
\hline $\mathrm{H}$ & -4.50656700 & 2.11305500 & -2.1259980 \\
\hline $\mathrm{H}$ & -3.34625400 & 2.33632400 & -3.4365790 \\
\hline $\mathrm{C}$ & -3.95753100 & 4.49122300 & -0.7502720 \\
\hline $\mathrm{H}$ & -4.66514700 & 5.15563100 & -1.25872200 \\
\hline $\mathrm{H}$ & -3.32388300 & 5.09158500 & -0.09048200 \\
\hline $\mathrm{H}$ & -4.52757300 & 3.78900800 & -0.13206000 \\
\hline $\mathrm{C}$ & 3.69301000 & 2.87171900 & 0.30434100 \\
\hline $\mathrm{H}$ & 4.03545700 & 2.24517200 & -0.52621700 \\
\hline $\mathrm{C}$ & 4.29072600 & 2.33333900 & 1.59820800 \\
\hline $\mathrm{H}$ & 5.38283800 & 2.41460300 & 1.57014200 \\
\hline $\mathrm{H}$ & 4.01997700 & 1.28315600 & 1.72835100 \\
\hline $\mathrm{H}$ & 3.92053400 & 2.90467900 & 2.45701400 \\
\hline $\mathrm{C}$ & 4.04639100 & 4.34018000 & 0.06095400 \\
\hline $\mathrm{H}$ & 5.13088300 & 4.45519200 & -0.04781300 \\
\hline $\mathrm{H}$ & 3.71833400 & 4.95148800 & 0.90922200 \\
\hline $\mathrm{H}$ & 3.57072500 & 4.72834700 & -0.84498500 \\
\hline $\mathrm{C}$ & 0.20327800 & -0.93939700 & 2.94761300 \\
\hline $\mathrm{H}$ & -0.47089000 & -0.83565200 & 3.80814100 \\
\hline $\mathrm{C}$ & 0.34583400 & -2.44656500 & 2.64101600 \\
\hline $\mathrm{H}$ & -0.61909000 & -2.88805100 & 2.36086200 \\
\hline $\mathrm{H}$ & 1.01184900 & -2.56127400 & 1.77678300 \\
\hline $\mathrm{C}$ & 0.93605300 & -3.19932900 & 3.84597900 \\
\hline $\mathrm{H}$ & 0.22807900 & -3.15342400 & 4.68744900 \\
\hline $\mathrm{H}$ & 1.05253800 & -4.26136400 & 3.59482100 \\
\hline $\mathrm{C}$ & 2.28169600 & -2.59967300 & 4.27839800 \\
\hline $\mathrm{H}$ & 3.02087500 & -2.76596300 & 3.48165700 \\
\hline $\mathrm{H}$ & 2.65931300 & -3.11683000 & 5.16995500 \\
\hline $\mathrm{C}$ & 2.15914800 & -1.09224500 & 4.54618200 \\
\hline $\mathrm{H}$ & 3.14125200 & -0.67017100 & 4.79503900 \\
\hline $\mathrm{H}$ & 1.52005100 & -0.93360700 & 5.42887700 \\
\hline $\mathrm{C}$ & 1.56839600 & -0.33760700 & 3.34186000 \\
\hline
\end{tabular}




\begin{tabular}{|c|c|c|c|}
\hline $\mathrm{H}$ & 2.23669600 & -0.40151400 & 2.47853400 \\
\hline $\mathrm{H}$ & 1.46213600 & 0.72390100 & 3.58834800 \\
\hline $\mathrm{C}$ & -2.48597100 & -0.51596000 & 1.69118500 \\
\hline $\mathrm{H}$ & -2.57081600 & -1.43028200 & 1.09406900 \\
\hline $\mathrm{C}$ & -2.99126100 & -0.85676600 & 3.11023900 \\
\hline $\mathrm{H}$ & -2.90156500 & 0.01948500 & 3.76575900 \\
\hline $\mathrm{H}$ & -2.39543000 & -1.65903300 & 3.55817700 \\
\hline $\mathrm{C}$ & -4.46745000 & -1.29627600 & 3.07389900 \\
\hline $\mathrm{H}$ & -4.54832400 & -2.23120500 & 2.50066600 \\
\hline $\mathrm{H}$ & -4.80417000 & -1.52394800 & 4.09359600 \\
\hline $\mathrm{C}$ & -5.36530600 & -0.22797600 & 2.43677100 \\
\hline $\mathrm{H}$ & -5.36390100 & 0.67087700 & 3.07125400 \\
\hline $\mathrm{H}$ & -6.40407300 & -0.57980200 & 2.39422200 \\
\hline $\mathrm{C}$ & -4.86463500 & 0.13942000 & 1.03414200 \\
\hline $\mathrm{H}$ & -5.46974800 & 0.95327600 & 0.61356700 \\
\hline $\mathrm{H}$ & -4.99615000 & -0.71928000 & 0.36251300 \\
\hline $\mathrm{C}$ & -3.38550400 & 0.56209400 & 1.04709900 \\
\hline $\mathrm{H}$ & -3.28653100 & 1.49663800 & 1.61315600 \\
\hline $\mathrm{H}$ & -3.05315200 & 0.78333500 & 0.03034200 \\
\hline $\mathrm{O}$ & -2.18701800 & 2.92108400 & -1.00457600 \\
\hline $\mathrm{O}$ & 2.26348400 & 2.71841200 & 0.44617300 \\
\hline $\mathrm{P}$ & -0.64726200 & -0.06114200 & 1.51369900 \\
\hline $\mathrm{Pd}$ & 0.36609900 & -0.88479700 & -0.58070500 \\
\hline $\mathrm{C}$ & -1.45889400 & -1.48589100 & -1.37368500 \\
\hline $\mathrm{C}$ & -2.04840200 & -0.64406300 & -2.24035200 \\
\hline $\mathrm{H}$ & -1.63663300 & 0.34904600 & -2.39369600 \\
\hline $\mathrm{C}$ & -3.25704600 & -0.88277700 & -3.09503800 \\
\hline $\mathrm{C}$ & -1.90470900 & -2.85288100 & -1.01312800 \\
\hline $\mathrm{C}$ & -0.97299400 & -3.89240600 & -0.81966300 \\
\hline $\mathrm{C}$ & -3.26786900 & -3.15593000 & -0.80889500 \\
\hline $\mathrm{C}$ & -1.38253300 & -5.17843300 & -0.47028800 \\
\hline $\mathrm{H}$ & 0.08639100 & -3.69173800 & -0.94257900 \\
\hline $\mathrm{C}$ & -3.67532600 & -4.43810400 & -0.44625900 \\
\hline $\mathrm{H}$ & -4.01290200 & -2.38087100 & -0.94158600 \\
\hline $\mathrm{C}$ & -2.73644000 & -5.45811700 & -0.27824200 \\
\hline $\mathrm{H}$ & -0.63887400 & -5.96027000 & -0.3415980 \\
\hline $\mathrm{H}$ & -4.73282200 & -4.63983400 & -0.29694900 \\
\hline $\mathrm{H}$ & -3.05598900 & -6.45764100 & 0.00357300 \\
\hline $\mathrm{F}$ & -3.38568700 & -2.14621500 & -3.54603600 \\
\hline $\mathrm{F}$ & -3.21440700 & -0.07762400 & -4.18731900 \\
\hline $\mathrm{F}$ & -4.42685100 & -0.57834000 & -2.45125000 \\
\hline $\mathrm{C}$ & 1.03262100 & -1.56026900 & -2.30226400 \\
\hline $\mathrm{O}$ & 1.32638600 & -1.95125100 & -3.33184600 \\
\hline $\mathrm{O}$ & 2.26387000 & -0.54884300 & 0.27118600 \\
\hline $\mathrm{C}$ & 3.42234000 & -0.82181600 & -0.32851800 \\
\hline $\mathrm{C}$ & 3.78415400 & -0.31825200 & -1.60057600 \\
\hline $\mathrm{C}$ & 4.38331500 & -1.62074200 & 0.34071300 \\
\hline $\mathrm{C}$ & 5.03093500 & -0.59429600 & -2.16617900 \\
\hline $\mathrm{H}$ & 3.07479700 & 0.31064800 & -2.13143000 \\
\hline $\mathrm{C}$ & 5.62601300 & -1.89266700 & -0.22490800 \\
\hline $\mathrm{H}$ & 4.12347000 & -2.02824200 & 1.31409500 \\
\hline $\mathrm{C}$ & 5.95835600 & -1.38232400 & -1.48440400 \\
\hline $\mathrm{H}$ & 5.29187200 & -0.20808700 & -3.14837400 \\
\hline $\mathrm{H}$ & 6.34047700 & -2.52453300 & 0.29747400 \\
\hline $\mathrm{O}$ & 7.17264700 & -1.68889800 & -2.08221100 \\
\hline $\mathrm{C}$ & 8.27570500 & -0.94879500 & -1.58063400 \\
\hline 1 & 9161137 & -1.29494400 & -2.1215650 \\
\hline
\end{tabular}



$\mathrm{H}$
$8.14664400 \quad 0.13058300 \quad-1.75046400$
$\mathrm{H}$
$8.42520000-1.11566000-0.50408100$

\section{TS9- OMe}

$\mathrm{E}(\mathrm{B} 3 \mathrm{LYP} / \mathrm{BS} 1)=-2968.428433$

$\mathrm{E}(\mathrm{PCM}($ dichloroethane $) / \mathrm{M} 06 / \mathrm{BS} 2 / / \mathrm{B} 3 \mathrm{LYP} / \mathrm{BS} 1)=-2968.641155$

$\begin{array}{ll}\text { Zero-point correction= } & 0.939518 \\ \text { Thermal correction to Energy= } & 0.997753 \\ \text { Thermal correction to Enthalpy= } & 0.998697 \\ \text { Thermal correction to Gibbs Free Energy= } & 0.841267\end{array}$

$\begin{array}{lrrr}\text { Charge }= & \text { Multiplicity }=1 & & \\ \mathrm{C} & -0.53276600 & 1.57973200 & 2.13130700 \\ \mathrm{C} & -0.69311400 & 1.80779500 & 3.51351200 \\ \mathrm{H} & -0.85027400 & 0.97128000 & 4.18408400 \\ \mathrm{C} & -0.64857900 & 3.08633000 & 4.06211000 \\ \mathrm{H} & -0.77463600 & 3.21996300 & 5.13306500 \\ \mathrm{C} & -0.44204500 & 4.18299400 & 3.22670600 \\ \mathrm{H} & -0.41035300 & 5.19021700 & 3.63354100 \\ \mathrm{C} & -0.25572800 & 3.97454600 & 1.86444000 \\ \mathrm{H} & -0.06382600 & 4.82056500 & 1.21077400 \\ \mathrm{C} & -0.28238600 & 2.68940200 & 1.29365200 \\ \mathrm{C} & 0.04781800 & 2.62669300 & -0.16563100 \\ \mathrm{C} & 1.39573800 & 2.45280400 & -0.54582700 \\ \mathrm{C} & 1.78036400 & 2.58300400 & -1.88891300 \\ \mathrm{H} & 2.81356100 & 2.44718300 & -2.18219700 \\ \mathrm{C} & 0.81746100 & 2.89883100 & -2.84363300 \\ \mathrm{H} & 1.1245900 & 2.99445900 & -3.88516300 \\ \mathrm{C} & -0.51772000 & 3.09925200 & -2.49428200 \\ \mathrm{H} & -1.24443800 & 3.34632000 & -3.25815500 \\ \mathrm{C} & -0.89839400 & 2.97844000 & -1.14949100 \\ \mathrm{C} & -3.07559200 & 4.03777400 & -1.45259900 \\ \mathrm{H} & -2.47829400 & 4.73980400 & -2.04852300 \\ \mathrm{C} & -3.96301200 & 3.19521100 & -2.36686900 \\ \mathrm{H} & -4.66563900 & 3.84188900 & -2.90549900 \\ \mathrm{H} & -4.54088700 & 2.47404800 & -1.78010500 \\ \mathrm{H} & -3.38617300 & 2.63335400 & -3.10544000 \\ \mathrm{C} & -3.88212700 & 4.81802300 & -0.41802500 \\ \mathrm{H} & -4.58046600 & 5.50065700 & -0.91459500 \\ \mathrm{H} & -3.21993500 & 5.40241700 & 0.22803200 \\ \mathrm{H} & -4.45941800 & 4.13261900 & 0.21215700 \\ \mathrm{C} & 3.65682000 & 2.54717300 & 0.37443300 \\ \mathrm{H} & 4.08055300 & 2.10263500 & -0.53168700 \\ \mathrm{C} & 4.32857500 & 1.91212600 & 1.58489300 \\ \mathrm{H} & 5.39919200 & 2.14291500 & 1.58089600 \\ \mathrm{H} & 4.21146300 & 0.82572100 & 1.55997600 \\ \mathrm{H} & 3.89093600 & 2.29855100 & 2.51237400 \\ \mathrm{C} & 3.80804500 & 4.06818700 & 0.34918900 \\ \mathrm{H} & 4.86799800 & 4.33887600 & 0.28613700 \\ \mathrm{H} & 3.39228100 & 4.50486500 & 1.26436200 \\ \mathrm{H} & 3.29439000 & 4.51096400 & -0.50993800 \\ \mathrm{C} & 0.12230700 & -1.20981400 & 2.81750700 \\ \mathrm{H} & -0.33092700 & -0.98801500 & 3.79294700 \\ \mathrm{C} & -0.07585600 & -2.71652600 & 2.53870000 \\ \mathrm{H} & -1.14173200 & -2.97405000 & 2.51064900 \\ & & & \end{array}$




\begin{tabular}{|c|c|c|c|}
\hline $\mathrm{H}$ & 0.32999900 & -2.95420800 & 1.54642200 \\
\hline & 0.64017400 & -3.57320600 & 3.59739500 \\
\hline $\mathrm{H}$ & 0.16558100 & -3.41247500 & 4.57734300 \\
\hline $\mathrm{H}$ & 0.50861800 & -4.63606800 & 3.35799300 \\
\hline $\mathrm{C}$ & 2.13260700 & -3.22469800 & 3.69017900 \\
\hline $\mathrm{H}$ & 2.62398300 & -3.49619800 & 2.74494700 \\
\hline $\mathrm{H}$ & 2.61255600 & -3.81809400 & 4.47899200 \\
\hline $\mathrm{C}$ & 2.33598300 & -1.72495400 & 3.94990100 \\
\hline $\mathrm{H}$ & 3.40597900 & -1.48166100 & 3.95986400 \\
\hline $\mathrm{H}$ & 1.94863500 & -1.47266300 & 4.94901700 \\
\hline $\mathrm{C}$ & 1.62571600 & -0.86393400 & 2.89128300 \\
\hline $\mathrm{H}$ & 2.08458300 & -1.04039900 & 1.91038900 \\
\hline $\mathrm{H}$ & 1.75984500 & 0.19895800 & 3.11979000 \\
\hline $\mathrm{C}$ & -2.59871200 & -0.49532100 & 1.76622700 \\
\hline $\mathrm{H}$ & -2.74758800 & -1.45761800 & 1.25903100 \\
\hline $\mathrm{C}$ & -3.07529800 & -0.64973500 & 3.22621500 \\
\hline $\mathrm{H}$ & -2.90708200 & 0.28782800 & 3.77065800 \\
\hline $\mathrm{H}$ & -2.50804300 & -1.43083200 & 3.74471300 \\
\hline $\mathrm{C}$ & -4.57651200 & -0.98635700 & 3.28784600 \\
\hline $\mathrm{H}$ & -4.74854500 & -1.96589900 & 2.81840300 \\
\hline $\mathrm{H}$ & -4.88627300 & -1.08244100 & 4.33653300 \\
\hline $\mathrm{C}$ & -5.41933500 & 0.07759900 & 2.57273900 \\
\hline $\mathrm{H}$ & -5.32428300 & 1.03415700 & 3.10780400 \\
\hline $\mathrm{H}$ & -6.48225500 & -0.19417900 & 2.60179900 \\
\hline $\mathrm{C}$ & -4.95195900 & 0.25514900 & 1.12237700 \\
\hline $\mathrm{H}$ & -5.51945600 & 1.05650400 & 0.63159200 \\
\hline $\mathrm{H}$ & -5.16719700 & -0.66536200 & 0.56260400 \\
\hline $\mathrm{C}$ & -3.44749200 & 0.56777900 & 1.03348100 \\
\hline $\mathrm{H}$ & -3.25618800 & 1.55213300 & 1.47828500 \\
\hline $\mathrm{H}$ & -3.14718400 & 0.63953700 & -0.01535200 \\
\hline $\mathrm{O}$ & -2.16770100 & 3.20185000 & -0.69759000 \\
\hline $\mathrm{O}$ & 2.25842200 & 2.17818200 & 0.47248400 \\
\hline $\mathrm{P}$ & -0.75091800 & -0.16383000 & 1.52141100 \\
\hline $\mathrm{Pc}$ & 0.20547200 & -1.03265600 & -0.57211400 \\
\hline $\mathrm{C}$ & -1.55899800 & -1.38264000 & -1.49729000 \\
\hline $\mathrm{C}$ & -1.96528700 & -0.43205700 & -2.35407200 \\
\hline $\mathrm{H}$ & -1.42497100 & 0.50644000 & -2.42904100 \\
\hline $\mathrm{C}$ & -3.13739600 & -0.48687300 & -3.28586100 \\
\hline $\mathrm{C}$ & -2.20653700 & -2.68682500 & -1.22769000 \\
\hline $\mathrm{C}$ & -1.45546000 & -3.87347800 & -1.12080500 \\
\hline $\mathrm{C}$ & -3.60228600 & -2.77525300 & -1.04545000 \\
\hline $\mathrm{C}$ & -2.07277000 & -5.09661600 & -0.86705200 \\
\hline $\mathrm{H}$ & -0.37919300 & -3.83579800 & -1.24655500 \\
\hline $\mathrm{C}$ & -4.21609300 & -3.99793600 & -0.77770700 \\
\hline $\mathrm{H}$ & -4.20800400 & -1.88103700 & -1.12809000 \\
\hline $\mathrm{C}$ & -3.45607500 & -5.16532400 & -0.68790000 \\
\hline $\mathrm{H}$ & -1.46837000 & -5.99759100 & -0.80462700 \\
\hline $\mathrm{H}$ & -5.29384000 & -4.03608800 & -0.64220900 \\
\hline $\mathrm{H}$ & -3.93562600 & -6.11782200 & -0.47929400 \\
\hline $\mathrm{F}$ & -3.36435600 & -1.69638600 & -3.83306600 \\
\hline $\mathrm{F}$ & -2.95366800 & 0.38781600 & -4.30818700 \\
\hline $\mathrm{F}$ & -4.30850200 & -0.10917300 & -2.68266400 \\
\hline $\mathrm{C}$ & 1.30553000 & -1.94998800 & -1.93153600 \\
\hline $\mathrm{O}$ & 1.79839700 & -2.48922600 & -2.82665700 \\
\hline $\mathrm{O}$ & 2.46208200 & -1.60251600 & -0.40450400 \\
\hline & 3.71700300 & -1.22702000 & -0.72232700 \\
\hline & 4.02833500 & -0.38455700 & -1.8105050 \\
\hline
\end{tabular}




$\begin{array}{lrrr}\mathrm{C} & 4.78911000 & -1.72400400 & 0.04027600 \\ \mathrm{C} & 5.34713700 & -0.05873400 & -2.11249100 \\ \mathrm{H} & 3.22410000 & 0.01236000 & -2.42190600 \\ \mathrm{C} & 6.11450300 & -1.38873900 & -0.25202300 \\ \mathrm{H} & 4.56847800 & -2.39072900 & 0.86917200 \\ \mathrm{C} & 6.40309400 & -0.55175700 & -1.33490600 \\ \mathrm{H} & 5.58310900 & 0.58074400 & -2.95871300 \\ \mathrm{H} & 6.90741300 & -1.79453000 & 0.36724600 \\ \mathrm{O} & 7.66504700 & -0.16020700 & -1.71479700 \\ \mathrm{C} & 8.76050100 & -0.66610000 & -0.97891000 \\ \mathrm{H} & 9.65725200 & -0.25146600 & -1.44495100 \\ \mathrm{H} & 8.72541000 & -0.35289700 & 0.07461600 \\ \mathrm{H} & 8.80775900 & -1.76359900 & -1.01974700\end{array}$

\section{IM10- OMe}

$\mathrm{E}(\mathrm{B} 3 \mathrm{LYP} / \mathrm{BS} 1)=-2968.44446$

$\mathrm{E}(\mathrm{PCM}$ (dichloroethane) $/ \mathrm{M} 06 / \mathrm{BS} 2 / / \mathrm{B} 3 \mathrm{LYP} / \mathrm{BS} 1)=-2968.658968$

$\begin{array}{ll}\text { Zero-point correction }= & 0.940940 \\ \text { Thermal correction to Energy= } & 0.999403 \\ \text { Thermal correction to Enthalpy= } & 1.000347 \\ \text { Thermal correction to Gibbs Free Energy= } & 0.839799\end{array}$

$\begin{array}{lrrr}\text { Charge }= & \text { Multiplicity }=1 & \\ \mathrm{C} & 1.87857400 & -1.74678000 & 1.78423700 \\ \mathrm{C} & 2.49876800 & -2.09820000 & 2.99875000 \\ \mathrm{H} & 2.64926600 & -1.34462400 & 3.76386800 \\ \mathrm{C} & 2.93078700 & -3.39641000 & 3.25924600 \\ \mathrm{H} & 3.40334200 & -3.63000400 & 4.20954600 \\ \mathrm{C} & 2.75295900 & -4.38399600 & 2.29168400 \\ \mathrm{H} & 3.08633700 & -5.40225700 & 2.47360500 \\ \mathrm{C} & 2.13259400 & -4.05871300 & 1.08945200 \\ \mathrm{H} & 1.97937000 & -4.82731800 & 0.33757100 \\ \mathrm{C} & 1.67689100 & -2.75750600 & 0.81376700 \\ \mathrm{C} & 0.96605800 & -2.58211800 & -0.49528000 \\ \mathrm{C} & -0.44048600 & -2.52754200 & -0.53686900 \\ \mathrm{C} & -1.13297600 & -2.55611400 & -1.75551800 \\ \mathrm{H} & -2.21384400 & -2.49619000 & -1.77728500 \\ \mathrm{C} & -0.41145600 & -2.63000200 & -2.94113400 \\ \mathrm{H} & -0.94134800 & -2.64117500 & -3.88951400 \\ \mathrm{C} & 0.98264600 & -2.66915100 & -2.93990400 \\ \mathrm{H} & 1.51372800 & -2.70999800 & -3.88179100 \\ \mathrm{C} & 1.67212200 & -2.65020100 & -1.71942800 \\ \mathrm{C} & 3.85295300 & -2.91748200 & -2.78069400 \\ \mathrm{H} & 3.34632800 & -3.63237900 & -3.44222100 \\ \mathrm{C} & 4.10045800 & -1.59990400 & -3.51784200 \\ \mathrm{H} & 4.70279000 & -1.78188600 & -4.41548800 \\ \mathrm{H} & 4.64824600 & -0.90364800 & -2.87463200 \\ \mathrm{H} & 3.17313000 & -1.10972500 & -3.82726200 \\ \mathrm{C} & 5.14415100 & -3.54858200 & -2.26913800 \\ \mathrm{H} & 5.82283400 & -3.75419000 & -3.10393100 \\ \mathrm{H} & 4.93551500 & -4.48806900 & -1.74824800 \\ \mathrm{H} & 5.64958200 & -2.87277700 & -1.57086200 \\ \mathrm{C} & -2.37100100 & -3.04769100 & 0.86309200 \\ \mathrm{H} & -3.06181700 & -2.66876000 & 0.10374300 \\ \mathrm{C} & -2.86372400 & -2.59636200 & 2.23034800\end{array}$




\begin{tabular}{lccc}
$\mathrm{H}$ & -3.84808800 & -3.03209800 & 2.43238800 \\
$\mathrm{H}$ & -2.95574600 & -1.50779100 & 2.26034400 \\
$\mathrm{H}$ & -2.17234800 & -2.91799000 & 3.01690700 \\
$\mathrm{C}$ & -2.23852000 & -4.56596600 & 0.75533900 \\
$\mathrm{H}$ & -3.21527400 & -5.03917600 & 0.90623000 \\
$\mathrm{H}$ & -1.54777000 & -4.94195800 & 1.51831400 \\
$\mathrm{H}$ & -1.86551400 & -4.86674700 & -0.22857300 \\
$\mathrm{C}$ & 0.85918400 & 0.65475400 & 3.17768500 \\
$\mathrm{H}$ & 1.65747900 & 0.45593700 & 3.90495400 \\
$\mathrm{C}$ & 0.62412600 & 2.18195200 & 3.14049700 \\
$\mathrm{H}$ & 1.53741200 & 2.71037500 & 2.84370100 \\
$\mathrm{H}$ & -0.12934300 & 2.41298900 & 2.37516500 \\
$\mathrm{C}$ & 0.14821300 & 2.70787800 & 4.50587900 \\
$\mathrm{H}$ & 0.95256400 & 2.57865200 & 5.24576300 \\
$\mathrm{H}$ & -0.04081500 & 3.78640500 & 4.43598900 \\
$\mathrm{C}$ & -1.10978900 & 1.97450800 & 4.99225800 \\
$\mathrm{H}$ & -1.94612800 & 2.20534200 & 4.31603400 \\
$\mathrm{H}$ & -1.40130600 & 2.33593300 & 5.98665200 \\
$\mathrm{C}$ & -0.88834400 & 0.45547300 & 5.01969100 \\
$\mathrm{H}$ & -1.81069300 & -0.06116300 & 5.31477700 \\
$\mathrm{H}$ & -0.13543700 & 0.21030700 & 5.78382600 \\
$\mathrm{C}$ & -0.41539400 & -0.07142000 & 3.65480400 \\
$\mathrm{H}$ & -1.21237100 & 0.08541200 & 2.91337300 \\
$\mathrm{H}$ & -0.24419400 & -1.15243900 & 3.70379900 \\
$\mathrm{C}$ & 3.03540000 & 0.90501800 & 1.16628100 \\
$\mathrm{H}$ & 2.72161200 & 1.93797700 & 0.96020700 \\
$\mathrm{C}$ & 4.03119800 & 0.92849600 & 2.34421900 \\
$\mathrm{H}$ & 4.32560600 & -0.09966500 & 2.59261100 \\
$\mathrm{H}$ & 3.56886600 & 1.35519100 & 3.24202100 \\
$\mathrm{C}$ & 5.29428300 & 1.73566600 & 1.99277900 \\
$\mathrm{C}$ & -0.7900 & 2.78920900 & 1.83732900 \\
$\mathrm{C}$ & -1.73969000 & 3.42031900 & -0.53009100 \\
$\mathrm{H}$ & 1.69342600 & 5.20502300 & -0.34202000 \\
$\mathrm{C}$ & 2.52036900 & 3.36325000 & -1.08179100 \\
$\mathrm{H}$ & 0.53601100 & 5.89246100 & 0.02437800 \\
$\mathrm{C}$ & 5.99007300 & 1.71659500 & 2.84171400 \\
$\mathrm{H}$ & 5.97445800 & 1.19592800 & 0.72766300 \\
$\mathrm{H}$ & 6.34972700 & 0.18061100 & 0.92488500 \\
$\mathrm{C}$ & 6.84824200 & 1.80957000 & 0.47358600 \\
$\mathrm{C}$ & 4.98887700 & 1.15415000 & -0.44741300 \\
$\mathrm{H}$ & 5.46897600 & 0.71848400 & -1.33298900 \\
$\mathrm{H}$ & 4.71032500 & 2.18048800 & -0.72540600 \\
$\mathrm{C}$ & 3.71763000 & 0.35750200 & -0.10554000 \\
$\mathrm{H}$ & 3.97539400 & -0.69859600 & 0.04393800 \\
$\mathrm{H}$ & 3.02541400 & 0.39733500 & -0.95077600 \\
$\mathrm{O}$ & 3.03073900 & -2.71727400 & -1.61108300 \\
$\mathrm{O}$ & -1.07658900 & -2.41424100 & 0.67748500 \\
$\mathrm{P}$ & 1.39582700 & 0.02297500 & 1.48806300 \\
$\mathrm{Pd}$ & -0.55792100 & 0.48932600 & -0.01078300 \\
$\mathrm{C}$ & 0.31433300 & 1.79665500 & -1.26469600 \\
$\mathrm{C}$ & 0.84199800 & 1.24265100 & -2.36655900 \\
\hline
\end{tabular}




$\begin{array}{llcc}\text { H } & -1.61017100 & 5.77382100 & 0.21936900 \\ \mathrm{H} & 2.66024800 & 5.70025800 & -0.29874400 \\ \mathrm{H} & 0.59425900 & 6.92464700 & 0.35967900 \\ \mathrm{~F} & 1.19714200 & 3.17141700 & -3.74614800 \\ \mathrm{~F} & 1.59217000 & 1.19672600 & -4.58151300 \\ \mathrm{~F} & 2.95339100 & 2.04998500 & -3.11975300 \\ \mathrm{C} & -2.32751000 & 0.83994800 & -0.93676700 \\ \mathrm{O} & -2.74594500 & 1.49719800 & -1.85261100 \\ \mathrm{O} & -3.20440300 & 0.01994300 & -0.16768000 \\ \mathrm{C} & -4.56764800 & -0.02114000 & -0.46743500 \\ \mathrm{C} & -5.04005500 & -0.36504000 & -1.73948600 \\ \mathrm{C} & -5.46677200 & 0.22318000 & 0.56529700 \\ \mathrm{C} & -6.40808700 & -0.46384000 & -1.96157800 \\ \mathrm{H} & -4.34057200 & -0.54092200 & -2.54828100 \\ \mathrm{C} & -6.84465000 & 0.11854600 & 0.34740200 \\ \mathrm{H} & -5.08605900 & 0.49255800 & 1.54592700 \\ \mathrm{C} & -7.32040800 & -0.22429500 & -0.92273300 \\ \mathrm{H} & -6.79635300 & -0.72447800 & -2.94122000 \\ \mathrm{H} & -7.52558000 & 0.31011800 & 1.16880800 \\ \mathrm{O} & -8.64213400 & -0.35339300 & -1.25194600 \\ \mathrm{C} & -9.60722800 & -0.09937700 & -0.24737200 \\ \mathrm{H} & -10.58033700 & -0.24635600 & -0.72023200 \\ \mathrm{H} & -9.50767200 & -0.79535600 & 0.59717100 \\ \mathrm{H} & -9.53985200 & 0.93070400 & 0.12859500\end{array}$

\section{TS10- OMe}

$\mathrm{E}(\mathrm{B} 3 \mathrm{LYP} / \mathrm{BS} 1)=-2968.43291$

$\mathrm{E}(\mathrm{PCM}$ (dichloroethane)/M06/BS2// B3LYP/BS1 $)=-2968.647214$

Zero-point correction= 0.940175

Thermal correction to Energy= 0.998056

Thermal correction to Enthalpy= 0.999000

Thermal correction to Gibbs Free Energy= 0.840029

$\begin{array}{lrrr}\text { Charge }= & \text { Multiplicity }=1 & \\ \mathrm{C} & 2.86906500 & 1.65349100 & 0.53918100 \\ \mathrm{C} & 3.79263100 & 2.70539800 & 0.38255000 \\ \mathrm{H} & 3.72392500 & 3.35713000 & -0.48141400 \\ \mathrm{C} & 4.80343900 & 2.94604200 & 1.30966600 \\ \mathrm{H} & 5.49720000 & 3.76811600 & 1.15444700 \\ \mathrm{C} & 4.91319600 & 2.12589900 & 2.43174600 \\ \mathrm{H} & 5.69556200 & 2.29671800 & 3.16663300 \\ \mathrm{C} & 4.00407300 & 1.08792100 & 2.61007200 \\ \mathrm{H} & 4.07638200 & 0.45316900 & 3.48882700 \\ \mathrm{C} & 2.97435500 & 0.83414500 & 1.68748200 \\ \mathrm{C} & 2.05087500 & -0.29575400 & 2.04247100 \\ \mathrm{C} & 0.91129800 & -0.04787600 & 2.84261500 \\ \mathrm{C} & 0.15253500 & -1.11079000 & 3.35372200 \\ \mathrm{H} & -0.72596500 & -0.93051900 & 3.95970800 \\ \mathrm{C} & 0.53309300 & -2.41932100 & 3.06858200 \\ \mathrm{H} & -0.07315200 & -3.23995200 & 3.43963500 \\ \mathrm{C} & 1.66214300 & -2.69817400 & 2.30251800 \\ \mathrm{H} & 1.93424600 & -3.72692400 & 2.10398900 \\ \mathrm{C} & 2.43294200 & -1.63488100 & 1.80733000 \\ \mathrm{C} & 4.25108700 & -3.07997100 & 1.06255400 \\ \mathrm{H} & 4.05856900 & -3.60104000 & 2.00910200 \\ \mathrm{C} & 3.73558800 & -3.91408400 & -0.11091300\end{array}$




\begin{tabular}{|c|c|c|c|}
\hline $\mathrm{H}$ & 4.20037100 & -4.90698300 & -0.10234200 \\
\hline $\mathrm{H}$ & 3.98317500 & -3.42750200 & -1.06009700 \\
\hline $\mathrm{H}$ & 2.64991100 & -4.04116800 & -0.07396800 \\
\hline $\mathrm{C}$ & 5.74363400 & -2.78174800 & 0.95495200 \\
\hline $\mathrm{H}$ & 6.31745600 & -3.71474500 & 0.93150000 \\
\hline $\mathrm{H}$ & 6.08018500 & -2.18444500 & 1.80772100 \\
\hline $\mathrm{H}$ & 5.95684700 & -2.22180300 & 0.03793500 \\
\hline $\mathrm{C}$ & -0.36634900 & 1.65484600 & 4.03098200 \\
\hline $\mathrm{H}$ & -1.26804400 & 1.05473600 & 3.85641400 \\
\hline $\mathrm{C}$ & -0.68679100 & 3.11372500 & 3.72825900 \\
\hline $\mathrm{H}$ & -1.45425000 & 3.48153800 & 4.41792300 \\
\hline $\mathrm{H}$ & -1.05638500 & 3.22071600 & 2.70499900 \\
\hline $\mathrm{H}$ & 0.20925200 & 3.73366900 & 3.84237400 \\
\hline $\mathrm{C}$ & 0.13971900 & 1.45458600 & 5.46066700 \\
\hline $\mathrm{H}$ & -0.63308800 & 1.74545200 & 6.18123400 \\
\hline $\mathrm{H}$ & 1.02557900 & 2.07568600 & 5.63389900 \\
\hline $\mathrm{H}$ & 0.40910400 & 0.41172700 & 5.65179700 \\
\hline $\mathrm{C}$ & 1.00849800 & 3.12022200 & -1.19531100 \\
\hline $\mathrm{H}$ & 1.90794300 & 3.71672400 & -1.40128700 \\
\hline $\mathrm{C}$ & 0.13149200 & 3.14055500 & -2.46628300 \\
\hline $\mathrm{H}$ & 0.67870900 & 2.72610900 & -3.32096100 \\
\hline $\mathrm{H}$ & -0.74803300 & 2.50108900 & -2.31843900 \\
\hline $\mathrm{C}$ & -0.31806700 & 4.57326400 & -2.80562300 \\
\hline $\mathrm{H}$ & 0.56315800 & 5.17542500 & -3.07468500 \\
\hline $\mathrm{H}$ & -0.96508900 & 4.55305800 & -3.69157500 \\
\hline $\mathrm{C}$ & -1.04587900 & 5.23919000 & -1.62981200 \\
\hline $\mathrm{H}$ & -1.99180700 & 4.70947200 & -1.44908300 \\
\hline $\mathrm{H}$ & -1.30936200 & 6.27442000 & -1.88262500 \\
\hline $\mathrm{C}$ & -0.18904400 & 5.20350700 & -0.35568300 \\
\hline $\mathrm{H}$ & -0.74397700 & 5.62843100 & 0.49130600 \\
\hline $\mathrm{H}$ & 0.69726200 & 5.84054600 & -0.49568600 \\
\hline $\mathrm{C}$ & 0.26364100 & 3.77362400 & -0.01384400 \\
\hline $\mathrm{H}$ & -0.61115600 & 3.15605000 & 0.23121000 \\
\hline $\mathrm{H}$ & 0.89985300 & 3.78218800 & 0.87848400 \\
\hline $\mathrm{C}$ & 2.46632600 & 0.78302700 & -2.29050200 \\
\hline $\mathrm{H}$ & 1.66247300 & 0.77474000 & -3.04110300 \\
\hline $\mathrm{C}$ & 3.60905700 & 1.67160400 & -2.81808300 \\
\hline $\mathrm{H}$ & 4.41106100 & 1.71396600 & -2.06986800 \\
\hline $\mathrm{H}$ & 3.26437300 & 2.70143600 & -2.97534700 \\
\hline $\mathrm{C}$ & 4.18506900 & 1.11778500 & -4.13434800 \\
\hline $\mathrm{H}$ & 3.41258100 & 1.16344700 & -4.91614000 \\
\hline $\mathrm{H}$ & 5.01125400 & 1.75615700 & -4.47373400 \\
\hline $\mathrm{C}$ & 4.65960400 & -0.33310500 & -3.97375000 \\
\hline $\mathrm{H}$ & 5.51010900 & -0.35900500 & -3.27606800 \\
\hline $\mathrm{H}$ & 5.02832800 & -0.72148000 & -4.93190000 \\
\hline $\mathrm{C}$ & 3.53245800 & -1.22420100 & -3.43298100 \\
\hline $\mathrm{H}$ & 3.89806200 & -2.24755100 & -3.27514200 \\
\hline $\mathrm{H}$ & 2.73229900 & -1.29449200 & -4.18427400 \\
\hline $\mathrm{C}$ & 2.94412200 & -0.67341300 & -2.12263200 \\
\hline $\mathrm{H}$ & 3.69820900 & -0.72170300 & -1.32665000 \\
\hline $\mathrm{H}$ & 2.10588300 & -1.30282400 & -1.79910800 \\
\hline $\mathrm{O}$ & 3.59533800 & -1.79610700 & 1.10901500 \\
\hline $\mathrm{O}$ & 0.64414900 & 1.26816200 & 3.07732500 \\
\hline $\mathrm{P}$ & 1.55404000 & 1.37656400 & -0.74566600 \\
\hline $\mathrm{Pd}$ & -0.18096400 & -0.18441100 & -0.25627300 \\
\hline $\mathrm{C}$ & -1.72749600 & -1.63558800 & -0.27991200 \\
\hline- & 50 & -1.89518100 & 0.844270 \\
\hline
\end{tabular}




\begin{tabular}{|c|c|c|c|}
\hline $\mathrm{H}$ & -2.63015100 & -1.09912900 & 1.54659800 \\
\hline $\mathrm{C}$ & -3.04558200 & -3.20181400 & 1.22583900 \\
\hline $\mathrm{C}$ & -1.33970600 & -2.63223400 & -1.30753900 \\
\hline $\mathrm{C}$ & -1.70412300 & -2.51194600 & -2.66232400 \\
\hline $\mathrm{C}$ & -0.55055400 & -3.73601600 & -0.92135300 \\
\hline $\mathrm{C}$ & -1.29845500 & -3.47117000 & -3.58988100 \\
\hline $\mathrm{H}$ & -2.30048600 & -1.66458400 & -2.97510700 \\
\hline $\mathrm{C}$ & -0.14891400 & -4.68937500 & -1.85533200 \\
\hline $\mathrm{H}$ & -0.26113400 & -3.83795600 & 0.11928200 \\
\hline $\mathrm{C}$ & -0.52040200 & -4.56184400 & -3.19554100 \\
\hline $\mathrm{H}$ & -1.59733000 & -3.36496600 & -4.62980300 \\
\hline $\mathrm{H}$ & 0.45244500 & -5.53618800 & -1.53353100 \\
\hline $\mathrm{H}$ & -0.20891500 & -5.30595100 & -3.92404900 \\
\hline $\mathrm{F}$ & -3.56108300 & -3.88420500 & 0.17850500 \\
\hline $\mathrm{F}$ & -4.06134800 & -2.99279700 & 2.09823500 \\
\hline $\mathrm{F}$ & -2.17694500 & -4.04687700 & 1.85048600 \\
\hline $\mathrm{C}$ & -2.09845100 & 0.20329500 & -0.86216900 \\
\hline $\mathrm{O}$ & -2.44802700 & 0.35517700 & -2.01076700 \\
\hline $\mathrm{O}$ & -2.74535500 & 0.85555100 & 0.19589600 \\
\hline $\mathrm{C}$ & -3.95391200 & 1.49895600 & -0.07435000 \\
\hline $\mathrm{C}$ & -5.11014200 & 0.74523900 & -0.36494700 \\
\hline $\mathrm{C}$ & -4.02515300 & 2.87930400 & 0.04464200 \\
\hline $\mathrm{C}$ & -6.32238700 & 1.41524600 & -0.55786600 \\
\hline $\mathrm{C}$ & -5.24271300 & 3.54436300 & -0.13748400 \\
\hline $\mathrm{C}$ & -6.38332400 & 2.80857900 & -0.44542200 \\
\hline $\mathrm{H}$ & -7.22169700 & 0.85568600 & -0.78950300 \\
\hline $\mathrm{H}$ & -7.33507800 & 3.31071900 & -0.59584800 \\
\hline $\mathrm{H}$ & -5.29004200 & 4.62506100 & -0.04256700 \\
\hline $\mathrm{H}$ & -3.11873100 & 3.42337600 & 0.28948700 \\
\hline $\mathrm{O}$ & -4.94575000 & -0.60203900 & -0.41620500 \\
\hline $\mathrm{C}$ & -6.06947300 & -1.42416000 & -0.68915400 \\
\hline $\mathrm{H}$ & -6.85697800 & -1.29067800 & 0.06440500 \\
\hline $\mathrm{H}$ & -6.48214600 & -1.22106500 & -1.68593300 \\
\hline $\mathrm{H}$ & -5.69533700 & -2.44771600 & -0.64705600 \\
\hline
\end{tabular}

\section{2,4,6-Cl $-\mathrm{PhO}^{-}$}

$\mathrm{E}(\mathrm{B} 3 \mathrm{LYP} / \mathrm{BS} 1)=-1685.704585$

$\mathrm{E}(\mathrm{PCM}($ dichloroethane $) / \mathrm{M} 06 / \mathrm{BS} 2 / / \mathrm{B} 3 \mathrm{LYP} / \mathrm{BS} 1)=-1685.646155$

Zero-point correction= 0.871061

Thermal correction to Energy= 0.928170

Thermal correction to Enthalpy= 0.929115

Thermal correction to Gibbs Free Energy=

0.774473

$\begin{array}{lrrr}\text { Charge }= & -1 \text { Multiplicity }=1 \\ \text { C } & -0.89046100 & -1.21128600 & -0.00007000 \\ \mathrm{C} & -1.58355200 & -0.00006500 & -0.00010600 \\ \mathrm{C} & -0.89056200 & 1.21121800 & -0.00007000 \\ \mathrm{C} & 0.49516300 & 1.19377500 & -0.00011400 \\ \mathrm{C} & 1.33076700 & 0.00004500 & -0.00037000 \\ \mathrm{C} & 0.49526900 & -1.19375400 & -0.00011400 \\ \mathrm{H} & -1.42536400 & 2.15518200 & 0.00003400 \\ \mathrm{O} & 2.57776300 & 0.00009100 & 0.00015600 \\ \mathrm{H} & -1.42517600 & -2.15523600 & 0.00003300 \\ \mathrm{Cl} & 1.34511600 & -2.75362400 & 0.00009700 \\ \mathrm{Cl} & 1.34488000 & 2.75373200 & 0.00009900\end{array}$




\section{IM6-Cl3}

$\mathrm{E}(\mathrm{B} 3 \mathrm{LYP} / \mathrm{BS} 1)=-4119.384715$

$\mathrm{E}(\mathrm{PCM}$ (dichloroethane $) / \mathrm{M} 06 / \mathrm{BS} 2 / / \mathrm{B} 3 \mathrm{LYP} / \mathrm{BS} 1)=-4119.662362$

$\begin{array}{ll}\text { Zero-point correction }= & 0.871061 \\ \text { Thermal correction to Energy= } & 0.928170 \\ \text { Thermal correction to Enthalpy= } & 0.929115 \\ \text { Thermal correction to Gibbs Free Energy= } & 0.774473\end{array}$

Charge $=0$ Multiplicity $=1$

$\begin{array}{llll}\text { C } & -2.86484700 & 0.81896200 & -1.52263300\end{array}$

C $\quad-3.55806700 \quad 1.78509400 \quad-2.27767800$

$\mathrm{H} \quad-3.25126200 \quad 2.82231800 \quad-2.24614100$

C $\quad-4.64819700 \quad 1.46408200 \quad-3.08350200$

$\mathrm{H} \quad-5.14754000 \quad 2.24546700 \quad-3.64971800$

C $\quad-5.08328100 \quad 0.14376700 \quad-3.15381700$

$\mathrm{H} \quad-5.92864200 \quad-0.13037100 \quad-3.77900500$

C $\quad-4.41690000 \quad-0.82811300 \quad-2.41556100$

$\mathrm{H} \quad-4.74655000 \quad-1.86083900 \quad-2.46785200$

C $\quad-3.31158900 \quad-0.52711500 \quad-1.60042400$

$\begin{array}{llll}\text { C } & -2.68347400 & -1.69886500 & -0.91318800\end{array}$

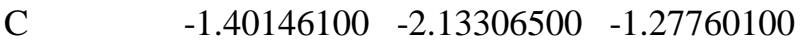

$\begin{array}{llll}\mathrm{C} & -0.82056400 & -3.28639400 & -0.74336300\end{array}$

$\begin{array}{llll}\mathrm{H} & 0.19787300 & -3.55176100 & -0.99792100\end{array}$

$\begin{array}{llll}\text { C } & -1.55944900 & -4.03836700 & 0.16332500\end{array}$

$\mathrm{H} \quad-1.12331000 \quad-4.93480000 \quad 0.59504500$

C $\quad-2.84563500 \quad-3.65292000 \quad 0.54548700$

$\mathrm{H} \quad-3.38977500 \quad-4.25594400 \quad 1.26135100$

$\begin{array}{llll}\text { C } & -3.41056600 & -2.48826500 & 0.01062100\end{array}$

$\begin{array}{llll}\text { C } & -5.58327600 & -2.84785800 & 1.07161500\end{array}$

$\begin{array}{llll}\mathrm{H} & -5.42722800 & -3.89922400 & 0.79774500\end{array}$

$\begin{array}{llll}\text { C } & -5.36640700 & -2.65570400 & 2.57286900\end{array}$

$\mathrm{H} \quad-6.05016000 \quad-3.29793300 \quad 3.13968800$

$\mathrm{H} \quad-5.56250400 \quad-1.61473700 \quad 2.85143800$

$\begin{array}{llll}\mathrm{H} & -4.34228500 & -2.89981600 & 2.86968600\end{array}$

$\begin{array}{lllll}\text { C } & -6.97773000 & -2.41830800 & 0.62643900\end{array}$

$\begin{array}{llll}\mathrm{H} & -7.74144700 & -2.99417500 & 1.16014500\end{array}$

$\mathrm{H} \quad-7.10832500 \quad-2.57812900 \quad-0.44816300$

$\mathrm{H} \quad-7.13427900 \quad-1.35506200 \quad 0.83778400$

C $\quad-0.00887200 \quad-1.93547500 \quad-3.30231600$

$\mathrm{H} \quad 0.85034000 \quad-2.46809000 \quad-2.88711600$

C $\quad 0.47665600 \quad-0.77731200 \quad-4.15878700$

$\mathrm{H} \quad 1.05440700 \quad-1.16529500 \quad-5.00477000$

$\mathrm{H} \quad 1.12687300 \quad-0.11718900 \quad-3.58155300$

$\mathrm{H} \quad-0.37142700 \quad-0.20392700 \quad-4.54993200$

$\begin{array}{llll}\text { C } & -0.93572200 & -2.87548400 & -4.06759700\end{array}$

$\mathrm{H} \quad-0.39350900 \quad-3.29570100 \quad-4.92182200$

$\mathrm{H} \quad-1.80823100 \quad-2.33144800 \quad-4.44672600$

$\mathrm{H} \quad-1.28357500 \quad-3.70742400 \quad-3.44907700$

$\begin{array}{llll}\text { C } & -0.94631700 & 3.06122500 & -1.14717300\end{array}$

$\mathrm{H} \quad \begin{array}{llll}\mathrm{H} & -1.86414100 & 3.63865700 & -1.31154700\end{array}$

$\begin{array}{llll}\text { C } & -0.08041500 & 3.89053600 & -0.17231600\end{array}$

$\begin{array}{llll}\mathrm{H} & -0.61433400 & 4.06123200 & 0.76958500\end{array}$

$\begin{array}{lllll}\mathrm{H} & 0.83062900 & 3.34091600 & 0.08149000\end{array}$ 


\begin{tabular}{|c|c|c|c|}
\hline $\mathrm{C}$ & 0.29648200 & 5.24764500 & -0.79401900 \\
\hline $\mathrm{H}$ & -0.61351300 & 5.85077900 & -0.93381600 \\
\hline $\mathrm{H}$ & 0.93366600 & 5.80144800 & -0.09352100 \\
\hline $\mathrm{C}$ & 1.00578400 & 5.07988500 & -2.14487600 \\
\hline $\mathrm{H}$ & 1.97122800 & 4.57780200 & -1.98764300 \\
\hline $\mathrm{H}$ & 1.23024200 & 6.06147300 & -2.58080100 \\
\hline $\mathrm{C}$ & 0.15516600 & 4.24762900 & -3.11423300 \\
\hline $\mathrm{H}$ & 0.69419600 & 4.08583400 & -4.05610000 \\
\hline $\mathrm{H}$ & -0.75844800 & 4.80590500 & -3.36902500 \\
\hline $\mathrm{C}$ & -0.23065100 & 2.88942300 & -2.50456800 \\
\hline $\mathrm{H}$ & 0.67391000 & 2.28543200 & -2.35232400 \\
\hline $\mathrm{H}$ & -0.86434100 & 2.33095800 & -3.20369100 \\
\hline $\mathrm{C}$ & -2.26714300 & 1.80462000 & 1.20432600 \\
\hline $\mathrm{H}$ & -1.43561100 & 2.17338500 & 1.82016200 \\
\hline $\mathrm{C}$ & -3.34341200 & 2.90796400 & 1.10703100 \\
\hline $\mathrm{H}$ & -4.15534700 & 2.56343100 & 0.45272900 \\
\hline $\mathrm{H}$ & -2.93623700 & 3.81884600 & 0.65381000 \\
\hline $\mathrm{C}$ & -3.92048300 & 3.24885000 & 2.49315200 \\
\hline $\mathrm{H}$ & -3.12948100 & 3.69502700 & 3.11355300 \\
\hline $\mathrm{H}$ & -4.70125900 & 4.01283300 & 2.38562100 \\
\hline $\mathrm{C}$ & -4.47662800 & 2.00489700 & 3.19852700 \\
\hline $\mathrm{H}$ & -5.34618200 & 1.63050000 & 2.63753300 \\
\hline $\mathrm{H}$ & -4.83910100 & 2.26758400 & 4.20063600 \\
\hline $\mathrm{C}$ & -3.41198100 & 0.90319100 & 3.28425500 \\
\hline $\mathrm{H}$ & -3.83224600 & -0.00038600 & 3.74374500 \\
\hline $\mathrm{H}$ & -2.59342100 & 1.23105300 & 3.93873600 \\
\hline $\mathrm{C}$ & -2.84141700 & 0.55265700 & 1.89858900 \\
\hline $\mathrm{H}$ & -3.64074800 & 0.12598200 & 1.28056500 \\
\hline $\mathrm{H}$ & -2.06580900 & -0.21271100 & 1.99972000 \\
\hline $\mathrm{O}$ & -4.66329900 & -2.03589700 & 0.31251900 \\
\hline $\mathrm{O}$ & -0.72354400 & -1.31556200 & -2.17449000 \\
\hline$P$ & -1.44746000 & 1.38865500 & -0.43762000 \\
\hline $\mathrm{Pd}$ & 0.40586700 & -0.07548200 & -0.50929500 \\
\hline $\mathrm{C}$ & 1.35777900 & 0.71518500 & 1.07237500 \\
\hline $\mathrm{C}$ & 2.42625000 & 1.48403700 & 0.81150800 \\
\hline $\mathrm{H}$ & 2.66960100 & 1.76670400 & -0.20690500 \\
\hline $\mathrm{C}$ & 3.41414500 & 2.03388800 & 1.79780800 \\
\hline $\mathrm{C}$ & 0.88366000 & 0.30190300 & 2.41031700 \\
\hline $\mathrm{C}$ & 0.59030100 & -1.04720700 & 2.68432400 \\
\hline $\mathrm{C}$ & 0.71412200 & 1.24679500 & 3.44156400 \\
\hline $\mathrm{C}$ & 0.16794200 & -1.43972200 & 3.95335300 \\
\hline $\mathrm{H}$ & 0.71963700 & -1.78614300 & 1.90079900 \\
\hline $\mathrm{C}$ & 0.28268000 & 0.85053400 & 4.70740200 \\
\hline $\mathrm{H}$ & 0.94852000 & 2.28926700 & 3.25300200 \\
\hline $\mathrm{C}$ & 0.00913700 & -0.49372400 & 4.96960400 \\
\hline $\mathrm{H}$ & -0.03353500 & -2.48954700 & 4.14938000 \\
\hline $\mathrm{H}$ & 0.16892800 & 1.59398300 & 5.49229400 \\
\hline $\mathrm{H}$ & -0.32183000 & -0.80169400 & 5.95791000 \\
\hline $\mathrm{F}$ & 3.65247700 & 1.23617100 & 2.85662100 \\
\hline $\mathrm{F}$ & 3.01300900 & 3.24185800 & 2.30252600 \\
\hline $\mathrm{F}$ & 4.59967300 & 2.25902100 & 1.18747600 \\
\hline $\mathrm{O}$ & 1.96869300 & -1.43137100 & -0.96577600 \\
\hline $\mathrm{C}$ & 3.24268300 & -1.44338800 & -0.69034500 \\
\hline $\mathrm{C}$ & 4.19899000 & -0.57204600 & -1.28662900 \\
\hline $\mathrm{C}$ & 3.81921600 & -2.40188100 & 0.19356900 \\
\hline $\mathrm{C}$ & 5.56222100 & -0.60919000 & -1.01136900 \\
\hline $\mathrm{C}$ & 5.17625900 & -2.47252600 & 0.485306 \\
\hline
\end{tabular}




\begin{tabular}{|c|c|c|c|}
\hline $\mathrm{C}$ & 6.04122400 & -1.56074700 & -0.11565100 \\
\hline $\mathrm{H}$ & 6.23647600 & 0.08815200 & -1.49387600 \\
\hline $\mathrm{H}$ & 5.55024600 & -3.22551700 & 1.16868300 \\
\hline $\mathrm{Cl}$ & 2.75566400 & -3.59483100 & 0.92856600 \\
\hline $\mathrm{Cl}$ & 3.64385600 & 0.59769200 & -2.48344600 \\
\hline $\mathrm{Cl}$ & 7.76179400 & -1.62580800 & 0.2470060 \\
\hline
\end{tabular}

\section{IM8- Cl3}

$\mathrm{E}(\mathrm{B} 3 \mathrm{LYP} / \mathrm{BS} 1)=-4232.692373$

$\mathrm{E}(\mathrm{PCM}$ (dichloroethane $) / \mathrm{M} 06 / \mathrm{BS} 2 / / \mathrm{B} 3 \mathrm{LYP} / \mathrm{BS} 1)=-4232.948067$

$\begin{array}{ll}\text { Zero-point correction }= & 0.878504 \\ \text { Thermal correction to Energy= } & 0.938669 \\ \text { Thermal correction to Enthalpy= } & 0.939613 \\ \text { Thermal correction to Gibbs Free Energy= } & 0.777212\end{array}$

\begin{tabular}{lrrr} 
Charge $=$ & M Multiplicity $=$ & & \\
$\mathrm{C}$ & -1.79053100 & 1.59509500 & 1.76830800 \\
$\mathrm{C}$ & -2.22872200 & 1.88686700 & 3.07823000 \\
$\mathrm{H}$ & -1.92013800 & 1.25851500 & 3.90368300 \\
$\mathrm{C}$ & -3.05106300 & 2.97169900 & 3.36598500 \\
$\mathrm{H}$ & -3.36040600 & 3.15663800 & 4.39098200 \\
$\mathrm{C}$ & -3.46654800 & 3.80879200 & 2.33249300 \\
$\mathrm{H}$ & -4.11279200 & 4.65934700 & 2.53190900 \\
$\mathrm{C}$ & -3.02694400 & 3.55230000 & 1.03936600 \\
$\mathrm{H}$ & -3.31938800 & 4.21546600 & 0.23040700 \\
$\mathrm{C}$ & -2.18500200 & 2.46712000 & 0.73013000 \\
$\mathrm{C}$ & -1.72939600 & 2.41734000 & -0.69381700 \\
$\mathrm{C}$ & -0.51860300 & 3.05425800 & -1.03543600 \\
$\mathrm{C}$ & -0.14776800 & 3.21063100 & -2.37863400 \\
$\mathrm{H}$ & 0.79229200 & 3.68062700 & -2.64019300 \\
$\mathrm{C}$ & -1.00601900 & 2.75800800 & -3.37759100 \\
$\mathrm{H}$ & -0.71914200 & 2.87441600 & -4.41926800 \\
$\mathrm{C}$ & -2.22875500 & 2.16045800 & -3.07003900 \\
$\mathrm{H}$ & -2.88184800 & 1.81784000 & -3.86390700 \\
$\mathrm{C}$ & -2.59581200 & 2.00416500 & -1.72547400 \\
$\mathrm{C}$ & -4.97231700 & 1.65162000 & -2.14345800 \\
$\mathrm{H}$ & -4.79395600 & 2.50353000 & -2.81174200 \\
$\mathrm{C}$ & -5.26849500 & 0.39721100 & -2.96112100 \\
$\mathrm{H}$ & -6.18430900 & 0.53898400 & -3.54676700 \\
$\mathrm{H}$ & -5.41103300 & -0.46371400 & -2.30042600 \\
$\mathrm{H}$ & -4.45648700 & 0.15285900 & -3.64996700 \\
$\mathrm{C}$ & -6.10229000 & 1.99473200 & -1.17619400 \\
$\mathrm{H}$ & -7.03402700 & 2.17223700 & -1.72474700 \\
$\mathrm{H}$ & -5.85966300 & 2.89116800 & -0.59763700 \\
$\mathrm{H}$ & -6.26674600 & 1.16896600 & -0.47529800 \\
$\mathrm{C}$ & 0.96961800 & 4.74008400 & -0.09279400 \\
$\mathrm{H}$ & 1.64707300 & 4.67792700 & -0.95226600 \\
$\mathrm{C}$ & 1.79851300 & 4.82208600 & 1.18208100 \\
$\mathrm{H}$ & 2.42914100 & 5.71759500 & 1.16551600 \\
$\mathrm{H}$ & 2.43983500 & 3.94170800 & 1.27334300 \\
$\mathrm{H}$ & 1.14357900 & 4.87297400 & 2.05894500 \\
$\mathrm{C}$ & 0.02458100 & 5.92933100 & -0.26541000 \\
$\mathrm{H}$ & 0.59863100 & 6.86061900 & -0.32902900 \\
$\mathrm{H}$ & -0.65670800 & 5.99821900 & 0.58985000 \\
$\mathrm{H}$ & -0.57206300 & 5.83657200 & -1.17867300 \\
& & & \\
\hline
\end{tabular}




\begin{tabular}{|c|c|c|c|}
\hline $\mathrm{C}$ & 0.26033800 & -0.02665900 & 3.10546400 \\
\hline $\mathrm{H}$ & -0.41128000 & -0.01896600 & 3.97371100 \\
\hline $\mathrm{C}$ & 1.08477400 & -1.32774100 & 3.16491400 \\
\hline $\mathrm{H}$ & 0.43270300 & -2.21001800 & 3.12354300 \\
\hline $\mathrm{H}$ & 1.73926100 & -1.36769000 & 2.29075300 \\
\hline $\mathrm{C}$ & 1.94448800 & -1.37526000 & 4.44021800 \\
\hline $\mathrm{H}$ & 1.28990700 & -1.43187800 & 5.32377900 \\
\hline $\mathrm{H}$ & 2.54766300 & -2.29174500 & 4.43798000 \\
\hline $\mathrm{C}$ & 2.85074600 & -0.14091000 & 4.55280600 \\
\hline $\mathrm{H}$ & 3.58152000 & -0.15836100 & 3.73311800 \\
\hline $\mathrm{H}$ & 3.42195300 & -0.17423400 & 5.48962300 \\
\hline $\mathrm{C}$ & 2.03343700 & 1.15657500 & 4.47253600 \\
\hline $\mathrm{H}$ & 2.69937500 & 2.02839200 & 4.50314100 \\
\hline $\mathrm{H}$ & 1.38113600 & 1.23118500 & 5.35681200 \\
\hline $\mathrm{C}$ & 1.17658800 & 1.21355600 & 3.19557300 \\
\hline $\mathrm{H}$ & 1.82415900 & 1.24322100 & 2.31321700 \\
\hline $\mathrm{H}$ & 0.57647800 & 2.13002300 & 3.19115900 \\
\hline $\mathrm{C}$ & -2.17214000 & -1.31469800 & 1.89013100 \\
\hline $\mathrm{H}$ & -1.72209200 & -2.23493700 & 1.49837400 \\
\hline $\mathrm{C}$ & -2.53763000 & -1.56705800 & 3.37000400 \\
\hline $\mathrm{H}$ & -2.96043600 & -0.65538900 & 3.81082500 \\
\hline $\mathrm{H}$ & -1.65042200 & -1.82873100 & 3.95533300 \\
\hline $\mathrm{C}$ & -3.57697200 & -2.69581000 & 3.50061100 \\
\hline $\mathrm{H}$ & -3.13723100 & -3.63676700 & 3.13921800 \\
\hline $\mathrm{H}$ & -3.81552900 & -2.84818200 & 4.56111900 \\
\hline $\mathrm{C}$ & -4.84822600 & -2.38584000 & 2.70077300 \\
\hline $\mathrm{H}$ & -5.33911900 & -1.49944900 & 3.12929500 \\
\hline $\mathrm{H}$ & -5.56446600 & -3.21311300 & 2.78512000 \\
\hline $\mathrm{C}$ & -4.50722900 & -2.12083000 & 1.22919200 \\
\hline $\mathrm{H}$ & -5.40881600 & -1.84015900 & 0.66947900 \\
\hline $\mathrm{H}$ & -4.13762500 & -3.05074200 & 0.77710700 \\
\hline $\mathrm{C}$ & -3.44934000 & -1.01362300 & 1.07143400 \\
\hline $\mathrm{H}$ & -3.87765800 & -0.06329700 & 1.41188100 \\
\hline $\mathrm{H}$ & -3.20959900 & -0.88034200 & 0.01338600 \\
\hline $\mathrm{O}$ & -3.78742200 & 1.46320200 & -1.32991900 \\
\hline $\mathrm{O}$ & 0.22271000 & 3.50675300 & 0.01579200 \\
\hline $\mathrm{P}$ & -0.83133500 & 0.00030900 & 1.57613300 \\
\hline $\mathrm{Pd}$ & 0.63399200 & -0.54652900 & -0.39211000 \\
\hline $\mathrm{C}$ & -0.74122300 & -1.83745000 & -1.22375900 \\
\hline $\mathrm{C}$ & -1.55514400 & -1.39094600 & -2.19253900 \\
\hline $\mathrm{H}$ & -1.61181200 & -0.33160800 & -2.41749800 \\
\hline $\mathrm{C}$ & -2.44401300 & -2.21019600 & -3.08122300 \\
\hline $\mathrm{C}$ & -0.57177000 & -3.23304000 & -0.76087200 \\
\hline $\mathrm{C}$ & 0.70481700 & -3.76054500 & -0.48100200 \\
\hline $\mathrm{C}$ & -1.68769900 & -4.07329900 & -0.56109500 \\
\hline $\mathrm{C}$ & 0.86108800 & -5.07401800 & -0.04099800 \\
\hline $\mathrm{H}$ & 1.58658200 & -3.14087600 & -0.60140600 \\
\hline $\mathrm{C}$ & -1.52860600 & -5.38330100 & -0.11496200 \\
\hline $\mathrm{H}$ & -2.68199800 & -3.69781900 & -0.76798000 \\
\hline $\mathrm{C}$ & -0.25422200 & -5.89176800 & 0.14673900 \\
\hline $\mathrm{H}$ & 1.85949400 & -5.45291300 & 0.15871700 \\
\hline $\mathrm{H}$ & -2.40576700 & -6.00912400 & 0.02665200 \\
\hline $\mathrm{H}$ & -0.13275500 & -6.91352000 & 0.49577100 \\
\hline $\mathrm{F}$ & -1.92718900 & -3.40146900 & -3.44006800 \\
\hline $\mathrm{F}$ & -2.69805200 & -1.52723100 & -4.22706500 \\
\hline $\mathrm{F}$ & -3.66345000 & -2.46855800 & -2.51933800 \\
\hline $\mathrm{C}$ & 1.56337300 & -0.76833600 & -2.11323500 \\
\hline
\end{tabular}




$\begin{array}{lrrr}\mathrm{O} & 2.05294300 & -0.82171600 & -3.14015300 \\ \mathrm{O} & 2.50154800 & 0.28691300 & 0.43022000 \\ \mathrm{C} & 3.68736300 & 0.13500300 & -0.09825900 \\ \mathrm{C} & 4.30096900 & 1.11648100 & -0.93445500 \\ \mathrm{C} & 4.52179600 & -1.00392500 & 0.12015900 \\ \mathrm{C} & 5.58712200 & 1.01557800 & -1.45384600 \\ \mathrm{C} & 5.81323700 & -1.13663800 & -0.38185200 \\ \mathrm{C} & 6.34265400 & -0.11743500 & -1.16706100 \\ \mathrm{H} & 5.98734200 & 1.80845100 & -2.07449400 \\ \mathrm{H} & 6.39259000 & -2.02556300 & -0.16231600 \\ \mathrm{Cl} & 7.97484700 & -0.26487900 & -1.80650200 \\ \mathrm{Cl} & 3.90501200 & -2.33962400 & 1.08744300 \\ \mathrm{Cl} & 3.36913300 & 2.54411900 & -1.34720200\end{array}$

\section{TS9- Cl3}

$\mathrm{E}(\mathrm{B} 3 \mathrm{LYP} / \mathrm{BS} 1)=-4232.688041$

$\mathrm{E}(\mathrm{PCM}$ (dichloroethane)/M06/BS2// B3LYP/BS1 $)=-4232.942755$

$\begin{array}{ll}\text { Zero-point correction }= & 0.877999 \\ \text { Thermal correction to Energy= } & 0.937457 \\ \text { Thermal correction to Enthalpy= } & 0.938401 \\ \text { Thermal correction to Gibbs Free Energy= } & 0.779050\end{array}$

$\begin{array}{lrrr}\text { Charge }= & \text { Multiplicity }= & \\ \mathrm{C} & 1.96236500 & -1.25774700 & 2.13799400 \\ \mathrm{C} & 2.44134200 & -1.29431200 & 3.46251400 \\ \mathrm{H} & 2.32504100 & -0.42997000 & 4.10585500 \\ \mathrm{C} & 3.06737400 & -2.42168500 & 3.98808600 \\ \mathrm{H} & 3.42067500 & -2.41380300 & 5.01553100 \\ \mathrm{C} & 3.23424000 & -3.54958100 & 3.18638800 \\ \mathrm{H} & 3.72429200 & -4.43734000 & 3.57698200 \\ \mathrm{C} & 2.75130100 & -3.53691400 & 1.88195900 \\ \mathrm{H} & 2.85519300 & -4.42050600 & 1.25933600 \\ \mathrm{C} & 2.10324500 & -2.41492700 & 1.33746500 \\ \mathrm{C} & 1.54475200 & -2.58136800 & -0.04033700 \\ \mathrm{C} & 0.16989700 & -2.86579000 & -0.18788000 \\ \mathrm{C} & -0.36482300 & -3.17829000 & -1.44806000 \\ \mathrm{H} & -1.42419000 & -3.36278400 & -1.56818300 \\ \mathrm{C} & 0.48565900 & -3.23069600 & -2.54790700 \\ \mathrm{H} & 0.07424600 & -3.46963400 & -3.52490200 \\ \mathrm{C} & 1.85354500 & -2.97953000 & -2.43017100 \\ \mathrm{H} & 2.48530200 & -3.02938300 & -3.30788300 \\ \mathrm{C} & 2.38474300 & -2.66099600 & -1.17195100 \\ \mathrm{C} & 4.70668800 & -2.76766000 & -1.92496700 \\ \mathrm{H} & 4.36445200 & -3.65997600 & -2.46483200 \\ \mathrm{C} & 4.93535500 & -1.61544200 & -2.90364300 \\ \mathrm{H} & 5.71146100 & -1.88834000 & -3.62811700 \\ \mathrm{H} & 5.26816100 & -0.72043400 & -2.36720700 \\ \mathrm{H} & 4.03236200 & -1.35411000 & -3.46108500 \\ \mathrm{C} & 5.96462800 & -3.11558200 & -1.13442200 \\ \mathrm{H} & 6.77103500 & -3.41289900 & -1.81349500 \\ \mathrm{H} & 5.76984100 & -3.93915100 & -0.44076900 \\ \mathrm{H} & 6.30298500 & -2.25018100 & -0.55421300 \\ \mathrm{C} & -1.80336000 & -3.56002300 & 1.07104400 \\ & & & \end{array}$




\begin{tabular}{|c|c|c|c|}
\hline $\mathrm{H}$ & -2.44475400 & -3.29277000 & 0.22991100 \\
\hline $\mathrm{C}$ & -2.46274100 & -3.07315600 & 2.35304000 \\
\hline $\mathrm{H}$ & -3.40989100 & -3.60164100 & 2.50650500 \\
\hline $\mathrm{H}$ & -2.67100400 & -2.00314500 & 2.28296100 \\
\hline $\mathrm{H}$ & -1.81701200 & -3.26028700 & 3.21881500 \\
\hline $\mathrm{C}$ & -1.51475600 & -5.06053500 & 1.08815100 \\
\hline $\mathrm{H}$ & -2.45287900 & -5.62036000 & 1.17301600 \\
\hline $\mathrm{H}$ & -0.87924900 & -5.31768100 & 1.94333500 \\
\hline $\mathrm{H}$ & -1.01070700 & -5.38524700 & 0.17231700 \\
\hline $\mathrm{C}$ & 0.37696700 & 1.09186400 & 2.97706600 \\
\hline $\mathrm{H}$ & 1.09426900 & 1.13791700 & 3.80673400 \\
\hline $\mathrm{C}$ & -0.09502900 & 2.53430500 & 2.69309400 \\
\hline $\mathrm{H}$ & 0.74104200 & 3.17083500 & 2.37975800 \\
\hline $\mathrm{H}$ & -0.80850200 & 2.52407900 & 1.85942800 \\
\hline $\mathrm{C}$ & -0.76384300 & 3.13726100 & 3.94233100 \\
\hline $\mathrm{H}$ & -0.00900100 & 3.24390100 & 4.73619300 \\
\hline $\mathrm{H}$ & -1.11975100 & 4.14986900 & 3.71432500 \\
\hline $\mathrm{C}$ & -1.92248400 & 2.26670800 & 4.45190800 \\
\hline $\mathrm{H}$ & -2.73809800 & 2.28394700 & 3.71748600 \\
\hline $\mathrm{H}$ & -2.32727900 & 2.68638300 & 5.38165800 \\
\hline $\mathrm{C}$ & -1.48115500 & 0.81238100 & 4.67488800 \\
\hline $\mathrm{H}$ & -2.34185700 & 0.19457800 & 4.95947500 \\
\hline $\mathrm{H}$ & -0.77297300 & 0.76842800 & 5.51640300 \\
\hline $\mathrm{C}$ & -0.81259700 & 0.21727300 & 3.42313400 \\
\hline $\mathrm{H}$ & -1.54526900 & 0.15889900 & 2.60745600 \\
\hline $\mathrm{H}$ & -0.48173700 & -0.80714600 & 3.62436200 \\
\hline $\mathrm{C}$ & 2.81430600 & 1.42572900 & 1.27872400 \\
\hline $\mathrm{H}$ & 2.43093000 & 2.31293400 & 0.75828200 \\
\hline $\mathrm{C}$ & 3.49636200 & 1.89499800 & 2.58239000 \\
\hline $\mathrm{H}$ & 3.85958500 & 1.02275800 & 3.14113300 \\
\hline $\mathrm{H}$ & 2.78918400 & 2.42239900 & 3.23157500 \\
\hline $\mathrm{C}$ & 4.68937300 & 2.82098100 & 2.28081400 \\
\hline $\mathrm{H}$ & 4.32126000 & 3.73949700 & 1.80069200 \\
\hline $\mathrm{H}$ & 5.15937200 & 3.12812400 & 3.22389200 \\
\hline $\mathrm{C}$ & 5.71538300 & 2.14199500 & 1.36451400 \\
\hline $\mathrm{H}$ & 6.16302400 & 1.28777100 & 1.89367200 \\
\hline $\mathrm{H}$ & 6.53599000 & 2.83290900 & 1.13321600 \\
\hline $\mathrm{C}$ & 5.05023000 & 1.64820400 & 0.07346100 \\
\hline $\mathrm{H}$ & 5.77578400 & 1.10845300 & -0.54859300 \\
\hline $\mathrm{H}$ & 4.72058900 & 2.51302600 & -0.51900100 \\
\hline $\mathrm{C}$ & 3.84647700 & 0.73397400 & 0.36089500 \\
\hline $\mathrm{H}$ & 4.19805100 & -0.18716300 & 0.84091200 \\
\hline $\mathrm{H}$ & 3.38128200 & 0.43485100 & -0.58099000 \\
\hline $\mathrm{O}$ & 3.70934300 & -2.42250200 & -0.93859200 \\
\hline $\mathrm{O}$ & -0.56042900 & -2.81366000 & 0.96116700 \\
\hline$P$ & 1.27492700 & 0.34935300 & 1.50664000 \\
\hline $\mathrm{Pd}$ & -0.33160200 & 0.30143200 & -0.35703600 \\
\hline $\mathrm{C}$ & 0.79005500 & 1.46698000 & -1.57665500 \\
\hline $\mathrm{C}$ & 1.51972200 & 0.80405000 & -2.48505900 \\
\hline $\mathrm{H}$ & 1.55422000 & -0.28054300 & -2.47685300 \\
\hline $\mathrm{C}$ & 2.36448100 & 1.39106400 & -3.57629600 \\
\hline $\mathrm{C}$ & 0.64541500 & 2.92353700 & -1.38572300 \\
\hline $\mathrm{C}$ & -0.61272900 & 3.50691200 & -1.13920200 \\
\hline C & 1.77311400 & 3.76986500 & -1.42804800 \\
\hline $\mathrm{C}$ & -0.74164700 & 4.88343100 & -0.96681900 \\
\hline $\mathrm{H}$ & -1.49417900 & 2.87811800 & -1.07861800 \\
\hline $\mathrm{C}$ & 1.64060200 & 5.14502500 & -1.24474800 \\
\hline
\end{tabular}




$\begin{array}{lrrr}\mathrm{H} & 2.75235300 & 3.34743900 & -1.62040700 \\ \mathrm{C} & 0.38405400 & 5.70882600 & -1.01475900 \\ \mathrm{H} & -1.72532800 & 5.30887000 & -0.78890400 \\ \mathrm{H} & 2.52385900 & 5.77690000 & -1.28437400 \\ \mathrm{H} & 0.28322300 & 6.78117300 & -0.87176500 \\ \mathrm{~F} & 1.86502600 & 2.51038700 & -4.13170400 \\ \mathrm{~F} & 2.52261900 & 0.48252000 & -4.57039600 \\ \mathrm{~F} & 3.62644500 & 1.69654700 & -3.14436100 \\ \mathrm{C} & -1.71906200 & 0.23329300 & -1.78109100 \\ \mathrm{O} & -2.36173600 & 0.32163300 & -2.72830700 \\ \mathrm{O} & -2.77612000 & -0.70283500 & -0.19252000 \\ \mathrm{C} & -4.03857400 & -0.42044900 & -0.35133500 \\ \mathrm{C} & -4.93764400 & -1.27229500 & -1.06167700 \\ \mathrm{C} & -4.66045500 & 0.76461500 & 0.14200400 \\ \mathrm{C} & -6.29499800 & -1.01370100 & -1.21788500 \\ \mathrm{C} & -6.01219600 & 1.05724800 & 0.00099300 \\ \mathrm{C} & -6.82637800 & 0.15497400 & -0.67842000 \\ \mathrm{H} & -6.92429300 & -1.71122600 & -1.75773200 \\ \mathrm{H} & -6.41967600 & 1.97375700 & 0.41085900 \\ \mathrm{Cl} & -8.54194700 & 0.49896500 & -0.86334500 \\ \mathrm{Cl} & -3.65262100 & 1.94210300 & 0.97733000 \\ \mathrm{Cl} & -4.30779400 & -2.75244000 & -1.77697000\end{array}$

\section{IM10- Cl3}

$\mathrm{E}(\mathrm{B} 3 \mathrm{LYP} / \mathrm{BS} 1)=-4232.696285$

$\mathrm{E}(\mathrm{PCM}$ (dichloroethane)/M06/BS2// B3LYP/BS1) $=-4232.949126$

Zero-point correction= 0.879460

Thermal correction to Energy= 0.938753

Thermal correction to Enthalpy= 0.939697

Thermal correction to Gibbs Free Energy= 0.779503

$\begin{array}{lrrr}\text { Charge }= & \text { Multiplicity }=1 & \\ \mathrm{C} & 2.71657900 & -0.71102400 & 1.96109500 \\ \mathrm{C} & 3.46813000 & -0.43646000 & 3.12061400 \\ \mathrm{H} & 3.52038200 & 0.57544900 & 3.50373000 \\ \mathrm{C} & 4.15936400 & -1.42831900 & 3.81187800 \\ \mathrm{H} & 4.72384100 & -1.17158000 & 4.70417100 \\ \mathrm{C} & 4.11600000 & -2.74223200 & 3.35040400 \\ \mathrm{H} & 4.64187400 & -3.53311300 & 3.87838900 \\ \mathrm{C} & 3.38969200 & -3.03446900 & 2.20128300 \\ \mathrm{H} & 3.35541700 & -4.05513500 & 1.83316300 \\ \mathrm{C} & 2.68511800 & -2.04622100 & 1.48996800 \\ \mathrm{C} & 1.94843800 & -2.52244500 & 0.27760600 \\ \mathrm{C} & 0.54715500 & -2.54788700 & 0.23035500 \\ \mathrm{C} & -0.14976100 & -3.13213000 & -0.83219900 \\ \mathrm{H} & -1.23232000 & -3.11235000 & -0.84659500 \\ \mathrm{C} & 0.57205400 & -3.69420100 & -1.87803800 \\ \mathrm{H} & 0.04414800 & -4.14032500 & -2.71606200 \\ \mathrm{C} & 1.96799700 & -3.67210500 & -1.88270400 \\ \mathrm{H} & 2.50238300 & -4.09919200 & -2.72177900 \\ \mathrm{C} & 2.65727900 & -3.09356900 & -0.81033200 \\ \mathrm{C} & 4.85840700 & -3.72436400 & -1.66646800 \\ \mathrm{H} & 4.37697900 & -4.67277800 & -1.93829200 \\ \mathrm{C} & 5.07857500 & -2.86704100 & -2.91323100 \\ \mathrm{H} & 5.70023300 & -3.40504300 & -3.63797700\end{array}$




\begin{tabular}{|c|c|c|c|}
\hline $\mathrm{H}$ & 5.59061700 & -1.93686300 & -2.64453100 \\
\hline $\mathrm{H}$ & 4.13456700 & -2.60635300 & -3.40036100 \\
\hline $\mathrm{C}$ & 6.15935000 & -4.02087000 & -0.92711000 \\
\hline $\mathrm{H}$ & 6.85763800 & -4.55033700 & -1.58418000 \\
\hline $\mathrm{H}$ & 5.97130700 & -4.64097900 & -0.04535700 \\
\hline $\mathrm{H}$ & 6.63131600 & -3.08892300 & -0.59796700 \\
\hline $\mathrm{C}$ & -1.13617100 & -2.70322100 & 2.00060500 \\
\hline $\mathrm{H}$ & -1.93422500 & -2.95938200 & 1.29817100 \\
\hline $\mathrm{C}$ & -1.68818700 & -1.78482200 & 3.07841400 \\
\hline $\mathrm{H}$ & -2.48175000 & -2.30294700 & 3.62817900 \\
\hline $\mathrm{H}$ & -2.11359500 & -0.88247200 & 2.63356300 \\
\hline $\mathrm{H}$ & -0.90272900 & -1.50456000 & 3.78814500 \\
\hline $\mathrm{C}$ & -0.50053000 & -3.96694700 & 2.57351600 \\
\hline $\mathrm{H}$ & -1.25549900 & -4.53226900 & 3.13123000 \\
\hline $\mathrm{H}$ & 0.31813200 & -3.71335400 & 3.25555700 \\
\hline $\mathrm{H}$ & -0.10968900 & -4.61732200 & 1.78510300 \\
\hline $\mathrm{C}$ & 1.49129400 & 1.90818300 & 2.49946500 \\
\hline $\mathrm{H}$ & 2.43825500 & 2.09921300 & 3.02046000 \\
\hline $\mathrm{C}$ & 0.98174900 & 3.26711700 & 1.97598400 \\
\hline $\mathrm{H}$ & 1.69386000 & 3.70845500 & 1.27031700 \\
\hline $\mathrm{H}$ & 0.04778400 & 3.12058100 & 1.42287600 \\
\hline $\mathrm{C}$ & 0.73279900 & 4.24745300 & 3.13539500 \\
\hline $\mathrm{H}$ & 1.68757500 & 4.48041200 & 3.63105100 \\
\hline $\mathrm{H}$ & 0.34979900 & 5.19273000 & 2.73170700 \\
\hline $\mathrm{C}$ & -0.24725500 & 3.66892600 & 4.16548800 \\
\hline $\mathrm{H}$ & -1.23557900 & 3.55466000 & 3.69703400 \\
\hline $\mathrm{H}$ & -0.37579800 & 4.36484100 & 5.00431000 \\
\hline $\mathrm{C}$ & 0.22998300 & 2.30175800 & 4.67540700 \\
\hline $\mathrm{H}$ & -0.50870200 & 1.86987600 & 5.36267700 \\
\hline $\mathrm{H}$ & 1.15641500 & 2.43206500 & 5.25495300 \\
\hline $\mathrm{C}$ & 0.49024400 & 1.32265000 & 3.51792200 \\
\hline $\mathrm{H}$ & -0.45552800 & 1.10960900 & 3.00150400 \\
\hline $\mathrm{H}$ & 0.85592600 & 0.36774100 & 3.91216800 \\
\hline $\mathrm{C}$ & 3.16774300 & 1.50897200 & 0.05864100 \\
\hline $\mathrm{H}$ & 2.62221400 & 2.31442000 & -0.45174700 \\
\hline $\mathrm{C}$ & 4.34361700 & 2.13528000 & 0.83394100 \\
\hline $\mathrm{H}$ & 4.88013300 & 1.34799400 & 1.38099100 \\
\hline $\mathrm{H}$ & 3.98439600 & 2.85417200 & 1.57959600 \\
\hline $\mathrm{C}$ & 5.31972600 & 2.84394500 & -0.12366800 \\
\hline $\mathrm{H}$ & 4.80777700 & 3.69834500 & -0.59018900 \\
\hline $\mathrm{H}$ & 6.16045500 & 3.25811300 & 0.44787000 \\
\hline $\mathrm{C}$ & 5.83030900 & 1.89876700 & -1.22011200 \\
\hline $\mathrm{H}$ & 6.44790800 & 1.11343900 & -0.75886600 \\
\hline $\mathrm{H}$ & 6.48276900 & 2.44246800 & -1.91517600 \\
\hline $\mathrm{C}$ & 4.66584500 & 1.24632300 & -1.97811600 \\
\hline $\mathrm{H}$ & 5.04775400 & 0.52471200 & -2.71197200 \\
\hline $\mathrm{H}$ & 4.11675300 & 2.00547600 & -2.55110000 \\
\hline $\mathrm{C}$ & 3.69062000 & 0.53864000 & -1.02202200 \\
\hline $\mathrm{H}$ & 4.19814400 & -0.30683500 & -0.53990900 \\
\hline $\mathrm{H}$ & 2.85251000 & 0.12392500 & -1.59063900 \\
\hline $\mathrm{O}$ & 4.01772500 & -3.03953700 & -0.71493300 \\
\hline $\mathrm{O}$ & -0.13500100 & -1.91685900 & 1.26765300 \\
\hline $\mathrm{P}$ & 1.83210500 & 0.68978100 & 1.10264900 \\
\hline $\mathrm{Pd}$ & -0.31710800 & 0.16598000 & -0.05596500 \\
\hline $\mathrm{C}$ & -0.08880900 & 1.53002800 & -1.53028400 \\
\hline $\mathrm{C}$ & 0.41899300 & 1.03021100 & -2.66858600 \\
\hline $\mathrm{H}$ & 0.67616200 & -0.02161000 & -2.745965 \\
\hline
\end{tabular}




$\begin{array}{lrrr}\mathrm{C} & 0.67595900 & 1.76536300 & -3.94945300 \\ \mathrm{C} & -0.45590300 & 2.94142400 & -1.28117100 \\ \mathrm{C} & -1.69910600 & 3.28790400 & -0.71969700 \\ \mathrm{C} & 0.44960000 & 3.97955600 & -1.57689400 \\ \mathrm{C} & -2.02669000 & 4.62004900 & -0.47532100 \\ \mathrm{H} & -2.41258900 & 2.50706200 & -0.48621400 \\ \mathrm{C} & 0.12434800 & 5.31089800 & -1.31801500 \\ \mathrm{H} & 1.40686600 & 3.73969400 & -2.02673200 \\ \mathrm{C} & -1.11442200 & 5.63861100 & -0.76462800 \\ \mathrm{H} & -2.99900600 & 4.86192600 & -0.05405600 \\ \mathrm{H} & 0.84102400 & 6.09319400 & -1.55495900 \\ \mathrm{H} & -1.36867800 & 6.67614200 & -0.56452900 \\ \mathrm{~F} & -0.21682900 & 2.73212900 & -4.23262300 \\ \mathrm{~F} & 0.67610400 & 0.89788200 & -4.98886500 \\ \mathrm{~F} & 1.91080100 & 2.36715100 & -3.96829100 \\ \mathrm{C} & -2.19080200 & -0.12436000 & -0.84702300 \\ \mathrm{O} & -2.76549700 & 0.17959700 & -1.84883900 \\ \mathrm{O} & -2.97747400 & -0.86260200 & 0.18739000 \\ \mathrm{C} & -4.32757100 & -0.92779500 & 0.01232200 \\ \mathrm{C} & -4.94590800 & -1.95976100 & -0.71266800 \\ \mathrm{C} & -5.17395200 & 0.02789600 & 0.60209500 \\ \mathrm{C} & -6.33047700 & -2.04671500 & -0.84729100 \\ \mathrm{C} & -6.56067400 & -0.03234200 & 0.48720000 \\ \mathrm{C} & -7.12384800 & -1.07491500 & -0.24427800 \\ \mathrm{H} & -6.77494600 & -2.85639400 & -1.41279300 \\ \mathrm{H} & -7.18299000 & 0.71922200 & 0.95704000 \\ \mathrm{Cl} & -8.86846500 & -1.16963100 & -0.40224100 \\ \mathrm{Cl} & -4.46984600 & 1.33557300 & 1.52847700 \\ \mathrm{Cl} & -3.96665400 & -3.20366500 & -1.45772000\end{array}$

\section{TS10- Cl3}

$\mathrm{E}(\mathrm{B} 3 \mathrm{LYP} / \mathrm{BS} 1)=-4232.681641$

$\mathrm{E}(\mathrm{PCM}$ (dichloroethane $) / \mathrm{M} 06 / \mathrm{BS} 2 / / \mathrm{B} 3 \mathrm{LYP} / \mathrm{BS} 1)=-4232.934281$

Zero-point correction $=$

Thermal correction to Energy=

Thermal correction to Enthalpy=

Thermal correction to Gibbs Free Energy=
0.877896

0.937123

0.938067

0.775757

\footnotetext{
Charge $=0$ Multiplicity $=1$

$\begin{array}{llll}\text { C } & 2.89746700 & -2.12372000 & 0.09018900\end{array}$

$\begin{array}{llll}\text { C } & 3.74784300 & -3.08985000 & 0.66327400\end{array}$

$\begin{array}{llll}\mathrm{H} & 3.65851100 & -3.32586000 & 1.71794100\end{array}$

C $\quad 4.70735000 \quad-3.76675400 \quad-0.08516600$

$\begin{array}{llll}\mathrm{H} & 5.34525500 & -4.50598300 & 0.39200400\end{array}$

C $\quad 4.83881200 \quad-3.48555900 \quad-1.44449000$

$\begin{array}{llll}\mathrm{H} & 5.58552700 & -3.99860100 & -2.04490300\end{array}$

C $\quad 3.99201800 \quad-2.55201200 \quad-2.03374600$

$\mathrm{H} \quad 4.07184300 \quad-2.34751600 \quad-3.09774000$

$\begin{array}{llll}\text { C } & 3.00965000 & -1.86810100 & -1.29674700\end{array}$

$\begin{array}{llll}\text { C } & 2.11203800 & -0.96441200 & -2.09030200\end{array}$

$\begin{array}{llll}\text { C } & 0.93309300 & -1.49427000 & -2.65806500\end{array}$

C $\quad 0.15294800 \quad-0.72727400 \quad-3.53514700$

$\mathrm{H} \quad-0.76092700 \quad-1.12306800 \quad-3.96010300$

C $\quad 0.57028100 \quad 0.55995300 \quad-3.86475700$

$\begin{array}{llll}\mathrm{H} & -0.03416900 & 1.15923500 & -4.54065300\end{array}$

$\begin{array}{lllll}\text { C } & 1.74200900 & 1.10239800 & -3.34257700\end{array}$
} 


\begin{tabular}{|c|c|c|c|}
\hline $\mathrm{H}$ & 2.02802400 & 2.11072200 & -3.61041300 \\
\hline $\mathrm{C}$ & 2.51743300 & 0.33485000 & -2.46087900 \\
\hline $\mathrm{C}$ & 4.39378200 & 1.89191500 & -2.48050000 \\
\hline $\mathrm{H}$ & 4.19225200 & 1.92342900 & -3.55912800 \\
\hline $\mathrm{C}$ & 3.93327900 & 3.19438100 & -1.82419500 \\
\hline $\mathrm{H}$ & 4.48056400 & 4.04418400 & -2.24856500 \\
\hline $\mathrm{H}$ & 4.13162500 & 3.16311300 & -0.74708400 \\
\hline $\mathrm{H}$ & 2.86452500 & 3.37260900 & -1.97212600 \\
\hline C & 5.87915300 & 1.62226300 & -2.25809000 \\
\hline $\mathrm{H}$ & 6.48149500 & 2.43349200 & -2.68141900 \\
\hline $\mathrm{H}$ & 6.17506000 & 0.68162300 & -2.73229600 \\
\hline $\mathrm{H}$ & 6.09937200 & 1.55098600 & -1.18731200 \\
\hline $\mathrm{C}$ & -0.30891800 & -3.55663800 & -3.05563000 \\
\hline $\mathrm{H}$ & -1.24629700 & -2.99152000 & -3.13621000 \\
\hline $\mathrm{C}$ & -0.56046200 & -4.81084600 & -2.22653400 \\
\hline $\mathrm{H}$ & -1.29884500 & -5.44984500 & -2.72248000 \\
\hline $\mathrm{H}$ & -0.93539600 & -4.55015300 & -1.23312100 \\
\hline $\mathrm{H}$ & 0.36776400 & -5.37993700 & -2.10538700 \\
\hline $\mathrm{C}$ & 0.23552800 & -3.88955900 & -4.44620600 \\
\hline $\mathrm{H}$ & -0.49550500 & -4.48503800 & -5.00473300 \\
\hline $\mathrm{H}$ & 1.15937800 & -4.47179700 & -4.35623400 \\
\hline $\mathrm{H}$ & 0.45270400 & -2.98729400 & -5.02477000 \\
\hline $\mathrm{C}$ & 0.98263500 & -2.54910000 & 2.28790200 \\
\hline $\mathrm{H}$ & 1.81724300 & -3.07853400 & 2.76867200 \\
\hline $\mathrm{C}$ & 0.10245900 & -1.93655400 & 3.39906500 \\
\hline $\mathrm{H}$ & 0.68927800 & -1.25290800 & 4.02456500 \\
\hline $\mathrm{H}$ & -0.69429300 & -1.33501100 & 2.94267900 \\
\hline $\mathrm{C}$ & -0.51927300 & -3.03068400 & 4.28521600 \\
\hline $\mathrm{H}$ & 0.27900600 & -3.54932500 & 4.83766000 \\
\hline $\mathrm{H}$ & -1.16810200 & -2.56511100 & 5.03770300 \\
\hline C & -1.30931400 & -4.05416100 & 3.45764000 \\
\hline $\mathrm{H}$ & -2.17690400 & -3.55710600 & 3.00064700 \\
\hline $\mathrm{H}$ & -1.70518300 & -4.84475900 & 4.10806100 \\
\hline $\mathrm{C}$ & -0.43567200 & -4.66081400 & 2.35047600 \\
\hline $\mathrm{H}$ & -1.02520500 & -5.35224800 & 1.73436500 \\
\hline $\mathrm{H}$ & 0.36855400 & -5.25825700 & 2.80601200 \\
\hline $\mathrm{C}$ & 0.18176100 & -3.57162500 & 1.45745700 \\
\hline $\mathrm{H}$ & -0.61767400 & -3.04288900 & 0.92271600 \\
\hline $\mathrm{H}$ & 0.82396800 & -4.02571300 & 0.69464300 \\
\hline $\mathrm{C}$ & 2.78710800 & -0.18362700 & 2.34384700 \\
\hline $\mathrm{H}$ & 2.03699100 & 0.29118500 & 2.99303300 \\
\hline $\mathrm{C}$ & 3.79234000 & -0.92821600 & 3.24306600 \\
\hline $\mathrm{H}$ & 4.54772100 & -1.42067400 & 2.61776700 \\
\hline $\mathrm{H}$ & 3.29331800 & -1.71397900 & 3.82373300 \\
\hline $\mathrm{C}$ & 4.50171000 & 0.04606100 & 4.20198700 \\
\hline $\mathrm{H}$ & 3.76239900 & 0.47860200 & 4.89197200 \\
\hline $\mathrm{H}$ & 5.22246200 & -0.50425800 & 4.82063500 \\
\hline $\mathrm{C}$ & 5.20585600 & 1.17506400 & 3.43643000 \\
\hline $\mathrm{H}$ & 6.02227200 & 0.74762000 & 2.83539800 \\
\hline $\mathrm{H}$ & 5.66953100 & 1.87977800 & 4.13855500 \\
\hline $\mathrm{C}$ & 4.22594100 & 1.91021500 & 2.51078700 \\
\hline $\mathrm{H}$ & 4.75695200 & 2.67191300 & 1.92419000 \\
\hline $\mathrm{H}$ & 3.48196700 & 2.44754500 & 3.11631900 \\
\hline $\mathrm{C}$ & 3.49620600 & 0.94275300 & 1.56364200 \\
\hline $\mathrm{H}$ & 4.21003100 & 0.50383100 & 0.85450800 \\
\hline $\mathrm{H}$ & 2.76502900 & 1.49862700 & 0.96369200 \\
\hline $\mathrm{O}$ & 3.70069700 & 0.75612300 & -1.92239800 \\
\hline
\end{tabular}




$\begin{array}{lrrr}\mathrm{O} & 0.64306000 & -2.77924000 & -2.30038600 \\ \mathrm{P} & 1.70199400 & -1.21211500 & 1.18201400 \\ \mathrm{Pd} & 0.15647200 & 0.43492500 & 0.30759400 \\ \mathrm{C} & -0.79871500 & 2.26341500 & -0.00750500 \\ \mathrm{C} & -1.04066800 & 2.49141500 & -1.30907700 \\ \mathrm{H} & -1.29628400 & 1.67326000 & -1.97206600 \\ \mathrm{C} & -0.96708600 & 3.81988300 & -2.00207300 \\ \mathrm{C} & -0.41509500 & 3.25730400 & 1.02084000 \\ \mathrm{C} & -1.22023500 & 3.52503500 & 2.14301000 \\ \mathrm{C} & 0.80416300 & 3.95168400 & 0.88837600 \\ \mathrm{C} & -0.81666100 & 4.46219800 & 3.09366700 \\ \mathrm{H} & -2.15775100 & 2.99824400 & 2.26559700 \\ \mathrm{C} & 1.20364500 & 4.88328500 & 1.84478000 \\ \mathrm{H} & 1.43215500 & 3.75954700 & 0.02495800 \\ \mathrm{C} & 0.39465300 & 5.14244600 & 2.95331100 \\ \mathrm{H} & -1.45631000 & 4.66131400 & 3.94956900 \\ \mathrm{H} & 2.14578900 & 5.41078700 & 1.71968800 \\ \mathrm{H} & 0.70420900 & 5.86998900 & 3.69898200 \\ \mathrm{~F} & -1.43044600 & 4.84601700 & -1.26454800 \\ \mathrm{~F} & -1.68037400 & 3.78970300 & -3.15200100 \\ \mathrm{~F} & 0.31318500 & 4.13920100 & -2.35900300 \\ \mathrm{C} & -1.83311100 & 0.65130400 & 0.69151900 \\ \mathrm{O} & -2.31170900 & 0.70412600 & 1.79650800 \\ \mathrm{O} & -2.61134400 & 0.20302600 & -0.41174100 \\ \mathrm{C} & -3.93265300 & -0.10015100 & -0.18107300 \\ \mathrm{C} & -4.91705000 & 0.89083500 & -0.02849000 \\ \mathrm{C} & -4.35692900 & -1.43710900 & -0.18250400 \\ \mathrm{C} & -6.26212900 & 0.56446700 & 0.14028700 \\ \mathrm{C} & -5.69615200 & -1.78897800 & -0.02332200 \\ \mathrm{C} & -6.63581300 & -0.77631200 & 0.14478000 \\ \mathrm{H} & -6.99981500 & 1.34853900 & 0.25819500 \\ \mathrm{H} & -5.99202900 & -2.83066400 & -0.02901900 \\ \mathrm{Cl} & -4.48199000 & 2.57872100 & -0.08343200 \\ \mathrm{Cl} & -3.18544200 & -2.71205600 & -0.40885600 \\ \mathrm{Cl} & -8.32480000 & -1.19750400 & 0.35341000\end{array}$

